\title{
Phasenaufgelöste Spektrophotometrie des magnetischen kataklysmischen Veränderlichen EX Hya
}

\author{
Dissertation \\ zur Erlangung des Doktorgrades \\ der Mathematisch-Naturwissenschaftlichen Fakultäten \\ der Georg-August-Universität zu Göttingen
}

vorgelegt von

Stephan F. Eisenbart, geb. Brandt aus Flensburg

Göttingen 2000 
D 7

Referent : Prof. Dr. K. Beuermann

Koreferent : Prof. Dr. F. Kneer

Tag der mündlichen Prüfung : 31. Oktober 2000 
Für meine Frau Bettina und meinen Sohn Alexander, der nur wenige Tage vor Fertigstellung dieser Arbeit das Licht der Welt erblickt hat (und eines Tages über das Licht der Sterne staunen wird).

"The great tragedy in science:

the slaying of a beautiful hypothesis by an ugly fact!"

(T.H. Huxley) 



\section{Zusammenfassung}

In der vorliegenden Arbeit werden zeitaufgelöste Beobachtungen des bedeckenden intermediären Polars EX Hya studiert, die im Jahr 1997 mit der ESO Faint Object Spectrographic Camera 2 am 2.2m-Teleskop der Max-Planck-Gesellschaft auf La Silla (Chile) und mit dem Infrared Spectrometer am 4m-Teleskop des Cerro Tololo Interamerican Observatory (Chile) durchgeführt wurden. Diese Daten werden durch weitere, ebenfalls zeitaufgelöste Spektren ergänzt, die mit dem Faint Object Spectrograph an Bord des Hubble Space Telescope und mit der Short Wave Prime Camera des International Ultraviolet Explorer aufgenommen und den jeweiligen Datenarchiven entnommen wurden. Zusammen mit Lichtkurven und Spektren, die von anderen Autoren für den für die Gesamtleuchtkraft wichtigen Energiebereich $\approx 0.08 \ldots 20 \mathrm{keV}$ (EUV- und Röntgenwellenlängen) angegeben wurden, ist dies das erste Mal, daß das System über den gesamten Energiebereich vom nahen Infrarot bis zu harter Röntgenstrahlung einer detaillierten Betrachtung unterzogen wird.

Aus der Analyse der Daten wird ein geometrisch und energetisch in sich konsistentes Modell für EX Hya abgeleitet. Dieses liefert neben Systemparametern wie den Massen des Weißen Zwergs und seines Begleiters auch die Geometrie und Ausdehnung der in den verschiedenen Energiebereichen emittierenden Gebiete und gibt alle wesentlichen in der Literatur berichteten Eigenschaften des Systems angemessen wieder. Die wichtigsten neuen Erkenntnisse sind: (1) Der erstmals einigermaßen sicher bestimmte Spektraltyp des Sekundärsterns entspricht mit M4.5 \pm 0.5 den Erwartungen für ein kurzperiodisches System wie EX Hya; (2) die Entfernung des Systems ist mit $66 \pm 9$ pc um einen Faktor $\approx 2$ kleiner als bisher angenommen und damit die geringste bisher für einen CV bestimmte Entfernung; (3) die Masse des Weißen Zwergs ist mit $1.2 \pm 0.1 M_{\odot}$ etwa doppelt so groß wie die aus früheren Beobachtungen bestimmte und zählt zu den größten bekannten Massen Weißer Zwerge überhaupt; und (4) die Akkretionsrate $\dot{M}=(1.0 \pm 0.5) \cdot 10^{15} \mathrm{~g} \mathrm{~s}^{-1}$ ist deutlich kleiner als die früher berichteten Werte von $(1 \ldots 3) \cdot 10^{16} \mathrm{~g} \mathrm{~s}^{-1}$, befindet sich aber in guter Übereinstimmung mit der aus der Abnahme der Spinperiode des Weißen Zwergs abgeleiteten Massentransferrate. Die hier ermittelte Akkretionsrate liegt im Gegensatz zu den früher bestimmten unter derjenigen, die aufgrund theoretischer Berechnungen des Drehimpulsverlusts durch Gravitationsstrahlung im langfristigen Mittel $\left(\approx 10^{9} \mathrm{yr}\right)$ für EX Hya erwartet wird.

Die Diskrepanz zwischen der hier durch ein rein geometrisches Verfahren ermittelten Masse des Weißen Zwergs und den Werten, die von einigen Autoren mit verschiedenen Instrumenten aus der Temperatur der thermischen Bremsstrahlungskomponente im Röntgenbereich bestimmt wurden $\left(M_{\mathrm{WD}} \approx 0.5 \pm 0.05 M_{\odot}\right)$, werte ich als starkes Indiz dafür, daß die letztgenannte Methode zur Bestimmung der Massen Weißer Zwerge in magnetischen CVs mit großen systematischen Unsicherheiten behaftet ist. 


\section{Inhaltsverzeichnis}

\begin{tabular}{lr}
\hline Zusammenfassung & 1
\end{tabular}

Inhaltsverzeichnis $\quad 2$

\begin{tabular}{lr}
\hline Abbildungsverzeichnis & 6
\end{tabular}

$\begin{array}{ll}\text { Tabellenverzeichnis } & 10\end{array}$

$\begin{array}{lll}1 & \text { Einleitung } & 11\end{array}$

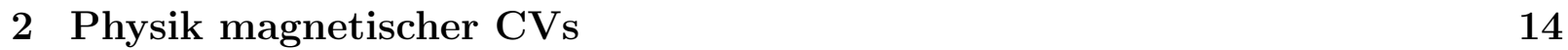

2.1 Enge Doppelsternsysteme . . . . . . . . . . . . . . . . . . . . . . . . . . . . 14

2.1.1Roche-Geometrie . . . . . . . . . . . . . . . . 16

2.2 Komponenten eines CVs . . . . . . . . . . . . . . . . . . . . . . . . 17

2.2 .1 Der Weilje Zwerg . . . . . . . . . . . . . . . . . . . . . . . 17

2.2.1.] Massen und Kernradien . . . . . . . . . . . . . . . . . 17

2.2.1.2 Temperatur und Leuchtkraft. . . . . . . . . . . . . . . . . . 18

2.2.1.3 Kühlung und Photosphärenradien . . . . . . . . . . . . . . . . 19

2.2.1.4 Magnetfeld . . . . . . . . . . . . . . . . . . . 20

2.2 .2 Der Sekundärstern . . . . . . . . . . . . . . . . . . . . . . . . . 20

2.2.2.1 Massen und Radien . . . . . . . . . . . . . . . . . . . . . . . 20

2.2 .2 .2 Ellipsoidale Modulation und Oberflächenhelligkeit . . . . . . . 22

2.2 .2 .3 Magnetfeld . . . . . . . . . . . . . . . . . . . . . . 24

2.2 .3 Der Akkretionsstrom . . . . . . . . . . . . . . . . . . . . . . . 24

2.2 .4 Die Akkretionsscheibe . . . . . . . . . . . . . . . . . . . . 24

2.3 Klassifikation von CVs . . . . . . . . . . . . . . . . . . 25

2.3 .1 Nichtmagnetische $\mathrm{CVS}$. . . . . . . . . . . . . . . . . 26

2.3 .2 Polare . . . . . . . . . . . . . . . . . . . . . 27

2.3 .3 nntermediäre Polare . . . . . . . . . . . . . . . . . . . . 28

2.4 Betrachtungen zur Energetik . . . . . . . . . . . . . . . . . . . . . . . . . . 30

2.4 .1 Energiequelle. . . . . . . . . . . . . . . . . . . . . . . . 30

2.4 .2 Strahlungsprozesse $\ldots \ldots \ldots$. . . . . . . . . . . . . . . 31

2.4.2.1 Aufheizung in einem starken hydrodynamischen Stoß] . . . . . 31

2.4 .2 .2 Thermische Bremsstrahlung . . . . . . . . . . . . 33

2.4.2.3 Zyklotronstrahlung . . . . . . . . . . . . . . . . 33

2.4.2.4 Reprozessierte Strahlung aus der Akkretionsregion . . . . . . 35

2.4.2.5 Photosphäre des Weißen Zwergs . . . . . . . . . . . . . . . . . 35 
2.4.2.6 Akkretionsscheibe, bulge und bright spot . . . . . . . . . 35

2.4 .2 .7 Angeregte Linienemission . . . . . . . . . . . . . . . . 36

2.4.2.8 Photosphäre des Sekundärsterns _ . . . . . . . . . . . 37

2.4.3 Einflußs von Magnetfeld und Akkretionsrate auf das Spektrum . . . . 38

2.5 Aspekte der Evolution von CVs $\ldots \ldots \ldots \ldots$

2.5 .1 Entstehung . . . . . . . . . . . . . . . . . . . . . . . . . 39

2.5 .2 Drehimpulsverlust und Orbitalperiode . . . . . . . . . . . . . . 40

2.5 .3 Spin-Bahn-Synchronisation in Polaren . . . . . . . . . . . . . . . 42

2.5 .4 Spinperiodenentwicklung in IPs . . . . . . . . . . . . . . 43

2.5.5 Weitere Entwicklung des Weil3en Zwergs $\ldots \ldots \ldots \ldots$

2.5.5.1 Massenentwicklung . . . . . . . . . . . . . . . . . 44

2.5 .5 .2 Wärmehaushalt . . . . . . . . . . . . . . . . . 46

3 EX Hya: Der Stand der Dinge 49

3.1 Einführung und Historie. . . . . . . . . . . . . . . . . . . . . . . . 49

3.2 Eigenschaften des Systems $\ldots \ldots \ldots \ldots$

3.2 .1 Ephemeriden für Orbit und Spin . . . . . . . . . . . . . . 51

3.2 .2 Entfernung und interstellare Absorption . . . . . . . . . . . . 51

3.2 .3 Massen und Systemgeometrie. . . . . . . . . . . . . . . . . . . . . 52

3.2 .4 Magnetfeld des Weil3en Zwergs . . . . . . . . . . . . . . . . . . 53

3.2.5 Spektrum, Leuchtkraft und Akkretionsrate . . . . . . . . . . . 54

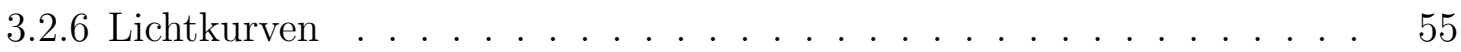

3.2 .6 .1 Variationen mit der Spinphase . . . . . . . . . . . . . 55

3.2.6.2 Eigenschaften der Bedeckungen . . . . . . . . . . . . . . 57

3.2.6.3 Weitere Variationen mit der Orbitalphase . . . . . . . . 58

3.2 .7 Akkretionsgeometrie . . . . . . . . . . . . . . . . . . . . 59

3.2.7.1 Entstehung der Spinmodulation . . . . . . . . . . . . . 61

3.2.7.2 Ursache des breiten Intensitätsminimums bei Orbitalphase 0.963

3.2.7.3 Grundsätzliche Geometrie der Bedeckungen . . . . . . . . . 64

4 Beobachtungen und Datenanalyse $\quad 67$

4.1 Datenmateriall . . . . . . . . . . . . . . . . . . . . . 67

4.1.1 IRS am 4m-Teleskop aut dem Cerro Tololo, Chile . . . . . . . . . . . 68

4.1 .2 EFOSC2 am 2.2m-Teleskop auf La Silla, Chile . . . . . . . . . . . 70

4.1 .3 International Ultraviolet Explorer (IUE) $\ldots \ldots \ldots \ldots$

4.1 .4 Hubble Space Telescope (HST) . . . . . . . . . . . . . . . . . . . . . 72

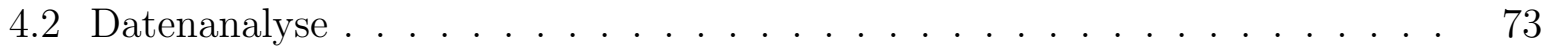

42.1 Verwendete Hard- und Software . . . . . . . . . . . . . 73

4.2 .2 Spektren der beobachteten M-Sterne . . . . . . . . . . . . . . . . 74

4.2 .3 IUE-Spektren von EX Hya . . . . . . . . . . . . . . . . . . . . 80

4.2 .4 HST-Spektren von EX Hya $\ldots \ldots \ldots$

4.2 .5 EFOSC2-Spektren von EX Hya $\ldots \ldots \ldots \ldots \ldots$

4.2 .6 IRS-Spektren von EX Hya $\ldots \ldots \ldots \ldots$ 
4.2 .7 Lichtkurvenanalyse $\ldots \ldots \ldots$

42.7 Konstruktion der lichtkurven . . . . . . . . . . . . . 93

4.2 .7 .2 Methodik der Modellierung . . . . . . . . . . . . . . . 95

4.2 .7 .3 Darstellungsformen der Modelle . . . . . . . . . . . . . . . 97

1.2.7.4 Modelldefinitionen für die verschiedenen Beobachtungen . . . 101

1.2.7.5 Ergebnisse der Lichtkurvenanpassungen . . . . . . . . . . . 102

4.2 .8 Gesamtspektrum von EX Hya . . . . . . . . . . . . . . . . . . 105

5 Interpretation der Ergebnisse 112

5.1 Systemparameter . . . . . . . . . . . . . . . . . . . . . . . . 112

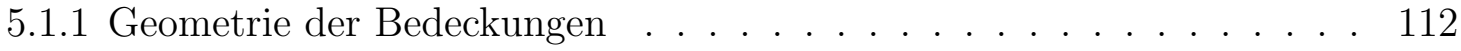

5.1 .2 Zusammenhang von Inklination und Massenverhältnis . . . . . . . . . 113

5.1.3 Massen des Weißen Zwergs und des Sekundärsterns . . . . . . . . . . 114

5.1 .4 Radius des lnnenrands der Akkretionsscheibe . . . . . . . . . . . . . 117

5.1.5 Ausdehnung der Röntgenstrahlung emittierenden Gebiete . . . . . . . 118

5.1 .6 Beitrag des Sekundärsterns zum beobachteten Flüs . . . . . . . . . . 119

5.1 .7 Spektraltyp des Sekundärsterns _. . . . . . . . . . . . . . . . . 121

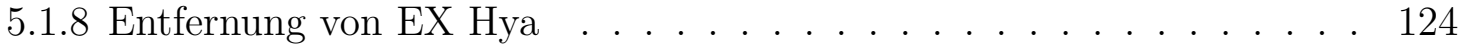

5.2 Bulge der Akkretionsscheibe . . . . . . . . . . . . . . . . . . . . . . . . . . 124

5.3 Bedeckungen der Akkretionsscheibe $\ldots \ldots \ldots \ldots \ldots$

5.4 Spinmodulation der TR-Emissionslinien $\ldots \ldots \ldots \ldots \ldots$

5.5 Beitrag der Akkretionsscheibe und des Weißßen Zwergs zum Flüs . . . . . . 127

5.6 Leuchtkraft und Akkretionsrate . . . . . . . . . . . . . . . . 131

5.6 .1 Röntgenspektrum . . . . . . . . . . . . . . . . . 131

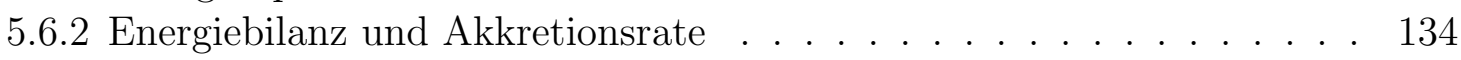

5.6 .3 EUV-Leuchtkraft nach der Zanstra-Methode . . . . . . . . . 136

5.6 .4 Beleuchteter Innenrand der Akkretionsscheibe . . . . . . . . . . 137

5.7 Magnetfeld des Weißßen Zwergs . . . . . . . . . . . . . . . . . 137

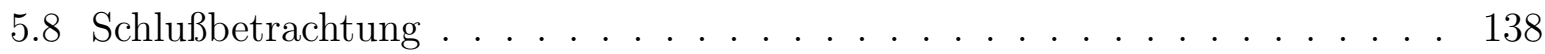

5.8 .1 Zusammenfassung der neuen Erkenntnisse . . . . . . . . . . . . . . 139

5.8 .2 Bewertung der Ergebnisse $\ldots \ldots \ldots \ldots \ldots . \ldots \ldots 14$

5.8 .3 Offene tragen $\ldots \ldots \ldots \ldots \ldots$

5.8 .4 Was ist noch zll tun $7 . \ldots \ldots \ldots$. . . . . . . . . . . . 142

\begin{tabular}{|lr}
\hline A Ergebnisse der Lichtkurven-Fits & 143
\end{tabular}

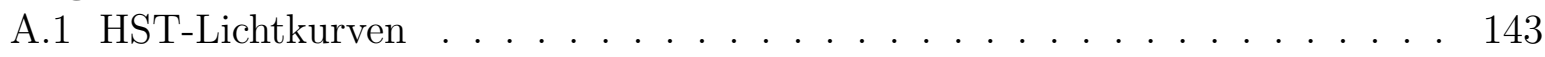

A.1.1 HST-Lichtkurven: Kontinuwmstlüsse . . . . . . . . . . . . . . . 143

A. 12 HST-Lichtkurven: Linientlüsse . . . . . . . . . . . . . . 159

A.2 TUE-Lichtkurven . . . . . . . . . . . . . . . . . . . . . . . . . . . . 174

A.2.1 TUE-Lichtkurven: Kontinumomstlüsse . . . . . . . . . . . . 174

A.2.2 TUE-Lichtkurven: Linientlüsse . . . . . . . . . . . . . . 183

A.3 EFOSC2-Lichtkurven . . . . . . . . . . . . . . . . . . . . . . . . . 188

A.3.1 EFOSC2-Lichtkurven:Kontinuwmstlïsse . . . . . . . . . . 188 
A.3.2 EFOSC2-Lichtkurven: Linientlüsse . . . . . . . . . . . . . . . 194

A.4 IRS-Lichtkurven . . . . . . . . . . . . . . . . . . . . . . . . . . . . . . . . 199

A.4.1 KRS-Lichtkurven: Kontinumenstiusse . . . . . . . . . . . . . . . . . . . 199

A.4.2 IRS-Lichtkurven: Linientlüsse . . . . . . . . . . . . . . . . . . . 209

\begin{tabular}{lr}
\hline B Reduktion von IRS-Spektren & 218
\end{tabular}

B.1 Struktur der 1 RS-Daten . . . . . . . . . . . . . . . . . . . . . . . . 218

B.2 IRS-spezifische Kalibrationsdaten . . . . . . . . . . . . . . . . . . . . 219

B.3 Beobachtungstechnik und Extraktion der Spektren . . . . . . . . . . . . . . 221

B.4 Kalibration der Spektren . . . . . . . . . . . . . . . . . . . . . 224

$\begin{array}{lc}\text { Literaturverzeichnis } & 233\end{array}$

\begin{tabular}{ll}
\hline Abkürzungsverzeichnis & 237
\end{tabular}

Naturkonstanten und astronomische Makeinheiten 239

$\begin{array}{ll}\text { Danksagungen } & 241\end{array}$

\begin{tabular}{ll}
\hline Lebenslauf & 243
\end{tabular} 


\section{Abbildungsverzeichnis}

2.1 Aqquipotentialflächen eines Doppelsternsystems . . . . . . . . . . . . . . . . 15

2.2 Masse-Radius-Relationen für Weiße Zwerge . . . . . . . . . . . . . . . . . . . 19

2.3 Periodenverteilung von $\mathrm{CVS}$. . . . . . . . . . . . . . . . . . . . . . . . . . . . . . . . . . . . .

2.4 Masse-Radius-Relationen für CV-Sekundärsterne . . . . . . . . . . . . . 23

2.5 Schematische Darstellung eines Polars . . . . . . . . . . . . . . . . . . . . . 27

2.6 Schematische Darstellung eines IPS . . . . . . . . . . . . . . . . . . . . . . 29

2.7 Akkretionsvorhänge eines IPS $\ldots \ldots \ldots$. . . . . . . . . . . . . . . . . . . . . . 29

2.8 Akkretionsregion eines magnetischen CVs . . . . . . . . . . . . . . . . . . . 31

2.9 Spektrum des Polars AMHer (Infrarot...30 keV) . . . . . . . . . . . . . . . 32

2.10 Spektrum des Polars RX J0531.5-4624 (3500-9000 A $)$. . . . . . . . . . . 34

2.11 Orbitale Variationen von Emissionslinien des Polars RX $1015.5+0904$. . . 37

2.12 Massenentwicklung Weiß3er Zwerge in CVs . . . . . . . . . . . . . . . . 45

2.13 Temperaturentwicklung Weißer Zwerge in CVs . . . . . . . . . . . . . . . . 47

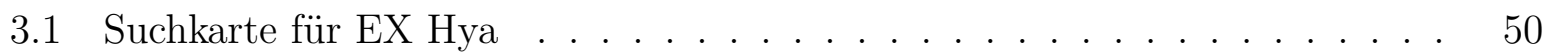

3.2 Akkretionsvorhang von EX Hya zu verschiedenen Spinphasen . . . . . . . . 60

3.3 Schema des zentralen Akkretionsgebiets von EX Hya . . . . . . . . . . . . . 61

3.4 Schematischer Blick in der Orbitalebene auf EX Hya . . . . . . . . . . . . . 62

3.5 Schematischer Blick auf die Orbitalebene von EX Hya . . . . . . . . . . . . 64

3.6 Zeit- und Längenverhältnisse der Bedeckungen . . . . . . . . . . . . . . . . 64

3.7 Spinphasenabhängigkeit der optischen Bedeckungszeitpunkte . . . . . . . . 65

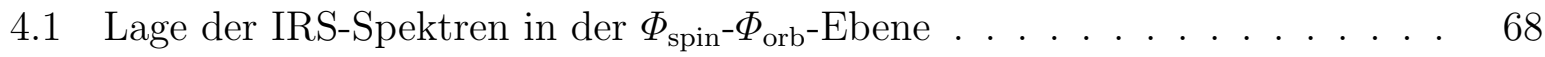

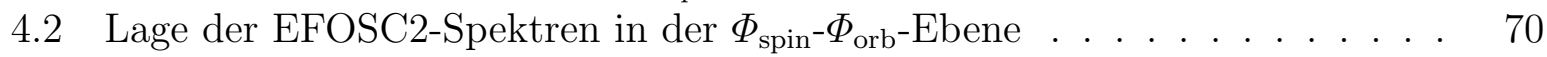

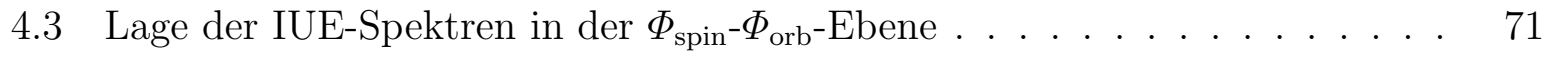

4.4 Lage der HST-Spektren in der $\Phi_{\text {spin }} \Phi_{\text {orb-Ebend }}$. . . . . . . . . . . . . . . 72

4.5 Transmissionsfunktionen der photometrischen Bänder . . . . . . . . . . . . 74

4.6 GI382: Verbindung von Infrarot- und optischem Spektrum . . . . . . . . . 76

4.7 G1443: Verbindung von Infrarot- und optischem Spektrum . . . . . . . . . 77

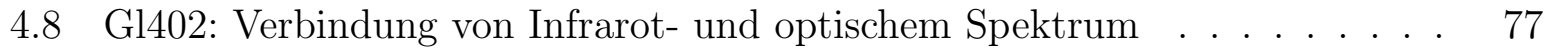

4.9 GI285: Verbindung von Infrarot- und optischem Spektrum . . . . . . . . . 78

4.10 G1473: Verbindung von Infrarot- und optischem Spektrum . . . . . . . . . 78

4.11 Spektren aller Sterne des Samples roter Zwerge . . . . . . . . . . . . . . . 79

4.12 Mittleres IUE/LWP-Spektrum von EXHya . . . . . . . . . . . . . . . . . . 80

4.13 Mittlere IUE/SWP-Spektren von EX Hya für verschiedene Spinphasen . . . 81

4.14 HST-Spektrum vor und nach der Bedeckung . . . . . . . . . . . . . . . . 85

4.15 Orbitalphasenaufgelöste HST-Spektren von EX Hya, Teil 1 . . . . . . . . . 86

4.16 Orbitalphasenaufgelöste HST-Spektren von EX Hya, Teil 2 . . . . . . . . . 87 


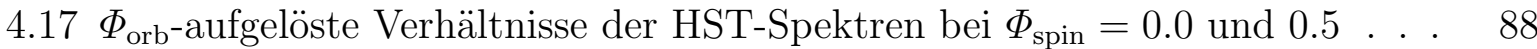
4.18 Mittlere HST-Spektren von EX Hya für verschiedene Spinphasen . . . . . . 89 4.19 Mittlere EFOSC2-Spektren von EX Hya für verschiedene Spinphasen . . 90 4.20 Mittlere IRS-Spektren von EX Hya für verschiedene Spinphasen . . . . . 92 4.21 Lichtkurvenmodellierung: ungefaltete Darstellung $f(t)$. . . . . . . . . . . . 98 4.22 Lichtkurvenmodellierung: gefaltete Darstellung $f\left(\Phi_{\text {spin }}\right), f\left(\Phi_{\text {orb }}\right)$. . . . . . 99 4.23 Mittleres Gesamtspektrum von EX Hya . . . . . . . . . . . . . . . . . . . . 107 4.24 Gesamtspektrum der spinmodulierten Komponente von EX Hya . . . . . . 108 4.25 Spinmodulationsgrad der Emissionslinien . . . . . . . . . . . . . . . . . . . 109 4.26 EX Hya: Uberlappbereich von EFOSC 2- und IRS-Spektrum . . . . . . . . 109 4.27 Walraven-Photometrie von EX Hya . . . . . . . . . . . . . . . . . . . . 110

5.1 Mögliche Bedeckungsszenarien von EX Hya . . . . . . . . . . . . . . . . . . 113

5.2 Inklination, Massenverhältnis und Bedeckungsdauer . . . . . . . . . . . . . 114

5.3 Bedeckungsgeometrie und -lichtkurven von EX Hya . . . . . . . . . . . . . 115

5.4 Flußbeitrag des Sekundärsterns im Infrarot . . . . . . . . . . . . . . . . . 120

5.5 Suche nach spektralen Merkmalen des M-Sterns (opt.) . . . . . . . . . . . 121

5.6 Suche nach spektralen Merkmalen des M-Sterns (Infrarot) . . . . . . . . . 122

5.7 Differenzspektren EX Hya - M-Stern für Spektraltypen M2.0 ... M5.5 . . . . 123

5.8 Reprozessierung energiereicher Strahlung im bulge der Akkretionsscheibe $\quad 125$

5.9 Spektrum der nicht spinmodulierten Komponenten von EX Hya . . . . . . 128

5.10 Konstruktion des Spektrums der nicht spinmodulierten Komponenten . . . 129

5.11 Beobachtetes Röntgen- und EUV-Photonenflulspektrum von EX Hya $\ldots 132$

A.1 HST-Lichtkurve $1255 \ldots 1285 \AA$ A, ungefaltet . . . . . . . . . . . . . . . . . . . 144

A.2 HST-Lichtkurve $1255 \ldots 1285 \AA$ A, über $\Phi_{67}$ bzw. $\Phi_{98}$ gefaltet . . . . . . . . . . 145

A.3 HST-Lichtkurve $1350 \ldots 1365 \AA$ A, ungefaltet . . . . . . . . . . . . . . . . . . . 146

A.4 HST-Lichtkurve $1350 \ldots 1365 \AA$ A, über $\Phi_{67}$ bzw. $\Phi_{98}$ gefaltet . . . . . . . . . . 147

A.5 HST-Lichtkurve $1350 \ldots 1380 \AA$ A, ungefaltet . . . . . . . . . . . . . . . . . . . 148

A.6 HST-Lichtkurve $1350 \ldots 1380 \AA$, über $\Phi_{67}$ bzw. $\Phi_{98}$ gefaltet . . . . . . . . . . 149

A.7 HST-Lichtkurve $1425 \ldots 1525 \AA$, ungefaltet . . . . . . . . . . . . . . . . . . . 150

A.8 HST-Lichtkurve $1425 \ldots 1525 \AA$, über $\Phi_{67}$ bzw. $\Phi_{98}$ gefaltet . . . . . . . . . . 151

A.9 HST-Lichtkurve $1570 \ldots 1600 \AA$, ungefaltet . . . . . . . . . . . . . . . . . . . 152

A.10 HST-Lichtkurve $1570 \ldots 1600 \AA$, über $\Phi_{67}$ bzw. $\Phi_{98}$ gefaltet . . . . . . . . . . 153

A.11 HST-Lichtkurve $1250 \ldots 1380 \AA$, ungefaltet . . . . . . . . . . . . . . . . . . . 154

A.12 HST-Lichtkurve $1250 \ldots 1380 \AA$, über $\Phi_{67}$ bzw. $\Phi_{98}$ gefaltet . . . . . . . . . . 155

A.13 HST-Lichtkurve 1230...1600A, ungefaltet . . . . . . . . . . . . . . . . . . 156

A.14 HST-Lichtkurve $1230 \ldots 1600 \AA$, über $\Phi_{67}$ bzw. $\Phi_{98}$ gefaltet . . . . . . . . . . 157

A.15 HST-Lichtkurve C III 1175, ungefaltet . . . . . . . . . . . . . . . 160

A.16 HST-Lichtkurve CIII 1175, über $\Phi_{67}$ bzw. $\Phi_{98}$ gefaltet . . . . . . . . . . . . 161

A.17 HST-Lichtkurve Ly $\alpha$, ungefaltet . . . . . . . . . . . . . . . . . . . . 162

A.18 HST-Lichtkurve Ly $\alpha$, über $\Phi_{67}$ bzw. $\Phi_{98}$ gefaltet . . . . . . . . . . . . . . . 163

A.19 HST-Lichtkurve NV 1240, ungefaltet . . . . . . . . . . . . . . . . . . . . . 164 
A.20 HST-Lichtkurve NV 1240, über $\Phi_{67}$ bzw. $\Phi_{98}$ gefaltet . . . . . . . . . . . . 165

A.21 HST-Lichtkurve Sill 1300, ungefaltet . . . . . . . . . . . . . . . . . . . . 166

A.22 HST-Lichtkurve Sill 1300, über $\Phi_{67}$ bzw. $\Phi_{98}$ gefaltet . . . . . . . . . . . 167

A.23 HST-Lichtkurve CII 1335, ungefaltet . . . . . . . . . . . . . . . . . 168

A.24 HST-Lichtkurve C II 1335, über $\Phi_{67}$ bzw. $\Phi_{98}$ gefaltet . . . . . . . . . . . . 169

A.25 HST-Lichtkurve SiIV 1400, ungefaltet . . . . . . . . . . . . . . . . . . . 170

A.26 HST-Lichtkurve SiIV 1400, über $\Phi_{67}$ bzw. $\Phi_{98}$ gefaltet . . . . . . . . . . . 171

A.27 HST-Lichtkurve CTV 1550, ungefaltet . . . . . . . . . . . . . . . . . 172

A.28 HST-Lichtkurve CIV 1550, über $\Phi_{67}$ bzw. $\Phi_{98}$ gefaltet . . . . . . . . . . . . 173

A.29 IUE-Lichtkurve $1255 \ldots 1285 \AA$, ungefaltet und über $\Phi_{67}$ bzw. $\Phi_{98}$ gefaltet . . 175

A.30 IUE-Lichtkurve $1350 \ldots 1380 \AA$ A, ungefaltet und über $\Phi_{67}$ bzw. $\Phi_{98}$ gefaltet . . 176

A.31 IUE-Lichtkurve $1425 \ldots 1525 \AA$ A, ungefaltet und über $\Phi_{67}$ bzw. $\Phi_{98}$ gefaltet . . 177

A.32 IUE-Lichtkurve $1575 \ldots 1625 \AA$, ungefaltet und über $\Phi_{67}$ bzw. $\Phi_{98}$ gefaltet . . 178

A.33 IUE-Lichtkurve $1680 \ldots 1780 \AA$, ungefaltet und über $\Phi_{67}$ bzw. $\Phi_{98}$ gefaltet . . 179

A.34 IUE-Lichtkurve $1875 \ldots 1975 \AA$, ungefaltet und über $\Phi_{67}$ bzw. $\Phi_{98}$ gefaltet . . 180

A.35 IUE-Lichtkurve $1250 \ldots 1380 \AA$, ungefaltet und über $\Phi_{67}$ bzw. $\Phi_{98}$ gefaltet . . 181

A.36 IUE-Lichtkurve $1250 \ldots 1950 \AA$ A, ungefaltet und über $\Phi_{67}$ bzw. $\Phi_{98}$ gefaltet . . 182

A.37 IUE-Lichtkurve Si IV 1400, ungefaltet und über $\Phi_{67}$ bzw. $\Phi_{98}$ gefaltet . . . 184

A.38 10E-Lichtkurve CIV 1550, ungefaltet und über $\Phi_{67}$ bzw. $\Phi_{98}$ gefaltet . . . 185

A.39 10E-Lichtkurve He 11 1640, ungefaltet und über $\Phi_{67}$ bzw. $\Phi_{98}$ gefaltet . . . 186

A.40 1UE-Lichtkurve AIII 1860, ungefaltet und über $\Phi_{67}$ bzW. $\Phi_{98}$ gefaltet . . . 187

A.41 EFOSC2-Lichtkurve $3600 \ldots 4000 \AA$, ungefaltet und über $\Phi_{67}$ bzw. $\Phi_{98}$ gefaltet 189 A.42 EFOSC2-Lichtkurve $3900 \ldots 5000 \AA$ A, ungefaltet und über $\Phi_{67}$ bzw. $\Phi_{98}$ gefaltet 190 A.43 EFOSC2-Lichtkurve 5000...6200 A, ungefaltet und über $\Phi_{67}$ bzw. $\Phi_{98}$ gefaltet 191 A.44 EFOSC2-Lichtkurve 5800...8000 A, ungefaltet und über $\Phi_{67}$ bzw. $\Phi_{98}$ gefaltet 192 A.45 EFOSC2-Lichtkurve $7500 \ldots 10000 \AA$, ungefaltet und über $\Phi_{67}$ bzw. $\Phi_{98}$ gefaltet 193 A.46 EFOSC2-Lichtkurve HeII 4686, ungefaltet und über $\Phi_{67}$ bzW. $\Phi_{98}$ gefaltet . 195 A.47 EFOSC2-Lichtkurve $\mathrm{H} \beta$, ungefaltet und über $\Phi_{67}$ bzw. $\Phi_{98}$ gefaltet . . . . 196 A.48 EFOSC2-Lichtkurve He 15875 , ungefaltet und über $\Phi_{67}$ bzW. $\Phi_{98}$ gefaltet . 197 A.49 EFOSC2-Lichtkurve $\mathrm{H} \alpha$, ungefaltet und über $\Phi_{67}$ bzw. $\Phi_{98}$ gefaltet . . . . 198 A.50 IRS-Lichtkurve 9650...10650 Á, ungefaltet . . . . . . . . . . . . . . . . . . 200 A.51 IRS-Lichtkurve $9650 \ldots 10650 \AA$ A, über $\Phi_{67}$ bzw. $\Phi_{98}$ gefaltet . . . . . . . . . . 201 A.52 IRS-Lichtkurve $11000 \ldots 13500 \AA$, ungefaltet . . . . . . . . . . . . . . . . 202 A.53 IRS-Lichtkurve $11000 \ldots 13500 \AA$, über $\Phi_{67}$ bzw. $\Phi_{98}$ gefaltet . . . . . . . . . 203 A.54 IRS-Lichtkurve $14500 \ldots 18000 \AA$ A, ungefaltet . . . . . . . . . . . . . . . . . . 204 A.55 IRS-Lichtkurve $14500 \ldots 18000 \AA$ A, über $\Phi_{67}$ bzw. $\Phi_{98}$ gefaltet . . . . . . . . . 205 A.56 IRS-Lichtkurve $20000 \ldots 24000 \AA$, ungefaltet . . . . . . . . . . . . . . . . . . 206 A.57 IRS-Lichtkurve $20000 \ldots 24000 \AA$, über $\Phi_{67}$ bzw. $\Phi_{98}$ gefaltet . . . . . . . . . 207 A.58 IRS-Lichtkurve $\mathrm{P} \delta$, ungefaltet . . . . . . . . . . . . . . . . 210 A.59 IRS-Lichtkurve P $\delta$, über $\Phi_{67}$ bzw. $\Phi_{98}$ gefaltet . . . . . . . . . . . . . . . . 211 A.60 IRS-Lichtkurve $\mathrm{P} \gamma+\mathrm{He} 1$ 10830, ungefaltet . . . . . . . . . . . . . . . 212 A.61 IRS-Lichtkurve $\mathrm{P} \gamma+$ He1 10830, über $\Phi_{67}$ bzw. $\Phi_{98}$ gefaltet . . . . . . . . 213 A.62 IRS-Lichtkurve $\mathrm{P} \beta$, ungefaltet . . . . . . . . . . . . . . . . . . . . . . 214 
A.63 IRS-Lichtkurve P $\beta$, über $\Phi_{67}$ bzw. $\Phi_{98}$ gefaltet . . . . . . . . . . . . . . 215 A.64 IRS-Lichtkurve $\mathrm{B} \gamma$, ungefaltet . . . . . . . . . . . . . . . . . . . 216 A.65 IRS-Lichtkurve B $\gamma$, über $\Phi_{67}$ bzw. $\Phi_{98}$ gefaltet . . . . . . . . . . . . 217

B.1 Lage der einzelnen Ordnungen der IRS-Spektren . . . . . . . . . . . . . . 228 B.2 $\quad$ Flatfieldspektrum des IRS . . . . . . . . . . . . . . . . . . . . . . . . . . . 228 B.3 Darkfieldspektrum des IRS . . . . . . . . . . . . . . . . . . . . . . . . . . 228 B.4 Flatfieldspektrum des IRS nach Abzug des Dunkelstroms . . . . . . . . . . 228 B.5 Karte defekter Pixel des 1 RS-Detektors . . . . . . . . . . . . . . . . 228 B.6 Hochfrequenz-Flatfieldspektrum des IRS . . . . . . . . . . . . . . . 228 B.7 Beobachtungstechnik: Objektspektrum an verschiedenen Positionen . . . . 229 B.8 Mittleres Himmelsspektrum einer Serie von Spektren . . . . . . . . . . . . 229 B.9 Objektspektrum nach Abzug des mittleren Himmelsspektrums . . . . . . . 229 B.10 Extraktion der Ordnungen und Abzug des Himmelshintergrunds . . . . . . 229 B.11 Auswahl der Spalten des Himmels- bzw. Objektspektrums . . . . . . . . . 230 B.12 Bestimmung der mechanischen Drift in Dispersionsrichtung . . . . . . . . . 230 B.13 Response-Funktionen für die Ordnungen der IRS-Spektren . . . . . . . . . 230 B.14 Verknüpfung der Ordnungen zum Gesamtspektrum . . . . . . . . . . . . . 231 B.15 Konstruktion einer atmosphärischen Korrekturfunktion . . . . . . . . . . . 232 B.16 Atmosphärische Korrektur eines IRS-Spektrums . . . . . . . . . . . . . . 232 


\section{Tabellenverzeichnis}

3.1 Mittlere photometrische Helligkeiten von EXHya . . . . . . . . . . . . . . 55

4.1 Eckdaten des Beobachtungsmaterials . . . . . . . . . . . . . . . . . 67

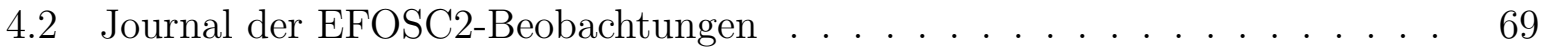

4.3 Photometrische Flußkonstanten und Effektivwellenlängen . . . . . . . . . . 74

4.4 Photometrische Helligkeiten der beobachteten M-Sterne . . . . . . . . . . . 75

4.5 Definition der Wellenlängenbereiche für die Lichtkurvenanalyse . . . . . . . 94

4.6 In der Lichtkurvenanalyse untersuchte Emissionslinien . . . . . . . . . . . . 95

4.7 Lokal gültige lineare Ephemeriden für die einzelnen Beobachtungen . . . 96

4.8 Ellipsoidale Modulation im Infrarot-Kontinuum . . . . . . . . . . . . . . . 102

4.9 Spinmodulation im Kontinuum . . . . . . . . . . . . . . . . . . . . . . 103

4.10 Spinmodulation der Emissionslinien . . . . . . . . . . . . . . . . . . . . . . 104

4.11 Eigenschaften des Walraven-Systems . . . . . . . . . . . . . . . . . . . . . 105

4.12 Walraven-Photometrie von EX Hya . . . . . . . . . . . . . . . . . . . . . . 106

5.1 Systemparameter von EXHya . . . . . . . . . . . . . . . . . . . . . . . . . 117

5.2 Flußbeitrag des Sekundärsterns im Intrarot . . . . . . . . . . . . . . . . . . 120

5.3 Röntgentlüsse von EX Hya . . . . . . . . . . . . . . . . . . . . . . . . 132

5.4 Integrale Flüsse der spektralen Komponenten von EX Hya . . . . . . . . . 134

A.1 Fits an HST-Kontinummslichtkurven: Übersicht . . . . . . . . . . . . . . . 143

A.2 Fits an HST-Emissionslinienlichtkurven: Ubersicht . . . . . . . . . . . . . . 159

A.3 Fits an IUE-Kontinummslichtkurven: Ubersicht . . . . . . . . . . . . . . . . 174

A.4 Fits an IUE-Fmissionslinienlichtkurven: Ubersicht . . . . . . . . . . . . . . 183

A.5 Fits an EFOSC2-Kontinummslichtkurven: Ubersicht . . . . . . . . . . . . . 188

A.6 Fits an EFOSC2-Emissionslinienlichtkurven: Übersicht . . . . . . . . . . . 194

A.7 Fits an IRS-Kontinummslichtkurven: Übersicht . . . . . . . . . . . . . . . . 199

A.8 Fits an IRS-Emissionslinienlichtkurven: Ubersicht . . . . . . . . . . . . . . 209

B.1 Wellenlängenbereiche der einzelnen Ordnungen des IRS . . . . . . . . . . . 224

B.2 Zur Flußkalibration der IRS-Spektren verwendete Sterne . . . . . . . . . . 225 


\section{Kapitel 1}

\section{Einleitung}

Kataklysmische Veränderliche (nach der englischen Bezeichnung cataclysmic variables meist kurz 'CVs' genannt) sind eine Klasse von engen Doppelsternsystemen mit Umlaufzeiten von nur wenigen Stunden, die aus einem Weißen Zwerg (Primärstern) und einem massearmen 'normalen' Stern (Sekundärstern) bestehen. Infolge der großen räumlichen Nähe der beiden Partner und des Volumens des Sekundärsterns, das gerade einem durch das Massenverhältnis der beiden Sterne bestimmten kritischen Volumen entspricht (dem sogenannten Roche-Volumen), wird von letzterem in beträchtlichem Umfang Masse auf den Weißen Zwerg transferiert他 (bei typischen CVs etwa $10^{15} \ldots 10^{18} \mathrm{~g} \mathrm{~s}^{-1}$ ). Massentransfer auf ein zentrales Objekt, Akkretion genannt, ist ein weitverbreitetes Phänomen im Universum und findet in so verschiedenen Objekten wie in entstehenden Sternen, Röntgendoppelsternen (hier ist das zentrale Objekt ein Neutronenstern oder ein stellares Schwarzes Loch), den Kernen aktiver Galaxien (die nach gängiger Vorstellung in ihrem Zentrum ein supermassives Schwarzes Loch enthalten) und eben CVs statt. Akkretierende Objekte besitzen oft um das Zentralobjekt herum eine ausgedehnte scheibenförmige Struktur, eine sogenannte Akkretionsscheibe, in der das Material allmählich von außen nach innen transportiert wird. In allen diesen Fällen führt der Transfer von Masse auf ein kompaktes Zentralobjekt zur Freisetzung von potentieller Energie (Gravitationsenergie), die letztlich in thermische Energie umgesetzt und abgestrahlt wird. Unabhängig davon, ob dies z.B. in der Akkretionsscheibe oder an anderen Orten im System geschieht, haben akkretierende Objekte daher in der Regel beträchtliche Leuchtkräfte; so kann ein einzelner akkretierender Neutronenstern ohne weiteres die 10000-fache Leuchtkraft der Sonne und mehr entfalten, und die Leuchtkraft des Quasars 3C 273 ist etwa so groß wie die von $10^{14}$ Sonnen, was etwa der 10000 -fachen Leuchtkraft der gesamten Milchstraße entspricht.

Das Studium akkretierender Objekte ist insofern auch abseits der Astronomie von Interesse, als die eben genannten Leuchtkräfte schon andeuten, daß in den Objekten physikalische Bedingungen herrschen, wie sie in keinem irdischen Labor erzeugt werden können. So können z.B. bei der Akkretion auf den Weißen Zwerg eines CVs durchaus Plasmatem-

1 Daher auch die Bezeichnung, die sich aus dem Griechischen ableitet: $\kappa \alpha \tau \alpha \kappa \lambda v \sigma \mu o ́ \varsigma=$ Überschwemmung, Sintflut. Im Gegensatz zum Deutschen ist das Wort im Englischen nicht ungebräuchlich. 
peraturen von einigen $10^{8} \mathrm{~K}$ erreicht werden (zum Vergleich: Die Temperatur der Sonnenoberfläche beträgt etwa $6000 \mathrm{~K}$ ); sie sind daher oft helle Röntgenquellen. Die Magnetfelder Weißer Zwerge in CVs können $10^{4} \mathrm{~T}$ überschreiten, was dazu führt, daß das einfallende Material Zyklotron-Strahlung erzeugt, deren Grundharmonische im Optischen liegen kann. (Als irdischer Vergleich sei das Magnetfeld der Erde angeführt, das selbst an den Polen nur $\approx 6 \cdot 10^{-5} \mathrm{~T}$ beträgt.) Akkretierende Objekte können damit als kosmische Plasma-Laboratorien betrachtet werden, in denen Materie unter extremen, auf der Erde nicht herstellbaren Bedingungen untersucht werden kann. CVs sind hierfür insofern besonders geeignet, als sie einerseits recht leuchtkräftige und andererseits relativ häufige (und damit nahe und helle) Objekte sind.

Gegenstand dieser Arbeit ist der CV EX Hydrae (üblicherweise mit EX Hya abgekürzt), der zu einer Unterklasse der CVs gehört, den sogenannten intermediären Polaren (meist kurz 'IPs' genannt). Bei diesen Systemen verfügt der Weiße Zwerg über ein Magnetfeld, das stark genug ist, die Akkretionsscheibe im Innenbereich aufzubrechen, so daß sich das einfallende Material auf dem letzten Stück des Weges entlang der magnetischen Feldlinien bewegt und folglich in eng begrenzten Gebieten in der Nähe der magnetischen Pole des Weißen Zwergs deponiert wird. Während in den sogenannten Polaren, einer anderen Unterklasse der CVs, das Magnetfeld stark genug ist, die Eigenrotation des Weißen Zwergs mit der Orbitalbewegung zu synchronisieren, rotiert der Weiße Zwerg eines IPs unabhängig davon. Da das Plasma in der Nähe des Weißen Zwergs an das Magnetfeld gekoppelt ist und dessen Achse in der Regel (wie auch bei der Erde) nicht identisch mit der Rotationsachse des Weißen Zwergs ist, zeigen IPs periodische Variationen der Helligkeit und des Spektrums sowohl mit der Orbitalphase als auch mit der Rotationsphase des Weißen Zwergs. Vermutlich ist es gerade diese 'Lebendigkeit' der Systeme gepaart mit dem Wissen um die in ihnen herrschenden extremen Bedingungen, die mich und viele andere Astronomen an Objekten wie EX Hya besonders fasziniert. Die verschiedenen Periodizitäten ermöglichen es im Prinzip, anhand zeitaufgelöster Beobachtungen in verschiedenen Wellenlängenbereichen die Akkretionsvorgänge in der Nähe des Weißen Zwergs und die dort herrschenden physikalischen Bedingungen einerseits und die Eigenschaften der Akkretionsscheibe und die Geometrie des Systems andererseits unabhängig voneinander zu studieren.

EX Hya ist für ein solches Vorhaben besonders geeignet, da die kurze Orbitalperiode des Systems von 98 min und die relativ lange Rotationsperiode des Weißen Zwergs von 67 min zum einen schon im Laufe einer Nacht die Beobachtung jeweils mehrerer Zyklen ermöglicht und zum anderen auch mit längeren Belichtungszeiten von z.B. 5 min eine gute Phasenauflösung bei gleichzeitig gutem Signal-zu-Rausch-Verhältnis der Daten erreicht werden kann. Im Sinne des letztgenannten Punkts ist es auch ausgesprochen förderlich, daß EX Hya mit einer visuellen Helligkeit $V \approx 13$ einer des hellsten (bekannten) CVs am Himmel ist. Als besonders fruchtbar bei der Analyse der Systemgeometrie wird sich ferner der Umstand erweisen, daß EX Hya infolge des flachen Blickwinkels auf das System bei jedem Umlauf eine kurze Bedeckung des Weißen Zwergs durch seinen Begleiter zeigt. Nur ein weiterer bekannter IP hat diese wertvolle Eigenschaft.

Um zu Aussagen über die physikalischen Bedingungen im Akkretionsplasma zu gelangen, ist eine möglichst detaillierte Kenntnis der Struktur des Systems erforderlich. Hier- 
zu gehören z.B. die Massen und Radien der beiden Partner, die Massentransferrate und Einzelheiten des Akkretionsprozesses wie die Größe einer eventuell vorhandenen Akkretionsscheibe oder die vorherrschenden Mechanismen der Strahlungserzeugung. Ziel dieser Arbeit ist es daher, aus zeitaufgelösten Beobachtungen von EX Hya vom Energiebereich harter Röntgenstrahlung bis hin zum nahen Infrarot diese Eigenschaften zu bestimmen.

Die Arbeit ist wie folgt gegliedert:

In Kapitel 2 werden die physikalischen Eigenschaften von CVs im allgemeinen und von intermediären Polaren im besonderen beschrieben. Kapitel 3 enthält eine Darstellung der Erkenntnisse, die bisher über das System EX Hya gewonnen wurden. Das für die Arbeit verwendete Beobachtungsmaterial und die bei seiner Auswertung angewandten Verfahren werden in Kapitel 4 beschrieben. Kapitel 5 beinhaltet die Interpretation der Ergebnisse der Datenanalyse und in Abschnitt 5.8 die Diskussion der dabei gewonnenen neuen Erkenntnisse. Der Anhang A enthält die bildlichen Darstellungen der Analysen der in den verschiedenen Energiebereichen gewonnenen Lichtkurven, die inhaltlich dem Kapitel 4 zuzuordnen sind, aufgrund des Seitenumfangs und der besseren Übersichtlichkeit wegen aber separat wiedergegeben werden. Aus demselben Grund erfolgt die Beschreibung des Verfahrens zur Reduktion von IRS-Spektren, das von mir eigens für diese Arbeit entwickelt wurde und z.T. erheblich von Standard-Verfahren abweicht, ebenfalls separat in Anhang B. Als Ergänzung finden sich am Ende noch ein Verzeichnis der in dieser Arbeit verwendeten Abkürzungen sowie eine Aufstellung der verwendeten Zahlenwerte von Naturkonstanten und astronomischen Maßeinheiten im SI- und cgs-System. 


\section{Kapitel 2}

\section{Physik magnetischer CVs}

In diesem Kapitel werden die grundlegenden physikalischen Zusammenhänge beschrieben, die das Verhalten und das Erscheinungsbild von kataklysmischen Veränderlichen (Cataclysmic Variables, ab jetzt kurz CVs) bestimmen, sowie wichtige Begriffe eingeführt. In den ersten drei Abschnitten werden die Kinematik von Doppelsternsystemen, die grundsätzliche Struktur aller CVs sowie darauf aufbauend die Klassifikation von CVs besprochen. Im Anschluß werden Fragen der Energieerzeugung sowie der Strahlungsprozesse diskutiert, die für die Interpretation der Beobachtungsergebnisse von Belang sind. Abschließend folgt ein kurzer Überblick über Entstehung und Evolution von CVs. Aspekte, die für die vorliegende Arbeit nicht direkt relevant sind, jedoch für eine Einordnung in den Gesamtkontext nützlich sind, werden nur kurz behandelt. Für eine weitaus detailliertere Darstellung sei auf die Literatur verwiesen (z.B. Warner 1995).

Zusätzlich zu den formalen Gleichungen gebe ich - wo möglich - auch numerische Formeln unter Bezug auf typische in CVs vorkommende Massen, Radien usw. an, um eine schnelle Abschätzung der resultierenden Werte zu erleichtern.

\section{$2.1 \quad$ Enge Doppelsternsysteme}

Die Kinematik von Doppelsternen wird im wesentlichen durch das dritte Keplersche Gesetz

$$
\begin{aligned}
a^{3} & =\frac{G}{4 \pi^{2}}\left(M_{1}+M_{2}\right) P_{\text {orb }}^{2} \quad \text { bzw } \\
a & =0.506 R_{\odot}\left(\frac{P_{\text {orb }}}{1 \mathrm{~h}}\right)^{\frac{2}{3}}\left(\frac{M_{1}}{M_{\odot}}\right)^{\frac{1}{3}}(1+q)^{\frac{1}{3}} \\
& =3.53 \cdot 10^{10} \mathrm{~cm}\left(\frac{P_{\text {orb }}}{1 \mathrm{~h}}\right)^{\frac{2}{3}}\left(\frac{M_{1}}{M_{\odot}}\right)^{\frac{1}{3}}(1+q)^{\frac{1}{3}}
\end{aligned}
$$

bestimmt ( $a=$ Länge der großen Halbachse, $P_{\text {orb }}=$ Orbitalperiode, $M_{1}, M_{2}=$ Massen der beiden Komponenten, $q=$ Massenverhältnis $M_{2} / M_{1}, G=$ Gravitationskonstante). Für CV-typische Verhältnisse $\left(P_{\text {orb }}=1.3 \ldots 10 \mathrm{~h}\right.$, vgl. Abbildung 2.3, und $\left.M_{1}=0.4 \ldots 1.3 M_{\odot}\right)$ 


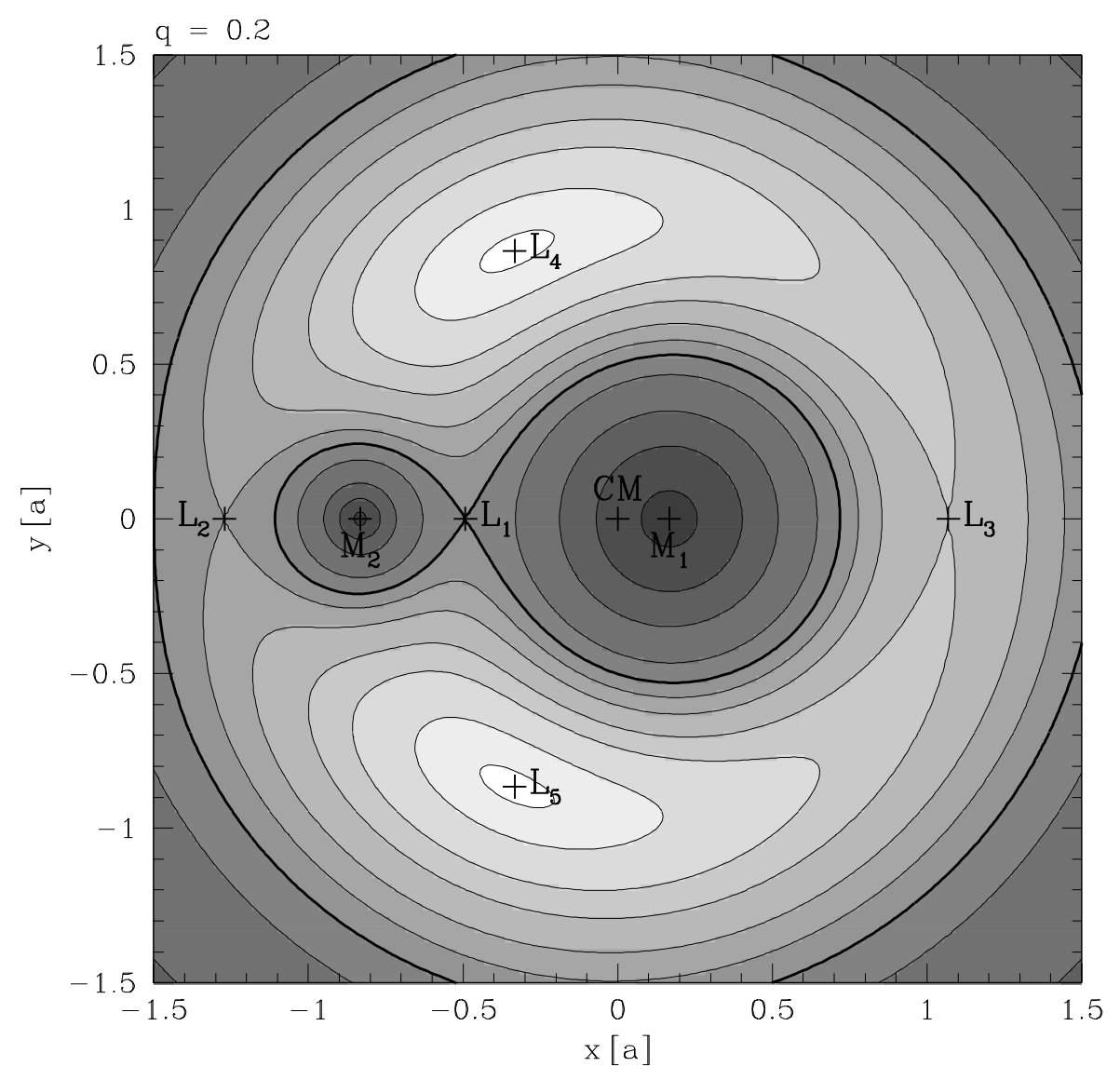

Abbildung 2.1: Schnitt durch die Äquipotentialflächen des Roche-Potentials $\Phi_{R}$ im mitrotierenden Koordinatensystem eines Doppelsternsystems (Abschnitt 2.1.1) mit $q=0.2$ in der Bahnebene. $C M$ bezeichnet den Schwerpunkt des Systems, $M_{1}$ und $M_{2}$ diejenigen der als Punktmassen betrachteten Komponenten, $L_{1}$ bis $L_{5}$ die fünf Lagrange-Punkte. $\Phi_{R}$ hat Minima an den Orten von $M_{1}$ und $M_{2}$, Sattelpunkte in $L_{1}, L_{2}$ und $L_{3}$ und Maxima in $L_{4}$ und $L_{5}$. Letztere bilden mit $M_{1}$ und $M_{2}$ gleichseitige Dreiecke; in ihrer Nähe sind stabile Orbits entlang der Äquipotentiallinien möglich, vgl. die Orbits der Trojaner im Sonne-Jupiter-System. Die dicke hantelförmige Kurve stellt die Roche-Volumina der beiden Massen dar.

erhält man in Abhängigkeit von $q$ Bahnhalbachsen $a$ von $\approx 0.5 \ldots 5 R_{\odot}$, was etwa dem einbis zehnfachen Erde-Mond-Abstand entspricht. Die zugehörigen Bahngeschwindigkeiten $v_{1}, v_{2}$ der beiden Komponenten hängen von deren Abständen $a_{1}, a_{2}$ vom Schwerpunkt des Systems ab und betragen

$$
v_{1,2}=\frac{2 \pi a_{1,2}}{P_{\text {orb }}}=1215 \mathrm{~km} \mathrm{~s}^{-1}\left(\frac{a_{1,2}}{R_{\odot}}\right)\left(\frac{P_{\text {orb }}}{1 \mathrm{~h}}\right)^{-1}
$$

mit $a_{1}+a_{2}=a$ und $a_{1} / a_{2}=M_{2} / M_{1}=q$. 


\subsubsection{Roche-Geometrie}

Im mitrotierenden Koordinatensystem eines Doppelsternsystems mit Ursprung im Schwerpunkt ist das effektive Gravitationspotential am Punkt $\vec{r}$ (Roche-Potential) durch

$$
\begin{aligned}
\Phi_{R}(\vec{r}) & =-\frac{G M_{1}}{\left|\vec{r}-\overrightarrow{r_{1}}\right|}-\frac{G M_{2}}{\left|\vec{r}-\overrightarrow{r_{2}}\right|}-\frac{1}{2}(\vec{\omega} \times \vec{r})^{2} \\
& =-\frac{G M_{1}}{a}\left(\frac{1}{\left|\vec{r}-\overrightarrow{r_{1}}\right| / a}+\frac{q}{\left|\vec{r}-\overrightarrow{r_{2}}\right| / a}+\frac{1+q}{2}\left(\frac{\vec{\omega}}{|\vec{\omega}|} \times \frac{\vec{r}}{a}\right)^{2}\right)
\end{aligned}
$$

gegeben $\left(\overrightarrow{r_{1}}, \overrightarrow{r_{2}}=\right.$ Orte der Massen $\left.M_{1}, M_{2} ; \omega=2 \pi / P_{\text {orb }}\right)$, wobei im zweiten Schritt (2.1) benutzt wurde. Die zusätzlich zu den Gravitationskräften auftretende Zentrifugalkraft wird dabei durch den zusätzlichen Potentialterm $-(\vec{\omega} \times \vec{r})^{2} / 2$ dargestellt. Da nach (2.5) $\Phi_{R}=\left(G M_{1} / a\right) \cdot F(\vec{r} / a, q)$, ist die Form der Äquipotentialflächen allein durch $q$, ihre Ausdehnung hingegen durch a bestimmt. Für jede der beiden Massen existiert eine größte geschlossene Äquipotentialfläche, die als ihre Roche-Fläche (engl.: Roche-lobe) bezeichnet wird; das von ihr eingeschlossene Volumen heißt Roche-Volumen. Die Roche-Flächen beider Massen berühren sich im inneren Lagrange-Punkt $L_{1}$. Abbildung 2.1 zeigt dies für ein System mit $q=0.2$.

Obwohl die genaue Form der Äquipotentialflächen nur numerisch zu bestimmen ist, können für einige wichtige Größen Näherungsformeln für bestimmte Bereiche von $q$ angegeben werden. So beträgt der Abstand des Primärsterns $\left(M_{1}\right)$ vom Lagrange-Punkt $L_{1}$ nach Plavec \& Kratchovil (1964)

$$
\frac{R_{L_{1}}}{a}=0.5-0.227 \log q \quad(\text { für } 0.1 \leq q \leq 10)
$$

$\approx 0.73 \ldots 0.50$ für $q=0.1 \ldots 1.0$.

Das Roche-Volumen $V_{R}$ des Sekundärsterns kann über seinen Volumenradius $R_{V}$ charakterisiert werden, d.h. den Radius einer Kugel mit $4 \pi R_{V}^{3} / 3=V_{R}$; für diesen gilt (Eggleton 1983, weniger als 1\% Fehler)

$$
\frac{R_{V}}{a}=\frac{0.49 q^{2 / 3}}{0.6 q^{2 / 3}+\ln \left(1+q^{1 / 3}\right)}
$$

oder in leichter handhabbarer Form (Paczyński 1971, weniger als 2\% Fehler)

$$
\begin{aligned}
\frac{R_{V}}{a} & =0.462\left(\frac{q}{1+q}\right)^{\frac{1}{3}} & & (\text { für } 0<q<0.3) \\
& =0.38+0.20 \log q & & (\text { für } 0.3<q<20)
\end{aligned}
$$

$\approx 0.21 \ldots 0.38$ für $q=0.1 \ldots 1^{2}$. Damit folgt mit (2.2) für $0<q<0.3$

$$
R_{V}=0.234 R_{\odot}\left(\frac{P_{\text {orb }}}{1 \mathrm{~h}}\right)^{\frac{2}{3}}\left(\frac{M_{2}}{M_{\odot}}\right)^{\frac{1}{3}} .
$$

\footnotetext{
2 Aus $R_{V}(q=1)<a / 2$ erkennt man übrigens deutlich die Abweichung des Roche-Volumens von der Kugelform.
} 
Da die Oberfläche eines Sterns in einem Doppelsternsystem (streng: eines orbitsynchron rotierenden Sterns) auf einer Fläche mit $\Phi_{R}=$ const liegt (s. z.B. Pringle 1985), definiert seine Roche-Fläche $\Phi_{R}=\Phi_{\text {crit }}$ seine maximal mögliche Ausdehnung. Ist der Radius eines Sterns hinreichend klein gegen $R_{V}$, kann er als sphärisch betrachtet werden (vgl. Abbildung 2.1). Ein System, in denen beide Komponenten kleiner als ihre Roche-Volumina sind, heißt getrennt. In einem halbgetrennten System füllt einer der Partner sein Roche-Volumen vollständig aus, mit der Folge, daß Masse über den $L_{1}$-Punkt in das Roche-Volumen des anderen Partners überströmt. Systeme, in denen beide Partner ihre Roche-Volumina ausfüllen (Kontaktsysteme), sollen hier nicht weiter behandelt werden.

\subsection{Komponenten eines CVs}

CVs sind enge Doppelsternsysteme, in denen der Primärstern ein Weißer Zwerg und der Sekundärstern ein Roche-lobe-füllender später Hauptreihenstern ist, der infolge der eben geschilderten Umstände Masse an den Weißen Zwerg verliert. Im folgenden werden die wichtigsten Eigenschaften derjenigen Komponenten vorgestellt, die allen CVs gemeinsam sind. Die Unterschiede zwischen den verschiedenen CV-Klassen und insbesondere die Eigenschaften einer eventuell vorhandenen Akkretionsscheibe werden in Abschnitt 2.3 beschrieben.

\subsubsection{Der Weiße Zwerg}

\subsubsection{Massen und Kernradien}

Weiße Zwerge sind das häufigste Endprodukt der Sternentwicklung. Sie haben Massen von typischerweise 0.4..1.3 $M_{\odot}$, wobei die mittlere Masse alleinstehender Weißer Zwerge mit $0.54 M_{\odot}$ (Bergereon et al. 1992) etwas niedriger liegt als die der in CVs beobachteten $\left(0.76 M_{\odot}\right.$, Ritter \& Kolb 1998). Da Weiße Zwerge keine Energie durch Kernfusion mehr erzeugen, werden sie durch ihre eigene Gravitation auf Dichten von $\approx 10^{5} \mathrm{~g} \mathrm{~cm}^{-3}$ komprimiert, bei denen Elektronenentartung eintritt. Der Entartungsdruck der Elektronen stabilisiert den Weißen Zwerg, solange seine Masse $M_{\mathrm{WD}}$ kleiner als die ChandrasekharGrenzmasse $M_{\mathrm{Ch}}=1.44 M_{\odot}$ ist. Der Radius $R_{\mathrm{WD}}$ eines Weißen Zwergs ist aufgrund der Entartung bis dicht unter die Oberfläche in erster Näherung nur von seiner Masse abhängig und kann mit der hier nötigen Genauigkeit - d.h. unter der Annahme $T=0$ und unter Vernachlässigung der Ausdehnung der nichtentarteten äußeren Hülle - mit

$$
\begin{aligned}
R_{\mathrm{WD}} & =1.125 \cdot 10^{-2} R_{\odot} \sqrt{\left(\frac{M_{\mathrm{WD}}}{M_{\mathrm{Ch}}}\right)^{-\frac{2}{3}}-\left(\frac{M_{\mathrm{WD}}}{M_{\mathrm{Ch}}}\right)^{\frac{2}{3}}} \\
& =7.83 \cdot 10^{8} \mathrm{~cm} \sqrt{\left(\frac{M_{\mathrm{WD}}}{M_{\mathrm{Ch}}}\right)^{-\frac{2}{3}}-\left(\frac{M_{\mathrm{WD}}}{M_{\mathrm{Ch}}}\right)^{\frac{2}{3}}}
\end{aligned}
$$

angegeben werden (Nauenberg 1972). Für die oben genannten Massen führt dies auf $R_{\mathrm{WD}}=$ $10 \ldots 5 \cdot 10^{8} \mathrm{~cm}$, was in der Größenordnung des Erdradius liegt. Einfachere Näherungen mit 
$<2 \%$ Abweichung von obiger Beziehung lauten

$$
\begin{aligned}
R_{\mathrm{WD}} & =9.1 \cdot 10^{-3} R_{\odot}\left(\frac{M_{\mathrm{WD}}}{M_{\odot}}\right)^{-\frac{3}{5}}=6.3 \cdot 10^{8} \mathrm{~cm}\left(\frac{M_{\mathrm{WD}}}{M_{\odot}}\right)^{-\frac{3}{5}}\left(0.4 \ldots 0.7 M_{\odot}\right) \\
& =8.0 \cdot 10^{-3} R_{\odot}\left(\frac{M_{\mathrm{WD}}}{M_{\odot}}\right)^{-1}=5.6 \cdot 10^{8} \mathrm{~cm}\left(\frac{M_{\mathrm{WD}}}{M_{\odot}}\right)^{-1}\left(0.7 \ldots 1 M_{\odot}\right) .
\end{aligned}
$$

Der tatsächlich beobachtete Radius ist allerdings der Photosphärenradius, der größer als der nach obigen Gleichungen berechnete Radius des entarteten Kerns ist. Hierauf werde ich kurz in Abschnitt 2.2.1.3 im Zusammenhang mit der Kühlung Weißer Zwerge eingehen. Die hohe Schwerebeschleunigung an der Oberfläche $\left(\approx 10^{5}\right.$-fache Erdbeschleunigung) führt zur Sedimentation der vorhandenen Elemente, so daß Weiße Zwerge meist eine reine Wasserstoff- oder eine reine Helium-Atmosphäre haben und in ihren Spektren stark druckverbreiterte Balmer- oder Helium-Absorptionslinien zeigen.

\subsubsection{Temperatur und Leuchtkraft}

Bei Photosphärentemperaturen von $T_{\text {eff }} \approx 5000 \ldots 60000 \mathrm{~K}$ haben Weiße Zwerge Leuchtkräfte von

$$
L_{\mathrm{WD}}=\sigma A T_{\mathrm{eff}}^{4} \approx 2 \cdot 10^{-3} L_{\odot}\left(\frac{R_{\mathrm{WD}}}{10^{9} \mathrm{~cm}}\right)^{2}\left(\frac{T_{\mathrm{eff}}}{10000 \mathrm{~K}}\right)^{4}
$$

( $L_{\odot}=$ Sonnenleuchtkraft, $A=$ Fläche des Weißen Zwergs, $\sigma=$ Strahlungskonstante). Da sie keine nennenswerte eigene Energieerzeugung mehr haben, steht ihnen nur ihre thermische Energie

$$
E_{\text {th }} \approx \frac{3}{2} k_{\mathrm{B}} T_{\mathrm{c}} \frac{M_{\mathrm{WD}}}{m_{\text {ion }}} \approx 1.5 \cdot 10^{7} L_{\odot} \mathrm{yr}\left(\frac{T_{\mathrm{c}}}{10^{8} \mathrm{~K}}\right)\left(\frac{M_{\mathrm{WD}}}{M_{\odot}}\right)
$$

zur Verfügung $\left(T_{\mathrm{c}}=\right.$ Temperatur des isothermen Kerns des Weißen Zwergs $\approx 10^{8} \mathrm{~K}$ bei der Entstehung, $\overline{m_{\text {ion }}}=$ mittlere Ionenmasse $=12 m_{\mathrm{u}}$ für Kohlenstoff). Die Ionen wurden dabei der Einfachheit halber als ideales Gas mit $c_{\mathrm{V}}=(3 / 2) k_{\mathrm{B}}$ betrachtet. Weiterhin wurde die spezifische Wärme des Elektronengases, die bei zunehmender Entartung abnimmt, vernachlässigt. Diese thermische Energie $E_{\text {th }}$ wird auf der thermischen Zeitskala

$$
\tau_{\text {cool }}=\frac{E_{\mathrm{th}}}{L_{\mathrm{WD}}} \approx 10^{10} \mathrm{yr}
$$

abgestrahlt. Geringe Energiemengen können zwar noch durch Freisetzung von Gravitationsenergie infolge von Kontraktion im Zuge der Abkühlung, durch Kristallisation des Kerns bei sinkenden Temperaturen und durch pyknonukleare Reaktionen[3 erzeugt werden. Auf der anderen Seite ist in den ersten $\approx 10^{7}$ Jahren nach Entstehung des Weißen Zwergs Kühlung durch Neutrinoverluste direkt aus dem heißen Kern bedeutsam. Abgesehen davon sind (2.14) und (2.15) jedoch adäquate Näherungen. 


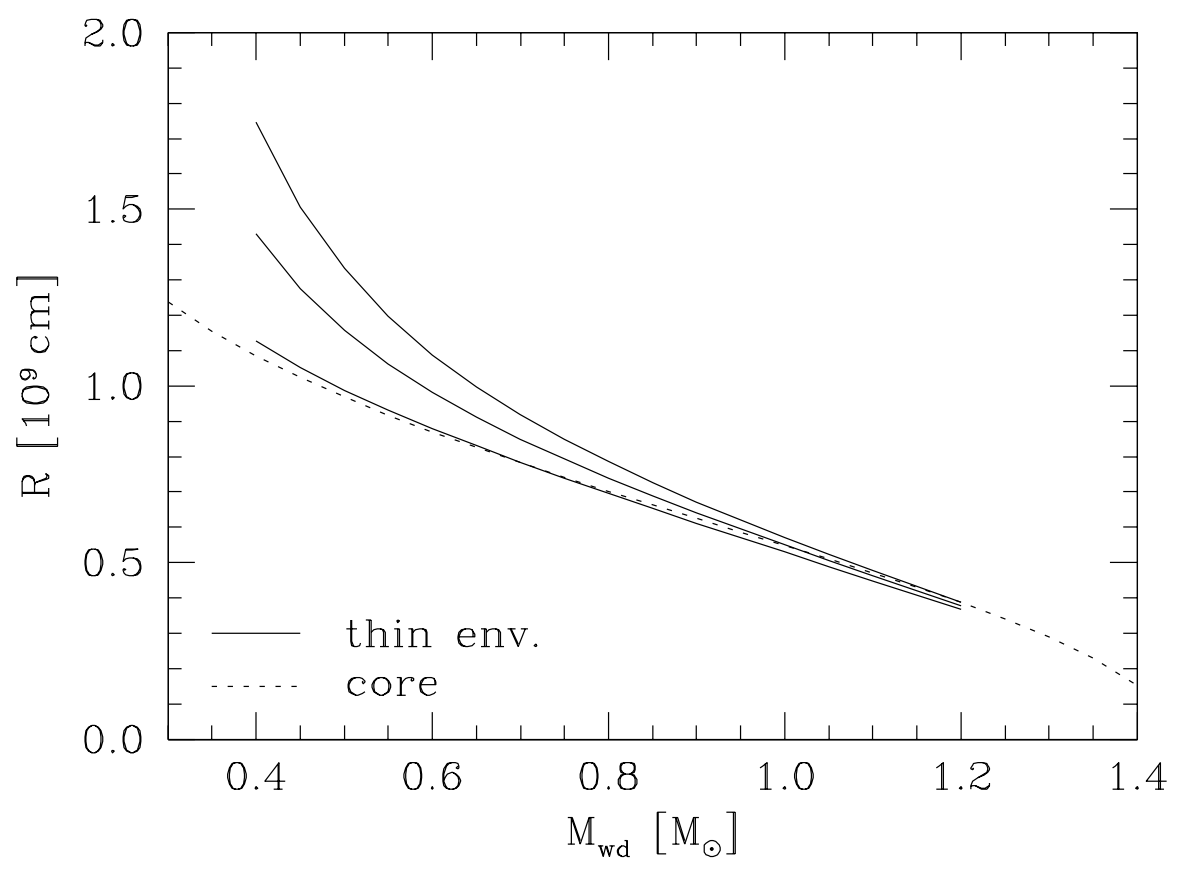

Abbildung 2.2: Masse-Radius-Relationen für Weiße Zwerge. Gestrichelt: Kernradius nach (2.11) (Nauenberg 1972); ausgezogen: photosphärischer Radius einer dünnen Hülle $\left(10^{-2} M_{\odot} \mathrm{H}, 10^{-4} M_{\odot} \mathrm{He}\right)$ über einem C-Kern mit - von unten nach oben $-T_{\text {eff }}=10000$, 30000 und $50000 \mathrm{~K}$ (parametrisiert gem. (2.18) nach Rechnungen von Wood 1994, s. Text).

\subsubsection{Kühlung und Photosphärenradien}

Benutzt man zur Bestimmung der Photosphärenleuchtkraft eine Hüllenlösung mit geeigneter Kramers-Opazität, ergeben sich genauere Abschätzungen für das Kühlungsalter $t_{\text {cool }}$ und die zum Zeitpunkt $t_{\text {cool }}$ beobachtete Temperatur $T_{\text {eff,WD }}=\left(L_{\mathrm{WD}} / 4 \pi \sigma R_{\mathrm{WD}}^{2}\right)^{1 / 4}$ :

$$
\begin{aligned}
t_{\mathrm{cool}} & =6.7 \cdot 10^{6} \mathrm{yr}\left(\frac{M_{\mathrm{WD}}}{M_{\odot}}\right)^{\frac{5}{7}}\left(\frac{L_{\mathrm{WD}}}{L_{\odot}}\right)^{-\frac{5}{7}}, \\
T_{\mathrm{eff}, \mathrm{WD}} & =8370 \mathrm{~K}\left(\frac{M_{\mathrm{WD}}}{M_{\odot}}\right)^{\frac{1}{4}}\left(\frac{R_{\mathrm{WD}}}{10^{9} \mathrm{~cm}}\right)^{-\frac{1}{2}}\left(\frac{t_{\mathrm{cool}}}{10^{9} \mathrm{yr}}\right)^{-\frac{7}{20}} .
\end{aligned}
$$

(2.17) eignet sich zur Bestimmung des Alters isolierter Weißer Zwerge aus der beobachteten Temperatur. (Für Weiße Zwerge in CVs gilt dies nicht, da ihr Wärmehaushalt durch die Akkretion beeinflußt wird, vgl. Abschnitt 2.5.5.2.) Hierbei ist aber zu beachten, daß für $R_{\mathrm{WD}}$ nicht der Radius des entarteten Kerns nach (2.11), sondern der Radius der Photosphäre des Weißen Zwergs zu benutzen ist. Dieser läßt sich ebenfalls aus der Hüllenlösung ableiten und ist besonders für kleine $M_{\mathrm{WD}}$ und große $T_{\text {eff }}$ deutlich größer als der Kernradius. Die

3 Von $\pi v \kappa \nu o ́ \varsigma=$ dicht, kompakt; langsame Kernreaktionen aufgrund der extrem hohen Dichten im Zentrum des Weißen Zwergs; im Gegensatz zu thermonuklearen Reaktionen ein reiner Dichteeffekt. 
Ergebnisse entsprechender aktueller Modellrechnungen von Wood (1994) für Weiße Zwerge mit einem Kohlenstoffkern und einer dünnen Hülle $\left(10^{-2} M_{\odot}\right.$ Helium, $10^{-4} M_{\odot}$ Wasserstoff $)$ lassen sich zwar nicht analytisch angeben, können aber für den Bereich $M_{\mathrm{WD}}=0.4 \ldots 1 M_{\odot}$ und $T_{\text {eff }}=4000 \ldots 50000 \mathrm{~K}$ näherungsweise mit

$$
R_{\mathrm{phot}}=1.3 \cdot 10^{9} \mathrm{~cm}\left(1-0.6\left(\frac{M_{\mathrm{WD}}}{M_{\odot}}\right)\right)\left(1+0.018\left(\frac{T_{\mathrm{eff}}}{10000 \mathrm{~K}}\right)^{1.05}\left(\frac{M_{\mathrm{WD}}}{M_{\odot}}\right)^{-2.25}\right)
$$

parametrisiert werden. Es sei darauf hingewiesen, daß (2.18) die Ergebnisse von Wood nur im genannten Wertebereich adäquat (d.h. mit Abweichungen von wenigen Prozent) wiedergibt. Für $T_{\text {eff }}>50000 \mathrm{~K}$ erhält man z.T. deutlich zu kleine und für $M_{\mathrm{WD}}>1 M_{\odot}$ etwas zu große Radien. Ferner ist zu beachten, daß Weiße Zwerge in CVs infolge der Akkretion vermutlich dickere Hüllen und in der Konsequenz noch etwas (z.B. für $M_{\mathrm{WD}}=$ $1 M_{\odot}$ und $T_{\text {eff }}=20000 \mathrm{~K}$ etwa $5 \%$ ) größere Radien als nach (2.18) haben. Die Masse-Radius-Relation des Kerns nach (2.11) und der Photosphäre nach (2.18) sind zur Übersicht in Abbildung 2.2 gezeigt.

\subsubsection{Magnetfeld}

Viele Weiße Zwerge haben Magnetfelder in der Größenordnung $1 \ldots 1000 \mathrm{MG}\left(1 \mathrm{G}=10^{-4} \mathrm{~T}\right)$ und entsprechende magnetische Momente

$$
\mu=B R^{3}=10^{33} \mathrm{Gcm}^{3}\left(\frac{B}{1 \mathrm{MG}}\right)\left(\frac{R}{10^{9} \mathrm{~cm}}\right)
$$

von $\mu=10^{33} \ldots 10^{36} \mathrm{G} \mathrm{cm}^{3}$. Diese sind vermutlich 'fossil', d.h. der auf die Größe eines Weißen Zwergs komprimierte magnetische Fluß eines Vorläufersterns mit einem Magnetfeld von einigen $100 \mathrm{G}$ (etwa eines Ap-Sterns). Die Stärke des Magnetfelds ist bei den Akkretionsvorgängen in CVs von entscheidender Bedeutung (s. Abschnitt 2.3).

\subsubsection{Der Sekundärstern}

\subsubsection{Massen und Radien}

Die beobachteten Bahnperioden von CVs (s. Abbildung 2.3) implizieren nach (2.10) für $M_{\mathrm{WD}}=0.4 \ldots 1.3 M_{\odot}$ Volumenradien des Sekundärsterns von $R_{V}=0.2 \ldots 1 R_{\odot}$, d.h. Radien, wie sie späte Hauptreihensterne mit Massen bis hinunter zum Limit für wasserstoffbrennende Sterne (ca. $0.08 M_{\odot}$ ) haben. Solche Sterne haben Oberflächentemperaturen von $\lesssim$ $4000 \mathrm{~K}$ und sind damit vom Spektraltyp K bis M. Für Einzelsterne mit $M=0.13 \ldots 0.6 M_{\odot}$ wurde von Caillault und Patterson (1990) die empirische Masse-Radius-Beziehung

$$
R_{2}=0.918 R_{\odot}\left(\frac{M_{2}}{M_{\odot}}\right)^{0.796}=6.39 \cdot 10^{10} \mathrm{~cm}\left(\frac{M_{2}}{M_{\odot}}\right)^{0.796}
$$




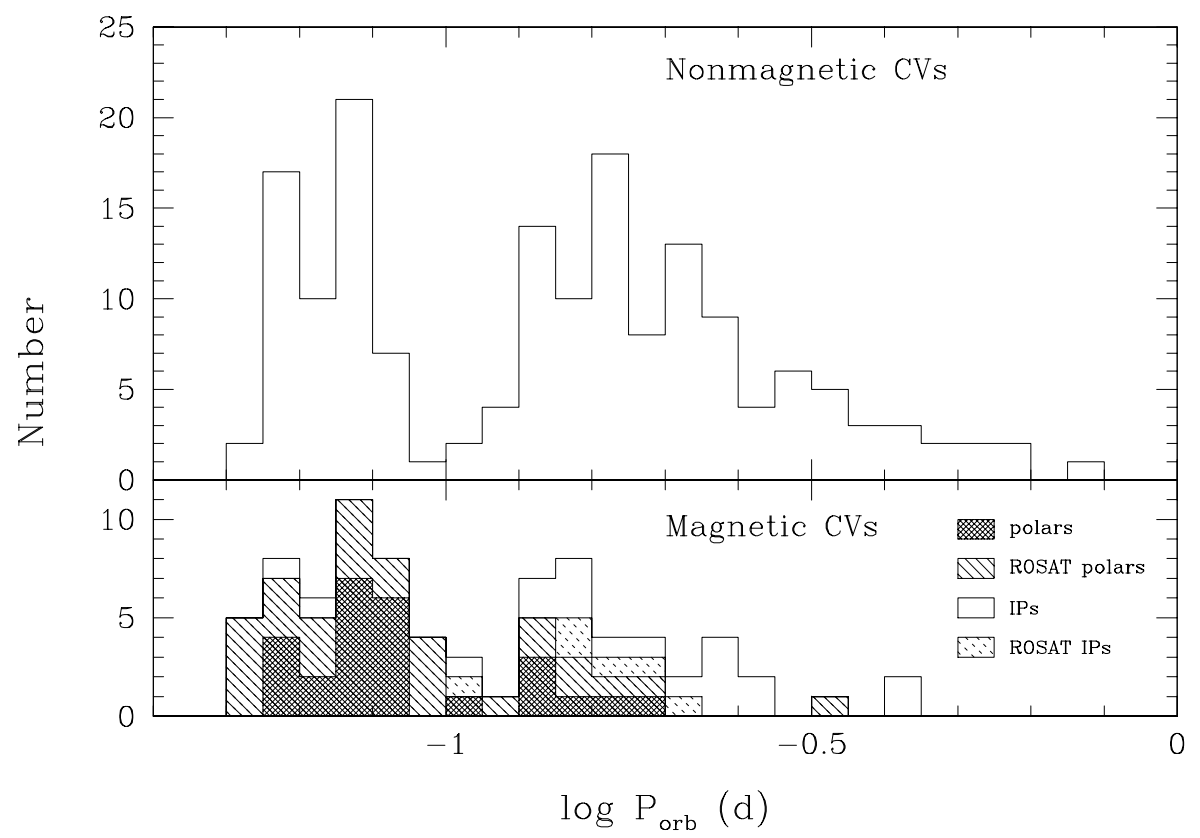

Abbildung 2.3: Periodenverteilung von CVs nach Daten von Ritter \& Kolb (1998), Beuermann \& Burwitz (1995) und Haberl \& Motch (1995) (Abbildung aus Burwitz 1997). Der Bereich von $2 \ldots 3 \mathrm{~h}$ wird aufgrund der geringen dort vorhandenen Anzahl (bekannter) Systeme als Periodenlücke bezeichnet.

angegeben円. Für Sekundärsterne in CVs ist jedoch vermutlich die von Patterson (1984) für ebensolche Sterne gefundene Beziehung

$$
R_{2}=1 R_{\odot}\left(\frac{M_{2}}{M_{\odot}}\right)^{0.88}=6.96 \cdot 10^{10} \mathrm{~cm}\left(\frac{M_{2}}{M_{\odot}}\right)^{0.88}
$$

zutreffender, obwohl ihr (und auch (2.20)) eine inzwischen als ungenau erkannte BarnesEvans-Relation (s. Abschnitt 2.2.2.2) zugrundeliegt. Für Roche-lobe-füllende Sekundärsterne ergeben sich dann mit (2.10) für $0<q<0.3$ folgende Beziehungen zwischen Periode und Masse bzw. Periode und Radius:

$$
\begin{aligned}
& M_{2}=0.0702 M_{\odot}\left(\frac{P_{\text {orb }}}{1 \mathrm{~h}}\right)^{1.220}, \\
& R_{2}=0.0965 R_{\odot}\left(\frac{P_{\text {orb }}}{1 \mathrm{~h}}\right)^{1.073}=6.72 \cdot 10^{9} \mathrm{~cm}\left(\frac{P_{\text {orb }}}{1 \mathrm{~h}}\right)^{1.073} .
\end{aligned}
$$

Dies setzt voraus, daß die Sekundärsterne in CVs abgesehen von ihrer Deformation immer noch als Hauptreihensterne betrachtet werden können. Dies ist zwar nicht selbstverständlich, da der Stern durch den permanenten Massenverlust aus dem thermischen Gleichgewicht getrieben wird, scheint aber nach neueren Befunden (Beuermann et al. 1998; Kolb

\footnotetext{
${ }^{4}$ Grob gilt theoretisch wie empirisch für die gesamte Hauptreihe $R \sim M^{0.8}$ für $M<M_{\odot}$ und $R \sim M^{0.57}$ für $M>M_{\odot}$, s. z.B. Kippenhahn \& Weigert (1991).
} 
\& Baraffe 2000) dennoch in guter Näherung gegeben zu sein. Weiterhin gelten die angegebenen Beziehungen nur im Mittel; so haben z.B. metallarme Sterne kleinere Radien als solche mit solarer Metallizität, während nuklear schon etwas entwickelte Sterne etwas größere haben. Vermutlich ist ein großer Teil der Sekundärsterne von CVs zumindest leicht nuklear entwickelt (vgl. Abschnitt 2.5.1).

Theoretische Modelle ergeben ebenfalls einen Zusammenhang zwischen Masse und Radius. Neuere Rechnungen von Baraffe et al. (1995) ergeben etwa für die Radien von Hauptreihensternen solarer Zusammensetzung des Alters $t=0$ (ZAMS-Modelle; ZAMS =Zero Age Main Sequence; Parametrisierungen von Beuermann 2000b)

$$
\begin{array}{rlr}
R_{2} & =1.163 R_{\odot}\left(\frac{M_{2}}{M_{\odot}}\right)^{0.973} & \left(M_{2}<0.11 M_{\odot}\right) \\
& =0.721 R_{\odot}\left(\frac{M_{2}}{M_{\odot}}\right)^{0.753} & \left(0.11 M_{\odot} \leq M_{2} \leq 0.4 M_{\odot}\right) \\
& =0.961 R_{\odot}\left(\frac{M_{2}}{M_{\odot}}\right)^{1.069} & \left(M_{2}>0.4 M_{\odot}\right) .
\end{array}
$$

Die verschiedenen angegebenen Masse-Radius-Relationen sind zum Vergleich in Abbildung 2.4 dargestellt. Speziell bei der Anwendung von (2.24) auf Sekundärsterne von CVs ist allerdings zu beachten, daß diese sich nicht im thermischen Gleichgewicht befinden (s.o.) und daher z.T. deutlich expandiert sein können. So beträgt etwa am oberen Ende der Periodenlücke $\left(P_{\text {orb }} \approx 3 \mathrm{~h}\right.$, vgl. Abbildung 2.3$)$ die Masse des Sekundärsterns $\approx 0.2 M_{\odot}$ (zum Grund hierfür s. Abschnitt 2.5.2), woraus sich nach (2.10) ergibt, daß der Sekundärstern da er ja sein Roche-Volumen ausfüllen muß, damit überhaupt Massentransfer stattfinden kann - um ca. 30\% gegenüber dem nach (2.24) berechneten ZAMS-Radius expandiert sein muß. Sekundärsterne von Systemen unterhalb der Periodenlücke sind jedoch vermutlich nur um einige Prozent expandiert.

\subsubsection{Ellipsoidale Modulation und Oberfächenhelligkeit}

Aufgrund der nicht-sphärischen Form des Roche-Volumens hängt der sichtbare Querschnitt des Sekundärsterns von der Orbitalphase ab, so daß sich eine charakteristische Modulation der beobachteten Helligkeit mit der halben Orbitalperiode ergibt (ellipsoidale Modulation). Für die volle Amplitude $\Delta \mathrm{m}$ der ellipsoidalen Modulation des Sekundärsterns eines Systems, das unter der Inklination $i$ gesehen wird, hat Warner (1995) die Beziehung

$$
\Delta \mathrm{m}=0.34 \frac{\sin ^{2} i}{1+q}
$$

angegeben. Diesen Sachverhalt kann man sich zunutze machen, um bei bekanntem $i$ und $q$ etwa aus der gemessenen absoluten Amplitude im K-Band die scheinbare Helligkeit $K$

des Sekundärsterns zu bestimmen. Kennt man weiterhin die von Bailey (1981) definierte Oberflächenhelligkeit

$$
S_{K}=K+5-5 \log d+5 \log \frac{R}{R_{\odot}}
$$




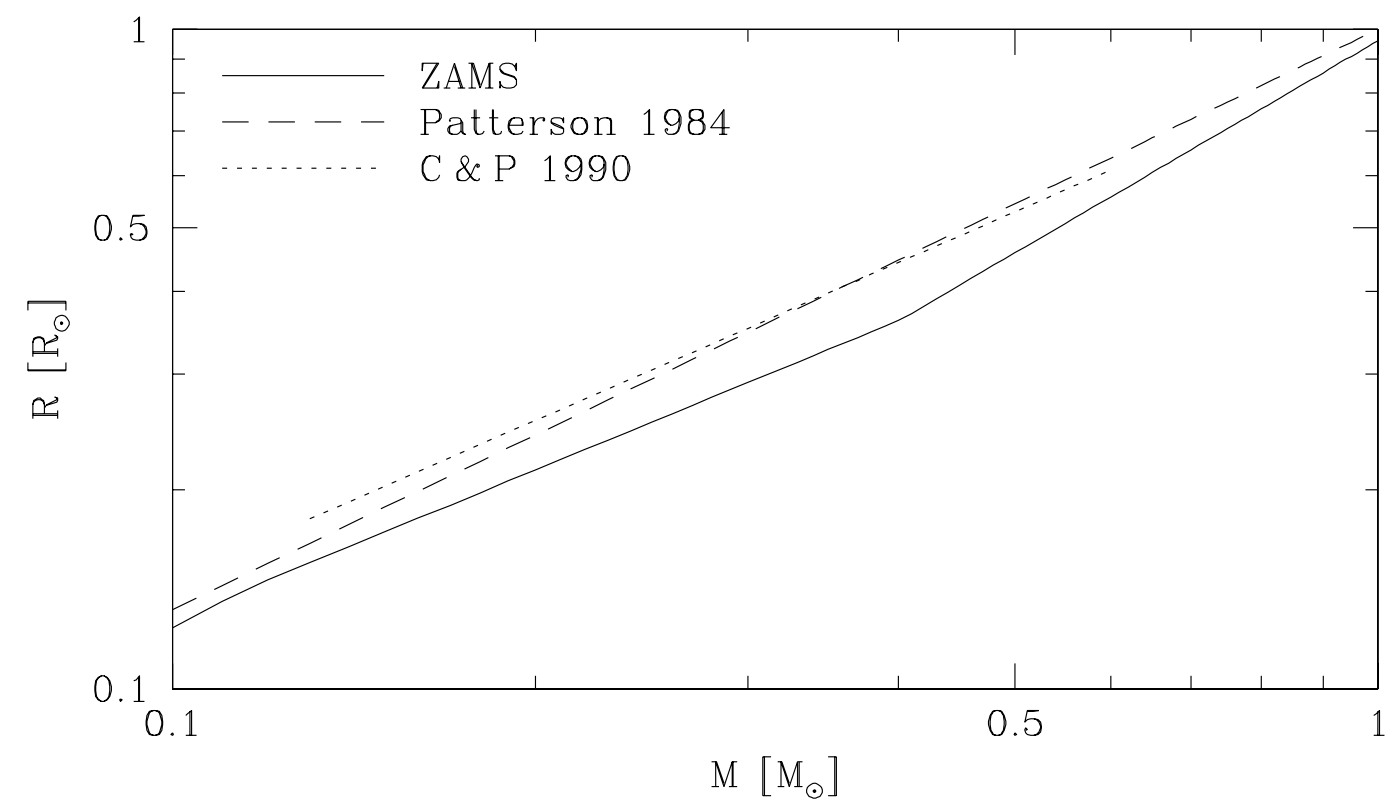

Abbildung 2.4: Masse-Radius-Relationen für potentielle CV-Sekundärsterne im Vergleich. Dargestellt sind die theoretische Beziehung (2.24) für Hauptreihensterne solarer Zusammensetzung mit dem Alter $t=0$ (Baraffe et al. 1995, ausgezogen) sowie die empirischen Relationen (2.20) (Caillault \& Patterson 1990; gepunktet) und (2.21) (Patterson 1984; gestrichelt).

des Sekundärsterns im K-Band ( $d=$ Entfernung in pc, $R=$ Radius des Sterns), so läßt sich bei bekanntem $R$ (etwa aus (2.10) oder (2.23)) aus der ellipsoidalen Modulation letztlich die Entfernung des Systems bestimmen. Barnes \& Evans (1976) stellten erstmals fest, daß allgemein die Flächenhelligkeit $S$ eines Sterns einen systematischen Gang mit seiner Effektivtemperatur und damit seinem Spektraltyp bzw. seiner Farbe aufweist und weitgehend von der Schwerebeschleunigung an der Oberfläche bzw. der Leuchtkraft unabhängig ist. Für Hauptreihensterne kann daher etwa $S_{K}$ durch eine Barnes-Evans-Relation, d.h. eine Funktion der Farbe $V-K(V=$ scheinbare Helligkeit im V-Band) ausgedrückt werden (z.B. Bailey 1981; Ramseyer 1994). Für Sekundärsterne von CVs wurde eine aktuelle BarnesEvans-Relation von Beuermann et al. (1999) angegeben:

$$
S_{K}=2.95( \pm 0.08)+0.264( \pm 0.017) \cdot(V-K)
$$

Wegen des Zusammenhangs von Spektraltyp und Farbe läßt sich diese Beziehung auch über den Spektraltyp ausdrücken (Beuermann \& Weichhold 1999; Beuermann 2000b):

$$
\begin{aligned}
& S_{K}=6.153-0.1501 X+0.001978 X^{2}, \quad \text { SpTyp G2-M3 } \\
& S_{K}=7.170-0.1294 X-0.002770 X^{2}, \quad \text { SpTyp > M3 },
\end{aligned}
$$

wobei der zu $X$ gehörige Spektraltyp $\mathrm{M}(20-X)$ ist (d.h. $X=30$ für K0, $X=20$ für M0, $X=10$ für L0 usw.). 


\subsubsection{Magnetfeld}

Man nimmt an, daß rote Zwerge Magnetfelder von $B=10^{2} \ldots 10^{3} \mathrm{G}$ haben. Die resultierenden magnetischen Momente sind dann mit denen eines magnetischen Weißen Zwergs (s. Abschnitt 2.2.1) vergleichbar, was bei der Frage nach der Ursache der Spin-BahnSynchronisation in Polaren von Bedeutung ist (s. Abschnitt 2.5.3).

\subsubsection{Der Akkretionsstrom}

Das Material, das den Sekundärstern am $L_{1}$-Punkt verläßt, tritt im mitrotierenden System etwa mit der (isothermen) Schallgeschwindigkeit

$$
c_{s}=\sqrt{\frac{k_{\mathrm{B}} T}{\bar{m}}} \approx 9 \mathrm{kms}^{-1}\left(\frac{\bar{m}}{m_{\mathrm{H}}}\right)^{-\frac{1}{2}}\left(\frac{T}{10000 \mathrm{~K}}\right)^{\frac{1}{2}}
$$

( $\bar{m}=$ mittlere Teilchenmasse, $m_{\mathrm{H}}=$ Masse des Wasserstoffatoms) in das Roche-Volumen des Primärsterns ein (für Wasserstoff bei $\lesssim 4000 \mathrm{~K}: c_{s} \lesssim 6 \mathrm{~km} \mathrm{~s}^{-1}$ ). Hingegen beträgt die orbitale Bahngeschwindigkeit des $L_{1}$-Punkts im raumfesten Koordinatensystem mit $a$ aus (2.2) und (2.6)

$$
v_{\text {orb }}\left(L_{1}\right)=\frac{2 \pi\left(R_{L_{1}}-a q /(1+q)\right)}{P_{\text {orb }}}=C \cdot\left(\frac{P_{\text {orb }}}{1 \mathrm{~h}}\right)^{-\frac{1}{3}}\left(\frac{M_{1}}{M_{\odot}}\right)^{\frac{1}{3}}
$$

mit $C \approx 410 \ldots 120 \mathrm{~km} \mathrm{~s}^{-1}$ für $q=0.1 \ldots 0.6$ bei annähernd linearem Verlauf. (Der Term $-a q /(1+q)$ im Zähler von (2.30) berücksichtigt dabei den Abstand $M_{1}-C M$ in Abbildung 2.1.) Der Akkretionsstrom ist also im raumfesten Koordinatensystem hoch supersonisch und bleibt darum stark kollimiert. Sein Verlauf kann daher zunächst durch die ballistische Trajektorie eines Partikels beschrieben werden, das im Gravitationsfeld des Weißen Zwergs auf diesen hin beschleunigt und durch die Corioliskraft von der Verbindungslinie $M_{1}-M_{2}$ weggelenkt wird.

\subsubsection{Die Akkretionsscheibe}

Wenn in einem CV der Massentransfer erstmals einsetzt, gerät das Material des Stroms aufgrund der Coriolis-Ablenkung zunächst auf eine exzentrische Bahn innerhalb des RocheVolumens des Weißen Zwergs. Da diese Bahn in der Orbitalebene des Systems liegt, kollidiert der Strom an einem gewissen Punkt mit sich selbst, wobei sich das Material dort aufheizt und einen Teil der kinetischen Energie $E_{\text {kin }}$ des Materials abstrahlt (bright spot). Da sein Drehimpuls $J$ dabei erhalten bleibt und kreisförmige Orbits das niedrigste Verhältnis $E_{\text {kin }} / J$ haben, resultiert eine zunehmende Zirkularisierung des Orbits, d.h. es bildet sich ein Ring um den Weißen Zwerg herum, in dem das Material mit der Kepler-Geschwindigkeit $v_{\text {kep }}(R)=\left(G M_{\mathrm{WD}} / R\right)^{1 / 2}$ differentiell rotiert. Viskose Reibung zwischen Material bei verschiedenen Radien führt zu dessen Aufheizung. Der damit einhergehende Energieverlust 
durch Abstrahlung wird dadurch kompensiert, daß die Materie tiefer in den Potentialtopf des Weißen Zwergs sinkt, d.h. nach innen transportiert wird. (Drehimpulserhaltung erfordert, daß gleichzeitig ein gewisser Teil der Materie nach außen transportiert wird, etwa durch einen Wind, der von der Scheibe ausgeht. Hierauf soll hier aber nicht weiter eingegangen werden.) Da stetig weiteres Material vom Sekundärstern überströmt, ist das letztendliche Resultat eine scheibenförmige Struktur (Akkretionsscheibe), deren Temperatur von außen nach innen auf bis zu $10^{4} \ldots 10^{5} \mathrm{~K}$ in der Nähe des Weißen Zwergs zunimmt (Frank et al. 1985), so daß die Scheibe vom Infraroten bis ins (ferne) UV emittiert. Die Ausdehnung der Akkretionsscheibe und damit das Erscheinungsbild des Systems hängt von der Magnetfeldstärke des Weißen Zwergs ab; dies wird im folgenden Abschnitt diskutiert.

Eine umfassende Darstellung der Struktur und Dynamik von Akkretionsscheiben ist im Rahmen dieser Arbeit nicht möglich, ich verweise daher an dieser Stelle auf die Literatur (z.B. Warner 1995 und Verweise ebd.). Es sei jedoch noch kurz auf drei Punkte hingewiesen:

- Nach dem Standard-Modell für geometrisch dünne, stationäre Scheiben (' $\alpha$-Scheiben'; $\alpha$ ist ein ad hoc-Parameter, der die Viskosität des Scheibenmaterials parametrisiert, dessen physikalischer Gehalt aber noch nicht sehr gut verstanden ist) gilt unter der Annahme $\alpha=$ const und bei Verwendung einer Kramers-Opazität für die Scheibendicke $h \mathrm{im} \mathrm{Ab-}$ stand $r$ vom Zentralobjekt $h \sim r^{9 / 8}$ (Shakura \& Sunyaev 1973; Frank et al. 1985), d.h. die Oberfläche der Scheibe ist konkav. Mithin kann Strahlung aus dem heißeren Innenbereich der Scheibe oder vom zentralen Weißen Zwerg die Außenbereiche der Scheibe beleuchten und heizen sowie durch Photoionisation Linienemission anregen (vgl. Abschnitt 2.4.2).

- Am bright spot, an dem der Akkretionsstrom auf die Scheibe trifft, wird das dort vorhandene Material durch Kompression geheizt und kann durch den Staudruck aus der Scheibenebene herausgehoben werden (bulge; vgl. die schematische Darstellung in Abbildung 2.6). Da dieser Punkt im mitrotierenden Koordinatensystem ortsfest ist, ergeben sich daraus Variationen der Helligkeit und des Spektrums mit der Orbitalperiode $P_{\text {orb }}$.

- Thermische Instabilitäten in der Scheibe können zu quasi-periodischen Helligkeitsausbrüchen mit $\Delta \mathrm{mag} \approx 2 \ldots 5$ von typischerweise $2 . .20$ Tagen Dauer führen. Dabei läuft eine Heizungswelle von außen nach innen durch die Akkretionsscheibe. Die Ausbrüche beginnen daher im Optischen und setzen sich zu kürzeren Wellenlängen hin fort. Dies ist der Mechanismus der sogenannten Zwergnova-Ausbrüche, die prinzipiell alle CVs mit einer Scheibe zeigen können, d.h. auch IPs (s. Abschnitt 2.3.3).

\subsection{Klassifikation von CVs}

In allen CVs wird die Gravitationsenergie, die von der mit der Rate $\dot{M}$ akkretierten Materie im Gravitationspotential des Weißen Zwergs freigesetzt wird, fast vollständig in Strahlung umgewandelt, die das Spektrum in weiten Spektralbereichen dominiert: $L \approx L_{\text {acc }} \equiv$ $\dot{M} \Phi_{\text {grav }}=\left(G M_{\mathrm{WD}} / R_{\mathrm{WD}}\right) \dot{M}$ (zu Details s. Abschnitt 2.4). Alle CVs zeigen Variationen der Helligkeit und des Spektrums mit der Orbitalperiode $P_{\text {orb }}$ sowie unperiodische Helligkeitsvariationen wie Flackern auf Zeitskalen von Sekunden bis Minuten oder moderaten 
Anstieg oder Abfall der Leuchtkraft im Verlauf von Tagen. Einige Merkmale der Spektren und Lichtkurven wie etwa Polarisation oder Auftreten einer zusätzlichen Periode in der Größenordnung $1 . . .60 \mathrm{~min}$, die mit der Spinperiode $P_{\text {spin }}$ des Weißen Zwergs identifiziert wird (s. Abschnitt 2.3.3), treten jedoch nur in manchen Systemen auf. Dies hat zu einer Einteilung der CVs in im wesentlichen zwei Klassen geführt: 'nichtmagnetische' Systeme, in denen der Weiße Zwerg kein oder nur ein sehr schwaches Magnetfeld hat, und magnetische. Die magnetischen Systeme zerfallen weiter in die Unterklassen der Polare und der intermediären Polare (IPs). Im folgenden stelle ich die Phänomenologie der drei Klassen und ihre physikalische Interpretation im Rahmen des heutigen Standardmodells dar. Die für diese Arbeit relevanten Strahlungsprozesse werden im Detail später in Abschnitt 2.4 behandelt.

\subsection{1 'Nichtmagnetische' CVs}

In CVs mit fehlendem oder einem nur schwachen Magnetfeld reicht die Akkretionsscheibe bis an den Weißen Zwerg heran, und die akkretierte Materie wird in einem 'Akkretionsgürtel' rund um seinen Äquator abgelagert. (Dies gilt unter der stillschweigenden Annahme, daß die Rotationsachse des Weißen Zwergs senkrecht auf der Scheibenebene steht, was nicht zwangsläufig der Fall ist, aber durch Gezeitenkräfte in der Regel herbeigeführt wird.) Aufgrund des Virialsatzes kann in der Scheibe nur die Hälfte der Gravitationsenergie thermalisiert und abgestrahlt werden: $L_{\text {disc }}=L_{\text {acc }} / 2$. Die andere Hälfte steckt noch in der kinetischen Energie der mit der Kepler-Geschwindigkeit

$$
v_{\text {kep }}=\sqrt{\frac{G M}{R}}=3650 \mathrm{~km} \mathrm{~s}^{-1}\left(\frac{M_{\mathrm{WD}}}{M_{\odot}}\right)^{\frac{1}{2}}\left(\frac{R_{\mathrm{WD}}}{10^{9} \mathrm{~cm}}\right)^{-\frac{1}{2}}
$$

kreisenden Materie, die erst in einer dünnen Grenzschicht (boundary layer) auf die äquatoriale Rotationsgeschwindigkeit des Weißen Zwergs

$$
v_{\text {spin }}=\frac{2 \pi R}{P_{\text {spin }}}=1050 \mathrm{~km} \mathrm{~s}^{-1}\left(\frac{R_{\mathrm{WD}}}{10^{9} \mathrm{~cm}}\right)\left(\frac{P_{\mathrm{spin}}}{1 \mathrm{~min}}\right)^{-1}
$$

abgebremst und dabei weiter aufgeheizt wird. Unter Berücksichtigung von Energie- und Drehimpulserhaltung wird dabei in der Grenzschicht die Leuchtkraft

$$
L_{\mathrm{BL}}=\frac{L_{\mathrm{acc}}}{2}\left(1-\frac{v_{\mathrm{spin}}}{v_{\mathrm{kep}}\left(R_{\mathrm{WD}}\right)}\right)^{2}
$$

freigesetzt (Kley 1991). Für $v_{\text {spin }} \ll v_{\text {kep }}$ ist $L_{\mathrm{BL}} \approx L_{\mathrm{disc}}$. Die Temperatur der Grenzschicht beträgt im optisch dicken Fall $10^{5} \ldots 10^{6} \mathrm{~K}$, so daß sie hauptsächlich im EUV und im Bereich weicher Röntgenstrahlung $(\$ 0.1 \mathrm{keV})$ emittiert. Eine optisch dünne Grenzschicht ist $\approx$ $10^{8} \mathrm{~K}$ heiß und emittiert entsprechend hauptsächlich harte Röntgenstrahlung $(\approx 10 \mathrm{keV})$ (Frank et al. 1985). 


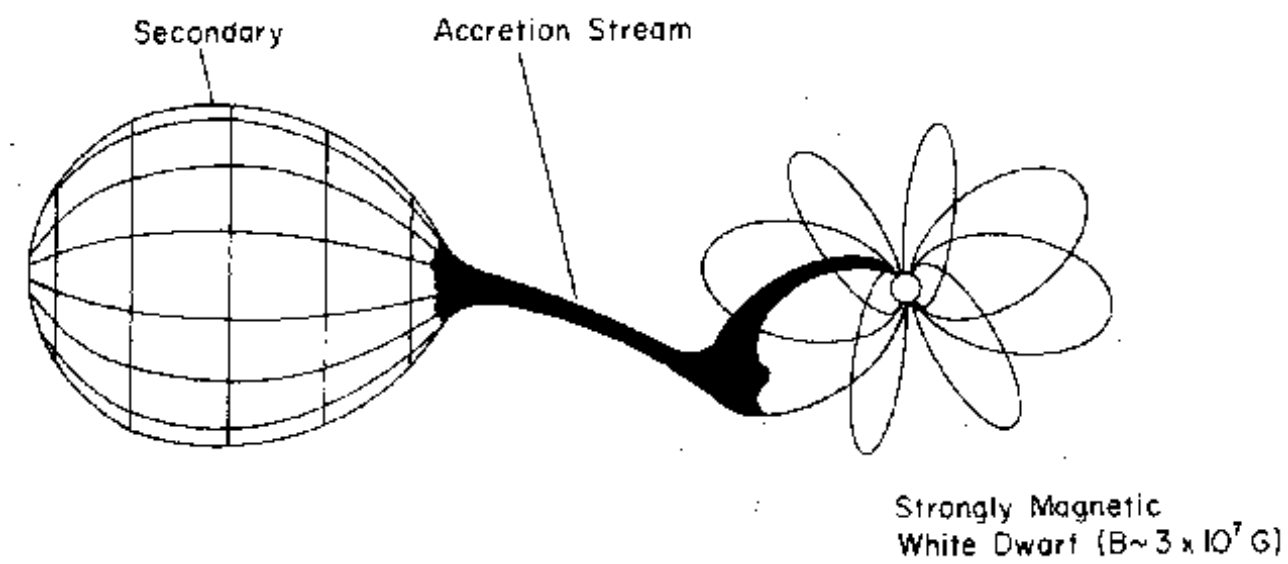

Abbildung 2.5: Schematische Darstellung eines Polars (aus Cropper, 1990). Der ballistisch fallende Akkretionsstrom koppelt an das Magnetfeld an, sobald magnetischer Druck und Staudruck gleich groß sind, und folgt ab da den magnetischen Feldlinien zum nähergelegenen Magnetpol des Weißen Zwergs.

\subsubsection{Polare}

Polare (nach dem Prototyp auch AM Her-Sterne) zeigen in ihren Spektren neben einer Bremsstrahlungskomponente im harten Röntgenbereich $(\approx 10 \ldots 30 \mathrm{keV})$ und einer starken Schwarzkörper-ähnlichen Komponente im EUV bis weichen Röntgenbereich $\left(k_{\mathrm{B}} T_{\mathrm{bb}} \lesssim\right.$ $50 \mathrm{eV}$ ) Anzeichen für ein Magnetfeld des Weißen Zwergs von 1...230 MG wie Zyklotronlinien im Optischen oder IR, bis zu 70\% zirkular polarisierte optische Strahlung (die den Systemen ihren Namen gab) oder Zeeman-Aufspaltung der photosphärischen Balmer-Linien des Weißen Zwergs. Durch das starke Magnetfeld ist die Spinperiode des Weißen Zwergs mit der Orbitalperiode synchronisiert: $P_{\text {spin }}=P_{\text {orb }}$ (zur Ursache s. Abschnitt 2.5.3). Dies definiert geradezu die Polare. Ihre Lichtkurven zeigen daher (abgesehen von Flackern und gelegentlichen 'low states', in denen die Leuchtkraft um bis zu mehrere Größenordnungen abfallen kann) nur Variationen mit der Orbitalperiode. Es gibt jedoch einige wenige Systeme, in denen die Synchronisation nicht perfekt ist und der Weiße Zwerg um einige Prozent asynchron rotiert, die man aber dennoch aus anderen Gründen als Polare klassifiziert, wie etwa V1500 Cyg, der seine Synchronisation im Nova-Ausbruch von 1975 verloren hat (Stockman et al. 1988), und BY Cam.

Das Magnetfeld hat auch Auswirkungen auf das ionisierte Material des Akkretionsstroms: Es fällt entlang einer Partikel-Trajektorie bis zu dem Abstand $r_{\mu}$ vom Weißen Zwerg, in dem der magnetische Druck den Staudruck übersteigt (Alfvén-Radius), d.h. in dem

$$
p_{\text {mag }}=\frac{B^{2}}{8 \pi} \gtrsim p_{\text {ram }}=\rho v^{2}=\frac{4 \dot{M}}{\sigma v} v^{2}
$$

gilt (mit der Querschnittsfläche $\sigma$ und der Geschwindigkeit $v$ des Akkretionsstroms). Ab da folgt es den magnetischen Feldlinien und trifft schließlich in der Nähe der magnetischen 
Pole in relativ kleinen Akkretionsflecken auf den Weißen Zwerg, wo es in einem starken hydrodynamischen Stoß auf hohe Temperaturen geheizt wird und seine kinetische Energie größtenteils in Strahlung umwandelt (s. Abschnitt 2.4). Da die magnetische Achse des Weißen Zwergs i.a. nicht senkrecht auf der Bahnebene steht, erfolgt die Akkretion meist bevorzugt auf den dem Ankoppelpunkt näherliegenden Pol, vgl. Abbildung 2.5. Der AlfvénRadius $r_{\mu}$ eines akkretierenden Objekts ist nach Hameury et al. (1986)

$$
r_{\mu}=\phi \cdot 0.39 R_{\odot}\left(\frac{B}{1 \mathrm{MG}}\right)^{\frac{4}{3}}\left(\frac{R_{\mathrm{WD}}}{10^{9} \mathrm{~cm}}\right)^{\frac{4}{3}}\left(\frac{M_{\mathrm{WD}}}{M_{\odot}}\right)^{-\frac{1}{7}}\left(\frac{\dot{M}}{10^{16} \mathrm{~g} \mathrm{~s}^{-1}}\right)^{-\frac{2}{7}} .
$$

Dabei ist $\phi$ ein Korrekturfaktor für den Fall nicht-sphärischer Akkretion mit dem Wert 0.5 für Akkretion über eine Scheibe und 0.4 für scheibenlose Akkretion wie im vorliegenden Fall. In CVs ist $r_{\mu}$ schon für sehr moderate Feldstärken vergleichbar mit dem Abstand $R_{L_{1}}$ zum $L_{1}$-Punkt (vgl. (2.6)), so daß sich auch weit vom Weißen Zwerg entfernt keine Scheibe ausbilden kann. Insgesamt ergibt sich das in Abbildung 2.5 dargestellte Bild. Einen weit detaillierteren Überblick über Polare gibt etwa Cropper (1990).

\subsubsection{Intermediäre Polare}

Intermediäre Polare (IPs; auch DQ Her-Sterne) ähneln beobachtungsseitig prinzipiell den Polaren, zeigen jedoch als definierendes Merkmal zusätzlich zur orbitalen Modulation Variationen mit der (meist wesentlich) kürzeren Spinperiode des Weißen Zwergs - bei den bekannten IPs $\approx 0.5-60$ min (Ritter \& Kolb 1998) - und gelegentlich auch mit den Schwebungsperioden $P_{\text {beat }}=\left(P_{\text {spin }}^{-1}-N P_{\text {orb }}^{-1}\right)^{-1}, N=1,2, \ldots$ (e.g. Warner 1986; zur Ursache s. Abschnitt 2.4.2.7). Es werden fast nie Zyklotronlinien beobachtet, oft jedoch zirkulare Polarisation, deren Grad mit der Spinperiode moduliert sein kann. Manche Systeme haben Zwergnova-Ausbrüche, was auf das Vorhandensein einer Akkretionsscheibe hindeutet (s. Abschnitt 2.2.4).

Nach dem erstmals von Warner (1983) vorgeschlagenen intermediate polar model (daher der Name IPs) sind diese Systeme CVs mit schwächeren Magnetfeldern als typische Polare (einige MG), in denen die Eigendrehung des Weißen Zwergs (noch) nicht mit der Orbitalbewegung synchronisiert ist. Das vom Sekundärstern überströmende Material bildet in den Außenbereichen eine Scheibe, im Innenbereich koppelt das Plasma wie bei den Polaren an das Magnetfeld an und folgt den Magnetfeldlinien (Abbildung 2.6). Dies geschieht jedoch anders als bei den Polaren entlang des gesamten Innenrands der Scheibe. Da magnetische und Rotationsachse des Weißen Zwergs (wie bei der Erde) meist nicht parallel zueinander sind, fließt das Material von jedem Punkt des Innenrands zum jeweils näherliegenden Magnetpol, so daß sich ober- und unterhalb der Scheibe je ein Akkretionsvorhang ausbildet (s. Abbildung 2.7). Folglich sind die Akkretionsregionen auf dem Weißen Zwerg in IPs im Gegensatz zu denen in Polaren nicht fleckförmig, sondern eher Ringe (oder Stücke von Ringen) um die Pole herum; direkt an den Polen findet (vermutlich) keine Akkretion statt. (Eine vergleichbare Situation liegt bei den Polarlichtregionen der Erde vor, in denen die geladenen Teilchen des Sonnenwinds entlang der Feldlinien des Erdmagnetfelds in die Atmosphäre eindringen: Auch sie umgeben die magnetischen Pole ringförmig.) 

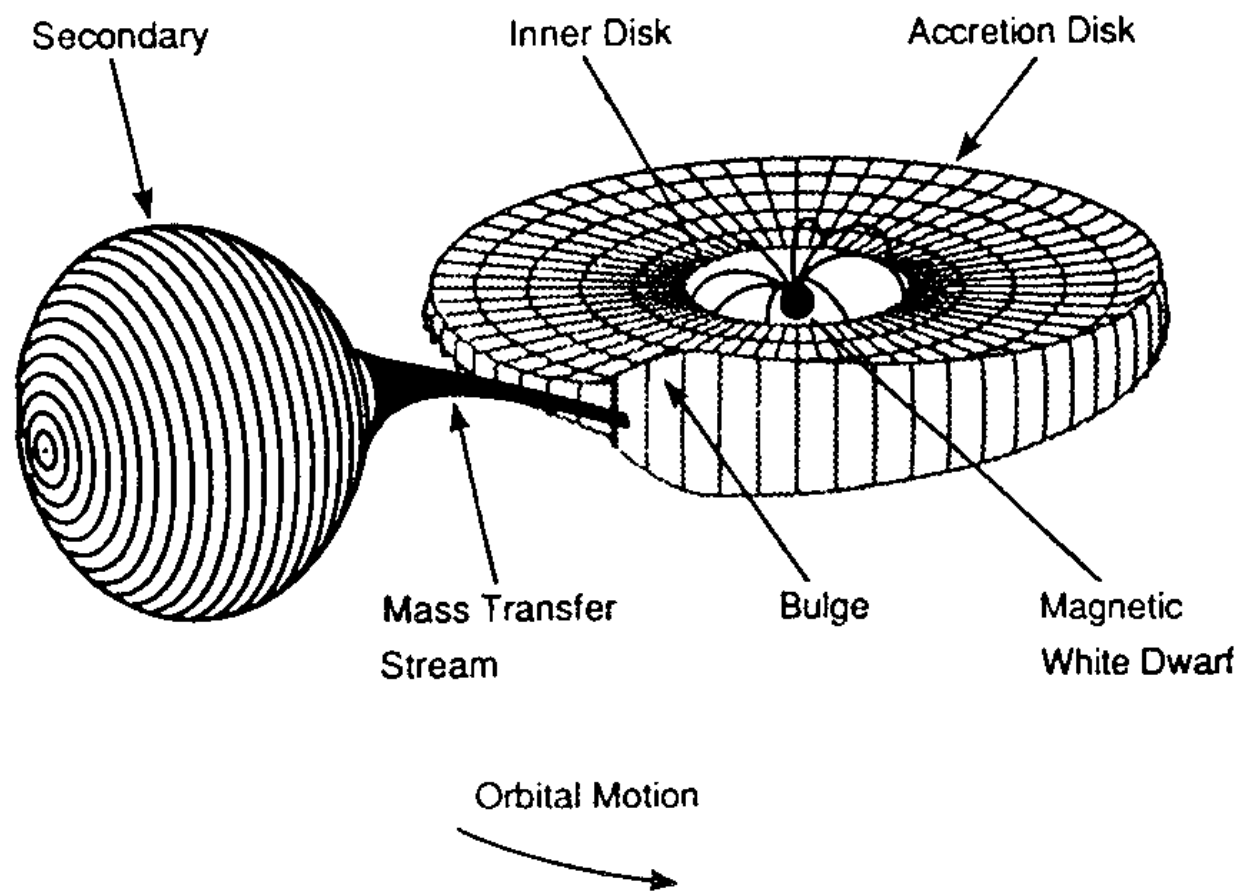

Abbildung 2.6: Schematische Darstellung eines intermediären Polars (aus Mason et al. 1988). Das akkretierte Material bildet eine Scheibe um den Weißen Zwerg, die im Innenbereich von dessen Magnetfeld aufgebrochen wird; die Akkretion erfolgt aus dem gesamten Innenrand der Scheibe auf ringförmige Regionen um die Magnetpole herum (vergleichbar den Polarlichtzonen der Erde). Dort, wo der Akkretionsstrom auf die Scheibe trifft, kann sich ein 'bulge' bzw. 'bright spot' bilden, s. Abschnitt 2.2.4

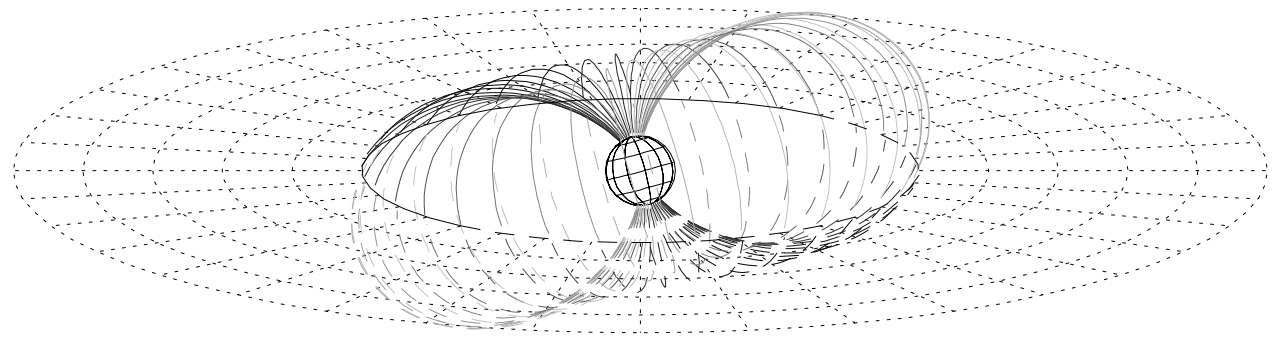

Abbildung 2.7: Schematische Darstellung der Akkretionsvorhänge eines IPs. Das Koordinatensystem auf dem Weißen Zwerg deutet seine magnetische Länge bzw. Breite an, seine Rotationsachse möge hier senkrecht auf der Scheibenebene stehen. Akkretion erfolgt ausgehend vom Innenrand der Scheibe entlang der Magnetfeldlinien bevorzugt auf den jeweils näherliegenden Magnetpol; ober- bzw. unterhalb der Scheibenebene bildet sich je ein Akkretionsvorhang (durchgezogene bzw. gestrichelte Kurven). Die Fußkurven der Vorhänge wandern mit der Rotation des Weißen Zwergs um den Scheibeninnenrand herum. 
Im Laufe einer Umdrehung wandern die Magnetpole um die Rotationsachse und damit die Fußkurven der Akkretionsvorhänge um den Innenrand der Scheibe herum, wodurch sich die Sichtbedingungen auf die in den inneren Bereichen der Akkretionsvorhänge und in den Akkretionsregionen nahe der Oberfläche des Weißen Zwergs erzeugte Strahlung (s. Abschnitt 2.4.2) periodisch verändern. Weiterhin wird diese Strahlung je nach Spinphase noch in unterschiedlichem Maße vom kühlen Material in den Außenbereichen der Vorhänge absorbiert; dies betrifft insbesondere EUV- und weiche Röntgenstrahlung (vgl. Abschnitt 2.4.2.4). Die in IPs zusätzlich auftretende Periode ist somit einfach ein Abbild der Rotation des Weißen Zwergs, wobei ein wesentlicher Teil der beobachteten Intensitätsmodulation auf den mitrotierenen Akkretionsvorhang als charakteristischer Komponente eines IPs zurückzuführen ist. (Vermutlich existieren aber auch scheibenlose IPs, die dann in ihren Eigenschaften bis auf die fehlende Spin-Orbit-Synchronisation eher den Polaren gleichen sollten.)

\subsection{Betrachtungen zur Energetik}

CVs emittieren Strahlung in einem weiten Wellenlängenbereich von harter Röntgenstrahlung bis hin zum Infrarot (Abbildung 2.9). In diesem Kapitel werden die wesentlichen Mechanismen erläutert, die die kinetische Energie des auf den Weißen Zwerg einfallenden Materials in Strahlung in jeweils charakteristischen Energiebereichen umwandeln.

\subsubsection{Energiequelle}

Die Leuchtkraft eines akkretierenden kompakten Objekts - hier eines Weißen Zwergs - beruht zum größten Teil auf der fast vollständigen Umwandlung der freigesetzten potentiellen Energie der mit der Rate $\dot{M}$ akkretierten Materie in Strahlung. Die Akkretionsleuchtkraft $L_{\text {acc }}$, die ein Weißer Zwerg bei einer Akkretionsrate $\dot{M}$ produziert, ist durch

$$
\begin{aligned}
L_{\mathrm{acc}} & =\frac{G M_{\mathrm{WD}}}{R_{\mathrm{WD}}} \dot{M}=0.34 L_{\odot}\left(\frac{M_{\mathrm{WD}}}{M_{\odot}}\right)\left(\frac{R_{\mathrm{WD}}}{10^{9} \mathrm{~cm}}\right)^{-1}\left(\frac{\dot{M}}{10^{16} \mathrm{~g} \mathrm{~s}^{-1}}\right) \\
& =1.33 \cdot 10^{33} \mathrm{erg} \mathrm{s}^{-1}\left(\frac{M_{\mathrm{WD}}}{M_{\odot}}\right)\left(\frac{R_{\mathrm{WD}}}{10^{9} \mathrm{~cm}}\right)^{-1}\left(\frac{\dot{M}}{10^{16} \mathrm{~g} \mathrm{~s}^{-1}}\right) .
\end{aligned}
$$

gegeben. Typische Akkretionsraten liegen im Bereich $10^{-11} \ldots 10^{-8} M_{\odot} \mathrm{yr}^{-1} \approx 10^{15} \ldots 10^{18} \mathrm{~g} \mathrm{~s}^{-1}$. $\left(10^{-10} M_{\odot} \mathrm{yr}^{-1} \simeq 6.3 \cdot 10^{15} \mathrm{~g} \mathrm{~s}^{-1}\right)$. (Zur Veranschaulichung der Größenordnung: Ein Massenfluß von $10^{16} \mathrm{~g} \mathrm{~s}^{-1}$ auf einen Akkretionsfleck von $1000 \mathrm{~km}$ Durchmesser entspricht ca. $12 \mathrm{~kg} \mathrm{~m}^{-2} \mathrm{~s}^{-1}$ oder, um einen irdischen Vergleich heranzuziehen, einem Dauerregen über ganz Deutschland, bei dem pro Minute $70 \mathrm{~cm}$ Wassersäule, d.h. etwa der Niederschlag eines ganzen Jahres niedergeht.) Typische akkretierende Weiße Zwerge können somit Leuchtkräfte bis zu $10^{35} \mathrm{erg} \mathrm{s}^{-1} \approx 25 L_{\odot}$ entwickeln, d.h. wesentlich mehr als ihre 'intrinsische' photosphärische Leuchtkraft (vgl. (2.13)). 


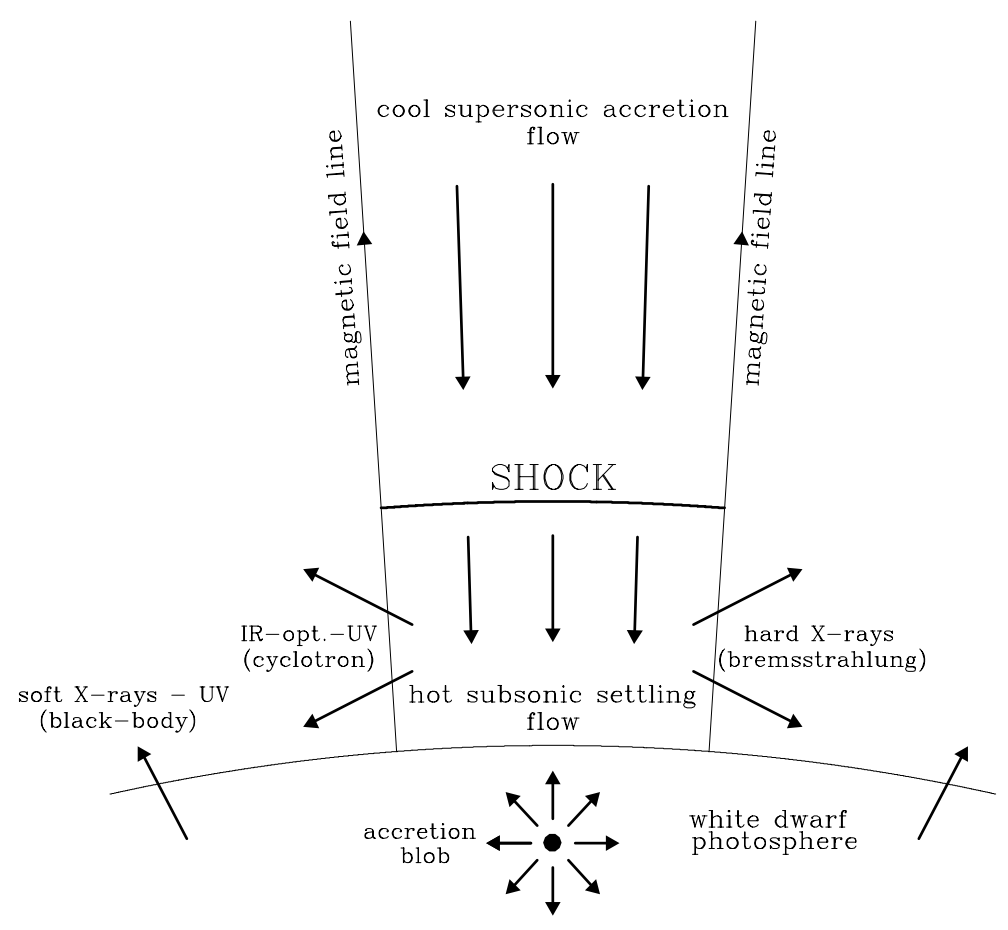

Abbildung 2.8: Schematische Darstellung der Akkretionsregion eines magnetischen CVs mit der Akkretionssäule bzw. dem Akkretionsvorhang, dem hydrodynamischen Stoß und den Entstehungsorten der verschiedenen Strahlungskomponenten (s. Text).

\subsubsection{Strahlungsprozesse}

Die verschiedenen Strahlungskomponenten, die in CVs beobachtet werden, haben ihren Ursprung an verschiedenen Stellen im System; die zugrundeliegenden Entstehungsmechanismen unterscheiden sich ebenfalls stark. Da nichtmagnetische Systeme für diese Arbeit nicht relevant sind, beziehen sich die folgenden Ausführungen zum größten Teil auf magnetische Systeme. Polare und IPs können dabei in vielerlei Hinsicht als gleich betrachtet werden; wo charakteristische Unterschiede bestehen, werde ich darauf hinweisen.

\subsubsection{Aufheizung in einem starken hydrodynamischen Stoß}

Das akkretierte Material fällt entlang der Feldlinien des Weißen Zwergs als Akkretionssäule (im Fall eines Polars) bzw. als Akkretionsvorhang (im Fall eines IPs, vgl. Abbildung 2.7) mit der Freifall-Geschwindigkeit

$$
v_{\mathrm{ff}}=\sqrt{\frac{2 G M_{\mathrm{WD}}}{R_{\mathrm{WD}}}}=5150 \mathrm{~km} \mathrm{~s}^{-1}\left(\frac{M_{\mathrm{WD}}}{M_{\odot}}\right)^{\frac{1}{2}}\left(\frac{R_{\mathrm{WD}}}{10^{9} \mathrm{~cm}}\right)^{-\frac{1}{2}}
$$

ein. Für typische Weiße Zwerge mit einer Masse von 0.4...1 $M_{\odot}$ ist knapp über der Oberfläche $v_{\mathrm{ff}} \approx 3000 \ldots 7000 \mathrm{~km} \mathrm{~s}^{-1}$, was weit über der lokalen Schallgeschwindigkeit (vgl. (2.29)) 


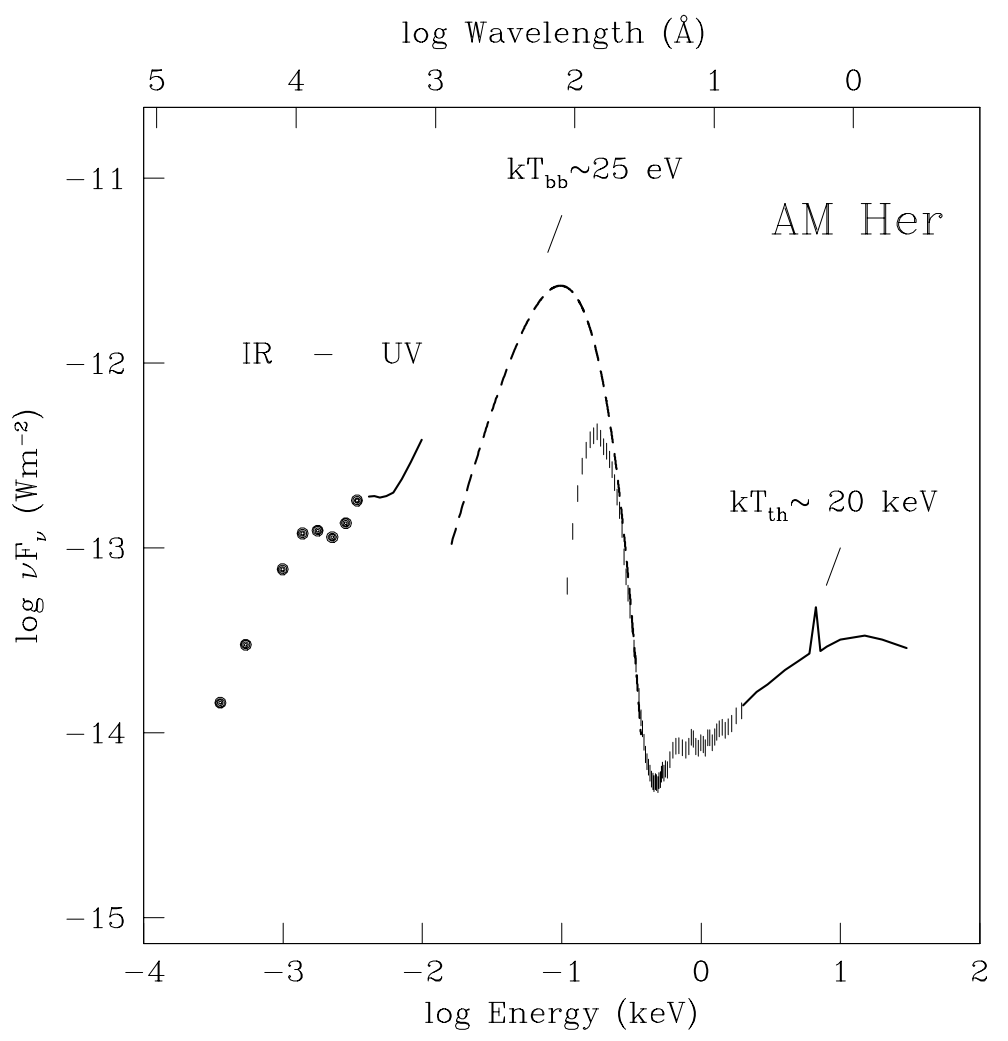

Abbildung 2.9: Spektrum des Polars AMHer vom Infrarot bis in den Bereich harter Röntgenstrahlung (aus Burwitz 1997). Von den in Abschnitt 2.4.2 diskutierten Strahlungskomponenten erkennt man hier thermische Bremsstrahlung $(E \gtrsim 0.5 \mathrm{keV})$, reprozessierte Strahlung aus dem Akkretionsfleck (EUV und weicher Röntgenbereich; die Punkte im Bereich $0.1 \ldots 2.4 \mathrm{keV}$ sind ROSAT-Meßwerte, die gestrichelte Kurve ein daran angepaßtes Schwarzkörpermodell ohne interstellare Absorption) und die Photosphäre des Weißen Zwergs (UV).

liegt. Unter diesen Bedingungen bildet sich nach dem Standardmodell für akkretierende magnetische Weiße Zwerge (King \& Lasota 1979; Lamb \& Masters 1979) dicht über der Oberfläche eine stehende adiabatische Stoßfront in der Akkretionssäule aus, in der das Material schlagartig auf etwa ein Viertel seiner Geschwindigkeit abgebremst und seine kinetische Energie in thermische Energie umgewandelt wird (Abbildung 2.8). Dabei wird es auf die Temperatur

$$
\begin{aligned}
T_{\mathrm{sh}} & =\frac{3}{16} \frac{\bar{m}}{k_{\mathrm{B}}} v_{\mathrm{ff}}^{2}=\frac{3}{8} \frac{\bar{m}}{k_{\mathrm{B}}} \frac{G M_{\mathrm{WD}}}{R_{\mathrm{WD}}} \\
& =6 \cdot 10^{8} \mathrm{~K}\left(\frac{\bar{m}}{m_{\mathrm{H}}}\right)\left(\frac{M_{\mathrm{WD}}}{M_{\odot}}\right)\left(\frac{R_{\mathrm{WD}}}{10^{9} \mathrm{~cm}}\right)^{-1}
\end{aligned}
$$

aufgeheizt $\left(\bar{m}=\right.$ mittlere Teilchenmasse). Für Weiße Zwerge von $0.4 \ldots 1 M_{\odot}$ erhält man so Temperaturen hinter der Stoßfront von $10^{8} \ldots 10^{9} \mathrm{~K}$ bzw. $k_{\mathrm{B}} T_{\mathrm{sh}}=10 \ldots 100 \mathrm{keV}$. 


\subsubsection{Thermische Bremsstrahlung}

Unterhalb der Stoßfront fällt das auf $T_{\text {sh }}$ aufgeheizte Material mit Unterschallgeschwindigkeit in einem sogenannten 'settling flow' weiter. Coulomb-Stöße zwischen den Protonen und Elektronen des Plasmas führen dazu, daß die im elektrischen Feld der Protonen beschleunigten Elektronen isotrope frei-frei-Strahlung (Bremsstrahlung) mit einer Temperatur von $10 \mathrm{keV} \lesssim k_{\mathrm{B}} T_{\mathrm{br}} \lesssim k_{\mathrm{B}} T_{\mathrm{sh}}$ (d.h. im harten Röntgenbereich) emittieren. Die Effektivität dieses Prozesses und die Form des emittierten Spektrums hängen von den Teilchenenergien und daher im vorliegenden Fall von $T_{\text {sh }}$ ab, daher spricht man hier auch von thermischer Bremsstrahlung. Gemessene Temperaturen liegen für Polare im Bereich 10...30 keV und für IPs bei etwa $10 \mathrm{keV}$. Bei den im settling flow vorherrschenden Dichten und Temperaturen ist das Plasma für Photonen mit Energien $\gtrsim 1 \mathrm{keV}$ optisch dünn, so daß die Strahlung ungehindert in alle Richtungen entweichen kann und das Spektrum praktisch aller magnetischen CVs oberhalb von $\approx 1 \mathrm{keV}$ dominiert (s. Abbildung 2.9). Thermische Bremsstrahlung ist daher einer der dominanten Kühlungsmechanismen in CVs.

Da $T_{\mathrm{sh}}$ nach (2.38) im wesentlichen nur von $M_{\mathrm{WD}}$ abhängt $\left(R_{\mathrm{WD}}=R_{\mathrm{WD}}\left(M_{\mathrm{WD}}\right)\right.$, vgl. (2.11)), eröffnet die thermische Bremsstrahlung zumindest prinzipiell die Möglichkeit, über die Messung ihrer Temperatur die Masse des Weißen Zwergs relativ frei von weiteren Annahmen zu bestimmen. In der Praxis besteht aber ein grundsätzliches Problem darin, daß z.B. Kühlung durch Zyklotronstrahlung (Abschnitt 2.4.2.3) die Temperatur des Plasmas im settling flow deutlich herabsetzen kann, was zur Folge hat, daß letztlich die Masse des Weißen Zwergs unterschätzt wird. Ähnliche Probleme treten z.B. dann auf, wenn die Stoßfront hoch über dem Weißen Zwerg steht und die höchste Plasmatemperatur daher nicht direkt hinter der Stoßfront, sondern im nachbeschleunigten Material dichter am Weißen Zwerg erreicht wird, oder wenn (unerkannte) lokale Absorption eine wesentliche Rolle spielt. Letztere wirkt bevorzugt bei niedrigen Photonenenergien und spiegelt daher ein härteres Spektrum als tatsächlich vorhanden vor. Beide Effekte können dazu führen, daß $T_{\mathrm{sh}}$ und $M_{\mathrm{WD}}$ überschätzt werden. Das Verfahren ist also in der Regel mit methodischen Unsicherheiten behaftet.

\subsubsection{Zyklotronstrahlung}

Im starken Magnetfeld des Weißen Zwergs $(B=1 \ldots 230 \mathrm{MG})$ kreisen die Elektronen mit der Zyklotronfrequenz $\omega_{\text {cyc }}=e B / m_{\mathrm{e}}$ um die magnetischen Feldlinien ( $e$ und $m_{\mathrm{e}}$ sind die Elektronenladung bzw. -masse). Dies führt zur Emission von Zyklotronstrahlung, die polarisiert ist und bei der Grundfrequenz $\omega_{\text {cyc }}$ und den höheren Harmonischen $N \omega_{\text {cyc }}(N=$ $1,2, \ldots)$ bzw. bei den Wellenlängen

$$
\lambda_{N} \equiv \frac{\lambda_{\text {cyc }}}{N}=\frac{2 \pi c}{N \omega_{\text {cyc }}}=\frac{107 \mu \mathrm{m}}{N}\left(\frac{B}{1 \mathrm{MG}}\right)^{-1} \quad, \quad N=1,2, \ldots
$$

auftritt. (Zyklotronstrahlung ist der nichtrelativistische Grenzfall der Synchrotronstrahlung.) Die Grundharmonische liegt damit bei Polaren in der Regel im nahen bis fernen IR. Da die Zyklotronlinien aufgrund der hohen Plasmatemperaturen enorm stark dopplerverbreitert sind, treten sie als charakteristische Folge von Buckeln im IR, im Optischen 


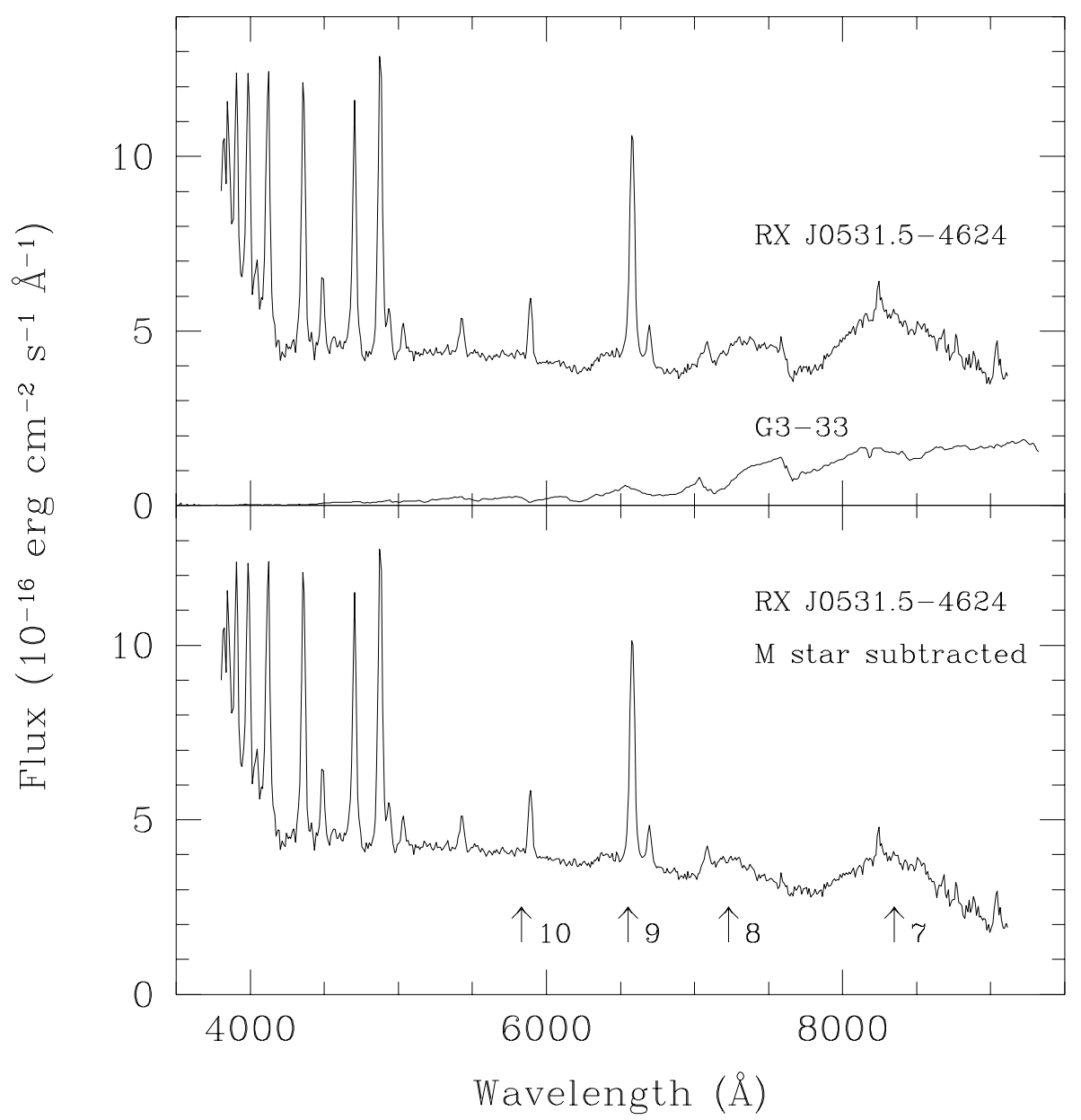

Abbildung 2.10: Spektrum des mit ROSAT entdeckten Polars RX J0531.5-4624 (= UW Pic) (aus Reinsch et al. 1994). Man erkennt den Beitrag der Photosphäre des Sekundärsterns mit seinen TiO-Banden (oberer Teil; zusätzlich ist das Spektrum des Sterns G3-33 gezeigt, der den gleichen Spektraltyp wie der Sekundärstern von UW Pic hat), Zyklotronemission aus dem settling flow (unten; die Pfeile deuten die 7. bis 10. ZyklotronHarmonische für eine Magnetfeldstärke von $19 \mathrm{MG}$ an) und Emissionslinien aus dem Akkretionsstrom und der beleuchteten Hemisphäre des Sekundärsterns.

oder sogar im UV auf. Aus ihren gegenseitigen Abständen kann nach (2.39) die Stärke des Magnetfelds bestimmt werden (s. Abbildung 2.10).

Obwohl IPs in ihren Spektren aufgrund des schwächeren Magnetfelds keine ausgeprägten Zyklotronlinien haben, sieht man die Zyklotronemission doch oft in Form eines polarisierten Strahlungsanteils. Da Zyklotronstrahlung hauptsächlich senkrecht zu den magnetischen Feldlinien emittiert wird, ist der beobachtete Polarisationsgrad oft mit der Spinperiode des Weißen Zwergs moduliert. Zyklotronstrahlung ist der zweite wichtige Kühlungsmechanismus; das Flußverhältnis $F_{\text {cyc }} / F_{\text {br }}$ ('br' für Bremsstrahlung) erlaubt Rückschlüsse auf die physikalischen Bedingungen im settling flow (vgl. Abschnitt 2.4.3). 


\subsubsection{Reprozessierte Strahlung aus der Akkretionsregion}

Ungefähr die Hälfte der Bremsstrahlung wird aus dem settling flow direkt in den Raum abgestrahlt, die andere Hälfte beleuchtet die Photosphäre des Weißen Zwergs in der unmittelbaren Nähe der Akkretionsregion. Da die Stoßfront meist sehr dicht über der Oberfläche liegt ( $h \lesssim 0.1 R_{\mathrm{WD}}$ ), gilt dies in guter Näherung auch für die anisotrope Zyklotronstrahlung. Der beleuchtete Teil der Photosphäre wird dadurch auf $T_{\mathrm{bb}} \approx 10^{5} \ldots 10^{6} \mathrm{~K}$ aufgeheizt, was zu Emission von EUV- und weicher Röntgenstrahlung mit einem (Quasi-)Schwarzkörperspektrum und typischen Photonenenergien von $k_{\mathrm{B}} T_{\mathrm{bb}} \approx 15 \ldots 60 \mathrm{eV}$ führt. Hiervon kann aber wegen der Absorption durch das kalte interstellare Medium nur der kleine höherenergetische Abteil oberhalb von $\approx 0.1 \mathrm{keV}$ beobachtet werden. Abbildung 2.9 zeigt dies am Beispiel des Polars AM Her. Während bei den meisten Polaren eine starke reprozessierte Komponente beobachtet wird und dazu benutzt werden kann, sie von anderen Arten von Röntgenquellen zu unterscheiden, ist ihre Existenz in IPs nicht gesichert. Die Ursache hierfür ist in der sehr viel höheren lokalen Absorption durch das Material des Akkretionsvorhangs (vgl. Abschnitt 2.3.3) zu suchen (z.B. Kim \& Beuermann 1995).

Da nach obiger Überlegung etwa die Hälfte der im settling flow generierten Strahlung reprozessiert wird, sollte die Beziehung

$$
L_{\mathrm{EUV} / \mathrm{SX}} \approx L_{\mathrm{br}}+L_{\mathrm{cyc}} \approx \frac{1}{2} L_{\mathrm{acc}}
$$

gelten. Bei den meisten Polaren ist der Anteil reprozessierter Strahlung jedoch deutlich größer (z.B. Beuermann 1997; Ramsay et al. 1994). Diese als soft X-ray puzzle bekannte Diskrepanz hat zur Erweiterung des Standard-Modells um die Regimes sehr hoher und sehr kleiner Akkretionsraten $\dot{M}$ geführt. Diese Erweiterungen werden kurz in Abschnitt 2.4.3 diskutiert.

\subsubsection{Photosphäre des Weißen Zwergs}

Die von Heizungseffekten unbeeinflußte Photosphäre des Weißen Zwergs emittiert bei Temperaturen von $5000 \ldots 60000 \mathrm{~K}$ vornehmlich im nahen UV und bei kurzen optischen Wellenlängen. Ihr Spektrum zeigt charakteristische breite Balmer- oder Helium-Absorptionslinien (s. Abschnitt 2.2.1). Gänsicke et al. (1995) haben gezeigt, daß die Photosphäre in manchen Fällen auch im weiteren Umkreis der Akkretionsregion um $\approx 10000 \mathrm{~K}$ geheizt ist. Ihre Emission tritt im UV als photosphärisches Spektrum eines Weißen Zwergs entsprechend höherer Temperatur auf. Die Größe dieser moderat geheizten Region hängt von der Höhe der Stoßfront über der Oberfläche und damit von $B$ und $\dot{M}$ ab (vgl. Abschnitt 2.4.3).

\subsubsection{Akkretionsscheibe, bulge und bright spot}

Die in IPs (und in nichtmagnetischen Systemen) vorhandene Akkretionsscheibe wird vor allem durch viskose Prozesse geheizt (s. Abschnitt 2.2.4) und hat typische Temperaturen von $\lesssim 10000 \mathrm{~K}$. In nichtmagnetischen CVs werden in den Innenbereichen der Scheibe auch deutlich höhere Temperaturen erreicht; in IPs fehlen diese Teile, da das Plasma vorher 
an das Magnetfeld ankoppelt. Die Scheibe eines IPs emittiert daher vornehmlich ein thermisches Spektrum, das kräftige Emissionslinien des Wasserstoffs sowie von He I und He II über einem optisch dünnen Kontinuum zeigt, das vom IR bis ins UV reicht. Je nach Dichte des Materials in verschiedenen Gebieten der Scheibe können auch Anteile eines optisch dicken Kontinuums auftreten. Die Emissionslinien haben ein charakteristisches Profil mit zwei Maxima, das auf die Rotation der Scheibe zurückzuführen ist: Die Emission des vom Beobachter weg- bzw. des auf ihn zu rotierenden Materials erscheint - sofern die Sichtlinie hinreichend gegen die Senkrechte auf die Scheibenebene geneigt ist, d.h. bei hinreichend hoher Inklination $i$ - rot- bzw. blauverschoben.

Die Emission einer 'ungestörten' radialsymmetrischen Scheibe ist nicht mit der Orbitalperiode moduliert. Der bulge und der bright spot, die durch das Auftreffen des Akkretionsstroms auf die Scheibe verursacht werden, stellen jedoch Asymmetrien dar. Die beobachtete Emission des bright spot variiert daher in der Regel mit dem Blickwinkel, d.h. mit der Orbitalphase; dasselbe gilt z.B. für die Absorption der reprozessierten Strahlung aus der Zentralregion (Abschnitt 2.4.2.4) durch das kühle Material des bulge bei hinreichend großer Inklination $i$, die sich etwa als tiefer Einbruch ('dip') in der EUV-Lichtkurve bemerkbar machen kann, während der bulge die Sichtlinie passiert.

\subsubsection{Angeregte Linienemission}

Die energiereiche Strahlung aus der heißen Akkretionsregion und der Photosphäre des Weißen Zwergs beleuchtet den Akkretionsstrom, die Akkretionsscheibe und die dem Weißen Zwerg zugewandte Seite des Sekundärsterns. Sie führt dort durch Photoionisation und Anregung der Materie zur Erzeugung zahlreicher Emissionslinien von den Bracketund Paschen-Linien im IR über Balmer-, He I- und HeII-Linien im Optischen (s. Abbildung 2.10) bis zur Lyman $\alpha$-Linie und Linien hochionisierter Atome (z.B. C IV, N V, O VI, Si IV, Al III) im UV. Letztere entstehen bevorzugt in Gebieten hoher Temperatur und Strahlungsdichte, d.h. in der Nähe der Akkretionsregion; ihre Intensität ist daher in IPs oft stark mit der Spinperiode des Weißen Zwergs moduliert. Hingegen hängt die beobachtete Strahlung des Akkretionsstroms hauptsächlich vom Blickwinkel auf den Strom und damit von der Orbitalphase ab. Allerdings kann die Beleuchtung des Akkretionsstroms sowie asymmetrischer Strukturen in der Akkretionsscheibe auch das Auftreten von Modulationen

mit der Schwebungsperiode $P_{\text {beat }}=\left(P_{\text {spin }}^{-1}-P_{\text {orb }}^{-1}\right)^{-1}$ verursachen (vgl. Abschnitt 2.3.3): Die Beleuchtungsstärke und damit die Stärke der angeregten Emission eines Punkts z.B. im Strom variieren mit der Spinphase, der hiervon beobachtbare Anteil kann wiederum mit $P_{\text {orb }}$ moduliert sein.

Aus den Emissionslinien lassen sich zahlreiche Schlüsse auf die physikalischen Bedingungen (z.B. Druck und Temperatur) in den jeweiligen Entstehungsgebieten ziehen. Ihre Radialgeschwindigkeitsvariationen betragen aufgrund der hohen orbitalen Bahngeschwindigkeiten bis zu einigen $100 \mathrm{~km} \mathrm{~s}^{-1}$ (vgl. (2.3)) und enthalten Informationen über den Ort der Entstehung selbst. So schließt man etwa, daß die schmale Komponente der Linien in Abbildung 2.11 in Material bei recht einheitlicher Geschwindigkeit entsteht; die orbitalen Variationen der Radialgeschwindigkeit und der Intensität legen als Quelle die beleuchtete 


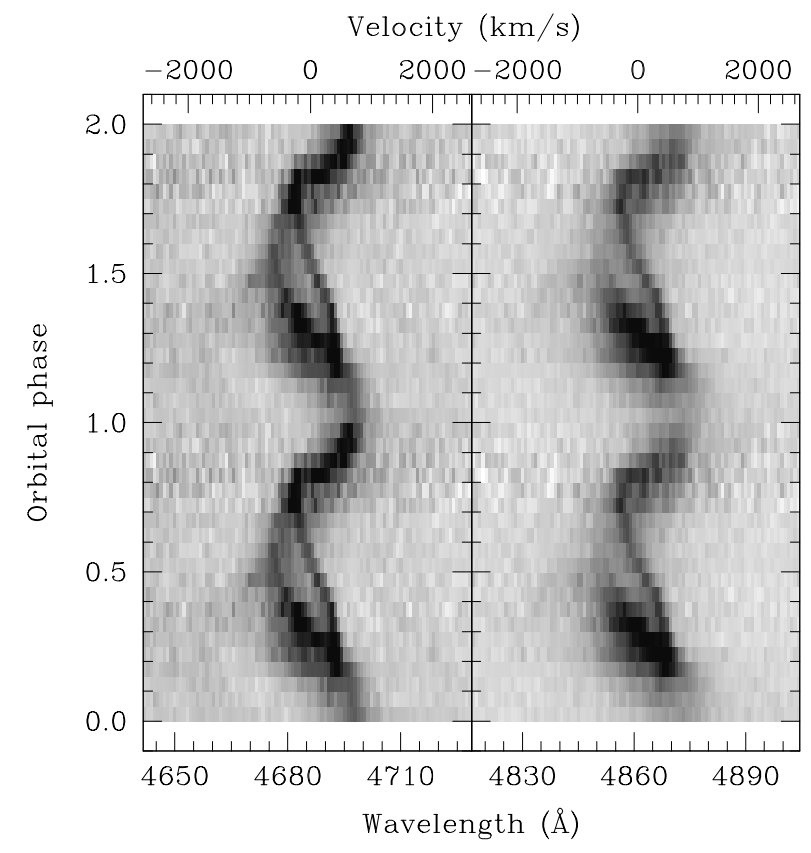

Abbildung 2.11: Orbitalphasenabhängige Variationen von Emissionslinen im Spektrum des Polars RX J1015.5+0904 (aus Burwitz 1997; links: He II 4686; rechts: H $\beta$ ). Die von Bahnphase $\approx 0.2-0.8$ sichtbare scharfe Komponente wird der von der Akkretionsregion beleuchteten Seite des Sekundärsterns zugeschrieben, die breite dem Akkretionsstrom. Bei dieser Interpretation markiert die 'Lücke' in der scharfen Komponente den Zeitpunkt der

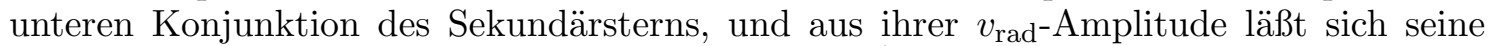
orbitale Bahngeschwindigkeit ablesen (329 $\pm 6 \mathrm{~km} \mathrm{~s}^{-1}$, obere $\mathrm{x}$-Achse).

Hemisphäre des Sekundärsterns nahe. Analog entsteht die breite Komponente in Material mit einer großen Geschwindigkeitsbandbreite, wie sie etwa entlang des Akkretionsstroms vorkommt; diese Vorstellung wird durch die Modulation der Intensität (beste Sichtbarkeit bei $\Phi_{\text {orb }} \approx 0.3$ und $\Phi_{\text {orb }} \approx 0.8$, d.h. bei annähernd senkrechter Sicht auf den Strom, vgl. Abbildung 2.6) und die Amplitude der Radialgeschwindigkeitsmodulation gestützt. Linien aus der Akkretionssäule nahe am Weißen Zwerg können auch Variationen von $v_{\text {rad }}$ in der Größenordnung von $v_{\text {ff }}$ zeigen, die allerdings stark vom Blickwinkel zur Akkretionssäule und vom Abstand des Entstehungsorts vom Weißen Zwerg abhängig sind $\left(v_{\mathrm{ff}}=v_{\mathrm{ff}}(R)\right)$. Insgesamt sind die Linienprofile oft sehr komplex (s. Abbildung 2.11). Damit ist der Informationsgehalt hoch, die Interpretation jedoch oft schwierig.

\subsubsection{Photosphäre des Sekundärsterns}

Da der Sekundärstern ein später Hauptreihenstern mit $T_{\text {eff }} \lesssim 4000 \mathrm{~K}$ ist, emittiert seine Photosphäre hauptsächlich im IR jenseits von $\approx 10000 \AA$. Charakteristische Eigenschaften seines Spektrums sind Absorptionsbanden von $\mathrm{TiO}, \mathrm{VO}, \mathrm{CO}$ und $\mathrm{H}_{2} \mathrm{O}$ sowie Absorptionslinien unionisierter Metallatome (z.B. Ca I-Linien, Na I-Dupletts). Sofern der 
Sekundärstern nicht von anderen Komponenten des Systems überstrahlt wird, können die genannten Merkmale dazu benutzt werden, seinen Spektraltyp, seinen Beitrag zum Fluß und daraus die ansonsten schwierig zu messende Entfernung des Systems zu bestimmen (z.B. Dhillon \& Marsh 1995; Beuermann 2000a; s.a. Abbildung 2.10). Dasselbe gilt für die ellipsoidale Modulation (Abschnitt 2.2.2), der allerdings noch eine Modulation mit der einfachen Orbitalperiode überlagert sein kann, die durch die Beleuchtung der dem Weißen Zwerg zugewandten Hemisphäre verursacht wird (s.o.).

\subsubsection{Einfluß von $B$ und $\dot{M}$ auf das Spektrum}

Gewisse Eigenschaften der Spektren und des Heizungsmechanismus magnetischer CVs hängen wesentlich von der Magnetfeldstärke $B$ des Weißen Zwergs und der Akkretionsrate $\dot{M}$ (bzw. genauer: der Massenflußdichte $\dot{m} \equiv \dot{M} / A, A=$ Fläche des Akkretionsflecks) ab.

- Bei niedrigen Massenflußdichten $\left(\dot{m} \lesssim 0.1 \mathrm{~g} \mathrm{~cm}^{-2} \mathrm{~s}^{-1}\right)$ kühlen die einfallenden Protonen durch Coulomb-Stöße innerhalb einer mittleren freien Weglänge ab. (Die kinetische Energie der einfallenden Elektronen fällt wegen ihrer viel geringeren Masse nicht ins Gewicht.) Statt eine Stoßfront auszubilden, diffundieren die Teilchen direkt in die äußere Photosphäre des Weißen Zwergs hinein ('bombardment solution', Kuijpers \& Pringle 1982), die dabei lediglich auf $\approx 10^{7} \mathrm{~K}$ aufgeheizt wird. Unter diesen Bedingungen ist Kühlung durch Zyklotronstrahlung weitaus effektiver als durch Bremsstrahlung. Strahlungstransport, Temperaturstruktur und emittierte Spektren solcher partikelgeheizter Atmosphären in Abhängigkeit von Akkretionsrate, Magnetfeld und Masse des Weißen Zwergs wurden von Woelk \& Beuermann $(1992,1993)$ numerisch beschrieben.

- Im Bereich $\dot{m} \approx 0.1 \ldots 10 \mathrm{~g} \mathrm{~cm}^{-2} \mathrm{~s}^{-1}$ bildet sich - wie oben beschrieben - über der Oberfläche des Weißen Zwergs ein starker hydrodynamischer Stoß aus, in dem die kinetische Energie der Protonen thermalisiert und als Zyklotron- und thermische Bremsstrahlung abgestrahlt wird. Die Höhe $h$ der Stoßfront über der Oberfläche und das Verhältnis $F_{\mathrm{cyc}} / F_{\mathrm{br}}$ von Zyklotron- und Bremsstrahlungsfluß hängen jedoch sowohl von $\dot{m}$ als auch von $B$ ab: - Mit wachsendem $B$ wird Kühlung durch Zyklotronstrahlung effektiver, wodurch die Maximaltemperatur des Materials in der Region hinter der Stoßfront sinkt. Dadurch wird zum einen Bremsstrahlung als Kühlungsmechanismus ineffektiver, d.h. $F_{\text {cyc }} / F_{\text {br }}$ steigt. (Indirekt wird also Bremsstrahlung durch starke Magnetfelder unterdrückt.) Zum anderen hat der geringere Druck hinter der Stoßfront eine geringere Höhe derselben zur Folge.

- Mit wachsendem $\dot{m}$ wächst der Staudruck der Akkretionssäule, was ebenfalls $h$ verringert; gleichzeitig erhöht sich die Temperatur im Gebiet des Stoßes und damit die Effektivität der Kühlung durch Bremsstrahlung, d.h. $F_{\mathrm{cyc}} / F_{\mathrm{br}}$ sinkt. Die Höhe der Stoßfront ist demnach bei niedrigen Akkretionsraten und kleinen Feldstärken am größten und kann bis $\mathrm{zu} \approx 0.1 R_{\mathrm{WD}}$ betragen (Beuermann \& Woelk 1996). Der Vollständigkeit halber sei darauf hingewiesen, daß $\dot{m}$ und $B$ nicht unabhängig voneinander sind: Bei gegebenem $\dot{M}$ hängt die Größe des Akkretionsflecks und damit $\dot{m}$ auch von $B$ ab. Für ein konkretes System ist allerdings $B$ gegeben und $\dot{m}$ dann im wesentlichen nur noch von $\dot{M}$ abhängig. Eine numerische Behandlung des Strahlungstransports durch einen starken hydrodynamischen 
Stoß hindurch findet sich bei Woelk \& Beuermann (1996), eine weitergehende Diskussion der Stoßfronthöhe bei Beuermann \& Woelk (1996), Beuermann (1997) und Fischer \& Beuermann (2000).

- Bei hohen Massenflußdichten $\left(\dot{m} \gtrsim 10 \mathrm{~g} \mathrm{~cm}^{-2} \mathrm{~s}^{-1}\right)$ treibt der Staudruck der Akkretionssäule die Stoßfront tief in die Photosphäre des Weißen Zwergs hinein ('accretion piston'). Die vom derart 'vergrabenen' Stoß produzierte Bremsstrahlung wird dann bereits tief im Inneren der Atmosphäre reprozessiert und tritt an der Oberfläche der Akkretionsregion als EUV- oder weiche Röntgenstrahlung aus. Dieser Mechanismus wurde bereits von Kuijpers \& Pringle (1982) als Lösung für das 'soft X-ray puzzle' vorgeschlagen (s. Abschnitt 2.4.2.4; s.a. Morfill et al. 1984 zu ähnlichen Phänomenen bei akkretierenden Neutronensternen). Eine Variation des Themas besteht darin, daß dichtere Materieklumpen ('blobs') eine über dem Weißen Zwerg stehende Stoßfront 'durchschlagen' und tief in die Atmosphäre eindringen können ('blobby accretion', s. Abbildung 2.8). Eine konsistente zeitabhängige numerische Simulation steht aber noch aus, mit Ausnahme einer näherungsweisen Lösung im Rahmen der Theorie der Wärmeleitung (Litchfield \& King 1990).

Beobachtungen legen nahe, daß $\dot{m}$ über die gesamte Akkretionsregion betrachtet um bis zu mehrere Größenordnungen variieren kann (z.B. Rousseau et al. 1996) und zudem durch kleinräumige Dichtevariationen zeitlichen Schwankungen auf Skalen bis hinunter in den Millisekundenbereich unterliegt, mit entsprechenden Auswirkungen auf Zyklotron- und Bremsstrahlung. Da die Stoßfronthöhe auch die Größe und Form der beleuchteten Fläche des Weißen Zwergs bestimmt, sind auch die Temperatur der im weiteren Umkreis geheizten Photosphäre des Weißen Zwergs (s. Abschnitt 2.4.2.5) und das von ihr emittierte Spektrum ebenfalls von $\dot{M}$ und $B$ abhängig. Letztlich hängt die genaue Form des Gesamtspektrums dadurch sehr stark von den individuellen Gegebenheiten jedes Systems ab und läßt sich nicht auf einfache Weise (etwa aus $\dot{M}$ und $B$ ) vorhersagen. Umgekehrt eröffnet dies aber die Möglichkeit, aus dem Spektrum und seiner zeitlichen Variation (etwa mit der Spinund/oder Orbitalphase oder bei Variation von $\dot{M}$ ) Informationen über ebendiese Gegebenheiten zu gewinnen.

\subsection{Aspekte der Evolution von CVs}

In diesem Abschnitt werden auch einige Aspekte der Entwicklung von CVs zur Sprache kommen, die für diese Arbeit nicht direkt relevant sind, jedoch der Abrundung des Verständnisses dieser Systeme dienen und für die Einordnung der Arbeit in den Gesamtkontext nützlich sind. Für eine vertiefte Diskussion der Evolution von CVs sei auf die Literatur verwiesen (z.B. King 1988; Kolb 1995; Kolb 1996).

\subsubsection{Entstehung}

CVs entwickeln sich aus Doppelsternsystemen, die aus einem massearmen Hauptreihenstern $\left(M_{\mathrm{sec}} \approx 0.1 \ldots 1.5 M_{\odot}\right)$ und einem massereicheren Primärstern $\left(M_{\text {prim }} \approx 1 \ldots 10 M_{\odot}\right)$ bestehen und Orbitalperioden $P_{\text {orb }} \gtrsim 10 \mathrm{~d}$ haben. (Masseärmere Primärsterne entwickeln sich 
nicht schnell genug zu Weißen Zwergen, massereichere entwickeln sich zwar schnell genug, enden aber als Neutronensterne oder Schwarze Löcher; in den letztgenannten Fällen kann ein Röntgendoppelstern entstehen.) Der Primärstern entwickelt sich auf seiner nuklearen Zeitskala

$$
\tau_{\text {nuc }} \approx 10^{10} \mathrm{yr}\left(\frac{M}{M_{\odot}}\right)^{-2}
$$

und wird schließlich zum Roten Riesen. Sobald er sein Roche-Volumen ausfüllt, setzt ein massiver, dynamisch instabiler Massentransfer ein, der schließlich zu einer ausgedehnten Hülle führt, in der beide Sterne eingehüllt sind und ihre Orbitalbewegung ausführen (common envelope phase). Die Reibung, die der Sekundärstern auf seiner Bahn durch die Hülle des Primärsterns erfährt, führt zur Übertragung von Drehimpuls aus der Bahnbewegung auf die Hülle, d.h. Bahnradius und Bahnperiode sinken. Schließlich wird die Hülle als planetarischer Nebel abgestoßen. Falls sich der Sekundärstern und der Kern des Primärsterns (der praktisch schon ein Weißer Zwerg ist) bei diesem Prozess nicht so nahe kommen, daß sie miteinander verschmelzen, ist das Resultat dieser Entwicklung ein enges Doppelsternsystem mit $P_{\text {orb }} \lesssim 1 \mathrm{~d}$, das aber noch getrennt ist, ein sogenannter Prä-CV. (Prä-CVs verraten sich dadurch, daß sie in ihren Spektren in den Zentren der breiten Absorptionslinien, die vom Weißen Zwerg stammen, schmale Emissionslinien haben, die auf der beleuchteten Seite des Sekundärsterns angeregt werden. Auch beobachtet man im Sichtbaren und IR das Spektrum des Sekundärsterns.) Damit Massentransfer auf den Weißen Zwerg eintreten und das System somit endlich ein CV werden kann, muß der Bahnabstand weiter reduziert werden, bis der Sekundärstern sein Roche-Volumen ausfüllt (Abschnitt 2.1).

Im Prinzip kann auch die Expansion des Sekundärsterns, die mit seiner nuklearen Entwicklung einhergeht, zum Ausfüllen seines Roche-Volumens und zum Einsetzen des Massentransfers führen. Die nukleare Entwicklungszeitskala für Sterne mit $M \lesssim 1.5 M_{\odot}$ ist jedoch so lang, daß dieser Mechanismus nur für wenige CVs eine Rolle spielen sollte (vgl. (2.41)). Sie ist jedoch bei (anfänglichen) Massen von $\gtrsim 1 M_{\odot}$ hinreichend kurz, daß der Stern bereits in gewissem Ausmaß nuklear entwickelt sein kann, bevor Massentransfer erstmalig einsetzt und der Stern sich in der Folge zu kleineren Massen hin entwickelt. Dies erklärt den scheinbaren Widerspruch, daß z.B. ein Sekundärstern mit einer (heutigen) Masse von $0.3 M_{\odot}$ trotz seiner (heute) sehr langen Entwicklungszeitskala von $\approx 10^{11} \mathrm{yr}-$ ein Mehrfaches des Alters des Universums - schon deutliche Anzeichen nuklearer Entwicklung wie z.B. Expansion zeigen kann.

\subsubsection{Drehimpulsverlust und Orbitalperiode}

Es sind zwei Mechanismen bekannt, die Drehimpuls aus dem System abführen und damit den Bahnabstand des Systems verringern können:

(1) ein Wind, der vom magnetisch aktiven Sekundärstern ausgeht, bis zu dessen AlfvénRadius mit ihm korotiert, schließlich aus dem System entweicht und seinen Drehimpuls dabei mitnimmt (Verbunt \& Zwaan 1981). Bei Einzelsternen wie der Sonne, deren Wind sich ebenso verhält, führt dieser Mechanismus allerdings zur Verringerung der Eigenrotation des 
Sterns. In CVs ist die Eigenrotation des Sekundärsterns durch die starken Gezeitenkräfte, die der Weiße Zwerg ausübt, mit der Orbitalperiode synchronisiert (vgl. die Verhältnisse im Erde-Mond-System), so daß der abgeführte Drehimpuls aus der Bahnbewegung entnommen wird.

(2) Emission von Gravitationsstrahlung (z.B. Kraft et al. 1962; Krzeminski \& Kraft 1964). Dies ist ein allgemein-relativistischer Effekt: Beschleunigte Massen emittieren Gravitationsstrahlung in Analogie zu beschleunigten elektrischen Ladungen, die elektromagnetische Strahlung (Bremsstrahlung) emittieren.

Die Zeitskalen $\tau_{\mathrm{MWB}}$ bzw. $\tau_{\mathrm{GR}}$, auf denen diese Mechanismen den gesamten Bahndrehimpuls $J$ des Systems abführen könnten, lassen sich über

$$
\begin{aligned}
\tau_{\mathrm{MWB}} & \equiv \frac{J}{\dot{J}_{\mathrm{MWB}}}=2.2 \cdot 10^{9} \mathrm{yr}\left(\frac{M_{\mathrm{WD}}}{M_{\odot}}\right)\left(\frac{M_{\mathrm{WD}}+M_{2}}{M_{\odot}}\right)^{-\frac{1}{3}}\left(\frac{R_{2}}{R_{\odot}}\right)^{-4}\left(\frac{P_{\mathrm{orb}}}{1 \mathrm{~d}}\right)^{\frac{8}{3}} \\
\tau_{\mathrm{GR}} & \equiv \frac{J}{\dot{J}_{\mathrm{GR}}}=3.8 \cdot 10^{11} \mathrm{yr}\left(\frac{M_{\mathrm{WD}}+M_{2}}{M_{\odot}}\right)^{\frac{1}{3}}\left(\frac{M_{\mathrm{WD}} M_{2}}{M_{\odot}^{2}}\right)^{-1}\left(\frac{P_{\mathrm{orb}}}{1 \mathrm{~d}}\right)^{\frac{10}{3}}
\end{aligned}
$$

abschätzen (z.B. Kolb \& Stehle 1996). Für CV-typische Verhältnisse ist stets $\tau_{\mathrm{GR}} \gg \tau_{\mathrm{MWB}}$, so daß zunächst Bremsung durch einen magnetischen Wind dominiert. Bei $P_{\text {orb }} \approx 10 \mathrm{~h}$ setzt Massentransfer ein (der den Sekundärstern leicht aus dem thermischen Gleichgewicht treibt), und das System tritt als CV in Erscheinung.

Bei Erreichen von $P_{\text {orb }} \approx 3 \mathrm{~h}$ nach $\approx 10^{8}$ Jahren ist die Masse des Sekundärsterns auf einen Wert gesunken $\left(M_{2} \approx 0.2 M_{\odot}\right)$, bei der er voll konvektiv wird. Nach gängigen, aber nicht endgültig akzeptierten Vorstellungen büßt er damit seine magnetische Aktivität ein. Die Abbremsung durch den Wind und damit der Massentransfer kommen zum Erliegen, der Sekundärstern findet zum thermischen Gleichgewicht zurück und kontrahiert dabei leicht. In der Folge ist das System wieder getrennt und mangels Massentransfer unbeobachtbar, verliert aber weiter Drehimpuls durch Gravitationsstrahlung und entwickelt sich weiter hin zu kürzeren Orbitalperioden.

Nach $\approx 10^{9}$ Jahren ist das System bei $P_{\text {orb }} \approx 2 \mathrm{~h}$ so eng geworden, daß der Sekundärstern sein Roche-Volumen wieder ausfüllt und Akkretion wieder einsetzt, deren Antrieb jetzt aber die weitaus schwächere Abbremsung durch Gravitationsstrahlung ist. Dieses als disrupted braking model bekannte Szenario (z.B. Rappaport et al. 1983; Verbunt 1984; McDermott \& Taam 1989) erklärt relativ zwanglos die beobachtete 'Lücke' in der Verteilung der Bahnperioden von CVs im Bereich 2...3 h (Abbildung 2.3). Da die Akkretionsrate durch den Drehimpulsverlust bestimmt ist und Gravitationsstrahlung der langsamere Mechanismus ist, sind die Akkretionsraten unterhalb der Periodenlücke kleiner $\left(\approx 10^{-11} \ldots 10^{-10} M_{\odot} \mathrm{yr}^{-1}\right)$ als oberhalb $\left(\approx 10^{-9} \ldots 10^{-8} M_{\odot} \mathrm{yr}^{-1}\right)$. Nach Patterson (1984) gilt beobachtungsseitig für den gesamten Periodenbereich im Mittel (aber mit großen Schwankungen)

$$
\dot{M}=5.1 \cdot 10^{-10} M_{\odot} \mathrm{yr}^{-1}\left(\frac{P_{\text {orb }}}{4 \mathrm{~h}}\right)^{3.2} .
$$

Nach einigen weiteren $10^{9}$ Jahren ist die Masse des Sekundärsterns auf $\approx 0.1 M_{\odot}$ gesunken, und seine Materie beginnt zunehmend zu entarten. Als Konsequenz ändert sich 
seine Masse-Radius-Beziehung allmählich von $R_{2} \sim M_{2}^{0.8}$ vor Beginn der Entartung (Abschnitt 2.2.2) auf $R_{2} \sim M_{2}^{-1 / 3}$ bei vollständiger (nichtrelativistischer) Entartung, so daß sein Radius mit sinkender Masse wächst. Da er weiter sein Roche-Volumen exakt ausfüllen muß und dessen Volumenradius $R_{V}$ proportional zu $M_{2}^{1 / 3} P_{\text {orb }}^{2 / 3}$ ist (s. (2.10)), muß dann bei weiter sinkender Masse die Orbitalperiode wieder ansteigen. Dieses an sich paradoxe Ergebnis kann man sich veranschaulichen, wenn man sich die Akkretion als zweistufigen Prozeß vorstellt: (1) Massentransfer auf den Weißen Zwerg beim Bahnradius $a_{0}$ unter Drehimpulserhaltung führt wegen $M_{2}<M_{\mathrm{WD}}$ zum neuen Bahnradius $a_{1}>a_{0}$, das System trennt sich. (2) Drehimpulsverlust durch Bremsung verringert den Bahnradius wieder bis auf den endgültigen Wert $a_{2}$, bei dem das System wieder in Kontakt tritt. Abhängig davon, wie der Radius $R_{2}$ des Sekundärstern auf den Massenverlust reagiert, ist $a_{2}<a_{0} \Rightarrow P_{\text {orb }, 2}<P_{\text {orb }, 0}$ (im nichtentarteten Fall) oder $a_{2}>a_{0} \Rightarrow P_{\text {orb }, 2}>P_{\text {orb }, 0}$ (im entarteten Fall). In Wirklichkeit ist der Prozeß natürlich kontinuierlich.

Numerisch findet man die minimal mögliche Periode bei $P_{\min } \approx 67$ min (Kolb \& Baraffe 1999), was allerdings nicht ganz zum beobachteten Wert von $\approx 78$ min paßt $(\mathrm{Ab}-$ bildung 2.3); der Sekundärstern hat dann eine Masse von $\approx 0.06 M_{\odot}$. Er ist infolge des Erlöschens des Wasserstoffbrennens bei $\approx 0.08 M_{\odot}$ inzwischen zum Braunen Zwerg geworden, der aber sehr wohl noch Masse transferieren und den CV 'füttern' kann. Zur Zeit wird intensiv nach CVs mit einem Braunen Zwerg als Massespender gesucht. Derzeit gibt es jedoch nur zwei ziemlich gute Kandidaten für solche Systeme (V 592 Her, van Teeseling et al. 1999; EF Eri, Beuermann et al. 2000), was aber angesichts der Schwierigkeiten, sie in den Spektren zu finden (extrem geringe Leuchtkraft), auch nicht allzu verwunderlich ist. Man kann aber unter gewissen Umständen aus der Not eine Tugend machen und gerade aus dem Fehlen jeglicher Signaturen des Sekundärsterns eines Systems nahe dem Periodenminimum auf einen Braunen Zwerg schließen, so geschehen im Fall von EF Eri.

\subsubsection{Spin-Bahn-Synchronisation in Polaren}

Das definierende Merkmal eines Polars ist die bahnsynchrone Rotation des Weißen Zwergs (Abschnitt 2.3.2). Abbildung 2.3 zeigt, daß die meisten Polare Systeme mit $P_{\text {orb }} \lesssim 4 \mathrm{~h}$ sind und die meisten IPs $P_{\text {orb }} \gtrsim 3 \mathrm{~h}$ haben. Da $P_{\text {orb }}$ im Laufe der Zeit abnimmt (s. Abschnitt 2.5.2), suggeriert dies einen Entwicklungseffekt: Magnetische Systeme könnten demnach als IPs mit $P_{\text {spin }} \ll P_{\text {orb }}$ entstehen und im Laufe ihrer Entwicklung durch Abbremsung der Eigenrotation des Weißen Zwergs synchronisieren. Gegen eine solche Vorstellung spricht allerdings, daß die Magnetfeldstärken von IPs - sofern sie überhaupt meßbar sind - sehr viel schwächer als diejenigen typischer Polare sind (z.B. Beuermann 1997). Umgekehrt wird aber ein Polar vor Eintritt der Synchronisation für eine gewisse Zeit als IP erschienen sein.

Damit ein System synchronisieren kann, muß dem Drehmoment $G_{\text {acc }}$, das die akkretierte Materie bewirkt und das die Eigenrotation des Weißen Zwergs (wie in den nichtmagnetischen $\mathrm{CVs}$ ) weiter beschleunigen würde (spin-up; s. Abschnitt 2.5.4), ein größeres synchronisierendes Drehmonent $G_{\text {sync }}$ gegenüberstehen. Ein einfaches Modell, das ein solches Drehmoment liefert, beruht auf der magnetostatischen Wechselwirkung zwischen dem magnetischen Moment des Sekundärsterns (vgl. Abschnitt 2.2.2) und dem des Weißen 
Zwergs (Campbell 1985, 1989, 1990). Eine grobe Abschätzung zeigt, daß Synchronisation in der Nähe derjenigen Periode $P_{\text {orb }}$ stattfindet, für die $B_{\mathrm{WD}} \approx 18 \mathrm{MG}\left(P_{\text {orb }} / 4 \mathrm{~h}\right)^{2.7}$ gilt; diejenigen Systeme, für die die magnetische Feldstärke $B_{\mathrm{WD}}$ des Weißen Zwergs gemessen werden konnte, scheinen dies zu bestätigen (Beuermann 1997).

Bei hinreichender Dichte des Plasmas zwischen den beiden Partnern erwartet man weiterhin ein magneto-hydrodynamisches Drehmoment. Es entsteht dadurch, daß asynchrone Rotation des Weißen Zwergs die magnetischen Feldlinien, an denen das Plasma angekoppelt ist, quasi 'aufwickelt' (Lamb et al. 1983; Lamb \& Melia 1988). Da dieser Mechanismus nur funktioniert, solange $P_{\text {spin }} \neq P_{\text {orb }}$ ist, kann er zwar Synchronisation herbeiführen, nicht aber erhalten; dies wird dann nach erfolgter Synchronisation von der magnetostatischen Wechselwirkung geleistet.

\subsubsection{Spinperiodenentwicklung in IPs}

Bei der Akkretion wird neben der Masse $M$ der Materie auch Drehimpuls auf den Weißen Zwerg übertragen. Der akkretierte Drehimpuls $J_{\text {acc }}$ ist bei Polaren durch den Bahndrehimpuls des Materials am $L_{1}$-Punkt bestimmt, d.h. $\dot{J}_{\text {acc }}=\dot{M} R_{L_{1}} v_{\text {orb }}\left(R_{L_{1}}\right)$ (vgl. (2.6), (2.30)). Bei nichtmagnetischen CVs und IPs verliert die Materie einen Teil ihres Drehimpulses in der Akkretionsscheibe (Abschnitt 2.2.4). In diesen Systemen ist $\dot{J}_{\text {acc }}$ daher durch den Drehimpuls bestimmt, den die Materie bei demjenigen Radius $r$ hat, an dem sie an den Weißen Zwerg ankoppelt, d.h.

$$
\dot{J}_{\mathrm{acc}}=\dot{M} r v_{\mathrm{kep}}(r)=\dot{M} \sqrt{r G M_{\mathrm{WD}}} .
$$

Bei nichtmagnetischen CVs ist $r$ der Radius $r_{\mathrm{BL}}$ des Außenrands der Grenzschicht zwischen Akkretionsscheibe und Weißem Zwerg (s. Abschnitt 2.3.1), bei IPs der Alfvén-Radius $r_{\mu}$ (vgl. (2.35)). In allen Fällen vergrößert sich der Eigendrehimpuls

$$
J_{\text {spin }}=\Theta \omega_{\text {spin }}=\frac{2 \pi \Theta}{P_{\text {spin }}}
$$

des Weißen Zwergs um den akkretierten Betrag, wobei

$$
\Theta=\zeta \cdot \frac{2}{5} M_{\mathrm{WD}} R_{\mathrm{WD}}^{2}
$$

sein Trägheitsmoment ist. Der zweite Teil der rechten Seite von (2.47) ist das Trägheitsmoment einer homogenen Kugel. Der Korrekturfaktor $\zeta$ berücksichtigt die zum Zentrum hin zunehmende Dichte des Weißen Zwergs. Er ist nach Ritter (1985) durch

$$
\zeta=\frac{5}{2}\left[0.452+0.0853 \log \left(1-\frac{M_{\mathrm{WD}}}{M_{\mathrm{Ch}}}\right)\right]^{2}
$$

gegeben $\left(M_{\mathrm{Ch}}=1.44 M_{\odot}\right.$, Chandrasekhar-Grenzmasse $)$ und hat bei $M_{\mathrm{WD}}=0.4,1.0$ bzw. $1.3 M_{\odot}$ die Werte $0.484,0.416$, bzw. 0.334 . Aus $\dot{J}_{\text {acc }}=\dot{J}_{\text {spin }}$ ergibt sich speziell für IPs 
aus den obigen drei Gleichungen eine Beziehung zwischen der Akkretionsrate $\dot{M}$ und der Abnahme der Spinperiode $P_{\text {spin }}$ :

$$
\begin{aligned}
\dot{M} & =-\frac{4 \pi \zeta}{5} R_{\mathrm{WD}}^{2} \sqrt{\frac{M_{\mathrm{WD}}}{G r_{\mu}}} \frac{\dot{P}_{\mathrm{spin}}}{P_{\mathrm{spin}}^{2}} \\
& =4.58 \cdot 10^{19} \mathrm{~g} \mathrm{~s}^{-1} \zeta\left(\frac{R_{\mathrm{WD}}}{10^{9} \mathrm{~cm}}\right)^{2}\left(\frac{M_{\mathrm{WD}}}{M_{\odot}}\right)^{\frac{1}{2}}\left(\frac{r_{\mu}}{R_{\odot}}\right)^{-\frac{1}{2}}\left(\frac{P_{\mathrm{spin}}}{1 \mathrm{~min}}\right)^{-2}\left(\frac{\dot{P}_{\mathrm{spin}}}{10^{-10} \mathrm{~s} \mathrm{~s}^{-1}}\right)
\end{aligned}
$$

Diese Beziehung zeigt zum einen, daß Akkretion ein Drehmoment erzeugt, das zu einer Abnahme der Spinperiode führt. Zum anderen erlaubt sie zumindest prinzipiell, bei bekanntem $M_{\mathrm{WD}}$ (und damit im wesentlichen auch $R_{\mathrm{WD}}$, s. (2.11)) die Akkretionsrate $\dot{M}$ unabhängig von der angenommenen Entfernung des Systems aus der Abnahme der Spinperiode zu bestimmen. (Andere Methoden basieren meist auf der Abschätzung der Gesamtleuchtkraft $L_{\text {bol }}$ aus dem beobachteten Röntgenfluß $f_{\mathrm{X}}$ und der Beziehung (2.36) und sind damit wegen $\dot{M} \sim L_{\text {bol }} \sim d^{2} f_{\mathrm{X}}$ entfernungsabhängig.)

In der Praxis ergeben sich Unsicherheiten z.B. daraus, daß $r_{\mu}-$ d.h. der Radius des Innenrands der Akkretionsscheibe - meist nicht gut bestimmt ist. Weiterhin tritt in den Fällen, in denen der Außenradius der Scheibe größer als der sog. Korotationsradius des Weißen Zwergs $r_{\text {cor }}$ ist, bei dem $\omega_{\text {kep }}=\omega_{\text {spin }}$ gilt, zusätzlich zum beschleunigenden Akkretionsdrehmoment ein Drehmoment auf, das die Eigenrotation des Weißen Zwergs zu ver-

langsamen sucht. (Außerhalb des Korotationsradius ist $\omega_{\text {spin }}>\omega_{\text {kep }}(R)$; der Weiße Zwerg zerrt also gewissermaßen seine Magnetfeldlinien über das langsamer rotierende Material der äußeren Scheibe hinweg, wodurch dieses beschleunigt und er selbst abgebremst wird.) Je größere Teile der Akkretionsscheibe außerhalb des Korotationsradius liegen, d.h. je größer ihr Außenradius und/oder je kleiner die Spinperiode des Weißen Zwergs ist, desto stärker ist also die Abbremsung. Das Verhältnis beider Drehmomente entscheidet letztlich darüber, ob der Weiße Zwerg einen spin-up oder einen spin-down erfährt. Da die obige einfache Ableitung kein abbremsendes Drehmoment berücksichtigt, wird (2.49) in den Fällen, in denen ein solches vorhanden ist, zu kleine Akkretionsraten liefern. Für eine detaillierte Diskussion des Themas sei z.B. auf Ritter (1985) verwiesen.

\subsubsection{Weitere Entwicklung des Weißen Zwergs}

\subsubsection{Massenentwicklung}

Bei typischen Akkretionsraten wird während der Entwicklungszeit eines CVs eine beträchtliche Menge Material (einige Zehntel der Sonnenmasse) auf der Oberfläche des Weißen Zwergs abgelagert, das im wesentlichen aus nuklear unverbranntem Wasserstoff besteht. Sobald sich eine kritische Menge davon nahe der Oberfläche angesammelt hat, zündet in ihm - wegen der Entartung praktisch bis an die Oberfläche explosives - Wasserstoffbrennen (thermonuclear runaway, TNR). Nach einer solchen Nova-Explosion setzt wieder Akkretion ein, und der Zyklus beginnt von Neuem. Lange Zeit glaubte man, daß nur nichtmagnetische 

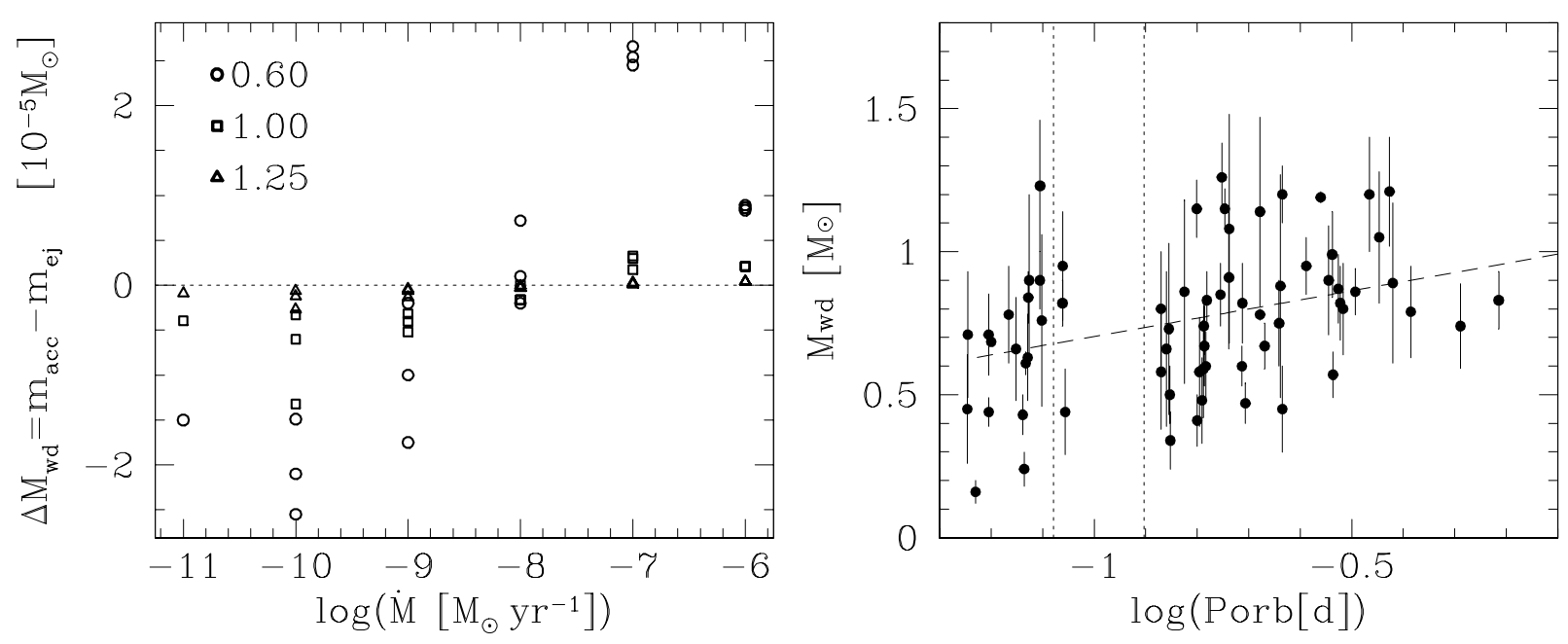

Abbildung 2.12: Links: Mittlerer Netto-Massegewinn $\Delta M_{\mathrm{WD}}$ Weißer Zwerge in CVs in einem Nova-Zyklus (s. Text) in Einheiten von $10^{-5} M_{\odot}$ für drei verschiedene Massen $M_{\mathrm{WD}}$ als Funktion der Akkretionsrate $\dot{M}$ (nach Tabelle 1 aus Prialnik \& Kovetz 1995). Für jede $\left(M_{\mathrm{WD}}, \dot{M}\right)$-Kombination wurden die Temperaturen $T_{\mathrm{eff}}=10000,30000,50000 \mathrm{~K}$ des Weißen Zwergs betrachtet. Rechts: Weiße-Zwerg-Massen der im Katalog von Ritter \& Kolb (1998) aufgeführten CVs als Funktion der Orbitalperiode $P_{\text {orb }}$. Die gepunkteten Senkrechten deuten die Periodenlücke an (vgl. Abbildung 2.3), die gestrichelte Linie stellt eine least-square-Anpassung an die Daten dar $\left(M_{\mathrm{WD}}=1.023+0.32 \log \left(P_{\mathrm{orb}} / 1 \mathrm{~d}\right)\right.$, Gänsicke 1997).

CVs Nova-Ausbrüche haben könnten. Die Nova V1500 Cyg von 1975 war zuvor jedoch vermutlich ein Polar, der allerdings bei dem Ausbruch seine Synchronisation verlor (Stockman et al. 1988), sie aber in $\approx 170 \mathrm{yr}$ wiedererlangt haben wird (Schmidt et al. 1995).

Die für eine Nova-Explosion erforderliche kritische Masse $\Delta M_{\mathrm{H}}$ (und damit auch die dabei freigesetzte Energie) ist sowohl von $M_{\mathrm{WD}}$ als auch von $\dot{M}$ abhängig (Nariai \& Nomoto 1979): Sie sinkt mit wachsendem $M_{\mathrm{WD}}$ (aufgrund der stärkeren Kompression und damit Aufheizung des akkretierten Materials) und steigt mit sinkendem $\dot{M}$ (Wärmeleitung ins Innere des Weißen Zwergs kühlt das akkretierte Material und erlaubt bei geringerem $\dot{M}$ durch längere Akkretionsphasen ein größeres $\Delta M_{\mathrm{H}}$, bevor die Bedingungen für das Zünden des Wasserstoffbrennens erreicht sind). Numerische Modelle ergeben für $M_{\mathrm{WD}}=0.7 \ldots 1.4 M_{\odot}$ und $\dot{M}=10^{-11} \ldots 10^{-7} M_{\odot} \mathrm{yr}^{-1}$ kritische Massen $\Delta M_{\mathrm{H}} \approx 3 \cdot 10^{-7} \ldots 3 \cdot 10^{-4} M_{\odot}$ und damit Zykluszeiten von $\approx 10^{1} \ldots 10^{7}$ yr (Nariai \& Nomoto 1979; Nomoto 1982).

Prinzipiell kann ein akkretierender Weißer Zwerg auf diese Weise schließlich über die Chandrasekhar-Grenzmasse $\left(1.44 M_{\odot}\right)$ getrieben werden. Dies würde eine Supernova-Explosion vom Typ Ia auslösen, bei der der Weiße Zwerg durch eine thermonukleare Explosion des entarteten $\mathrm{CO}-$ Kerns $(\mathrm{CO}=$ Kohlenstoff-Sauerstoff $)$ restlos zerrissen wird. Bei jeder Nova-Explosion wird jedoch auch Masse abgestoßen, so daß die entscheidende Frage ist, ob der Netto-Massegewinn in einem Nova-Zyklus $\Delta M_{\mathrm{WD}} \equiv \Delta M_{\mathrm{acc}}-\Delta M_{\mathrm{ej}} \mathrm{im}$ 
Mittel $>0$ ist $\left(\Delta M_{\mathrm{acc}}=\right.$ akkretierte Masse, $\Delta M_{\mathrm{ej}}=\mathrm{im}$ Novaereignis abgestoßene Masse). Neuere Evolutionsrechnungen über viele Novazyklen für $M_{\mathrm{WD}}=0.65 \ldots 1.4 M_{\odot}$ und $\dot{M}=10^{-11} \ldots 10^{-6} M_{\odot} \mathrm{yr}^{-1}$ von Prialnik \& Kovetz (1995) deuten darauf hin, daß $\Delta M_{\mathrm{WD}}<0$ für $\dot{M} \lesssim 10^{-9} M_{\odot}$ und $\Delta M_{\mathrm{WD}}>0$ für $\dot{M} \gtrsim 10^{-8} M_{\odot}$ ist, wobei massereiche Weiße Zwerge $\left(M_{\mathrm{WD}} \geq 1.25 M_{\odot}\right)$ praktisch keine Masseänderung zeigen (Abbildung 2.12). Demnach muß man für typische CVs $\left(\dot{M}=10^{-11} \ldots 10^{-8} M_{\odot} \mathrm{yr}^{-1}\right)$ wohl davon ausgehen, daß die Masse des Weißen Zwergs im Zuge seiner Entwicklung allmählich abnimmt. Die beobachteten Massen in Abhängigkeit von der Orbitalperiode unterstützen diese Vorstellung (Abbildung 2.12).

Bei hohen Akkretionsraten in einem engen, von $M_{\mathrm{WD}}$ fast unabhängigen Bereich (einige $10^{-7} M_{\odot} \mathrm{yr}^{-1}$ ) kann der akkretierte Wasserstoff auf dem Weißen Zwerg in einer Schicht dicht unter der Oberfläche auch stationär brennen (z.B. Nomoto 1982) und seine Photosphäre auf hohe Temperaturen $\left(10^{5}-10^{6} \mathrm{~K}\right)$ heizen. Dies ist das heute gängige Modell für die mit ROSAT entdeckten sogenannten superweichen Röntgenquellen (supersoft X-ray sources, SUSOs, SSS; neuere Übersichtsartikel z.B. Kahabka \& van den Heuvel 1997, van Teeseling 1998). Da solche Systeme keine Nova-Ausbrüche mit entsprechendem Massenverlust erleiden, sind sie nach heutigem Wissen die wahrscheinlichsten Vorläufersterne der Supernovae vom Typ Ia. Bei noch höheren Akkretionsraten $\left(\dot{M} \gtrsim 10^{-6} M_{\odot} \mathrm{yr}^{-1}\right)$ brennt der Wasserstoff ebenfalls stationär, es bildet sich jedoch eine sehr ausgedehnte Hülle, die das gesamte System einhüllt und wie einen Roten Riesen erscheinen läßt. Auch diese Systeme sind vermutlich SN Ia-Kandidaten.

Abschließend sei bemerkt, daß eine Veränderung der Masse des Weißen Zwergs - so sie denn stattfindet - auch direkte Auswirkungen auf beobachtbare Größen hat. Nach (2.36) und (2.11) steigt die Akkretionsleuchtkraft $L_{\mathrm{acc}}$ mit $M_{\mathrm{WD}}$ an. Die Temperatur in der Region hinter der Stoßfront eines magnetischen CVs und damit die Form des emittierten Spektrums sind nach (2.38) ebenso von $M_{\mathrm{WD}}$ abhängig. Entsprechendes gilt für nichtmagnetische CVs, in denen Temperatur und Leuchtkraft der Grenzschicht (Abschnitt 2.2.4) mit $M_{\mathrm{WD}}$ steigen. In beiden Fällen verändern sich auch Form und Größe der von Beleuchtung betroffenen Gebiete (Akkretionsscheibe und/oder Photosphäre des Weißen Zwergs), was gleichfalls das Spektrum verändert.

\subsubsection{Wärmehaushalt}

Der gesamte Akkretionsprozess in CVs beeinflußt auf diverse Weise auch den Wärmehaushalt des Weißen Zwergs. Einige Aspekte werden hier kurz angesprochen; mangels Relevanz für die vorliegende Arbeit verweise ich für eine umfangreichere Darstellung auf die Literatur (z.B. Gänsicke 1997, 2000, mit Vergleich mit Beobachtungen).

- Wie bereits früher angesprochen wurde (Abschnitt 2.4.2.4), führt Reprozessierung der Strahlung, die bei magnetischen CVs durch den hydrodynamischen Stoß (und bei nichtmagnetischen CVs in der Grenzschicht der Akkretionsscheibe) erzeugt wird, zur Heizung der Photosphäre des Weißen Zwergs.

- Das akkretierte Material wird auf der Hülle des Weißen Zwergs abgelagert. Diese wird dadurch zum einen komprimiert und damit geheizt, zum anderen aber auch tiefer in das Gravitationspotential des Weißen Zwergs hineingedrückt. Dabei wird Gravitationsenergie 


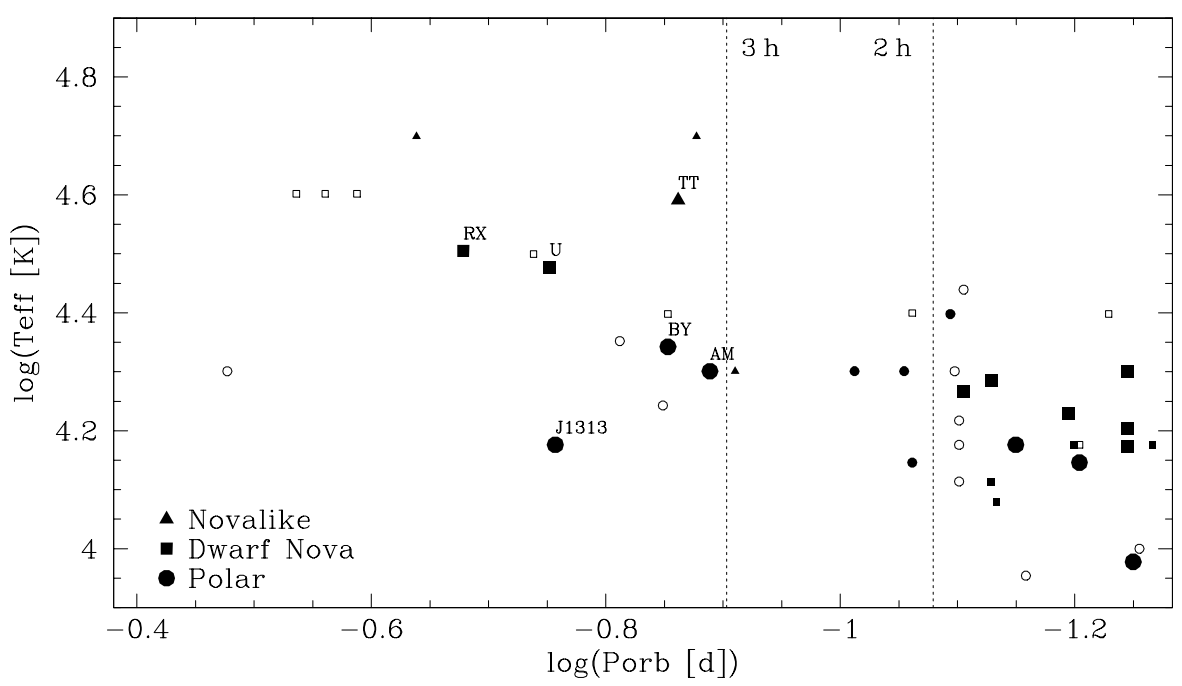

Abbildung 2.13: Photosphärentemperaturen $T_{\text {eff }}$ Weißer Zwerge in CVs gegen die Orbitalperiode $P_{\text {orb }}$ (aus Gänsicke 2000). Ausgefüllte Symbole: Messungen (groß = sicher, klein $=$ unsicher); offene Symbole: Abschätzungen. Tendenziell sinkt $T_{\text {eff }}$ mit kürzer werdendem $P_{\text {orb }}$ (in der Abbildung nach rechts hin), d.h. mit zunehmendem Alter. Die Ursache hierfür sind die dort vorherrschenden geringeren Akkretionsraten (s. Text).

frei, die zu weiterer Heizung führt. Da die bis dicht unter die Oberfläche entartete Materie sehr gut Wärme leitet, gelangt ein nennenswerter Teil der freigesetzten Energie in das Innere des Weißen Zwergs.

- Durch die Zunahme der Masse des Weißen Zwergs kontrahiert dieser adiabatisch (s. (2.11)), was wiederum zur Freisetzung von Gravitationsenergie und Aufheizung des gesamten Sterninneren führt. Umgekehrt führt der Massenverlust bei einem Nova-Ereignis zu adiabatischer Kühlung.

- Nach einem Nova-Ausbruch sind die äußeren Schichten des Weißen Zwergs stark aufgeheizt (einige $10^{5} \mathrm{~K}$ in der Photosphäre) und kühlen im Laufe einiger Jahrzehnte wieder auf die ursprüngliche Temperatur ab. Ein Teil der thermischen Energie wandert auch hier in das Innere des Weißen Zwergs.

- Aus Beobachtungen weiß man, daß bei Zwergnovae-Ausbrüchen (s. Abschnitt 2.2.4) der Weiße Zwerg um einige $1000 \mathrm{~K}$ über seine Ruhephasen-Temperatur aufgeheizt wird. Der Mechanismus, der hierfür verantwortlich ist, ist noch nicht eindeutig bestimmt. Vorgeschlagen wurden u.a.:

- Beleuchtung aus einer heißen $\left(\approx 10^{6} \mathrm{~K}\right)$, optisch dicken Grenzschicht (Pringle 1988). (Ein Zwergnova-Ausbruch hat durch die höhere Viskosität des Materials im Bereich der Heizungswelle eine zeitweilig stark erhöhte radiale Massenflußrate durch die Scheibe zur Folge; dadurch kann z.B. auch eine ansonsten optisch dünne Grenzschicht während eines Ausbruchs optisch dick werden.) Eine neuere Arbeit von Popham (1997) deutet jedoch darauf hin, daß Beleuchtung außer bei massiven Weißen Zwergen vernachlässigbar ist; hingegen kann die direkte Ablagerung des heißen Materials der Grenzschicht auf den Weißen Zwerg zu substantieller Heizung führen. 
- Heizung durch die periodische Kompression, die mit der erhöhten Masseablagerung bei jedem Ausbruch einhergeht (Sion 1995). Sion findet aus Simulationen, daß die Photosphäre während eines Ausbruchs um 5000...10000 K über ihre Gleichgewichtstemperatur geheizt wird und letztere um 10000...20000 K über der Temperatur liegt, die der Weiße Zwerg bei $\dot{M}=0$ hätte.

Aus dem Gesagten wird klar, daß sich das Alter Weißer Zwerge in CVs - im Gegensatz zur Situation bei isolierten Weißen Zwergen - nicht einfach nach (2.17) aus seiner beobachteten Temperatur $T_{\text {eff }}$ bestimmen läßt. Gleichwohl legen die (leider noch nicht allzu zahlreichen) Beobachtungen nahe, daß $T_{\text {eff }}$ im Laufe der Zeit abnimmt (Abbildung 2.13; s.a. Sion (1991)). Es zeichnet sich jedoch ab, daß der entscheidende Faktor die Akkretionsrate ist, die eine minimal mögliche Photosphärentemperatur des Weißen Zwergs festlegt (Gänsicke 2000). In diesem Sinne ist der in Abbildung 2.13 erkennbare Trend dann im wesentlichen ein Spiegelbild der mit $P_{\text {orb }}$ abnehmenden Akkretionsraten, vgl. (2.44). Das Alter von CVs und damit die Längen der einzelnen Evolutionsphasen entziehen sich somit der direkten Beobachtung. 


\section{Kapitel 3}

\section{EX Hya: Der Stand der Dinge}

\subsection{Einführung und Historie}

EX Hya (RA $2000=12^{\mathrm{h}} 52^{\mathrm{m}} 25.5$, DEC $2000=-29^{\circ} 14^{\prime} 58^{\prime \prime}$, Suchkarte s. Abbildung 3.1) ist ein IP mit einer mittleren Helligkeit $V \approx 13$, der im Laufe seiner nun fast 40-jährigen Erforschung immer wieder für Überraschungen gesorgt hat, in einigen Aspekten immer noch Rätsel aufgibt und vermutlich gerade deshalb Astronomen besonders fasziniert.

EX Hya ist in vielerlei Hinsicht ein außergewöhnlicher IP:

- Seine Orbitalperiode ist mit $P_{\text {orb }} \approx 98$ min ungewöhnlich kurz; nur ein weiterer bekannter IP (SW Uma) liegt unterhalb der Periodenlücke von $2 \ldots 3 \mathrm{~h}$ 卢.

- Die Spinperiode von EX Hya ist mit $P_{\text {orb }} \approx 67 \mathrm{~min} \approx(2 / 3) P_{\text {orb }}$ vergleichsweise lang; für die meisten IPs gilt grob $P_{\text {spin }} \approx 0.07 P_{\text {orb }}$ (King \& Wynn 1999; s.a. King 1993, Wynn \& King 1995).

- Außer DQ Her ist EX Hya der einzige bekannte IP, der infolge seiner hohen Inklination Bedeckungen des Weißen Zwergs durch den Sekundärstern zeigt. Dies macht ihn insofern zu einem Glücksfall, als sich dadurch zum einen (zumindest prinzipiell) die Massen beider Komponenten recht gut bestimmen lassen, und zum anderen die Bedeckung von Gebieten sehr dicht am Weißen Zwerg und die damit einhergehenden spektralen Variationen Rückschlüsse auf die Struktur der Akkretionsregion zulassen.

- EX Hya zeigt Zwergnovaausbrüche $(\Delta m \approx 3.5)$, die sehr selten sind und in unregelmäßigen Abständen von einigen Jahren auftreten (15 Ausbrüche in 44 Jahren; letzter Ausbruch im August 1998). Sie sind von vergleichsweise kurzer Dauer (2...3 Tage), und die Anstiegsund Abklingzeiten variieren beträchtlich. (Für einen aktuellen Überblick über die Ausbrüche von EX Hya s. Hellier et al. 2000.) Wegen der jahreszeitlich bedingten zeitweiligen Unbeobachtbarkeit von EX Hya dürften etliche Ausbrüche unbeobachtet geblieben sein, das wahre mittlere Intervall zwischen zwei Ausbrüchen sollte aber immer noch bei $\approx 1.5 \mathrm{yr}$ liegen. Ob die Ausbrüche von EX Hya tatsächlich durch eine thermische Instabilität der Akkretionsscheibe (s. Abschnitt 2.2.4) oder durch eine Instabilität des Massentransfers vom Sekundärstern verursacht werden, ist noch nicht abschließend geklärt (Hellier et al. 2000).

5 Fast alle magnetischen CVs mit $P_{\text {orb }}<3 \mathrm{~h}$ sind Polare, vgl. Abbildung 2.3 und Abschnitt 2.5.3. 


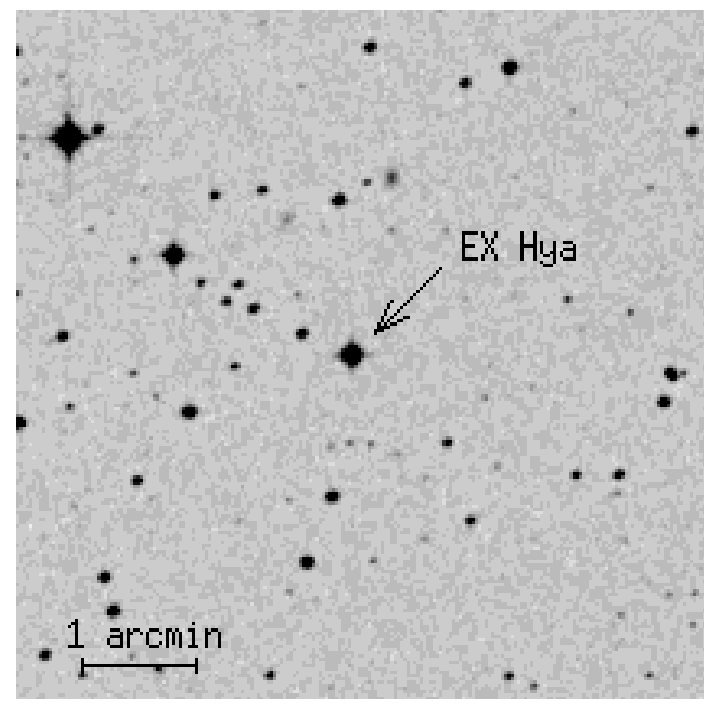

Abbildung 3.1: Suchkarte für EX Hya (RA 2000 $=12^{\mathrm{h}} 52^{\mathrm{m}} 25.5$, DEC $2000=-29^{\circ} 14^{\prime} 58^{\prime \prime}$; Norden ist oben, Osten ist links).

Historisch gesehen war EX Hya zunächst nur als Objekt mit moderaten Helligkeitsausbrüchen ('Zwergnova') und als unregelmäßig angesehenen Helligkeitsvariationen von $\Delta \mathrm{mag} \approx 1$ auf Zeitskalen von $10 \ldots 1000 \mathrm{~s}$ in den Zeiträumen zwischen den Ausbrüchen bekannt. Erst die Entdeckung von Emissionslinien mit zwei Maxima (Kraft 1962) sowie periodischer Bedeckungen in den Lichtkurven (Kraft \& Krzeminski 1962) führte dazu, daß EX Hya als Doppelsternsystem mit einer Orbitalperiode von $P_{\text {orb }} \approx 98$ min erkannt wurde, das fast genau 'von der Seite' gesehen wird und eine Akkretionsscheibe besitzt. Die erste veröffentlichte und lange Zeit benutzte Ephemeride stammt von Mumford (1967). Die nächste wichtige Entdeckung war die Identifikation von EX Hya mit der mit HEAO1 entdeckten Röntgenquelle 4U1249-28 (Schwartz et al. 1979), was auf hochenergetische Prozesse hindeutete. Schließlich fanden Vogt et al. (1980) bei der Analyse optischer Lichtkurven aus 14 Jahren zum einen eine Abnahme der Orbitalperiode, zum anderen aber eine weitere kohärente, grob sinusförmige Modulation mit einer Periode von $\approx 67$ min mit $\Delta \mathrm{mag}=0.4 \ldots 0.9$, die von den Autoren jedoch nicht als Spinperiode des Weißen Zwergs erkannt wurde. Stattdessen nahmen sie einen modulierten Massentransfer als Ursache an, vermutlich aufgrund des 'verführerischen' $\approx 2: 3$-Verhältnisses zur Bahnperiode und der Tatsache, daß in anderen Systemen, in denen die Rotation des Weißen Zwergs als Ursache der zweiten Periode vermutet wurde, diese mit $\approx 30 \ldots 1000$ s sehr viel kürzer war.

Weitere Meilensteine waren der definitive Nachweis von Röntgenemission auch zwischen den Ausbrüchen (Watson et al. 1978, < 2 keV; Córdova \& Riegler 1979, > 2 keV), das Auffinden der 67-Minuten-Modulation auch im weichen Röntgenbereich (Kruszewski et al. 1981), die Entdeckung der Abnahme der Spinperiode (Gilliland 1982; Sterken et al. 1983; später von Jablonski \& Busko (1985) sowie Bond \& Freeth (1988) als stabil bestätigt) und schließlich die Entdeckung einer partiellen Bedeckung im harten Röntgen- 
bereich (Beuermann \& Osborne 1985). Von Warner (1983) kam der Vorschlag, EX Hya und vergleichbare Systeme durch Akkretion auf einen schwach magnetischen, asynchron rotierenden Weißen Zwerg über eine mitrotierende Akkretionssäule zu erklären (intermediate polar model, s. Abschnitt 2.3.3). Nicht zuletzt aufgrund der ausgiebigen Forschung über EX Hya in den darauf folgenden Jahren - EX Hya ist wohl der bestuntersuchte IP, was sich in entsprechend umfangreicher Literatur niedergeschlagen hat - wird dieses Modell heute als allgemein zutreffend betrachtet. Im folgenden gebe ich einen Überblick über den aktuellen Kenntnisstand zu EX Hya.

\subsection{Eigenschaften des Systems}

\subsubsection{Ephemeriden für Orbit und Spin}

Wie oben erwähnt, zeigt EX Hya Bedeckungen durch den Sekundärstern sowie eine Modulation mit der Spinphase mit säkular abnehmender Periode. Die derzeit besten verfügbaren Ephemeriden für den Zeitpunkt TDB $_{\text {ecl }}$ der Mitte der Bedeckung des Weißen Zwergs durch

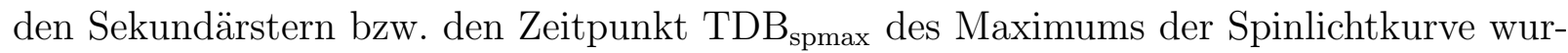
den von Hellier \& Sproats (1992) zu

$$
\begin{aligned}
\mathrm{TDB}_{\text {ecl }}= & 2437699.94152(3)+0.068233846(2) N \\
& +0.00028(4) \sin \left(360^{\circ} \frac{N}{93500 \pm 6500}+(7 \pm 24)^{\circ}\right) \\
\mathrm{TDB}_{\text {spmax }}= & 2437699.8914(5)+0.046546504(9) N-7.9(4) \cdot 10^{-13} N^{2}
\end{aligned}
$$

angegeben (für ganzzahlige $N$ ). (TDB ist die baryzentrische dynamische Zeit, die an die Einheit der Atomzeit angeschlossen und seit 1984 Nachfolger der baryzentrischen Ephemeridenzeit ETB ist, welche an das tropische Jahr 1900 angeschlossen war. Beide unterscheiden sich von der Weltzeit UT um einen Betrag der Größenordnung 1 min, der nur im Nachhinein bestimmbar ist und bei Bedarf z.B. dem Astronomical Almanach entnommen werden kann.) Der quadratische Term der Spinephemeride entspricht $\dot{P}_{\text {spin }}=-3.4 \cdot 10^{-11}$. Der Sinus-Term in der Orbitalephemeride impliziert eine Variation um \pm 0.004 Zyklen mit einer Periode von $\approx 17.5 \mathrm{yr}$, ist aber nicht allgemein anerkannt. Die Zeitpunkte der optischen Bedeckungen wandern abhängig von der Spinphase um ca. \pm 20 s um den nach (3.1) berechneten Zeitpunkt (Hill \& Watson 1984; Jablonski \& Busko 1985; Siegel et al. 1989).

Neuere Messungen der Bedeckungszeitpunkte (Mukai et al. 1998, RXTE: $\Phi_{\text {ecl,obs }}=0.98$ ) sowie der Lage der Spinmaxima (Mauche 1999, EUVE: $\Phi_{\text {spmax }, \text { obs }}=0.04$ ) deuten darauf hin, daß beide Ephemeriden eventuell aktualisiert werden müßten.

\subsubsection{Entfernung und interstellare Absorption}

Die Entfernung von EX Hya ist wie diejenige vieler anderer CVs nicht sehr gut bestimmt. Die Literaturangaben sind entsprechend uneinheitlich und variieren beträchtlich mit der angewandten Methode. Sherrington et al. (1980) paßten an ihre photometrischen Daten 
im $V$ - und $K$-Band und ein mittleres Spektrum im Bereich 1200...7000 $\AA$ von Bath et al. (1980) ein Akkretionsscheibenmodell an (zur Erinnerung: das intermediate polar model wurde erst 1983 von Warner vorgeschlagen) und schlossen daraus auf eine Entfernung von $d=76 \ldots 190$ pc. Aus Spektrophotometrie im Bereich 3200...10000 A ermittelte Wade (1982) $d>60$ pc. Berriman et al. (1985) geben eine weitere, aus $J H K$-Photometrie abgeleitete untere Grenze an: $d>130$ pc. Schließlich leitete Warner (1987) aus der $K$-Helligkeit von Berriman et al. (1985) unter Benutzung einer empirischen Perioden-Radius-Beziehung für den Sekundärstern (vgl. (2.23)) und seiner nach der Methode von Bailey (1981) abgeschätzten Oberflächenhelligkeit (vgl. Abschnitt 2.2.2.2) den Wert $d=105 \mathrm{pc}$ ab.

Wesentlich mehr Einigkeit herrscht bezüglich der Absorption durch das interstellare Medium (ISM): Sie ist gering. Aus dem Fehlen eines charakteristischen Absorptionsmerkmals bei $2200 \AA$ schließt Verbunt (1987) auf $E(B-V)=0.0 \pm 0.05$, was konsistent ist mit der absorbierenden Säulendichte $N_{H} \approx 2 \cdot 10^{19} \mathrm{~cm}^{-2}$, die sich aus der H I-Karte von Fruscione (1994) abschätzen läßt. Säulendichten, die sich aus Anpassungen an EUVund Röntgenspektren ergeben, deuten ebenfalls auf einen vergleichsweise geringen Beitrag $\left(\lesssim 10^{20} \mathrm{~cm}^{-2}\right.$ ) des ISM zur Gesamtabsorption hin (z.B. Córdova et al. 1985; Heise et al. 1987; Rosen et al. 1988; Singh \& Swank 1993; Hurwitz et al. 1997).

\subsubsection{Massen und Systemgeometrie}

Die Tatsache, daß die Bedeckungen von EX Hya von kurzer Dauer sind (s. Abschnitt 3.2.6.2), schränkt die Inklination $i$ bereits auf den Bereich $\approx 75 \ldots 80^{\circ}$ ein. Unter der Annahme $i=75^{\circ}$ wurde die erste Massenbestimmung der Komponenten von EX Hya anhand der Messung der Radialgeschwindigkeitsvariationen von Emissionslinien von Cowley et al. (1981) durchgeführt und lieferte die Werte $M_{1}=M_{\mathrm{WD}}=0.7 \pm 0.25 M_{\odot}$ und $M_{2}=0.16 \pm 0.05 M_{\odot}$. Eine weitere Untersuchung optischer Emissionslinien von Hellier et al. (1987) ergab $i=78 \pm 1^{\circ}$, $M_{1}=0.78 \pm 0.17 M_{\odot}$ und $M_{2}=0.128 M_{\odot}$, wobei der letztere Wert in Ermangelung einer Messung der Radialgeschwindigkeitsamplitude $K_{2}$ des Sekundärsterns aus der PeriodenRadius-Beziehung für Roche-lobe-füllende Sterne (s. (2.23)) und der empirischen MasseRadius-Beziehung für späte Hauptreihensterne von Patterson (1984; s. (2.21)) bestimmt wurde. Eine indirekte Messung von $K_{2}=356 \pm 4 \mathrm{~km} \mathrm{~s}^{-1}$ von Smith et al. (1993) (bzw. nach einer Reanalyse der Daten $K_{2}=357 \pm 44 \mathrm{~km} \mathrm{~s}^{-1}$, Smith 2000) zusammen mit dem von Hellier et al. (1987) gemessenen Wert $K_{1}=69 \pm 9 \mathrm{~km} \mathrm{~s}^{-1}$ ergibt mit $i=78^{\circ}$ die Werte $M_{1}=0.49 \pm 0.03 M_{\odot}$ und $M_{2}=0.095 \pm 0.013 M_{\odot}$ (Hellier 1996) (bzw. $M_{1}=0.49 \pm 0.19 M_{\odot}$ und $M_{2}=0.095 \pm 0.037 M_{\odot}$ mit dem aktuelleren Wert für $\left.K_{2}\right)$. Mit einem anderen Wert $K_{1}=85 \pm 9 \mathrm{~km} \mathrm{~s}^{-1}$, der aus den Variationen der schmalen Komponente der O VI-Linie in ORFEUS-Spektren gewonnen wurde (Mauche 1999) und dem neueren Wert für $K_{2}$ erhält $\operatorname{man} M_{1}=0.53 \pm 0.20 M_{\odot}$ und $M_{2}=0.13 \pm 0.05 M_{\odot}$.

Zur von Smith et al. (1993) angewandten Methode ('skew map method') ist noch eine Bemerkung angebracht. Sie beruht auf Korrelation von zu verschiedenen Orbitalphasen beobachteten Objektspektren mit dem Musterspektrum eines M-Sterns in einem Wellenlängenbereich, in dem dieser charakteristische Merkmale (etwa Na I-Absorptionslinien) hat. Das Spektrum des Mustersterns wird entsprechend der Orbitalphase des Objektspek- 
trums wellenlängenverschoben. Durch Variation der für die Verschiebung benutzten Radialgeschwindigkeitsamplitude $K_{2}$ und durch Ausprobieren von Mustersternen verschiedenen Spektraltyps sind dadurch im Prinzip gleichzeitig $K_{2}$ und der Spektraltyp des Sekundärsterns bestimmbar. Letzterer kann jedoch unsicher sein, wenn z.B. die Metallizität von Sekundärstern und Musterstern deutlich verschieden sind oder Beleuchtung eine nennenswerte Rolle spielt. Weiterhin hängen der ermittelte Wert der Radialgeschwindigkeitsamplitude und sein formaler Fehler vom verwendeten Musterstern ab und sind somit mit einer systematischen Unsicherheit behaftet.

Neuere Bestimmungen der Masse des Weißen Zwergs über die Messung der Temperatur in der Schockregion (s. Abschnitt 2.4.2.2) liefern Werte von $M_{\mathrm{WD}} / M_{\odot}=0.48_{-0.06}^{+0.10}$ (Fujimoto \& Ishida 1997, ASCA), 0.46 \pm 0.04 (Cropper et al. 1999, GINGA), $0.48_{-0.10}^{+0.06}$ (Ezuka \& Ishida 1999, ASCA) und $0.45 \pm 0.03$ (Ramsay 2000, RXTE). Allerdings sind die angewandten Methoden (Messung der Temperatur der Bremsstrahlungskomponente oder Bestimmung der Ionisations- bzw. Anregungstemperatur für Emissionslinien im Röntgenbereich) ebenfalls anfällig für systematische Fehler (s. Abschnitt 2.4.2.2). Von der theoretischen Seite her ist noch anzumerken, daß die Minimalmasse eines Weißen Zwergs mit Kohlenstoffkern $0.45 M_{\odot}$ beträgt, die Masse aber möglicherweise nachträglich durch Nova-Explosionen infolge der Akkretion noch weiter reduziert werden könnte (s. Abschnitt 2.5.5.1).

Für den Sekundärstern erwartet man nach (2.22) $M_{2}=0.128 M_{\odot}$. Die $M-R$-Beziehung (2.24) für die ZAMS (Baraffe et al. 1995; s. Abschnitt 2.2.2) liefert unter der Annahme $M_{\mathrm{WD}}=0.5 M_{\odot}$ für einen Roche-lobe-füllenden Sekundärstern den Wert $M_{2}=0.147 M_{\odot}$, der jedoch aufgrund der in (2.24) nicht berücksichtigten Expansion des Sekundärsterns geringfügig zu groß sein dürfte. Die Gesamtheit der vorliegenden Informationen über die Masse legt mithin $M_{2} \approx 0.13 \pm 0.01 M_{\odot}$ nahe. Dieser Wert impliziert einen recht späten Spektraltyp $(\approx \mathrm{M} 4.5)$, was zwar zu den Spektraltypen paßt, die in CVs mit $P_{\text {orb }} \approx 1.6 \mathrm{~h}$ typischerweise beobachtet werden (M4...5, Beuermann 2000a), aber sowohl mit dem Befund $\mathrm{Sp} \approx \mathrm{M} 3$ von Dhillon et al. (1997) als auch mit dem Spektraltyp M1.5 des von Smith (2000) zur Bestimmung von $K_{2}$ benutzten Mustersterns (s.o.) inkonsistent ist. Die vorliegende Arbeit wiederum unterstützt einen etwas späteren Spektraltyp M4.5 \pm 0.5 (s. Kapitel 5.1.7), der recht gut zu einer Masse von $\approx 0.13 M_{\odot}$ paßt.

\subsubsection{Magnetfeld des Weißen Zwergs}

Über die Magnetfeldstärke von EX Hya ist wie für viele IPs nur wenig bekannt. Für den Anteil $V$ zirkular polarisierter Strahlung im Bereich 5900...8600 A geben Stockman et al. (1992) eine obere Grenze von $0.3 \%$ an. Eine frühere Messung von Cropper (1986) im Band $5700 \ldots 9200 \AA$ ergab $V=(-0.02 \pm 0.04) \%$ und eine obere Grenze für die Variabilität von $\Delta V / V \leq 0.3 \%$. Im optischen Spektrum sind keine Zyklotronlinien sichtbar; Veröffentlichungen über eine Suche nach IR-Zyklotronlinien in EX Hya existieren meines Wissens nicht. 


\subsubsection{Spektrum, Leuchtkraft und Akkretionsrate}

Im Röntgenbereich kann das mittlere Spektrum[ von EX Hya gut durch thermische Bremsstrahlung mit $k_{\mathrm{B}} T_{\mathrm{br}} \approx 10 \mathrm{keV}$ beschrieben werden (z.B. Rosen et al. 1988; Rosen et al. 1991; Singh \& Swank 1993). Bei niedrigeren Energien tritt eine zusätzliche optisch dünne Bremsstrahlungskomponente mit $k_{\mathrm{B}} T_{\mathrm{br}} \approx 2 \mathrm{keV}$ (Ishida et al. 1994; EXOSAT) bzw. $k_{\mathrm{B}} T_{\mathrm{br}} \approx 0.75 \mathrm{keV}$ (Singh \& Swank 1993; EINSTEIN) auf; Singh \& Swank geben die Emissionsmaße der heißen bzw. kühlen Komponente mit $E M \approx 10^{55} \mathrm{~cm}^{-3}$ bzw. $10^{54} \mathrm{~cm}^{-3}$ an. Ferner wurden $\mathrm{K} \alpha$-Linien diverser H- und He-ähnlicher Ionen (z.B. Rosen et al. 1991; Fujimoto \& Ishida 1997) und (erstmals in einem magnetischen CV) die K $\beta$-Emissionslinie des Fe XXVI beobachtet (Ishida et al. 1994). Der beobachtete Röntgenfluß beträgt 1...2 . $10^{-10} \mathrm{erg} \mathrm{cm}^{-2} \mathrm{~s}^{-1}$ im Bereich $1 \ldots 10 \mathrm{keV}$ (Watson et al. 1978; Córdova et al. 1985; Rosen et al. 1988; Rosen et al. 1991; Allan et al. 1998), was einer (unabsorbierten) Leuchtkraft von $L=1.2 \ldots 2.4 \cdot 10^{32}(d / 100 \mathrm{pc})^{2} \mathrm{erg} \mathrm{s}^{-1}$ im genannten Bereich entspricht. Oberhalb von $10 \mathrm{keV}$ wird nach Rosen et al. (1991) unter der Annahme $k_{\mathrm{B}} T_{\mathrm{br}}=10 \mathrm{keV}$ (s.o.) ein vergleichbarer Fluß erwartet; für den Bereich $0.7 \ldots 2 \mathrm{keV}$ geben Córdova \& Riegler (1979) $8.6 \cdot 10^{-11} \mathrm{erg} \mathrm{cm}^{-2} \mathrm{~s}^{-1}$ an.

Im UV und FUV zeigt EX Hya ein Kontinuum, das im Bereich 912...1400 $\AA$ bei einem Fluß von $\approx 2 \cdot 10^{-13} \mathrm{erg} \mathrm{cm}^{-2} \mathrm{~s}^{-1} \AA^{-1}$ flach ist, bei größeren Wellenlängen leicht bis auf $\approx$ $10^{-13} \mathrm{erg} \mathrm{cm}^{-2} \mathrm{~s}^{-1} \AA^{-1}$ bei $3000 \AA$ abfällt (z.B. Bath et al. 1980; Greeley et al. 1997; Mauche $1999)$ und von zahlreichen breiten $\left(F W H M \approx 2500 \mathrm{~km} \mathrm{~s}^{-1}\right)$ Emissionslinien (Ly $\alpha / \beta$, He II, C II/III/IV, N IV/V, O V/VI, Si III/IV, Al III, Mg II u.a.) überlagert ist (z.B. Greeley et al. 1997; Mauche 1999). Unterhalb der Lyman-Kante bei $912 \AA$ fällt der beobachtete Fluß aufgrund der interstellaren Absorption schlagartig auf Null und steigt erst im EUV wieder auf beobachtbare Werte an. Das O VI-Duplett bei $\lambda \lambda 1032,1038 \AA$ zeigt zusätzlich schmale Komponenten $\left(\mathrm{FWHM} \approx 200 \mathrm{~km} \mathrm{~s}^{-1}\right.$ ), die vermutlich nahe am oder auf dem Weißen Zwerg entstehen (Mauche 1999). Im EUV finden sich hauptsächlich schmale Emissionslinien von hochionisiertem Eisen, Neon und Nickel, die nach Hurwitz et al. (1997) in optisch dünnem Plasma mit $T \approx 10^{7} \mathrm{~K}, n_{e} \gtrsim 10^{13} \mathrm{~cm}^{-3}$ und einem Emissionsmaß $E M \approx 3 \cdot 10^{54} \mathrm{~cm}^{-3}$ entstehen; des weiteren finden die genannten Autoren einen Beitrag von Plasma mit $T \approx$ $10^{6} \mathrm{~K}$. Das mit dem Hopkins Ultraviolet Telescope (HUT) beobachtete FUV-Kontinuum läßt sich durch Emission eines Schwarzkörpers mit $T \approx 25000 \mathrm{~K}$ und einem Radius der emittierenden Fläche von $1.1 \cdot 10^{9}(d / 100 \mathrm{pc}) \mathrm{cm}$ modellieren, was eine Leuchtkraft von $3.6 \cdot 10^{32} \mathrm{erg} \mathrm{s}^{-1}$ impliziert (Greeley et al. 1997). Dieselben Autoren geben als alternatives Modell optisch dünne thermische Bremsstrahlung mit $T \approx 75000 \mathrm{~K}$, einem Emissionsmaß $E M=2.7 \cdot 10^{57} \mathrm{~cm}^{-3}$ und einer Leuchtkraft von $1.1 \cdot 10^{33} \mathrm{erg} \mathrm{s}^{-1}$ an und weisen darauf hin, daß es sich in diesem Fall nicht um das von Hurwitz et al. (1997) beobachtete Plasma handeln kann (s.o.).

Das optische Spektrum ist durch kräftige Emissionslinien des Wasserstoffs sowie von He I und He II geprägt, die bis auf He II das für Akkretionsscheiben typische Doppelhöckerprofil zeigen (z.B. Cowley et al. 1981; Gilliland 1982; Kaitchuck et al. 1987; Hellier et al.

6 In diesem Abschnitt ist stets das Spektrum bzw. der Fluß außerhalb der Ausbrüche (d.h. 'in quiescence') gemeint; das Ausbruchsspektrum soll hier nicht behandelt werden. 
Tabelle 3.1: Mittlere photometrische Helligkeiten $\bar{m}$ und Amplituden $\Delta m$ der 67 minbzw. 49 min-Modulation von EX Hya (Beuermann 1982; unveröffentlicht). Die statistischen Fehler der Magnituden betragen jeweils $\approx 0.009$, diejenigen der Amplituden jeweils $\approx$ 0.017 (67 min) bzw. $\approx 0.019$ (49 min); systematische Fehler können einige Hundertstel Magnituden betragen. Die Werte von $R$ und $I$ sind im Kron-Cousins-System gemessen, alle anderen im Johnson-System.

\begin{tabular}{lllllllll}
\hline Band & $U_{J}$ & $B_{J}$ & $V_{J}$ & $R_{K C}$ & $I_{K C}$ & $J_{J}$ & $H_{J}$ & $K_{J}$ \\
\hline $\bar{m}$ & 12.33 & 13.39 & 13.37 & 13.18 & 12.97 & 12.45 & 12.05 & 11.81 \\
$\Delta m(67)$ & 0.20 & 0.15 & 0.12 & 0.13 & 0.09 & 0.09 & 0.07 & 0.05 \\
$\Delta m(49)$ & 0.03 & 0.04 & 0.03 & 0.03 & 0.04 & 0.07 & 0.09 & 0.09 \\
\hline
\end{tabular}

1987). Auch das Infrarot-Spektrum zeigt diese Emissionslinien (z.B. Dhillon et al. 1997). Von Beuermann (1982; unveröffentlicht) beobachtete mittlere photometrische Helligkeiten sind in Tabelle 3.1 aufgeführt; sie können als typisch gelten. Allerdings zeigt EX Hya im Infraroten Helligkeitsschwankungen um einige Zehntel Magnituden auf Zeitskalen von Minuten (z.B. Sherrington et al. 1980). Die Form des Spektrums im Optischen und nahen UV kann nach Bath et al. (1980; IUE+AAT) im Bereich 2500...7000 $\AA$ durch ein Potenzgesetz mit einem Spektralindex $\alpha=0.2 \pm 0.1$ beschrieben werden. (Dieses Ergebnis ist jedoch korrekturbedürftig.) Der beobachtete Fluß im Bereich 1260...7000 ̊ beträgt $\approx 1.4 \cdot 10^{-10} \mathrm{erg} \mathrm{cm}^{-2} \mathrm{~s}^{-1}$ (Bath et al. 1980).

Die Akkretionsrate $\dot{M}$, die sich prinzipiell nach (2.36) aus der Summe der oben angeführten Flüsse bzw. Leuchtkräfte in den einzelnen Energiebereichen ermitteln läßt, ist bisher - nicht zuletzt aufgrund der unsicheren Entfernung - nicht sehr gut bestimmt, liegt aber vermutlich bei $\approx 10^{16} \mathrm{~g} \mathrm{~s}^{-1}$. Beispielhaft seien hier einige Werte (alle in $\mathrm{g} \mathrm{s}^{-1}$ ) sowie die zugrundeliegenden Methoden genannt: Rappaport et al. (1983) aus Evolutionsrechnungen für CVs: $6 \cdot 10^{15}$; Ritter (1985) aus der beobachteten Abnahme der Spinperiode (entfernungsunabhängig, vgl. Abschnitt 2.5.4): $3 \cdot 10^{15}$; Córdova et al. (1985) sowie Mason et al. 1988, beide aus dem beobachteten Röntgenspektrum: 10 ${ }^{16}$; Hurwitz et al. (1997), aus dem beobachteten EUV-Spektrum: $3 \cdot 10^{16}$.

\subsubsection{Lichtkurven}

\subsubsection{Variationen mit der Spinphase}

Die wesentlichen Eigenschaften der von Vogt et al. (1980) im Optischen entdeckten und von Kruszewski et al. (1981) erstmals auch im weichen Röntgenbereich $(0.1 \ldots 2 \mathrm{keV})$ nachgewiesenen 67-Minuten-Modulation lassen sich wie folgt zusammenfassen:

\section{Optisch und Infrarot}

- Im Optischen sind die Lichtkurven sowohl des Kontinuums als auch der Emissionslinien von H, He I und He II grob sinusförmig (z.B. Siegel et al. 1989; Gilliland 1982; Hellier et al. 1987 ); alle Lichtkurven sind in Phase und haben ihre Maxima bei $\Phi_{\text {spin }} \approx 0$ (z.B. Hellier et al. 1987). 
- Die volle Amplitude der Kontinuumsmodulation ist wellenlängenabhängig; sie beträgt im U-, B- bzw. V-Band 0m55, 0m 40 bzw. 0. 34 (Siegel et al. 1989) (entsprechend Modulationsgraden $\left(f_{\max }-f_{\min }\right) / f_{\max }$ von $40 \%, 31 \%$ bzw. $\left.27 \%\right)$.

- Die Linienflüsse sind stärker moduliert als das unterliegende Kontinuum: He II 4686 mit $\approx 50 \%$, He I 4471 mit fast 100\% und die Balmerlinien mit dazwischenliegenden Werten (z.B. Hellier et al. 1987; Kaitchuck et al. 1987).

- Im Infrarot bietet sich im wesentlichen dasselbe Bild: Die Modulation nimmt vom I- bis zum K-Band kontinuierlich bis auf $10 \%$ ab; die Emissionslinien sind auch hier mit bis zu $\approx 30 \%$ stärker moduliert als das Kontinuum (diese Arbeit).

\section{Röntgen}

Die Röntgenlichtkurven sind ebenfalls grob sinusförmig und in Phase mit den optischen Lichtkurven. Der Modulationsgrad (s.o.) nimmt von $\gtrsim 50 \%$ bei Energien $<0.28 \mathrm{keV}$ auf $\approx 20 \%$ bei $5 \mathrm{keV}$ ab und ist oberhalb von $\approx 10 \mathrm{keV}$ im wesentlichen konsistent mit Null (Beuermann \& Osborne 1985; Córdova et al. 1985; Rosen et al. 1988; Norton \& Watson 1989; Rosen et al. 1991; Allan et al. 1998).

\section{UV}

Auch die Lichtkurven im UV (Mauche 1999, IUE; diese Arbeit, IUE, HST), FUV (Greeley et al. 1997, HUT; Mauche 1999, ORFEUS II) und EUV (Hurwitz et al. 1997, EUVE) zeigen im Kontinuum wie in den Linien eine grob sinusförmige Modulation in Phase mit den optischen und Röntgenlichtkurven. Im Wellenlängenbereich $80 . . .180 \AA$ ist der Fluß mit einem Faktor 3.7 noch stärker moduliert als im weichen Röntgenbereich; die Lichtkurve kann durch ein einfaches Modell mit einer konstanten Komponente und einer Komponente mit sinusförmig modulierter optischer Tiefe modelliert werden (Hurwitz et al. 1997). Bei größeren Wellenlängen fallen die Modulationsgrade des Kontinuums wieder moderater aus ( $\approx 40 \%$ bei $1000 \pm 5 \AA$, Mauche $1999 ; 25-40 \%$ an verschiedenen Stellen im Bereich $1200 \ldots 2000 \AA$, diese Arbeit). Hingegen sind die Emissionslinien in allen genannten Bändern deutlich stärker moduliert; viele Linien verschwinden im Spinminimum völlig (alle oben genannten Autoren).

\section{Zusammenfassung}

Das oben Gesagte läßt sich wie folgt zusammenfassen: (1) Die Spinmodulation ist vom Bereich harter Röntgenstrahlung bis in das Infrarot zu beobachten. (2) Die Emissionslinien sind generell stärker moduliert als das Kontinuum. (3) Der Modulationsgrad des Kontinuums ist im EUV $(\approx 100 \AA)$ maximal und fällt zu höheren wie zu niedrigeren Photonenenergien ab. (4) Die Lichtkurven sind in allen Wellenlängenbereichen näherungsweise sinusförmig (mit Abweichungen im harten Röntgenbereich). (5) Die Lichtkurven haben in allen Wellenlängenbereichen im Kontinuum wie in den Linien im Rahmen der Meßgenauigkeit ihr Maximum bei $\Phi_{\text {spin }}=0$. 


\subsubsection{Eigenschaften der Bedeckungen}

Die Bedeckungen von EX Hya wurden von Kraft \& Krzeminski (1962) im Optischen entdeckt. Ihre wesentlichen Merkmale sollen hier zusammengefaßt werden.

\section{Optisch}

- Die Bedeckungen dauern im Optischen ca. 3...4 min; die Ein- und Austrittsphase dauern je etwa 1 min (z.B. Warner \& McGraw 1981). Sie haben im B-Band eine Tiefe von 0. 3...0.7 (Siegel et al. 1989). Die Bedeckung wird (zumindest im B-Band) von einer wesentlich schwächeren Einsenkung in der Lichtkurve von 10..15 min Dauer begleitet (Siegel et al. 1989).

- Die Tiefe einer Bedeckung (d.h. das Flußdefizit gegenüber Zeiten kurz vor oder nach der Bedeckung) variiert mit der Spinphase: Sie ist im Maximum der Spinlichtkurve am größten und umgekehrt. Der in der Mitte der Bedeckung noch vorhandene Restfluß ist ungefähr konstant (d.h. insbesondere unabhängig von der Spinphase) und beträgt $\approx 2 / 3$ des mittleren Flusses (Warner \& McGraw 1981).

- Der Zeitpunkt der Bedeckungsmitte variiert ungefähr sinusförmig mit der Spinphase; gegenüber (3.1) tritt die Bedeckung bei $\Phi_{\text {spin }}=0.25$ um ca. $20 \mathrm{~s}$ (Hill \& Watson 1984) bzw. $35 \mathrm{~s}$ (Jablonski \& Busko 1985) zu spät und bei $\Phi_{\text {spin }}=0.75$ um denselben Betrag zu früh ein (s.a. Siegel et al. 1989).

\section{Röntgen}

Beuermann \& Osborne $(1984 ; 1985)$ entdeckten, daß EX Hya im harten Röntgenbereich (1...9 keV) ebenfalls partielle Bedeckungen zeigt, die mit $\approx 3$ min von vergleichbarer Dauer wie die Bedeckungen im Optischen sind und ähnliche Ein- und Austrittszeiten wie diese haben. Sie haben eine Reihe bemerkenswerter Eigenschaften:

- Sie erfolgen im Rahmen der Meßgenauigkeit zeitgleich mit den optischen Bedeckungen; Länge sowie Ein- und Austrittszeiten sind im wesentlichen unabhängig von der Photonenenergie (z.B. Beuermann \& Osborne 1985, 1988; Rosen et al. 1988; Rosen et al. 1991).

- Die Tiefe der Bedeckung steigt mit der Photonenenergie von $\approx 30 \%$ bei $2 \mathrm{keV}$ bis auf $\gtrsim 60 \%$ bei $10 \mathrm{keV}$ an (Beuermann \& Osborne 1988; Rosen et al. 1988; Rosen et al. 1991). Im weichen Röntgenbereich gibt es nur schwache Evidenz für eine Bedeckung (Córdova et al. 1985; Rosen et al. 1988); oberhalb von $15 \mathrm{keV}$ läßt der geringe Fluß von Ex Hya mit den bisher verwendeten Instrumenten keine eindeutigen Schlüsse zu (z.B. Rosen et al. 1991).

- Die Tiefe der Bedeckungen hängt im gleichen Sinne wie die der optischen Bedeckungen von der Spinphase ab; im Spinminimum sind die Bedeckungen kaum noch nachweisbar (Beuermann \& Osborne 1985, 1988). Wie im Optischen ist der unbedeckte Restfluß im Zentrum der Bedeckung bei gegebener Photonenenergie ungefähr konstant (Beuermann \& Osborne 1988; Rosen et al. 1988).

- Nach einer neueren Untersuchung mit RXTE dauert die Bedeckung im Energiebereich $4 \ldots 6 \mathrm{keV} 157 \pm 4 \mathrm{~s}$ (FWHM). Ein- und Austritt dauern jeweils $21 \pm 4 \mathrm{~s}$; der Restfluß von $\approx 64 \%$ bleibt während der zentralen $\approx 135$ s etwa konstant, wobei die Bedeckungen möglicherweise bei $\Phi_{\text {spin }} \approx 0.25$ am ausgeprägtesten sind (Mukai et al. 1998). Der Zeitpunkt der Bedeckungsmitte von $\Phi_{\text {orb }}=0.98$ legt vermutlich nahe, daß die Orbitalephemeride (3.1) aktualisierungsbedürftig ist (vgl. Abschnitt 3.2.1). 


\section{UV}

Im EUV $(80 \ldots 180 \AA)$ erfolgt die Bedeckung ebenfalls bei $\Phi_{\text {orb }}=0$, ist aber total und hat eine Dauer von nur $41 \pm 2 \mathrm{~s}$. Ein- und Austritt sind mit $<3 \mathrm{~s}$ Dauer sehr scharf. Der Zeitpunkt der Bedeckung ist anders als im Optischen innerhalb von \pm 3 s unabhängig von der Spinphase (Hurwitz et al. 1997). Abgesehen davon ist EX Hya im UV erst relativ wenig untersucht worden. Das Verhalten bei Wellenlängen zwischen 180 und $912 \AA$ konnte noch nicht untersucht werden. Auch über Bedeckungen bei noch größeren UV-Wellenlängen gibt es meines Wissens bisher keine Veröffentlichungen.

\section{Zusammenfassung}

Auch hier gebe ich eine knappe Zusammenfassung der wesentlichen Eigenschaften. (1) Die Bedeckungen sind zeitgleich im Optischen, im harten und ansatzweise auch im weichen Röntgenbereich sowie im EUV zu beobachten; über das Verhalten im längerwelligen UV ist noch nichts bekannt. (2) Sie sind im Optischen und harten Röntgenbereich partiell und im EUV total. (3) Die Dauer beträgt jeweils 3...4 min mit deutlich sichtbarem Ein- und Austritt im Optischen und harten Röntgenbereich und 41 s mit scharfem Ein- und Austritt im EUV. (4) Die Tiefe der Bedeckungen im Optischen und harten Röntgenbereich ist im Spinmaximum am größten und im Spinminimum am kleinsten; der unbedeckte Restfluß ist in beiden Fällen ungefähr konstant. (5) Die Tiefe der Bedeckungen steigt im Röntgenbereich zumindest bis $\approx 10 \mathrm{keV}$ mit der Photonenenergie an. (6) Der Zeitpunkt der optischen Bedeckungen variiert mit der Spinphase; es ist nicht bekannt, ob dies auch im harten Röntgenbereich der Fall ist.

\subsubsection{Weitere Variationen mit der Orbitalphase}

Abgesehen von den oben besprochenen Bedeckungen weist die Orbitallichtkurve von EX Hya folgende Merkmale auf:

- Im harten Röntgenbereich ( $\gtrsim 1 \mathrm{keV}$ ) ist die Lichtkurve (bis auf die Bedeckungen) flach (z.B. Beuermann \& Osborne 1984, 1985; Rosen et al. 1988).

- Im weichen Röntgenbereich erscheint bei $\Phi_{\text {orb }} \approx 0.7$ ein ausgeprägter Einbruch ('dip') in der Lichtkurve, dessen tiefster Punkt mit ca. $50 \%$ Restfluß bei $\Phi_{\text {orb }} \approx 0.85$ liegt. Der dip ist für ca. $1 / 3$ des Orbitalzyklus sichtbar (Córdova et al. 1985; Rosen et al. 1988) und oberhalb von $2 \mathrm{keV}$ nicht mehr nachweisbar (Hellier et al. 1993).

- Die Situation im EUV ist ähnlich wie im weichen Röntgenbereich: Lage, Dauer und Form des dips sind vergleichbar, allerdings bricht der Fluß im dip bis auf $\approx 1 / 8$ ein (Hurwitz et al. 1997; Mauche 1999). Die relative Tiefe des dips ist unabhängig von der Spinphase (Hurwitz et al. 1997). Hurwitz et al. (1997) bemerken, daß der EUV-dip durch Absorption in Material mit einer Säulendichte von $N_{H} \approx 1.3 \cdot 10^{20} \mathrm{~cm}^{-2}$ bei solarer Zusammensetzung erklärt werden kann. Eine solche Säulendichte hätte bei $0.22 \mathrm{keV}$ eine optische Tiefe von $\tau \approx 0.35$; dies ist konsistent mit dem Befund von Hellier et al. (1993), die aus den Beobachtungen mit EXOSAT (s.o.) analog eine effektive optische Tiefe von 0.35 im Band $0.05 \ldots 2 \mathrm{keV}$ ableiten.

-Während der Dauer des EUV-dips erscheinen in den meisten FUV-Emissionslinien schmale, schwache Absorptionslinien (Mauche 1999). Gleichzeitig sinkt die Helligkeit aller UV- 
Emissionslinien (außer He II 1640) um 30-40\% ab, während das UV-Kontinuum im wesentlichen unbeeinflußt bleibt (Mauche 1999, IUE-SWP).

- Im längerwelligen UV $(2000 \ldots 3300 \AA)$ treten keine systematischen Variationen mit der Orbitalphase mehr auf (Krautter \& Buchholz 1990).

- Im Optischen erscheint im B-Band bei $\Phi_{\text {orb }} \approx 0.8$ (und schwächer ausgeprägt auch bei $\left.\Phi_{\text {orb }} \approx 0.3\right)$ für die Dauer von $\approx 0.2$ Orbitalzyklen ein $\approx 0 . \mathrm{m} 1$ hohes Maximum in der Lichtkurve (Siegel et al. 1989).

\subsubsection{Akkretionsgeometrie}

Ein Modell der Akkretionsgeometrie von ExHya, das beschreibt, auf welche Weise die Materie akkretiert wird, wo und nach welchen Mechanismen Strahlung in den einzelnen Wellenlängenbereichen emittiert wird und welche Ausdehnung die jeweiligen Gebiete haben, muß unter anderem folgende Phänomene erklären können:

- das Auftreten der Helligkeitsmodulation mit der Spinphase vom Optischen bis in den Röntgenbereich

- das zeitliche Zusammentreffen des Zeitpunkts des Maximums der Spinlichtkurve vom Optischen bis in den Röntgenbereich

— den Abfall der Amplitude der Spinmodulation mit steigender Photonenenergie im Röntgenbereich

- den Unterschied im Modulationsgrad zwischen Kontinuum und Emissionslinien im EUV, FUV und UV

- Auftreten und Dauer der partiellen Bedeckungen im harten Röntgenbereich und im Optischen sowie den allmählichen Ein- und Austritt

- Totalität und Dauer der Bedeckungen im EUV sowie die Schärfe des Ein- und Austritts

- den Anstieg der Tiefe der Bedeckungen mit der Energie im Röntgenbereich

- die Spinphasenabhängigkeit der Tiefe der Bedeckungen im Röntgenbereich

— die Spinphasenabhängigkeit des Zeitpunkts der Bedeckungsmitte im Optischen

- die Unabhängigkeit der Bedeckungszeitpunkte von der Spinphase im EUV

- Auftreten, Phasenlage und Dauer des breiten Minimums in der Orbitallichtkurve bei $\Phi_{\text {orb }} \approx 0.9 \mathrm{im}$ EUV und im weichen Röntgenbereich

Weiterhin muß es einen Satz von Werten für die Massen der beiden Sterne, die Inklination $i$ usw. enthalten, der mit der Modellgeometrie und den sonstigen Eigenschaften des Systems konsistent ist. Teile eines solchen Modells können bereits an dieser Stelle dargestellt werden. Andere Aspekte - insbesondere Details der Bedeckungen sowie die Sternmassen - werden erst in Kapitel 5 im Rahmen der Interpretation der Ergebnisse dieser Arbeit diskutiert. 


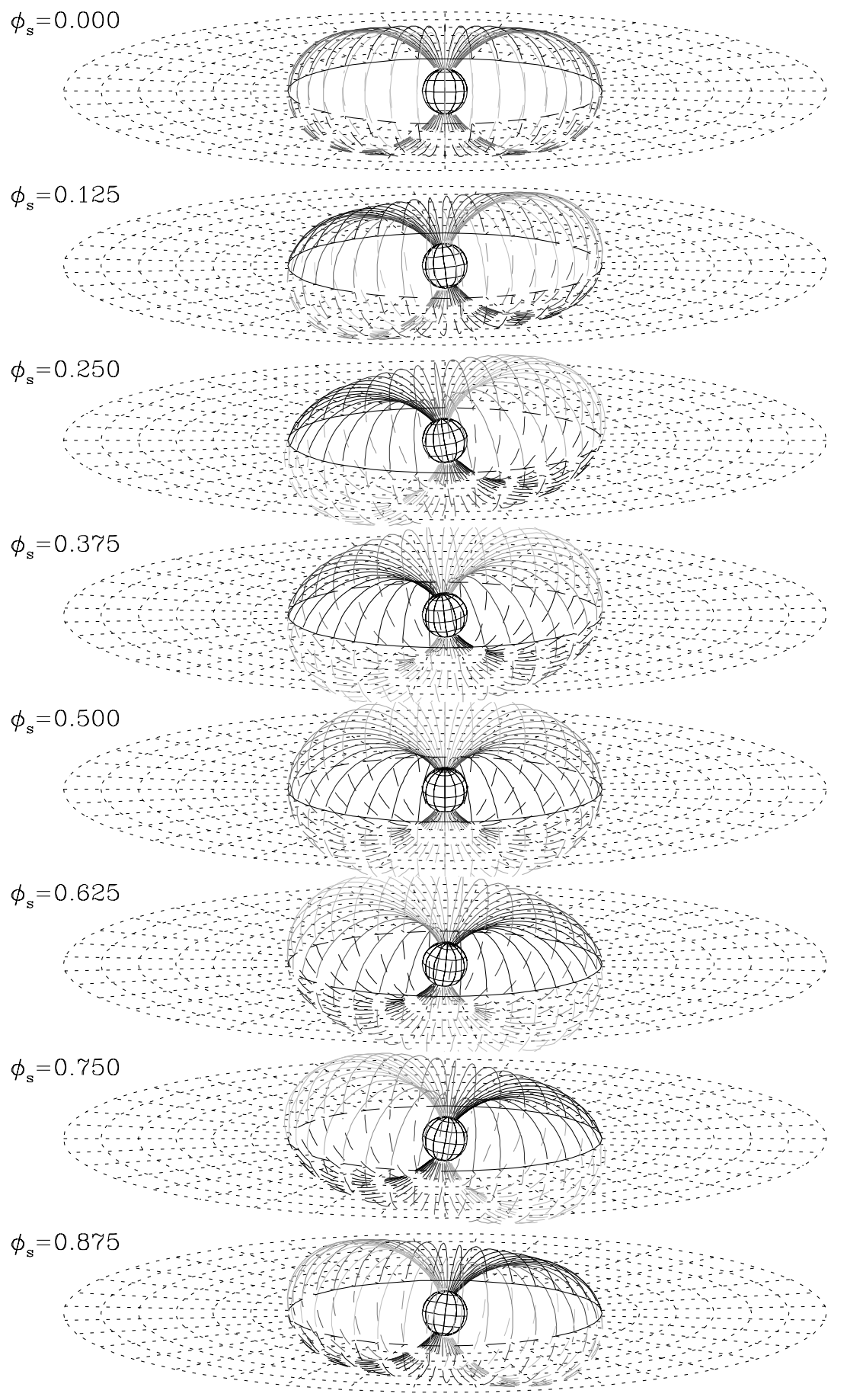

Abbildung 3.2: Akkretionsvorhänge von EX Hya zu verschiedenen Spinphasen für eine Inklination $i=78^{\circ}$, eine Neigung der magnetischen Achse des Weißen Zwergs gegen seine Rotationsachse von $\delta=10^{\circ}$ und einen angenommenen Radius des Innenrands der Akkretionsscheibe von $R_{i}=7 R_{\mathrm{WD}}$. Die Grautöne der Dipolfeldlinien symbolisieren die jeweilige Massenflußdichte $\dot{m}$ bzw. die Dichte $\rho$ der Materie. 


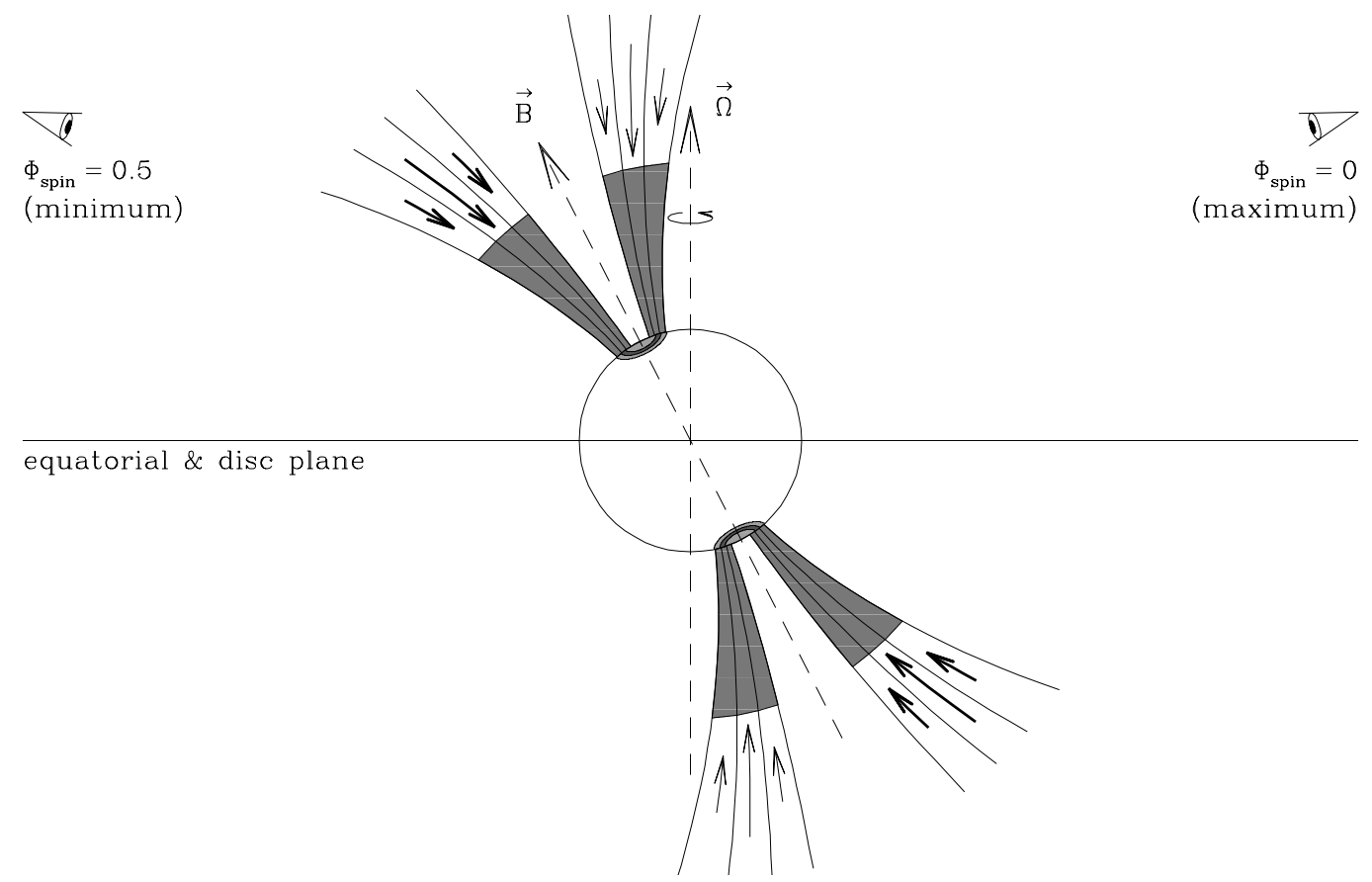

Abbildung 3.3: Schema des zentralen Akkretionsgebiets von EX Hya mit dem entlang der Feldlinien einfallenden kühlen Material (Pfeile; Dicke und Länge deuten die Massenflußdichte $\dot{m}$ an), dem heißen settling flow unterhalb der Stoßfront (grau) und den geheizten Teilen der Photosphäre des Weißen Zwergs (Ringe). Die Dipolfeldlinien sind rotationssymmetrisch um die Magnetfeldachse zu denken.

\subsubsection{Entstehung der Spinmodulation}

Die Eigenschaften der in allen Wellenlängenbereichen beobachtbaren Helligkeitsmodulation mit der Spinphase lassen sich im Rahmen des intermediate polar models (s. Abschnitt 2.3.3) verstehen. Ausgangspunkt ist dabei das in Abbildung 3.2 schematisch dargestellte Erscheinungsbild von EX Hya zu verschiedenen Spinphasen.

Die beobachtete Röntgenemission entsteht im heißen Plasma unterhalb des hydrodynamischen Stoßes (Abschnitt 2.4.2.2). Aufgrund der Geometrie der Feldlinien wird das Emissionsgebiet im Spinmaximum $\left(\Phi_{\text {spin }}=0\right)$ frontal und ohne absorbierendes Material im Vordergrund gesehen (Abbildung 3.3). Bei $\Phi_{\text {spin }}=0.5$ (Spinminimum) wird die Strahlung hingegen durch das vergleichsweise kühle einfallende Material oberhalb der Stoßfront teilweise absorbiert; bei optischen Tiefen $\tau \gtrsim 1$ erscheint das Emissionsgebiet zudem perspektivisch verkürzt. Dieses Szenario, in dem das Röntgenmaximum auftritt, wenn der obere Magnetpol des Weißen Zwergs vom Beobachter wegzeigt, wurde bereits von Rosen et al. (1988) und Beuermann \& Osborne (1988) vorgeschlagen.

Da der entscheidende Punkt die verstärkte Absorption durch den Akkretionsvorhang im Spinminimum ist, ist auch die Abhängigkeit des Modulationsgrades von der Photonenenergie prinzipiell leicht verständlich: während weiche Röntgenstrahlung stark absorbiert 


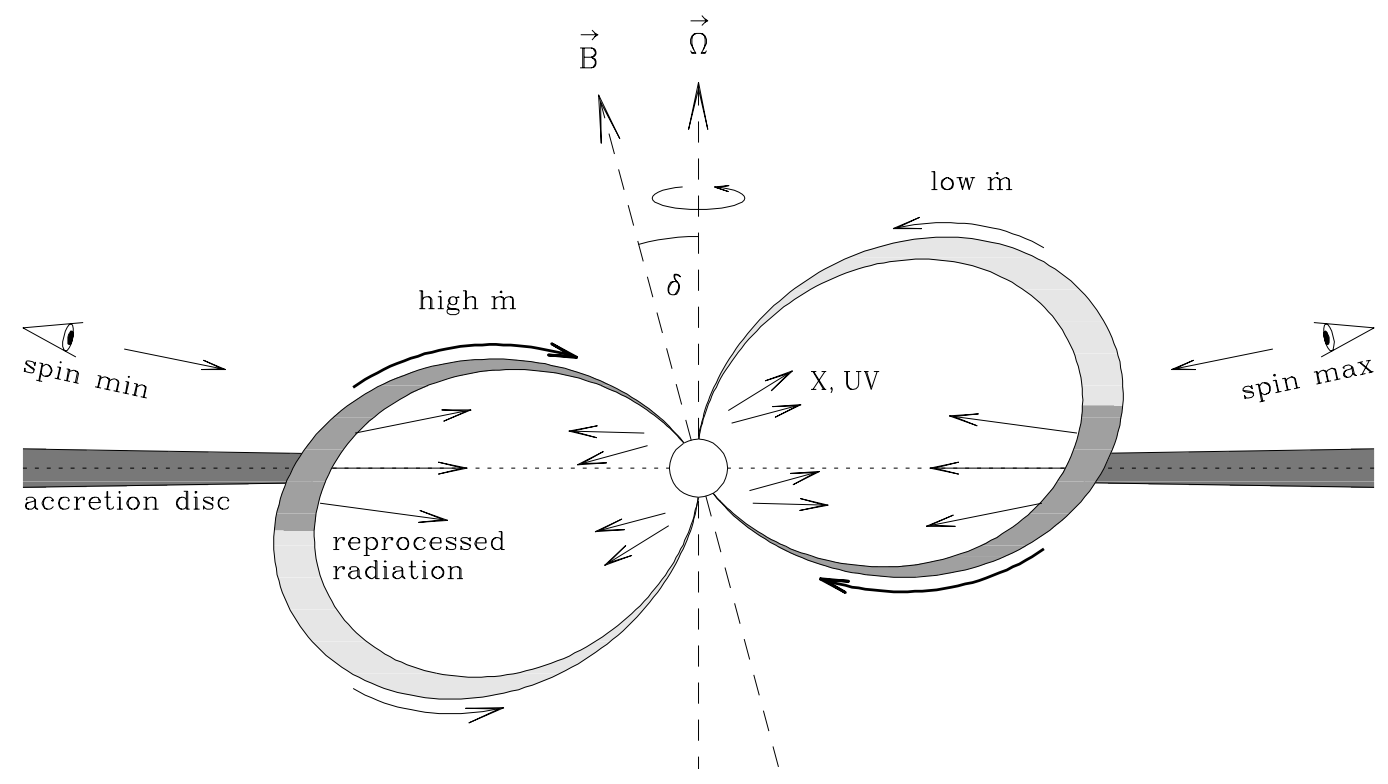

\begin{abstract}
Abbildung 3.4: Schematischer Blick in der Orbitalebene auf das Zentralgebiet von EX Hya. Die Grautöne symbolisieren die unterschiedlichen Massenflußraten in den verschiedenen Teilen der Akkretionsvorhänge (vgl. Abbildung 3.2). Energiereiche Strahlung von der Zentralquelle wird an der Innenseite der Akkretionsvorhänge reprozessiert und vor allem als optische Strahlung emittiert (gerade Pfeile). Man beachte, daß die äußeren Teile des unteren Akkretionsvorhangs aufgrund der hohen Inklination teilweise von der Akkretionsscheibe verdeckt werden. Ferner kann Material zunächst ein Stück weit den längeren Ast entlanglaufen, bevor es in die Scheibenebene zurückfällt und schließlich den Weg zum näherliegenden Magnetfeld einschlägt (Kube et al. 2000; s.a. Abbildung 2.5). In diesem Gebiet ist entsprechend mit turbulenten Bewegungen des Plasmas zu rechnen.
\end{abstract}

wird, wird das Material des Akkretionsvorhangs für Photonen größerer Energie zunehmend transparent. Im Detail ist jedoch die Form der Lichtkurven bei verschiedenen Energien und insbesondere der im Spinminimum nicht verschwindende Fluß im weichen Röntgenbereich - nur zu verstehen, wenn man annimmt, daß das Material in den Akkretionsvorhängen nicht homogen ist, sondern in 'Klumpen' vorliegt, die einen Bruchteil $f$ der beobachteten projizierten Fläche einnehmen ('partial absorber'). Mit der lokalen absorbierenden Säulendichte $N_{\mathrm{H} \text {,loc }}$ im Akkretionsvorhang und der Säulendichte $N_{\mathrm{H}, \mathrm{ISM}}$ des kalten interstellaren Mediums läßt sich dann der beobachtete Fluß $F_{\text {obs }}(E)$ aus dem ursprünglich emittierten Fluß $F_{\text {emi }}(E)$ bestimmen:

$$
F_{\text {obs }}(E)=F_{\text {emi }}(E) \cdot\left[(1-f)+f \cdot e^{-\sigma_{\mathrm{w}}(E) N_{\mathrm{H}, \mathrm{loc}}}\right] \cdot e^{-\sigma_{\mathrm{c}}(E) N_{\mathrm{H}, \mathrm{ISM}}} .
$$

Dabei sind $\sigma_{\mathrm{w}}(E)$ bzw. $\sigma_{\mathrm{c}}(E)$ die Absorptionskoeffizienten des warmen lokalen bzw. des kalten interstellaren Mediums. Der Term $(1-f)$ repräsentiert denjenigen Anteil des emittierten Flusses, der ungehindert 'zwischen den Klumpen hindurchschlüpft' und nur der interstellaren Absorption unterworfen ist (d.h. $f=1$ entspricht einem homogenen und $f=0$ einem nicht vorhandenen lokalen Medium) und legt somit den minimal möglichen Fluß 
fest. Die Spinphasenabhängigkeit von $N_{\mathrm{H} \text {,loc }}$ und die Energieabhängigkeit von $\sigma_{\mathrm{w}}$ erzeugen schließlich im Zusammenspiel die unterschiedlich großen Modulationsgrade bei verschiedenen Photonenenergien. Entsprechende Modellrechnungen von Kim \& Beuermann (1995) zeigen, daß schon bei recht moderaten Neigungen der magnetischen Achse des Weißen

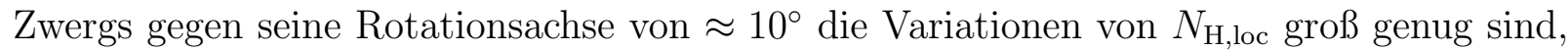
um die beobachteten Lichtkurven gut wiederzugeben.

Die optische Emission entsteht nach weitgehend akzeptierter Vorstellung durch Reprozessierung energiereicher Strahlung (Röntgen und UV) aus der Nähe des Weißen Zwergs auf der konkaven Innenseite des Akkretionsvorhangs, und zwar bevorzugt in Gebieten mit hoher Materiedichte und Beleuchtungsstärke (z.B. Hellier et al. 1987). Die besten Bedingungen hierfür sind am Rand der Akkretionsscheibe sowie im äußeren Teil des Akkretionsvorhangs in dem Bereich gegeben, wo der magnetische Pol der Scheibe am nächsten kommt (Abbildung 3.4; s.a. Abschnitt 2.4.2.7). Die besten Sichtbedingungen auf dieses Gebiet hat der Beobachter bei $\Phi_{\text {spin }}=0$, d.h. wiederum dann, wenn der obere Magnetpol des Weißen Zwergs vom Beobachter wegzeigt und die Sichtlinie auf den jenseits des Weißen Zwergs liegenden Rand der Akkretionsscheibe nur durch dünnes Material führt (vgl. Abbildung 3.2). Bei $\Phi_{\text {spin }}=0.5$ hingegen wird die reprozessierte Strahlung zu einem großen Teil wieder im nun in der Sichtlinie liegenden dichten Teil des Akkretionsvorhangs absorbiert. Dies erklärt das zeitgleiche Auftreten der Maxima und Minima der Spinlichtkurve im Optischen und Röntgenbereich, obwohl die Entstehungsgebiete der Strahlung und die der Spinmodulation zugrundeliegenden Mechanismen sehr verschieden sind. Numerische Rechnungen von Kim \& Beuermann (1996) zeigen, daß sich in diesem Szenario die Lichtkurven und die Intensitätsvariationen der Balmerlinie $\mathrm{H} \beta$ zu verschiedenen Spinphasen befriedigend modellieren lassen.

\subsubsection{Ursache des breiten Intensitätsminimums bei $\Phi_{\text {orb }} \approx 0.9$}

Der Einbruch in der Lichtkurve im EUV und weichen Röntgenbereich sowie das Auftreten von Absorption in den UV-Emissionslinien bei $\Phi_{\text {orb }} \approx 0.9$ (Abschnitt 3.2.6.3) lassen sich einheitlich durch das Vorhandensein eines bulge mit einem bright spot am äußeren Rand der Akkretionsscheibe erklären (s. Abschnitt 2.2.4). Aufgrund der hohen Inklination befindet sich der bulge von $\Phi_{\text {orb }} \approx 0.75$ bis $\Phi_{\text {orb }} \approx 0.9$ vom Beobachter aus gesehen vor dem Zentralgebiet (Abbildung 3.5) und verursacht Absorption vor allem im dafür besonders anfälligen weichen Röntgenbereich und EUV. Die schmalen Absorptionskerne der FUV-Linien und der Intensitätsabfall der mit IUE beobachtbaren Emissionslinien werden ebenfalls vom kühlen bulge-Material verursacht. Die Intensitätsmaxima im Optischen bei $\Phi_{\text {orb }} \approx 0.8$ und $\Phi_{\text {orb }} \approx 0.3$ (Abschnitt 3.2.6.3) gehen auf das Konto der Emission des bright spot, der zu diesen Phasen frontal zu sehen ist. Das Maximum bei $\Phi_{\text {orb }} \approx 0.3$ fällt dabei schwächer aus, weil die Emission des bright spot vornehmlich am äußeren Rand des bulge dort entsteht, wo der Akkretionsstrom die Scheibe trifft, und bei dieser Orbitalphase auf dem Weg zum Beobachter selbst das Material des bulge durchqueren muß (s.a. Abschnitt 2.4.2.6). 


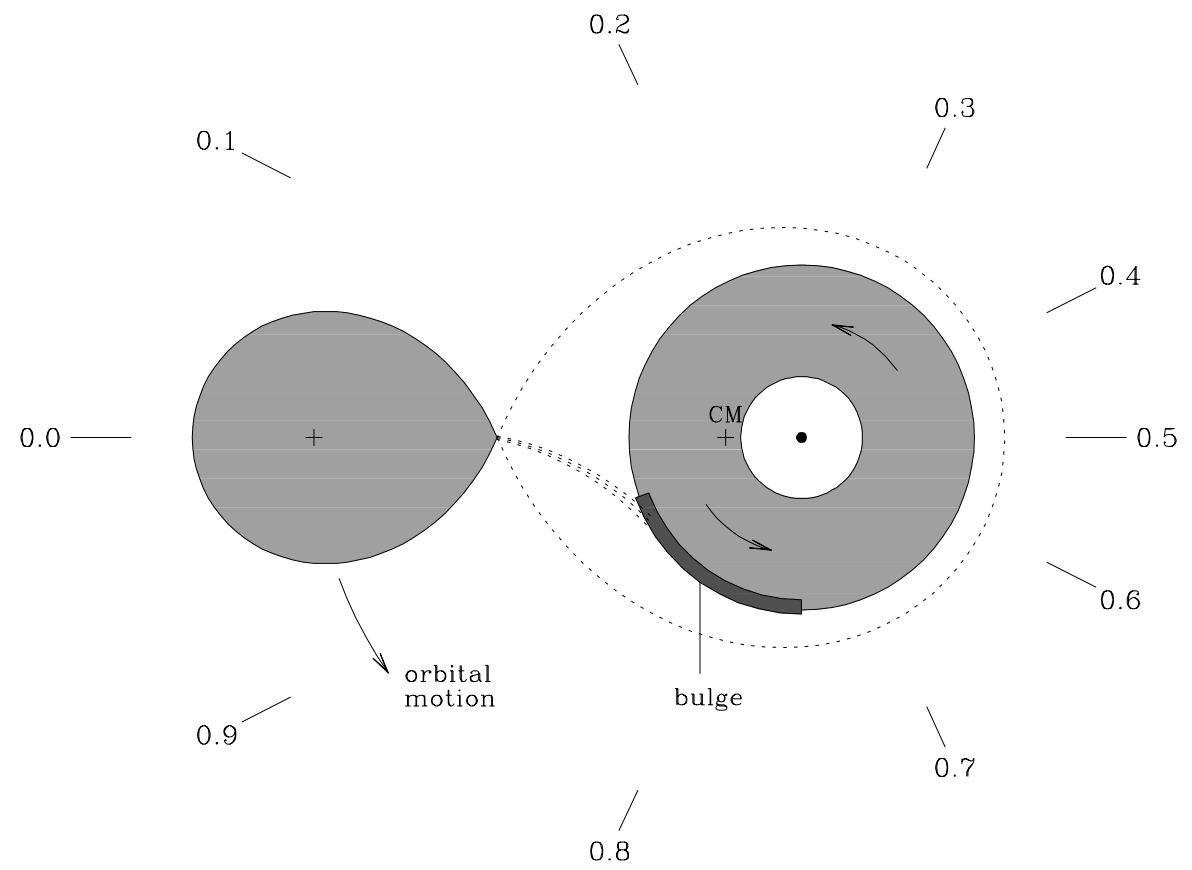

Abbildung 3.5: Schematischer Blick auf die Orbitalebene von EX Hya. Der bulge am äußeren Rand der Akkretionsscheibe befindet sich in dieser Darstellung ca. von $\Phi_{\text {orb }} \approx 0.75$ bis $\Phi_{\text {orb }} \approx 0.9$ zwischen dem Beobachter und dem Zentralgebiet.

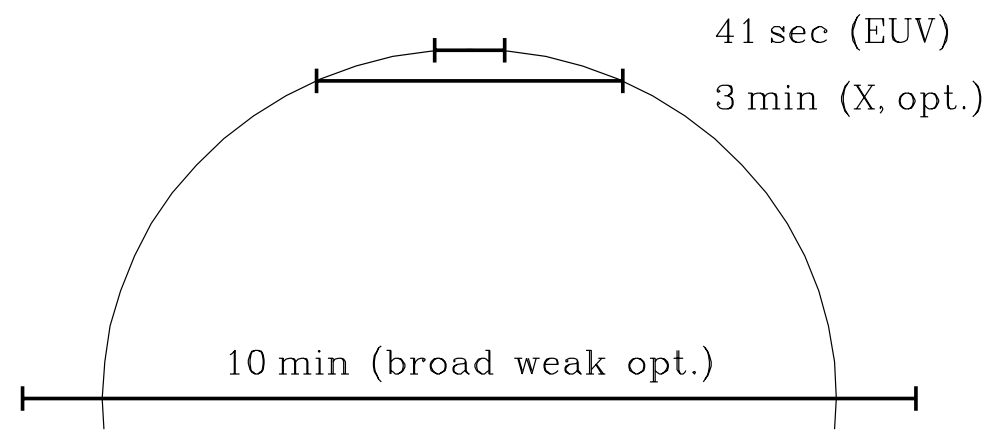

Abbildung 3.6: Zeit- und Längenverhältnisse der Bedeckungen unter den Annahmen $M_{2}=0.13 M_{\odot}$ und $M_{\mathrm{WD}}=1 M_{\odot}$ (s. Text). Dargestellt sind die Silhouette des Sekundärsterns sowie die den verschiedenen Bedeckungslängen entsprechenden Strecken als Kreissehnen.

\subsubsection{Grundsätzliche Geometrie der Bedeckungen}

An dieser Stelle sollen nur Sachverhalte erläutert werden, die sich bereits hier aus den in Abschnitt 3.2.6.2 dargestellten Befunden ableiten lassen. Eine Diskussion der hier noch unbehandelten Fragen findet in Kapitel 5 statt. 

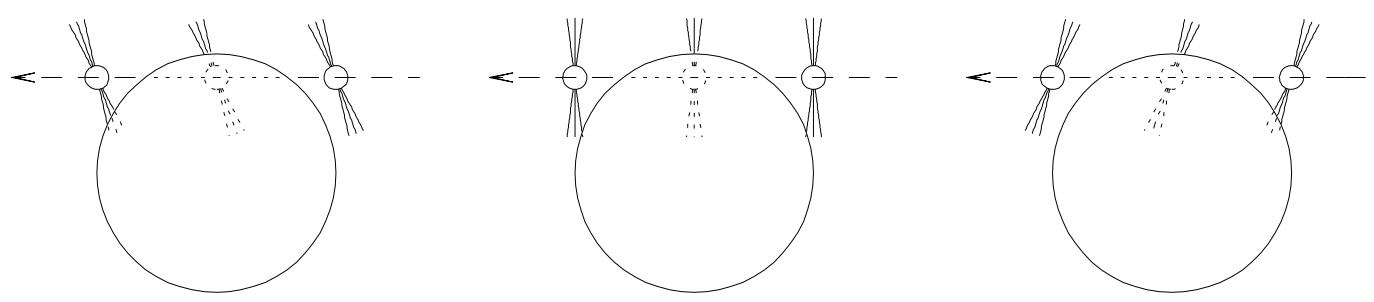

Abbildung 3.7: Veranschaulichung der Spinphasenabhängigkeit der Bedeckungszeitpunkte. Die Emissionsgebiete sind an das Magnetfeld des Weißen Zwergs gekoppelt, dessen Achse gegen seine senkrecht auf der Orbitalebene stehende Rotationsachse geneigt ist, und befinden sich daher zu verschiedenen Spinphasen an unterschiedlichen Orten relativ zum Zentrum des Weißen Zwergs. Im linken bzw. rechten Teilbild erfolgt daher die Bedeckung später bzw. früher als im mittleren Teilbild. Die Darstellung ist nur als Prinzipskizze zu verstehen, da Lage, Form und Größe der Emissionsgebiete willkürlich sind und nicht unbedingt einen Bezug zur Situation in EX Hya haben müssen.

Unter den Annahmen $M_{2}=0.13 M_{\odot}$ (s. Abschnitt 3.2.3) und $M_{\mathrm{WD}}=1 M_{\odot}$ beträgt nach (2.1) die relative Bahngeschwindigkeit der beiden Komponenten $v_{\text {rel }}=2 \pi a / P_{\text {orb }} \approx$ $530 \mathrm{~km} \mathrm{~s}^{-1}$ und (unter Benutzung von (2.10)) die Dauer einer zentralen Bedeckung $t_{\mathrm{ecl}} \approx$ $2 R_{V} / v_{r e l} \approx 7$ min. (Beide genannten Werte sind nur schwach von der tatsächlichen Masse des Weißen Zwergs abhängig, vgl. (2.1).) Dies zeigt bereits, daß bei den nur jeweils $\approx 3$ min dauernden Bedeckungen im Optischen und im Röntgenbereich die jeweils bedeckte Quelle im Zentrum der Bedeckung nicht sehr weit vom projizierten oberen Rand des Sekundärsterns entfernt sein kann, d.h. die Bedeckungen streifend sind (s. Abbildung 3.6). Der allmähliche Ein- und Austritt legen weiterhin eine gewisse Ausdehnung der bedeckten Quellen in Richtung der Bahnbewegung und/oder senkrecht dazu nahe.

Die Kürze der EUV-Bedeckung (41s) und die Schärfe von Ein- und Austritt zeigen ferner, daß die EUV-Quelle zum einen vermutlich noch näher am projizierten Rand des Sekundärsterns liegt und zum anderen sowohl in Richtung der Orbitalbewegung als auch senkrecht dazu sehr klein sein muß $\left(\lesssim 2 \cdot 10^{8} \mathrm{~cm}\right.$ parallel zur Bahnebene, $\lesssim 10^{7} \mathrm{~cm}$ senkrecht dazu; Hurwitz et al. 1997). Analog legt die breite schwache Bedeckung, die im B-Band mit den $\approx 3$-minütigen Bedeckungen einhergeht, als Quelle eine Struktur nahe, deren Ausdehnung in Richtung der Bahnbewegung mit dem Durchmesser des Sekundärsterns vergleichbar oder größer ist. Der naheliegendste Kandidat hierfür ist die Akkretionsscheibe (vgl. Abbildung 3.5). Als weitere Folgerung ergibt sich dann aus der Schwäche der breiten Bedeckung, daß der Beitrag der Akkretionsscheibe zur optischen Emission zumindest im B-Band klein im Vergleich zu dem des Akkretionsvorhangs ist.

Die Spinphasenabhängigkeit der Zeitpunkte der Bedeckungen im Optischen läßt sich nach den Ausführungen in Abschnitt 3.2.7.1 prinzipiell ebenfalls leicht verstehen. Da das dominierende optische Emissionsgebiet im Akkretionsvorhang liegt, hat dessen Wanderung um den Innenrand der Scheibe infolge der Rotation des Weißen Zwergs auch eine periodische Wanderung des Helligkeitsschwerpunkts zur Folge. Dadurch tritt die in Abbildung 3.7 
schematisch dargestellte Situation ein. Der Zeitpunkt der Bedeckung des Helligkeitsschwerpunkts legt dann den Zeitpunkt der Bedeckungsmitte fest. Aus der Amplitude der Wanderbewegung läßt sich im Prinzip die maximal mögliche Größe des emittierenden Gebiets, d.h. der Radius des Innenrands der Scheibe ableiten (vgl. Abbildung 2.7). Dies wird in Kapitel 5 durchgeführt. Umgekehrt läßt sich jedoch aus der Tatsache, daß die Bedeckungszeitpunkte im EUV im Rahmen der Meßgenauigkeit nicht von der Spinphase abhängen, auf eine Entstehung der Emission in einer kleinen Region sehr nahe am Weißen Zwerg in der Nähe seiner Rotationspole schließen, vermutlich sogar auf seiner Oberfläche. Dies wurde bereits von Hurwitz et al. (1997) konstatiert.

Schließlich läßt sich aus der Beobachtung, daß die Bedeckungen sowohl im Optischen als auch im Röntgenbereich partiell mit etwa spinphasenunabhängigem Restfluß sind, folgern, daß sich in beiden Energiebereichen die beobachtete Gesamtemission aus zwei Anteilen zusammensetzt, von denen einer im wesentlichen konstant ist und der andere mit der Spinphase moduliert ist und bedeckt wird. Für die Röntgenstrahlung kommt für den konstanten Anteil der durch den Term $(1-f)$ in (3.3) repräsentierte Bruchteil (oder zumindest ein Teil davon) in Frage. Im Falle der optischen Bedeckungen ist die Emission des über den Rand des Sekundärsterns herausragenden Teils des Akkretionsvorhangs ein naheliegender Kandidat für den konstanten Restfluß. Die Details sowie die Energieabhängigkeit der Bedeckungstiefe im Röntgenbereich hängen wiederum von Lage und Form der bedeckten und unbedeckten Emissionsgebiete ab und werden ebenfalls in Kapitel 5 diskutiert. 


\section{Kapitel 4}

\section{Beobachtungen und Datenanalyse}

Dieses Kapitel enthält im ersten Teil eine Beschreibung des für diese Arbeit verwendeten Beobachtungsmaterials. Der zweite Teil befaßt sich mit der Analyse der Daten und der dafür verwendeten Software. Die Interpretation der Ergebnisse erfolgt anschließend in Kapitel 5 .

\subsection{Datenmaterial}

Für diese Arbeit wurde EX Hya in drei Nächten mit dem Infrared Spectrometer (IRS) am 4 m-Teleskop des Cerro Tololo Interamerican Observatory (CTIO) sowie während einer Nacht mit der ESO Faint Object Spectrograph and Camera 2 (EFOSC 2) am 2.2 mTeleskop der Max-Planck-Gesellschaft auf La Silla beobachtet. Zusätzlich wurden Spektren verwendet, die von anderen Beobachtern mit der Short Wave Prime Camera (SWP) des International Ultraviolet Explorer (IUE) sowie mit dem Faint Object Spectrograph (FOS) an Bord des Hubble Space Telescope (HST) gewonnen und den jeweiligen Datenarchiven

Tabelle 4.1: Eckdaten des Beobachtungsmaterials. Die Spalte $t_{\text {int }}$ gibt die Belichtungszeit pro Spektrum und die Spalte $t_{\text {res }}$ die mittleren zeitlichen Abstände zweier nacheinander aufgenommener Spektren (unter Berücksichtigung der Auslesezeiten der Detektoren) an (beide in Sekunden). Zur tatsächlichen zeitlichen Überdeckung der angegebenen Beobachtungszeiträume (insbesondere für IUE und HST) s. Text.

\begin{tabular}{lllllll}
\hline Instrum. & $\lambda$-Bereich $[\AA]$ & Datum & $\mathrm{UT}$ & $t_{\text {int }}$ & $t_{\text {res }}$ & Beobachter \\
\hline IRS & $8200-24200$ & $22 .-23.02 .1997$ & $05: 55-09: 55$ & 60 & $\lesssim 65$ & K. Reinsch, \\
& & $23 .-24.02 .1997$ & $07: 35-09: 20$ & & & S. Eisenbart \\
& & $24 .-25.02 .1997$ & $07: 00-09: 55$ & & & \\
\hline EFOSC2 & $3600-10200$ & $28.02 .-01.03 .1997$ & $06: 50-08: 50$ & 240 & $\approx 360$ & (s. IRS) \\
\hline IUE SWP & $1150-1980$ & $23.06 .1995,13: 50-24.06 .1995,20: 30$ & 600 & $\approx 2400$ & K. Mukai \\
\hline HST FOS & $1160-1600$ & 12.02 .1995 & $04: 05-14: 20$ & 3.34 & $\approx 13.3$ & S. Rosen \\
& & 15.02 .1995 & $12: 30-22: 40$ & & & \\
\hline
\end{tabular}




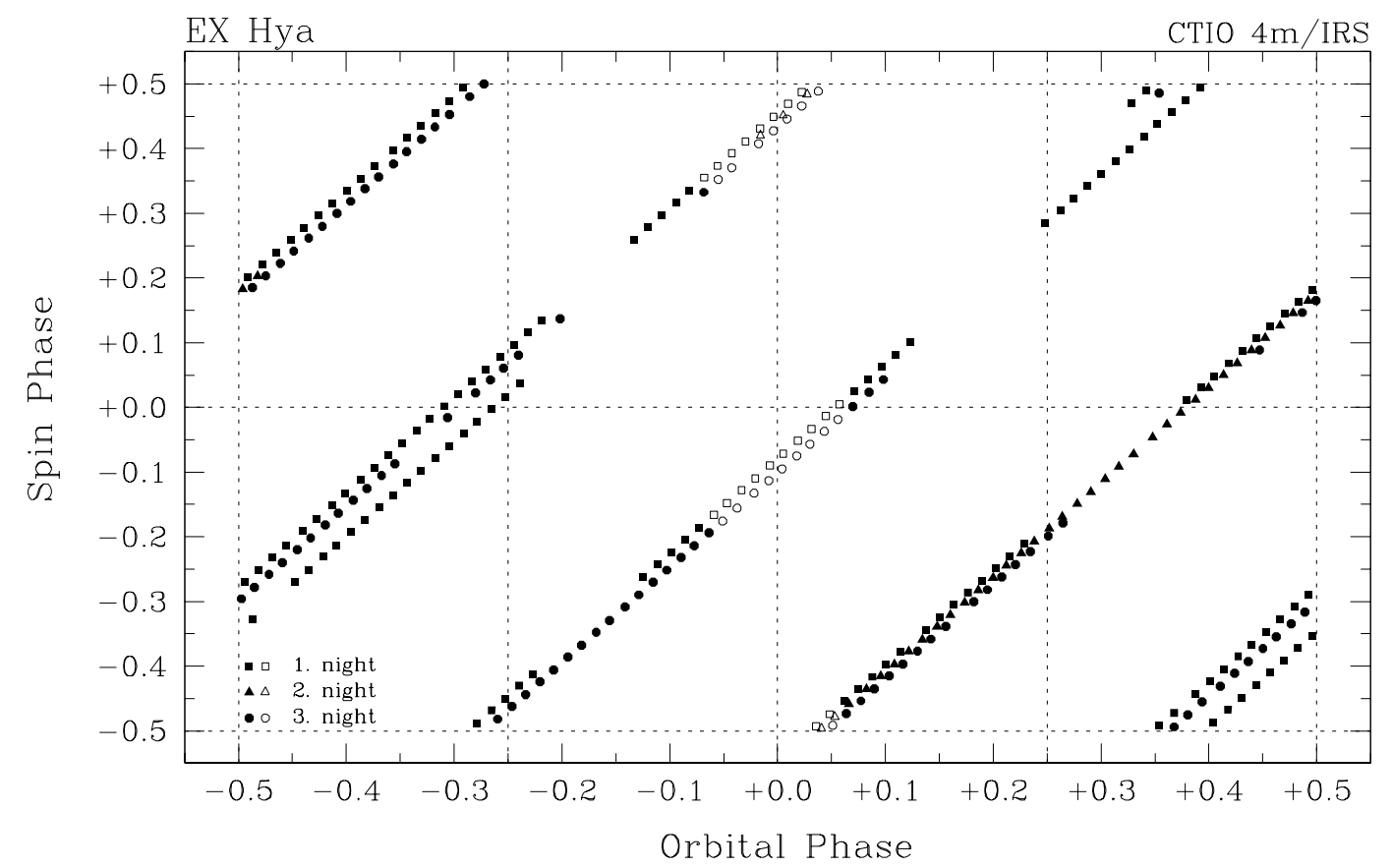

Abbildung 4.1: Lage der IRS-Spektren in der $\Phi_{\text {spin }}-\Phi_{\text {orb }}$-Ebene nach den Ephemeriden von Hellier \& Sproats (1992) (s. Abschnitt 3.2.1). Quadrate, Dreiecke bzw. Kreise: Daten der ersten, zweiten bzw. dritten Nacht (vgl. Tabelle 4.1); offene bzw. ausgefüllte Symbole: Spektrum innerhalb bzw. außerhalb der Dauer der Bedeckung im Infrarot.

entnommen wurden. Alle oben genannten Spektren wurden zeitaufgelöst aufgenommen; EX Hya befand sich dabei in allen Fällen im Ruhezustand ('in quiescence'). In den folgenden Abschnitten werden die einzelnen Datensätze näher beschrieben; ihre Eckdaten sind in Tabelle 4.1 zusammengefasst. Die dort in der Spalte $t_{\text {int }}$ angegebenen Belichtungszeiten der Einzelspektren entsprechen dabei Intervallen der Rotationsphase $\Phi_{\text {spin }}$ des Weißen Zwergs im Bereich $\Delta \Phi_{\text {spin }} \approx 0.015$ (IRS) bis $\Delta \Phi_{\text {spin }} \approx 0.15$ (IUE).

\subsubsection{IRS am 4m-Teleskop auf dem Cerro Tololo, Chile}

Das IRS (für eine Beschreibung des Instruments s. Depoy et al. 1990) ist ein gekühltes Vielzweck-Gitterspektrometer mit einem zweidimensionalen InSb-Detektorarray, das für die Beobachtung des nahen Infrarots im Wellenlängenbereich $\approx 0.8 \ldots 5 \mu \mathrm{m}$ entworfen wurde und spektrale Auflösungen $\lambda / \Delta \lambda$ von $\approx 150 \ldots 3000$ erlaubt. Zur Zeit der Beobachtung war abweichend von der Beschreibung von Depoy et al. (1990) bereits ein neuerer InSb-Detektor der Santa Barbara Research Corporation (SBRC) mit $256 \times 256$ Pixeln sowie ein anderes Reflexionsgitter im Einsatz.

Für die Beobachtungen, die in den drei Nächten vom 22. bis 25.02.1997 durchgeführt wurden, wurde eine Prisma-Gitter-Kombination benutzt, mit der Spektren niedriger Auflösung $(\lambda / \Delta \lambda \approx 560)$ im Wellenlängenbereich $\approx 0.8 \ldots 2.4 \mu \mathrm{m}$ gewonnen werden können. Die Belichtungszeit pro Spektrum betrug $60 \mathrm{~s}$, die Auslesezeiten für jedes Spektrum wenige Sekunden. Insgesamt wurden dabei 324 Spektren gewonnen (156, 56 bzw. 112 in der 
Tabelle 4.2: Journal der EFOSC2-Beobachtungen von EX Hya. Die Werte in den Spalten $\mathrm{HMJD}_{\text {cen }}$ beziehen sich auf den zeitlichen Mittelpunkt der einzelnen Belichtungen.

\begin{tabular}{llll|llll}
\hline Nr. & HMJD $_{\text {cen }}$ & $\Phi_{\text {orb }}$ & $\Phi_{\text {spin }}$ & Nr. & HMJD $_{\text {cen }}$ & $\Phi_{\text {orb }}$ & $\Phi_{\text {spin }}$ \\
\hline 1 & 50508.28982 & $0.843-0.884$ & $0.228-0.287$ & 11 & 50508.33102 & $1.447-1.488$ & $1.113-1.173$ \\
2 & 50508.29434 & $0.909-0.950$ & $0.325-0.385$ & 12 & 50508.33506 & $1.506-1.547$ & $1.200-1.259$ \\
3 & 50508.29838 & $0.969-1.009$ & $0.412-0.472$ & 13 & 50508.33909 & $1.565-1.606$ & $1.286-1.346$ \\
4 & 50508.30242 & $1.028-1.068$ & $0.499-0.558$ & 14 & 50508.34313 & $1.624-1.665$ & $1.373-1.433$ \\
5 & 50508.30646 & $1.087-1.128$ & $0.585-0.645$ & 15 & 50508.34715 & $1.683-1.724$ & $1.460-1.519$ \\
6 & 50508.31050 & $1.146-1.187$ & $0.672-0.732$ & 16 & 50508.35119 & $1.743-1.783$ & $1.546-1.606$ \\
7 & 50508.31454 & $1.205-1.246$ & $0.759-0.819$ & 17 & 50508.35522 & $1.802-1.842$ & $1.633-1.693$ \\
8 & 50508.31858 & $1.265-1.305$ & $0.846-0.905$ & 18 & 50508.35926 & $1.861-1.901$ & $1.720-1.779$ \\
9 & 50508.32261 & $1.324-1.364$ & $0.932-0.992$ & 19 & 50508.36332 & $1.920-1.961$ & $1.807-1.867$ \\
10 & 50508.32663 & $1.383-1.423$ & $1.019-1.078$ & 20 & 50508.36744 & $1.981-2.021$ & $1.896-1.955$ \\
\hline
\end{tabular}

ersten, zweiten bzw. dritten Nacht), von denen aufgrund technischer Probleme allerdings nur 293 brauchbar sind. Von diesen sind wiederum $\approx 15 \%$ wegen durchziehender Wolkenfelder (hauptsächlich in der zweiten und dritten Nacht) von minderer Qualität. Das Seeing betrug 1.0...1.3" in der ersten Nacht und $\approx 1.2 \ldots 1.8^{\prime \prime}$ in der zweiten und dritten Nacht, wobei die höheren Werte jeweils gegen Ende der Nacht erreicht wurden. Die verwendete Spaltbreite betrug in allen Fällen $2^{\prime \prime}$.

Da Spin- und Orbitalperiode mit einem Verhältnis von $\approx 2 / 3$ fast kommensurabel sind, liegen die Spektren einer Nacht in der $\Phi_{\text {spin }}-\Phi_{\text {orb }}$-Ebene alle in einem eng begrenzten, streifenförmigen Gebiet. Da beide Perioden zudem fast kommensurabel mit der Tageslänge sind, sind die Streifen von Spektren, die in aufeinanderfolgenden Nächten gewonnen wurden, nur wenig gegeneinander verschoben. Für die vorliegenden Beobachtungen ergibt sich somit die in Abbildung 4.1 dargestellte Situation, daß zwar Spin- und Orbitalphase jede für sich fast gleichmäßig in den Daten repräsentiert sind, die gesamte $\Phi_{\text {spin }}-\Phi_{\text {orb }}$-Ebene jedoch nur zu einem geringen Teil überdeckt ist, d.h. daß gewisse $\Phi_{\text {spin }}-\Phi_{\text {orb }}-$ Kombinationen also nicht einmal näherungsweise vorkommen.

Die Transparenz der Erdatmosphäre hängt im nahen Infrarot besonders im Bereich der Wasserabsorptionsbanden stark vom Wasserdampfgehalt der Luft in mittleren Atmosphärenschichten ab. Da dieser während einer Nacht und auch räumlich beträchtlich schwanken kann, wurden in jeder Nacht vor Beginn der ersten und nach Ende der letzten Beobachtung von EX Hya sowie nach jeweils $\approx 1 \mathrm{~h} 10 \ldots 20$ Spektren des im selben Himmelsgebiet stehenden Sterns SAO 181198 mit einer Belichtungszeit von 1s aufgenommen. Dieser ist vom Spektraltyp G5 und hat daher im nahen Infrarot außer Wasserstoffabsorptionslinien fast keine prominenten intrinsischen Absorptionsmerkmale. Diese Vergleichsspektren wurden zur Korrektur der atmosphärischen Absorption benutzt (zum Verfahren s. Anhang B.4).

Weiterhin wurden Spektren einer Reihe ausgewählter M-Sterne verschiedener Spektraltypen (s. Tabelle 4.4 in Abschnitt 4.2.2) mit derselben spektralen Auflösung wie EX Hya aufgenommen. Auch hier wurde unmittelbar vor oder nach jedem Objektspektrum das Spektrum eines G-Sterns im selben Himmelsareal zum Vergleich aufgenommen. Diese Sterne sind ebenfalls in Tabelle 4.4 angegeben. Schließlich wurden noch die Spektren der A0- 


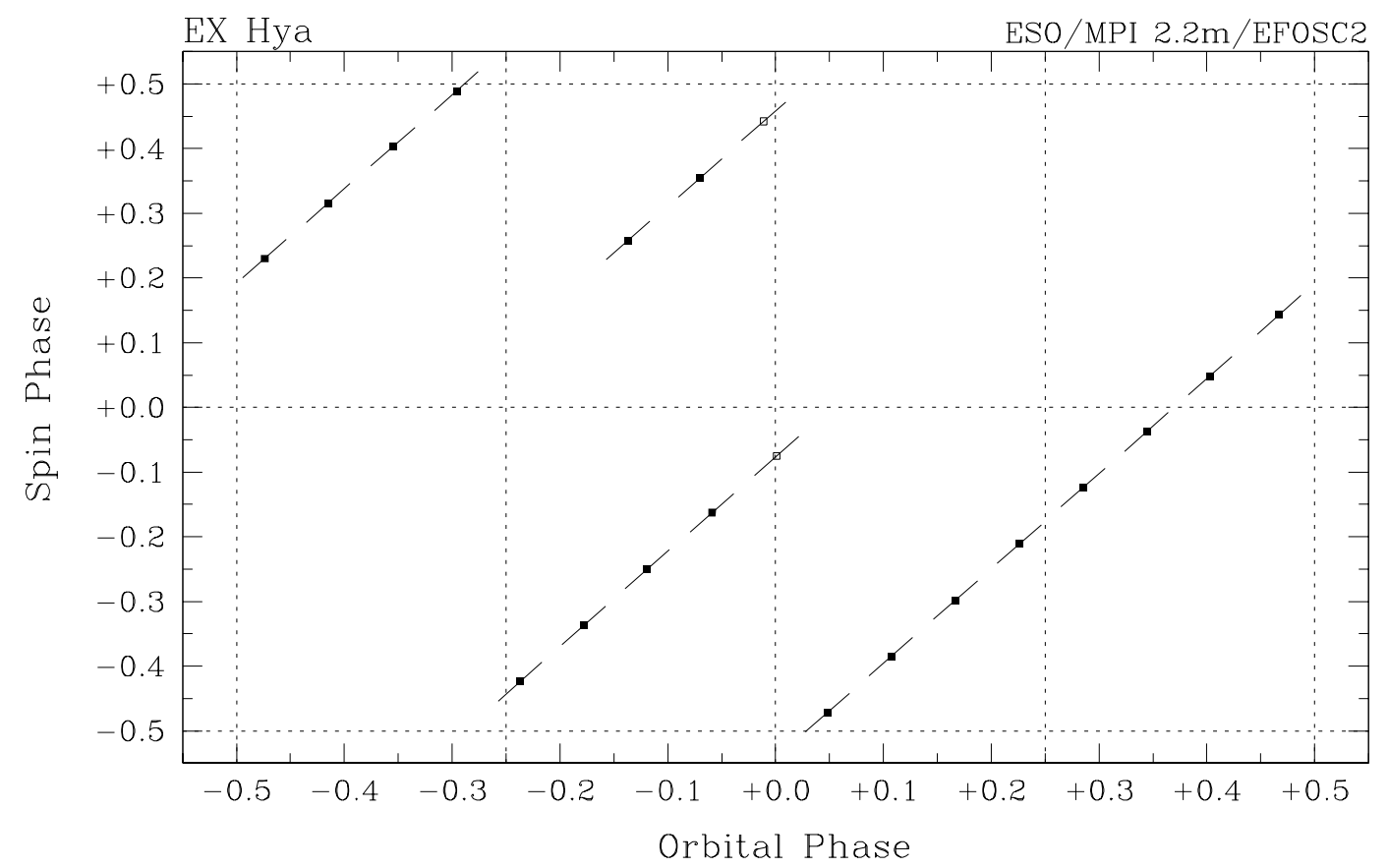

Abbildung 4.2: Lage der EFOSC2-Spektren in der $\Phi_{\text {spin }}-\Phi_{\text {orb }}$-Ebene nach den Ephemeriden von Hellier \& Sproats (1992) (s. Abschnitt 3.2.1). Offenes bzw. ausgefülltes Quadrat: Spektrum während bzw. außerhalb der Bedeckung. Die Punkte geben den zeitlichen Mittelpunkt der einzelnen Beobachtungen an.

Sterne HD 40335 und HD 130163 sowie des A2-Sterns HD 77281 aufgenommen, die zusammen mit den JHK-Helligkeiten dieser Sterne, die in der Liste photometrischer IRStandardsterne von Elias et al. (1982) aufgeführt sind, eine absolute Flußkalibration aller Spektren ermöglichen (s. Anhang B.4).

\subsubsection{EFOSC 2 am 2.2m-Teleskop auf La Silla, Chile}

In der Nacht vom 28.02. auf den 01.03.1997 wurde EX Hya mit EFOSC 2 bei niedriger spektraler Auflösung (G1-Gitter, d.h. $\lambda / \Delta \lambda \approx 150)$ im Wellenlängenbereich 3600...10200 spektrographiert. Abgesehen von einigen leichten Cirrus-Wolken vor allem in Horizontnähe war der Himmel wolkenfrei. Das Seeing betrug zur Zeit der Beobachtung 1.7" und die verwendete Spaltbreite 2". Die so gewonnenen 20 Spektren decken bei einer Belichtungszeit von 4 min pro Spektrum und unter Berücksichtigung der Auslesezeit des Detektors etwa 1.2 Bahnperioden (einschließlich zweier Bedeckungen) bzw. 1.7 Spinzyklen ab (s. Tabelle 4.2 und Abbildung 1.2); die effektive Zeitauflösung beträgt dabei $\approx 6 \mathrm{~min}$ (vgl. Tabelle 4.1). Für die Überdeckung der gesamten $\Phi_{\text {spin }}-\Phi_{\text {orb }}$-Ebene gilt das oben in Abschnitt 4.1.1 Gesagte. 


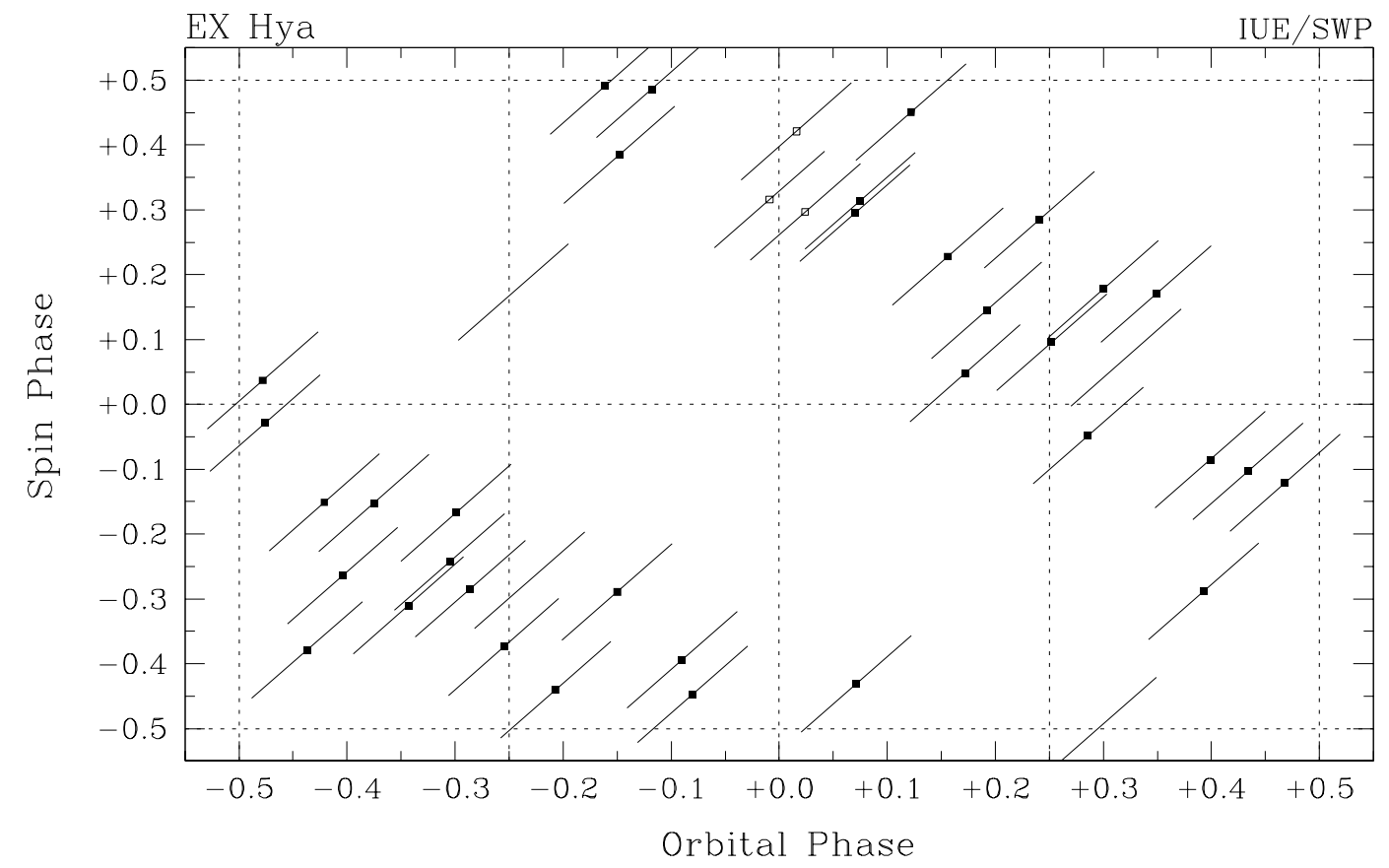

Abbildung 4.3: Lage der IUE-Spektren in der $\Phi_{\text {spin }}-\Phi_{\text {orb }}$-Ebene nach den Ephemeriden von Hellier \& Sproats (1992) (s. Abschnitt 3.2.1). Die Länge der Striche gibt die Integrationszeit von $600 \mathrm{~s}$ wieder. Mit offenem bzw. ausgefülltem Quadrat: Zeitpunkt der Mitte der Aufnahme liegt innerhalb bzw. außerhalb des Zeitraums der optischen bzw. Röntgenbedeckung; ohne Symbol: unbrauchbares Spektrum.

\subsubsection{International Ultraviolet Explorer (IUE)}

Im IUE-Datenarchiv finden sich 174 Spektren von EX Hya, von denen 124 mit der Short Wave Prime Camera (SWP; Wellenlängenbereich 1150...1980 A) durch die große Apertur aufgenommen wurden, d.h. photometrisch sind. Unter letzteren gibt es eine kontinuierliche Serie von 45 Spektren, die von K. Mukai über einen Zeitraum von $\approx 1.3$ Tagen hinweg aufgenommen wurden. Die Belichtungszeit beträgt 600 s pro Spektrum. Aufgrund der langen Auslesedauer des Detektors von $\approx 30$ min sind jedoch entsprechende Lücken zwischen den einzelnen Aufnahmen unvermeidbar, so daß die effektive Zeitauflösung $\approx 40 \mathrm{~min}$ beträgt (s. Tabelle 4.1). Eine Tabelle mit den Startzeiten und überdeckten Phasenintervallen jedes einzelnen Spektrums findet sich bei Mauche (1999). Insgesamt ergibt sich die in Abbildung 4.3 dargestellte Überdeckung der $\Phi_{\text {spin }}-\Phi_{\text {orb }}$-Ebene, wobei der Abstand der beiden prominenten 'Bänder' im wesentlichen der effektiven Zeitauflösung entspricht; die relativ gute Überdeckung entlang der Bänder hat ihren Grund in der langen kontinuierlichen Beobachtungszeit. Bei manueller Durchsicht der Daten wurden vier Spektren gefunden, in denen - vermutlich aufgrund technischer Probleme - nur ein um einen Faktor 5...10 gegenüber allen anderen Spektren reduzierter Fluß vorhanden war. Diese Spektren sind in Abbildung 4.3 als Striche ohne zusätzliches Symbol dargestellt und wurden bei der Datenanalyse nicht verwendet.

Leider enthält das IUE-Archiv keine Serie von vergleichbar gut zeitaufgelösten Spektren 


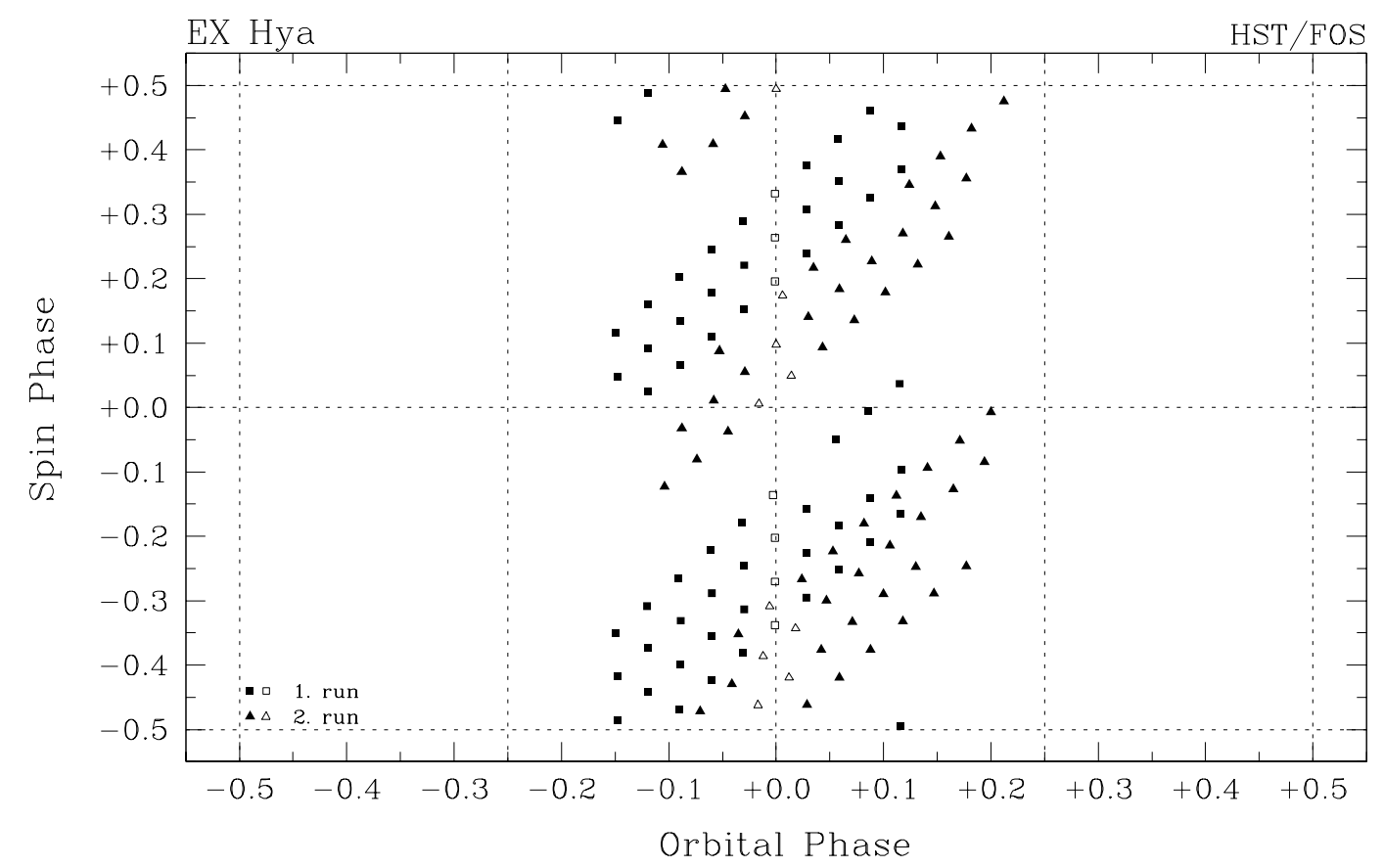

Abbildung 4.4: Lage der HST-Spektren in der $\Phi_{\text {spin }}-\Phi_{\text {orb }}$-Ebene nach den Ephemeriden von Hellier \& Sproats (1992) (s. Abschnitt 3.2.1). Quadrate bzw. Dreiecke: Daten vom 12. bzw. 15.02.1995 (vgl. Tabelle 4.1); offene bzw. ausgefüllte Symbole: Spektrum innerhalb bzw. außerhalb der Dauer der optischen bzw. Röntgenbedeckung. Die Datenpunkte repräsentieren die Phasenlagen der bereits gruppenweise zusammengefassten Spektren (s. Text).

im Wellenlängenbereich der LWP bzw. LWR (Long Wave Prime bzw. Redundant Camera; $1850 \ldots 3350 \AA$ ), jedoch finden sich dort genügend Spektren, um zumindest ein gut definiertes mittleres Spektrum von EX Hya zu konstruieren.

\subsubsection{Hubble Space Telescope (HST)}

EX Hya wurde am 12. sowie am 15. Februar 1995 von S. Rosen mit dem Faint Object Spectrograph (FOS) des HST beobachtet; bislang wurden jedoch noch keine aus diesen Daten gewonnenen Ergebnisse veröffentlicht. Bei der Beobachtung wurden jeweils für eine Zeit von $1600 \mathrm{~s}\left(\approx P_{\text {orb }} / 4\right)$ um die Zeitpunkte von jeweils sieben aufeinanderfolgenden Bedeckungen herum zeitaufgelöste Spektren aufgenommen. Der FOS wurde dabei mit einer Belichtungszeit von $3.3 \mathrm{~s}$ und im schnellen Auslesemodus des Detektor-CCDs (rapid readout mode) betrieben, so daß die effektive Zeitauflösung bei $\lesssim 13.5$ s liegt. Insgesamt liegen somit $14(=2 \times 7)$ Datensätze vor, die aus jeweils 120 Spektren um den Zeitpunkt einer Bedeckung herum bestehen.

Sowohl die kurze Belichtungszeit als auch das schnelle Auslesen des Detektors haben zur Folge, daß das Signal-zu-Rausch-Verhältnis ( $S / N$, signal-to-noise) der einzelnen Spektren nur bei $S / N \approx 2 \ldots 2.5$ liegt. Um dieses zu verbessern und die Anzahl der zu bearbeitenden Spektren in überschaubarem Rahmen zu halten, wurden - da die hohe Zeitauflösung dieser 
Daten für die vorliegende Arbeit ohnehin nicht erforderlich ist - in jedem Datensatz jeweils zwölf aufeinanderfolgende Spektren gemittelt, so daß letztlich $2 \times 7 \times 10$ Spektren mit einer effektiven Zeitauflösung von $160 \mathrm{~s}$ und $S / N \approx 7 \ldots 9$ zur Verfügung standen. Ich teile dies im Vorgriff auf die Beschreibung der Datenanalyse in Abschnitt 4.2 .4 bereits hier mit, da sich die in Abbildung 1.4 dargestellten Phasenlagen der Spektren im Gegensatz zu den in Tabelle 4.1 angegebenen Werte auf diese bereits zusammengefassten Spektren beziehen. Abbildung 1.4 zeigt, daß die verschiedenen Spinphasen in diesen Daten relativ gleichmäßig vertreten sind, wohingegen die Orbitalphasen auf den Bereich 0.85...1.20 beschränkt sind, diesen aber auch für die meisten Spinphasen fast gleichmäßig abdecken.

\subsection{Datenanalyse}

\subsubsection{Verwendete Hard- und Software}

Die Analyse der in den vorigen Abschnitten vorgestellten Daten wurde zu einem großen Teil auf einer DEC AXP 3000-Workstation der Universitätssternwarte Göttingen mit dem Betriebssystem Open VMS unter Verwendung des von der ESO entwickelten Programmpakets MIDAS (Munich Image Data Analysis System; Version November 1995) durchgeführt. Die unreduzierten IRS-Spektren haben - bedingt durch die Bauart des Instruments - allerdings Eigenschaften, aufgrund derer sie den MIDAS-Standardroutinen, die primär für die Reduktion optischer Spektren geschrieben wurden, nur schwer oder gar nicht zugänglich sind. Aus diesem Grund habe ich für fast jeden Schritt der Reduktion der IRS-Spektren eigene Programme in der Sprache C entwickelt, die den Eigenarten der Daten Rechnung tragen und eine effiziente und differenziert steuerbare Bearbeitung erlauben, und diese Routinen unter Benutzung der C-Programmierschnittstelle des MIDAS-Pakets in das System eingebunden. Die besagten Eigenarten der Rohdaten und die Verfahrensschritte ihrer Reduktion werden ausführlich in Anhang B dargestellt.

Für die Analyse der Lichtkurven, die in Abschnitt 4.2.7 beschrieben wird, habe ich hingegen das kommandozeilenorientierte Datenvisualisierungsprogramm 'Supermongo' eingesetzt, das neben seinen graphischen Fähigkeiten auch recht komfortable Mechanismen der Datenmanipulation z.B. zur Anpassung von Modellen bereitstellt. Mit Hilfe eines von mir hierfür entwickelten Makro-Pakets können Modelle gleichzeitig angepaßt und zusammen mit den Daten graphisch dargestellt werden. Eine Auswahl der anzupassenden Daten z.B. nach Phasenintervallen sowie ein schneller Wechsel des anzupassenden Modells bzw. seiner Komponenten und der Darstellungsart sind damit ebenfalls möglich, was es erlaubt, die Daten flexibel unter verschiedenen Aspekten zu untersuchen, ohne daß hierfür die Dateien, in denen sie gespeichert sind, verändert oder gar neu angelegt werden müßten. Letzteres hält vor allem die Anzahl anzupassender Datensätze überschaubar und diese untereinander konsistent; nicht zuletzt erhöht es wesentlich die Geschwindigkeit, mit der mit verschiedenen Modellvarianten, Untermengen der Daten usw. experimentiert werden kann. 


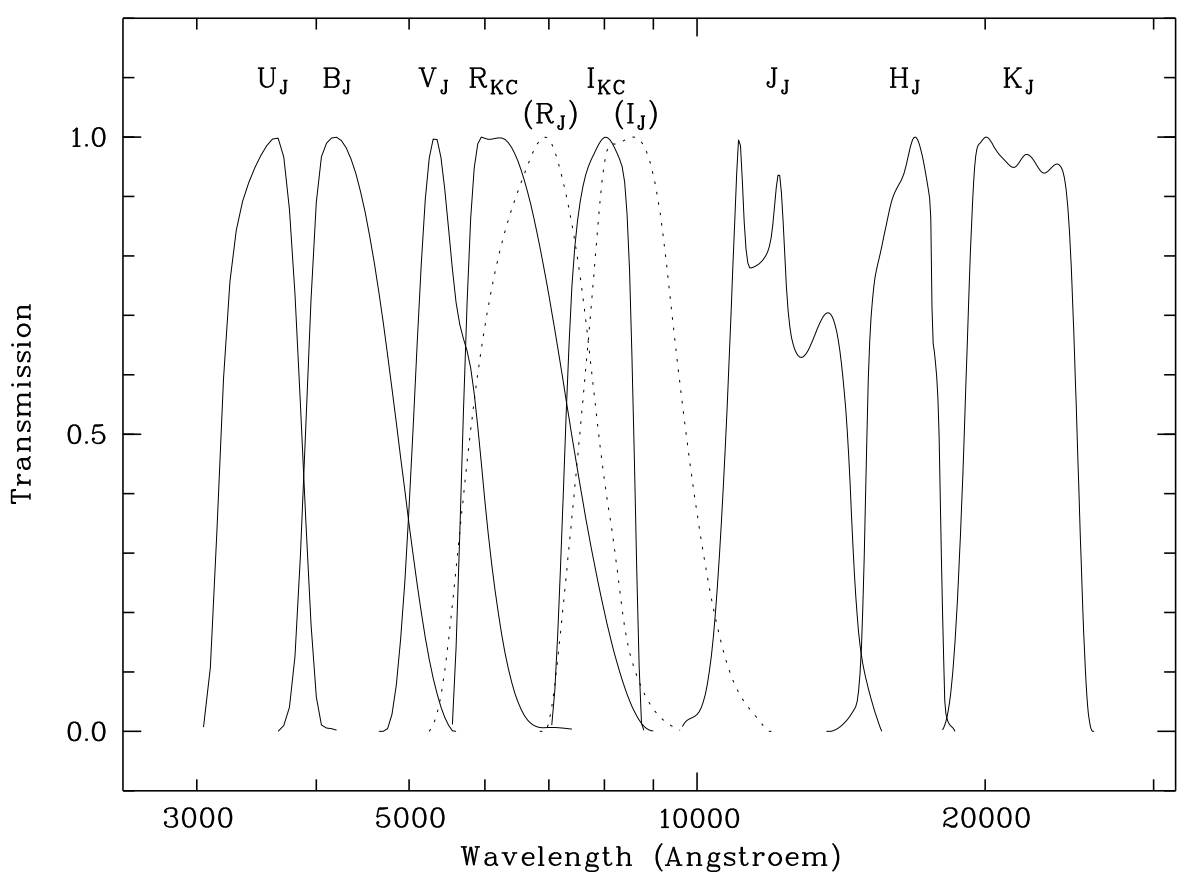

Abbildung 4.5: Verwendete Transmissionsfunktionen der photometrischen Bänder (auf 1 normiert). $\mathrm{R}_{\mathrm{KC}}$ und $\mathrm{I}_{\mathrm{KC}}$ beziehen sich auf das Kron-Cousins-System, alle anderen auf das Johnson-System. $\mathrm{R}_{\mathrm{J}}$ und $\mathrm{I}_{\mathrm{J}}$ (gepunktete Kurven) werden heute nicht mehr verwendet.

\subsubsection{Spektren der beobachteten M-Sterne}

Die Spektren der in Tabelle 4.4 aufgeführten M-Sterne wurden zunächst mit dem in Anhang B beschriebenen Verfahren reduziert. Als Vergleichssterne für die atmosphärische Korrektur dienten die in der Tabelle angegebenen Objekte. Anschließend wurden aus den fertig kalibrierten IRS-Spektren und optischen Spektren der Objekte, die im Rahmen diverser anderer Beobachtungen in unserer Gruppe gewonnen wurden, zusammengesetzte Spektren erzeugt, die den Bereich $\approx 5000 \ldots 25000 \AA$ umfassen. Für die Sterne Gl382 bzw. Gl443 waren keine optischen Spektren verfügbar; in diesen beiden Fällen wurden auf die Spektren der Sterne Gl393 bzw. Gl207 1 zurückgegriffen, die nach Leggett (1992) vom selben Spektraltyp wie Gl382 bzw. Gl443 sind. Die vorhandenen optischen Spektren sind zwar auf atmosphärische Absorption korrigiert, jedoch wurde die Absorption durch Was-

Tabelle 4.3: Flußkonstanten $f_{0}$ der verwendeten photometrischen Bänder (in Einheiten von $10^{-9} \mathrm{erg} \mathrm{cm}^{-2} \mathrm{~s}^{-1} \AA^{-1}$ ) und Effektivwellenlängen $\lambda_{\text {eff }}$ für ein A0V-Stern-Spektrum (Leggett et al. 1996) sowie Halbwertsbreiten der Transmissionsfunktionen aus Abbildung 4.5 .

\begin{tabular}{lrrrrrrrr}
\hline Band & \multicolumn{1}{c}{$\mathrm{U}_{\mathrm{J}}$} & \multicolumn{1}{c}{$\mathrm{B}_{\mathrm{J}}$} & \multicolumn{1}{c}{$\mathrm{V}_{\mathrm{J}}$} & $\mathrm{R}_{\mathrm{KC}}$ & $\mathrm{I}_{\mathrm{KC}}$ & $\mathrm{J}_{\mathrm{J}}$ & \multicolumn{1}{c}{$\mathrm{H}_{\mathrm{J}}$} & $\mathrm{K}_{\mathrm{J}}$ \\
\hline$\lambda_{\text {eff }}[\AA]$ & 3650 & 4360 & 5450 & 6410 & 7990 & 12210 & 16310 & 21890 \\
FWHM $[\AA]$ & 685 & 970 & 870 & 1720 & 1300 & 3640 & 2860 & 5950 \\
$f_{0}$ & 4.27 & 5.99 & 3.65 & 2.23 & 1.13 & 0.325 & 0.120 & 0.0423 \\
\hline
\end{tabular}


Tabelle 4.4: Photometrische Helligkeiten der beobachteten M-Sterne. Die gemessenen Werte wurden nach (4.1) unter Verwendung der Transmissionskurven in Abbildung 4.5 aus den aus optischen und IRS-Spektren zusammengesetzten Spektren bestimmt (s. Text). Die B-Magnituden (und im Fall von Gl382 die V-Magnitude) sind aufgrund der nötigen Extrapolation der Spektren zu kurzen Wellenlängen hin unsicher (vgl. Abbildung 4.11). Die Literaturwerte und Spektraltypen sind aus Leggett (1992) entnommen, der Spektraltyp von Gl443 stammt von Beuermann (2000c). Der in Klammern unter jedem Objektnamen angegebene Stern diente jeweils als Vergleichsstern für die Korrektur der atmosphärischen Extinktion, s. Text.

\begin{tabular}{|c|c|c|c|c|c|c|c|c|}
\hline \multirow{2}{*}{$\begin{array}{l}\text { Objekt SpTyp } \\
\text { (atm. Vergl.stern) }\end{array}$} & & \multicolumn{7}{|c|}{ Band } \\
\hline & & $\mathrm{B}_{\mathrm{J}}$ & $\mathrm{V}_{\mathrm{J}}$ & $\mathrm{R}_{\mathrm{KC}}$ & $\mathrm{I}_{\mathrm{KC}}$ & $\mathrm{J}^{(2)}$ & $\mathrm{H}^{(2)}$ & $\mathrm{K}^{(2)}$ \\
\hline \multirow{3}{*}{$\begin{array}{l}\mathrm{Gl}^{(1 a)} \quad \mathrm{dM} 2.0 \\
(\mathrm{SAO} 137387, \mathrm{G} 0)\end{array}$} & $m_{\mathrm{obs}}$ & & $(9.36)$ & 8.27 & 7.00 & 5.93 & 5.34 & 5.04 \\
\hline & $m_{\text {Lit }}$ & 10.76 & 9.26 & 8.27 & 7.09 & 5.93 & 5.29 & 5.09 \\
\hline & $\Delta m_{\text {obs-Lit }}$ & & $(0.10)$ & 0.00 & -0.09 & 0.00 & 0.05 & -0.05 \\
\hline \multirow{3}{*}{$\begin{array}{l}\operatorname{Gl}_{443}^{(1 b)} \quad \text { dM3.0 } \\
\text { SAO 156870, G0) }\end{array}$} & $m_{\mathrm{obs}}$ & $(13.20)$ & 11.70 & 10.64 & 9.31 & 7.93 & 7.33 & 7.10 \\
\hline & $m_{\mathrm{Lit}}$ & 13.19 & 11.69 & 10.64 & 9.28 & 7.92 & 7.35 & 7.12 \\
\hline & $\Delta m_{\mathrm{obs}-\mathrm{Lit}}$ & $(0.01)$ & 0.01 & 0.00 & 0.03 & 0.01 & -0.02 & 0.02 \\
\hline \multirow{3}{*}{$\begin{array}{l}\text { Gl402 } \\
\text { (SAO 118548, G5) }\end{array}$} & $m_{\mathrm{obs}}$ & $(13.21)$ & 11.65 & 10.44 & 8.89 & 7.31 & 6.70 & 6.41 \\
\hline & $m_{\text {Lit }}$ & 13.30 & 11.65 & 10.44 & 8.86 & 7.30 & 6.69 & 6.42 \\
\hline & $\Delta m_{\text {obs-Lit }}$ & $(-0.09)$ & 0.00 & 0.00 & 0.03 & 0.01 & 0.01 & -0.01 \\
\hline \multirow{3}{*}{$\begin{array}{l}\text { Gl285 dM4.5e } \\
(\text { SAO 115869, G5) }\end{array}$} & $m_{\mathrm{obs}}$ & $(12.69)$ & 11.16 & 9.89 & 8.23 & 6.59 & 6.01 & 5.72 \\
\hline & $m_{\mathrm{Lit}}$ & 12.76 & 11.15 & 9.89 & 8.20 & 6.59 & 5.99 & 5.73 \\
\hline & $\Delta m_{\mathrm{obs}}$ & $(-0.07)$ & 0.01 & 0.00 & 0.03 & 0.00 & 0.02 & -0.01 \\
\hline \multirow{3}{*}{$\begin{array}{l}\text { G1473AB dM5.5e } \\
\text { (SAO 119471, G5) }\end{array}$} & $m_{\text {obs }}$ & $(14.22)$ & 12.51 & 10.90 & 8.98 & 6.97 & 6.37 & 6.06 \\
\hline & $m_{\text {Lit }}$ & 14.30 & 12.46 & 10.90 & 8.92 & 6.96 & 6.39 & 6.06 \\
\hline & $\Delta m_{\text {obs-Lit }}$ & $(-0.08)$ & 0.05 & 0.00 & 0.06 & 0.01 & -0.02 & 0.00 \\
\hline
\end{tabular}

$(1 a),(1 b)$ vgl. Abbildung 4.6 bzw. Abbildung 4.7 und Text.

(2) gemessene Werte: Johnson-System; Literaturwerte: CIT-System

serdampf, die im Gegensatz zur Absorption durch Sauerstoff nicht streng mit der absorbierenden Säulendichte entlang des Sehstrahls skaliert, offenbar nur unvollständig erfasst. (Bei $\approx 9300 \AA$ beginnt eine besonders starke Absorptionsbande des Wasserdampfs, die ein plötzliches Abfallen des Flusses verursacht, vgl. Abbildungen 4.8 und 4.9.) Aus diesem Grund wurde von den optischen Spektren jeweils nur der Teil unterhalb von $\lambda \approx 9300 \AA$ verwendet und ggf. vorhandene längerwellige Teile verworfen. Es sei noch angemerkt, daß im optischen Spektrum des Sterns Gl2071 (Abbildung 4.7) die Absorption des atmosphärischen Sauerstoffs bei $\approx 7600 \AA$ ('A-Band') noch deutlich zu erkennen ist, d.h. dieses Spektrum gar nicht oder unzureichend korrigiert wurde.

Innerhalb des Bereichs, in dem die Teilspektren überlappen, wurden diese anschließend gemittelt. Um einen 'weichen' Übergang zu erzeugen, wurde dabei eine lineare Gewichtung der Teilspektren mit einem Gewicht von 1 bzw. 0 am 'inneren' Ende des Übergangsbereichs bzw. am Ende des Spektrums durchgeführt. Dies ist in den Abbildungen 4.6 bis 4.10 durch die Funktionen am oberen Rand der Abbildungen angedeutet. Für die Flußanpassung der beiden Teilspektren wurden die von Leggett (1992) angegeben photometrischen Helligkeiten 


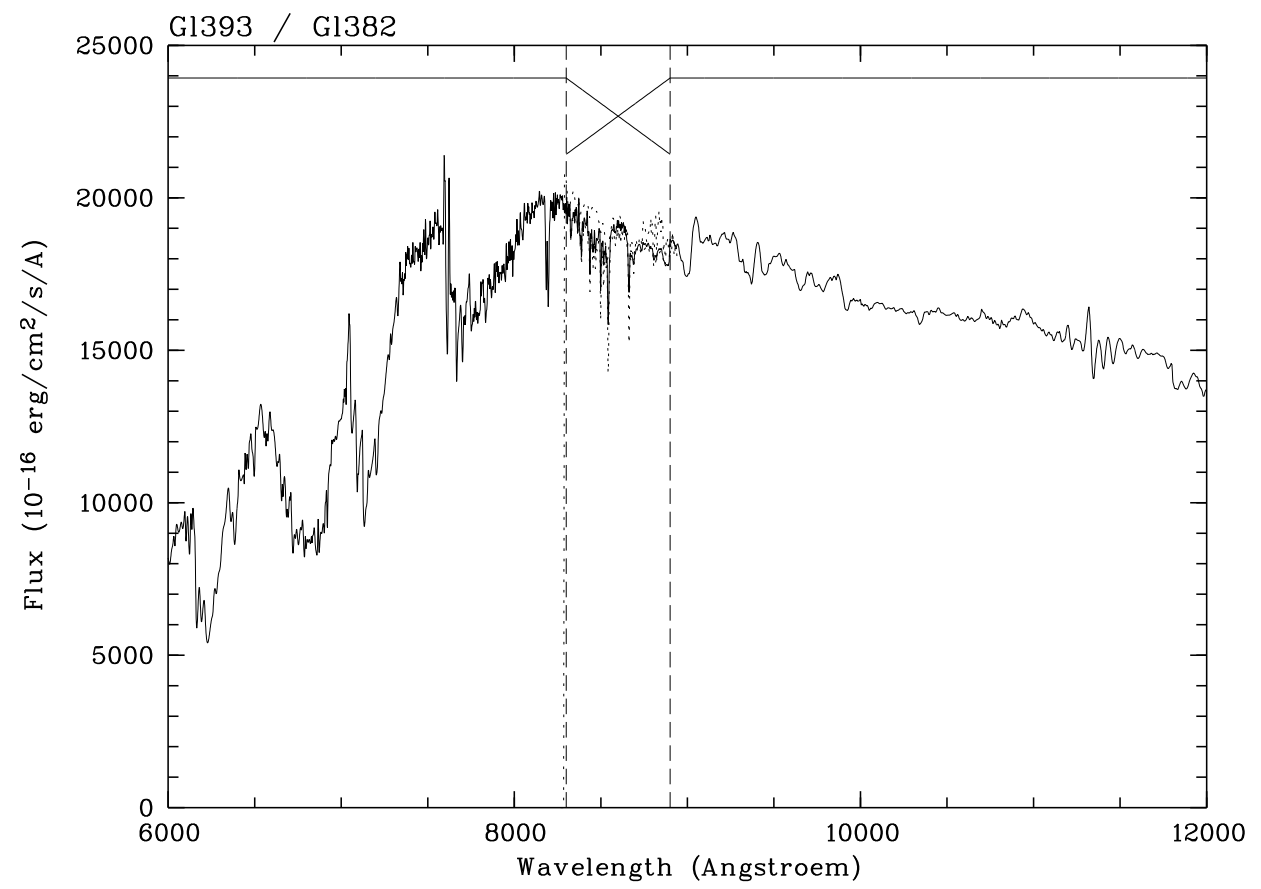

Abbildung 4.6: Verbindung von Infrarot- und optischem Spektrum des M2.0-Sterns Gl382 (s. Text). Für den optischen Teil wurde das Spektrum des Sterns Gl393 verwendet. Die senkrechten gestrichelten Linien geben die Grenzen des Mittelungsbereichs an. Die Gewichtungsfunktionen für die Teilspektren werden durch die ausgezogenen Linien am oberen Bildrand angedeutet; sie haben jeweils den Wert 1 außerhalb des Mittelungsbereichs und sinken zum Ende des Spektrums auf den Wert 0 ab. Das ausgezogene Spektrum ist das resultierende Gesamtspektrum. Die gepunkteten Kurven im Mittelungsbereich stellen die beiden Teilspektren dar; eventuell über den Mittelungsbereich hinausragende Teile der Teilspektren wurden im Gesamtspektrum nicht berücksichtigt.

der Objekte benutzt (s. Tabelle 4.4). Allgemein ist die photometrische Magnitude X eines Objekts mit dem spektralen Fluß $f(\lambda)$ gegeben durch

$$
\operatorname{mag}_{\mathrm{X}}=-2.5 \cdot \log \frac{f_{\mathrm{X}}}{f_{0, \mathrm{X}}} \equiv-2.5 \cdot \log \left(\frac{1}{f_{0, \mathrm{X}}} \frac{\int f(\lambda) T_{\mathrm{X}}(\lambda) d \lambda}{\int T_{\mathrm{X}}(\lambda) d \lambda}\right)
$$

mit der Transmissionsfunktion $T_{\mathrm{X}}(\lambda)$ des Filter-Photometer-Systems im Band $\mathrm{X}$ und dem zugehörigen Magnitude-0-Fluß $f_{0, \mathrm{X}}$ (vgl. Tabelle 4.3). Anwendung dieser Rechenvorschrift auf die Spektren der M-Sterne unter Verwendung der in Abbildung 4.5 gezeigten Transmissionsfunktionen $T_{\mathrm{X}}(\lambda)$ lieferte Magnituden $m_{\mathrm{obs}}$, die mit den Literaturwerten $m_{\text {Lit }}$ von Leggett (1992) verglichen wurden. Aus dem Vergleich wurden dann Skalierungsfaktoren für die Teilspektren derart bestimmt, daß eine möglichst gute Übereinstimmung beider Werte erzielt wurde. Die nach Anwendung der gesamten Prozedur resultierenden Helligkeiten $m_{\mathrm{obs}}$ sowie die noch verbleibenden Abweichungen $\Delta m_{\mathrm{obs}-\mathrm{Lit}}$ sind ebenfalls in Tabelle 4.4 aufgeführt. Die Spektren aller fünf Sterne im Spektralbereich $\approx 5000-25000 \AA$ sowie ein Überblick über die spektralen Merkmale, die für M-Sterne charakteristisch sind, sind in Abbildung 4.11 dargestellt. Man beachte dort vor allem die systematische Veränderung der Form der Spektren mit dem Spektraltyp im Bereich $\lambda \gtrsim 17000 \AA$. 


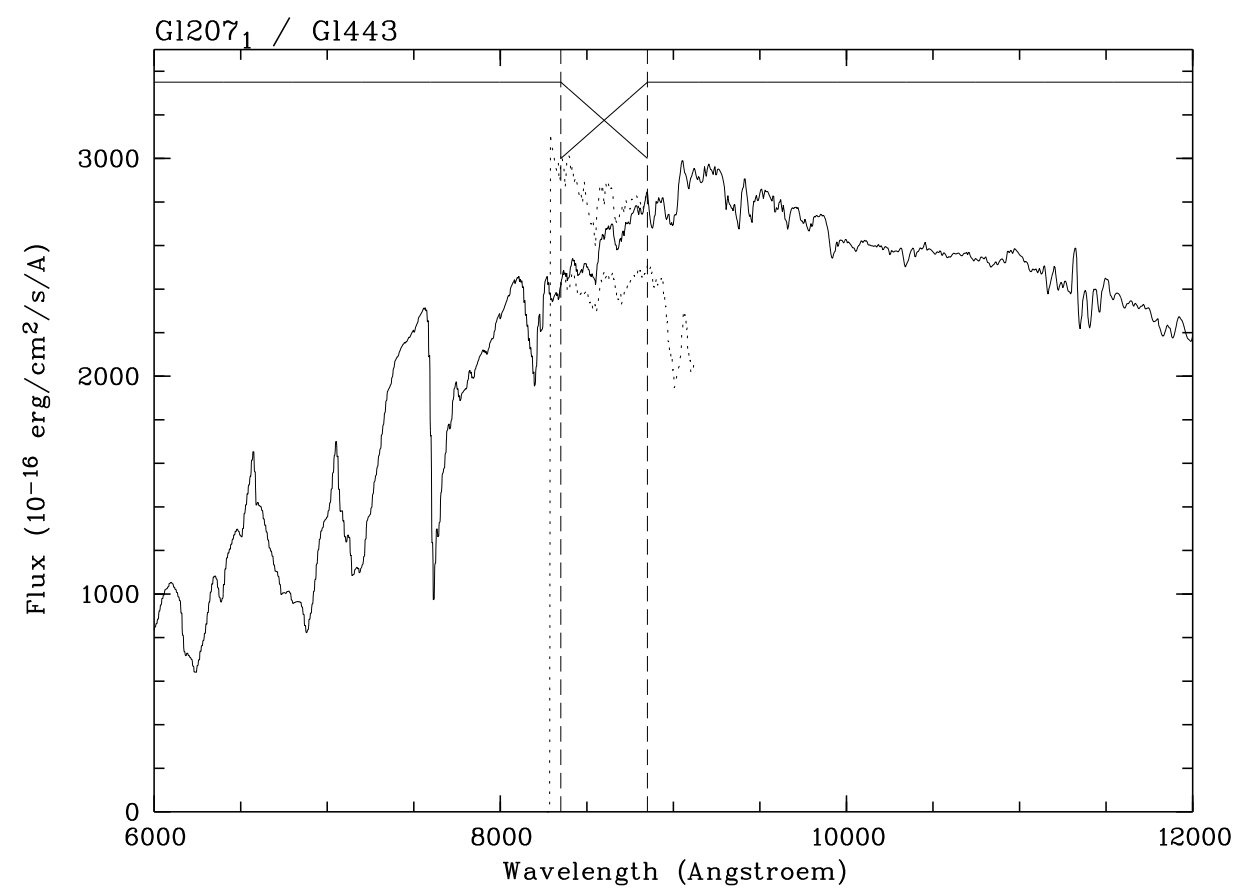

Abbildung 4.7: Verbindung von Infrarot- und optischem Spektrum des M3.0-Sterns G1443 (s. Text). Für den optischen Teil wurde das Spektrum des Sterns G1393 verwendet. Zur Bedeutung der Linien und Kurven s. Bildunterschrift zu Abbildung 4.6.

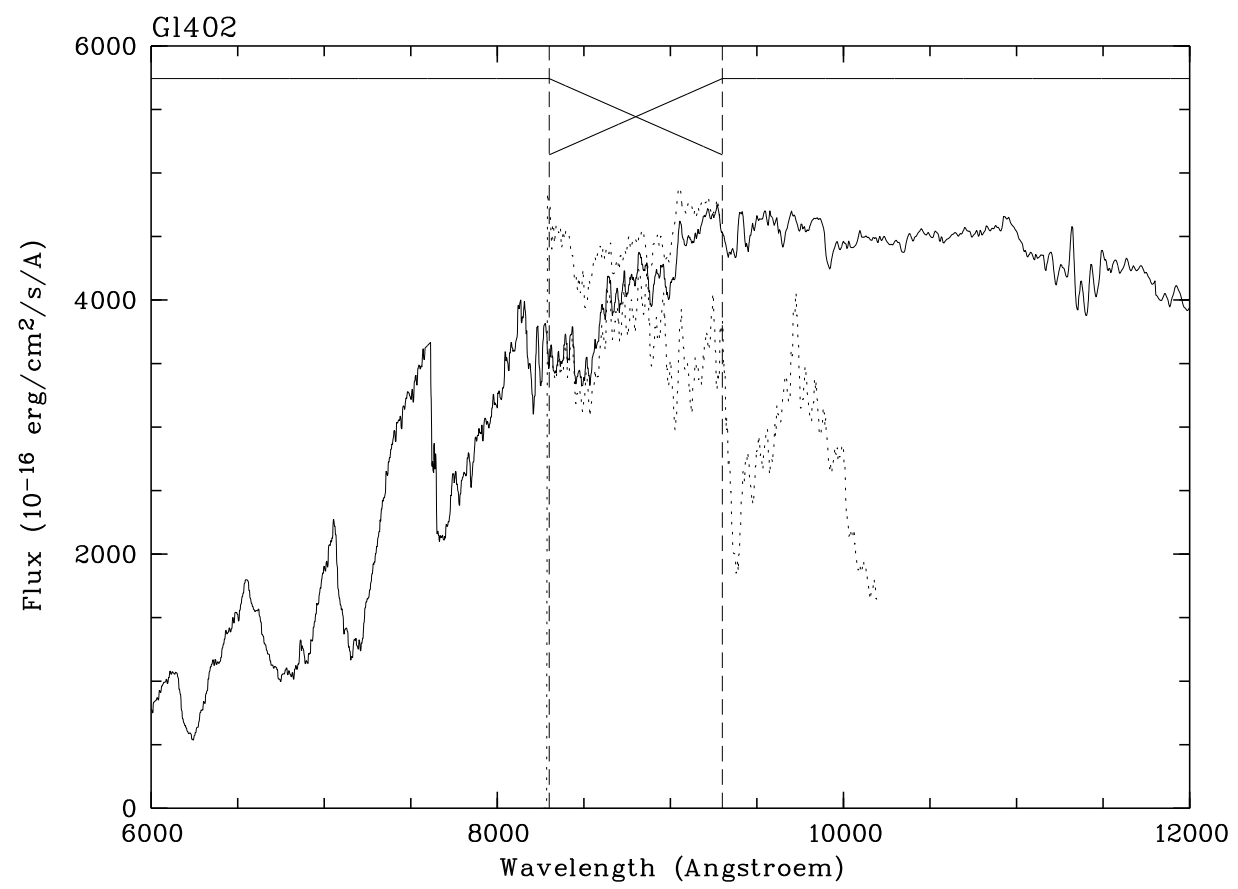

Abbildung 4.8: Verbindung von Infrarot- und optischem Spektrum des M4.0-Sterns Gl402. Der Teil des optischen Spektrums mit $\lambda>9300 \AA$ wurde verworfen (s. Text). Zur Bedeutung der Linien und Kurven s. Bildunterschrift zu Abbildung 4.6. 


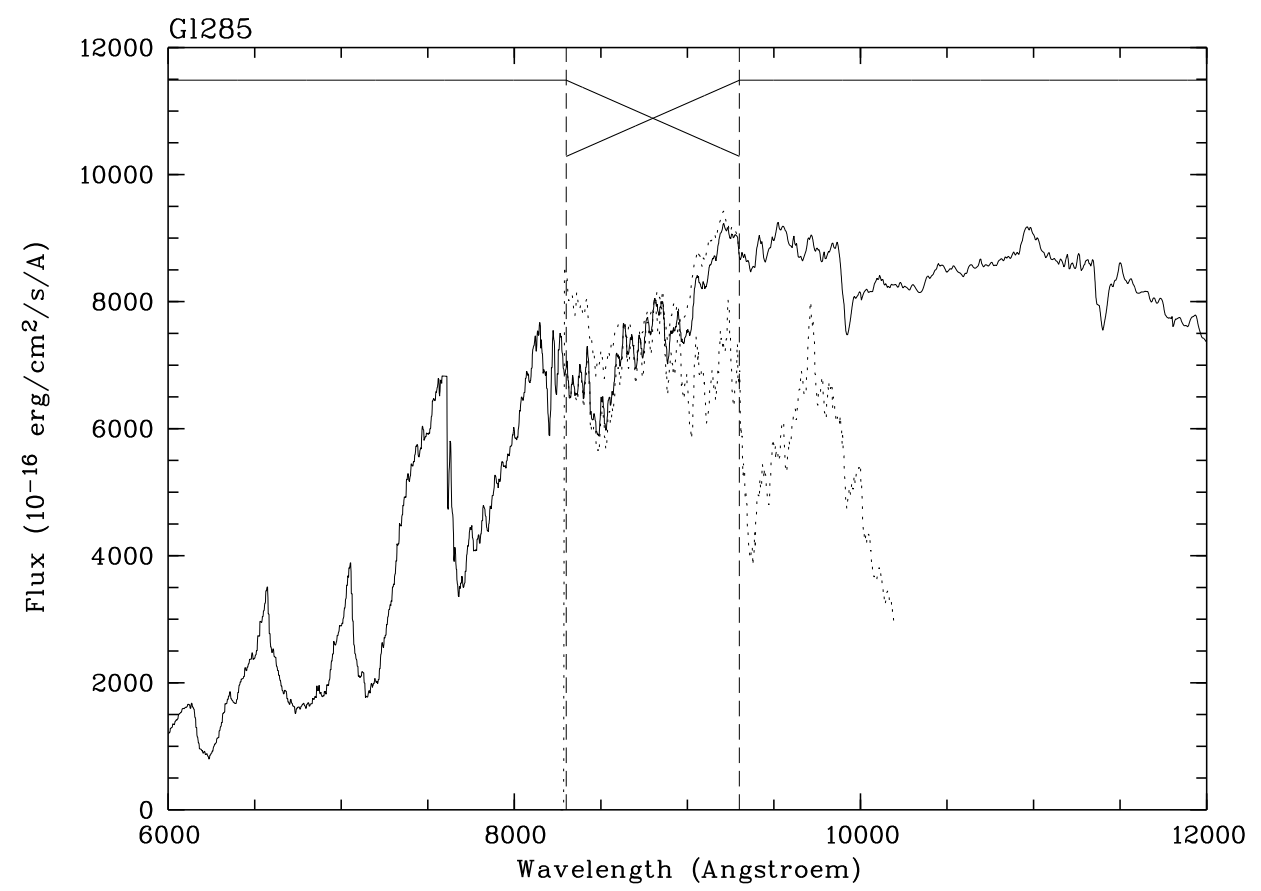

Abbildung 4.9: Verbindung von Infrarot- und optischem Spektrum des M4.5e-Sterns Gl285. Der Teil des optischen Spektrums mit $\lambda>9300 \AA$ wurde verworfen (s. Text). Zur Bedeutung der Linien und Kurven s. Bildunterschrift zu Abbildung 4.6.

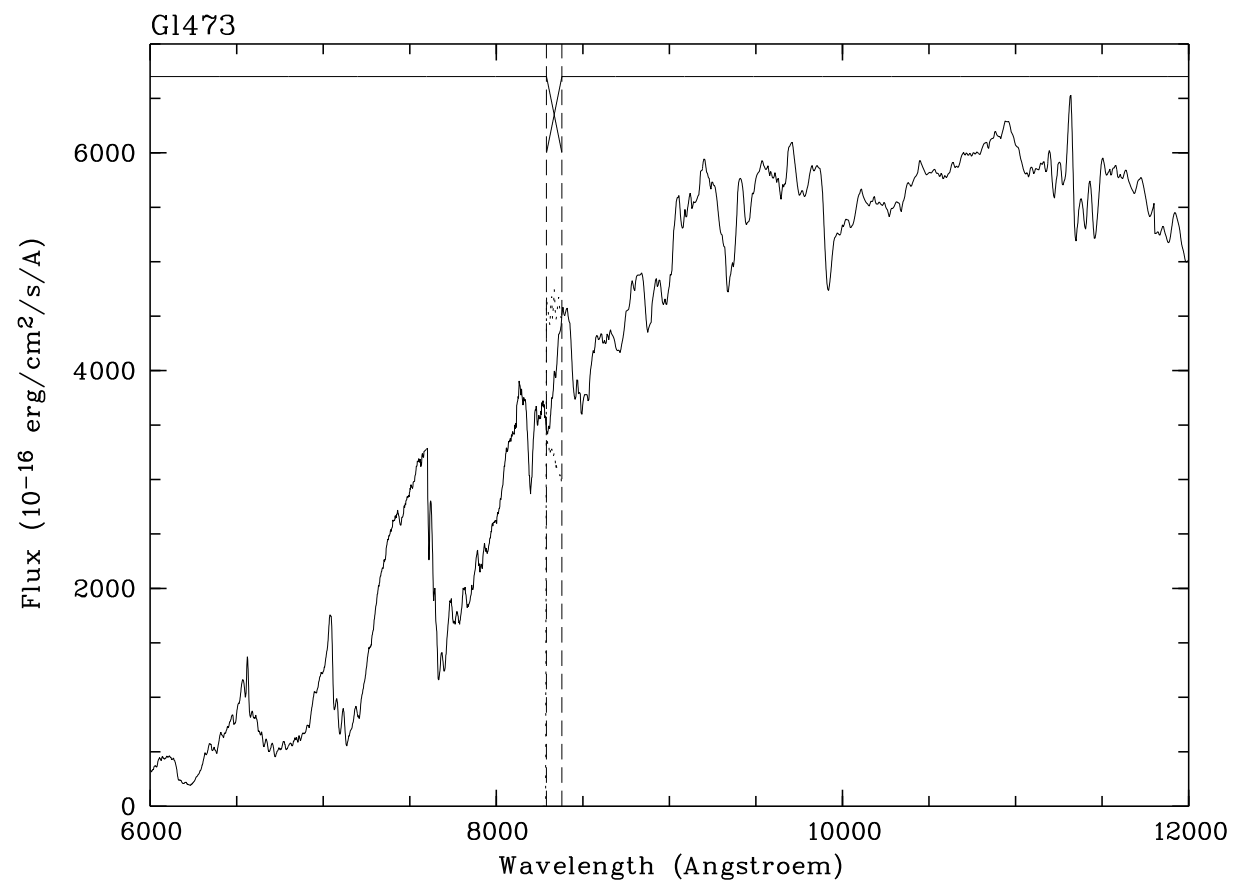

Abbildung 4.10: Verbindung von Infrarot- und optischem Spektrum des M5.5e-Sterns G1473 (s. Text). Zur Bedeutung der Linien und Kurven s. Bildunterschrift zu Abbildung 4.6. 


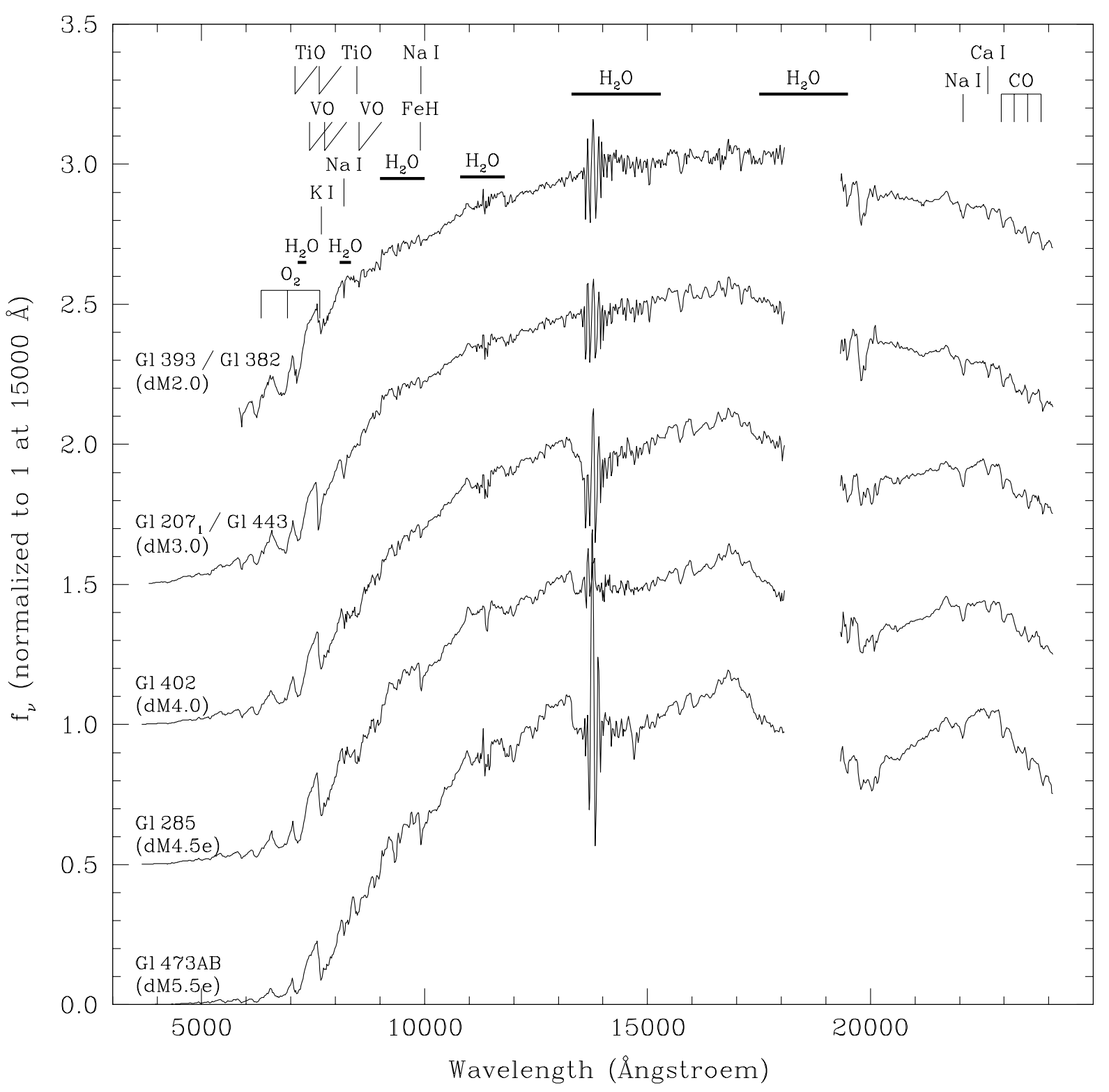

Abbildung 4.11: Spektren der untersuchten roten Zwerge aus Tabelle 4.4. Die Flüsse wurden auf den Wert 1 bei $\lambda=15000 \AA$ normiert und der Übersichtlichkeit halber um jeweils 0.5 Einheiten vertikal versetzt. Am oberen Bildrand ist die Lage charakteristischer Merkmale von M-Sternen (Absorptionsbanden von TiO und VO, Absorptionslinien von $\mathrm{CO}, \mathrm{FeH}$ und neutralen Metallen) sowie der atmosphärischen Sauerstoff- und der Wasserdampfabsorptionsbanden dargestellt. Das starke Rauschen bei $\approx 14000 \AA$ ist ein Artefakt, das durch die Korrektur der dort besonders starken Extinktion durch atmosphärischen Wasserdampf entsteht (s. Anhang B.4). Die zu späten Spektraltypen hin stärker werdenden Einsenkungen bei $\approx 20000 \AA$ und $\approx 25000 \AA$ werden durch Wassermoleküle in den Sternatmosphären verursacht. Der Bereich 18000...19000 A ist mit dem IRS in der benutzten Konfiguration nicht beobachtbar (s. Anhang B.1). 


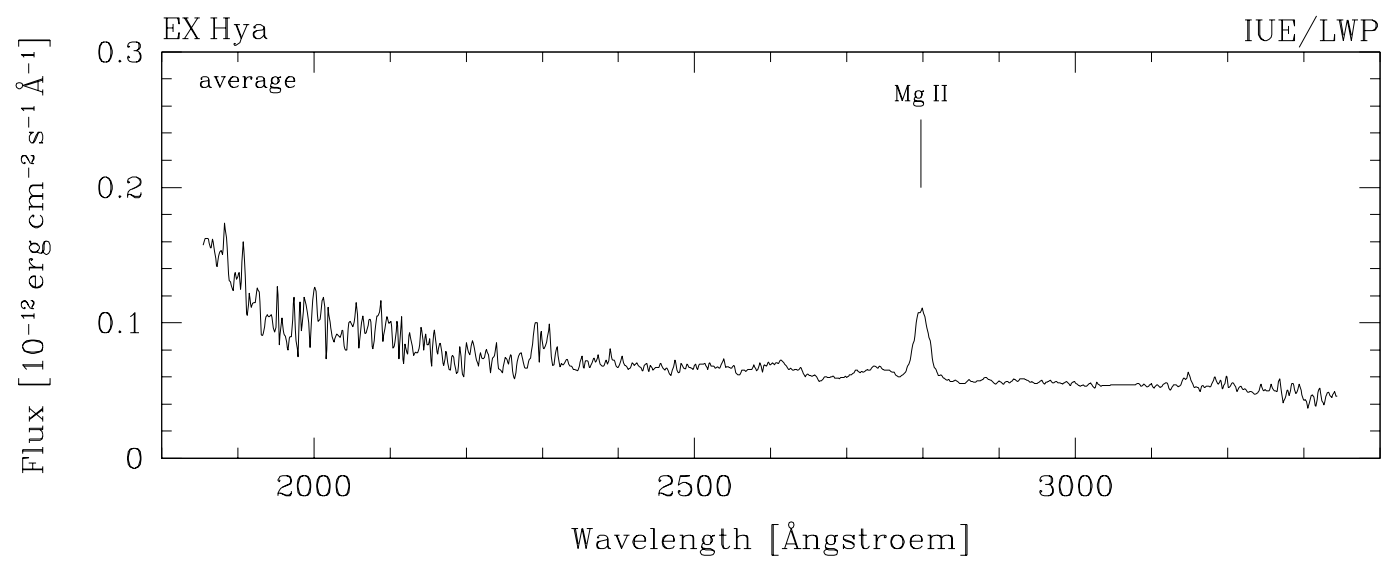

Abbildung 4.12: Mittleres IUE/LWP-Spektrum von EX Hya.

\subsubsection{IUE-Spektren von EX Hya}

Die Spektren im IUE-Datenarchiv liegen dort in fertig reduzierter und kalibrierter Form vor und erfordern daher keine weitere vorbereitende Bearbeitung mehr. In Ermangelung von hinreichend gut zeitaufgelösten LWP- oder LWR-Spektren konnte für den Wellenlängenbereich 1850-3350 A nur ein mittleres Spektrum hergestellt werden, wobei aus den gesamten verfügbaren Archivdaten nur LWP-Spektren mit hinreichendem Signal-zu-Rausch-Verhältnis für die Mittelung benutzt wurden. Einige Spektren lassen aufgrund des hohen Flusses vermuten, daß sie während eines vom Normalen abweichenden Zustands von EX Hya aufgenommen wurden, und wurden daher nicht verwendet. Abbildung 4.12 zeigt das sich ergebende Spektrum.

Aus den 41 SWP-Spektren, die nicht offensichtlich defekt sind (vgl. Abschnitt 4.1.3), wurden ein mittleres Spektrum sowie je ein Spektrum für EX Hya während des Maximums bzw. Minimums der Spinlichtkurve konstruiert. Hierbei gilt es, systematische Verfälschungen dieser Spektren zu korrigieren, die durch einen Auswahleffekt hervorgerufen werden. Da das Problem in mehr oder weniger derselben Form auch bei der Analyse der Spektren der anderen drei Instrumente (HST, EFOSC2, IRS) auftritt, werde ich es hier zusammen mit einer Methode zu seiner Lösung etwas ausführlicher besprechen.

Die erwähnte Verfälschung hat ihre Wurzel darin, daß zum Zwecke der anschließenden Mittelung aus der Menge aller Einzelspektren diejenigen ausgewählt werden (müssen), die bei Spinphasen $\Phi_{\text {spin }}$ aus einem gewissen endlichen Intervall aufgenommen wurden. Unter den so ausgewählten Spektren befinden sich unvermeidlich auch solche (sehr wahrscheinlich die meisten), die nicht exakt im Zentrum des betrachteten Phasenintervalls liegen. Diese haben jedoch Flüsse, die aufgrund der Intensitätsmodulation systematisch von dem Fluß abweichen, die ein genau im Zentrum des Phasenintervalls aufgenommenes Spektrum hätte, und verfälschen somit das Ergebnis der anschließenden Mittelung. Dieser Effekt spielt besonders dann eine Rolle, wenn man repräsentative Spektren für das Maximum oder das Minimum der Spinlichtkurve konstruieren möchte. (Zur Illustration ein Beispiel: Die Intensität sei maximal bei $\Phi_{\text {spin }}=0$. Zur Herstellung des Spektrums im Spinmaximum mögen 


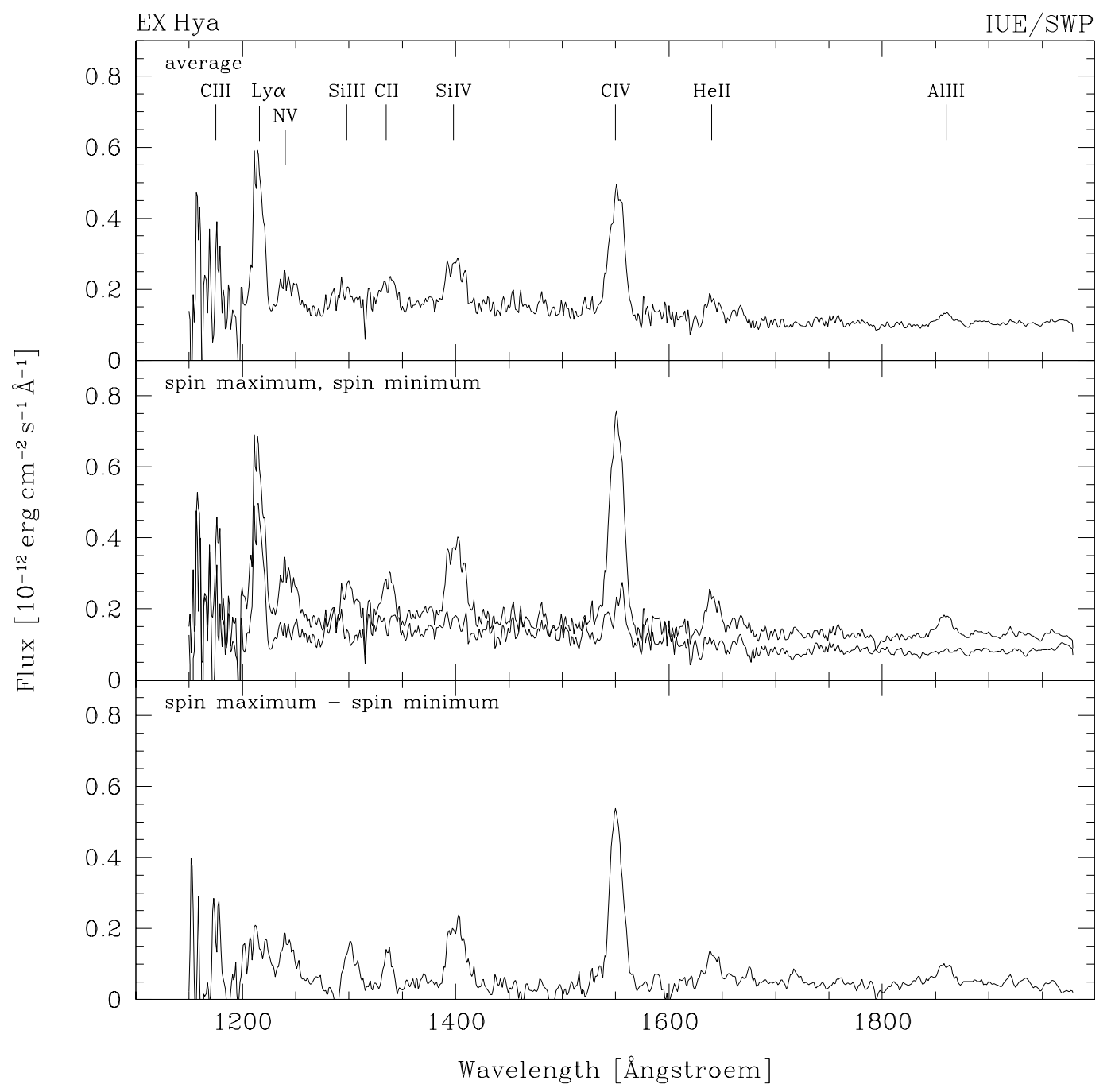

Abbildung 4.13: IUE/SWP-Spektren von EX Hya für verschiedene Spinphasen. Oben: über alle Spinphasen gemittelt; Mitte: im Spinmaximum bzw. -minimum; unten: Differenz von Spinmaximums- und -minimumsspektrum. Die Linie Lyman $\alpha$ ist in starkem Maße geokoronalen Ursprungs. Zur Konstruktion der Spektren s. Text.

Einzelspektren aus dem Intervall $\Phi_{\text {spin }}=-0.1 \ldots+0.1$ ausgewählt werden. Alle (oder fast alle) diese Spektren haben daher kleinere Flüsse als ein Spektrum bei $\Phi_{\text {spin }}=0$, ihr Mittel hat also ebenfalls einen systematisch zu kleinen Fluß. Für das Spinminimum erhielte man analog ein Spektrum mit zu hohem Fluß.)

Um diese Verfälschung zumindest weitgehend zu kompensieren, wende ich ein Verfahren an, das darauf aufbaut, daß sich im Fall von EX Hya die Modulation des Flusses $f$ bei jeder Wellenlänge - oder ab jetzt kurz 'die Modulation des Spektrums f' - in guter Näherung durch $f=f_{\text {av }}+f_{\text {mod }} \cos \left(\Phi_{\text {spin }}\right)$ beschreiben läßt. Dabei ist $f_{\text {av }}$ das mittlere und $f_{\text {mod }}$ das modulierende Spektrum (d.h. $f$ variiert über einen ganzen Zyklus um $f_{\max }-f_{\min }=2 f_{\bmod }$ ). 
Das Verfahren umfaßt die folgenden Schritte:

(1) Aus allen Einzelspektren in den Phasenintervallen $0.0 \pm 0.1$ (6 Spektren) bzw. $0.5 \pm 0.1$ (7 Spektren) werden durch Mittelung vorläufige Spektren $f_{\max , 0}$ bzw. $f_{\min , 0}$ für das Spinmaximum bzw. -minimum hergestellt. Durch Mittelung aller 41 Einzelspektren wird weiterhin ein ebenfalls vorläufiges mittleres Spektrum $f_{\mathrm{av}, 0}$ erzeugt.

(2) Für jeden der drei Sätze von Einzelspektren aus (1) wird aus den Spinphasen der in ihm enthaltenen Spektren der Mittelwert $C \equiv\left\langle\cos \left(\Phi_{\text {spin }}\right)\right\rangle$ berechnet. Die berechneten Werte $C\left(C_{\max }=0.9249, C_{\min }=-0.9443, C_{\mathrm{av}}=-0.0497\right)$ lassen sich als Kosinus der effektiven Spinphase interpretieren: $\left\langle\cos \left(\Phi_{\text {spin }}\right)\right\rangle \bumpeq \cos \left(\Phi_{\text {spin,eff }}\right)$. Ihre Abweichungen von den Erwartungswerten $(C=+1$ bzw. $C=-1$ für das Spektrum im Spinmaximum bzw. -minimum, $C=0$ für das wahre mittlere Spektrum) sind daher ein Maß für die Fehler der drei in (1) erzeugten vorläufigen Spektren.

(3) Die tatsächliche (d.h. vom Auswahleffekt freie) Modulationsamplitude $f_{\text {mod }}$ wird nun nach der Vorschrift $f_{\bmod }=\left(f_{\max , 0}-f_{\min , 0}\right) /\left(C_{\max }-C_{\min }\right)$ berechnet. Analog erhält man das korrigierte mittlere Spektrum durch $f_{\mathrm{av}}=f_{\mathrm{av}, 0}-C_{\mathrm{av}} f_{\mathrm{mod}}$.

(4) Die korrigierten Spektren $f_{\max }$ und $f_{\min }$ im Spinmaximum bzw. -minimum schließlich lassen sich nach $f_{\max }=f_{\mathrm{av}}+f_{\text {mod }}$ bzw. $f_{\min }=f_{\mathrm{av}}-f_{\text {mod }}$ berechnen. Diese Spektren enthalten zudem noch weniger Rauschen als die in Schritt (1) erzeugten 'rohen' Spektren $f_{\max , 0}$ und $f_{\min , 0}$, da hier als Ausgangspunkt das in Schritt (3) berechnete mittlere Spektrum $f_{\text {av }}$ verwendet wird. Dieses ist in der Regel durch deutlich mehr Spektren bestimmt als $f_{\max , 0}$ und $f_{\min , 0}$ (im vorliegenden Fall: 41 gegenüber 6 bzw. 7, s.o.) und entsprechend besser definiert, was sich auf die hier daraus berechneten 'endgültigen' Spektren überträgt. Die Resultate der Schritte (1) bis (4) sind in Abbildung 4.13 dargestellt. Auffällig ist das fast vollständige Verschwinden der Emissionslinien im Spinminimum (bis auf Lyman $\alpha$, deren Emission zum großen Teil aus der Geokorona stammt), wohingegen das Kontinuum lediglich um einen Faktor $\lesssim 2$ schwächer als im Spinmaximum ist. Dies entspricht den Befunden von Mauche (1999) aus der Analyse derselben Spektren.

Das vorgestellte Verfahren unterstellt implizit, daß keine analogen systematischen Fehler dadurch verursacht werden, daß durch die Auswahl der zu mittelnden Spektren allein anhand der Spinphasen automatisch die verschiedenen Orbitalphasen in der Regel nur ungleichmäßig repräsentiert sind (vgl. Abbildung 4.3). Diese Annahme ist dann erfüllt, wenn das Spektrum nicht mit der Orbitalphase variiert. Die Untersuchung der Lichtkurven in Abschnitt 4.2.7 wird zeigen, daß dies für die IUE-Spektren in guter Näherung gegeben ist.

\subsubsection{HST-Spektren von EX Hya}

Wie die IUE-Spektren liegen auch die HST-Spektren im Archiv in fertig reduzierter und kalibrierter Form vor. Abgesehen von dem bereits in Abschnitt 4.1.4 erwähnten Zusammenfassen mehrerer Spektren zu einem mit dem Ziel, das Verhältnis von Signal und Rauschen zu verbessern, waren daher auch hier keine weiteren vorbereitenden Bearbeitungsschritte erforderlich.

Bei der Analyse der HST-Lichtkurven, die weiter unten in Abschnitt 4.2 .7 beschrieben wird, fiel auf, daß der Fluß einiger Emissionslinien - insbesondere der Linien C IV $\lambda 1550$, 
N V $\lambda 1240$ und Ly $\alpha$ sowie in schwächerem Ausmaß Si IV $\lambda 1400$ - bei Orbitalphasen $\Phi_{\text {orb }}<$ 0 , d.h. vor dem Zeitpunkt der Bedeckung, signifikant niedriger ist als bei $\Phi_{\text {orb }}>0$, d.h. nach der Bedeckung. In den IUE-Spektren hingegen tritt eine solche Variation der Linienflüsse nicht auf (vgl. z.B. die aus den IUE-Spektren gewonnene Lichtkurve der in den HST-Spektren besonders stark betroffenen Linie C IV $\lambda 1550$ in Abbildung A.2.2). Um dem nachzugehen, habe ich zunächst jeweils alle Spektren mit $\Phi_{\text {orb }}<-0.05$ bzw. $\Phi_{\text {orb }}>+0.05$ gemittelt. Die Darstellung dieser beiden gemittelten Spektren sowie ihrer Differenz im oberen Teil von Abbildung 4.14 macht deutlich, daß die Veränderung der Linienflüsse nicht auf verstärkte Emission nach dem Zeitpunkt der Bedeckung, sondern vielmehr durch das Auftreten schmaler Absorptionskerne in den breiten Emissionslinien vor der Bedeckung verursacht wird. Die z.T. doppelt vorhandenen Absorptionslinien wurden anhand der von Wiese et al. $(1966,1969)$ tabellierten Wellenlängen als Multiplettkomponenten der in der Abbildung genannten Linien identifiziert.

Der untere Teil von Abbildung 4.14 zeigt anhand des Flußverhältnisses der beiden gemittelten Spektren, daß die Intensität des Kontinuums nur um $\lesssim 10 \%$ variiert und die Variation im wesentlichen wellenlängenunabhängig ist, möglicherweise mit Ausnahme des Bereichs $\lambda \lesssim 1250 \AA$. Im Gegensatz dazu beträgt der Flußverlust in den Zentren der Absorptionskerne bis zu 50\%. Man erkennt hier auch deutlicher als im oberen Teil der Abbildung, daß auch die Linien Si II $\lambda 1190, \lambda 1260$ und Si III $\lambda 1300$ von Absorption betroffen sind.

Die Tatsache, daß die Absorptionslinien so scharf sind, daß selbst die Multiplettkomponenten von C IV $\lambda 1550$ zumindest ansatzweise aufgelöst werden können (s. Abbildung 4.14 unten), erlaubt eine Vermessung der Verschiebung der Wellenlängen der Linien gegen die Ruhewellenlänge. Für letztere wurden statt der oben genannten Tabellen die neueren Werte von Kurucz \& Bell (1995) benutzt. In allen Fällen ist die daraus bestimmte Radialgeschwindigkeit des absorbierenden Materials gering $\left(0 \ldots+160 \mathrm{~km} \mathrm{~s}^{-1}\right)$. Der Mittelwert unter Berücksichtigung der Unsicherheiten der Einzelmessungen beträgt $+75 \pm 46 \mathrm{~km} \mathrm{~s}^{-1}$.

Die Abbildungen 4.15 und 4.16 zeigen die zeitliche Entwicklung der beobachteten Absorption im Bereich $\Phi_{\text {orb }}=-0.125 \ldots+0.175$. Im oberen Teil von Abbildung 4.15 wurden dabei sämtliche Spektren verwendet, im unteren nur solche, die bei Spinphasen in den Bereichen $\Phi_{\text {spin }}=0.25 \pm 0.1$ und $\Phi_{\text {spin }}=0.75 \pm 0.1$ aufgenommen wurden, d.h. außerhalb der Spinmaxima und -minima. Abgesehen vom besseren Signal-zu-Rausch-Verhältnis der Spektren im oberen Teil sind die Spektren in den beiden Teilen der Abbildung zu gleichen Orbitalphasen weitestgehend identisch. Abbildung 4.16 enthält im oberen bzw. unteren Teil die Spektren aus dem Bereich $\Phi_{\text {spin }}=0.00 \pm 0.15$ bzw. $\Phi_{\text {spin }}=0.50 \pm 0.15$, also aus den Zeiten um das Spinmaximum bzw. -minimum herum. In allen vier Fällen ist die Tiefe der beobachteten Absorptionslinien im jeweils untersten Spektrum bei $\Phi_{\text {orb }}=-0.125$ maximal und sinkt mit zunehmender Orbitalphase stetig ab; bei $\Phi_{\text {orb }}=+0.175$ ist die Absorption fast vollständig verschwunden. Vermutlich tritt das tatsächliche Absorptionsmaximum noch vor $\Phi_{\text {orb }}=-0.125$ auf; dies läßt sich anhand der vorliegenden Daten jedoch nicht entscheiden.

In Abbildung 1.16 fällt ferner auf, daß die Absorptionslinien im Spinminimum länger zu beobachten sind als im Spinmaximum; selbst bei $\Phi_{\text {orb }}=+0.175$ ist dort z.B. an der linken 
Flanke der Linie C IV $\lambda 1550$ noch Absorption zu erkennen, während die Linie bei gleicher Orbitalphase im Spinmaximum wieder ein fast symmetrisches Profil hat. Abbildung 4.17 zeigt das Verhältnis der Spektren im Spinmaximum und -minimum zu verschiedenen Orbitalphasen, d.h. die Verhältnisse der in Abbildung 4.16 in den beiden Bildteilen getrennt dargestellten Spektren. Wie in den IUE-Spektren (Abschnitt 4.2.3) sind die Emissionslinien stärker mit der Spinphase moduliert als das Kontinuum. Erstere weisen Intensitätsvariationen um einen Faktor 2...4 auf und sind zudem der oben beschriebenen orbitalphasenabhängigen Absorption unterworfen, letzteres variiert weitgehend wellenlängen- und orbitalphasenunabhängig um einen Faktor $\lesssim 1.5$.

Die Abbildung 4.18 schließlich zeigt das mittlere HST-Spektrum von EX Hya über alle Spinphasen, die mittleren Spektren im Spinmaximum bzw. -minimum und die Differenz der beiden letztgenannten. Die dargestellten Spektren wurden dabei mit demselben Verfahren wie die entsprechenden IUE-Spektren konstruiert (vgl. Abschnitt 4.2.3). Um die dort diskutierten systematischen Verfälschungen durch die hier gefundene orbitalphasenabhängige Absorption in den Linien möglichst gering zu halten, wurden dabei für die Mittelungen nur diejenigen Spektren verwendet, die im weniger stark von der Absorption betroffenen Orbitalphasenbereich $\Phi_{\text {orb }}>0.05$ aufgenommen wurden. (Um gerade diese Wahl zu rechtfertigen, muß ich hier auf die Interpretation der besagten Absorption in Kapitel 5.2 vorgreifen, derzufolge sie durch den bulge am äußeren Rand der Akkretionsscheibe verursacht wird und somit in diesem Zusammenhang als Störeffekt zu bewerten ist.) In das Verfahren gingen unter Beachtung dieser Randbedingung 50 Einzelspektren für das mittlere Spektrum und je 12 für das Spinmaximums- bzw. -minimumsspektrum ein. Es sei noch angemerkt, daß das Fehlen einer offensichtlichen orbitalphasenabhängigen Absorption in den IUE-Spektren (s.o.) dort eine entsprechende Vorauswahl der in die Mittelungen eingehenden Spektren unnötig macht.

Bis auf die geringere geokoronale Lyman $\alpha$-Emission gleichen die HST-Spektren aus Abbildung $4.18 \mathrm{im}$ wesentlichen den entsprechenden IUE-Spektren aus Abbildung 4.13; insbesondere zeigen die Emissionslinien auch hier eine deutlich stärkere Variation mit der Spinphase als das Kontinuum. In den HST-Spektren für das Spinmaximum bzw. minimum findet sich jedoch zusätzlich eine Andeutung einer breiten Lyman $\alpha$-Emission bzw. -absorption im Bereich $\approx 1190 \ldots 1240 \AA$, die in den korrespondierenden IUE-Spektren aufgrund des schlechteren Signal-zu-Rausch-Verhältnisses am kurzwelligen Ende nicht zu erkennen ist (vgl. mittlere Teilbilder von Abbildung 4.13 und 4.18). Die Differenzspektren hingegen (jeweils untere Teilbilder) sind wieder sehr ähnlich; insbesondere zeigen beide die besagte Lyman $\alpha$-Emission in etwa gleicher Breite sowie mit etwa gleicher Zentralintensität relativ zu den übrigen Linien. Ich weise im Vorgriff auf die Interpretation in Kapitel 5.5 bereits hier darauf hin, daß eine derartige Verbreiterung der Lyman $\alpha$-Linie, die durch den Stark-Effekt verursacht wird, die Dichte photosphärischen Plasmas erfordert, wohingegen der Ursprung der deutlich schmaleren übrigen Emissionslinien im sehr viel weniger dichten Material des Akkretionsvorhangs zu suchen ist. 

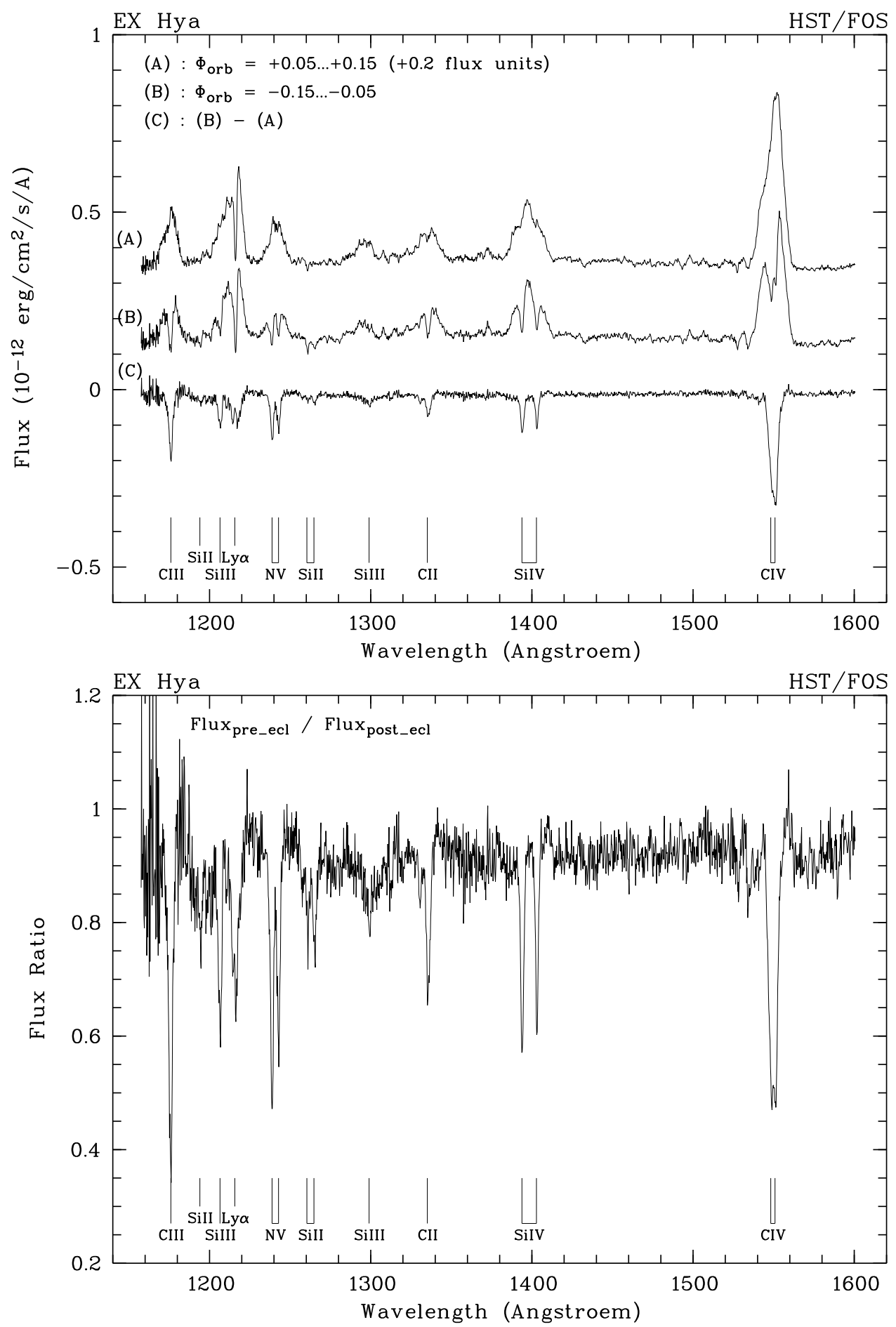

Abbildung 4.14: HST-Spektrum vor und nach der Bedeckung. Oben: $(\mathrm{B})=$ Spektrum vor der Bedeckung; $(\mathrm{A})$ = Spektrum nach der Bedeckung, um 0.2 Flußeinheiten nach oben versetzt; $(\mathrm{C})=$ Differenzspektrum $(\mathrm{B})-(\mathrm{A})$. Unten: Verhältnis der Flüsse vor und nach der Bedeckung $(\hat{=}(\mathrm{B}) /(\mathrm{A}))$. In beiden Abbildungen sind am unteren Bildrand die Wellenlängen der beobachteten Linien bzw. ihrer Multiplettkomponenten gekennzeichnet. 

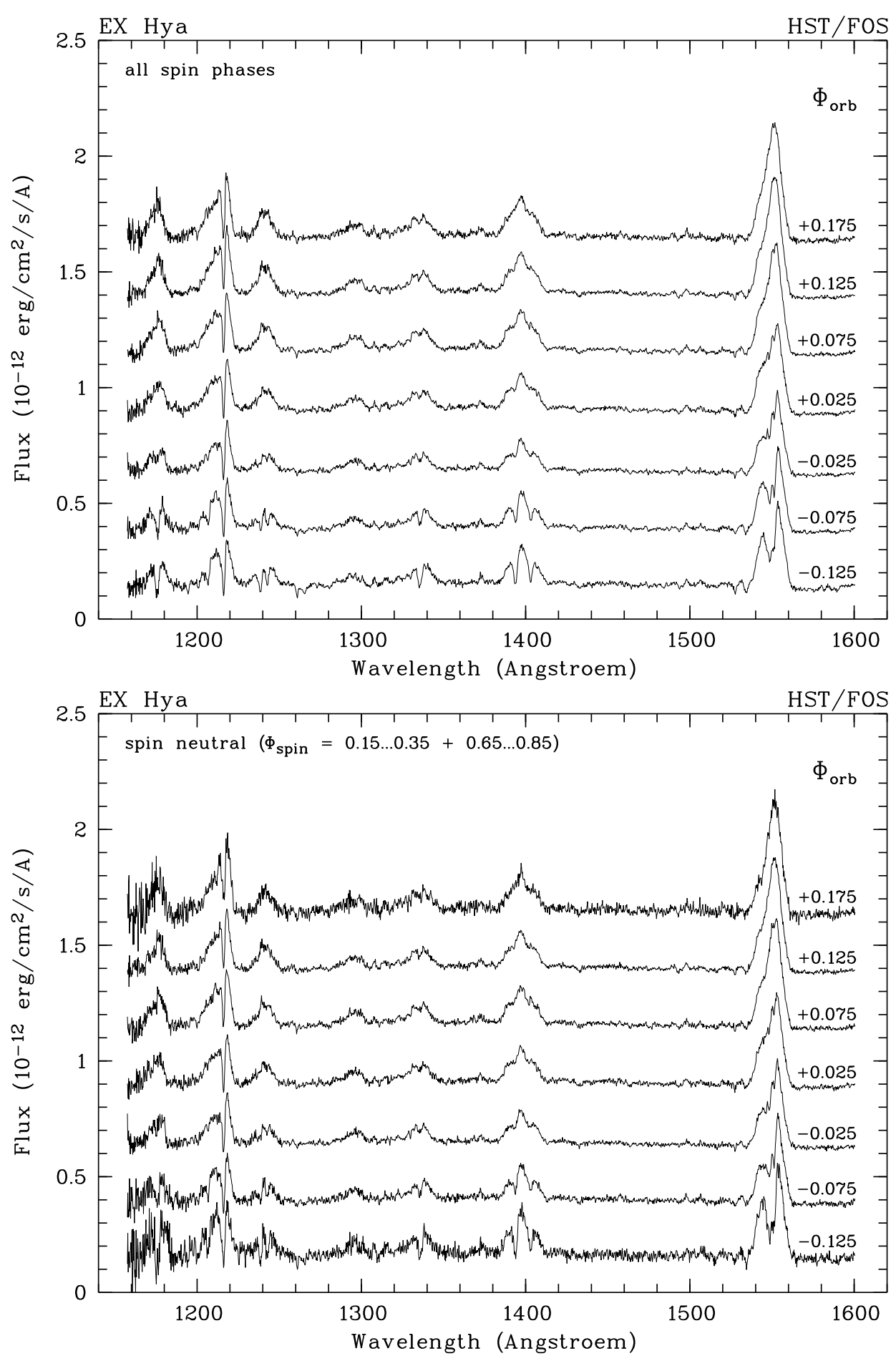

Abbildung 4.15: Orbitalphasenaufgelöste HST-Spektren von EX Hya im Intervall $\Phi_{\text {orb }}=$ $-0.125 \ldots+0.175$, Teil 1. Oben: gemittelt über alle Spinphasen. Unten: gemittelt über $\Phi_{\text {spin }}=0.25 \pm 0.1$ und $\Phi_{\text {spin }}=0.75 \pm 0.1$, d.h. über die Phasen außerhalb der Spinmaxima und -minima. Alle Spektren bis auf das jeweils unterste sind um Vielfache von 0.25 Einheiten vertikal versetzt dargestellt. 

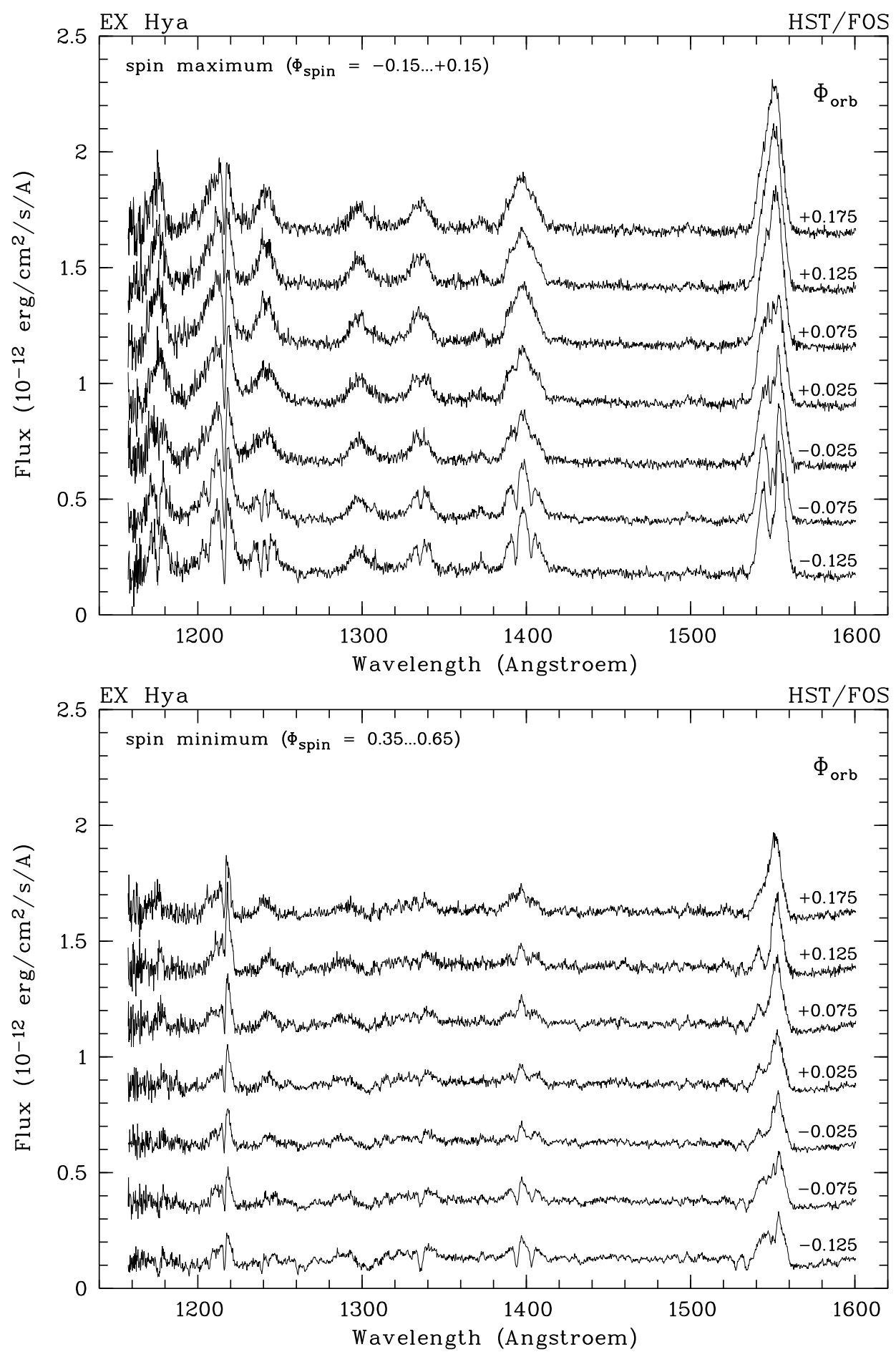

Abbildung 4.16: Orbitalphasenaufgelöste HST-Spektren von EX Hya im Intervall $\Phi_{\text {orb }}=$ $-0.125 \ldots+0.175$, Teil 2. Oben: gemittelt über $\Phi_{\text {spin }}=0.00 \pm 0.15$, d.h. über die Phasen um die Spinmaxima herum. Unten: gemittelt über $\Phi_{\text {spin }}=0.50 \pm 0.15$, d.h. über die Phasen um die Spinminima herum. Alle Spektren bis auf das jeweils unterste sind um Vielfache von 0.25 Einheiten vertikal versetzt dargestellt. 


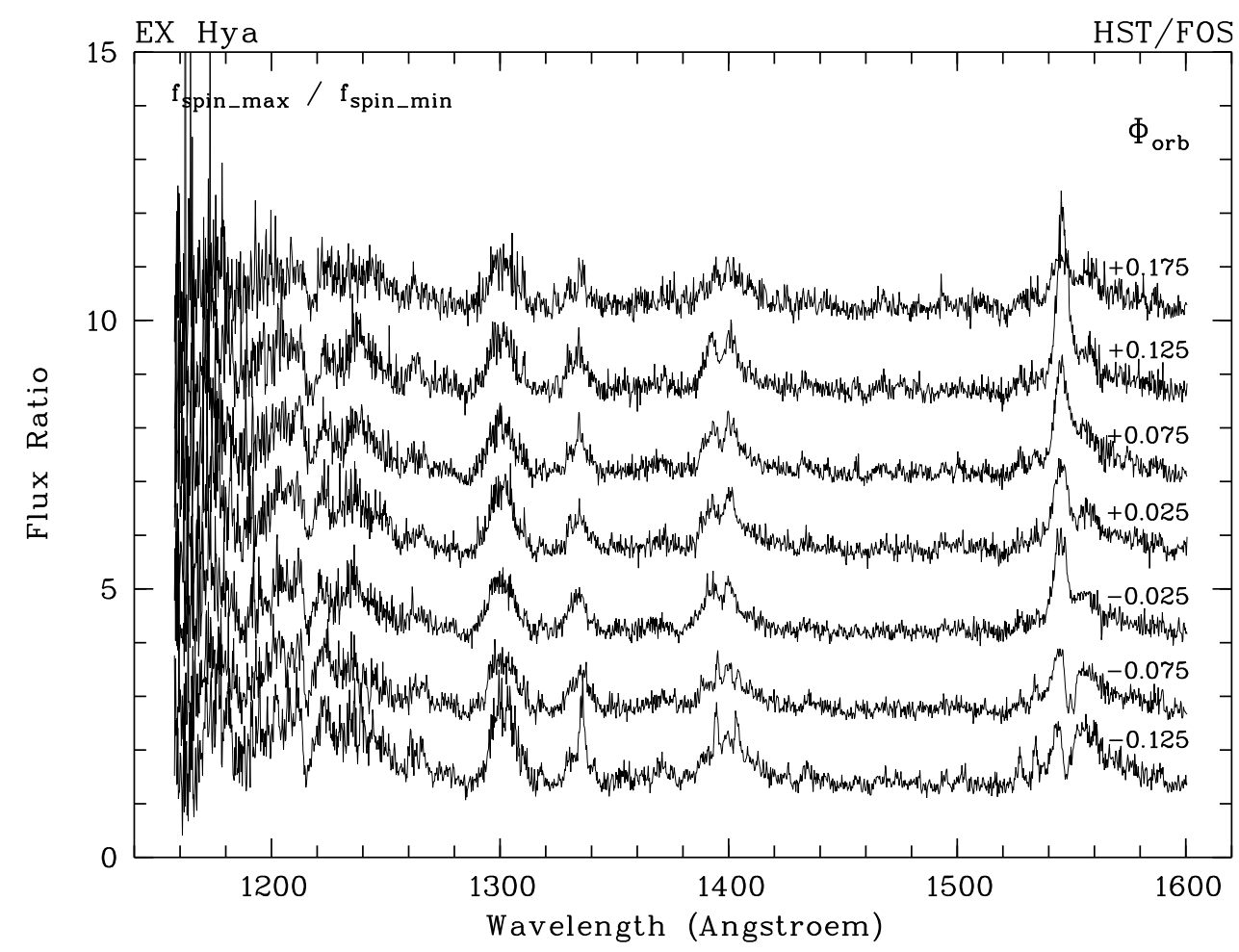

Abbildung 4.17: Orbitalphasenaufgelöste Flußverhältnisse der HST-Spektren von EX Hya im Spinmaximum bzw. -minimum. Alle Spektren bis auf das unterste sind um Vielfache von 1.5 vertikal versetzt dargestellt. Man beachte, daß aufgrund des gewählten Verhältnisses max/min Absorptionslinien, deren relative Tiefe im Minimum größer als im Maximum ist, in dieser Darstellung positiv erscheinen. 


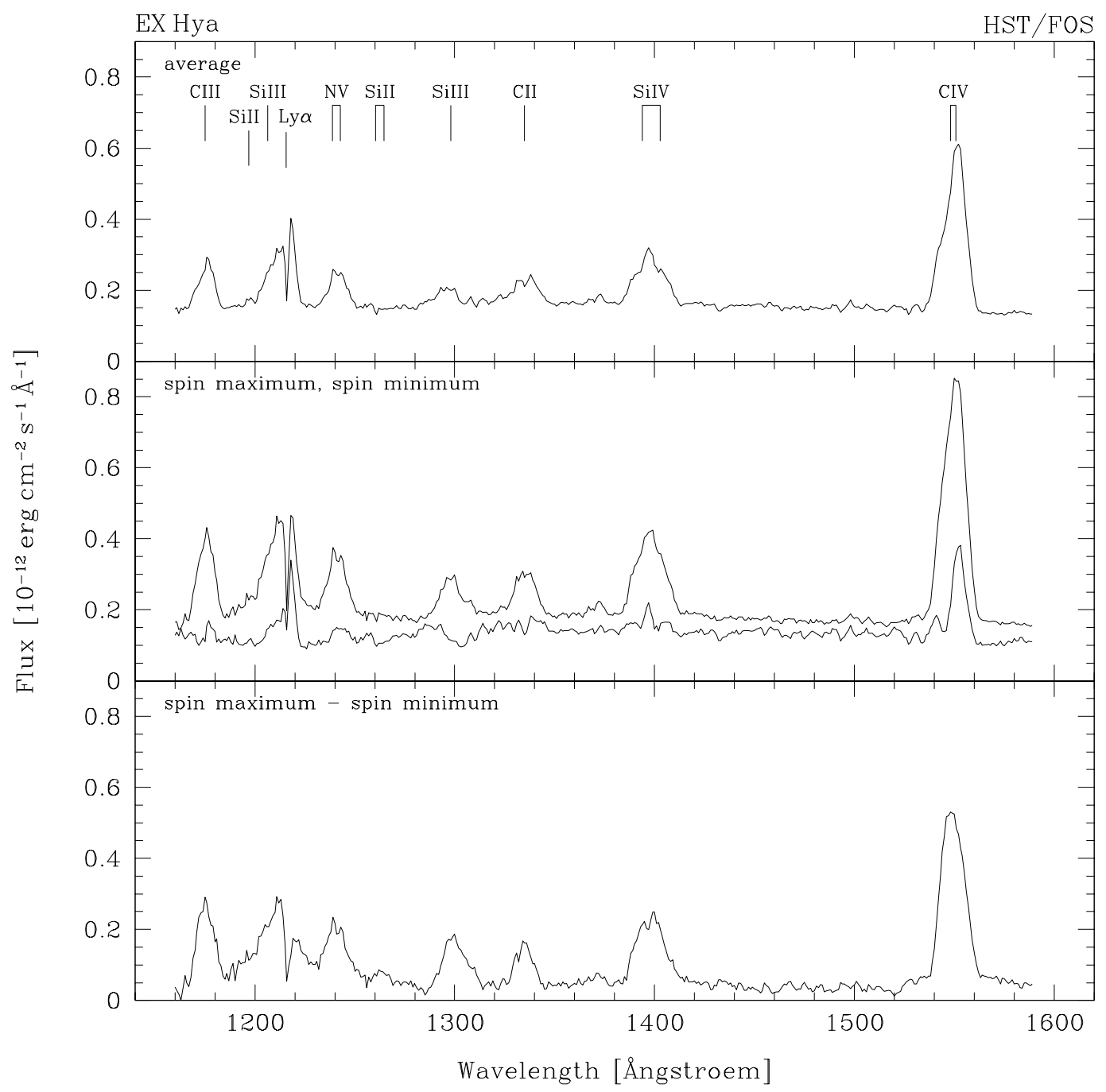

Abbildung 4.18: Mittlere HST-Spektren von EX Hya für verschiedene Spinphasen. Oben: über alle Spinphasen gemittelt; Mitte: im Spinmaximum bzw. -minimum; unten: Differenz von Spinmaximums- und -minimumsspektrum. In allen drei Fällen wurden nur Spektren verwendet, die bei Orbitalphasen $\Phi_{\text {orb }}>0.05$ aufgenommen wurden (s. Text). Die Linie Lyman $\alpha$ ist (anders als in den IUE-Spektren, vgl. Abbildung 4.13) höchstens zu einem geringen Teil geokoronal. Man beachte die Andeutung breiter Emission bzw. Absorption der Lyman $\alpha$-Linie $(\approx 1190 \ldots 1240 \AA$ ) im Spinmaximum bzw. -minimum (Mitte) sowie die große Ähnlichkeit des Differenzspektrums (unten) mit dem IUE-Pendant in Abbildung 4.13. Zur Konstruktion der Spektren s. Text. 


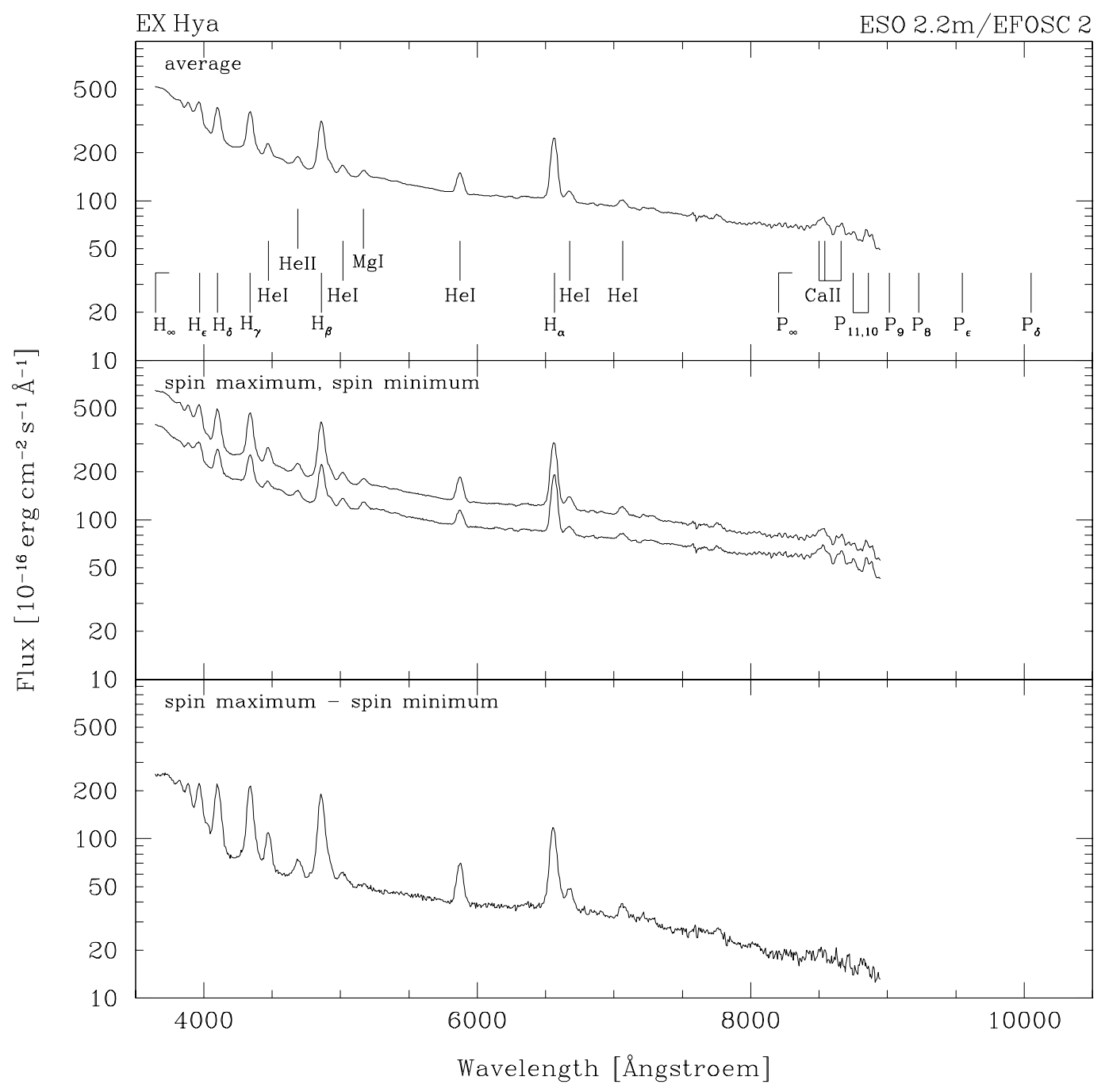

Abbildung 4.19: Mittlere EFOSC2-Spektren von EX Hya für verschiedene Spinphasen. Oben: über alle Spinphasen gemittelt; Mitte: im Spinmaximum bzw. -minimum; unten: Differenz von Spinmaximums- und -minimumsspektrum. Man beachte, daß im Gegensatz zu Abbildung 4.13 und 4.18 der Fluß hier der besseren Darstellbarkeit zuliebe logarithmisch aufgetragen ist.

\subsubsection{EFOSC2-Spektren von EX Hya}

Die Reduktion der EFOSC2-Daten erfolgte mit den dafür vorgesehenen Standard-Prozeduren des Programmpakets MIDAS (s. Abschnitt 4.2.1); ich verzichte daher auf eine nähere Beschreibung der einzelnen Schritte. Für die Korrektur der atmosphärischen Absorption durch $\mathrm{O}_{2}$ (A-, B- und C-Band bei $\approx 7600 \AA, \approx 6900 \AA$ und $\approx 6600 \AA$ ) und Wasserdampf (Zentren der Bänder bei $\approx 7250 \AA, \approx 8250 \AA$ sowie $\approx 9500 \AA$ ) wurden die Spektren der auch für die Flußkalibration verwendeten spektrophotometrischen Standardsterne Hiltner 600 (Spektraltyp B1V) und CD-32 9927 (Spektraltyp A0) verwendet. 
Angesichts der geringen Anzahl der Spektren gestaltete sich die Auswahl von Einzelspektren für die Erzeugung der Spektren im Spinmaximum bzw. -minimum sowie des mittleren Spektrums schwierig, zumal feststeht, daß im Optischen neben der Bedeckung noch weitere Modulationen mit der Orbitalphase auftreten (s. Kapitel 3.2.6.3). Diese können bei unsorgfältiger Wahl der Einzelspektren die am Ende von Abschnitt 4.2.3 erwähnte Bedingung für die Anwendbarkeit des verwendeten Mittelungs- und Korrekturverfahrens verletzen. Erschwerend kommt hinzu, daß - zumindest am langwelligen Ende des optischen Spektrums - die ellipsoidale Modulation des Flusses vom Sekundärstern (s. Abschnitt 2.2.2.2) ebenfalls einen spürbaren Einfluß hat. Die in Abschnitt 4.2.7 beschriebene Lichtkurvenanalyse zeigt, daß dies im Infrarot selbst bei den kürzesten dort beobachteten Wellenlängen der Fall ist; diese liegen z.T. schon im mit EFOSC2 beobachteten Bereich (vgl. Tabelle 4.1). Motiviert durch die Ergebnisse der Analyse der EFOSC2-Lichtkurven (Abschnitt 4.2.7) wurden schließlich für das Spinmaximum die Spektren Nr. 9 und 11 aus Tabelle 4.2 ausgewählt; Spektrum Nr. 10 wurde nicht verwendet, da die Lichtkurven hier einen zu starken Einfluß orbitaler Variationen nahelegen. Für das Spinminimum konnte aus ähnlichen Gründen nur das Spektrum Nr. 14 verwendet werden. Zur Konstruktion des mittleren Spektrums wurden nur Spektren aus einem Spinzyklus verwendet (Nr. 6...18 aus Tabelle 4.2), um eine Überrepräsentierung bestimmter Spinphasen zu vermeiden (vgl. Abbildung 4.2). Die resultierenden Spektren sind in Abbildung 4.19 gezeigt.

\subsubsection{IRS-Spektren von EX Hya}

Die mit dem IRS aufgenommenen Spektren von EX Hya wurden wie die in Abschnitt 4.2.2 beschriebenen Spektren der M-Sterne mit dem in Anhang B beschriebenen Verfahren reduziert. Sie gliedern sich in sieben Serien von je $\approx 1 \mathrm{~h}$ Dauer (drei in der ersten und je zwei in der zweiten und dritten Nacht), während derer EX Hya kontinuierlich beobachtet wurde. Jede Serie umfaßt ca. 50 Spektren, mit Ausnahme der zweiten Serie der zweiten Nacht

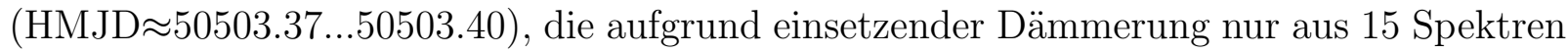
besteht. Letztere ist zudem noch stark durch Wolken beeinträchtigt; ich habe daher auf ihre Verwendung in der Datenanalyse verzichtet.

Die Spektren des Vergleichssterns SAO 181198 (vgl. Abschnitt 4.1.1) wurden unmittelbar vor der ersten und nach der letzten Serie einer Nacht sowie zwischen jeweils zwei Serien einer Nacht aufgenommen. Die aus diesen Spektren gewonnenen Korrekturfunktionen umgeben somit zeitlich paarweise jede Serie von Spektren von EX Hya und erlauben somit ein (zumindest grobes) Verfolgen der sich im Laufe der Nacht ändernden Transparenz der Atmosphäre. Für die Korrektur der Einzelspektren einer Serie wurden die beiden Korrekturfunktionen, die die Serie umgeben, auf den Zeitpunkt der individuellen Belichtungen interpoliert. Dieses Verfahren hat sich als deutlich besser erwiesen als die Verwendung einer einzigen gemittelten Korrekturfunktion für alle Spektren einer Serie oder gar alle Spektren einer Nacht. Da die Absorption im IR im wesentlichen durch Wasserdampf verursacht wird, dessen Säulendichte entlang der Sichtlinie auch auf Zeitskalen von Minuten (z.B. aufgrund von durchziehenden dünnen Wolkenschleiern oder auch nur feuchterer Luft) schwanken kann, können einzelne Spektren durch dieses Verfahren aber durchaus unteroder überkorrigiert worden sein. 


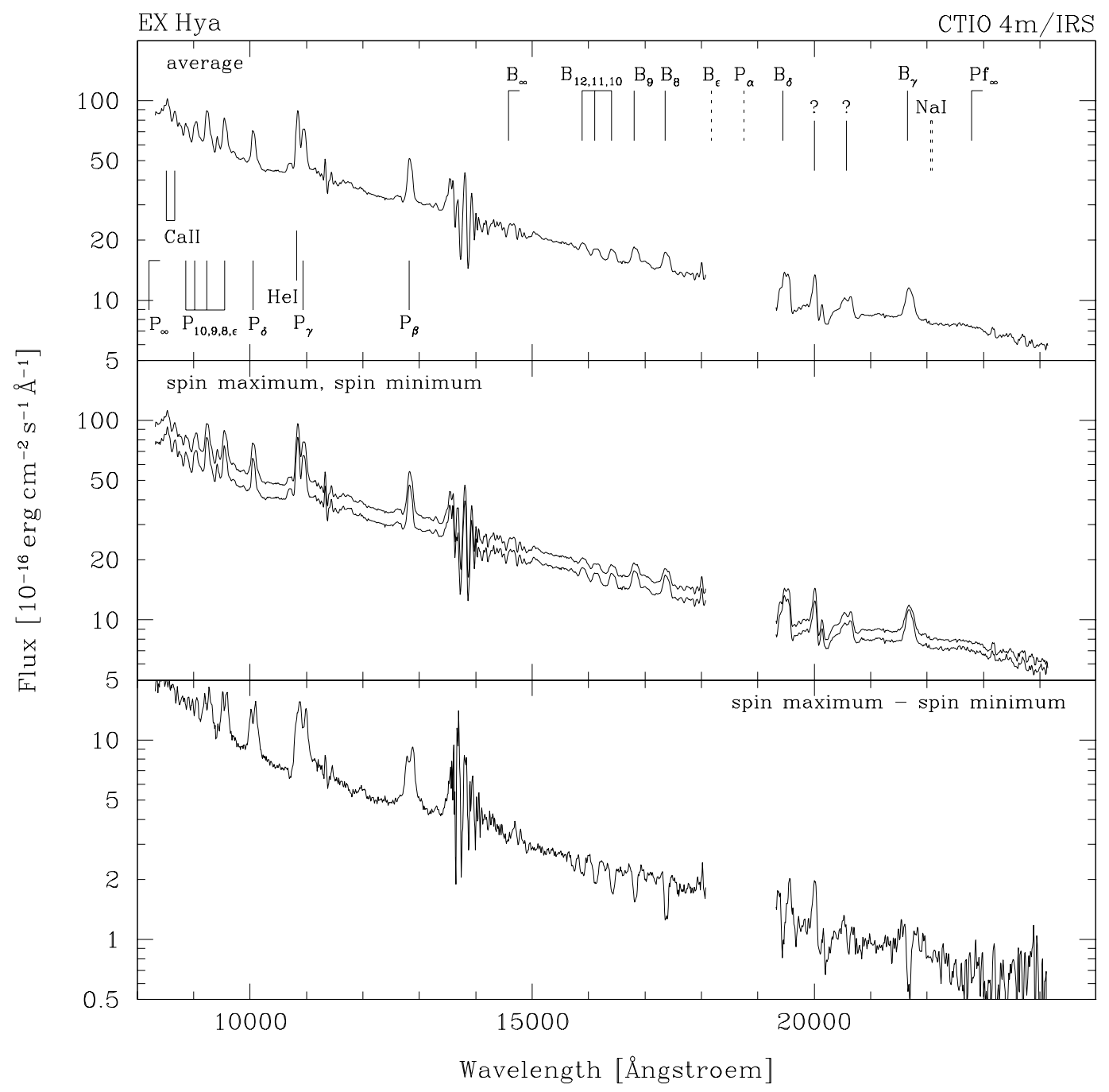

Abbildung 4.20: Mittlere IRS-Spektren von EX Hya für verschiedene Spinphasen. Oben: über alle Spinphasen gemittelt; Mitte: im Spinmaximum bzw. -minimum; unten: Differenz von Spinmaximums- und -minimumsspektrum. Wie in Abbildung 4.19 ist auch hier der Fluß logarithmisch aufgetragen. Die Wasserstofflinien Paschen $\alpha$ und Brackett $\epsilon$ liegen im Bereich $\lambda=18000 . . .19000$, der mit dem IRS in der benutzten Konfiguration nicht beobachtbar ist. Ferner ist die Lage des Na I-Dupletts $\lambda \lambda 22062 / 22090 \AA$ angegeben.

Bei der Konstruktion des mittleren Spektrums und der Spektren im Spinmaximum bzw. -minimum, die wieder nach der Methode aus Abschnitt 4.2.3 durchgeführt wurde, tritt wie bei den optischen Spektren das Problem auf, daß orbitale Variationen bei ungeschickter Wahl der verwendeten Einzelspektren deutliche Verfälschungen verursachen können (vgl. Abschnitt 4.2.5). Die volle Amplitude der ellipsoidalen Modulation mit einer Periode von $P_{\text {orb }} / 2 \approx 49$ min und dem Minimum bei $\Phi_{\text {orb }}=0$ macht z.B. im K-Band $\approx 13 \%$ des mittleren Flusses aus, s. Abschnitt 4.2.7; es ist hier also darauf zu achten, ihre Phasen $\Phi_{49}$ 
gleichmäßig (oder zumindest 'wirkungsneutral', d.h. mit $\left\langle\cos \left(\Phi_{49}\right)\right\rangle \approx 0$ ) zu repräsentieren. Ferner zeigen die Lichtkurven im Infrarot bei $\Phi_{\text {orb }}=0$ ausgeprägte Bedeckungen von $\approx 10$ min Gesamtdauer und einer relativen Tiefe von $10 \ldots 15 \%$. Aus diesem Grund wurden Spektren aus dem Phasenintervall $\Phi_{\text {orb }}=-0.06 \ldots+0.06$ nicht berücksichtigt. Weiterhin lassen die Lichtkurven in allen benutzten Bändern vermuten, daß EX Hya in der ersten Nacht während der zweiten Hälfte der ersten Serie (HMJD $50502.28 . .50502 .31$ ) einen milden Helligkeitsausbruch ('flare') gehabt hat, in dem der Fluß um $\approx 10 \%$ erhöht war (vgl. Abbildung 4.21). Weiterhin zeigen am Beginn der dritten Nacht aufgenommenen Spektren (HMJD $\approx 50504.29 . . .50504 .33)$ starke Intensitätsvariationen aufgrund eines durchziehenden Wolkenfelds (vgl. wiederum Abbildung 4.21). Auch diese beiden Gruppen von Spektren blieben hier unberücksichtigt.

Alle verbliebenen 222 Spektren wurden zur Erzeugung des mittleren Spektrums benutzt; der für sie berechnete Wert $C=\left\langle\cos \left(\Phi_{49}\right)\right\rangle=0.0135$ läßt vermuten, daß die ellipsoidale Modulation das Ergebnis nicht spürbar verfälscht. Für die Berechnung des Spektrums im Spinmaximum bzw. -minimum wurden die Spektren in den Phasenintervallen $\Phi_{\text {spin }}=0.0 \pm 0.075$ bzw. $\Phi_{\text {spin }}=0.5 \pm 0.075$ ausgewählt (32 bzw. 34 Spektren). Die Werte $C=-0.166$ bzw. $C=+0.182$ der beiden so definierten Sätze von Einzelspektren ließen ein weiteres manuelles 'Ausdünnen' mit dem Ziel einer weiteren Annäherung der Werte an 0, die nur um den Preis eines schlechteren Signal-zu-Rausch-Verhältnisses möglich gewesen wäre, unzweckmäßig erscheinen. Ich möchte jedoch anmerken, daß das so erhaltene Spinmaximums- bzw. -minimumsspektrum einen geringfügig zu hohen bzw. zu niedrigen Fluß enthalten kann. (Zur Erläuterung der Bedeutung des Vorzeichens von $C$ im vorliegenden Fall: Bei $\phi_{49}=0 \bumpeq \widehat{=}=+1$ hat die Lichtkurve der ellipsoidalen Modulation ein Minimum; $C<0$ bzw. $C>0$ entsprechen also einem positiven bzw. negativen Beitrag zum Fluß.) Da der relative Beitrag des Sekundärsterns zum Spektrum mit der Wellenlänge zunimmt, gilt dies auch für die Größe der Verfälschung, die aber selbst im K-Band betragsmäßig nur $\approx(13 \% / 2) \cdot 0.18 \approx 1.2 \%$ (= halbe Modulationsamplitude $C$ ) betragen sollte. Eine Darstellung der besprochenen Spektren findet sich in Abbildung 1.20.

\subsubsection{Lichtkurvenanalyse}

\subsubsection{Konstruktion der Lichtkurven}

Um die zeitlichen Intensitätsmodulationen von EX Hya näher untersuchen zu können, wurden aus den zeitaufgelösten Spektren Lichtkurven sowohl des Kontinuums in verschiedenen Wellenlängenbereichen als auch des Flusses von Emissionslinien hergestellt. Für die Kontinuumslichtkurven wurde für jedes einzelne Spektrum der mittlere Fluß in den in Tabelle 4.5 angegebenen Wellenlängenbereichen (ab jetzt kurz 'Bändern') bestimmt. Hierbei wurde keinerlei Gewichtung (etwa mit den Transmissionskurven der photometrischen Bänder aus Abbildung 4.5) durchgeführt. Die Bänder wurden jedoch im Optischen und Infrarot so gewählt, daß sie grob den photometrischen (Johnson-)Bändern $\mathrm{U}, \mathrm{B}, \ldots \mathrm{K}$ entsprechen; sie enthalten daher auch den Fluß der darin liegenden Emissionslinien. Es sei darauf hingewiesen, daß das in Tabelle 4.5 definierte "U"-Band nur den langwelligen Bereich des entsprechenden Johnson-Bandes mit $\lambda>=3600 \AA$ und das "K"-Band nur den 
Tabelle 4.5: Definition der für die Analyse der Kontinuumslichtkurven verwendeten Wellenlängenbereiche ('Bänder'). $\lambda_{c}$ gibt die Zentralwellenlänge der Bänder an. Ein 'EL' in der Spalte 'Bemerkung' im oberen Teil der Tabelle bedeutet, daß das entsprechende UV-Band Emissionslinien enthält. Die Bezeichnungen "U", "B" usw. im unteren Teil der Tabelle deuten grob die Lage der Bänder in bezug auf die in Abbildung 4.5 dargestellten photometrischen Bänder an und dienen nur der Orientierung. Alle Bänder im unteren Teil der Tabelle enthalten auch Emissionslinien.

\begin{tabular}{lccll|lllll}
\hline Instr. & Nr. & $\lambda \lambda[\AA]$ & $\lambda_{\mathrm{c}}[\AA]$ & Bem. & Instr. & Nr. & $\lambda \lambda[\AA]$ & $\lambda_{\mathrm{c}}[\AA]$ & Bem. \\
\hline HST/FOS & 1 & $1255-1285$ & 1270 & & IUE/SWP & 1 & $1255-1285$ & 1270 & \\
& 2 & $1350-1365$ & 1357.5 & EL? & & 2 & $1350-1380$ & 1365 & \\
& 3 & $1350-1380$ & 1365 & & & 3 & $1425-1525$ & 1475 & \\
& 4 & $1425-1525$ & 1475 & & & 4 & $1575-1625$ & 1600 & \\
& 5 & $1570-1600$ & 1585 & & & 5 & $1680-1780$ & 1730 & \\
& 6 & $1250-1380$ & 1315 & EL & & 6 & $1875-1975$ & 1925 & \\
& 7 & $1230-1600$ & 1415 & EL & & 7 & $1250-1380$ & 1315 & EL \\
& & & & & & 8 & $1250-1950$ & 1600 & EL \\
\hline ESO 2.2m $/ ~$ & $3600-4000$ & 3800 & "U" & CTIO 4m/ & 1 & $9650-10650$ & 10150 & "I" \\
EFOSC2 & 2 & $3900-5000$ & 4450 & "B" & IRS & 2 & $11000-13500$ & 12250 & "J" \\
& 3 & $5000-6200$ & 5600 & "V" & & 3 & $14500-18000$ & 16250 & "H" \\
& 4 & $5800-8000$ & 6900 & "R" & & 4 & $20000-24000$ & 22000 & "K" \\
& 5 & $7500-10000$ & 8750 & "I" & & & & & \\
\hline
\end{tabular}

kurzwelligen Teil des $\mathrm{K}_{\mathrm{J}}$-Bandes mit $\lambda<=24000 \AA$ umfaßt (vgl. Abbildung 4.5). Entsprechend decken das EFOSC2-"I"-Band bzw. das IRS-"I"-Band jeweils nur den kurz- bzw. langwelligen Teil des $\mathrm{I}_{\mathrm{J}}$-Bandes ab. Insofern sind die Benennungen der verwendeten optischen und Infrarot-Bänder in Tabelle 4.5 nur als Orientierungshilfe zu verstehen. Im UV wurden die Bänder so gewählt, daß sie jeweils zwischen zwei Emissionslinien liegen (vgl. die Spektren in Abbildung 4.13 und 4.18). Hiervon ausgenommen sind nur die Breitbandlichtkurven, die in Tabelle 4.5 durch den Eintrag 'EL' in der letzten Spalte gekennzeichnet sind. Weiterhin ist das HST-Band Nr. 2 möglicherweise durch die Linienflügel der das Band flankierenden Emissionslinien C II 1335 und Si IV 1400 beeinflußt (vgl. Abbildung 4.18).

Die Emissionslinienlichtkurven wurden durch Integration der Spektren in den in Tabelle 4.6 in der Spalte $\lambda \lambda_{\text {line }}$ angegebenen Bereichen und Abzug des Beitrags des Kontinuums gewonnen. Um letzteren zu bestimmen, wurden die Kontinuumsflüsse links und rechts der Linie durch Median-Bildung in den Bereichen $\lambda \lambda_{\text {cont }, 1 / 2}$ aus Tabelle 4.6 ermittelt und über den Bereich der Linie linear interpoliert. Mit der Ausnahme der Linien Paschen $\gamma$ und He I 10830, deren Flüsse mit dem IRS in der verwendeten Konfiguration nicht getrennt werden können, sowie der Linie Lyman $\alpha$, die in den HST-Spektren mit benachbarten SiLinien verschmilzt (s. Abbildung 4.18), wurden nur isolierte Linien verwendet, bei denen der Beitrag des Kontinuums hinreichend gut bestimmt werden konnte. Aus diesem Grund wurden z.B. in den IUE-Spektren die Linien im Bereich $\lambda<1350 \AA$ (vgl. Abbildung 4.13) nicht ausgewertet.

Im Fall der HST-Daten wurden nur diejenigen Spektren benutzt, die bei Orbitalphasen $\Phi_{\text {orb }}>0.05$ aufgenommen wurden, da die Emissionslinien in den restlichen Spektren stark 
Tabelle 4.6: In der Lichtkurvenanalyse untersuchte Emissionslinien. Die Spalte $\lambda \lambda_{\text {line }}$ gibt den Bereich an, über den der Fluß der Linie integriert wurde. Der Fluß des Kontinuums links und rechts der Linie wurde in den Wellenlängenbereichen $\lambda \lambda_{\text {cont,1 }}$ und $\lambda \lambda_{\text {cont,2 }}$ bestimmt.

\begin{tabular}{llrrr}
\hline Instr. & Linie & $\lambda \lambda_{\text {cont }, 1}[\AA]$ & $\lambda \lambda_{\text {line }}[\AA]$ & $\lambda \lambda_{\text {cont }, 2}[\AA]$ \\
\hline HST/FOS & C III 1175 & $1160-1165$ & $1165-1185$ & $1185-1195$ \\
& Ly $\alpha$ & $1185-1195$ & $1195-1225$ & $1225-1230$ \\
& N V 1240 & $1225-1230$ & $1230-1255$ & $1255-1285$ \\
& Si III 1300 & $1255-1285$ & $1285-1310$ & $1310-1320$ \\
& C II 1335 & $1310-1320$ & $1320-1350$ & $1350-1380$ \\
& Si IV 1400 & $1350-1385$ & $1385-1425$ & $1425-1525$ \\
& C IV 1550 & $1475-1525$ & $1525-1570$ & $1570-1600$ \\
\hline IUE/SWP & Si IV 1400 & $1350-1385$ & $1385-1425$ & $1425-1525$ \\
& C IV 1550 & $1425-1525$ & $1525-1575$ & $1575-1625$ \\
& He II 1640 & $1575-1625$ & $1625-1660$ & $1660-1760$ \\
& Al III 1860 & $1825-1845$ & $1845-1875$ & $1875-1975$ \\
\hline ESO 2.2m/ & He II 4686 & $4590-4640$ & $4640-4740$ & $4740-4790$ \\
EFOSC2 & H $\beta$ & $4740-4790$ & $4790-4950$ & $4950-4990$ \\
& He I 5875 & $5600-5800$ & $5800-5950$ & $5950-6150$ \\
& H $\alpha$ & $5975-6475$ & $6475-6650$ & $6650-7150$ \\
\hline CTIO 4m/ & P $\delta$ & $9650-9900$ & $9900-10150$ & $10150-10400$ \\
/IRS & P $\gamma /$ He I 10830 & $10200-10750$ & $10750-11050$ & $11050-11600$ \\
& P $\beta$ & $12400-12700$ & $12700-12950$ & $12950-13250$ \\
& B $\gamma$ & $20750-21500$ & $21500-21800$ & $21800-22550$ \\
\hline
\end{tabular}

durch zusätzliche Absorption beeinflußt sind (vgl. Abschnitt 4.2.4); eine entsprechende Vorauswahl der IUE-Spektren wurde nicht getroffen, da die Linien wie dort erwähnt keine offensichtliche Variation des Flusses mit der Orbitalphase zeigen.

Von den IRS-Daten habe ich die in Abschnitt 4.2.6 erwähnten 15 letzten Spektren der zweiten Nacht (HMJD $\approx 50503.37 . .50503 .40$ ) aus den dort genannten Gründen weder für die Kontinuums- noch für die Linienflußlichtkurven verwendet, so daß aus der zweiten Nacht nur für einen Zeitraum von $\approx 1 \mathrm{~h}$ Daten zur Verfügung stehen. Die auf das Baryzentrum des Sonnensystems korrigierten Zentralzeitpunkte HMJD aller verwendeten Spektren eines Instruments zusammen mit den oben bestimmten Flüssen je einer Emissionslinie bzw. in je einem Kontinuumsband bilden die Ausgangsdaten für die nachfolgend beschriebene Modellierung der Lichtkurven.

\subsubsection{Methodik der Modellierung}

Für die Modellierung der Lichtkurven habe ich das in Abschnitt 1.2 .1 erwähnte Programm 'Supermongo' nebst einem von mir für diesen Zweck entwickelten Makropaket benutzt. Bevor ich die Ergebnisse der Analyse zusammenfasse, gebe ich hier einen kurzen Überblick über die Arbeitsweise der Routinen, beschreibe die für die einzelnen Lichtkurventypen angesetzten Modelle und erläutere anhand eines Beispiels die beiden Darstellungsarten einer 
Modellanpassung sowie die Bedeutung der verschiedenen Parameter, die das Programm in den Darstellungen ausgibt.

Das Paket erlaubt die Anpassung von linearen Modellen $F=\Sigma_{i}\left(a_{i} F_{i}\right)$, wobei 'linear' bedeutet 'linear in den anzupassenden Koeffizienten $a_{i}$ '; die Komponentenfunktionen $F_{i}$ können hingegen beliebig gewählt werden. Im vorliegenden Fall wurden als mögliche und zur Laufzeit beliebig 'zuschaltbare' Komponenten gewählt:

(1) ein konstanter mittlerer Fluß $\left(F_{i}=1\right)$ sowie eine lineare Variation mit der Zeit $\left(F_{i}=t\right)$, zusammengenommen also ein linearer Trend. Falls die Lichtkurven wie im Fall von IRS und HST größere zeitliche Lücken aufweisen, können pro Segment (d.h. pro Nacht bzw. pro Datenlauf) bei Bedarf auch separate Trends angepaßt werden. Die Anpassung einer linearen Variation kann für einzelne oder für alle Segmente auch vollständig unterbleiben.

(2) periodische Variationen der Form $F_{i, \mathrm{NN}}= \pm \cos \left(\Phi_{\mathrm{NN}}-\Delta_{\Phi \mathrm{NN}}\right)$, wobei der Index NN verschiedene Periodizitäten symbolisiert. Das Vorzeichen legt fest, ob die betrachtete Komponente bei $\Phi=0$ bei positivem Koeffizienten $a_{i}$ ein Maximum oder ein Minimum hat; für die Spinmodulation wurde entsprechend '+' und für die ellipsoidale Modulation '-' gewählt. Die Amplituden dieser Modulationen werden immer für alle Datensegmente gemeinsam bestimmt; eine Differenzierung wie im Fall der Trends (s.o.) ist nicht vorgesehen. Die Phasenverschiebungen $\Delta_{\Phi \mathrm{NN}}$ müssen aufgrund der Beschränktheit der Methode auf lineare Modelle entweder im Voraus bekannt sein bzw. festgelegt werden oder aber iterativ durch Variation des Wertes mit dem Ziel z.B. einer Minimierung von $\chi_{\text {red }}^{2}$ bestimmt werden; eine gleichzeitige Anpassung mit den Koeffizienten $a_{i}$ ist nicht möglich.

Die Berechnung der Phasen $\Phi$ für die Komponenten unter (2) erfolgt zur Laufzeit aus den Zeiten HMJD. Da die Ephemeriden von Hellier \& Sproats (1992) hierfür etwas unhandlich sind (vgl. (3.1) und (3.2)), habe ich für die verschiedenen Beobachtungszeiträume lokal gültige lineare Ephemeriden benutzt (s. Tabelle 4.7). Diese wurden berechnet, indem für Anfang und Ende jedes Zeitraums die Zyklusnummern $N$ nach (3.1) bzw. (3.2) bestimmt wurden; aus diesen zusammen mit der Länge des Zeitraums lassen sich die zu dieser Zeit gültige Periode und ein Zeitpunkt $\mathrm{HMJD}_{0}$ bestimmen, an dem $\Phi=0$ ist. An dieser Stelle wurde auch die in Abschnitt 3.2.1 erwähnte Differenz zwischen Ephemeridenzeit TDB und baryzentrischer Zeit HJD berücksichtigt; aus diesem Grund beziehen sich die Ephemeriden in Tabelle 4.7 statt auf TDB jetzt auf HMJD $\equiv$ HJD-2400000.5.

Tabelle 4.7: Lokal gültige lineare Ephemeriden $\operatorname{HMJD}(\Phi=0)=\operatorname{HMJD}_{0}+N \cdot P$ für die Zeiträume der Beobachtungen mit den einzelnen Instrumenten (s. Text). (HMJD $\equiv$ HJD2400000.5).

\begin{tabular}{ll|ll|ll}
\hline Instrument & HMJD-Bereich & HMJD $_{0, \text { orb }}$ & $P_{\text {orb }}$ & HMJD $_{0, \text { spin }}$ & $P_{\text {spin }}$ \\
\hline HST & $49760.0-49764.0$ & 49760.04659 & 0.068233862 & 49760.00301 & 0.046546095 \\
IUE & $49891.5-49893.0$ & 49890.03209 & 0.068233863 & 49890.00625 & 0.046546090 \\
IRS, EFOSC2 & $50502.0-50508.5$ & 50500.04284 & 0.068233865 & 50500.03917 & 0.046546069 \\
\hline
\end{tabular}




\subsubsection{Darstellungsformen der Modelle}

Die Darstellung der Ergebnisse jeder Modellanpassung erfolgt in jeweils zwei verschiedenen Formen, deren Inhalt ich im folgenden kurz erläutern werde. Hierfür gebe ich als Beispiel in den Abbildungen 4.21 und 4.22 eine Anpassung an die aus den IRS-Spektren gewonnene "K"-Band-Lichtkurve wieder. (Es sei darauf hingewiesen, daß für dieses Beispiel anders als für die später durchgeführten 'echten' Anpassungen auch die Datenpunkte vom Ende der zweiten Nacht verwendet wurden.)

Abbildung 4.21 repräsentiert die erste Darstellungsart und zeigt in je einem Teilbild die ungefalteten Lichtkurven für jedes Datensegment (d.h. jede Nacht im Fall des IRS bzw. jeden der beiden Datenläufe für das HST) als Funktion der Zeit (Datenpunkte) zusammen mit dem angepaßten Gesamtmodell (gestrichelte Kurve) und den Beiträgen der einzelnen Komponenten (gepunktete Kurven). Der lineare Trend ist dabei wertgetreu über die Datenpunkte gelegt; die modulierten Komponenten befinden sich der Übersichtlichkeit zuliebe am oberen (spinmodulierte) bzw. unteren (orbitalmodulierte) Rand jedes Teilbilds. Die helleren Datenpunkte wurden anhand ihrer Orbitalphase als bedeckungsnah qualifiziert und nicht für die Anpassung der Parameter verwendet. Die Maßstäbe der Ordinaten aller Teilbilder sind identisch.

In Abbildung 4.22 sind dieselben Daten über die Spin- bzw. Orbitalphase gefaltet dargestellt, wobei der Beitrag einer eventuell angepaßten linearen Komponente bereits von jedem Datenpunkt abgezogen wurde. Die Darstellungen dieses Typs umfassen drei oder vier Teilbilder mit identischen Ordinatenmaßstäben, wobei die bedeckungsnahen Datenpunkte in der Regel nur in den über $\Phi_{\text {orb }}$ gefalteten Teilbildern erscheinen:

- Im obersten Teilbild wurden von den Datenpunkten zusätzlich zum linearen Trend die Beiträge aller angepaßten orbitalphasenabhängigen Komponenten abgezogen und das Ergebnis über die Spinphase gefaltet dargestellt. Die angepaßten spinphasenabhängigen Komponenten sind den Datenpunkten als ausgezogene Kurven überlagert.

- Das zweite Teilbild enthält entsprechend die Datenpunkte nach Abzug aller spinphasenabhängigen Komponenten über die Orbitalphase gefaltet sowie überlagert die angepaßten orbitalphasenabhängigen Modellkomponenten.

- Die unteren ein bis zwei Teilbilder schließlich geben die Residuen der Datenpunkte nach Abzug aller angepaßten Komponenten über $\Phi_{\text {spin }}$ bzw. $\Phi_{\text {orb }}$ gefaltet (drittes bzw. viertes Teilbild) wieder. Falls keine orbitalphasenabhängigen Komponenten angepaßt wurden, wären das zweite und das vierte Teilbild identisch, und letzteres entfällt. (Analog entfiele das dritte Teilbild, falls keine spinphasenabhängigen Komponenten angepaßt würden; dieser Fall tritt in dieser Arbeit aber nicht auf.)

Einige der in beiden Darstellungsarten zusätzlich vorhandenen Angaben bedürfen weiterer Erläuterung.

- Die Kopfzeile enthält neben der Identifikation der Lichtkurve die Angabe $\sigma_{\mathrm{i}}=\mathrm{x} *\langle\mathrm{f}\rangle$. Diese gibt den angenommenen statistischen Fehler $\sigma_{i}$ der einzelnen Datenpunkte wieder, der im vorliegenden Fall als Bruchteil $x$ des mittleren Wertes $\langle f\rangle$ aller für die Anpassung verwendeten Datenpunkte definiert wurde und somit für alle Datenpunkte identisch ist. Diese Methode wurde auch bei allen 'echten' Anpassungen benutzt. Der Wert $x$ wurde jeweils nach Augenschein so gewählt, daß $\sigma_{i}$ die beobachteten Wert-zu-Wert-Variationen 

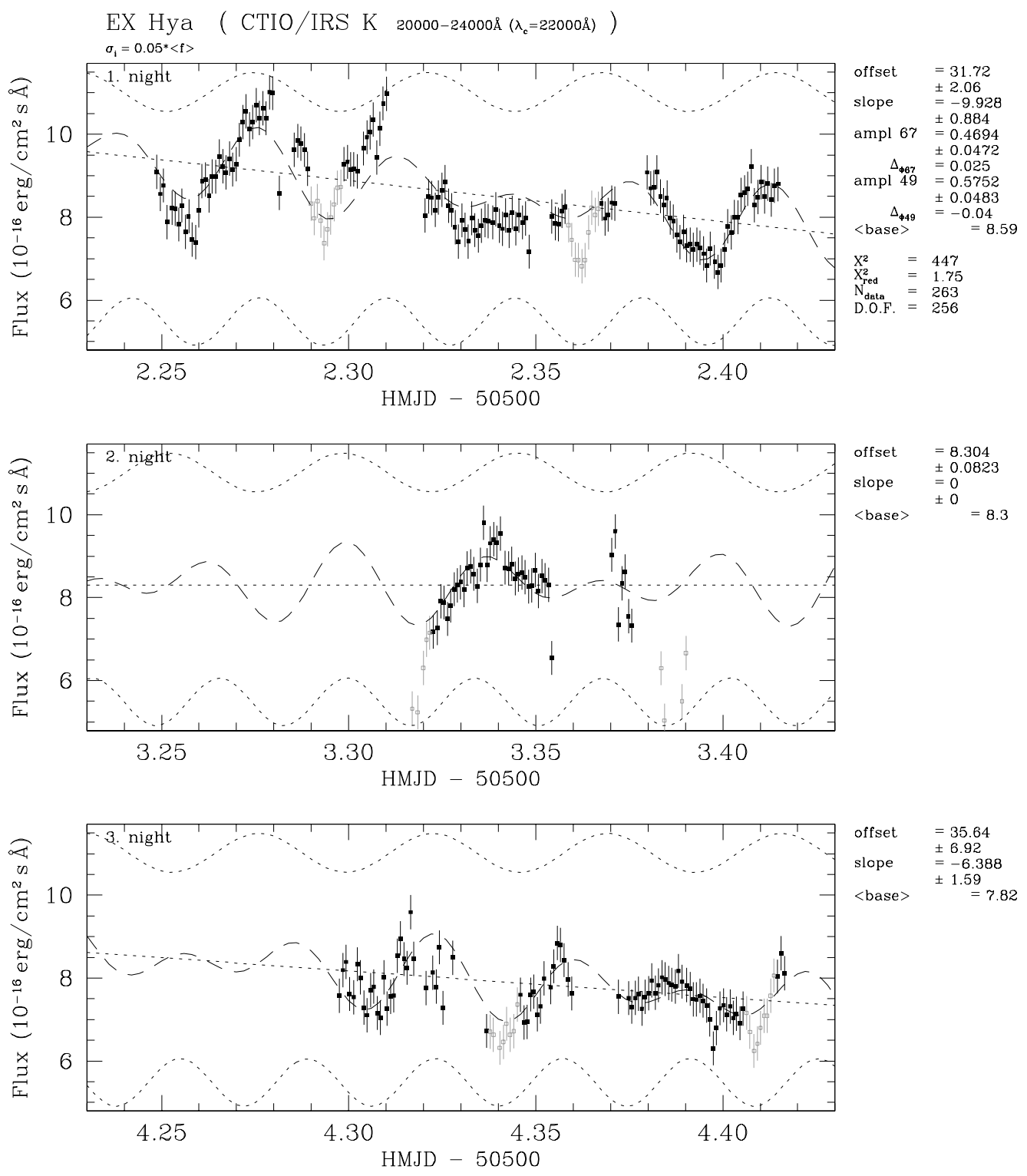

Abbildung 4.21: Beispiel für die ungefaltete Darstellung eines Lichtkurvenmodells: KBand. Abweichend von den tatsächlich durchgeführten Anpassungen wurden hier auch die Datenpunkte vom Ende der zweiten Nacht verwendet. Die Bedeutung der Kurven und der ausgegebenen Parameter werden im Text erläutert.

adäquat wiedergibt, die zum Teil in den intrinsischen Helligkeitsfluktuationen von EX Hya auf Zeitskalen von 10...100 s begründet liegen. Das resultierende $\sigma_{i}$ wurde auch für die Darstellung der bedeckungsnahen Datenpunkte verwendet. Es sei angemerkt, daß weder die Werte noch die Fehler der angepaßten Parameter von der konkreten Wahl von $x$ bzw. dem resultierenden $\sigma_{i}$ abhängen, wohl aber der Wert von $\chi^{2}$ bzw. $\chi_{\text {red }}^{2}$. Insofern sollte es nicht verwundern, daß für alle durchgeführten Anpassungen grob $\chi_{\text {red }}^{2} \approx 1$ ist; dies ist gerade auf die Art und Weise der Wahl von $x$ zurückzuführen. 


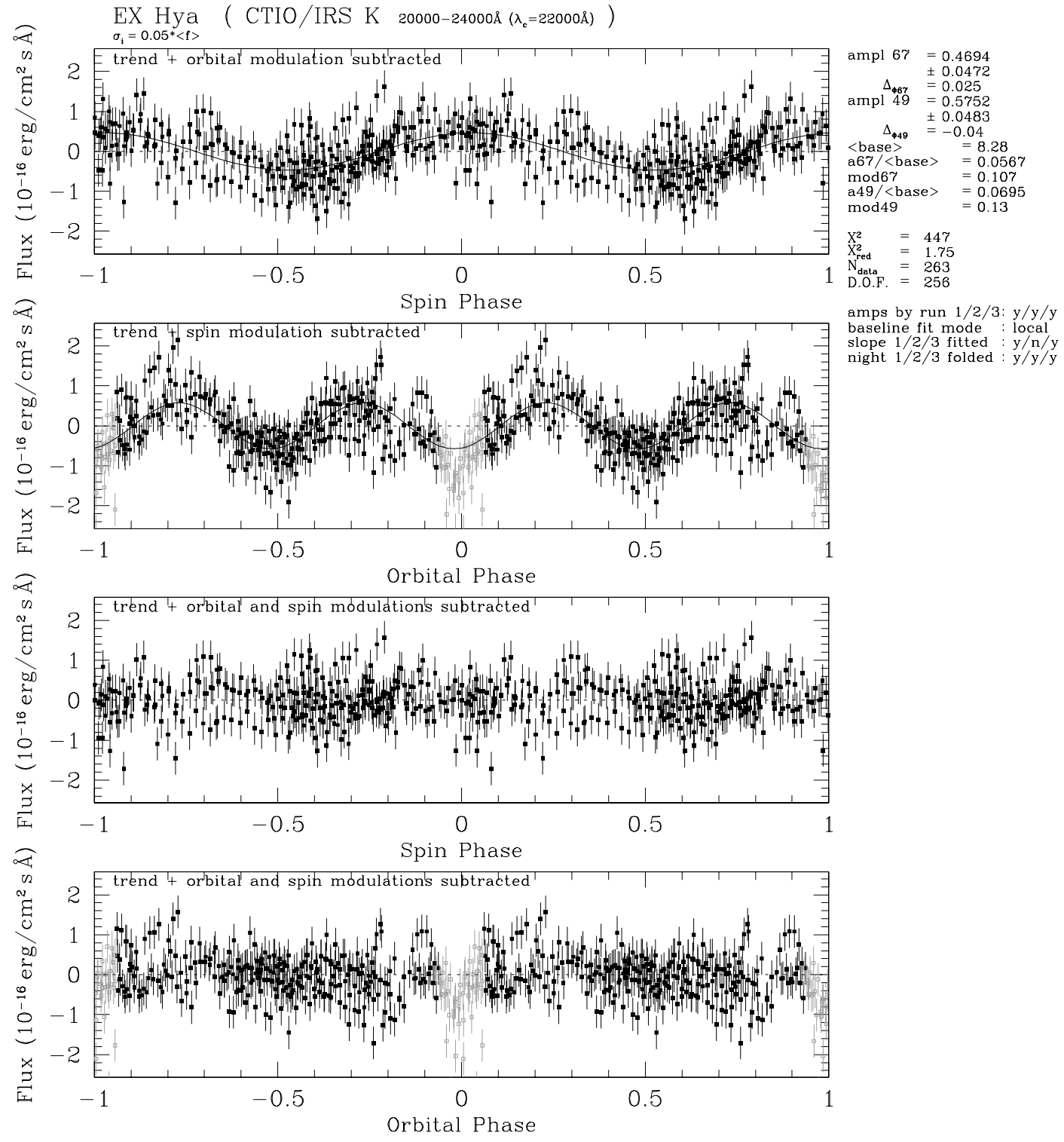

Abbildung 4.22: Beispiel für die über die Spin- bzw. Orbitalphase gefaltete Darstellung eines Lichtkurvenmodells, basierend auf denselben Daten wie Abbildung 4.21. Zur Bedeutung der Kurven und Parameter s. Text.

- In beiden Darstellungsarten sind neben dem jeweils obersten Teilbild die Amplituden der verschiedenen modulierten Komponenten als ampl NN zusammen mit ihren Fehlern angegeben, wobei NN die jeweilige Periodizität in Minuten symbolisiert (d.h. NN=98 für die orbitale, $\mathrm{NN}=49$ für die ellipsoidale und $\mathrm{NN}=67$ für die Spinmodulation). Die Einheiten sind dieselben wie die an den Ordinaten angegebenen. Der Wert $\Delta_{\Phi \mathrm{NN}}$ ist die vorgegebene Phasenverschiebung für die jeweilige Komponente (vgl. Punkt (2) in Abschnitt 4.2.7.2). 
- Die Werte offset und slope neben jedem Teilbild der ungefalteten Darstellung (Abbildung 4.21) repräsentieren den konstanten bzw. linearen Teil des Trends für die jeweilige Nacht, wobei für letzteren als unabhängige Koordinate der dargestellte Abszissenwert verwendet wurde; im Beispiel ist der Trend der ersten Nacht also durch 31.72 - 9.928(HMJD 50500) gegeben. Die Angabe slope $=0 \pm 0$ neben dem zweiten Teilbild symbolisiert, daß die Anpassung des Wertes für diese Nacht unterbunden wurde. Falls - wie im Fall der HST-Lichtkurven - nur ein einziger Trend für alle Datensegmente gemeinsam angepaßt wurde, erscheinen die Werte nur neben dem obersten Teilbild.

- Der Wert <base> ist der über alle Datenpunkte gemittelte Wert des Trends pro Nacht (in der ungefalteten Darstellung) bzw. für die gesamte Beobachtungszeit (in der gefalteten Darstellung); er gibt somit jeweils den mittleren Fluß nach Abzug der angepaßten Modulationen wieder.

- In der gefalteten Darstellung werden für jede angepaßte periodische Modulation die abgeleiteten Größen aNN/<base> (Verhältnis von Modulationsamplitude und mittlerem Fluß; ab jetzt 'relative Amplitude') und modNN (Modulationsgrad) angegeben. Letzteren habe ich als

$$
\bmod \equiv \frac{f_{\max }-f_{\min }}{f_{\max }}=1-\frac{f_{\min }}{f_{\max }}
$$

definiert ( $f_{\max }$ bzw. $f_{\min }=$ Maximal- bzw. Minimalfluß während eines Zyklus); für $f_{\max }=f_{\min }$ bzw. $f_{\min }=0$ ist damit $\bmod =0$ bzw. $\bmod =1$. Die relative Amplitude $a /\langle$ base $\rangle$ und der Modulationsgrad mod können über die Beziehungen

$$
\begin{aligned}
\frac{a}{\langle\text { base }\rangle} & =\frac{\bmod }{2-\bmod } \quad \text { bzw. } \\
\bmod & =\frac{2 a}{\langle\text { base }\rangle+a}=2 \frac{a}{\langle\text { base }\rangle} /\left(1+\frac{a}{\langle\text { base }\rangle}\right)
\end{aligned}
$$

ineinander umgerechnet werden.

- Die gefalteten Darstellungen der Anpassungen der HST- und IRS-Lichtkurven (vgl. Abbildung 4.22) enthalten noch weitergehende Angaben zur Behandlung der einzelnen Datensegmente:

- amps by run bezeichnet die Segmente, die zur Anpassung der Modulationsamplituden verwendet wurden $(\mathrm{y}=\mathrm{ja}, \mathrm{n}=$ nein); bei den endgültigen Anpassungen wurden stets alle Segmente verwendet, so daß hier immer y/y bzw. y/y/y steht.

- baseline fit mode gibt an, ob pro Segment ein separater Trend (local) oder ein gemeinsamer Trend für alle Segmente (global) angepaßt wurde. Ersteres ist bei den IRSLichtkurven und letzteres bei den HST-Lichtkurven der Fall.

- slope fitted identifiziert die Segmente, für die der Trend einen linearen Term beinhaltet (y) bzw. für die nur ein konstanter Beitrag angepaßt wurde (n). Im letzteren Fall ist für das entsprechende Segment in der ungefalteten Darstellung slope $=0 \pm 0$ (vgl. Abbildung 4.21).

- night folded bzw. run folded gibt die Segmente an, deren Daten in der gefalteten Darstellung gezeigt sind. Da bei den tatsächlichen Anpassungen stets alle Daten verwendet wurden, steht hier immer y/y bzw. y/y/y. 


\subsubsection{Modelldefinitionen für die verschiedenen Beobachtungen}

In diesem Abschnitt beschreibe ich die für die Anpassungen der verschiedenen Lichtkurven angesetzten Modelle sowie die Gründe, die im Einzelfall zu der getroffenen Wahl der Komponenten geführt haben. Generell wurde versucht, eine adäquate Beschreibung der Daten mit möglichst wenigen Modellparametern zu erreichen. Ferner wurde für alle gleichartigen Lichtkurven (z.B. alle IRS-Kontinuums- oder alle HST-Linien-Lichtkurven) stets das gleiche Modell benutzt. Wenn möglich, habe ich auch für die Kontinuums- und LinienLichtkurven desselben Instruments das gleiche Modell verwendet. Die graphischen Darstellungen aller Modelle jeweils in den beiden im vorigen Abschnitt diskutierten Formen sowie Tabellen mit den angepaßten Parametern werden aufgrund des beträchtlichen Seitenumfangs nicht hier, sondern separat in Anhang $\mathrm{A}$ gegeben.

IUE: Trend + spinmodulierte Komponente, d.h. $f=c+b \cdot t+a_{67} \cdot \cos \left(\Phi_{67}-\Delta_{\Phi 67}\right)$. Der lineare Term erwies sich als nötig, da die Lichtkurven sowohl der Kontinuumsbänder als auch der Emissionslinienflüsse einen Anstieg des mittleren Flusses um bis zu $\approx 30 \% \mathrm{im}$ Verlauf der Beobachtung zeigen (s. Abbildungen in Anhang A.2). Dieser ist bei der Analyse derselben Daten durch Mauche (1999) offensichtlich übersehen worden, da die dort angegebenen Anpassungen deutlich größere Streuungen als die in dieser Arbeit vorgestellten haben und sich durch eine versuchsweise durchgeführte Anpassung ohne den linearen Term nahezu exakt reproduzieren ließen. In den phasengefalteten Darstellungen wurden auch die bedeckungsnahen Punkte mit angegeben, da sie kein signifikant anderes Verhalten als die übrigen Punkte aufweisen. Die Anpassung erfolgte jedoch auch hier nur an die bedeckungsfernen Punkte.

HST: globaler Trend + spinmodulierte Komponente, d.h. $f=c+b \cdot t+a_{67} \cdot \cos \left(\Phi_{67}-\Delta_{\Phi 67}\right)$. Für die Anpassung der Lichtkurven der Emissionslinien wurden dabei aufgrund der störenden Absorption bei Orbitalphasen $\Phi_{\text {orb }} \lesssim 0.1$ (s. Abschnitt 4.2.4) nur die Datenpunkte mit $\Phi_{\text {orb }}>=0.05$ benutzt. Um konsistente und vergleichbare Ergebnisse zu erhalten, habe ich diese Einschränkung auch auf diejenigen Linien ausgedehnt, die in den Spektren keine auffällige Absorption zeigen. Versuchsweise wurden auch Anpassungen mit lokalen Trends durchgeführt. Aufgrund der auch innerhalb der beiden Datensegmente recht ungleichmäßigen zeitlichen Verteilung der Daten (s. Abbildungen in Anhang A.1) schwankten die angepaßten Steigungen verschiedener Lichtkurven jedoch in beiden Segmenten zwischen positiven und negativen Werten, wobei sich kein systematischer Gang etwa mit der Zentralwellenlänge des Kontinuumsbands oder dem Ionisierungspotential der Emissionslinie feststellen ließ. Insofern sind diese Werte - wenngleich für die einzelne Lichtkurve formal signifikant - im Rahmen einer Gesamtbetrachtung als unsinnig anzusehen und Anpassungen mit globalem Trend eindeutig vorzuziehen.

EFOSC2: Konstante + spinmodulierte Komponente, d.h. $f=c+a_{67} \cdot \cos \left(\Phi_{67}-\Delta_{\Phi 67}\right)$. Aufgrund der kurzen Zeitbasis $\left(\approx 1 P_{\text {orb }}\right.$ bzw. $\left.\approx 1.5 P_{\text {spin }}\right)$ und angesichts der intrinsischen Helligkeitsfluktuationen von EX Hya erschien die Anpassung eines linearen Terms nicht ratsam. Entsprechende Versuche lieferten dann auch ähnlich erratische Werte wie die aus den Anpassungen der HST-Lichtkurven mit lokalen Trends erhaltenen (s.o.). Die über die Orbitalphase gefalteten Residuen der Kontinuumslichtkurven (s. Abbildungen in Anhang A.3) 
zeigen möglicherweise eine verbleibende Modulation mit etwa der halben Orbitalperiode. Da aber nur Daten für einen einzigen Orbitalzyklus zur Verfügung stehen und die Variationen daher auch durch zufällige Intensitätsschwankungen hervorgerufen sein könnten, habe ich auf die Anpassung einer entsprechenden Komponente verzichtet.

IRS: lokale Trends + spinmodulierte Komponente + ellipsoidale Komponente, d.h. $f_{\text {cont }}=\Sigma_{i=1}^{3}\left(c_{i}+b_{i} t\right)+a_{67} \cdot \cos \left(\Phi_{67}-\Delta_{\Phi 67}\right)-a_{49} \cdot \cos \left(\Phi_{49}-\Delta_{\Phi 49}\right)$, für die Kontinuumslichtkurven; für die Emissionslinienlichtkurven zusätzlich eine mit der Periode $P_{\text {orb }}$ modulierte Komponente, d.h. $f_{\mathrm{el}}=f_{\text {cont }}+a_{98} \cdot \cos \left(\Phi_{98}-\Delta_{\Phi 98}\right)$. Wie bereits erwähnt, wurden die Daten vom Ende der zweiten Nacht aufgrund ihrer wetterbedingt großen Streuung (vgl. Abbildung 4.21) bei den Anpassungen nicht verwendet. Aufgrund der Kürze des verbleibenden Datensegments wurde für den Trend des zweiten Segments die Anpassung eines linearen Terms unterbunden $\left(b_{2}=0\right)$. Die Modulationsamplitude $a_{98}$ der zusätzlichen Komponente für die Emissionslinienlichtkurven ist in allen Fällen vergleichbar mit der Amplitude $a_{49}$ der ellipsoidalen Komponente (vgl. Abbildungen in Anhang A.4), so daß die Daten ohne diese Komponente nicht adäquat modelliert werden können. Die Interpretation dieses Sachverhalts erfolgt später.

\subsubsection{Ergebnisse der Lichtkurvenanpassungen}

Die Darstellungen der Anpassungen der im vorigen Abschnitt definierten Modelle an die Lichtkurven sowie die vollständigen Tabellen mit den Modellparametern finden sich wie bereits angekündigt in Anhang A. In allen Fällen wurden dabei die Phasenverschiebungen $\Delta \Phi$ aller modulierten Komponenten in Schritten von 0.005 variiert, um eine bestmögliche Anpassung (d.h. minimales $\chi_{\text {red }}^{2}$ ) zu erhalten. Die Fehler der $\Delta \Phi$ sind aufgrund dieses Verfahrens nicht unmittelbar bestimmbar, lassen sich jedoch für jede Lichtkurve aus dem Verlauf von $\chi_{\text {red }}^{2}(\Delta \Phi)$ zumindest abschätzen und sollten demzufolge für alle Anpassungen etwa $\sigma_{\Delta \Phi} \lesssim 0.03$ betragen. Die wesentlichen Ergebnisse der Anpassungen fasse ich hier kurz zusammen.

- Die Modulation mit der Spinphase ist im Kontinuum vom UV bis ins IR vorhanden; die relevanten Parameter für alle betrachteten Bänder sind in Tabelle 4.9 angegeben. Der Modulationsgrad (nach der Definition in (4.2)) beträgt im UV im Bereich 1250...1600 A ca. 0.30 und nimmt bis auf 0.43 bei $1925 \AA$ zu. Im Optischen und Infrarot nimmt er kontinu-

Tabelle 4.8: Mittlerer Fluß 〈base〉 und Amplitude $a_{49}$ der ellipsoidalen Modulation im Kontinuum der IRS-Spektren in Einheiten von $10^{-16} \mathrm{erg} \mathrm{cm}^{-2} \mathrm{~s}^{-1} \AA^{-1}$, Phasenverschiebung $\Delta_{\Phi 49}$ und Modulationsgrad $\bmod _{49}$ (s. Definition in (4.2)).

\begin{tabular}{rllccl}
\hline \multicolumn{1}{c}{$\lambda \lambda[\AA]$} & $\lambda_{\text {cen }}[\AA]$ & $\langle$ base $\rangle$ & $a_{49}$ & $\Delta_{\Phi 49}$ & $\bmod _{49}$ \\
\hline $9650-10650$ & 10150 & 52.0 & $2.39 \pm 0.34$ & -0.005 & 0.0879 \\
$11000-13500$ & 12250 & 37.6 & $2.16 \pm 0.23$ & -0.020 & 0.109 \\
$14500-18000$ & 16250 & 18.5 & $1.21 \pm 0.11$ & -0.030 & 0.123 \\
$20000-24000$ & 22000 & 8.30 & $0.564 \pm 0.047$ & -0.040 & 0.127 \\
\hline
\end{tabular}


Tabelle 4.9: Mittlerer Fluß 〈base $\rangle$ und Amplitude $a_{67}$ der Spinmodulation im Kontinuum in Einheiten von $10^{-16} \mathrm{erg} \mathrm{cm}^{-2} \mathrm{~s}^{-1} \AA^{-1}$, Phasenverschiebung $\Delta_{\Phi 67}$ und Modulationsgrad $\bmod _{67}$ (s. Definition in (4.2)).

\begin{tabular}{lcllccc}
\hline Instr. & $\lambda \lambda[\AA]$ & $\lambda_{\text {cen }}[\AA]$ & $\langle$ base $\rangle$ & $a_{67}$ & $\Delta_{\Phi 67}$ & $\bmod _{67}$ \\
\hline HST & $1255-1285$ & 1270 & 1480 & $319 \pm 24$ & -0.010 & 0.355 \\
& $1350-1365$ & 1358 & 1590 & $276 \pm 22$ & +0.005 & 0.296 \\
& $1350-1380$ & 1365 & 1630 & $304 \pm 23$ & +0.005 & 0.314 \\
& $1425-1525$ & 1475 & 1500 & $221 \pm 19$ & +0.005 & 0.256 \\
& $1570-1600$ & 1585 & 1360 & $261 \pm 17$ & +0.015 & 0.323 \\
\hline IUE & $1255-1285$ & 1270 & 1450 & $264 \pm 40$ & +0.050 & 0.308 \\
& $1350-1380$ & 1365 & 1560 & $356 \pm 60$ & +0.015 & 0.372 \\
& $1425-1525$ & 1475 & 1440 & $211 \pm 52$ & +0.020 & 0.256 \\
& $1575-1625$ & 1600 & 1220 & $250 \pm 49$ & +0.035 & 0.341 \\
& $1680-1780$ & 1730 & 1030 & $262 \pm 35$ & +0.025 & 0.407 \\
& $1875-1975$ & 1925 & 1030 & $283 \pm 39$ & +0.025 & 0.430 \\
\hline EFOSC2 & $3600-4000$ & 3800 & 431 & $112 \pm 14$ & +0.030 & 0.411 \\
& $3900-5000$ & 4450 & 229 & $47.3 \pm 8.4$ & +0.035 & 0.342 \\
& $5000-6200$ & 5600 & 124 & $20.7 \pm 4.5$ & +0.050 & 0.287 \\
& $5800-8000$ & 6900 & 96.5 & $17.8 \pm 3.2$ & +0.040 & 0.312 \\
& $7500-10000$ & 8750 & 59.5 & $9.09 \pm 1.9$ & +0.040 & 0.265 \\
\hline IRS & $9650-10650$ & 10150 & 52.0 & $5.28 \pm 0.32$ & +0.035 & 0.184 \\
& $11000-13500$ & 12250 & 37.6 & $3.31 \pm 0.22$ & +0.030 & 0.162 \\
& $14500-18000$ & 16250 & 18.5 & $1.34 \pm 0.10$ & +0.030 & 0.135 \\
& $20000-24000$ & 22000 & 8.30 & $0.470 \pm 0.047$ & +0.025 & 0.107 \\
\hline
\end{tabular}

ierlich von $\approx 0.41$ bei $3800 \AA$ bis auf $\approx 0.11$ bei $22000 \AA$ ab. Das Maximum der Lichtkurven tritt in allen Fällen im Rahmen der Fehler bei der Phase $\Phi_{\text {spin }}=0$ ein.

- Sämtliche untersuchte Emissionslinien mit Ausnahme von Brackett $\gamma$ sind ebenfalls spinmoduliert. Die entsprechenden Parameter in Tabelle 4.10 zeigen, daß der Modulationsgrad der Linien generell deutlich höher als der des benachbarten Kontinuums ist (vgl. Tabelle 4.9); er erreicht bei den UV-Linien Werte nahe 1. Auch hier ist die Lage des Maximums der Lichtkurve in allen Fällen konsistent mit $\Phi_{\text {spin,max }}=0$.

- Im Infrarot-Kontinuum tritt eine deutliche Modulation mit der halben Orbitalperiode auf, deren nahezu gleich hohe Maxima im Rahmen der Fehler bei $\Phi_{49}=0.5$ (d.h. bei $\Phi_{\text {orb }}=0.25$ und $\left.\Phi_{\text {orb }}=0.75\right)$ auftreten und deren Modulationsgrad stetig von $\approx 0.09$ bei $10150 \AA$ auf $\approx 0.13$ bei $22000 \AA$ zunimmt (Tabelle 4.8). Die Modulation ist in guter Näherung sinusförmig (s. Abbildung A.50 bis A.57 in Anhang A.4.1).

- Möglicherweise tritt eine Modulation mit der halben Orbitalperiode auch in den optischen Kontinuumslichtkurven auf (vgl. Abbildung A.41 bis A.3.1 in Anhang A.3.1); die Residuen der Anpassungen haben ihre Maxima allerdings bei $\Phi_{\text {orb }} \approx 0.35$ und $\Phi_{\text {orb }} \approx 0.85$ und somit um ca. $\Delta \Phi_{\text {orb }}=0.1$ später als die entsprechenden Maxima im Infrarot (s.o.). Zudem implizieren die Residuen einen Modulationsgrad im Optischen, der fast wellenlängenunabhängig $\approx 0.27$ beträgt, was nicht recht zu den aus den IRS-Lichtkurven gewonnenen Modulations- 
Tabelle 4.10: Mittlerer Fluß 〈base $\rangle$ und Amplitude $a_{67}$ der Spinmodulation der Emissionslinien in Einheiten von $10^{-16} \mathrm{erg} \mathrm{cm}^{-2} \mathrm{~s}^{-1}$, Phasenverschiebung $\Delta_{\Phi 67}$ und Modulationsgrad $\bmod _{67}$ (s. Definition in (4.2)).

\begin{tabular}{llcccc}
\hline Instr. & Linie & $\langle$ base $\rangle$ & $a_{67}$ & $\Delta_{\Phi 67}$ & $\bmod _{67}$ \\
\hline HST & C III 1175 & 13000 & $11900 \pm 810$ & +0.045 & 0.954 \\
& Ly $\alpha$ & 27300 & $13000 \pm 1000$ & +0.035 & 0.646 \\
& N V 1240 & 10300 & $6990 \pm 480$ & +0.005 & 0.807 \\
& Si III 1300 & 6190 & $7720 \pm 260$ & +0.040 & 1.11 \\
& C II 1335 & 9400 & $4870 \pm 390$ & +0.035 & 0.682 \\
& Si IV 1400 & 23300 & $17300 \pm 660$ & +0.020 & 0.853 \\
& C IV 1550 & 56400 & $37500 \pm 1400$ & +0.000 & 0.798 \\
\hline IUE & Si IV 1400 & 21300 & $14100 \pm 1800$ & +0.015 & 0.796 \\
& C IV 1550 & 51600 & $39400 \pm 3000$ & +0.005 & 0.865 \\
& He II 1640 & 6740 & $8490 \pm 980$ & +0.045 & 1.11 \\
& Al III 1860 & 4960 & $4560 \pm 720$ & +0.030 & 0.958 \\
\hline EFOSC2 2 He II 4686 & 1130 & $362 \pm 46$ & +0.025 & 0.484 \\
& H $\beta$ & 9030 & $4108 \pm 280$ & +0.010 & 0.625 \\
& He I 5875 & 1830 & $891 \pm 58$ & -0.005 & 0.654 \\
& H $\alpha$ & 8090 & $2770 \pm 190$ & +0.010 & 0.510 \\
\hline IRS & P $\delta$ & 2060 & $412 \pm 14$ & +0.020 & 0.333 \\
& P $\gamma /$ He I 10830 & 6260 & $755 \pm 39$ & +0.020 & 0.215 \\
& P $\beta$ & 2080 & $334 \pm 15$ & +0.020 & 0.277 \\
& B $\gamma$ & 585 & $7.64 \pm 4.8$ & +0.000 & 0.0258 \\
\hline
\end{tabular}

graden paßt. Ich vermute daher, daß die beobachtete 'Modulation' nicht real ist, sondern durch intrinsische Helligkeitsfluktuationen von EX Hya oder durch anderweitige Einflüsse während der recht kurzen Beobachtungsdauer vorgetäuscht werden. Dies läßt sich anhand der vorliegenden Daten aber nicht zweifelsfrei klären (s.a. den entsprechenden Absatz in Abschnitt 4.2.7.4).

- Während die Emissionslinien im UV und Optischen außer der oben beschriebenen Variation mit der Spinphase keine weitere signifikante Modulation aufweisen (möglicherweise mit Ausnahme der optischen Emissionslinien, die ein ähnliches Verhalten wie das optische Kontinuum aufweisen, das aber ebenso vorsichtig beurteilt werden sollte, s.o.), zeigen die Emissionslinien im Infrarot eine deutliche Variation mit der Orbitalphase. Diese ist durch ein ausgeprägtes Maximum bei $\Phi_{\text {orb }} \approx 0.3$ und teilweise ein zusätzliches, deutlich schwächeres Maximum bei $\Phi_{\text {orb }} \approx 0.8$ gekennzeichnet und läßt sich formal durch Überlagerung zweier Modulationen mit der vollen bzw. halben Orbitalperiode und entsprechenden Phasenverschiebungen $\Delta \Phi$ beschreiben (s. Abbildung A.58 bis A.65 sowie Tabelle A.8 in Anhang A.4.2). Der Modulationsgrad der 98-min-Variation liegt für die verschiedenen Linien bei $\approx 0.11 \ldots 0.14$ und ist stets größer als derjenige der 49 -min-Variation von $\approx 0.05 \ldots 0.10$. 


\subsubsection{Gesamtspektrum von EX Hyа}

Der besseren Darstellbarkeit in Abbildungen zuliebe werden im folgenden Flüsse oft nicht wie bisher als $f_{\lambda}$ (d.h. Fluß pro Wellenlängenintervall), sondern als $f_{\nu}$ (d.h. Fluß pro Frequenzintervall) angegeben. $f_{\lambda}$ und $f_{\nu}$ hängen über die Beziehung

$$
f_{\nu}=f_{\lambda} \frac{d \lambda}{d \nu}=f_{\lambda} \frac{\lambda^{2}}{c}
$$

miteinander zusammen ( $c=$ Lichtgeschwindigkeit). Die Einheit Jy (Jansky) von $f_{\nu}$ ist definiert durch $1 \mathrm{Jy} \equiv 10^{-26} \mathrm{~W} \mathrm{~m}^{-2} \mathrm{~Hz}^{-1}$; für die Umrechnung von $f_{\lambda}$ in $f_{\nu}$ gilt mithin

$$
\begin{aligned}
f_{\nu} & =\frac{10^{5}}{3} \operatorname{Jy}\left(\frac{\lambda}{1 \AA}\right)^{2}\left(\frac{f_{\lambda}}{1 \operatorname{erg~cm}{ }^{-2} \mathrm{~s}^{-1} \AA^{-1}}\right) \\
& =\frac{1}{3 \cdot 10^{8}} \operatorname{mJy}\left(\frac{\lambda}{1 \AA}\right)^{2}\left(\frac{f_{\lambda}}{10^{-16} \mathrm{erg} \mathrm{cm}^{-2} \mathrm{~s}^{-1} \AA^{-1}}\right) .
\end{aligned}
$$

In Abbildung 4.23 und 4.24 sind die spinphasengemittelten Spektren bzw. die Differenzen der Spinmaximums- und -minimumsspektren aus den Abbildungen 4.12, 4.13, 1.18, 4.19 und 4.20 gemeinsam gezeigt. Abbildung 4.25 gibt den Modulationsgrad der Emissionslinien (s. Tabelle 4.10) als Funktion der Linienenergie $E=h c / \lambda$ wieder.

Im unteren Teil von Abbildung 4.23 ist außer den bisher besprochenen Spektren schematisch das mittlere ORFEUS II- und das mittlere HUT-Spektrum (HUT=Hopkins Ultraviolet Telescope) gezeigt. Letztere wurden per Hand aus Abbildung 2 von Mauche (1999) bzw. Abbildung 1 von Greeley et al. (1997) abgelesen. Die Kontinua wurden dabei durch einen linearen (ORFEUS II) bzw. einen in zwei Abschnitten linearen Verlauf (HUT) abstrahiert. Die Emissionslinien wurden jeweils durch überlagerte Gauss-Profile repräsentiert, deren Zentralintensität und FWHM so geschätzt wurden, daß die Linien adäquat wiedergegeben wurden. Das gestrichelt dargestellte IUE/LWP-Spektrum in Abbildung 4.24 wurde, da zeitaufgelöste LWP-Spektren nicht verfügbar sind (vgl. Abschnitt 4.2.3), aus dem mittleren LWP-Spektrum (Abbildung 4.12) erzeugt, indem sein Fluß im Bereich 1930...1955 A an den Fluß des SWP-Spektrums im selben Bereich angepaßt wurde. Dieses Vorgehen unterstellt implizit, daß sich mit $\Phi_{\text {spin }}$ nur die Intensität des LWP-Spektrums, nicht aber seine Form ändert, was sicher nur näherungsweise richtig ist. Obwohl sich die Größe der

Tabelle 4.11: Effektivwellenlängen $\lambda_{\text {eff }}$, Halbwertsbreiten der Transmissionsfunktionen und Flußkonstanten $c_{\lambda}$ (vgl. (4.7)) der Bänder des photometrischen Walraven-Systems (Lub \& Pel 1977).

\begin{tabular}{llllll}
\hline Band & $\mathrm{V}$ & $\mathrm{B}$ & $\mathrm{L}$ & $\mathrm{U}$ & $\mathrm{W}$ \\
\hline$\lambda_{\text {eff }}[\AA]$ & 5467 & 4325 & 3838 & 3633 & 3255 \\
FWHM $[\AA]$ & 740 & 430 & 235 & 235 & 135 \\
$c_{\lambda}$ & -11.174 & -10.912 & -10.806 & -10.826 & -10.683 \\
\hline
\end{tabular}


Tabelle 4.12: Mittlerer Fluß $\langle f\rangle$ und Amplitude $a_{67}$ (beide in $10^{-16} \mathrm{erg} \mathrm{cm}^{-2} \mathrm{~s}^{-1} \AA^{-1}$ ) und Modulationsgrad $\bmod _{67}$ (s. (4.2)) der Spinmodulation in den WalravenPhotometerbändern (vgl. Tabelle 4.11). Die Werte entstammen den in Abbildung 4.27 dargestellten Anpassungen.

\begin{tabular}{lccccc}
\hline Band & $\mathrm{V}$ & $\mathrm{B}$ & $\mathrm{L}$ & $\mathrm{U}$ & $\mathrm{W}$ \\
\hline$\langle f\rangle$ & $168 \pm 5.6$ & $304 \pm 11$ & $467 \pm 17$ & $492 \pm 22$ & $605 \pm 29$ \\
$a_{67}$ & $37.6 \pm 3.2$ & $76.8 \pm 6.3$ & $132 \pm 9.8$ & $138 \pm 13$ & $180 \pm 17$ \\
$\bmod _{67}$ & 0.366 & 0.403 & 0.441 & 0.438 & 0.459 \\
\hline
\end{tabular}

möglichen Abweichungen am langwelligen Ende des LWP-Spektrums nicht ohne weiteres abschätzen läßt, kann das dargestellte Spektrum aber als Orientierungshilfe dienen.

Die in Abbildung 4.23 und 4.24 dargestellten Quadrate geben die mittleren Flüsse bzw. die vollen Amplituden der Spinmodulation (ab jetzt kurz 'vollen Spinamplituden') aus der Anpassung der Lichtkurven wieder (vgl. Tabelle 4.9). Der ausgefüllte Kreis in Abbildung 4.24 entspricht der vollen Spinamplitude im Bereich 1005...1015 $\AA$, die von Mauche (1999) aus den ORFEUS II-Spektren bestimmt wurde. Die offenen Quadrate repräsentieren die in Tabelle 3.1 angegebenen photometrischen Helligkeiten, die nach

$$
f_{\lambda}\left(\lambda_{\text {eff }}\right)=f_{0} \cdot 10^{-\mathrm{mag} / 2.5}
$$

unter Benutzung der Flußkonstanten $f_{0}$ aus Tabelle 4.3 in Flüsse bei der Effektivwellenlänge $\lambda_{\text {eff }}$ umgerechnet wurden. Die Erläuterung der offenen Kreise erfordert einen kurzen Exkurs. Ihnen liegt ein Teil der photometrischen Daten zugrunde, die 1985 von K. Reinsch mit dem Walraven-Photometer am 0.9m-Dutch-Teleskop auf La Silla gewonnen und von Siegel et al. (1989) veröffentlicht wurden. Das Walraven-System (für eine umfangreiche Beschreibung s. Lub \& Pel 1977) besteht aus fünf schmalen Bändern (VBLUW), von denen zwei (U und W) über den Balmersprung hinausreichen (s. Tabelle 4.11). Aus der in einem gegebenen Band beobachteten Walraven-Helligkeit $m_{\lambda}$ wird der Fluß $f_{\lambda}$ bei der Effektivwellenlänge $\lambda_{\text {eff }}$ nach

$$
f_{\lambda}\left(\lambda_{\text {eff }}\right)=1 \mathrm{erg} \mathrm{cm}^{-2} \mathrm{~s}^{-1} \AA^{-1} \cdot 10^{m_{\lambda}+c_{\lambda}}
$$

berechnet. Die hierin eingehenden Flußkonstanten $c_{\lambda}$ der einzelnen Bänder sind in Tabelle 4.11 angegeben. Die offenen Kreise in den Abbildungen entsprechen den mittleren Flüssen bzw. Spinamplituden, die an die nach (4.7) aus den Helligkeiten berechneten Flüsse angepaßt wurden (s. Abbildung 4.27). Die einzelnen Werte sind in Tabelle 4.12 nochmals zusammengefaßt.

In Abbildung 4.23 ist eine gute Übereinstimmung der mit ORFEUS II, HUT, HST und IUE gewonnenen mittleren Spektren festzustellen; speziell die IUE/SWP- und HSTSpektren sind fast nicht zu unterscheiden. Ob allerdings die sägezahnartige Struktur des Spektrums im Bereich $\approx 1700 \ldots .2500 \AA$ real ist oder ihre Ursache etwa in unsicheren Kalibrationen der SWP- bzw. LWP-Spektren an den Grenzen der jeweiligen Wellenłängenbereichen haben, ist nicht ohne weiteres zu entscheiden. Die aus der Analyse der Lichtkurven 

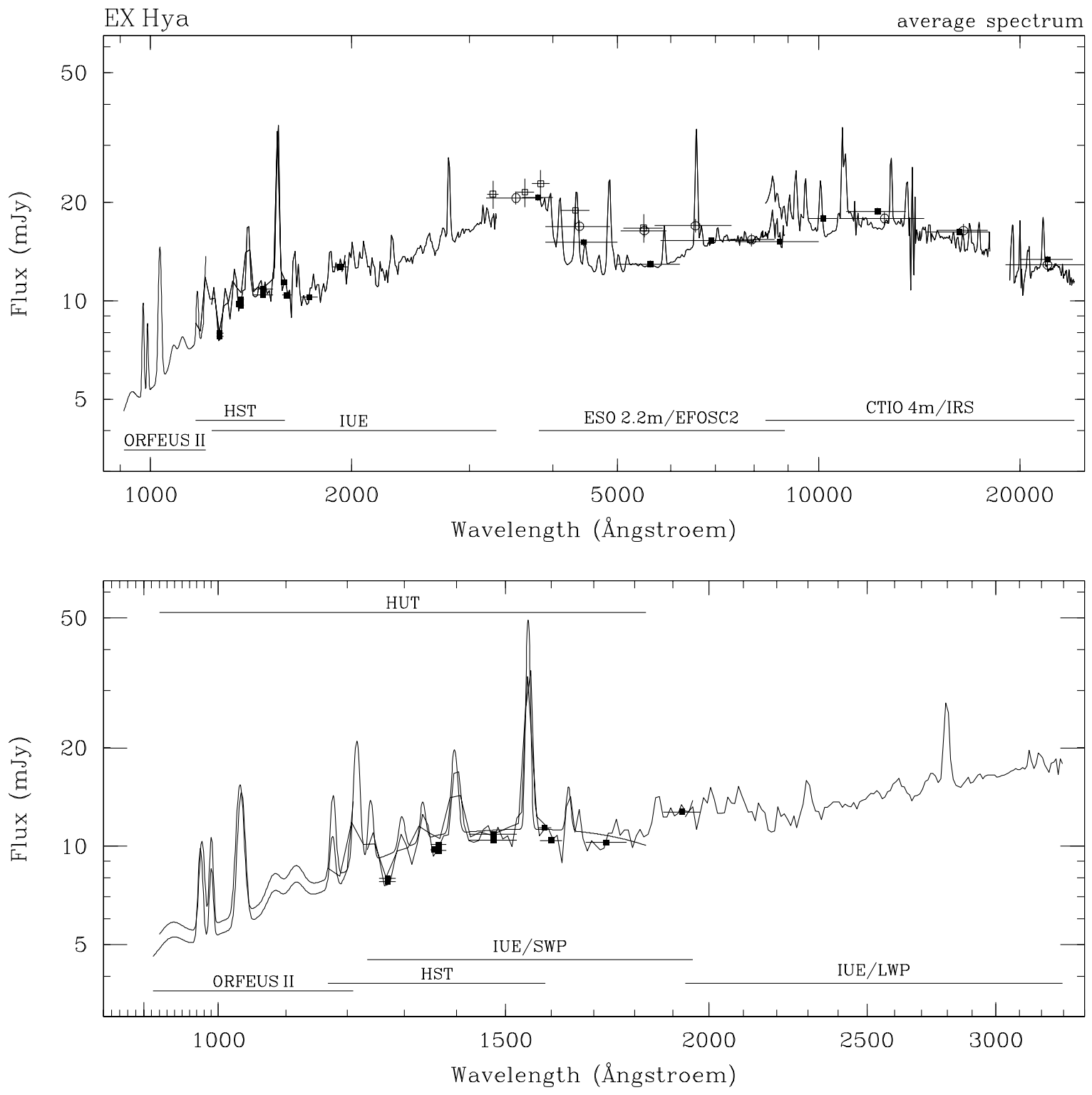

Abbildung 4.23: Mittleres Gesamtspektrum von EX Hya im Bereich 912...24000 Å Oben: mittlere Spektren aus den Abschnitten 4.2.3 bis 4.2.6 und mittleres ORFEUS II-Spektrum (aus Mauche 1999, s. Text); unten: vergrößerte Darstellung des UV-Bereich, zusätzlich mit mittlerem HUT-Spektrum (aus Greeley et al. 1997, s. Text). Ausgefüllte Quadrate: mittlerer Fluß aus der Modellierung der Lichtkurven (Werte 〈base〉 aus Tabelle 4.9); offene Quadrate: mittlere Flüsse in den Walraven-Bändern (Tabelle 4.12); offene Kreise: mittlere Flüsse entsprechend den photometrischen Helligkeiten in Tabelle 3.1. Die Fehlerbalken aller Symbole sind kleiner als die Symbolgröße.

gewonnenen mittleren Flüsse stimmen gut mit den Flüssen der gemittelten Spektren überein; dasselbe gilt im IR auch für die photometrischen Daten aus Tabelle 3.1. Es fällt jedoch auf, daß das mittlere optische Spektrum im Überlappbereich $\approx 8300 \ldots 9000 \AA$ nur $\approx 80 \%$ des 


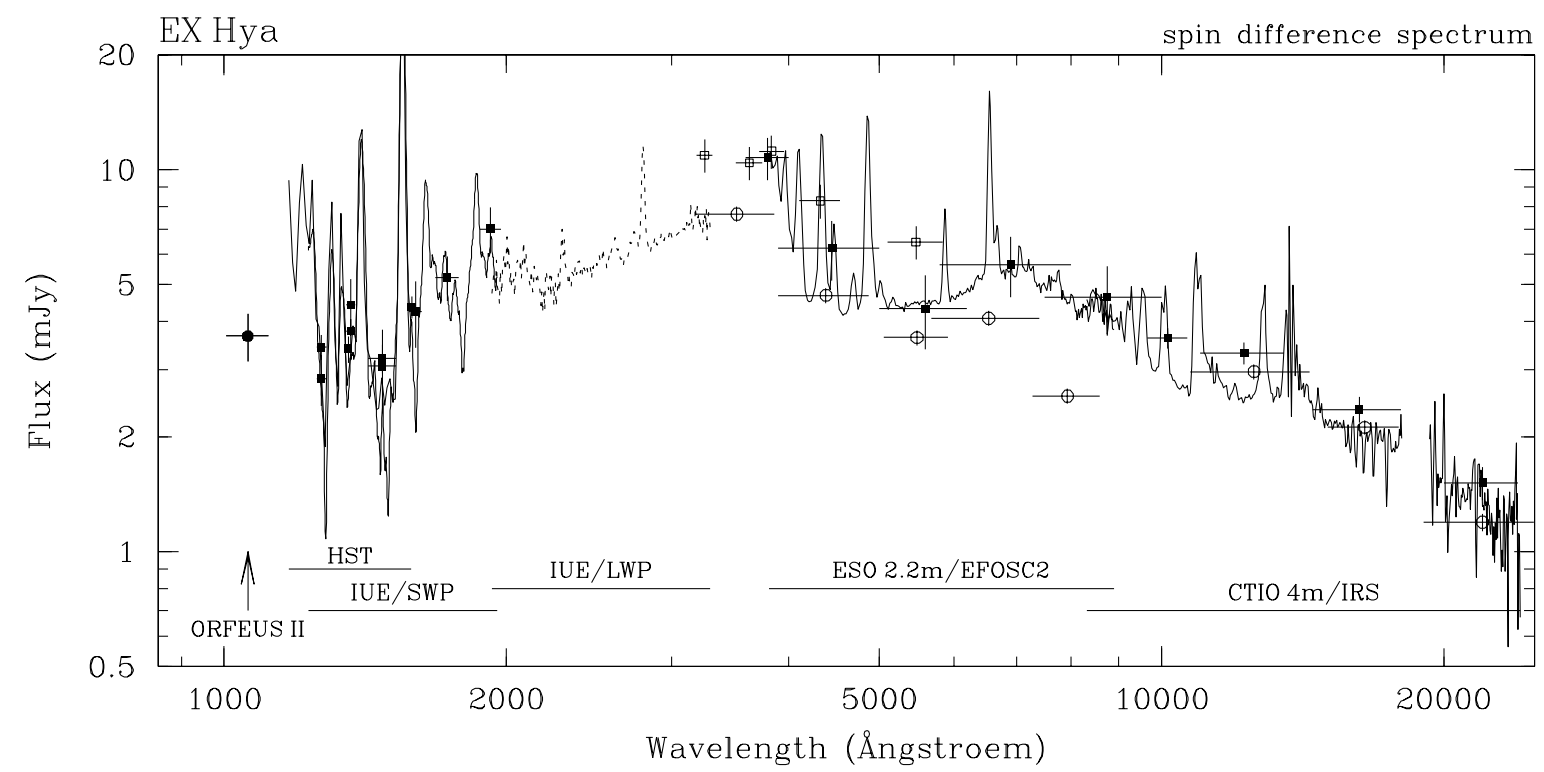

Abbildung 4.24: Gesamtspektrum der spinmodulierten Komponente von EX Hya im Bereich 1010...24000 Å. Ausgezogen: Spindifferenzspektren aus den Abschnitten 4.2.3 bis 4.2.6; gepunktet: an den Fluß des IUE/SWP-Spektrums im Bereich 1930...1955 A angepaßtes IUE/LWP-Spektrum. Die Symbole geben die volle Amplitude der Spinmodulation aus der Modellierung der Lichtkurven (ausgefüllte Quadrate; s. Tabelle 4.9), in den WalravenBändern (s. Tabelle 4.12; offene Quadrate), aus den photometrischen Daten in Tabelle 3.1 (offene Kreise) bzw. im Bereich 1005...1015 A (ORFEUS II, Mauche 1999; ausgefüllter Kreis) wieder.

Flusses des IRS-Spektrums aufweist, während das spinmodulierte Spektrum kein solches Defizit zeigt. Die Form der Spektren im genannten Bereich ist allerdings nahezu identisch (s. Abbildung 4.26). Die photometrischen Daten (offene Symbole) legen nahe, daß der Fluß des mittleren optischen Spektrums aus ungeklärten Gründen insgesamt um einen entsprechenden Faktor zu niedrig liegt. Zwar sind Ungenauigkeiten von $\pm 20 \%$ durchaus typisch für Schmalspalt-Spektrophotometrie. Nimmt man aber z.B. einen Flußverlust von 20\% - etwa aufgrund eines zu schmal gewählten Spektrographenspalts - an, sollten davon alle Einzelspektren und damit auch das mittlere wie das Spinmaximums- bzw. -minimumsspektrum in gleicher Weise betroffen sein, so daß dann wiederum die gute Übereinstimmung der Flüsse der spinmodulierten Spektren schwer verständlich ist (vgl. Abbildung 4.24). Eine mögliche Erklärung für diese Diskrepanz könnte darin bestehen, daß die für die Konstruktion der optischen Spinmaximums- und -minimumsspektren verwendeten Spektren (vgl. Abschnitt 4.2.5) bei $\Phi_{\text {orb }}=0.345$ und $\Phi_{\text {orb }}=0.47$ bzw. $\Phi_{\text {orb }}=0.645$ liegen (s. Tabelle 4.2) ) Bei dieser Phasenauswahl kann eine Beeinflussung der Spektren durch die am Ende von Abschnitt 3.2.6.3 erwähnten Maxima in der orbitalen Lichtkurve in dem Sinne entstehen, daß das Spinmaximumsspektrum 'zu hell' und das Minimumsspektrum 'zu schwach' erscheint. Eine weitere Fehlerquelle, die ebenfalls den Fluß des Differenzspektrums erhöht, liegt darin, daß die für das Maximumsspektrum ausgewählten Spektren beide etwas heller sind als es den Modellichtkurven entspricht (s. etwa Abbildung A.43). Möglicherweise 


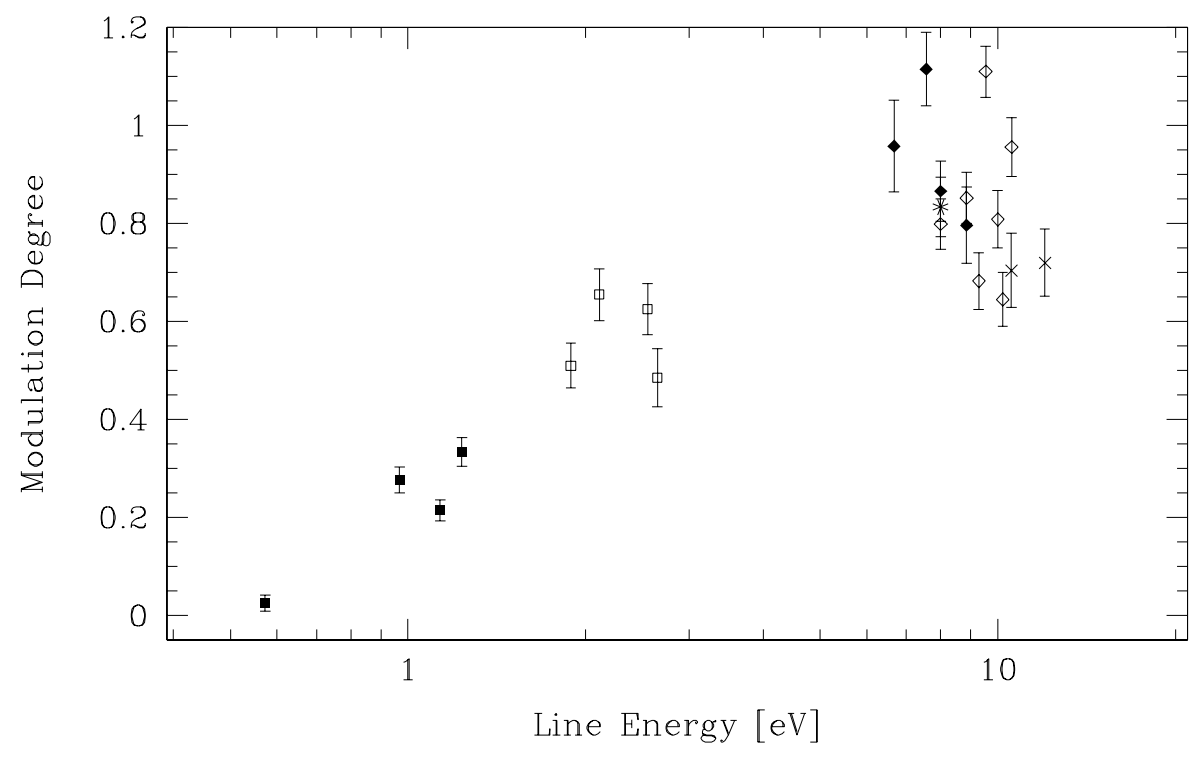

Abbildung 4.25: Nach (4.2) berechneter Spinmodulationsgrad der Emissionslinien als Funktion der Linienenergie $E=h c / \lambda$. Ausgefüllte bzw. offene Quadrate: IRS bzw. EFOSC 2; ausgefüllte bzw. offene Rauten: IUE bzw. HST (Daten aus Tabelle 4.10). Stern bzw. Kreuze: IUE bzw. ORFEUS II aus Mauche (1999).

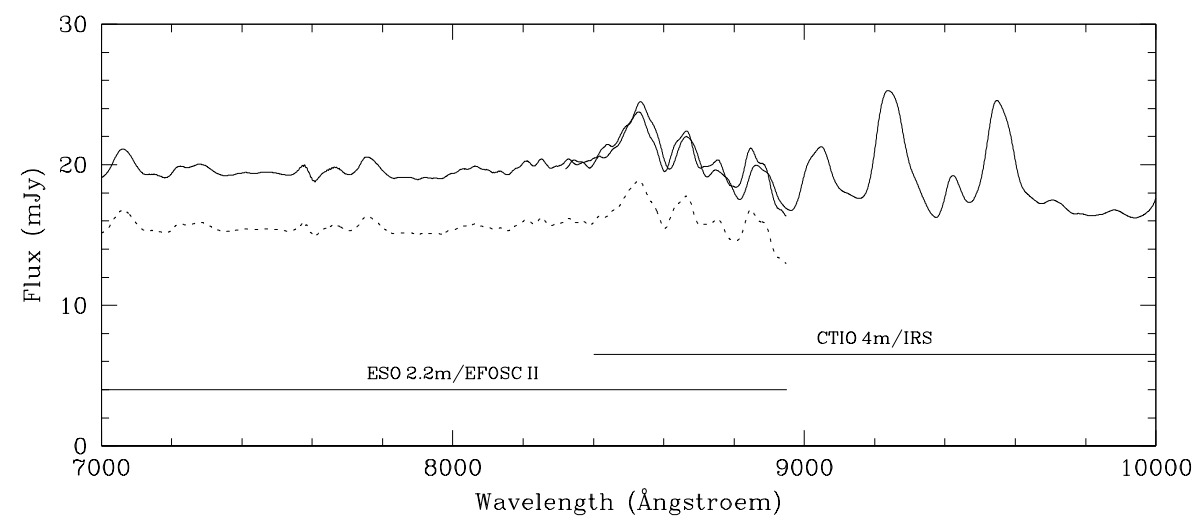

Abbildung 4.26: Mittleres EFOSC 2- und IRS-Spektrum von EX Hya im Überlappbereich. Das durchgezogene optische Spektrum wurde gegenüber dem gepunkteten 'Original' um den Faktor 1.25 hochskaliert, um die Übereinstimmung der Form zu demonstrieren.

spielen beide Effekte eine Rolle, so daß das 'wahre' spinmodulierte Spektrum ein ähnliches Flußdefizit gegenüber dem entsprechenden IRS-Spektrum aufwiese wie das mittlere und dann auch konsistenter mit den photometrischen Daten wäre. Ohne Daten mit einer längeren Zeitbasis, die auch das Anpassen und Abseparieren orbitaler Variationen zuläßt, ist diese Frage jedoch nicht abschließend zu klären.

Abschließend soll noch kurz auf einige weitere Punkte hingewiesen werden.

- Die mittleren Flüsse aus der Johnson- und Walraven-Photometrie (offene Symbole in 


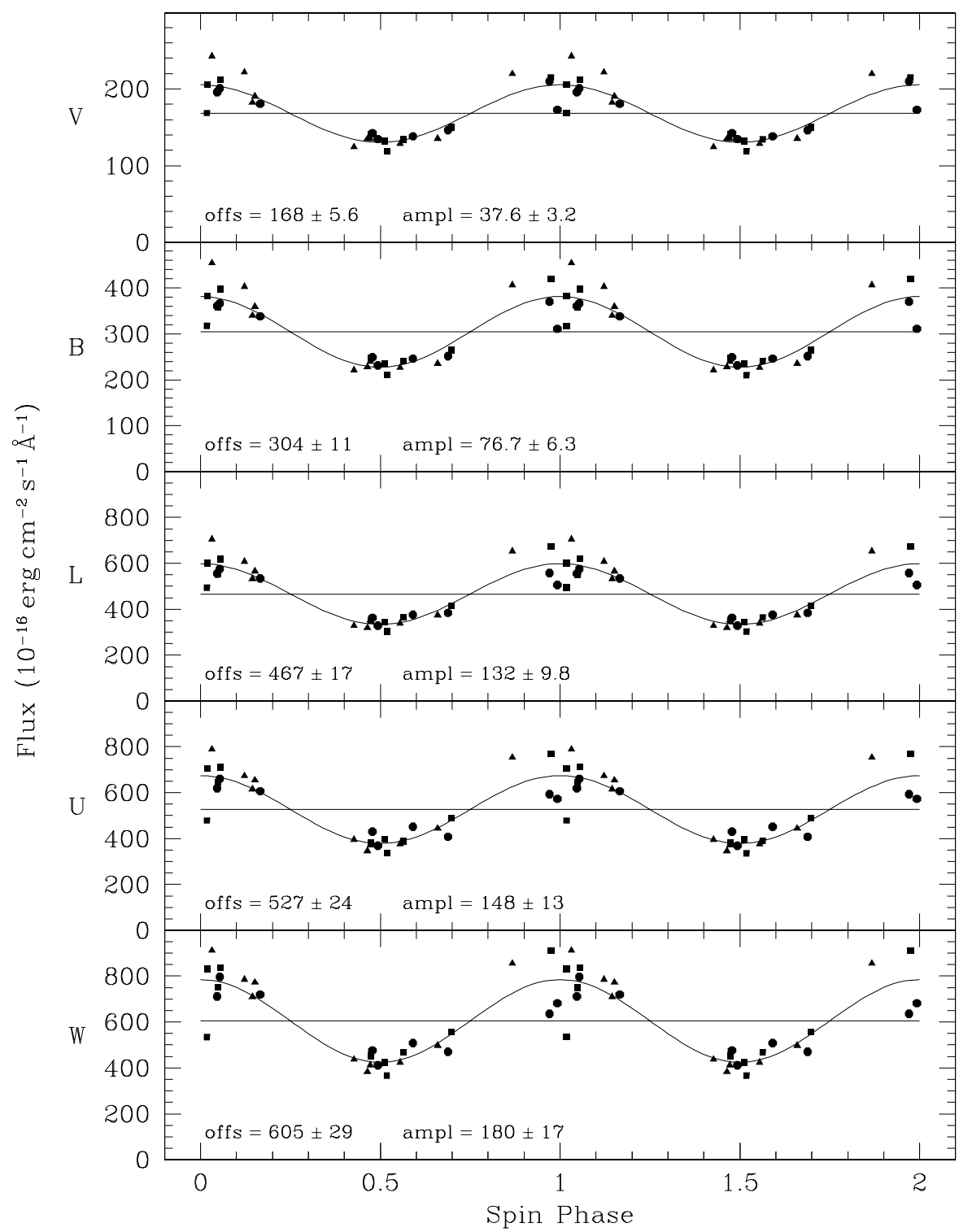

Abbildung 4.27: Walraven-VBLUW-Photometrie von EX Hya und Anpassung von mittlerem Fluß und Modulationsamplitude. Der Abbildung liegen Teile der von Siegel et al. (1989) verwendeten Daten aus dem Jahr 1985 zugrunde. Quadrate, Dreiecke bzw. Kreise symbolisieren Datenpunkte vom 23., 24. bzw. 25.04.1985. Die angepaßten Werte finden sich zusammengefaßt in Tabelle 4.12.

Abbildung 4.23) stimmen gut miteinander überein; hingegen ist die Spinmodulation in den Walraven-Daten deutlich ausgeprägter (vgl. Abbildung 4.24).

- Der Punkt für das $I_{\mathrm{KC}}$-Band bei $\approx 8000 \AA$ scheint in beiden Abbildungen zu niedrig zu liegen. Umgekehrt erscheint der Punkt für $W_{\mathrm{W}}$ bei $\approx 3200 \AA \mathrm{zu}$ hoch, zumal auf der kurzwelligen Seite des Balmersprungs ein schnell abfallender Fluß erwartet wird.

- Die Intensität des spinmodulierten Spektrums beträgt im Optischen $\approx 1 / 3$ derjenigen 
des mittleren Spektrums; es hat jedoch einen höheren Balmersprung als dieses und fällt bei Wellenlängen $\gtrsim 8000 \AA ̊$ deutlich schneller ab. Im UV steigt es im Gegensatz zum mittleren Spektrum unterhalb von $\approx 1500 \AA$ wieder an.

— Der 'Buckel' bei $\approx 6000 \ldots 8000 \AA$, der im mittleren und stärker noch im spinmodulierten Spektrum vorhanden ist, spiegelt sich in beiden Fällen auch in den photometrischen Daten wieder, ist also wahrscheinlich real. Seinen physikalischen Ursprung habe ich nicht klären können; insbesondere ist er zu schmal, um durch $\mathrm{H}^{-}$-Emission erklärt werden zu können. (Letztere entsteht bei der Anlagerung eines Elektrons an ein neutrales Wasserstoffatom und erstreckt sich über den Bereich $\approx 3000 \ldots 15000 \AA$, vgl. Abbildung IV.14 in Scheffler \& Elsässer 1990.) 


\section{Kapitel 5}

\section{Interpretation der Ergebnisse}

\section{$5.1 \quad$ Systemparameter}

Aus den vorliegenden Daten - insbesondere den Eigenschaften der Bedeckungen in den verschiedenen Energiebereichen - lassen sich weitreichende Schlüsse auf verschiedene Systemparameter von EX Hya ziehen. Dabei ist es allerdings nicht möglich, die einzelnen Parameter unabhängig voneinander zu bestimmen; vielmehr muß ein in sich konsistenter Parametersatz gesucht werden, der die Beobachtungen befriedigend erklärt. Aus diesem Grund werde ich im folgenden verschiedentlich auf weiter unten stehende Abschnitte vorgreifen müssen. Zunächst leite ich aus den Eigenschaften der Bedeckungen das Bedeckungsszenario und die grundsätzliche Lage der jeweils bedeckten Gebiete ab. Anschließend wird das Szenario quantifiziert. Die zugehörigen Rechnungen wurden dabei numerisch unter Benutzung der exakten Roche-Geometrie (s. Kapitel 2.1.1) durchgeführt.

\subsubsection{Geometrie der Bedeckungen}

Zunächst muß geklärt werden, wo genau im System sich die Strahlungsquellen befinden, deren Bedeckungen in den verschiedenen Energiebereichen beobachtet werden. Die kurzen Bedeckungen im Optischen wie im Röntgenbereich (s. Kapitel 3.2.6.2) erfordern zunächst, daß die Bedeckungen der jeweiligen Strahlungsquellen streifend sind (vgl. Abbildung 3.6 in Kapitel 3.2.7.3). Im intermediate polar model (Kapitel 2.3.3) entsteht die Röntgenemission in den nahe am Weißen Zwerg liegenden Bereichen der Akkretionsvorhänge durch Aufheizung des Plasmas in einem starken hydrodynamischen Stoß. Speziell im Röntgenbereich sind daher drei verschiedene Bedeckungsszenarien möglich, in denen einer, beide oder keiner der zwei Pole des Weißen Zwergs bedeckt wird (s. Abbildung 5.1). Sofern Röntgenemission von beiden Polen beobachtet wird und die emittierenden Gebiete hinreichend ausgedehnt sind, können alle drei Szenarien prinzipiell erklären, warum die Bedeckung im Röntgenbereich lediglich partiell ist.

Falls der Stoß hoch über dem Weißen Zwerg steht, kann das Material hinter der Stoßfront im Gravitationsfeld des Weißen Zwergs nachbeschleunigt werden und erreicht seine höchste Temperatur dann dichter am Weißen Zwerg (Kapitel 2.4.2.2). In jedem Fall führen 

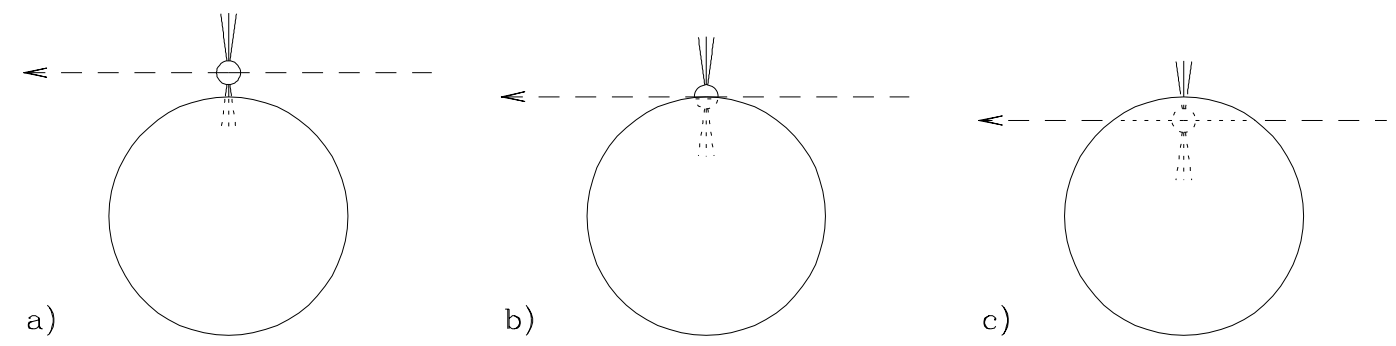

Abbildung 5.1: Mögliche Bedeckungsszenarien von EX Hya (nicht maßstabsgerecht) mit Bedeckung keines (links), eines (Mitte) oder beider Pole des Weißen Zwergs (rechts) (nach einer Abbildung aus Rosen et al. 1988). Prinzipiell können alle drei Szenarien die beobachtete partielle Bedeckung im harten Röntgenbereich erklären; die Totalität der Bedeckung im EUV legt jedoch c) als richtiges Szenario nahe (vgl. Abschnitt 3.2.6.2).

Brems- und Zyklotronstrahlung jedoch anschließend zu einer Abkühlung des weiter fallenden Plasmas, so daß sich der Schwerpunkt des emittierten Spektrums mit abnehmender Entfernung von der Oberfläche des Weißen Zwergs zunehmend zu niedrigeren Photonenenergien hin verschiebt. Die niedrigsten Temperaturen, bei denen hauptsächlich EUVStrahlung emittiert wird, finden sich daher bei $r \approx R_{\text {WD }}$ in der Nähe der magnetischen Pole. Die Totalität und Kürze der Bedeckung im EUV und der zeitlich nicht aufgelöste Ein- und Austritt sowie die Spinphasenunabhängigkeit der Bedeckungszeitpunkte (Kapitel 3.2.6.2) favorisieren daher klar das Szenario in rechten Teilbild von Abbildung 5.1, in dem die gesamte EUV-Emission vom oberen Pol des Weißen Zwergs stammt und vollständig bedeckt wird, während der untere Pol nicht beobachtbar ist. Prinzipiell wäre zwar auch die umgekehrte Situation denkbar, in der stets nur der untere Pol sichtbar ist, jedoch ist schwer vorstellbar, auf welche Weise ausschließlich die Emission des oberen Pols - und zwar vollständig - absorbiert werden könnte.

Da in dieser Situation die Trajektorie des oberen Pols des Weißen Zwergs in vertikaler Richtung sehr dicht unter dem Rand des Sekundärsterns verläuft (vgl. Abbildung 3.6), bleiben die Röntgenstrahlung emittierenden Teile des oberen Akkretionsvorhangs zum weitaus größten Teil unbedeckt. Wie auch schon von Beuermann \& Osborne (1988) und Mukai et al. (1998) vermutet, muß für die partielle Bedeckung im Röntgenbereich also die Bedeckung des unteren Akkretionsvorhangs verantwortlich sein, während der obere Akkretionsvorhang (zumindest größtenteils) permanent sichtbar bleibt. Insbesondere ist also außerhalb der Bedeckung die Röntgenemission beider Pole sichtbar, was bei der Bestimmung der Leuchtkraft und damit der Akkretionsrate $\dot{M}$ nach (2.36) von Bedeutung ist.

\subsubsection{Zusammenhang von Inklination und Massenverhältnis}

Wenn man die im EUV bedeckte Quelle mit dem oberen Pol des Weißen Zwergs identifiziert, läßt sich aus der Bedeckungsdauer von $41 \mathrm{~s}$ die Inklination $i$ als Funktion des Massenverhältnisses $q$ bestimmen. Abbildung 5.2 zeigt, daß bei gegebenem $q$ die erforder- 


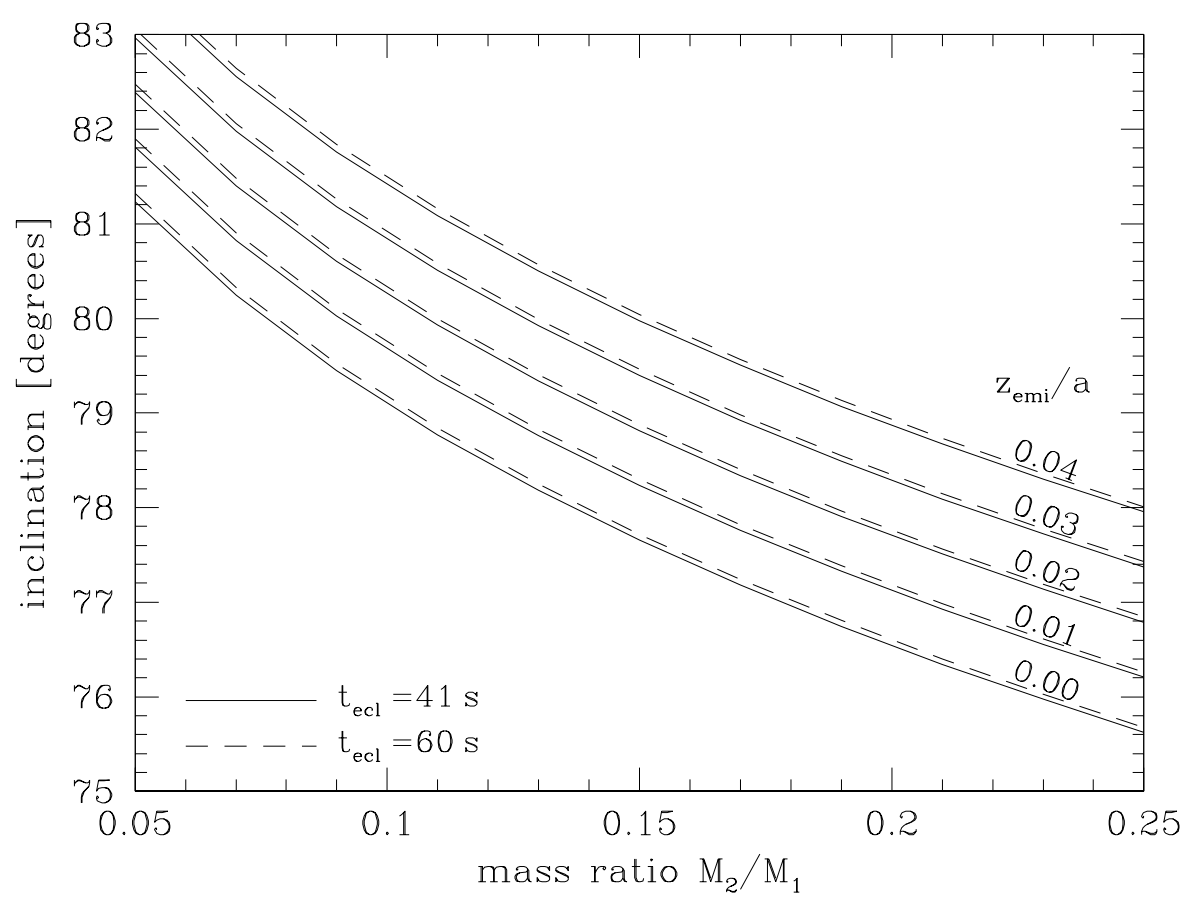

Abbildung 5.2: Notwendige Inklination $i$ als Funktion des Massenverhältnisses $q$ für eine Bedeckungsdauer von $41 \mathrm{~s}$ (ausgezogen) bzw. $60 \mathrm{~s}$ (gestrichelt). Die bedeckte Punktquelle befindet sich in der Orbitalebene am Ort des Weißen Zwergs; der Kurvenparameter $z_{\text {emi }}$ gibt die Höhe der Quelle über der Orbitalebene an (in Einheiten der Länge $a$ der Bahnhalbachse).

liche Inklination $i$ nur wenig von der tatsächlichen Bedeckungsdauer, aber stark von der Höhe $z_{\text {emi }}$ der bedeckten Quelle über der Orbitalebene abhängt, wobei letztere aufgrund des obigen Szenarios im wesentlichen mit dem Radius $R_{\mathrm{WD}}$ des Weißen Zwergs identisch ist. Da die Ausdehnung der Äquipotentialflächen des Roche-Potentials durch die Länge $a$ der Bahnhalbachse des Systems bestimmt ist (vgl. Kapitel 2.1.1), ist in der Abbildung der Kurvenparameter $z_{\mathrm{emi}}$ in Einheiten von $a$ angegeben.

\subsubsection{Massen des Weißen Zwergs und des Sekundärsterns}

Zur Bestimmung der Massen des Weißen Zwergs und des Sekundärsterns wurde zunächst die im Röntgenbereich bedeckte Quelle mit dem nahe am Weißen Zwerg liegenden Teil des unteren Akkretionsvorhangs identifiziert. Die Bedeckungsdauer, für die der von Mukai et al. (1998) genannte Wert 156s (FWHM) angenommen wurde (bzw. 136s für die Dauer der totalen Bedeckung des unteren Akkretionsvorhangs, entsprechend dem flachen Teil der Lichtkurve zwischen Ein- und Austritt, vgl. Kapitel 3.2.6.2), erlaubt dann die Bestimmung der Trajektorie des unteren Pols des Weißen Zwergs. Der Abstand zur Trajektorie des oberen Pols senkrecht zur Bahnebene liefert dann direkt den Durchmesser des Weißen Zwergs; seine Masse erhält man dann aus einer geeigneten Masse-Radius-Relation. Für letztere 


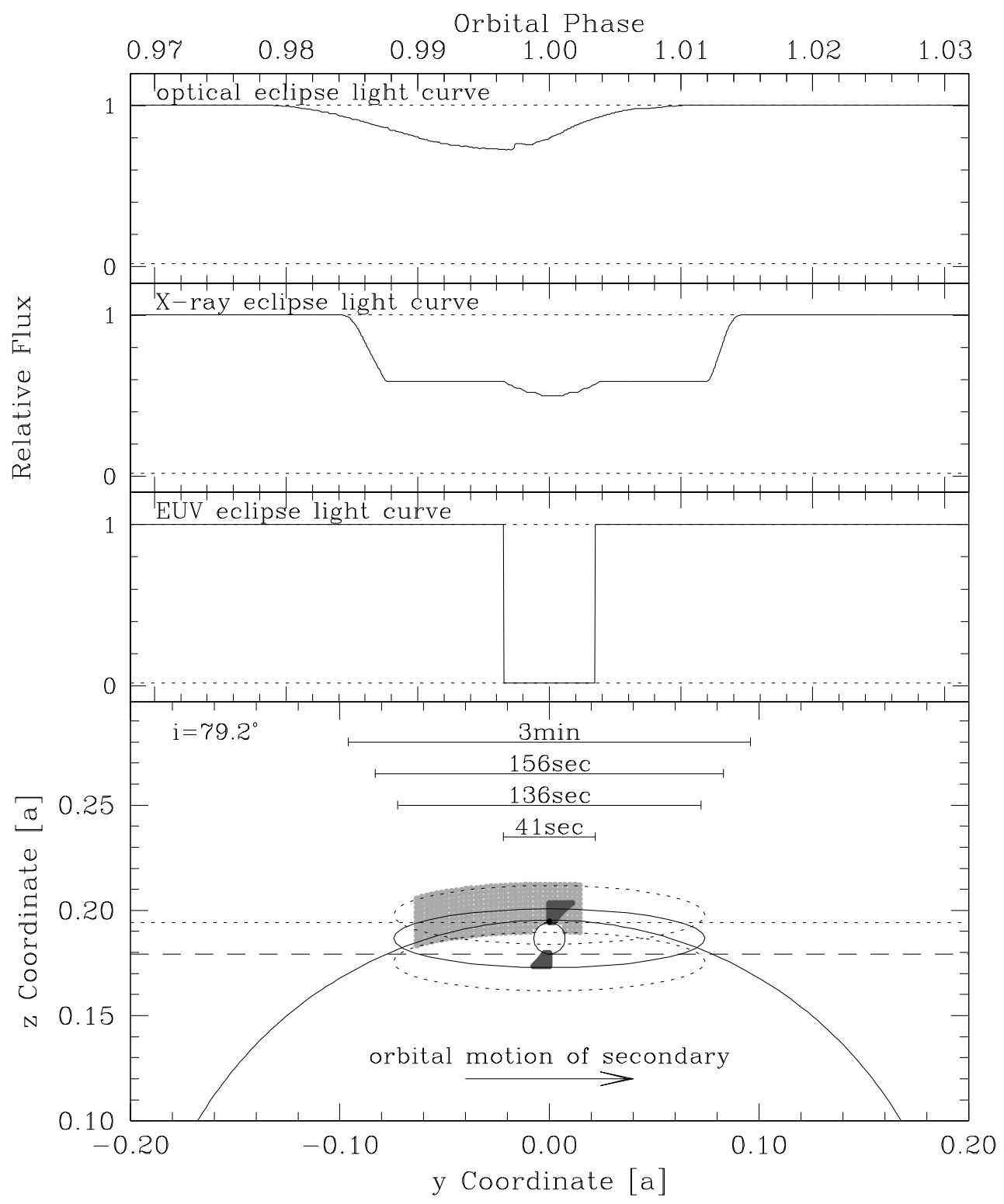

Abbildung 5.3: Bedeckungsgeometrie von EX Hya (unteres Teilbild) und synthetische Lichtkurven im Optischen, EUV und Röntgenbereich (obere Teilbilder) für ein Szenario mit $M_{\mathrm{WD}}=1.2 M_{\odot}$ und $q=0.11$ bei einer angenommenen Spinphase $\Phi_{\text {spin }} \approx 0.75$. Für den Radius $R_{i}$ des Innenrands der Akkretionsscheibe wurde $R_{i}=10 R_{\mathrm{WD}}$ angenommen. Für Details des dargestellten Szenarios und die Erläuterung der im unteren Teilbild dargestellten Linien und Kurven s. Text. Der 'Zacken' in der optischen Lichtkurve ist ein Artefakt des Verfahrens ihrer Konstruktion.

wurde eine Temperatur von $20000 \mathrm{~K}$ des Weißen Zwergs angesetzt und berücksichtigt, daß der Weiße Zwerg infolge der Akkretion vermutlich eine dicke Hülle und folglich einen etwas größeren Radius als nach (2.18) berechnet hat (vgl. Kapitel 2.2.1.3). 
Im Detail bin ich wie folgt vorgegangen:

- Es werden versuchsweise die Masse $M_{\mathrm{WD}}$ des Weißen Zwergs und das Massenverhältnis $q$ vorgegeben. Hieraus folgen unmittelbar die Masse $M_{2}$ des Sekundärsterns und mit der Orbitalperiode $P_{\text {orb }}$ die Länge der Bahnhalbachse $a$. Damit sind auch Form und Ausdehnung des Roche-Volumens des Sekundärsterns festgelegt. Ferner folgt nach der MasseRadius-Relation (2.24) der Hauptreihenradius des Sekundärsterns. Falls dieser größer oder wesentlich kleiner als der nach (2.7) berechnete Volumenradius $R_{V}$ des Roche-Volumens ist, kann das gewählte Wertepaar bereits an dieser Stelle ausgeschlossen werden.

- Mit Hilfe der eingangs erwähnten Masse-Radius-Relation für den Weißen Zwerg wird dessen Radius bestimmt, der gleichzeitig (zusammen mit der inzwischen ebenfalls bekannten Länge $a$ der Bahnhalbachse) die Höhe $z_{\text {emi }}$ der bedeckten EUV-Quelle über der Orbitalebene festlegt. Diese liefert aufgrund des in Abschnitt 5.1.2 dargestellten Zusammenhangs zwischen Massenverhältnis $q$ und Inklination $i$ auch den Wert für letztere, der für eine Bedeckung von $41 \mathrm{~s}$ Dauer erforderlich ist. Im unteren Teilbild von Abbildung 5.3 entspricht die gepunktete Linie der Trajektorie des oberen Pols; die Länge desjenigen Teils davon, der innerhalb der als Kreisbogen dargestellten Silhouette des Sekundärsterns liegt, entspricht dieser Bedeckungslänge.

- Aus der Roche-Geometrie wird nun die Bedeckungsdauer des unteren Pols berechnet; seine Trajektorie ist in Abbildung 5.3 unten als gestrichelte Gerade dargestellt. Falls die so ermittelte Bedeckungsdauer deutlich größer oder kleiner als der oben zitierte Wert von $136 \mathrm{~s}$ ist, kann das gewählte Wertepaar verworfen werden.

Bei Variation der gewählten Eingangsparameter $M_{\mathrm{WD}}$ und $q$ stellt sich heraus, daß die verschiedenen Randbedingungen den möglichen Werten sehr enge Grenzen setzen. So führen Massen des Weißen Zwergs von $M_{\mathrm{WD}} \lesssim 1.0 M_{\odot}$ bzw. $\gtrsim 1.3 M_{\odot}$ zu Bedeckungszeiten des unteren Pols (flacher, zentraler Teil der Lichtkurve) von $\gtrsim 180 \mathrm{~s}$ bzw. $\lesssim 120 \mathrm{~s}$ und sind somit unwahrscheinlich. Bei $M_{\mathrm{WD}}=1.1 M_{\odot}$ bzw. $1.2 M_{\odot}$ ergeben sich Bedeckungszeiten von $160 \mathrm{~s}$ bzw. $140 \mathrm{~s}$. Bemerkenswerterweise ist dabei zur Vermeidung einer Über- oder einer deutlichen Unterfüllung des Roche-Volumens des Sekundärstern nahezu unabhängig vom gewählten Wert für $M_{\mathrm{WD}}$ stets ein derartiges Massenverhältnis $q$ erforderlich, daß die resultierende Masse $M_{2}$ des Sekundärsterns bei $0.13 M_{\odot}$ liegt. Massen $M_{2} \gtrsim 0.14 M_{\odot}$ führen stets zu einer Überfüllung des Roche-Volumens, während zu kleineren Massen hin die Unsicherheit im noch akzeptierten Unterfüllungsgrad des Roche-Volumens besteht. Bei Massen $M_{2} \lesssim 0.12 M_{\odot}$ müsste der Sekundärstern bereits um $\gtrsim 10 \%$ gegenüber seinem Hauptreihenradius nach (2.24) expandiert sein, um sein Roche-Volumnen ausfüllen zu können, was unterhalb der Periodenlücke unwahrscheinlich erscheint.

In ihrer Gesamtheit deuten die Befunde auf die in Tabelle 5.1 angegebenen Werte der Systemparameter hin. (Dort aufgeführte und noch nicht diskutierte Parameter werden in den folgenden Abschnitten hergeleitet.) Die Masse des Weißen Zwergs liegt damit um einen Faktor $\approx 2$ über den bisher angenommenen Werten (vgl. Kapitel 3.2.3), wohingegen die Masse $M_{2}$ des Sekundärsterns recht gut sowohl mit dem nach (2.22) erwarteten Wert als auch mit verschiedenen Werten aus der Literatur übereinstimmt. Sie impliziert aber einen Spektraltyp des Sekundärsterns, der deutlich später als der von Dhillon et al. (1997; $\approx$ M.3) bzw. Smith (2000; M1.5) genannte ist (s. Kapitel 3.2.3). Diese Diskrepanz wird 
Tabelle 5.1: Systemparameter von EX Hya aus den Ableitungen in Abschnitt 5.1. Die Fehler der Sternmassen und der Inklination wurden großzügig abgeschätzt (s. Text).

\begin{tabular}{ll}
\hline$M_{\mathrm{WD}}$ & $1.2 \pm 0.1 M_{\odot}$ \\
$M_{2}$ & $0.13 \pm 0.01 M_{\odot}$ \\
$i$ & $79.2^{\circ} \pm 0.5^{\circ}$ \\
$a$ & $(5.38 \pm 0.15) \cdot 10^{10} \mathrm{~cm}=0.77 \pm 0.02 R_{\odot}$ \\
$K_{1}$ & $56 \pm 5 \mathrm{~km} \mathrm{~s}^{-1}$ \\
$K_{2}$ & $507 \pm 20 \mathrm{~km} \mathrm{~s}^{-1}$ \\
$R_{\mathrm{WD}}$ & $(4.0 \pm 0.8) \cdot 10^{8} \mathrm{~cm}$ \\
$R_{V, \text { Roche }}$ & $(1.15 \pm 0.03) \cdot 10^{10} \mathrm{~cm}=0.165 \pm 0.005 R_{\odot}$ \\
$R_{2, \mathrm{ZAMS}}$ & $(1.09 \pm 0.07) \cdot 10^{10} \mathrm{~cm}=0.157 \pm 0.010 R_{\odot}$ \\
$R_{\mathrm{i,disc}}$ & $10 \pm 2 R_{\mathrm{WD}}=(4.0 \pm 0.8) \cdot 10^{9} \mathrm{~cm}$ \\
$R_{\mathrm{a}, \mathrm{disc}}$ & $\approx 2.5 \cdot 10^{10} \mathrm{~cm}$ \\
$\operatorname{SpTyp}(2)$ & $\mathrm{M} 4.0 \ldots \mathrm{M} 5.0$ \\
$S_{K}(2)$ & $4.5 \pm 0.2$ \\
$\left\langle\operatorname{mag}_{K}(2)\right\rangle$ & $12.5 \pm 0.2$ \\
$d$ & $66 \pm 9 \mathrm{pc}$ \\
\hline
\end{tabular}

weiter unten geklärt werden. Auch impliziert die hohe Masse des Weißen Zwergs, daß die beobachtete Orbitalgeschwindigkeit des Sekundärsterns von $K_{2} \approx 360 \mathrm{~km} \mathrm{~s}^{-1}$ (Smith et al. 1993, Smith 2000; s. Kapitel 3.2.3) fehlerhaft sein muß, wie auch bereits von Allan et al. (1998) vermutet wurde. Aus den hier bestimmten Massen und ihren Unsicherheiten ergeben sich die Werte $K_{2}=507 \pm 20 \mathrm{~km} \mathrm{~s}^{-1}$ für den Sekundärstern und $K_{1}=56 \pm 5 \mathrm{~km} \mathrm{~s}^{-1}$ für den Weißen Zwerg, wobei der letztgenannte Wert innerhalb der Fehler mit den von Gilliland (1982) bzw. Hellier et al. (1987) angegebenen Werten $\left(K_{1}=58 \pm 9 \mathrm{~km} \mathrm{~s}^{-1}\right.$ bzw. $\left.K_{1}=69 \pm 9 \mathrm{~km} \mathrm{~s}^{-1}\right)$ übereinstimmt und noch marginal mit dem Wert $K_{1}=85 \pm 9 \mathrm{~km} \mathrm{~s}^{-1}$ von Mauche (1999) verträglich ist (vgl. Kapitel 3.2.3).

\subsubsection{Radius des Innenrands der Akkretionsscheibe}

Um zu prüfen, inwieweit die in diesem Szenario auftretenden Bedeckungslichtkurven mit den tatsächlich beobachteten konsistent sind, habe ich synthetische Lichtkurven berechnet. Hierfür habe ich schematisch die EUV bzw. Röntgenstrahlung emittierenden Gebiete durch eine Punktquelle am oberen Pol des Weißen Zwergs bzw. Punktquellen entlang der Dipolfeldlinien im inneren Teil der Akkretionsvorhänge repräsentiert. Weiterhin wurde die Quelle der optischen Emission am Innenrand der Akkretionsscheibe (ausgezogene Ellipse in Abbildung 5.3) durch ein Band von Punktquellen mit dem Radius $R_{i}$ und einer Ausdehnung $h_{\text {rim }}$ senkrecht zur Orbitalebene dargestellt (die gepunkteten Ellipsen in der Abbildung symbolisieren den oberen bzw. unteren Rand dieses Gebiets). Ferner habe ich angenommen, daß diejenigen Röntgenpunktquellen, die in der Projektion durch die Akkretionsscheibe verdeckt werden, nicht zum beobachteten Röntgenfluß beitragen. Die so definierten Emissionsgebiete sind im unteren Teilbild von Abbildung 5.3 als schwarzer Punkt bzw. schattierte Flächen dargestellt. In der Abbildung wurde eine Spinphase von 
$\Phi_{\text {spin }} \approx 0.75$ angenommen und der Einfachheit halber unterstellt, daß nur diejenigen Teile des Innenrands der Akkretionsscheibe, die nicht vom dichten Teil des oberen Akkretionsvorhangs verdeckt werden, wesentlich zur beobachteten optischen Emission beitragen (vgl. Abbildung 3.2).

Die resultierenden Bedeckungslichtkurven im Optischen, EUV und Röntgenbereich in den oberen Teilbildern von Abbildung 5.3 geben die wesentlichen Merkmale der beobachteten Lichtkurven, insbesondere Form und Tiefe der Röntgenlichtkurve sowie das $\approx 20 \ldots 30 \mathrm{~s}$ 'zu frühe' Eintreten der optischen Bedeckung bei $\Phi_{\text {spin }}=0.75$ (s. Kapitel 3.2.6.2 und 3.2.7.3), gut wieder. Die Form der optischen Lichtkurve wird in diesem einfachen Szenario stark durch die willkürlich gewählte Form des Emissionsgebiets (s.o.) bestimmt und ist entsprechend vorsichtig zu beurteilen; allerdings führen wesentlich kleinere vertikale Ausdehnungen als $h_{\text {rim }} \approx 3 R_{\mathrm{WD}}$ zu deutlich flacheren und kürzeren Bedeckungen, was ich als Hinweis darauf werte, daß EX Hya tatsächlich einen 'aufgeblähten' Innenrand der Scheibe besitzt, wie er in Abbildung 3.4 dargestellt ist. Da $h_{\text {rim }}$ aber hier nicht genauer bestimmt werden kann, erscheint der Wert nicht in Tabelle 5.1.

Der gewählte Radius $R_{i}$ des Innenrands der Akkretionsscheibe bestimmt unter den oben gemachten Annahmen bezüglich der Sichtbarkeit des unteren Akkretionsvorhangs wesentlich die Tiefe der Röntgenbedeckung: Größere Radien führen dazu, daß größere Teile des unteren Akkretionsvorhangs sichtbar sind, was eine größere Bedeckungstiefe zur Folge hat. Aus diesem Grund können deutlich kleinere oder größere Radien als $R_{i} \approx 10 R_{\mathrm{WD}}$ vermutlich ausgeschlossen werden. Die Zunahme der Bedeckungstiefe mit der Photonenenergie ist ebenfalls konsistent mit der Vorstellung, daß $R_{i}$ die Bedeckungstiefe wesentlich bestimmt. Bei größeren Photonenenergien steigt die Transparenz des absorbierenden Materials am Innenrand der Scheibe; da seine Dichte mit wachsendem Abstand von der Orbitalebene abnimmt, ist daher bei größeren Energien effektiv ein größerer Teil des unteren Akkretionsvorhangs sichtbar, so daß die Bedeckungstiefe steigt. Eine Bedeckungstiefe von $\approx 60 \%$, wie sie bei $10 \mathrm{keV}$ beobachtet wird (vgl. Kapitel 3.2.6.2), wäre in diesem Szenario unter entsprechenden Annahmen für die relevanten Modellparameter ohne weiteres zu reproduzieren.

\subsubsection{Ausdehnung der Röntgenstrahlung emittierenden Gebiete}

Eine wesentliche Folgerung aus dem Szenario ist, daß die Röntgenstrahlung emittierenden Gebiete eine relativ große vertikale Ausdehnung von $\approx 3 \ldots 4 \cdot 10^{8} \mathrm{~cm} \approx 1 R_{\mathrm{WD}}$ haben müssen, um die beobachtete Tiefe der Bedeckung und insbesondere die Länge der Ein- und Austrittsphase erklären zu können. Da die sichtbare Fläche des unbedeckt bleibenden oberen Emissionsgebiets die Bedeckungstiefe bestimmt, führen wesentlich geringere bzw. größere vertikale Ausdehnungen schnell zu Bedeckungstiefen von $>60 \%$ bzw. $<30 \%$, was im Widerspruch zu den Beobachtungen steht (vgl. Abschnitt 3.2.6.2). Die Ausdehnung des bedeckten unteren Emissionsgebiets in Richtung der Orbitalbewegung bestimmt die Länge der Ein- und Austrittsphase der Bedeckung. Im Szenario ist sie durch die Dipolgeometrie der magnetischen Feldlinien, den Radius des Weißen Zwergs $R_{\mathrm{WD}}$ und den Radius des Innenrands der Akkretionsscheibe $R_{i}=10 R_{\mathrm{WD}}$ bestimmt. Sie beträgt 
$\left(\left(R_{\mathrm{WD}} / R_{i}\right)^{1 / 2} \approx 0.3\right) R_{\mathrm{WD}}=1.2 \cdot 10^{8} \mathrm{~cm}$ an der Oberfläche des Weißen Zwergs (unter Berücksichtigung der Sichtbedingungen bei der für das Szenario angenommenen Spinphase $\Phi_{\text {spin }} \approx 0.75$ ) und kann die beobachteten Ein- und Austrittszeiten von $21 \pm 4 \mathrm{~s}$ (Mukai et al. 1998) gut reproduzieren. Eine vertikale Ausdehnung der Röntgenemissionsgebiete in der Größenordnung des Radius des Weißen Zwergs impliziert eine entsprechend hoch über dem Weißen Zwerg stehende Stoßfront, was wiederum eine relativ niedrige Massenflußdichte $\dot{m}$ (in $\mathrm{g} \mathrm{cm}^{2} \mathrm{~s}^{-1}$ ) erfordert (vgl. Abschnitt 2.4.3). Ob eine so hoch stehende Stoßfront dynamisch stabil sein kann, soll hier nicht weiter diskutiert werden. Auf jeden Fall ist aber damit zu rechnen, daß Nachbeschleunigung des einfallenden Materials unterhalb der Stoßfront stattfindet und zu weiterer Aufheizung des Plasmas führt, so daß die in Kapitel 2.4.2.2 erwähnte Methode der Massenbestimmung des Weißen Zwergs aus der Temperatur der Bremsstrahlung zumindest im Fall von EX Hya schon aus diesem Grund nur unsichere Ergebnisse liefern wird. Ob es auf diesem Wege möglich ist, die niedrige Temperatur der thermischen Bremsstrahlung von ca. $9 \mathrm{keV}$ zu erklären, bleibt abzuwarten.

Abschließend sei noch auf die kurze Einsenkung in der Mitte der Röntgenbedeckung hingewiesen. Im dargestellten Szenario hat sie ihre Ursache darin, daß die emittierenden Gebiete als bis auf die Oberfläche des Weißen Zwergs hinunterreichend angenommen wurden. Interessanterweise zeigt sich ein vergleichbares Merkmal in der mittleren Bedeckungslichtkurve im Energiebereich 1.5 ...10 keV (Rosen et al. 1988). Die Autoren haben dieses Merkmal offenbar als statistische Schwankung betrachtet und daher nicht weiter erwähnt. Die Tatsache, daß es exakt bei $\Phi_{\text {orb }}=0$ auftritt, legt jedoch nahe, daß eine kurze, $\lesssim 40$ s dauernde Bedeckung der untersten Teile des oberen Röntgenemissionsgebiets auftreten kann. Die Annahme, daß Röntgenstrahlung bis hinunter zur Oberfläche des Weißen Zwergs emittiert wird, ist sicher nur für nicht zu hohe Röntgenenergien richtig. Harte Röntgenstrahlung entsteht in Gebieten hoher Temperatur, d.h. in größerer Entfernung vom Weißen Zwerg; die Bedeckungslichtkurven bei höheren Photonenenergien sollten daher keine solche zentrale Einsenkung zeigen. In der Tat ist sie in der von Mukai et al. (1998) gezeigten Bedeckung im Bereich 4...6keV nicht vorhanden. Im Modellszenario genügt bereits eine untere Grenze des Röntgenemissionsgebiets von $\approx 0.2 R_{\mathrm{WD}} \approx 8 \cdot 10^{7} \mathrm{~cm}$ über der Oberfläche des Weißen Zwergs, um die zentrale Einsenkung in der Röntgenlichtkurve zum Verschwinden zu bringen.

\subsubsection{Beitrag des Sekundärsterns zum beobachteten Fluß}

Aus den in Kapitel 4.2.7 bestimmten Amplituden $a_{49}$ der Helligkeitsmodulation von EX Hya mit der halben Orbitalperiode im Infrarot (s. Tabelle 4.8), die hier als Amplitude $a_{\text {ell }}$ der ellipsoidalen Modulation des Sekundärsterns interpretiert wird, läßt sich nach (2.25) der mittlere Fluß $\langle f\rangle$ des Sekundärsterns in den in Tabelle 4.5 definierten Infrarot-Bändern bestimmen. Für $q=0.10 \ldots 0.12$ und $i=78 \ldots 80^{\circ}$ erhält man aus (2.25) die Helligkeitsdifferenz zwischen ellipsoidalem Maximum und Minimum $\Delta \mathrm{m}=0.29 \ldots 0.30$ und unter Benutzung der Beziehungen

$$
\frac{f_{\min }}{f_{\max }}=10^{-0.4 \Delta \mathrm{m}} \quad, \quad\langle f\rangle=\frac{f_{\max }+f_{\min }}{2} \quad \text { und } \quad a_{\mathrm{ell}}=\frac{f_{\max }-f_{\min }}{2}
$$


Tabelle 5.2: Flußbeitrag des Sekundärsterns im Infrarot. Die angegeben Fehler setzen sich aus den in Tabelle 4.8 angegebenen Fehlern der Modulationsamplituden und dem des Skalierungsfaktors aus (5.1) zusammen. $a_{\text {ell }}$ und $\left\langle f_{\lambda}\right\rangle$ sind in Einheiten von $10^{-16} \mathrm{erg} \mathrm{cm}^{-2} \mathrm{~s}^{-1} \AA^{-1}$ angegeben, $\left\langle f_{\nu}\right\rangle$ in mJy (vgl. (4.5)).

\begin{tabular}{crcccc}
\hline Band & \multicolumn{1}{c}{$\lambda \lambda[\AA]$} & $\lambda_{\text {cen }}[\AA]$ & $a_{\text {ell }}$ & $\left\langle f_{\lambda}\right\rangle$ & $\left\langle f_{\nu}\right\rangle$ \\
\hline "I" & $9650-10650$ & 10150 & $2.39 \pm 0.34$ & $17.7 \pm 2.5$ & $6.08 \pm 0.86$ \\
"J" & $11000-13500$ & 12250 & $2.16 \pm 0.23$ & $16.0 \pm 1.7$ & $8.00 \pm 0.85$ \\
"H" & $14500-18000$ & 16250 & $1.21 \pm 0.11$ & $8.95 \pm 0.83$ & $7.88 \pm 0.73$ \\
"K" & $20000-24000$ & 22000 & $0.564 \pm 0.047$ & $4.17 \pm 0.36$ & $6.73 \pm 0.58$ \\
\hline
\end{tabular}

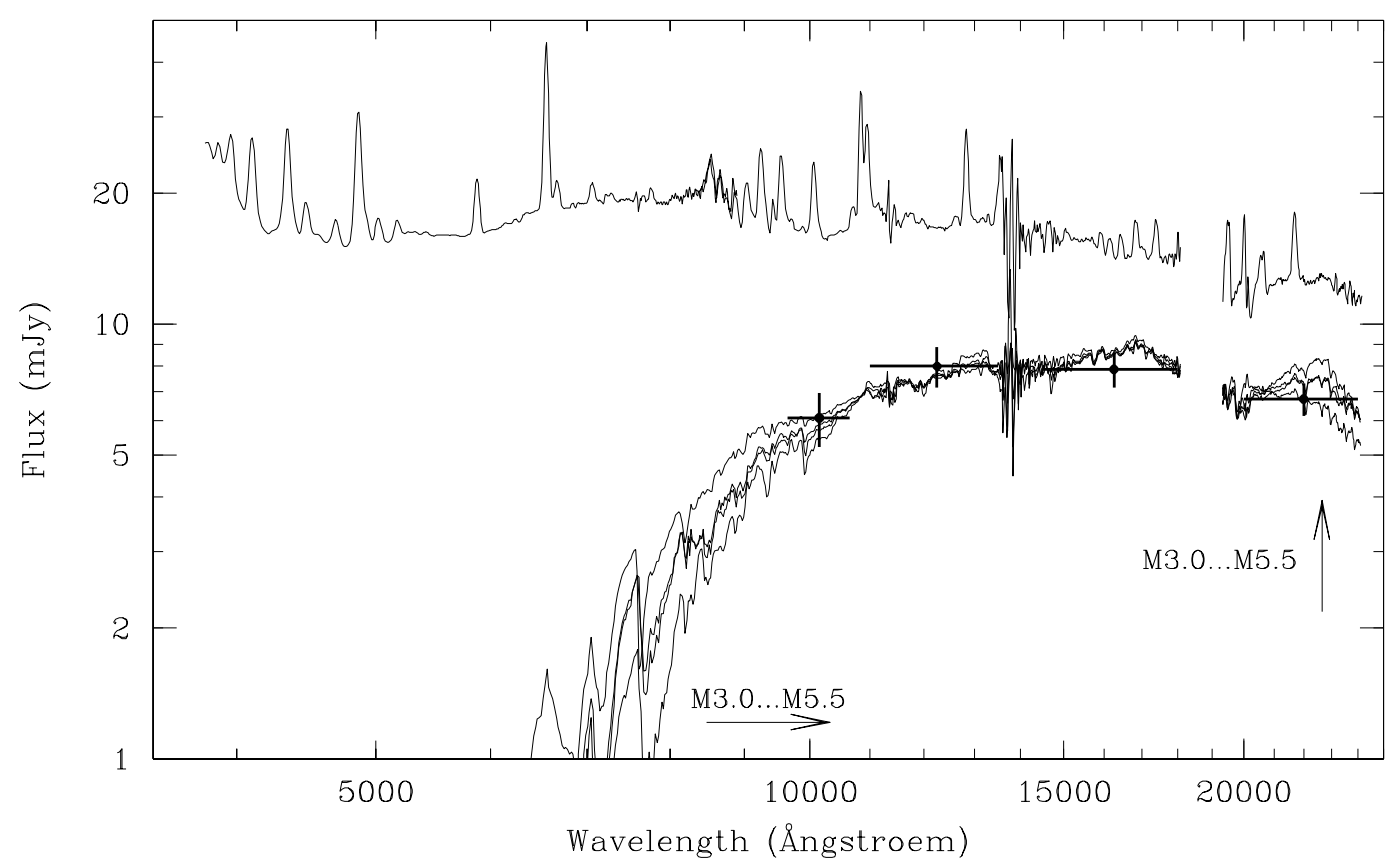

Abbildung 5.4: Mittleres Spektrum von EX Hya aus Kapitel 4.2.8 (obere Kurve; optisches Spektrum auf Niveau des Infrarotspektrums angehoben), Werte der aus den Amplituden $a_{\text {ell }}$ der ellipsoidalen Modulation abgeleiteten mittleren Flüsse des M-Sterns (Symbole; vgl. Tabelle 5.2) und auf die mittleren Flüsse skalierte Spektren der M-Sterne aus Abbildung 4.11 im Spektraltypenbereich M3.0...M5.5. Die Pfeile an den Bildrändern deuten den Gang der Spektraltypen an.

für den mittleren Fluß $\langle f\rangle$

$$
\langle f\rangle=a_{\mathrm{ell}} \frac{1+f_{\min } / f_{\max }}{1-f_{\min } / f_{\max }}=(7.40 \pm 0.15) a_{\mathrm{ell}}
$$

Für die vier betrachteten Wellenlängenbereiche ergeben sich die in Tabelle 5.2 angegebenen Werte für $\langle f\rangle$; sie sind in Abbildung 5.4 zusammen mit dem mittleren Spektrum von 


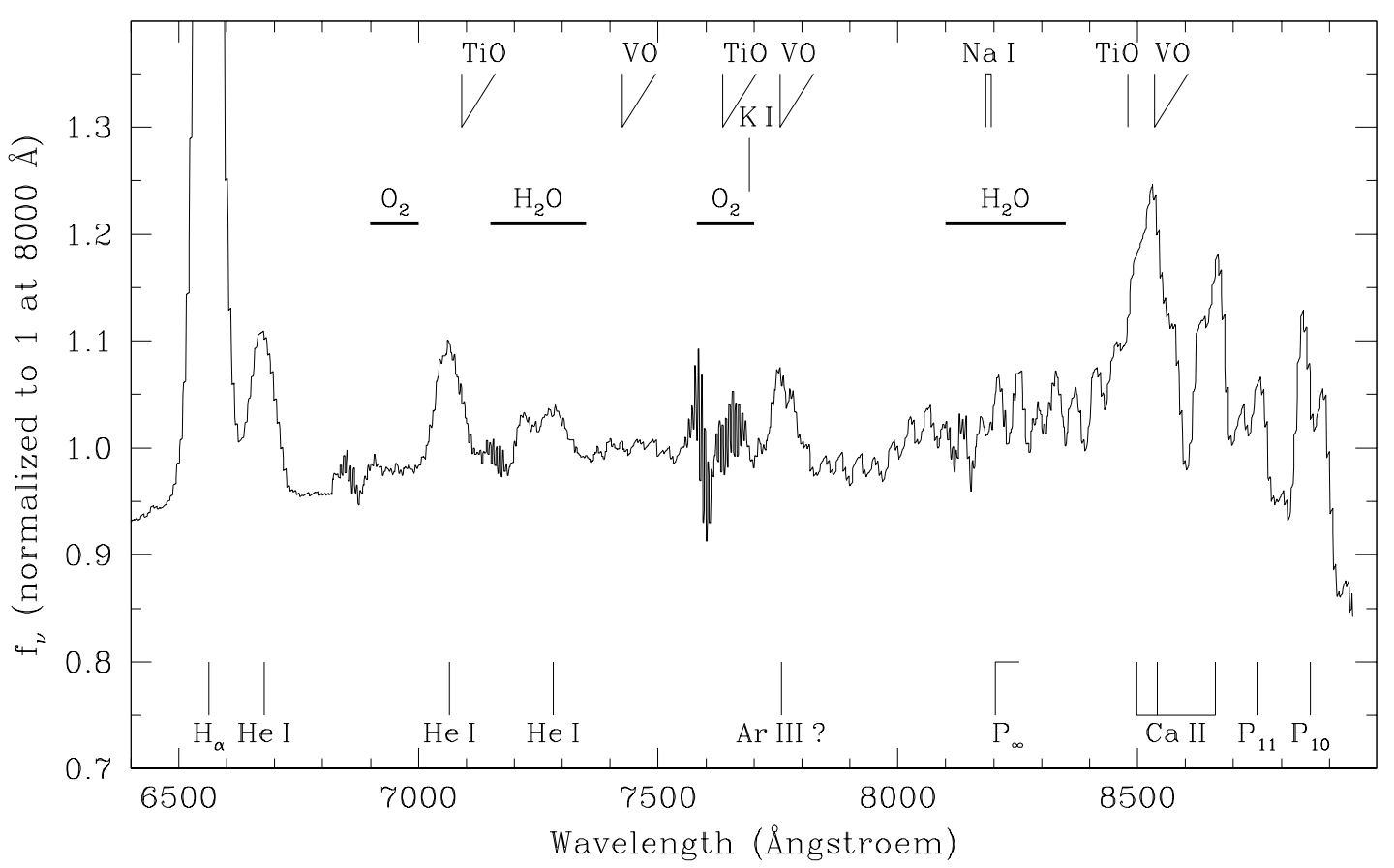

Abbildung 5.5: Mittleres optisches Spektrum von EX Hya und Lage der typischen spektralen Merkmale von M-Sternen, der atmosphärischen Absorptionsbänder und der identifizierten Emissionslinien.

EX Hya und den entsprechend skalierten Spektren der M-Sterne aus Abbildung $4.11 \mathrm{im}$ Spektraltypenbereich M3.0...M5.5 dargestellt. (Der Skalierungsfaktor wurde dabei durch Mittelung der Faktoren für die einzelnen Bänder bestimmt.) Das optische Spektrum von EX Hya wurde dabei auf das Flußniveau im Infrarot angehoben (vgl. Kapitel 4.2.8). Im wesentlichen sind alle gezeigten Spektraltypen mit dem Verlauf der aus den Amplituden bestimmten mittleren Flüsse verträglich, so daß sich hieraus noch keine Einschränkung hinsichtlich des Spektraltyps des Sekundärsterns von EX Hya ergibt. Allenfalls das Spektrum des M5.5-Sterns liegt in den Bereichen $\lambda \lesssim 10000 \AA$ bzw. $\lambda \gtrsim 20000 \AA$ spürbar zu niedrig bzw. zu hoch, wenngleich die Abweichungen immer noch im Rahmen der geschätzten Ungenauigkeiten bleiben.

\subsubsection{Spektraltyp des Sekundärsterns}

Zur Bestimmung des Spektraltyps des Sekundärsterns von EX Hya bietet es sich zunächst an, im Spektrum von EX Hya direkt nach typischen spektralen Merkmalen von M-Sternen zu suchen (vgl. Abbildung 4.11). Die Abbildungen 5.5 und 5.6 zeigen jedoch, daß keine auffälligen Merkmale vorhanden sind. Allenfalls in den mittleren beiden Spektren im rechten Teilbild von Abbildung 5.6, die jeweils das Spektrum im Spinminimum während der Bedeckung repräsentieren (also die unbeleuchtete Hemisphäre des Sekundärsterns zeigen, 


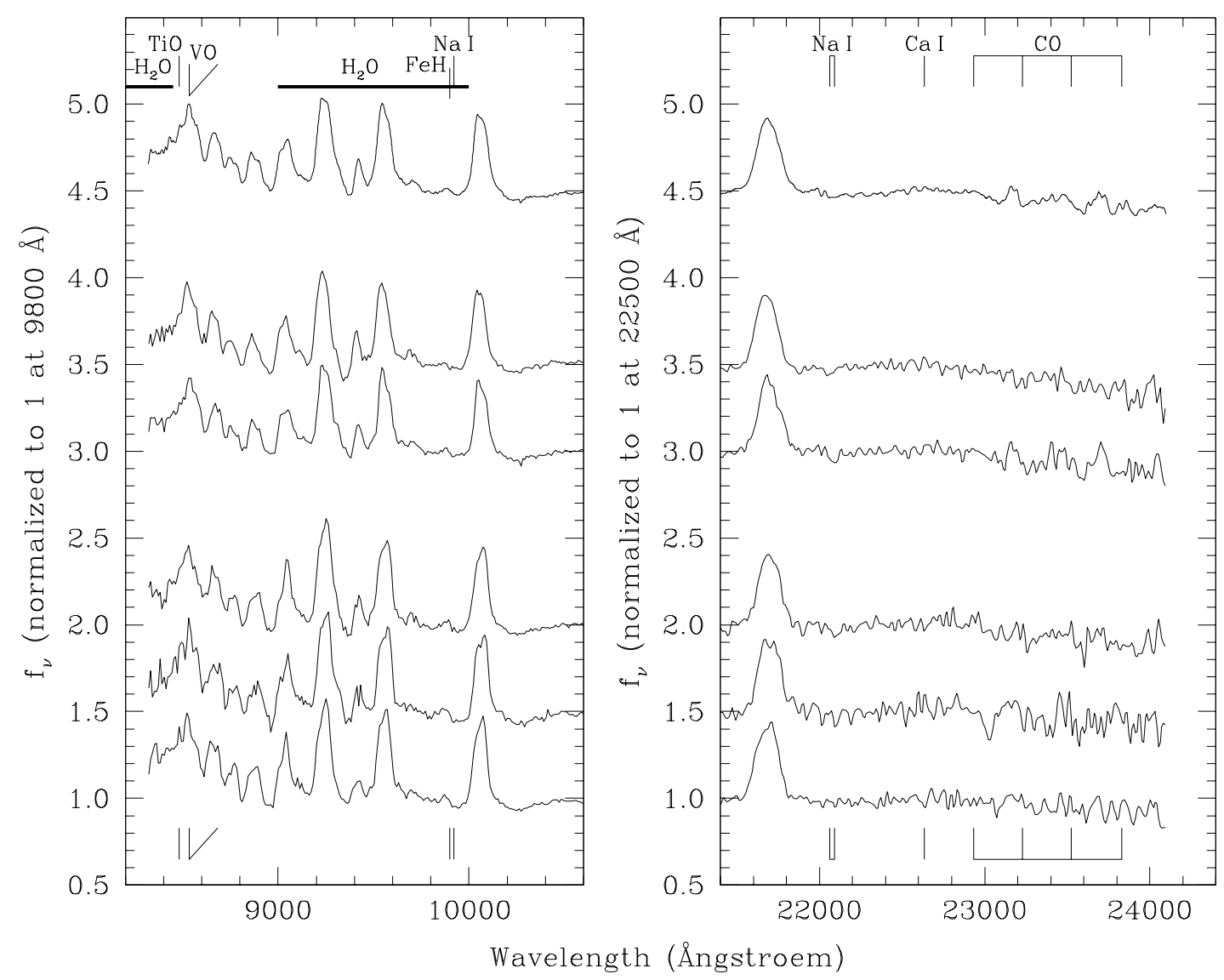

Abbildung 5.6: IR-Spektrum von EX Hya und Lage der typischen spektralen Merkmale von M-Sternen und der atmosphärischen Absorptionsbänder. Das jeweils oberste Spektrum ist das über alle Spin- und Orbitalphasen gemittelte Spektrum von EX Hya. Die Spektren der mittleren bzw. unteren Gruppe wurden durch Mittelung von jeweils 3...7 Einzelspektren aus jeweils einer Serie von Beobachtungen (s. Kapitel 4.2.6) erzeugt. Alle Einzelspektren liegen nahe am Minimum der Spinlichtkurven; die Spektren der mittleren Gruppe wurden während der Bedeckung im IR, die der unteren Gruppe um das Maximum der ellipsoidalen Modulation herum aufgenommen. Alle Spektren sind auf den Wert 1 bei $9800 \AA$ (links) bzw. $22500 \AA$ (rechts) normiert und um Vielfache von 0.5 vertikal gegeneinander versetzt.

auf der dessen Signaturen am ausgeprägtesten sein sollten), ist schwache Absorption des Na-Dupletts bei $\lambda \lambda 22062,22090 \AA$ festzustellen. Der Mangel an prominenten spektralen Signaturen des Sekundärsterns entspricht zwar der Situation in vielen anderen IPs, verhindert hier jedoch eine Bestimmung des Spektraltyps des Sekundärsterns von EX Hya auf diesem Wege.

Ein anderer Weg besteht darin, sich die charakteristische Änderung der Form der M-Stern-Spektren mit dem Spektraltyp bei Wellenlängen $\lambda \gtrsim 15000 \AA$ zunutze zu machen (vgl. Abbildung 4.11). Das Absorptionsband des Wassers in der Sternatmosphäre bei 


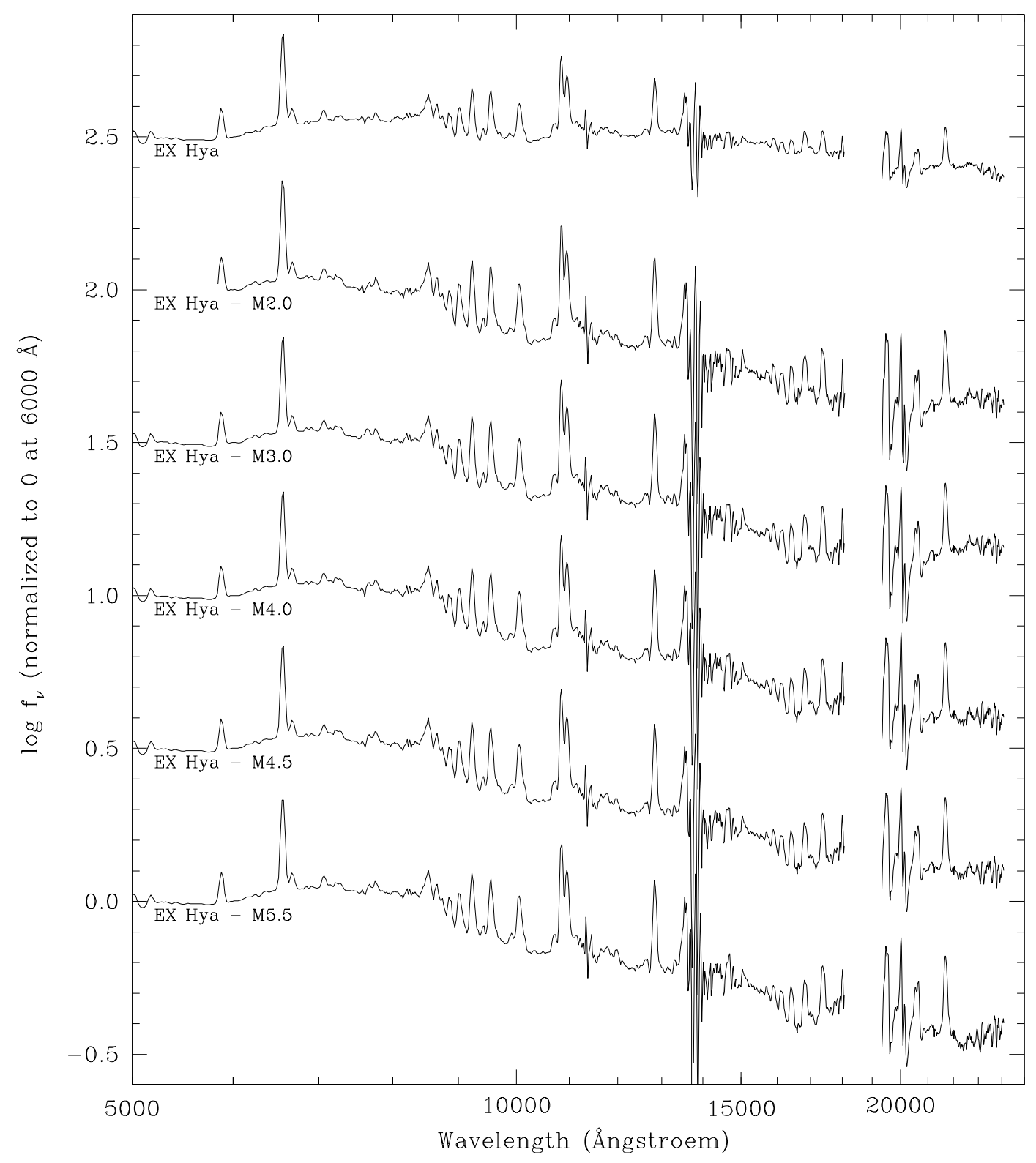

Abbildung 5.7: Mittleres Spektrum von EX Hya vor (oberstes Spektrum) und nach (untere Spektren) Abzug der auf den mittleren Fluß des Sekundärsterns skalierten Spektren der M-Sterne aus Abbildung 5.4. Die Spektren sind auf den Wert $f=1(\log (f)=0)$ bei $6000 \AA$ normiert und um Vielfache von 0.5 vertikal gegeneinander versetzt.

$\lambda \gtrsim 22900 \AA$ ist dabei ein besonders geeigneter Indikator (z.B. Dhillon \& Marsh 1995). Abbildung 5.7 zeigt oben das mittlere Spektrum von EX Hya und darunter dasselbe Spektrum nach Abzug jeweils eines der in Abbildung 5.4 dargestellten, auf den mittleren Fluß des Sekundärsterns skalierten M-Stern-Spektren (vgl. Abschnitt 5.1.6). Die Abbildung zeigt, daß ein Spektraltyp von M3.0 oder früher zu einem deutlichen Anstieg des Differenzspek- 
trums im Bereich $\lambda \gtrsim 19000 \AA$ und ein Spektraltyp von M5.5 zu einem 'Buckel' im Bereich $17000 . .22000 \AA$ führt. Die Spektraltypen M4.0 und M4.5 führen hingegen im langwelligen Bereich zu einem im wesentlichen glatten Differenzspektrum, wie man es auch erwartet, und kommen daher als mögliche Spektraltypen des Sekundärsterns in Betracht. Auch der Spektraltyp M5.0, für den kein Spektrum zur Verfügung stand, erscheint möglich. Hingegen halte ich angesichts des schon beim M4.0-Spektrum andeutungsweise zu erkennenden und beim Spektraltyp M3.0 stark ausgeprägten Anstieg des Spektrum am langwelligen Ende einen früheren Spektraltyp als M4.0 für unwahrscheinlich. Mithin kann der Spektraltyp des Sekundärsterns anhand der vorliegenden Spektren auf den Bereich M4.5 \pm 0.5 eingegrenzt werden. Dies ist konsistent sowohl mit dem bei einer Orbitalperiode $P_{\text {orb }} \approx 1.6 \mathrm{~h}$ erwarteten Spektraltyp (M4...M5, Beuermann 2000a) als auch mit der in Abschnitt 5.1.3 bestimmten Masse $M_{2}=0.13 \pm 0.01 M_{\odot}$. Der von Dhillon et al. (1997) angegebene Spektraltyp $\approx$ M3.0 hingegen kann mit einiger Gewißheit ausgeschlossen werden.

\subsubsection{Entfernung von EX Hya}

Aus den bisher gewonnenen Erkenntnissen lassen sich alle Größen ableiten, die für eine Bestimmung der Entfernung des Systems nach (2.26) nötig sind. Der Spektraltyp des Sekundärsterns liefert nach (2.28) seine Oberflächenhellgkeit $S_{K}$ im K-Band; für M3.0...M5.5 (entsprechend $X=17 \ldots 14.5$ in $(2.28)$ ) ergibt sich $S_{K}=4.17 \ldots 4.71$. Aus den skalierten Spektren aus Abbildung 5.4 im Spektraltypenbereich M3.0...M5.5 erhält man nach (4.1) mit den Transmissionskurven aus Abbildung 4.5 und den Flußkonstanten aus Tabelle 4.3 die K-Helligkeiten $K=12.6 \ldots 12.4$. Dabei wurden die Flüsse der Spektren im Bereich 18000...19000 $\AA$ interpoliert und für den Bereich $\lambda>25000 \AA$ extrapoliert. Für den Radius des Sekundärsterns wird der Volumenradius $R_{V}$ seines Roche-Volumens angenommen; nach (2.10) beträgt dieser bei der Orbitalperiode $P_{\text {orb }}=1.638 \mathrm{~h}$ und für $M_{2}=0.12 \ldots 0.14 M_{\odot} R_{V}=0.16 \ldots 0.17 R_{\odot}$. Hieraus ergibt sich aus (2.26) die Entfernung von EX Hya zu $d=68 \pm 14$ pc. Dabei geht der angegebene Fehler zum großen Teil auf die Unsicherheit von $S_{K}$ zurück; beschränkt man sich auf den Spektraltypenbereich M4.0...M5.0, entsprechend $S_{K}=4.39 \ldots 4.61$, erhält man $d=66 \pm 9$ pc. Diese Entfernung liegt um einen Faktor 1.5...2 unter den Werten aus früheren Bestimmungen (vgl. Kapitel 3.2.2), was entsprechende Auswirkungen auf die von anderen Autoren aus dem beobachteten Fluß bestimmte Leuchtkraft und die daraus abgeleitete Akkretionsrate $\dot{M}$ hat (vgl. Kapitel 3.2.5).

\subsection{Bulge der Akkretionsscheibe}

Das in Kapitel 4.2 .4 erwähnte Auftreten von scharfen Absorptionskernen in den breiten UV-Emissionslinien, die in den HST-Spektren bei Orbitalphasen $\Phi_{\text {orb }} \approx 0.85 \ldots 1$ auftreten und sich mit den Multiplettkomponenten der jeweiligen Linien identifizieren lassen (s. Abbildung 4.14), findet eine einfache Erklärung, wenn man annimmt, daß die Linienemission aus dem Zentralgebiet im kühlen Material des bulge teilweise absorbiert wird (s. Abbildung 5.8). Diese Vorstellung ist konsistent mit den geringen gemessenen Radialgeschwin- 


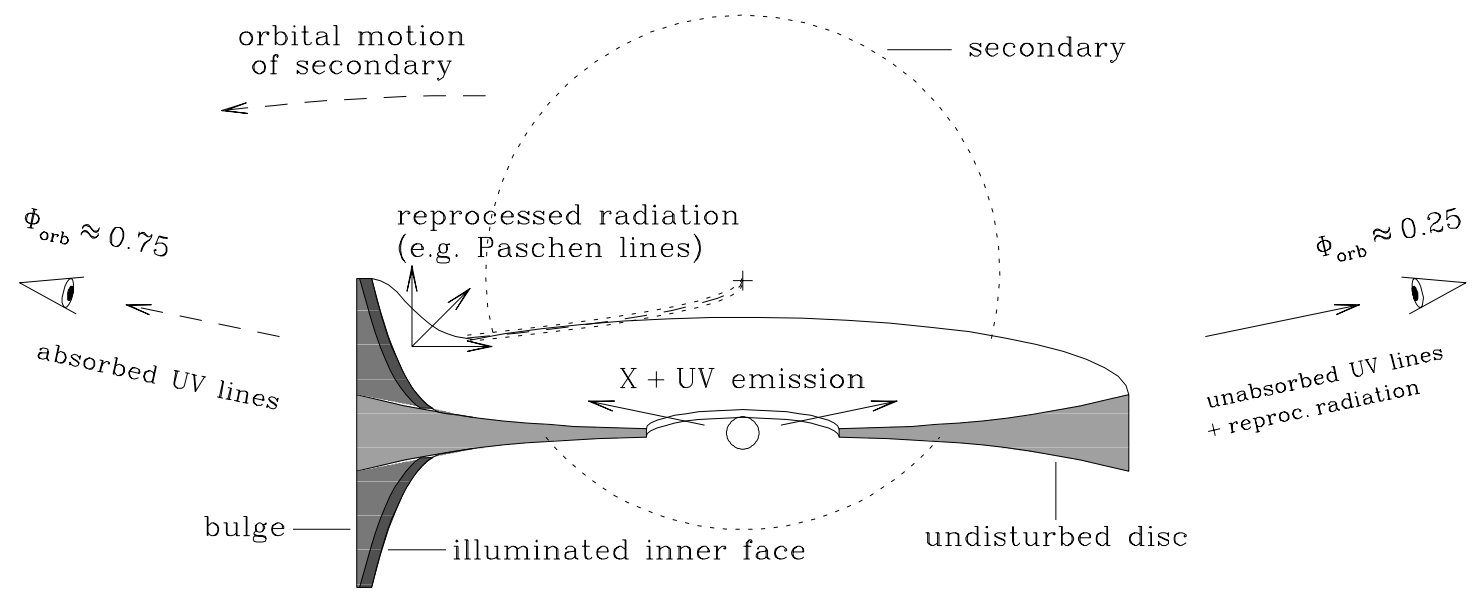

Abbildung 5.8: Schematische Darstellung des bulge am Außenrand der Akkretionsscheibe (stark überhöht). Energiereiche Strahlung aus dem Zentralgebiet wird im kühlen Material des bulge reprozessiert und regt dort u.a. Emissionslinien im Infrarot an. Die breiten Emissionslinien im UV werden teilweise absorbiert und erhalten dadurch schmale Absorptionskerne.

digkeiten der Absorptionskerne von $75 \pm 46 \mathrm{~km} \mathrm{~s}^{-1}$ (Kapitel 4.2.4) und impliziert, daß die Absorption bei Orbitalphasen $\Phi_{\text {orb }} \lesssim 0.7$ wieder verschwindet (vgl. Abbildung 3.5). Da die vorliegenden HST-Daten nur den Bereich $\Phi_{\text {orb }} \approx 0.85 \ldots 0.2$ überdecken (s. Abbildung 4.4), läßt sich letzteres allerdings derzeit nicht überprüfen. Das Fehlen ähnlich ausgeprägter Absorptionskerne in den IUE-Spektren, das sich auch in fehlender Modulation der Linienintensitäten mit der Orbitalperiode wiederspiegelt (vgl. die Abbildungen in Anhang A.2.2), beruht wahrscheinlich auf unterschiedlichen Ausdehnungen des bulge senkrecht zur Orbitalebene zu den Zeiten der HST- bzw. IUE-Beobachtungen, wie sie etwa durch zeitlich variable Massenflußraten im Akkretionsstrom verursacht werden könnten.

Reprozessierung energiereicher Strahlung aus dem Zentralgebiet im bulge ist vermutlich auch die Ursache für Modulation der Intensität der Emissionslinien im Infrarot mit einem Hauptmaximum bei $\Phi_{\text {orb }} \approx 0.3$ und einem Nebenmaximum bei $\Phi_{\text {orb }} \approx 0.8$ (vgl. die Abbildungen in Anhang A.4.2). Die unterschiedliche Höhe der beiden Maxima der Lichtkurven impliziert, daß die reprozessierte Strahlung hauptsächlich von der beleuchteten Innenseite des bulge beobachtet wird und nur ein geringerer Teil davon den bulge durchdringt, d.h. daß das dort befindliche Material für die IR-Linien eine nicht zu vernachlässigende optische Tiefe hat. Die Modulation des Kontinuums mit der Orbitalphase ist hingegen durch zwei etwa gleich hohe Maxima bei $\Phi_{\text {orb }} \approx 0.25$ und $\Phi_{\text {orb }} \approx 0.75$ gekennzeichnet. Ein Beitrag reprozessierter Strahlung aus dem bulge im Kontinuum kann zwar nicht ausgeschlossen werden, sollte aber aufgrund der obigen Betrachtung gering sein. Der größte Anteil der Intensitätsvariationen im Kontinuum wird daher auf der ellipsoidalen Modulation des Sekundärsterns beruhen, wie es in Abschnitt 5.1.6 bei der Bestimmung seines mittleren Flusses auch stillschweigend unterstellt wurde.

Abschließend sei noch angemerkt, daß die von Siegel et al. (1989) in den Daten aus dem 
Jahr 1985 festgestellte Modulation der optischen Emission mit $\Phi_{\text {orb }}$ ähnliche Eigenschaften hat wie diejenige der eben diskutierten IR-Linien (s. Kapitel 3.2.6.3), während eine entsprechende Modulation in den Daten aus dem Jahr 1983 nicht festzustellen war. Es ist naheliegend, dies ebenfalls durch Reprozessierung energiereicher Strahlung im einem bulge variabler Ausdehnung senkrecht zur Orbitalebene zu deuten. Im Detail müßten die hier präsentierten Vorstellungen anhand von Modellrechnungen für den Strahlungstransport überprüft werden, was im Rahmen dieser Arbeit aber nicht geschehen kann.

\subsection{Bedeckungen der Akkretionsscheibe}

Die Bedeckungen haben im Infrarot eine volle Breite von $\approx 10$ min (vgl. die Abbildungen in Anhang A.4). Abbildung 3.6 verdeutlicht, daß die bedeckte Quelle eine Ausdehnung haben muß, die mit dem Durchmesser des Sekundärsterns vergleichbar ist. Die einzige Quelle im System, die diese Bedingung erfüllt, ist die Akkretionsscheibe. Auch die Emissionslinien zeigen Bedeckungen, wenngleich mit deutlich geringerer Tiefe als das Kontinuum. Dies deutet darauf hin, daß ein wesentlicher Teil der Linienemission im IR nicht aus der Akkretionsscheibe, sondern aus den Außenbereichen der Akkretionsvorhänge stammen muß, von denen aufgrund der Systemgeometrie im wesentlichen nur die oberhalb der Orbitalebene liegenden Teile beobachtbar sind, die nicht bedeckt werden. Diese Vorstellung wird durch die Beobachtung gestützt, daß die Intensität der IR-Emissionslinien stark mit der Spinphase moduliert ist (vgl. Tabelle 4.10).

Im Optischen ist die Bedeckung der Akkretionsscheibe nur als schwache Einsenkung in der Lichtkurve von $\approx 10$ min voller Länge zu erkennen, die der deutlich tieferen Bedeckung des Zentralgebiets von $\approx 3$ min Länge überlagert ist (Siegel et al. 1989). Die daraus folgende geringe Leuchtkraft der Akkretionsscheibe im Optischen ist konsistent mit dem in Abschnitt 5.1.4 abgeleiteten Radius ihres Innenrands von $R_{i} \approx 4 \cdot 10^{9} \mathrm{~cm}$; die außerhalb dieses Radius liegenden Teile typischer Akkretionsscheiben sind kühl und emittieren hauptsächlich im Infrarot, während die heißeren Innenteile, in denen die optische und UVEmission dominiert, weiter innen liegen, hier aber fehlen.

\subsection{Spinmodulation der IR-Emissionslinien}

Die Intensitätsmodulation der Infrarot-Emissionslinien mit der Spinphase weist zwei bemerkenswerte Eigenschaften auf. Zum einen zeigt die Brackett-Linie B $\gamma$ im Gegensatz zu den Paschen-Linien keine signifikante Modulation mit der Spinphase (s. Abbildung A.65). Dies spiegelt sich in der Differenz der Spektren im Spinmaximum bzw. -minimum in Abbildung 4.20 darin wieder, daß einer Emissionskomponente von $\approx 800 \AA$ Gesamtbreite ein tiefer, schmaler Absorptionskern überlagert ist, dessen negativer Beitrag zum Fluß den der Emissionskomponente gerade aufwiegt. Die Linie B $\delta$ bei $19446 \AA$ zeigt ein ähnliches Verhalten, wenn auch die breite Emissionskomponente hier nicht so deutlich in Erscheinung tritt. Von den höheren Brackett-Linien ist im Differenzspektrum nur der Absorptionskern sicht- 
bar, es ist aber zu vermuten, daß auch dort eine unterliegende breite Emissionskomponente vorhanden ist.

Zum anderen haben die Paschen-Linien im Differenzspektrum in Abbildung 4.20 durchweg eine Doppelhöckerstruktur, die im Fall von $\mathrm{P} \gamma$ allerdings durch die benachbarte Linie He I $\lambda 10830$ verursacht wird. Interpretiert man die Separation der beiden Höckerspitzen der Linien $\mathrm{P} \beta, \mathrm{P} \delta, \mathrm{P} \epsilon$ und $\mathrm{P} 8$ als Radialgeschwindigkeitseffekt, ergibt sich als Mittel eine Geschwindigkeitsseparation von $2350 \pm 100 \mathrm{~km} \mathrm{~s}^{-1}$, was durchaus typischen Geschwindigkeiten in den Akkretionsvorhängen entsprechen kann. Diese Interpretation bereitet aber insofern Probleme, als man dazu annehmen müßte, daß der spinmodulierte Anteil des Spektrums vergleichbare Emissionsbeiträge von Material enthält, das vom Betrachter wegbzw. auf ihn zufällt. Es ist schwer vorstellbar, wo im Akkretionsvorhang diese Strahlung entstehen könnte (vgl. die Teilbilder für $\Phi_{\text {spin }}=0$ bzw. $\Phi_{\text {spin }}=0.5$ in Abbildung 3.2). Es erscheint mir wahrscheinlicher, daß das Phänomen wie bei den Brackett-Linien durch eine schmale Absorptionskomponente hervorgerufen wird, die bei den Paschen-Linien lediglich schwächer ausfällt als bei den Brackett-Linien. Diese Interpretation wird dadurch gestützt, daß die Linie $\mathrm{P} \beta$ (und möglicherweise auch die höheren Paschen-Linien) im spinmodulierten Spektrum genauso wie B $\gamma$ eine breite Emissionskomponente besitzt. Die beobachteten Linienprofile im spinmodulierten Spektrum im IR (Abbildung 4.20) könnten dann einheitlich so erklärt werden, daß die breite Emissionskomponente durch die große Bandbreite der beobachteten Radialgeschwindigkeiten an verschiedenen Stellen des Akkretionsvorhangs hervorgerufen wird; im Spinmaximum wird die Strahlung teilweise im Material des im Vordergrund liegenden Teils des Akkretionsvorhangs absorbiert, das sich bei $\Phi_{\text {spin }}=0$ in den Außenbereichen vorwiegend senkrecht zum Sehstrahl bewegt (vgl. Abbildung 3.2) und entsprechend schmalbandig absorbiert. Diese Hypothese kann aber nur durch detaillierte Modellrechnungen überprüft werden, die z.B. Aussagen über die Stärke der Absorption in den verschiedenen Linien(serien) machen (s. z.B. Kim \& Beuermann 1996). Dies kann im Rahmen dieser Arbeit nicht durchgeführt werden.

\subsection{Beitrag der Akkretionsscheibe und des Weißen Zwergs zum Fluß}

Das mittlere Spektrum von EX Hya aus Abbildung 4.23 kann nach Abzug des Beitrags des Sekundärsterns, für den hier der Spektraltyp M4.5 angesetzt wurde (vgl. Abbildung 5.7), unter Zuhilfenahme der Differenz der Spektren im Spinmaximum bzw. -minimum aus Abbildung 4.24 in das Spektrum der mit der Spinphase modulierten Quellen (Akkretionsvorhänge und geheizte Polkappen des Weißen Zwergs) und das der unmodulierten Quellen (Akkretionsscheibe und ungeheizte Photosphäre des Weißen Zwergs) zerlegt werden. Hierzu wird vom mittleren Spektrum $f_{\text {av }}$ ein so bestimmtes Vielfaches $\phi$ des halben Spindifferenzspektrums (d.h. der einfachen Amplitude der Spinmodulation) abgezogen, daß in der Differenz die UV-Emissionslinien im Mittel gerade verschwinden. Hinter diesem Vorgehen steht die Vorstellung, daß sich der Gesamtfluß der spinphasenmodulierten Quellen aus einem 


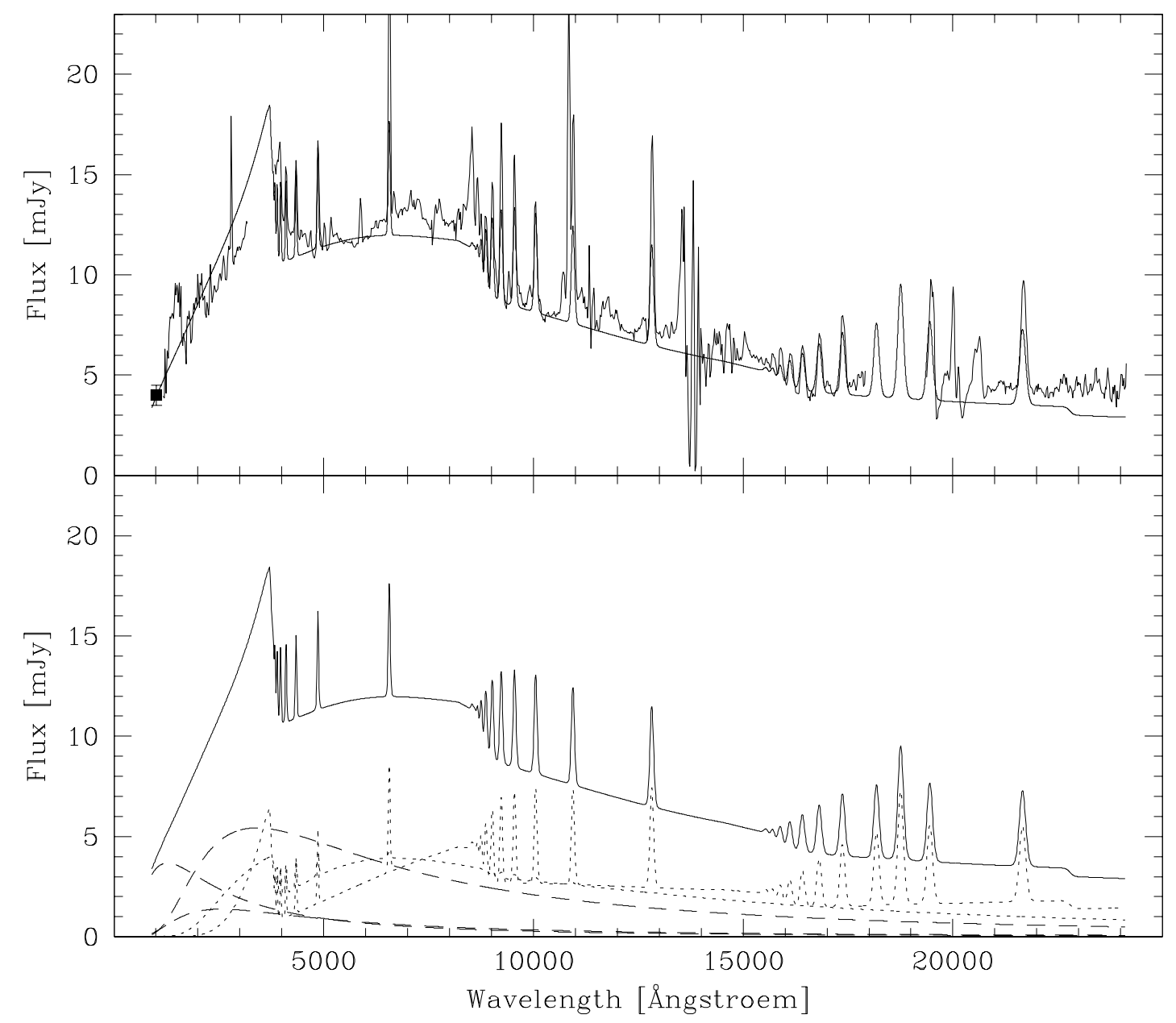

Abbildung 5.9: Spektrum der nicht spinmodulierten Strahlungsquellen von EX Hya. Oben: beobachtetes Spektrum (geglättet) und Gesamtmodellspektrum. Der Punkt bei $1010 \AA$ gibt den mit ORFEUS II beobachteten Fluß wieder (Mauche 1999) und wurde genauso wie das Spektrum konstruiert (s. Text). Unten: Gesamtmodellspektrum aus dem oberen Teilbild (ausgezogen) und seine Komponenten (gepunktet: optisch dünne Emission aus isothermem Plasma; gestrichelt: Schwarzkörperspektren). Für Details der Modellkomponenten und deren Interpretation verweise ich auf den Text.

modulierten und einem konstanten Anteil zusammensetzt, d.h. daß der Modulationsgrad ihres Spektrum $<1$ ist, so daß das in Abbildung 4.24 dargestellte Differenzspektrum nicht den gesamten Fluß dieser Quellen wiedergibt. Dieser Ansatz setzt stillschweigend voraus, daß (1) die Emissionslinien im UV ausschließlich in einer der modulierten Quellen (nämlich dem Akkretionsvorhang) und nicht etwa zum Teil auch in der Akkretionsscheibe entstehen, und daß (2) der unterliegende konstante Flußanteil der modulierten Quellen dasselbe Spektrum hat wie der modulierte. Während (1) vermutlich zutrifft, zeigen schon die in Tabelle 4.10 angegebenen Modulationsgrade $\bmod _{67}$ der UV-Emissionslinien, daß (2) nur eine 


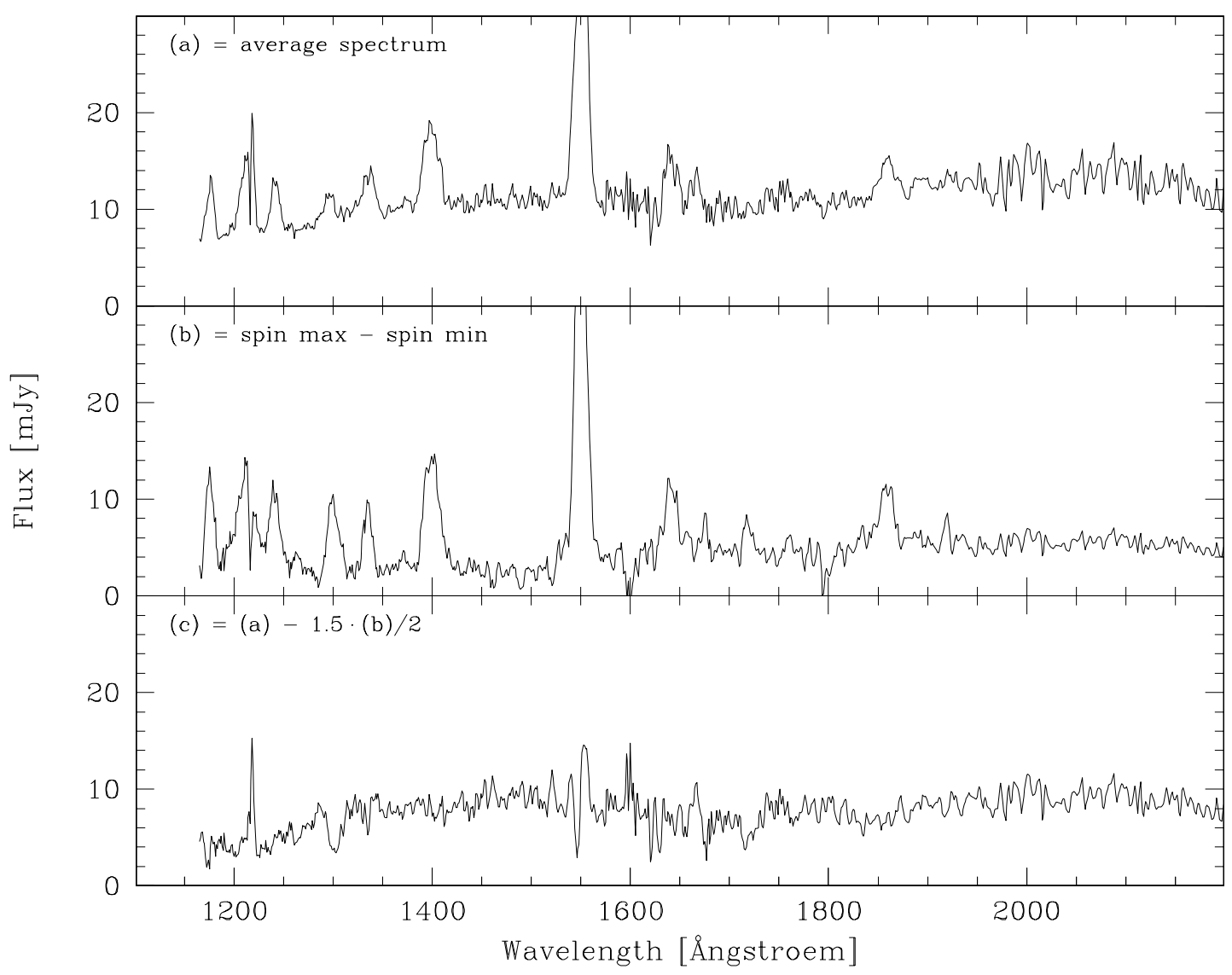

Abbildung 5.10: Konstruktion des Spektrums der nicht spinmodulierten Komponenten von EX Hya. Oben: mittleres Spektrum aus Abbildung 4.23; Mitte: Differenz der Spektren im Spinmaximum bzw. -minimum aus Abbildung 4.24; unten: mittleres Spektrum nach Abzug des $\phi=1.5$-fachen der einfachen Amplitude des Spinmodulationsspektrums. Man beachte, daß die Absorption in der Linie Si III 1300 durch die Konstruktion bedingt und nicht real ist.

Näherung sein kann; so implizieren etwa die Modulationsgrade der Linien Si IV 1400 und C IV 1550 von $\approx 80 \%$ einen Faktor $\phi \approx 1.5$, während die Linien C III 1175 und Si III 1300 $\left(\bmod _{67} \approx 100 \%\right) \phi \approx 1.0$ und die Linie CII $1335\left(\bmod _{67} \approx 68 \%\right) \phi \approx 2.0$ erfordern. Abbildung 5.9 zeigt im oberen Teil das mittlere Spektrum von EX Hya nach Abzug des $\phi=1.5$-fachen der einfachen Amplitude des Spinmodulationsspektrums; der Datenpunkt bei $1010 \AA$ wurde aus dem mittleren Fluß bzw. der Spinamplitude aus der Beobachtung mit ORFEUS II (Mauche 1999) auf dieselbe Weise bestimmt. Die vergrößerte Darstellung des Bereichs 1200...2200 $\AA$ in Abbildung 5.10 illustriert zum einen das Verfahren und zeigt zum anderen, daß hierdurch in der Tat die meisten Linien gut erfaßt und eliminiert werden.

Das so konstruierte Spektrum aller nicht mit der Spinphase modulierten Quellen wird nun als Summe verschiedener Komponenten interpretiert, die wie folgt physikalisch motiviert sind: 
1) Photosphäre des ungeheizten Weißen Zwergs. Das Spektrum wurde dabei als Schwarzkörperspektrum idealisiert. Für Radius und Temperatur wurden die Werte $R_{\mathrm{WD}}=4 \cdot 10^{8} \mathrm{~cm}$ und $T_{\mathrm{WD}}=20000 \mathrm{~K}$ aus Abschnitt 5.1 .3 angesetzt. Um Konsistenz mit den Ableitungen in Abschnitt 5.1 zu gewährleisten, wurden diese Werte nicht variiert.

2) Emission des permanent sichtbaren Teils der geheizten Polkappen des Weißen Zwergs. Der Anteil der Polkappen an der projizierten Querschnittsfläche $A$ des Weißen Zwergs läßt sich aus der Höhe der Röntgenstrahlung emittierenden Gebiete (einige $10^{8} \mathrm{~cm}$, vgl. Abschnitt 5.1.5) abschätzen; sie variiert mit der Spinphase im Bereich $10 \ldots 40 \%$, so daß für den mittleren Beitrag ein Flächenanteil von $g_{A} \approx 25 \%$ angenommen wird. (Entsprechend muß natürlich der Flächenanteil der ungeheizten Photosphäre (s. Punkt 1) auf den Wert $1-g_{A}$ reduziert werden.) Auch hier wurde ein Schwarzkörperspektrum angesetzt, wobei die Temperatur im Bereich $30000 . . .70000 \mathrm{~K}$ variiert wurde.

3) Strahlung aus einer optisch dünnen Akkretionsscheibe. Hierfür wurden Modellspektren für ein isothermes Wasserstoffplasma verwendet, die mit einem von B. Gänsicke in unserer Arbeitsgruppe entwickelten Programm berechnet wurden. Für den Radius der Scheibe habe ich $2.5 \cdot 10^{10} \mathrm{~cm}$ angenommen, was ca. $80 \%$ des Radius des Roche-Volumens des Weißen Zwergs entspricht; für den Radius des Innenrands wurde der Wert $4 \cdot 10^{9} \mathrm{~cm}$ aus Abschnitt 5.1.4 benutzt. In optisch dünnen Akkretionsscheiben ist die Temperatur des Materials in den Außenbereichen der Scheibe bis hin zu einem $(\dot{M}$-abhängigen) Abstand $R \approx 10^{10} \mathrm{~cm}$ vom Weißen Zwerg etwa konstant und beträgt dort $T \approx 6000 \mathrm{~K}$ (z.B. Williams 1980; Tylenda 1981, 1988); erst weiter innen steigt sie auf höhere Werte. Die Standardtheorie für Akkretionsscheiben sagt für den radialen Temperaturverlauf in der Scheibe $T \sim\left(M_{\mathrm{WD}} \dot{M} / R^{3}\right)^{1 / 4}$ voraus (s. z.B. Warner 1995); für $M_{\mathrm{WD}}=1.2 M_{\odot}$ und eine angenommene Akkretionsrate $\dot{M}=3 \cdot 10^{15} \mathrm{~g} \mathrm{~s}^{-1}$ erhält man für die Temperatur am Innenrand der Scheibe von EX Hya den Wert $T \approx 10000 \mathrm{~K}$. Es wurden daher zwei optisch dünne Emissionskomponenten angesetzt: eine kühle $(T=6000 \ldots 7000 \mathrm{~K})$, die über die gesamte Scheibe reicht, und eine heißere $(T \approx 10000 \mathrm{~K})$, die nur einige Prozent der Scheibenfläche bedeckt. Die Länge der Sichtlinie durch die Scheibe (unter Berücksichtigung der Inklination) und der thermische Druck des Materials sind hier weitere freie Parameter. Es sei noch angemerkt, daß sich die beobachteten Linienflüsse und -verhältnisse sowie die Höhe des Balmer-Sprungs mit nur einer optisch dünnen Komponente nicht befriedigend reproduzieren lassen.

Angesichts der großen Zahl freier Parameter und der hier verfolgten Absicht wurde keine regelrechte Anpassung des beobachteten Spektrums versucht; vielmehr wurde 'nach Augenschein' ein plausibler Satz von Komponenten gesucht, mit dem sich das Spektrum angemessen wiedergeben läßt. Das Ergebnis läßt sich wie folgt zusammenfassen:

- Mit den oben dargestellten Komponenten allein ist eine befriedigende Wiedergabe des beobachteten Spektrums nicht zu erreichen; in allen Fällen verbleibt ein Flußdefizit im Bereich $\approx 2000 \ldots 10000 \AA$. Es kann durch eine zusätzliche optisch dicke Emissionskomponente (d.h. ein Schwarzkörperspektrum) mit $T \approx 15000 \mathrm{~K}$ aufgefüllt werden, wobei die projizierte Fläche des Emissionsgebiets $\approx 4 \cdot 10^{18} \mathrm{~cm}^{2}$ betragen muß (d.h. $\approx 7 \times$ mehr als die Querschnittsfläche des Weißen Zwergs). Diese Komponente, deren integraler beobachteter Fluß $\approx 7.8 \cdot 10^{-11} \mathrm{erg} \mathrm{cm}^{-2} \mathrm{~s}^{-1}$ beträgt, interpretiere ich als Emission des beleuchteten Innenrands der Akkretionsscheibe, der aufgrund der dort herrschenden höheren Dichte des 
Materials vermutlich optisch dick ist. Dies impliziert, daß die gesamte vertikale Ausdehnung $h_{\text {rim }}$ dieses Rands $\approx 10^{9} \mathrm{~cm} \approx 2.5 R_{\text {WD }}$ betragen muß. Ein sehr ähnlicher Wert wurde bereits in Abschnitt 5.1.4 aus den optischen Bedeckungskurven abgeleitet, was ein gewisses Vertrauen in diese Interpretation einflößt. Eine solche Komponente wurde auch von Hurwitz et al. (1997) herangezogen, um das EUV-Spektrum von EX Hya im Bereich 80...180 ̊ zu deuten. In Abschnitt 5.6.4 wird noch geprüft werden, inwieweit diese Vorstellung auch energetisch konsistent ist.

- Unter Hinzunahme dieser zusätzlichen Komponente ist eine gute Wiedergabe des beobachteten Spektrums möglich. Das resultierende Modellspektrum ist in Abbildung 5.9 zusammen mit dem beobachteten Spektrum (oben) bzw. mit seinen Einzelkomponenten (unten) dargestellt. Die Temperaturen der optisch dünnen Komponenten betragen dabei $7000 \mathrm{~K}$ bzw. $10000 \mathrm{~K}$. Die Längen der Sichtlinen bzw. die Drücke betragen jeweils $10^{8} \mathrm{~cm}$

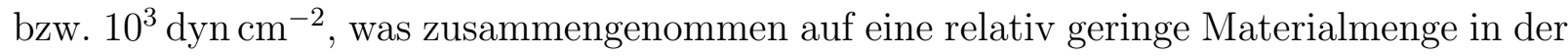
Scheibe hindeutet. Dies werte ich als weiteres Indiz für eine geringe Akkretionsrate. Diese wird in Abschnitt 5.6.2 quantitativ bestimmt; dort werden auch die integralen Flüsse aller in diesem Abschnitt diskutierten spektralen Komponenten von EX Hya angegeben.

- Der Flußverlauf am kurzwelligen Ende des Spektrums erfordert unter der Annahme eines Flächenanteils $g_{A}=0.25$ der heißen Polkappenkomponente (s.o.) eine Temperatur von $\approx 40000 \mathrm{~K}$. Auch etwas höhere bzw. niedrigere Temperaturen bei gleichzeitig etwas kleinerem bzw. größerem Flächenanteil $g_{A}$ sind möglich, jedoch erreicht $g_{A}$ für $T \gtrsim 50000 \mathrm{~K}$ oder $T \lesssim 30000 \mathrm{~K}$ schnell unsinnige Werte.

Abschließend sei noch bemerkt, daß der im Bereich $\lambda \gtrsim 17000 \AA$ etwas zu niedrige Fluß des Modells ein Hinweis darauf sein könnte, daß der Spektraltyp M4.5 des für den Abzug des Beitrags des Sekundärsterns verwendeten M-Stern-Spektrums sogar noch etwas zu früh ist. Die hier durchgeführte Zerlegung des unmodulierten Anteils des Spektrums in mehrere Komponenten sollte jedoch weniger als Anpassung, sondern eher als heuristische Betrachtung angesehen werden, die zeigen soll, daß eine Zerlegung der angegebenen Art möglich und physikalisch sinnvoll ist, und die eine Abschätzung der von den verschiedenen Quellen produzierten Leuchtkräfte ermöglichen soll. Letztere werden für die im folgenden Abschnitt durchgeführte gesamtenergetische Betrachtung benötigt. Da es dabei nicht so sehr auf die exakten Werte der einzelnen Parameter ankommt, die durchaus beträchtliche Unsicherheiten aufweisen können, ist die obige Aussage über den Spektraltyp entsprechend mit Vorsicht zu genießen.

\subsection{Leuchtkraft und Akkretionsrate}

\subsubsection{Röntgenspektrum}

Zur Bestimmung der Akkretionsrate von EX Hya ist die Kenntnis der Gesamtleuchtkraft $L$ erforderlich, zu der wesentlich die Röntgenleuchtkraft $L_{\mathrm{X}}$ beiträgt. Alle Leuchtkraftabschätzungen werden aus den beobachteten oder implizierten Flüssen $F$ unter Annahme isotroper Emission berechnet, d.h. es wird $L=4 \pi d^{2} F$ angesetzt, wobei $d$ die Entfernung des 


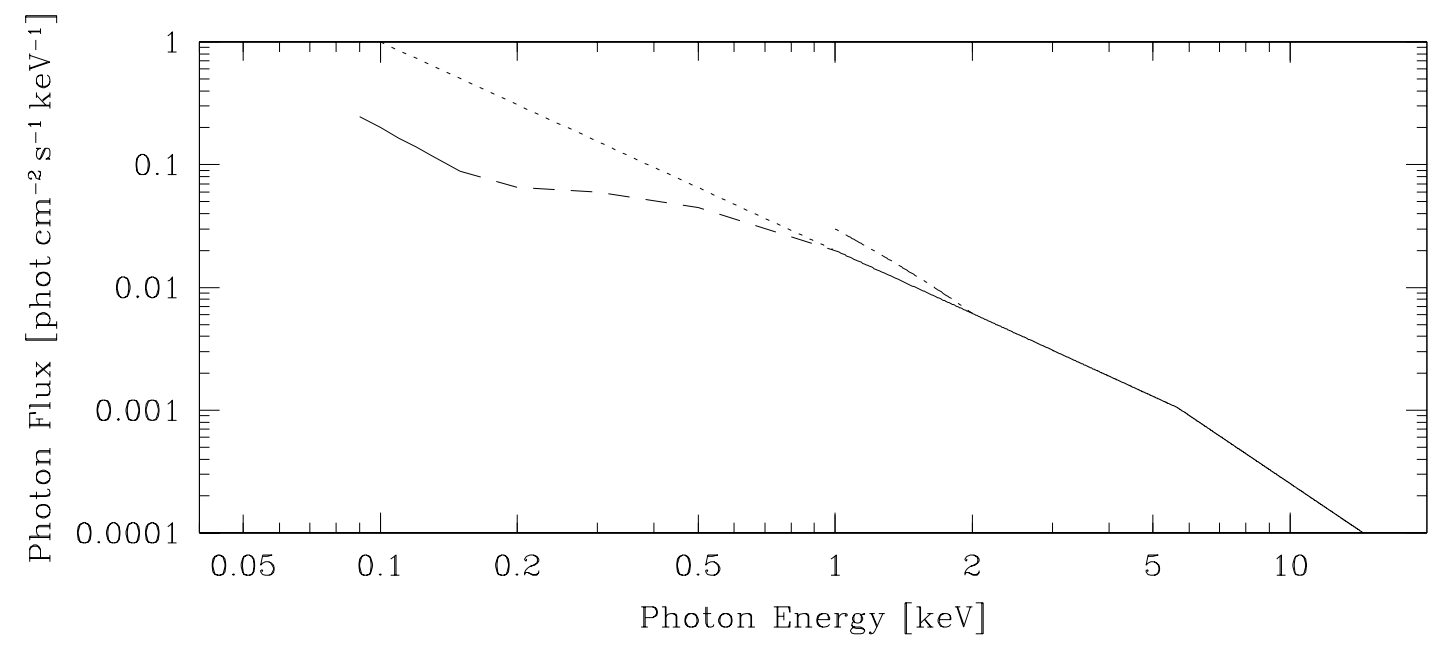

Abbildung 5.11: Beobachtetes Röntgen- und EUV-Photonenflußspektrum von EX Hya (schematisch). Ausgezogen: EXOSAT-Spektrum im Bereich 1...15 keV (Beuermann \& Osborne 1988) und EUVE-Spektrum im Bereich 90...150 eV (Hurwitz et al. 1997). Der zwischen den Bereichen der beiden Spektren liegende gestrichelte Bereich gibt schematisch die von Córdova et al. (1985) angegebenen Werte wieder.

Tabelle 5.3: Röntgenflüsse $F_{\mathrm{X}}$ von EX Hya in verschiedenen Energiebereichen. Den 'beobachteten' Werten liegt die ausgezogene und gestrichelte Kurve aus Abbildung 5.11 zugrunde; die 'intrinsischen' Werte beinhalten Korrekturen für die Absorption (s. Text).

\begin{tabular}{crr}
\hline$E[\mathrm{keV}]$ & \multicolumn{2}{c}{$F_{\mathrm{X}}\left[10^{-11} \mathrm{erg} \mathrm{cm}^{-2} \mathrm{~s}^{-1}\right]$} \\
beobachtet & intrinsisch \\
\hline $0.055 \ldots 0.28$ & 0.5 & 2.8 \\
$0.28 \ldots 0.5$ & 0.8 & $\approx 3.3$ \\
$0.5 \ldots 1.0$ & 3.4 & 4.8 \\
$1 \ldots 2$ & 3.0 & 3.9 \\
$2 \ldots 5$ & 4.2 & 4.8 \\
$5 \ldots 20$ & 5.7 & 6.3 \\
$20 \ldots \infty$ & $\approx 2.0$ & $\approx 2.0$ \\
\hline
\end{tabular}

Objekts ist. Im allgemeinen ist die Emission eines CVs als extrem nichtsphärischem Objekt jedoch frequenzabhängig anisotrop. Auch bei Anwendung dieses Berechnungsschemas auf den orbital gemittelten Fluß kann man daher systematische Fehler von der Größenordnung einiger $10 \%$ nicht ausschließen.

Die Leuchtkraft eines Objekts im Röntgenbereich wird meist unter Ansatz des Photonenflußspektrums (in Photonen $\mathrm{cm}^{-2} \mathrm{~s}^{-1} \mathrm{keV}^{-1}$ ) bestimmt, das auf einem angenommenen Emissionsmechanismus (z.B. thermischer Bremsstrahlung) basiert und an das beobachtete Zählratenspektrum (in counts s ${ }^{-1}$ ) angepaßt wird. Aus den so bestimmten Parametern (z.B. Temperatur und absorbierende Säulendichte $N_{\mathrm{H}}$ ) läßt sich dann der intrinsische, unabsorbierte Fluß der Quelle berechnen. Die so erhaltenen Werte sind stark von den Modellan- 
nahmen abhängig, z.B. von der Anzahl der thermischen Komponenten und - besonders bei niedrigen Photonenenergien - den Annahmen zur interstellaren und intrinsischen Absorption. Die Literaturangaben zu den (unabsorbierten) Röntgenflüssen von EX Hya wurden unter z.T. sehr verschiedenen Modellannahmen gewonnen und beinhalten Normalisierungen, die sich zum großen Teil aus den Veröffentlichungen nicht mehr nachvollziehen lassen. Als Konsequenz sind die implizierten Flüsse nicht ohne weiteres in konsistenter Weise miteinander vergleichbar. Das mit der größten Zuverlässigkeit angebbare Spektrum ist das auf den Detektor einfallende Photonenflußspektrum, das sich durch den Einfluß der interstellaren und intrinsischen Absorption von dem sogenannten Quellspektrum unterscheidet. Letzteres ist das Photonenflußspektrum, das ohne diese Absorption am Ort des Detektors beobachtet würde.

Abbildung 5.11 zeigt schematisch die mit EXOSAT und EUVE beobachteten Photonenflußspektren $N_{\mathrm{E}}(E)$ von EX Hya im Röntgen- und EUV-Bereich (Beuermann \& Osborne 1984; Cordóva et al. 1985; Hurwitz et al. 1997). Die ausgezogene Kurve bei $E>1 \mathrm{keV}$ stellt das beobachtete, also nicht auf Absorption korrigierte Photonenflußspektrum dar, bei dem die strichpunktierte Linie eine vermutlich zusätzlich vorhandene niederenergetische Komponente darstellt. Neuere Messungen mit GINGA und ASCA ergeben nahezu identische Ergebnisse (Rosen et al. 1991; Allan et al. 1998). Die berichteten Spektren liegen allerdings aufgrund der jeweiligen Annahmen über die interstellare und intrinsische Absorption um $10 \ldots 30 \%$ höher. Die gepunktete Linie stellt eine nicht physikalisch begründete Extrapolation des beobachteten EXOSAT-Spektrums zu niedrigen Energien hin dar. Die einzige energetisch hinreichend gut aufgelöste Beobachtung im Bereich $\approx 0.15 \ldots 1 \mathrm{keV}$ wurde mit dem Gitterspektrographen an Bord von EXOSAT durchgeführt (Córdova et al. 1985). Das resultierende Spektrum ist in der Abbildung schematisch durch die gestrichelte Linie wiedergegeben. Schließlich ist - wiederum schematisch - bei noch niedrigeren Energien das mit dem Gitterspektrographen an Bord von EUVE gewonnene Spektrum (Hurwitz et al. 1997) als ausgezogene Linie dargestellt. Während das Röntgenspektrum im keV-Bereich im wesentlichen ein Kontinuumsspektrum mit überlagerten Linien hochionisierter Atome ist, ist das Spektrum im EUVE-Bereich $(0.1 \mathrm{keV})$ durch Emissionslinien von Ionen mittlerer Ionisationsstufen dominiert. Das Photonenspektrum $N_{\mathrm{E}}(E)$ (in Einheiten von Photonen $\mathrm{cm}^{-2} \mathrm{~s}^{-1} \mathrm{keV}^{-1}$ ) läßt sich stückweise durch die Potenzgesetze $N_{\mathrm{E}}=2 \cdot 10^{-3} E^{-2}$ (EUVE), $N_{\mathrm{E}}=2 \cdot 10^{-2} E^{-1.7}(\mathrm{EXOSAT}, E \lesssim 6 \mathrm{keV})$ und $N_{\mathrm{E}}=8 \cdot 10^{-2} E^{-2.5}$ (EXOSAT, $E \gtrsim 6 \mathrm{keV}$ ) beschreiben, wobei $E$ die Photonenenergie in $\mathrm{keV}$ ist.

In Abschnitt 5.6 .3 wird gezeigt, daß der Fluß im Bereich 55..280 eV mindestens 2.4 . $10^{-11} \mathrm{erg} \mathrm{cm}^{-2} \mathrm{~s}^{-1}$ betragen muß, was dem Fluß des in Abbildung 5.11 dargestellten extrapolierten Spektrums (gepunktete Linie) entspricht (vgl. Tabelle 5.3). Dies bedeutet aber nicht, daß auch die Form des tatsächlichen Spektrums durch die Extrapolation richtig wiedergegeben wird; dies ist sogar mit großer Wahrscheinlichkeit nicht der Fall, s. unten. Da interstellare Absorption vermutlich nur eine geringe Rolle spielt (vgl. Kapitel 3.2.2), läßt der um einen Faktor $\approx 10$ geringere beobachtete Fluß den Schluß zu, daß intrinsische Absorption in EX Hya eine wesentliche Rolle spielen muß. Da sich ihr Ausmaß aber nicht ohne weiteres erschließen läßt, läßt sich auch die intrinsische Form des Spektrums nicht näher bestimmen. Im EXOSAT-Spektrum gibt es Anzeichen für einen Flußanstieg am kurwelligen 
Tabelle 5.4: Integrale Flüsse der spektralen Komponenten von EX Hya. Der Wert für die harte Röntgenkomponente $(E>=0.5 \mathrm{keV})$ entspricht der Summe der Werte für die entsprechenden Energiebereiche aus Tabelle 5.3; der Fluß der weichen Röntgenkomponente $(E=0.28 \ldots 0.5 \mathrm{keV})$ ist ein interpolierter Schätzwert, da das intrinsische Spektrum dort nicht bekannt ist (s. Abschnitt 5.6.1).

\begin{tabular}{lll}
\hline Komponente & $\begin{array}{l}\text { Energie- bzw. } \\
\text { Wellenlängenbereich }\end{array}$ & $\begin{array}{l}\text { Fluß } \\
{\left[10^{-11} \mathrm{erg} \mathrm{cm}^{-2} \mathrm{~s}^{-1}\right]}\end{array}$ \\
\hline EUV-Emission & $E=55 \ldots 280 \mathrm{eV}$ & 2.8 \\
weiche Röntgenemission & $E=0.28 \ldots .5 \mathrm{keV}$ & $\approx 3.3$ \\
harte Röntgenemission & $E>0.5 \mathrm{keV}$ & 21.8 \\
Akkretionsvorhänge & $\lambda=912 \ldots 25000 \AA$ & 11.9 \\
Akkretionsscheibe (optisch dünn) & $\lambda=912 \ldots 25000 \AA$ & $2.92(7000 \mathrm{~K})$ \\
desgl., heißere Komponente & $\lambda=912 \ldots 25000 \AA$ & $3.73(10000 \mathrm{~K})$ \\
Innenrand der Akkretionsscheibe & $\lambda=0 \ldots \infty$ & $7.75(15000 \mathrm{~K})$ \\
geheizte Polkappen des Weißen Zwergs & $\lambda=0 \ldots \infty$ & $13.1(40000 \mathrm{~K})$ \\
'ungeheizter' Weißer Zwerg & $\lambda=0 \ldots \infty$ & $3.49(20000 \mathrm{~K})$ \\
\hline
\end{tabular}

Ende (schematisch dargestellt durch das strichpunktierte Geradenstück; vgl. Beuermann \& Osborne 1985), der jedoch stark vom angenommenen Wert für $N_{\mathrm{H}}$ abhängig ist. Es ist deshalb nicht ausgeschlossen, daß der unabsorbierte Fluß von EX Hya bei niedrigen Energien noch oberhalb der gepunkteten Linie liegt.

Die integralen Röntgenflüsse von EX Hya in verschiedenen Energiebereichen sind in Tabelle 5.3 angegeben. Dabei enthält die zweite Spalte die beobachteten Flüsse, die in Abbildung 5.11 der ausgezogenen und gestrichelten Linie entsprechen. Die dritte Spalte enthält Schätzungen des tatsächlichen Quellflusses unter Berücksichtigung des wahrscheinlichen Einflusses der Absorption. Für deren Ausmaß ist im niederenergetischen Bereich $(55 \ldots 280 \mathrm{eV})$ die in Abschnitt 5.6 .3 beschriebene Zanstra-Methode und im keV-Bereich die aus der Amplitude der Spinmodulation abgeleitete intrinsische Absorption maßgeblich (zu den systematischen Unsicherheiten dieses Zugangs s. Allan et al. 1998).

\subsubsection{Energiebilanz und Akkretionsrate}

Die im vorigen Abschnitt ermittelten Röntgenflüsse und die integralen Flüsse der in Abschnitt 5.5 diskutierten Komponenten des nicht mit der Spinphase modulierten Anteils des Spektrums, das die Emission der Akkretionsscheibe und des Weißen Zwergs repräsentiert (s. Abbildung 5.9), sind in Tabelle 5.4 zusammengefaßt. Ferner findet sich dort der integrale Fluß der spinmodulierten Komponente, die in Abschnitt 5.5 bei der Konstruktion des unmodulierten Spektrums vom mittleren Spektrum abgezogen wurde und hier mit der Emission der Akkretionsvorhänge identifiziert wird. Bis auf den Beitrag des 'ungeheizten' Weißen Zwergs gehen alle aufgeführten Flüsse letztlich auf die Akkretion als Energiequelle zurück.

Vermutlich ist sogar ein wesentlicher Teil des Flusses des 'ungeheizten' Weißen Zwergs 
ebenfalls auf Akkretionsheizung zurückzuführen. Dabei spielen zwei Effekte eine Rolle, zum einen die adiabatische Kompression des langsam an Masse zunehmenden und dabei kontrahierenden Weißen Zwergs (s. Kapitel 2.5.5.2) und zum anderen die Strahlungsheizung durch die auf Teile der Oberfläche fallende Röntgenstrahlung. Die typische Temperatur eines Weißen Zwergs in einem kurzperiodischen CV mit einer Akkretionsrate $\dot{M} \approx 2$. $10^{-11} M_{\odot} \mathrm{yr}^{-1} \approx 10^{15} \mathrm{~g} \mathrm{~s}^{-1}$ sollte aufgrund des erstgenannten Prozesses bei $10000 \ldots 15000 \mathrm{~K}$ liegen (Gänsicke 2000). Dieser Prozess umfaßt den gesamten Weißen Zwerg und heizt daher seine gesamte Oberfläche. Heizung durch Bestrahlung aus der Stoßregion heizt hingegen nur die polnahen Gebiete.

Bei einer Stoßhöhe von $0.5 R_{\mathrm{WD}}=2 \cdot 10^{8} \mathrm{~cm}$ (vgl. Abschnitt 5.1.5) machen die bestrahlten Polkalotten $\approx 25 \%$ der Oberfläche des Weißen Zwergs aus. Bei einer Abschätzung der Temperatur dieser Gebiete muß man berücksichtigen, daß ein Teil der einfallenden Röntgenstrahlung durch Compton-Prozesse wieder aus der Atmosphäre herausgestreut wird. Nach van Teeseling et al. (1996) erreicht der Rückstreufaktor $r$ ein Maximum bei einer Photonenenergie $E \approx 30 \mathrm{keV}$, liegt jedoch unterhalb von $\approx 10 \mathrm{keV}$, wo EX Hya den größten Teil des Flusses aufweist (vgl. Tabelle 5.3), bei $r \lesssim 0.1$. Der weitaus größte Teil der den Weißen Zwerg treffenden Röntgenstrahlung sollte daher reprozessiert werden können. Für eine Röntgenleuchtkraft von $1.5 \cdot 10^{32} \mathrm{erg} \mathrm{s}^{-1}$ (basierend auf einem Gesamtfluß von $2.8 \cdot 10^{-10} \mathrm{erg} \mathrm{cm}^{-2} \mathrm{~s}^{-1}$ aus Tabelle 5.3) und zwei bestrahlte Polkappen von je $3 \cdot 10^{8} \mathrm{~cm}$ Radius ergibt sich deren mittlere Temperatur $\mathrm{zu} \approx 45000 \mathrm{~K}$. Wegen der stark vom Zentrum zum Rand der Polkappen hin abfallenden Intensität der Strahlung wird man erwarten, daß die Temperatur im Zentrum um mindestens einen Faktor zwei höher liegt und nach außen bis auf die Temperatur des 'ungeheizten' Zwergs abfällt. Eine eindeutige Trennung dieser Gebiete ist deshalb nicht möglich, und die in Abschnitt 5.5 angenommene Temperatur des 'ungeheizten' Weißen Zwergs erfaßt daher auch die äußeren Teile der geheizten Gebiete.

Die Summe aller auf die Akkretion zurückzuführenden Flüsse beträgt somit $F_{\text {acc }} \approx$ $6.7 \cdot 10^{-10} \mathrm{erg} \mathrm{cm}^{-2} \mathrm{~s}^{-1}$, wovon auf die Zentralquelle $F_{\text {cen }} \approx 5.3 \cdot 10^{-10} \mathrm{erg} \mathrm{cm}^{-2} \mathrm{~s}^{-1}$, auf den geheizten Innenrand der Akkretionsscheibe $F_{\text {rim }} \approx 0.8 \cdot 10^{-10} \mathrm{erg} \mathrm{cm}^{-2} \mathrm{~s}^{-1}$ und die eigentliche Akkretionsscheibe $F_{\text {disc }} \approx 0.7 \cdot 10^{-10} \mathrm{erg} \mathrm{cm}^{-2} \mathrm{~s}^{-1}$ entfallen. Bei der Leuchtkraft der Akkretionsscheibe ist noch zu berücksichtigen, daß ein Anteil $\approx 10^{-11} \mathrm{erg} \mathrm{cm}^{-2} \mathrm{~s}^{-1}$ durch Bestrahlung und nicht durch die viskose Freisetzung von Gravitationsenergie bedingt ist. Nach einer solchen Korrektur ist die Energiebilanz damit verträglich, daß bei einem Radius des Innenrands der Scheibe von $R_{i} \approx 10 R_{\mathrm{WD}}$ etwa $10 \%$ der gesamten Akkretionsleuchtkraft in der Scheibe freigesetzt wird.

Aus $F_{\text {acc }} \approx 6.7 \cdot 10^{-10} \mathrm{erg} \mathrm{cm}^{-2} \mathrm{~s}^{-1}$ ergibt sich zusammen mit der Entfernung von EX Hya von $d=66 \mathrm{pc}$ (Abschnitt 5.1.8) und unter Annahme isotroper Abstrahlung $(\Omega=4 \pi)$ die Akkretionsleuchtkraft $L_{\text {acc }}=\Omega d^{2} F_{\text {acc }}=3.5 \cdot 10^{32} \mathrm{erg} \mathrm{s}^{-1}$ (mit einem Anteil der Zentralquelle von $L_{\mathrm{cen}}=2.8 \cdot 10^{32} \mathrm{erg} \mathrm{s}^{-1}$ ) und mit $M_{\mathrm{WD}}$ und $R_{\mathrm{WD}}$ aus Tabelle 5.1 nach (2.36) die Akkretionsrate $\dot{M}=8.8 \cdot 10^{14} \mathrm{~g} \mathrm{~s}^{-1}$. Die entfernungsunabhängige Bestimmung von $\dot{M}$ aus der Abnahme der Spinperiode (s. Kapitel 2.5.4) liefert nach (2.49) - wieder mit den Parametern aus Tabelle 5.1 sowie $\dot{P}_{\text {spin }}$ aus Kapitel $3.2 .1-\dot{M}=9.4 \cdot 10^{14} \mathrm{~g} \mathrm{~s}^{-1}$ in guter Übereinstimmung mit dem eben ermittelten Wert. Nach den Ausführungen in Kapitel 2.5.4 wird bei Anwendung dieser Methode $\dot{M}$ unterschätzt, wenn Teile der Akkretionsscheibe außerhalb 
des Korotationsradius $R_{\text {cor }}$ liegen, an dem $\omega_{\text {kep }}=\omega_{\text {spin }}$ gilt. Für EX Hya mit $M_{\mathrm{WD}}=1.2 M_{\odot}$ und $P_{\text {spin }} \approx 4020 \mathrm{~s}$ berechnet man mit $R_{\text {cor }} \approx 4 \cdot 10^{10} \mathrm{~cm}$ einen Wert, der deutlich größer als der in Abschnitt 5.5 angesetzte Radius der Scheibe von $2.5 \cdot 10^{10} \mathrm{~cm}$ ist, so daß die aus $\dot{P}_{\text {spin }}$ abgeleitete Akkretionsrate keinen wesentlichen systematischen Fehler aufweisen sollte. Die relative Unsicherheit $\sigma_{\dot{M}} / \dot{M}$ der Akkretionsrate $\dot{M}=\Omega d^{2} F_{\text {acc }}\left(R_{\mathrm{WD}} / G M_{\mathrm{WD}}\right)$ läßt sich durch quadratische Addition der folgenden Fehler abschätzen: $d: 15 \%$ bzw. $d^{2}: 30 \%$; $M_{\mathrm{WD}}, R_{\mathrm{WD}}$ : je $10 \%$ bzw. $R_{\mathrm{WD}} / M_{\mathrm{WD}}: 20 \% ; F_{\mathrm{acc}}: 20 \% ; \Omega: 20 \%$; daraus $\dot{M}: \approx 50 \%$. Somit erhält man für $\dot{M}$ den (gerundeten) Wert $\dot{M}=(1.0 \pm 0.5) \cdot 10^{15} \mathrm{~g} \mathrm{~s}^{-1}$.

\subsubsection{EUV-Leuchtkraft nach der Zanstra-Methode}

Bei der Rekombination eines zweifach ionisierten Helium-Ions mit einem Elektron entsteht ein einfach ionisiertes HeII-Ion in einem angeregten Zustand. Die Abregung erfolgt auf verschiedenen möglichen Pfaden unter Aussendung eines oder mehrerer Photonen. Etwa $26 \%$ der Rekombinationen führen zur Emission eines Photons der Wellenlänge $\lambda=4686 \AA$ bzw. der Energie $2.65 \mathrm{eV}$. Zur Erzeugung eines $\mathrm{He}^{2+}$-Ions aus einem $\mathrm{He}^{+}$-Ion ist die Energiemenge $55 \mathrm{eV}$ nötig, sie erfolgt daher in nicht zu heißem Plasma fast ausschließlich durch Photoionisation. Aufgrund des Wirkungsquerschnitts des $\mathrm{He}^{+}$-Ions für Photoabsorption sind hierfür Photonen im Energiebereich $55 \ldots \approx 280 \mathrm{eV}$ erforderlich, d.h. EUVund weiche Röntgenstrahlung (z.B. Patterson \& Raymond 1985). Der beobachtete Gesamtfluß $F_{4686}$ der HeII-Linie von $1.65 \cdot 10^{-13} \mathrm{erg} \mathrm{cm}^{-2} \mathrm{~s}^{-1}$ (Patterson \& Raymond 1985) bzw. $1.13 \cdot 10^{-13} \mathrm{erg} \mathrm{cm}^{-2} \mathrm{~s}^{-1}$ (diese Arbeit, vgl. Tabelle 4.10, wobei dieser Wert wie die Flüsse aller optischen Spektren aus Tabelle 4.1 aufgrund der Unsicherheiten in der absoluten Flußkalibration um $\approx 20 \%$ zu niedrig sein kann, vgl. Kapitel 4.2.8) wird also durch Reprozessierung eines Flusses $F_{\mathrm{EUV}, \mathrm{SX}}^{\prime}$ energiereicher Photonen erzeugt, der intrinsisch vorhanden ist, im beobachteten Spektrum im EUV und weichen Röntgenbereich aber nicht mehr erscheint. Die mittlere Energie der ionisierenden Photonen im Bereich $55 \ldots 280 \mathrm{eV}$ liegt bei dem gepunkteten Spektrum aus Abbildung 5.11 bei $\approx 120 \mathrm{eV}$. Damit läßt sich nun der Fluß

$$
\begin{aligned}
F_{\mathrm{EUV}, \mathrm{SX}}^{\prime} & \approx F_{4686} \frac{1}{0.26} \frac{120 \mathrm{eV}}{2.65 \mathrm{eV}}\left(\frac{\Omega}{4 \pi}\right)^{-1} \\
& \approx 1.74 \cdot 10^{-11} \mathrm{erg} \mathrm{cm}^{-2} \mathrm{~s}^{-1}\left(\frac{\Omega}{4 \pi}\right)^{-1}\left(\frac{F_{4686}}{10^{-13} \mathrm{erg} \mathrm{cm}^{-2} \mathrm{~s}^{-1}}\right)
\end{aligned}
$$

berechnen, wobei $\Omega$ der Raumwinkel ist, den das Entstehungsgebiet der He II-Linie von der beleuchtenden Quelle aus gesehen einnimmt (z.B. Patterson et al. 1984)【. Man beachte, daß zur Erzeugung eines HeII 4686-Photons mit $E_{\text {out }}=2.65 \mathrm{eV}$ im Mittel die Energie $E_{\text {in }}=120 \mathrm{eV} / 0.26 \approx 460 \mathrm{eV}$ aufgewendet werden muß, der Wirkungsgrad also $<1 \%$ ist. Die übrigen $>99 \%$ treten als reprozessierte Strahlung bei anderen Wellenlängen auf. Für $F_{4686}=1.65 \cdot 10^{-13} \mathrm{erg} \mathrm{cm}^{-2} \mathrm{~s}^{-1}$ und $\Omega=4 \pi$ erhält man aus (5.2) den

7 Diese Methode, den Fluß ionisierender Photonen aus dem der durch sie erzeugten reprozessierten Photonen zu erschließen, wird häufig bei planetarischen Nebeln angewandt und ist als Zanstra-Methode bekannt (nach dem Niederländer Herman Zanstra). 
Wert $F_{\mathrm{EUV}, \mathrm{SX}}^{\prime}=2.87 \cdot 10^{-11} \mathrm{erg} \mathrm{cm}^{-2} \mathrm{~s}^{-1}$. Dieser Fluß entspricht zufällig dem des in Abbildung 5.11 gestrichelt dargestellten extrapolierten EXOSAT-Spektrums im interessierenden Energiebereich $55 \ldots 280 \mathrm{eV}$ (s. Tabelle 5.3) und impliziert eine Leuchtkraft in diesem Bereich, die um einen Faktor $\approx 10$ über derjenigen liegt, die aus dem beobachteten EUV-Fluß erschlossenen wird. Für Raumwinkel $\Omega<4 \pi$ wird der benötigte Fluß $F_{\mathrm{EUV}, \mathrm{Sx}}^{\prime}$ und damit auch die implizierte Leuchtkraft noch größer. Dies wäre konsistent mit einem Spektrum, das zu niedrigen Energien hin schneller ansteigt als die punktierte Linie in Abbildung [5.11, wie man es aufgrund des beobachteten Spektrums und unter Berücksichtigung der Wellenlängenabhängigkeit der interstellaren Absorption (Massenabsorptionskoeffizient $\sigma \sim \lambda^{3}$ ) erwarten würde.

\subsubsection{Beleuchteter Innenrand der Akkretionsscheibe}

In Abschnitt 5.5 wurde postuliert, daß der Innenrand der Akkretionsscheibe, für den bei einem Radius von $R_{i}=4 \cdot 10^{9} \mathrm{~cm}$ eine vertikale Ausdehnung $h_{\text {rim }} \approx 10^{9} \mathrm{~cm}$ angenommen wurde, zum Gesamtspektrum in Form einer Schwarzkörper-Komponente mit $T_{\text {rim }}=15000 \mathrm{~K}$ und einem integralen beobachteten Fluß von $F_{\text {rim }}=7.75 \cdot 10^{-11} \mathrm{erg} \mathrm{cm}^{-2} \mathrm{~s}^{-1}$ beiträgt. Unter der vereinfachten Annahme, daß die Heizung des Materials am Innenrand der Scheibe von der Temperatur $T_{\text {disc }} \approx 10000 \mathrm{~K}$ im unbeleuchteten Fall auf die Temperatur $T_{\text {rim }}$ ausschließlich durch vollständige Reprozessierung der Strahlung einer isotrop strahlenden, punktförmigen Zentralquelle erfolgt, läßt sich deren hierfür benötigte Leuchtkraft $L_{\text {ill }} \mathrm{zu}$

$$
\begin{aligned}
L_{\text {ill }} & =4 \pi R_{i}^{2} \sigma\left(T_{\text {rim }}^{4}-T_{\text {disc }}^{4}\right) \\
& =7.13 \cdot 10^{30} \operatorname{erg~s}^{-1}\left(\frac{R_{i}}{10^{9} \mathrm{~cm}}\right)^{2}\left(\left(\frac{T_{\text {rim }}}{10000 \mathrm{~K}}\right)^{4}-\left(\frac{T_{\text {disc }}}{10000 \mathrm{~K}}\right)^{4}\right) .
\end{aligned}
$$

Umgekehrt läßt sich aus der in Abschnitt 5.6.2 bestimmten Leuchtkraft der Zentralquelle $L_{\text {cen }}=2.8 \cdot 10^{32} \mathrm{erg} \mathrm{s}^{-1}$ durch Gleichsetzen mit $L_{\text {ill }}$ die am Innenrand der Akkretionsscheibe resultierende Temperatur zu $T_{\text {rim }} \approx 13500 \mathrm{~K}$ berechnen, was angesichts der Unsicherheiten z.B. von $R_{i}$ voll konsistent mit der in Abschnitt 5.5 benutzten Temperatur $T_{\text {rim }}=15000 \mathrm{~K}$ ist.

Hurwitz et al. (1997) finden im Spinminimum ein EUV-Spektrum, das weitgehend von den im Maximum dominierenden Emissionslinien hochangeregter Ionen frei ist. Die Autoren ordnen diese Komponente ebenfalls dem beleuchteten Innenrand der Akkretionsscheibe zu. Die erforderliche Plasmatemperatur beträgt $\approx 10^{6} \mathrm{~K}$, viel höher als die o.a. Schwarzkörpertemperatur. Dies muß keinesfalls inkonsistent sein, da die Quasi-Atmosphäre der beleuchteten Scheibe einen großen Temperaturbereich umfassen kann.

\subsection{Magnetfeld des Weißen Zwergs}

Die ursprüngliche Planung für diese Arbeit sah vor, EX Hya auch mit dem niedrigauflösenden Spektrophotometer PHOT-S an Bord des raumgestützten Infrarotteleskops ISO im 
Wellenlängenbereich $2.4 \ldots 12 \mu \mathrm{m}$ phasenaufgelöst zu untersuchen. Aufgrund technisch bedingter Einschränkungen bezüglich der Zeiten, zu denen bestimmte Himmelsgebiete während der ISO-Mission beobachtet werden konnten, wurde die bereits genehmigte Beobachtung schließlich doch nicht mehr durchgeführt. Dies ist aus zwei Gründen bedauerlich. Zum einen hätten die mit ISO gewonnenen Daten das Spektrum von EX Hya in den Bereich hinein erweitert, in dem der Sekundärstern die dominierende Strahlungsquelle ist, was eine sicherere Bestimmung seines Spektraltyps ermöglicht hätte. Zum anderen hätten sich in diesem Spektralbereich möglicherweise höhere Harmonische der Zyklotronstrahlung (s. Kapitel 2.4.2.3) finden lassen, falls das Magnetfeld des Weißen Zwergs stärker als $\approx 1 \mathrm{MG}$ ist (vgl. (2.39)); Abwesenheit von Zyklotronlinien wäre umgekehrt ein Indiz für ein schwächeres Magnetfeld gewesen.

Obwohl somit die aktuelle Datenlage eine direkte Aussage zur Magnetfeldstärke des Weißen Zwergs nicht zuläßt, erlauben die in diesem Kapitel abgeleiteten Werte der verschiedenen Systemparameter jedoch eine grobe Abschätzung nach (2.35), wenn man für den Alfvén-Radius $r_{\mu}$ des Weißen Zwergs (s. Kapitel 2.3.2) den Radius des Innenrands der Akkretionsscheibe $R_{\mathrm{i} \text {,disc }}$ ansetzt. Mit den Zahlenwerten für $R_{\mathrm{WD}}, M_{\mathrm{WD}}$ und $R_{\mathrm{i}, \mathrm{disc}}$ aus Tabelle 5.1 und $\dot{M}=10^{15} \mathrm{~g} \mathrm{~s}^{-1}$ (vgl. Abschnitt 5.6.2) erhält man $B \approx 0.6 \mathrm{MG}$, ein Wert, der in IPs vermutlich durchaus realisiert sein kann. Unter diesen Bedingungen sollte thermische Bremsstrahlung im Röntgenbereich der dominante Kühlungsmechanismus sein (vgl. Kapitel 2.4.3). Der Beitrag der Zyklotronstrahlung, die vornehmlich im Optischen und Infrarot auftritt, sollte entsprechend gering ausfallen und spielt vermutlich gegenüber den übrigen Strahlungsquellen in diesem Wellenlängenbereich nur eine untergeordnete Rolle. Insofern ist auch der bislang nicht gelungene Nachweis polarisierter Strahlung von EX Hya (s. Kapitel 3.2.4) nicht sonderlich überraschend.

Insgesamt ergeben sich aus den hier durchgeführten einfachen Betrachtungen also keine Hinweise auf grobe Inkonsistenzen des in dieser Arbeit entwickelten Modells für EX Hya. Eine genauere Prüfung dieser Aussage erfordert eine detailliertere Abschätzung des erwarteten Anteils der Zyklotronstrahlung durch numerische Berechnungen, die jedoch im Rahmen dieser Arbeit nicht durchgeführt werden können.

\subsection{Schlußbetrachtung}

EX Hya ist der Prototyp einer Klasse von kataklysmischen Veränderlichen, in denen ein frei rotierender magnetischer Weißer Zwerg Materie von seinem Begleiter akkretiert (intermediäre Polare). Die Bedeutung von EX Hya besteht darin, daß es sich hierbei um das weitaus hellste System dieser Art am Himmel handelt, das sich aufgrund seiner großen Nähe selbst in dem durch interstellare Absorption besonders stark beeinträchtigten EUVBereich beobachten läßt. Kein anderes bekanntes System dieser Klasse ist in diesem Detail beobachtbar. Da die Emission in den verschiedenen Energiebereichen durch verschiedene Mechanismen dominiert wird, ist es erforderlich, Beobachtungen vom Infrarot bis in den harten Röntgenbereich durchzuführen, um die komplizierte Phänomenologie korrekt beschreiben zu können. Diese Arbeit basiert auf eigenen spektroskopischen Beobachtungen 
im Optischen und nahen Infrarot. Diese wurden ergänzt durch Archivdaten von Satelliten, die im UV, EUV und Röntgenbereich operieren. Aufgrund dieser Daten gelang es erstmals, eine Gesamtanalyse des Systems durchzuführen, die wesentliche neue Ergebnisse hinsichtlich seiner Entfernung und Energetik ergeben hat.

\subsubsection{Zusammenfassung der neuen Erkenntnisse}

In dieser Arbeit wurde aus den Beobachtungsdaten ein Modell für EX Hya abgeleitet, das geometrisch und energetisch in sich konsistent ist und alle wesentlichen in der Literatur beschriebenen Eigenschaften des Systems erklären kann. Die sich aus dem Modell ergebenden neuen Erkenntnisse lassen sich wie folgt zusammenfassen]:

- Die Massen des Weißen Zwergs und des Sekundärsterns betragen $M_{\mathrm{WD}}=1.2 \pm 0.1 M_{\odot}$ bzw. $M_{2}=0.13 \pm 0.01 M_{\odot}$. Der Weiße Zwerg ist damit um einen Faktor $\approx 2$ massiver als bisher angenommen (vgl. Kapitel 3.2.3) und hat eine der größten überhaupt für Weiße Zwerge bestimmten Massen. Insbesondere ist die Masse deutlich größer als die in letzter Zeit von einigen Autoren mit verschiedenen Instrumenten aus der Temperatur der thermischen Bremsstrahlung im Röntgenbereich ermittelten Werte von $\approx 0.5 \pm 0.05 M_{\odot}$. In dieser Arbeit wurde die Masse des Weißen Zwergs über eine rein geometrische Radius-Bestimmung anhand der Analyse der Bedeckungsdauern und -lichtkurven im EUV und im Röntgenbereich und unter Benutzung einer Masse-Radius-Relation ermittelt (Abschnitt 5.1.3). Hierbei gingen lediglich die Annahmen ein, daß während der totalen Bedeckung im EUV von 41s Dauer der obere und während der partiellen Bedeckung im Röntgenbereich von 136 s Dauer der untere Pol des Weißen Zwergs von der Silhouette des Sekundärsterns bedeckt wird. Radius und Masse des Sekundärsterns ergeben sich dann unmittelbar und unabhängig voneinander aus der Roche-Geometrie des Systems und dem 3. Keplerschen Gesetz (vgl. Kapitel 2.1) und implizieren, daß der Sekundärstern sehr nahe der Hauptreihe stehen muß. Aus der Analyse der Infrarot-Spektren ergibt sich unabhängig davon ein Spektraltyp des Sekundärsterns von M4.0 bis M5.0 (Abschnitt 5.1.7), was gut sowohl mit dem bei der Orbitalperiode von EX Hya erwarteten Spektraltyp (Beuermann 2000a) als auch mit der geometrisch bestimmten Masse verträglich ist.

- Die Entfernung des Systems beträgt $d=66 \pm 9 \mathrm{pc}$ und ist damit um einen Faktor $\approx 2$ kleiner als nach früheren Bestimmungen (s. Kapitel 3.2.2). Dies macht EX Hya zum sonnennächsten CV mit bekannter Entfernung. Die Entfernungsbestimmung beruht auf der Interpretation der beobachteten Intensitätsmodulation mit der halben Orbitalperiode im nahen Infrarot (s. Tabelle 4.8) als der ellipsoidalen Modulation des Sekundärsterns (vgl. Kapitel 2.2.2.2). Der Fluß des letzteren wurde hiermit und mit der oben angesprochenen Analyse der Infrarot-Spektren erstmals direkt und quantitativ nachgewiesen. Der angegebene Fehler der Entfernung ist zum größten Teil auf die Unsicherheiten in der beobachteten K-Helligkeit und in der spektraltypabhängigen Oberflächenhelligkeit $S_{K}$ des Sekundärsterns zurückzuführen, für die jeweils $\pm 0.2 \mathrm{mag}$ angesetzt wurde. Speziell für $S_{K}$

${ }^{8}$ Der Kürze halber wird im folgenden für nähere Erläuterungen mit ggf. benötigten Literaturnachweisen meist auf die jeweils relevanten Abschnitte dieser Arbeit verwiesen. 
ist dies vermutlich sogar noch etwas zu großzügig, da dies einen Bereich möglicher Spektraltypen des Sekundärsterns von $\approx$ M3.0...5.5 implizieren würde, wohingegen das beobachtete Spektrum von EX Hya mit Spektraltypen früher als M4.0 oder später als M5.0 nicht recht verträglich ist (s. Abschnitt 5.1.7). Eine wirklich annahmenfreie Bestimmung der Entfernung ist nur durch Triangulation möglich (s. Abschnitt 5.8.4).

- Aus der Entfernung des Systems und dem akkretionsbedingten Anteil des Gesamtflusses ergibt sich für EX Hya eine Akkretionsleuchtkraft von $\approx 3 \cdot 10^{32} \mathrm{erg} \mathrm{s}^{-1}$ und zusammen mit der Masse des Weißen Zwergs eine Akkretionsrate von $\dot{M}=(1.0 \pm 0.5) \cdot 10^{15} \mathrm{~g} \mathrm{~s}^{-1}$ (Abschnitt 5.6.2). Der gegenüber früheren Bestimmungen von $3 \cdot 10^{15} \ldots 3 \cdot 10^{16} \mathrm{~g} \mathrm{~s}^{-1}$ (s. Kapitel 3.2.5) deutlich kleinere Wert ist im wesentlichen auf die geringere Entfernung des Systems und die größere Masse (und damit auch kleineren Radius) des Weißen Zwergs zurückzuführen. Der angegebene Fehler beruht auf den Unsicherheiten der oben genannten Größen sowie dem möglichen Fehler des akkretionsbedingten Flusses. Der aus den Daten gewonnene Wert entspricht recht genau demjenigen, den man aufgrund der beobachteten Abnahme der Spinperiode des Weißen Zwergs erwartet (vgl. Kapitel 2.5.4).

Für kataklysmische Veränderliche mit Orbitalperioden unterhalb von $2 \mathrm{~h}$ ist Gravitationsstrahlung des Massendipols der anerkannt dominante Mechanismus, der Drehimpuls aus dem System abführt und so die Akkretion treibt (s. Kapitel 2.5.2). Gravitationsstrahlung ist nach der Einsteinschen Gleichung für Quadrupolstrahlung mit einer definierten Abnahme des Bahnabstands der Sterne verknüpft, die zusammen mit der bekannten äußeren Struktur des Begleitsterns zu einer Massentransferrate von $\dot{M} \approx 3 \cdot 10^{15} \mathrm{~g} \mathrm{~s}^{-1}$ in sehr engen Grenzen und kaum abhängig von der Masse des Weißen Zwergs führt (z.B. Patterson 1984). Die hier für EX Hya ermittelte Akkretionsrate von $\dot{M}=(1.0 \pm 0.5) \cdot 10^{15} \mathrm{~g} \mathrm{~s}^{-1}$ liegt deutlich unter dem so erwarteten Wert. Damit ist die frühere Annahme widerlegt, daß die Akkretionsrate von EX Hya über dem für Gravitationsstrahlung vorhergesagten Wert liegt. Der hier abgeleitete niedrigere Wert stellt keine ernstzunehmende Diskrepanz dar, da Gravitationsstrahlung nur die mittlere Massentransferrate auf Zeitskalen von $\approx 10^{9} \mathrm{yr}$ vorhersagt. Auf kürzeren Zeitskalen treten bekanntermaßen Fluktuationen um diesen Mittelwert auf, deren Ursprung nicht vollständig geklärt ist.

- Die Akkretionsscheibe hat einen wahrscheinlichen Radius von $R_{a} \approx 2.5 \cdot 10^{10} \mathrm{~cm}$. Ihr Innenrand, der bei einem Radius von $\approx 4 \cdot 10^{9} \mathrm{~cm} \approx 10 R_{\mathrm{WD}}$ liegt, ist vermutlich 'aufgebläht'. Hinweise auf eine Ausdehnung senkrecht zur Orbitalebene von $\approx 10^{9} \mathrm{~cm} \approx 2.5 R_{\mathrm{WD}}$ ergeben sich unabhängig voneinander aus der Form und Tiefe der Bedeckungslichtkurven sowie aus der Analyse der Spektren (Abschnitte 5.1.4, 5.5). Letztere enthalten eine optisch dicke Komponente mit einer Temperatur von $\approx 15000 \mathrm{~K}$, die unter Zugrundelegung der oben angegebenen Akkretionsleuchtkraft und Ausdehnung durch Reprozessierung energiereicher Strahlung aus dem Zentralgebiet am Innenrand der Scheibe erklärt werden kann (Abschnitt 5.6.4). 


\subsubsection{Bewertung der Ergebnisse}

EX Hya ist vermutlich der am besten untersuchte intermediäre Polar. Trotzdem fehlte bisher eine Gesamtanalyse des Systems, und etliche der Diskussionen zu bestimmten Parametern in der Literatur sind widersprüchlich. Ein Modell, das in konsistenter Weise die System- und Akkretionsgeometrie sowie die energetischen Verhältnisse beschreibt und die beobachteten Eigenschaften erklären kann, existierte bisher nicht. Mit den in Tabelle 5.1 angegebenen Systemparametern und der in Abschnitt 5.1 entwickelten Vorstellung von den geometrischen Verhältnissen des Systems ist nun ein Modell vorhanden, das diese Anforderungen bis auf die in Abschnitt 5.8.3 geschilderten Punkte erfüllt. Maßgeblich bei der Entwicklung des Modells waren die glücklichen Umstände, daß EX Hya zum einen wegen der geringen Entfernung und damit geringen interstellaren Absorption auch im EUV sichtbar ist und zum anderen die Inklination gerade einen solchen Wert hat, daß beide Pole des Weißen Zwergs für kurze Zeit bedeckt werden. Dies ermöglichte eine etwas unkonventionelle Methode der Bestimmung der Masse des Weißen Zwergs über eine rein geometrische Radiusbestimmung, wobei sowohl die Bedeckung im Röntgenbereich als auch die im EUV herangezogen wurde.

Da es nur eine Handvoll kataklysmischer Variabler gibt, die so detailliert untersucht werden können, ist jedes die Gesamtheit aller Informationen umfassende Modell von grundsätzlicher Bedeutung für ein Verständnis dieser Systeme und der Eigenschaften der Klasse insgesamt.

\subsubsection{Offene Fragen}

Aus den in dieser Arbeit bestimmten Massen des Weißen Zwergs und des Sekundärsterns sowie der Inklination ergeben sich deren Radialgeschwindigkeitsamplituden zu $K_{1}=56 \pm$ $5 \mathrm{~km} \mathrm{~s}^{-1}$ und $K_{2}=506 \pm 20 \mathrm{~km} \mathrm{~s}^{-1}$. Der erstgenannte Wert stimmt innerhalb der Fehlergrenzen mit den von Gilliland (1982) bzw. Hellier et al. (1987) angegebenen Werten $\left(K_{1}=58 \pm 9 \mathrm{~km} \mathrm{~s}^{-1}\right.$ bzw. $\left.K_{1}=69 \pm 9 \mathrm{~km} \mathrm{~s}^{-1}\right)$ überein und ist noch marginal mit dem Wert $K_{1}=85 \pm 9 \mathrm{~km} \mathrm{~s}^{-1}$ von Mauche (1999) verträglich. Der o.a. Wert für $K_{2}$ ist jedoch nicht mit dem Wert $K_{2}=357 \pm 44 \mathrm{~km} \mathrm{~s}^{-1}$ von Smith (2000) zu vereinbaren. Schon Allen et al. (1998) äußerten die Vermutung, daß zumindest eine der beiden früher gemessenen Radialgeschwindigkeitsamplituden $K_{1}$ und $K_{2}$ falsch sein könnte. Angesichts der systematischen Unsicherheiten der von Smith (2000) angewandten Methode (s. Kapitel 3.2.3) scheint mir dies eher für $K_{2}$ der Fall zu sein. Diese Frage kann anhand der vorliegenden Spektren, die nur sehr schwache spektrale Signaturen des Sekundärsterns zeigen (vgl. Abschnitt 5.1.7), leider nicht geklärt werden. Die Angaben von Smith sind bisher nur als private Mitteilung verfügbar, die zugrundeliegenden Daten wurden bisher noch nicht veröffentlicht.

Die hier bestimmte Masse des Weißen Zwergs von $M_{\mathrm{WD}}=1.2 \pm 0.1 M_{\odot}$ steht in deutlichem Widerspruch zu den Werten, die von Fujimoto \& Ishida (1997), Cropper et al. (1999), Ezuka \& Ishida (1999) und Ramsay (2000) mit ASCA, GINGA und RXTE über die Messung der Temperatur der aus der Stoßregion im Röntgenbereich emittierten thermischen Bremsstrahlung und der Linienemission des $\mathrm{Fe}^{24+}$ und $\mathrm{Fe}^{25+}$ ermittelt wurden und sich mit 
$M_{\mathrm{WD}}=0.5 \pm 0.05 M_{\odot}$ zusammenfassen lassen. Dies verstärkt Zweifel an der Brauchbarkeit dieser Meßmethode, weil für die tatsächliche Stoßtemperatur nur eine untere Grenze bekannt ist (Allan et al. 1998). Sollte die hohe Masse des Weißen Zwergs in EX Hya auf anderem Wege bestätigt werden, müßte der Wert aller aus der Bremsstrahlungstemperatur bestimmten Massen Weißer Zwerge in IPs und Polaren generell in Zweifel gezogen werden.

\subsubsection{Was ist noch zu tun?}

Offensichtlich ist das wichtigste zu klärende Problem die Masse des Weißen Zwergs. Der erfolgsversprechendste und direkteste Weg dazu dürfte die zuverlässige Bestimmung der Radialgeschwindigkeitsamplituden $K_{1}$ und $K_{2}$ sein. Wie Mauche (1999) gezeigt hat, läßt sich erstere vermutlich aus den Radialgeschwindigkeitsvariationen der scharfen Komponenten der O VI-Emissionslinie bei $\lambda \approx 1035 \AA$ ermitteln, wenngleich nach meinem Empfinden die Unsicherheit des von ihm bestimmten Werts für $K_{1}$ größer ist als es der angegebene formale Fehler suggeriert. Den Wert von $K_{2}$ konnte ich aus den vorliegenden IR-Spektren, die mit einem 4m-Teleskop gewonnen wurden, nicht ermitteln. Da aber schwache spektrale Signaturen des Sekundärsterns auch in diesen Spektren vorhanden sind, sollte die Bestimmung von $K_{2}$ mit einem Teleskop der 8m-Klasse (VLT) und/oder leistungsfähigeren Detektoren ohne größere Schwierigkeiten möglich sein.

Weiterhin wäre eine Bestätigung des in Abschnitt 5.1.5 verwendeten Modells der Bedeckung des Weißen Zwergs im EUV und Röntgenbereich wichtig. Genauere Messungen (wie sie z.B. unpubliziert vom RXTE-Satelliten vorliegen, Mukai et al. 1998) können auch die Bestimmung des Radius des Weißen Zwergs noch deutlich verbessern. Es ist nicht ausgeschlossen, daß sich diese Messungen so weit verfeinern lassen, daß sich hier exemplarisch für einen sehr schweren Weißen Zwerg die Masse-Radius-Beziehung für elektronenentartete Sterne (s. Abschnitt 2.2.1.1ff.) prüfen läßt.

Schließlich sollte die in dieser Arbeit auf spektroskopischem Wege gefundene Entfernung von EX Hya sicher durch Triangulation überprüft werden. Wegen der Nähe des Systems ist dies mit dem Hubble Space Telescope ohne Schwierigkeiten möglich. Zur Zeit ist für unsere Arbeitsgruppe bereits der erste Teil einer entsprechenden Beobachtung mit HST zur Messung der Parallaxe durchgeführt worden, so daß diese Frage demnächst geklärt sein dürfte. Hiermit bliebe dann als einziger wesentlicher Unsicherheitsfaktor bei der Bestimmung der Leuchtkraft (bzw. zusammen mit einer gesicherten Masse des Weißen Zwergs auch bei der Bestimmung der Akkretionsrate) die Unsicherheit des tatsächlichen Gesamtflusses, die im wesentlichen durch das nicht hinreichend genau bekannte Ausmaß der Absorption im weichen Röntgenbereich und EUV hervorgerufen wird. Grundsätzlich verbesserte Beobachtungsdaten von EX Hya im EUV und Röntgenbereich sind mit den derzeit existierenden und geplanten Satellitenmissionen nicht zu erwarten. 


\section{Anhang A}

\section{Ergebnisse der Lichtkurven-Fits}

Dieser Anhang enthält die Darstellungen der Anpassungen an die Lichtkurven, die aus den mit den verschiedenen Instrumenten zeitaufgelöst aufgenommenen Spektren gewonnen wurden. Die Konstruktion der Lichtkurven aus den Spektren ist in Kapitel 1.2.7.1 und die generelle Methodik ihrer Modellierung in Kapitel 4.2.7.2 beschrieben. Der Aufbau der Darstellungen und die Bedeutung der in ihnen enthaltenen zusätzlichen Informationen ist Kapitel 4.2.7.3 erläutert. Die Gründe für die Auswahl der jeweils verwendeten Modellkomponenten für die verschiedenen Lichtkurventypen sind in Kapitel 4.2.7.4 dargelegt.

Die einzelnen Abschnitte dieses Anhangs enthalten am Anfang jeweils eine Tabelle, in denen die Modellparameter für die auf den darauf folgenden Seiten dargestellten Anpassungen zusammengefaßt sind. Um die zwei Darstellungsformen jeder Anpassung im Sinne einer besseren Überschaubarkeit jeweils auf gegenüberliegenden Seiten präsentieren zu können, bleiben einzelne Seiten in diesem Anhang absichtlich leer.

\section{A.1 HST-Lichtkurven}

\section{A.1.1 HST-Lichtkurven: Kontinuumsflüsse}

Tabelle A.1: Übersicht über die Anpassungen an die HST-Kontinuumslichtkurven: Definition der Bänder, best-fit-Amplituden, mittlerer Fluß und Modulationsgrad. $A_{67}$ und 〈base sind in Einheiten von $10^{-12} \mathrm{erg} \mathrm{cm}^{-2} \mathrm{~s}^{-1} \AA^{-1}$ angegeben.

\begin{tabular}{|c|c|c|c|c|c|c|c|}
\hline Nr. & $\lambda \lambda(\AA)$ & $\lambda_{\text {cen }}(\AA)$ & \multicolumn{2}{|c|}{ Abbildungen } & $N_{\text {data }}$ & \multicolumn{2}{|c|}{ Bemerkungen } \\
\hline 1 & $1255-1285$ & 1270 & \multicolumn{2}{|c|}{ A.1, A.2 } & 112 & & \\
\hline 2 & $1350-1365$ & 1357.5 & \multirow{2}{*}{\multicolumn{2}{|c|}{\begin{tabular}{|l|l|} 
A.3. \\
A.5.4 \\
A.6 \\
\end{tabular}}} & 112 & & \\
\hline 3 & $1350-1380$ & 1365 & & & 112 & & \\
\hline 4 & $1425-1525$ & 1475 & \multicolumn{2}{|c|}{ A.7, A.8 } & 112 & & \\
\hline 5 & $1570-1600$ & 1585 & \multicolumn{2}{|c|}{ A.9, A.10 } & 112 & & \\
\hline 6 & $1250-1380$ & 1315 & \multicolumn{2}{|c|}{ A.11, A.12 } & 112 & \\
\hline 7 & $1230-1600$ & 1415 & A.13, $\mathrm{A}$ & .14 & 112 & \multicolumn{2}{|c|}{ enthält Fmissionslinien } \\
\hline & $A_{67}$ & & $\Delta_{\Phi 67}$ & $\chi_{\mathrm{red}}^{2}$ & $\langle$ base $\rangle$ & $A_{67} /\langle$ base $\rangle$ & $\bmod _{67}$ \\
\hline 1 & $0.0319=$ & 0.0024 & -0.010 & 1.36 & 0.148 & 0.216 & 0.355 \\
\hline 2 & $0.0276=$ & $=0.0022$ & +0.005 & 1.05 & 0.159 & 0.174 & 0.296 \\
\hline 3 & $0.0304=$ & $=0.0023$ & +0.005 & 1.02 & 0.163 & 0.186 & 0.314 \\
\hline 4 & $0.0221=$ & $=0.0019$ & +0.005 & 1.30 & 0.150 & 0.147 & 0.256 \\
\hline 5 & $0.0261=$ & $=0.0017$ & +0.015 & 1.29 & 0.136 & 0.193 & 0.323 \\
\hline 6 & $0.0401=$ & $=0.0024$ & +0.005 & 1.03 & 0.170 & 0.236 & 0.381 \\
\hline 7 & $0.0453=$ & $=0.0023$ & +0.005 & 0.92 & 0.178 & 0.254 & 0.405 \\
\hline
\end{tabular}



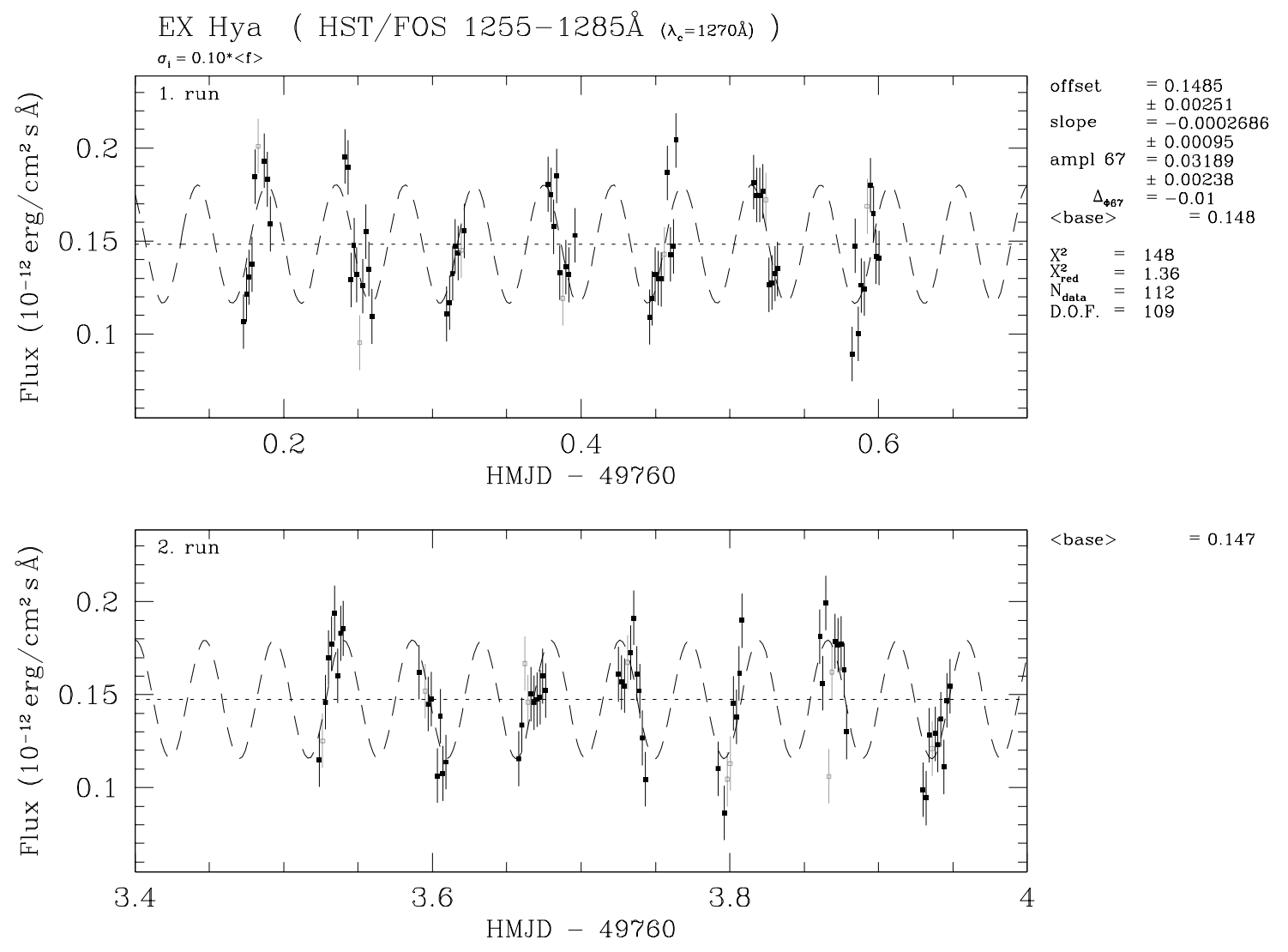

Abbildung A.1: Fit an HST-Lichtkurve 1255...1285 $\AA\left(\lambda_{\text {cen }}=1270 \AA\right)$;

Modell: $\left\langle f_{\lambda}\right\rangle_{\text {band }}=$ base $+A_{67} \cdot \cos \left(\Phi_{67}-\Delta_{\Phi 67}\right) \quad$ mit $\quad$ base $\equiv C+B \cdot($ HMJD -49760$)$. 


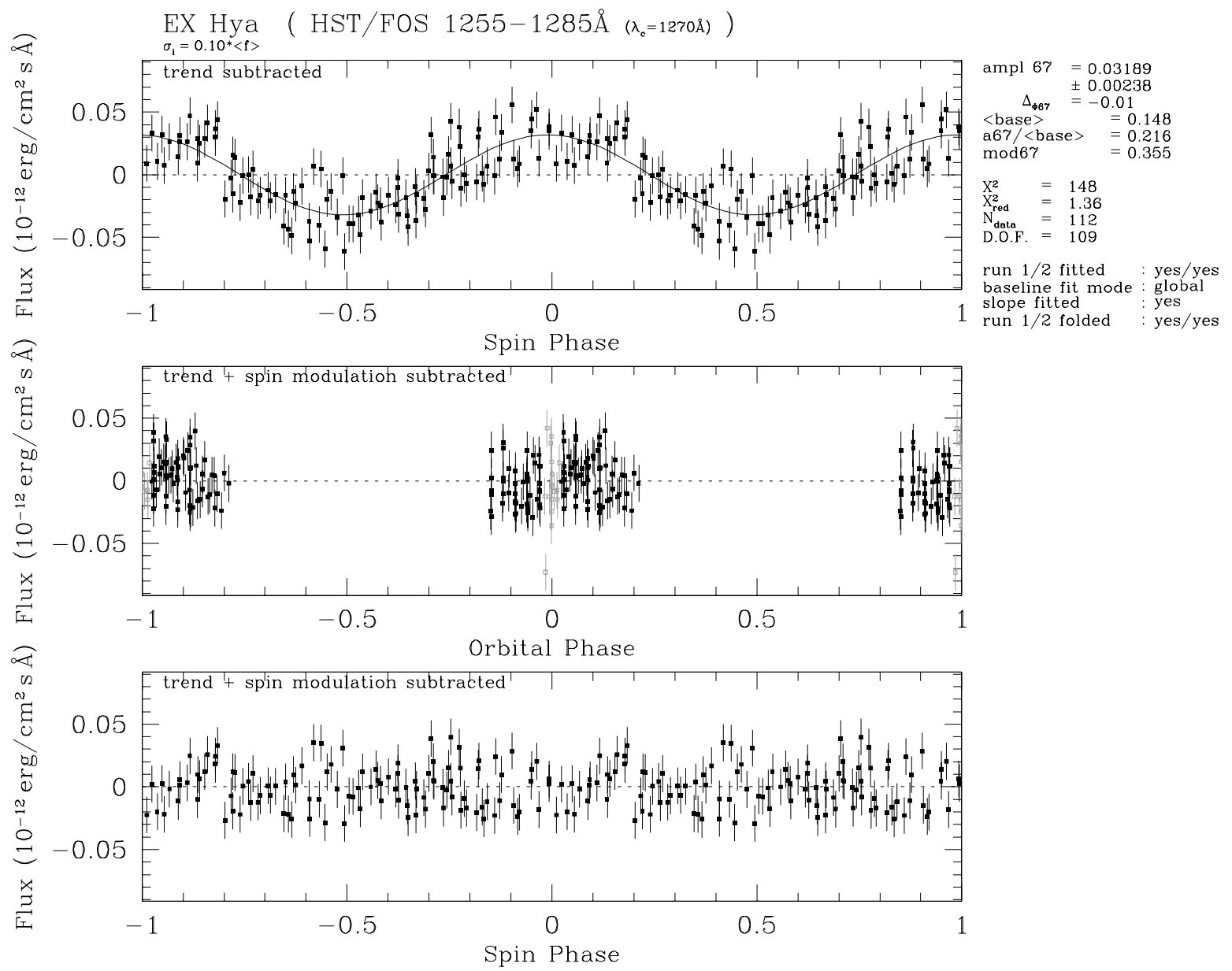

Abbildung A.2: Fit aus Abbildung A.1 über $\Phi_{67}$ bzw. $\Phi_{98}$ gefaltet, lineare Komponente abgezogen. 

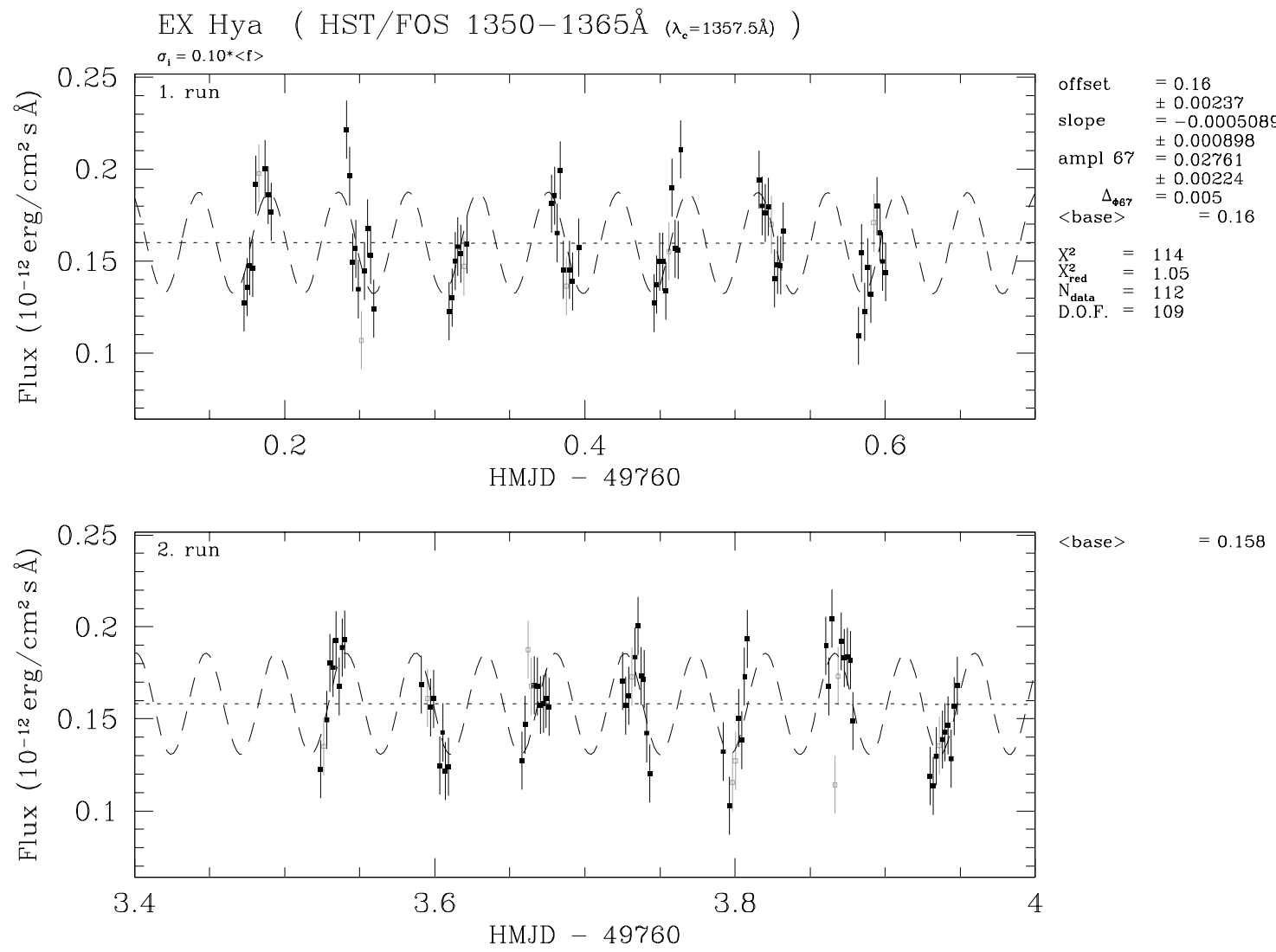

$<$ base $>\quad=0.158$

Abbildung A.3: Fit an HST-Lichtkurve $1350 \ldots 1365 \AA\left(\lambda_{\text {cen }}=1357 \AA\right)$;

Modell: $\left\langle f_{\lambda}\right\rangle_{\text {band }}=$ base $+A_{67} \cdot \cos \left(\Phi_{67}-\Delta_{\Phi 67}\right) \quad$ mit $\quad$ base $\equiv C+B \cdot($ HMJD -49760$)$. 


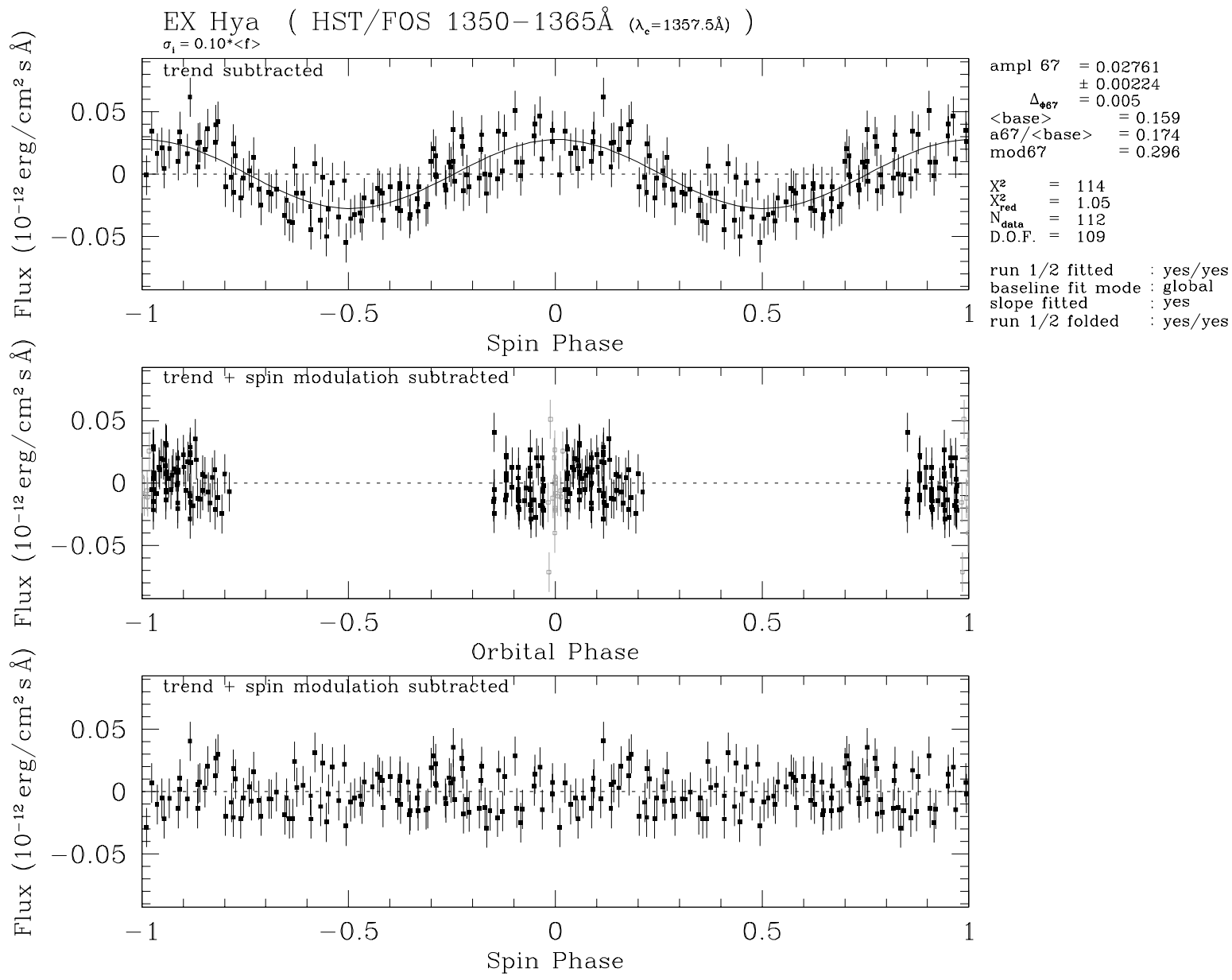

Abbildung A.4: Fit aus Abbildung A.3 über $\Phi_{67}$ bzw. $\Phi_{98}$ gefaltet, lineare Komponente abgezogen. 

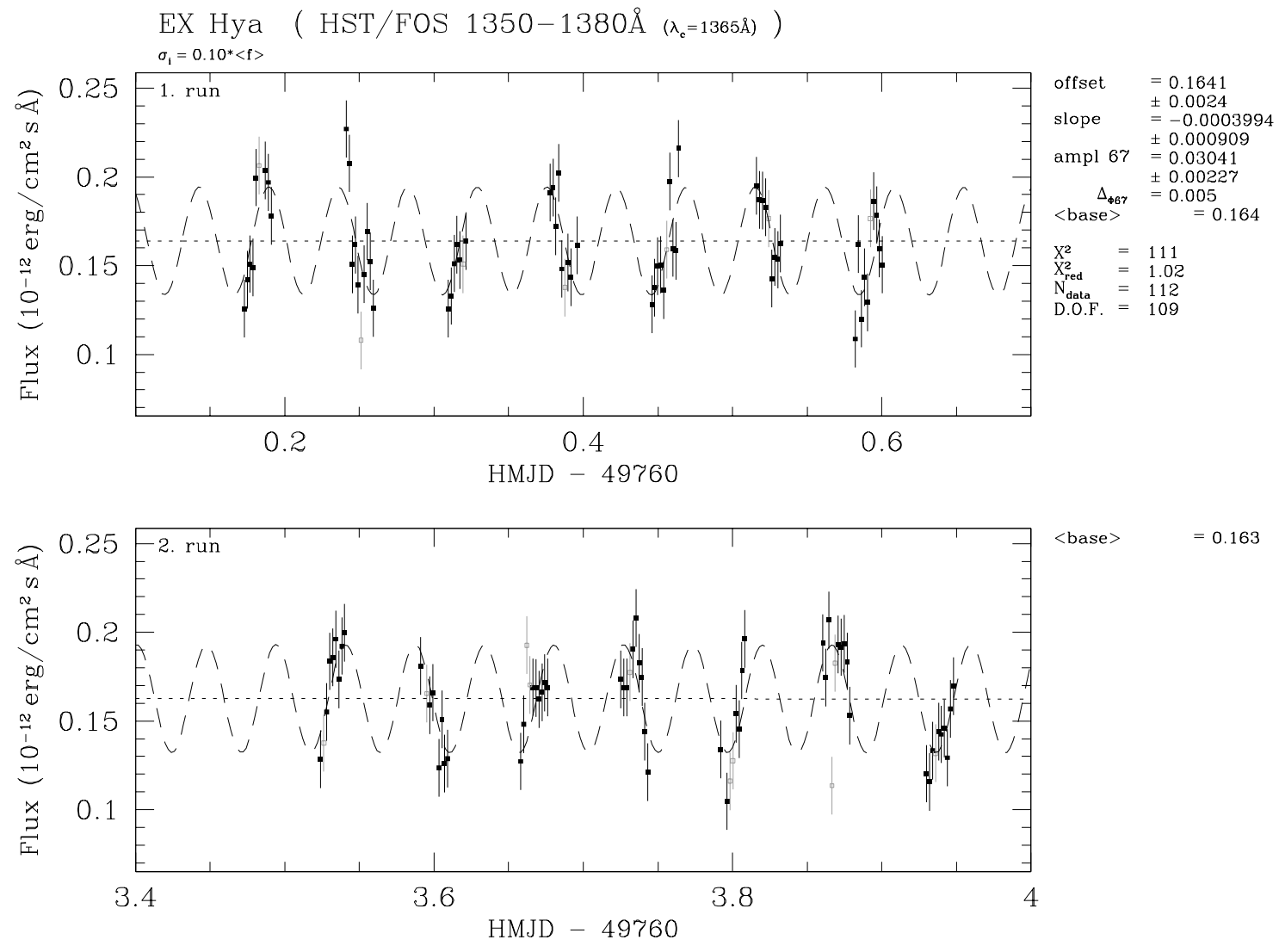

<base $>\quad=0.163$

Abbildung A.5: Fit an HST-Lichtkurve $1350 \ldots 1380 \AA\left(\lambda_{\text {cen }}=1365 \AA\right)$;

Modell: $\left\langle f_{\lambda}\right\rangle_{\text {band }}=$ base $+A_{67} \cdot \cos \left(\Phi_{67}-\Delta_{\Phi 67}\right) \quad$ mit $\quad$ base $\equiv C+B \cdot($ HMJD -49760$)$. 


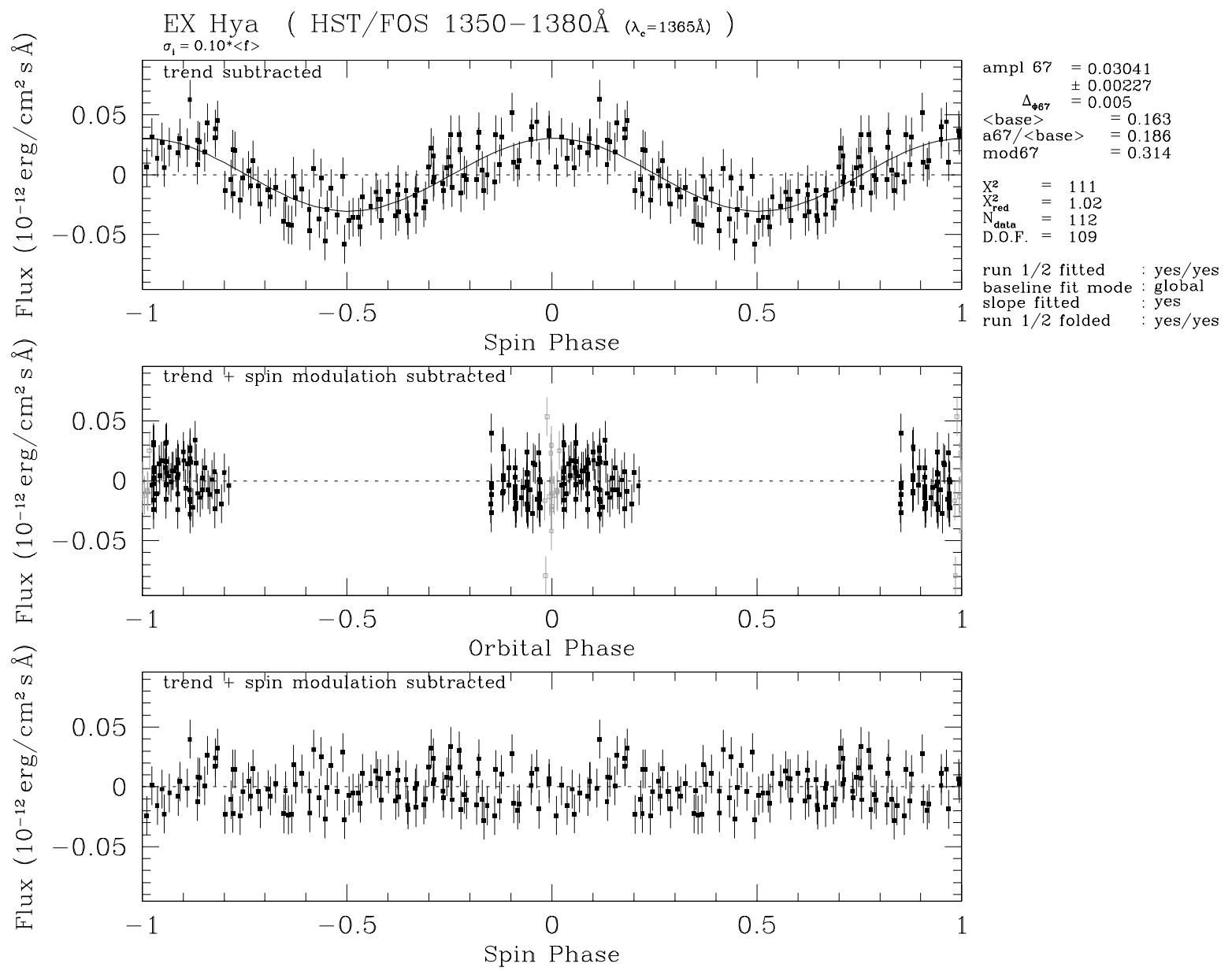

Abbildung A.6: Fit aus Abbildung $A .5$ über $\Phi_{67}$ bzw. $\Phi_{98}$ gefaltet, lineare Komponente abgezogen. 

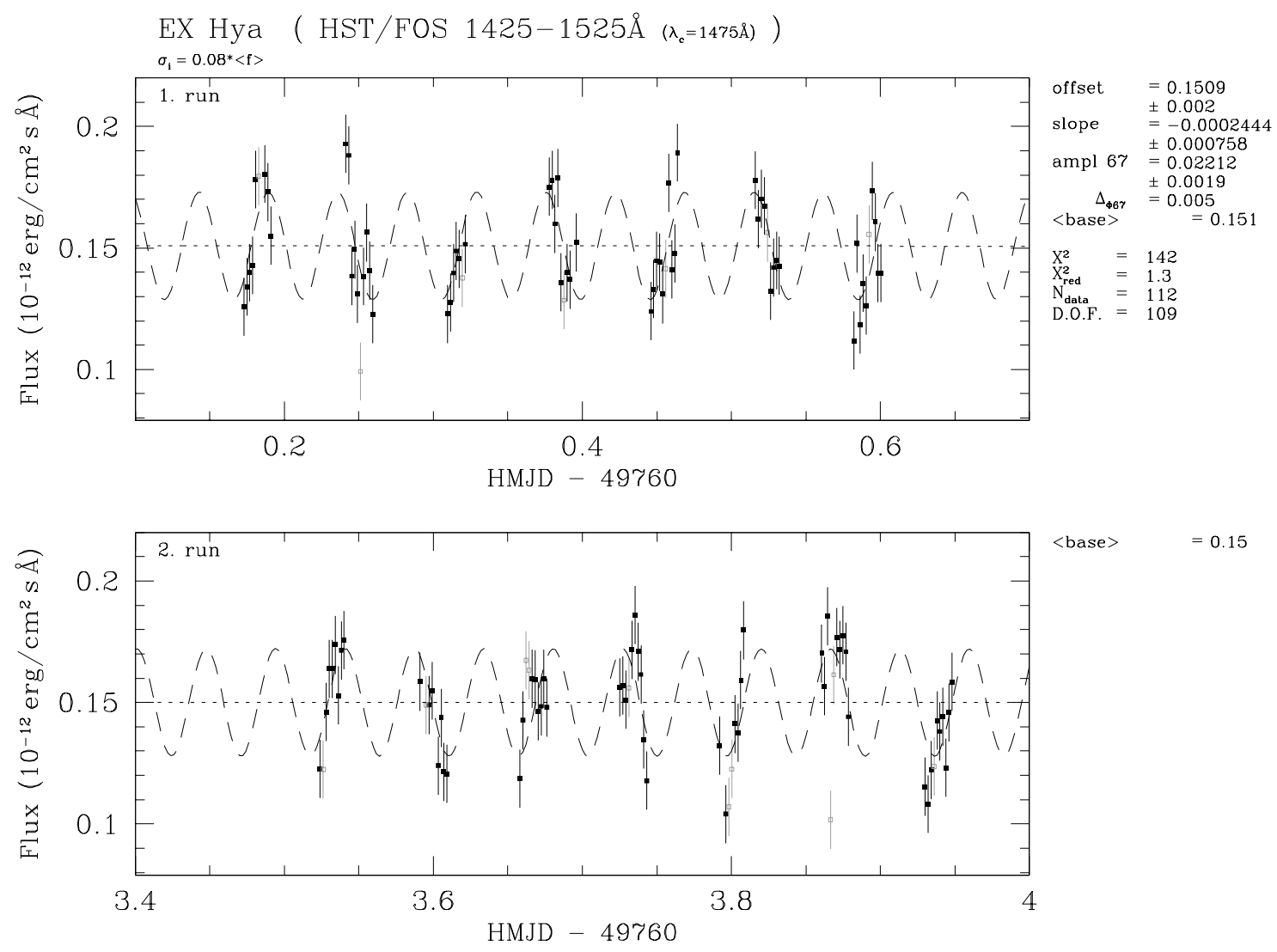

Abbildung A.7: Fit an HST-Lichtkurve 1425...1525 $\AA\left(\lambda_{\text {cen }}=1475 \AA\right)$;

Modell: $\left\langle f_{\lambda}\right\rangle_{\text {band }}=$ base $+A_{67} \cdot \cos \left(\Phi_{67}-\Delta_{\Phi 67}\right) \quad$ mit $\quad$ base $\equiv C+B \cdot($ HMJD -49760$)$. 


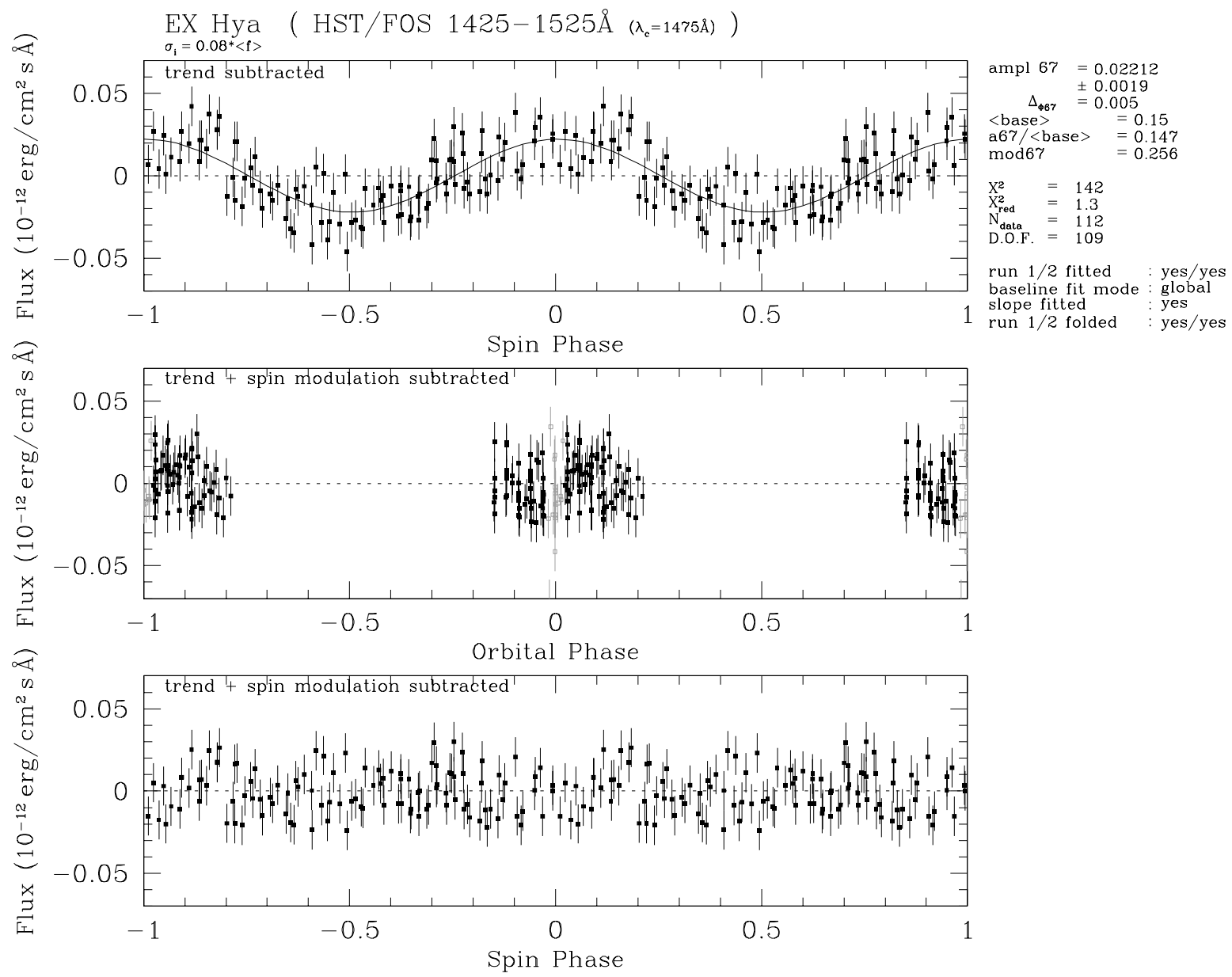

Abbildung A.8: Fit aus Abbildung A.7 über $\Phi_{67}$ bzw. $\Phi_{98}$ gefaltet, lineare Komponente abgezogen. 

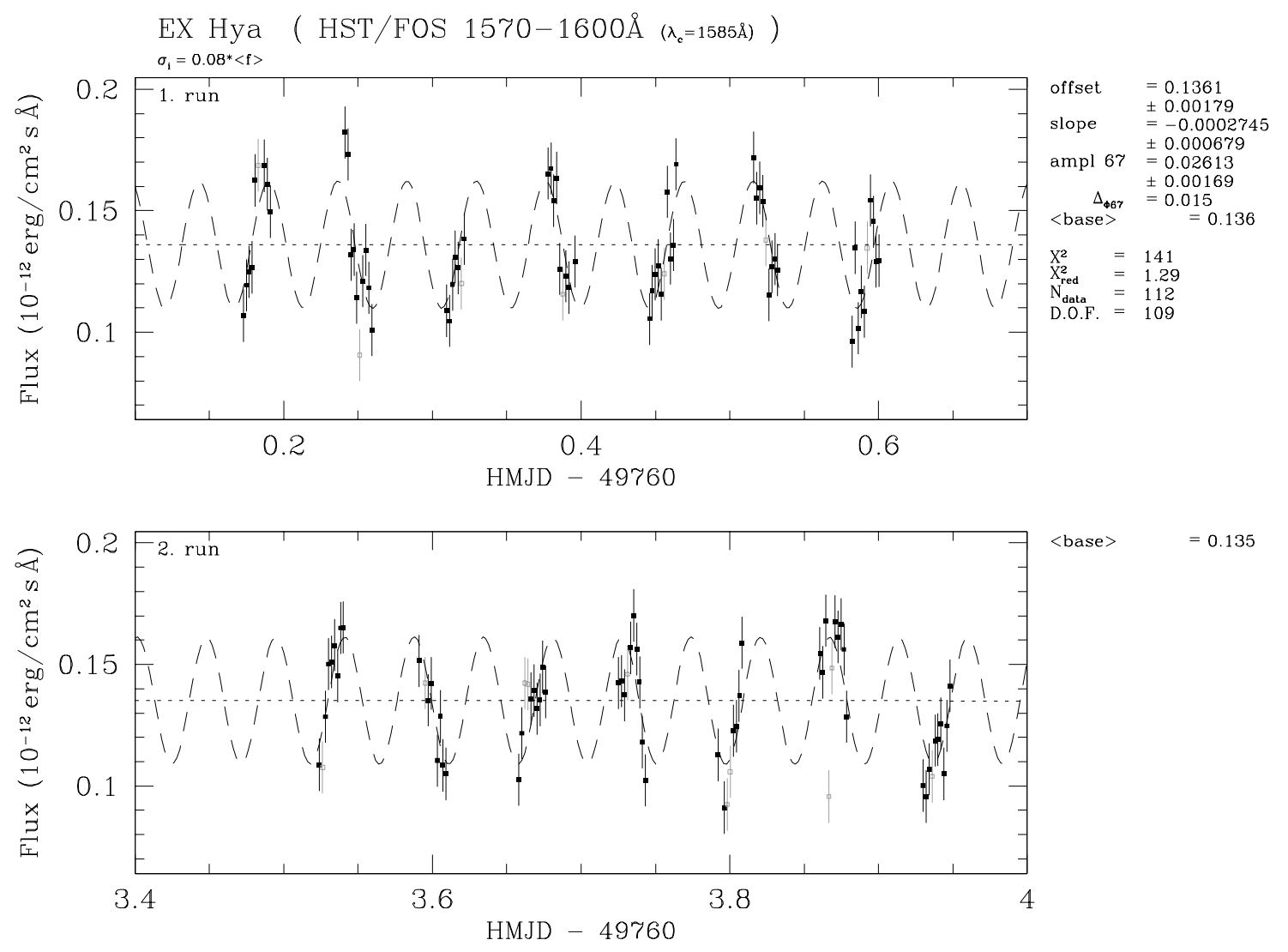

Abbildung A.9: Fit an HST-Lichtkurve 1570...1600 $\AA\left(\lambda_{\text {cen }}=1585 \AA\right)$;

Modell: $\left\langle f_{\lambda}\right\rangle_{\text {band }}=$ base $+A_{67} \cdot \cos \left(\Phi_{67}-\Delta_{\Phi 67}\right) \quad$ mit $\quad$ base $\equiv C+B \cdot($ HMJD -49760$)$. 


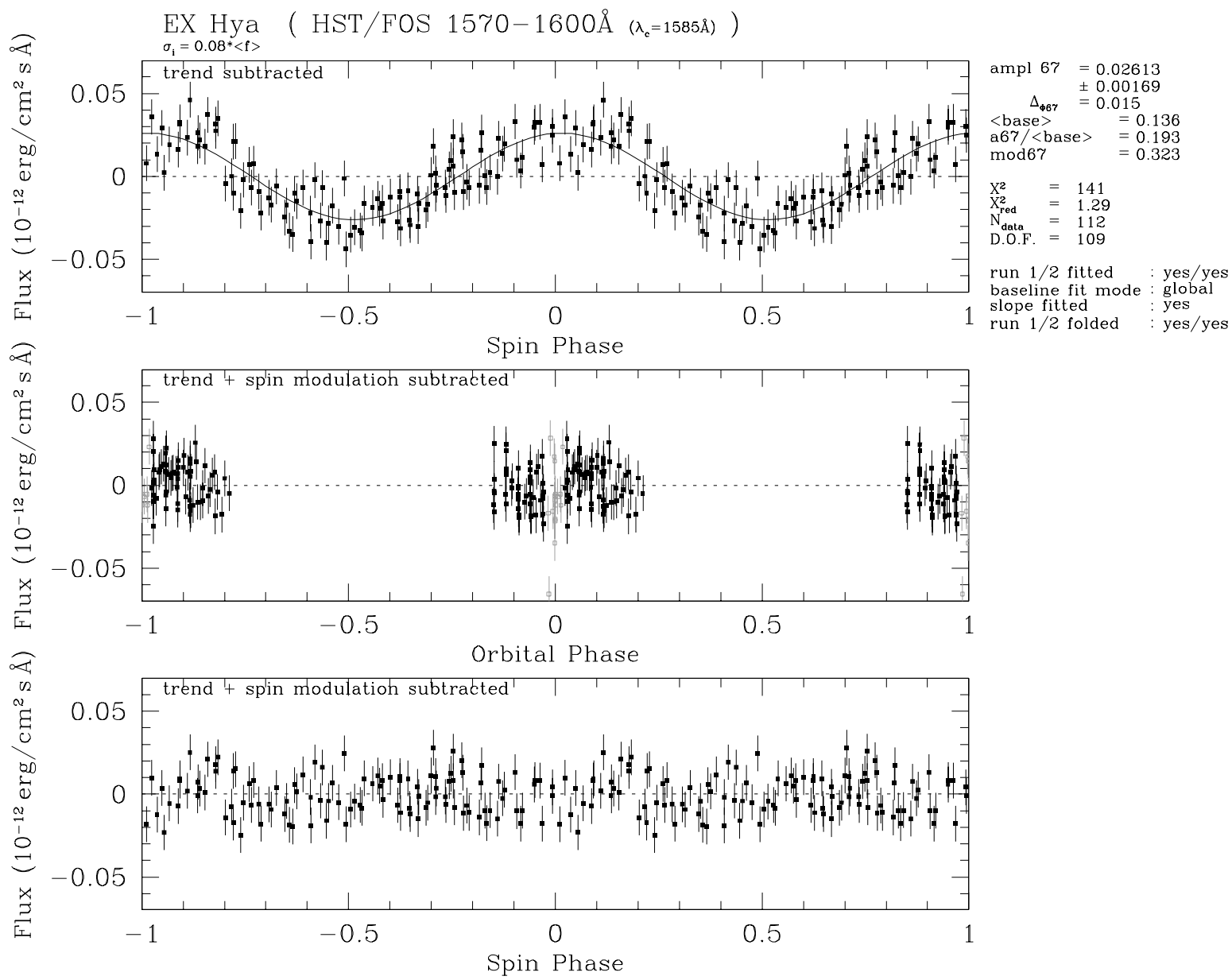

Abbildung A.10: Fit aus Abbildung A.9 über $\Phi_{67}$ bzw. $\Phi_{98}$ gefaltet, lineare Komponente abgezogen. 

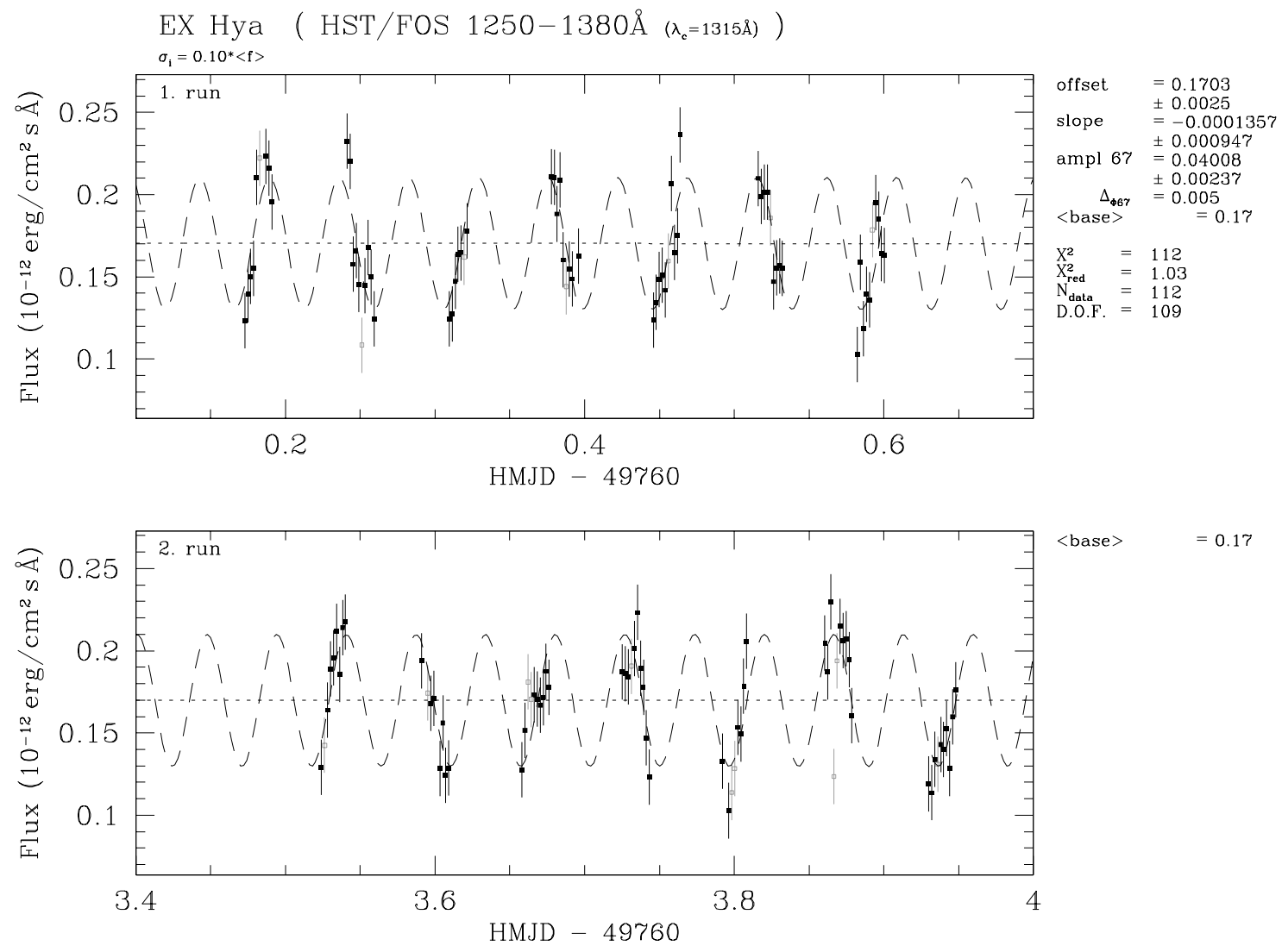

<base $>\quad=0.17$

Abbildung A.11: Fit an HST-Lichtkurve $1250 \ldots 1380 \AA\left(\lambda_{\text {cen }}=1315 \AA\right)$;

Modell: $\left\langle f_{\lambda}\right\rangle_{\text {band }}=$ base $+A_{67} \cdot \cos \left(\Phi_{67}-\Delta_{\Phi 67}\right) \quad$ mit $\quad$ base $\equiv C+B \cdot($ HMJD -49760$)$. 


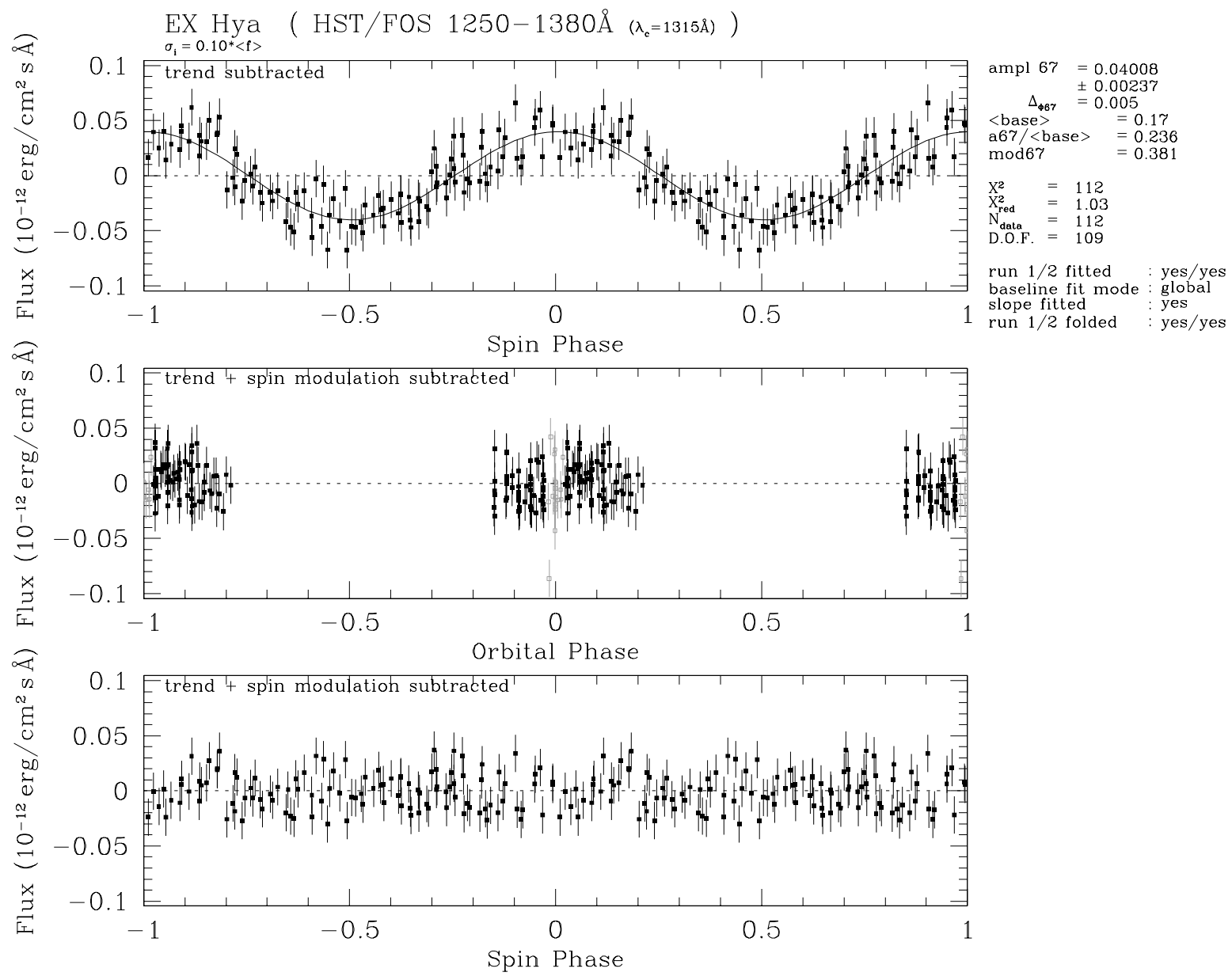

Abbildung A.12: Fit aus Abbildung A.11 über $\Phi_{67}$ bzw. $\Phi_{98}$ gefaltet, lineare Komponente abgezogen. 

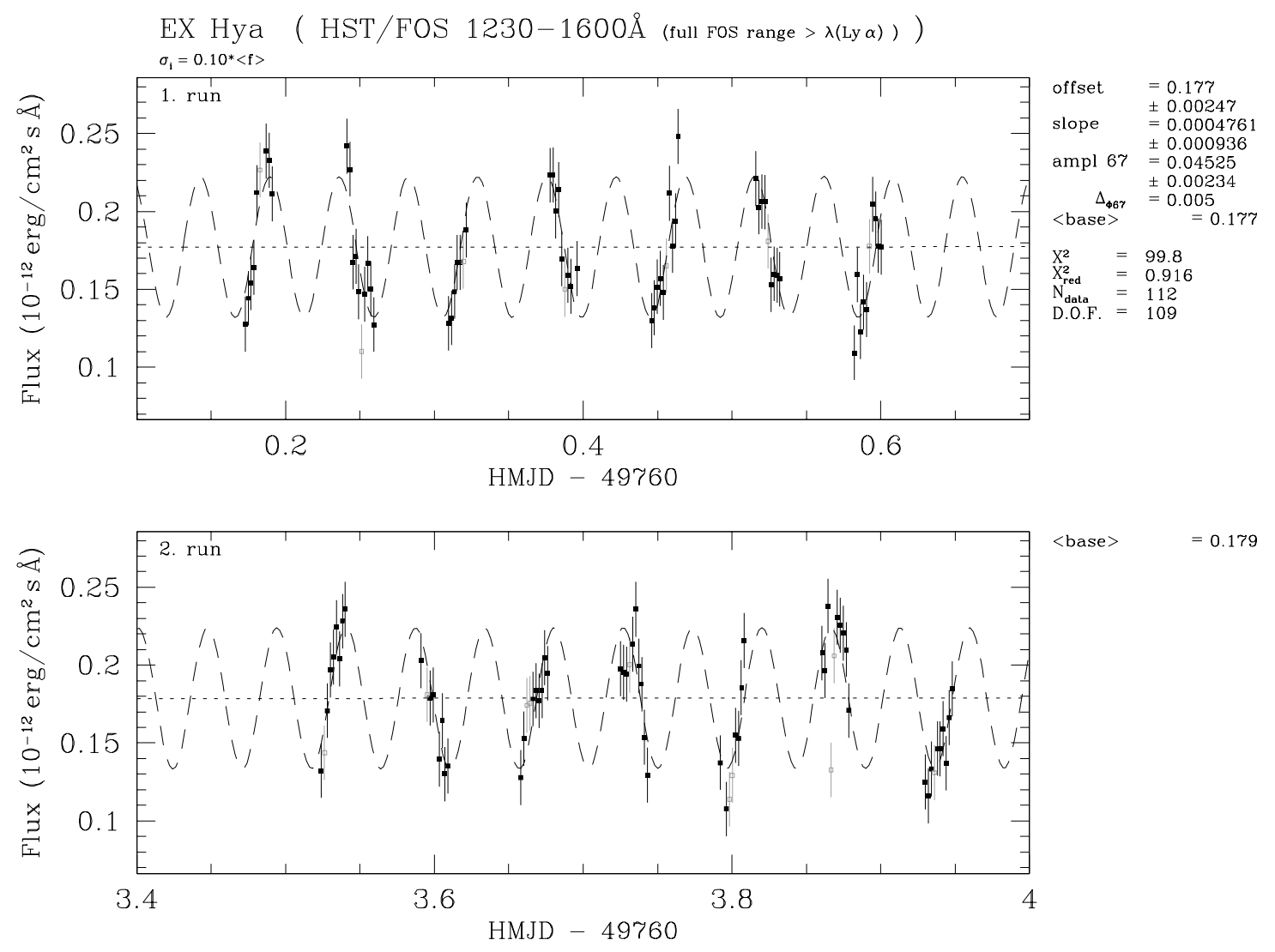

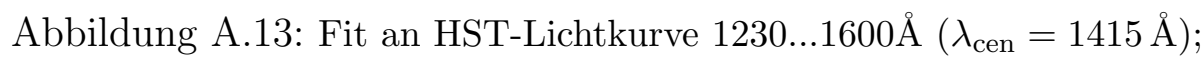

Modell: $\left\langle f_{\lambda}\right\rangle_{\text {band }}=$ base $+A_{67} \cdot \cos \left(\Phi_{67}-\Delta_{\Phi 67}\right) \quad$ mit $\quad$ base $\equiv C+B \cdot($ HMJD -49760$)$. 


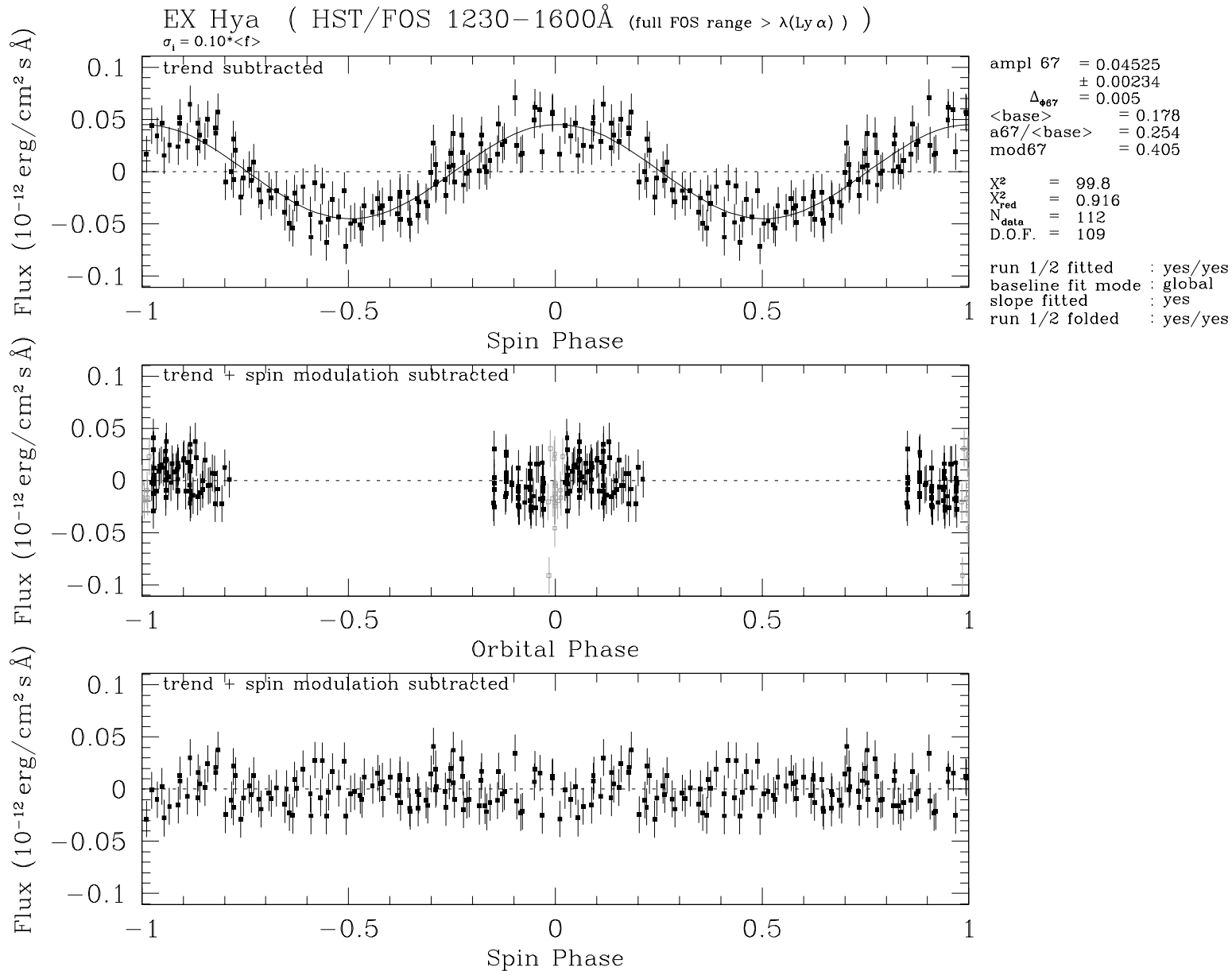

Abbildung A.14: Fit aus Abbildung A.13 über $\Phi_{67}$ bzw. $\Phi_{98}$ gefaltet, lineare Komponente abgezogen. 


\section{A.1.2 HST-Lichtkurven: Linienflüsse}

Tabelle A.2: Übersicht über die Anpassungen an die HST-Emissionslinienlichtkurven: best-fit-Amplituden, mittlerer Fluß und Modulationsgrad. $A_{67}$ und $\langle$ base $\rangle$ sind in Einheiten von $10^{-12} \mathrm{erg} \mathrm{cm}^{-2} \mathrm{~s}^{-1}$ angegeben.

\begin{tabular}{lll|lll}
\hline Nr. & Linie & Abbildungen & $N_{\text {data }}$ & Bemerkungen \\
\hline 1 & C III 1175 & A.15, & A.16 & 50 & $\Phi_{\text {orb }} \geq 0.05$ \\
2 & Ly $\alpha$ & A.17 & A.18 & 50 & $(-$ dito -$)$ \\
3 & N V 1240 & A.19, & A.20 & 50 & $(-$ dito -$)$ \\
4 & Si III 1300 & A.21, & A.22 & 50 & $(-$ dito -$)$ \\
5 & C II 1335 & A.23, & A.24 & 50 & $(-$ dito -$)$ \\
6 & Si IV 1400 & A.25, & A.26 & 50 & $(-$ dito -$)$ \\
7 & C IV 1550 & A.27, & A.28 & 50 & $(-$ dito -$)$ \\
\hline
\end{tabular}

\begin{tabular}{|c|c|c|c|c|c|c|}
\hline Nr. & $A_{67}$ & $\Delta_{\Phi 67}$ & $\chi_{\text {red }}^{2}$ & $\langle$ base $\rangle$ & $A_{67} /\langle$ base $\rangle$ & $\bmod _{67}$ \\
\hline 1 & $1.19 \pm 0.081$ & +0.045 & 1.05 & 1.30 & 0.912 & 0.954 \\
\hline 2 & $1.30 \pm 0.10$ & +0.035 & 0.92 & 2.73 & 0.477 & 0.646 \\
\hline 3 & $0.699 \pm 0.048$ & +0.005 & 0.79 & 1.03 & 0.677 & 0.807 \\
\hline 4 & $0.772 \pm 0.026$ & +0.040 & 0.68 & 0.619 & 1.25 & 1.11 \\
\hline 5 & $0.487 \pm 0.039$ & +0.035 & 0.92 & 0.940 & 0.518 & 0.682 \\
\hline 6 & $1.73 \pm 0.066$ & +0.020 & 0.83 & 2.33 & 0.743 & 0.853 \\
\hline 7 & $3.75 \pm 0.14$ & +0.000 & 0.75 & 5.64 & 0.663 & 0.798 \\
\hline
\end{tabular}



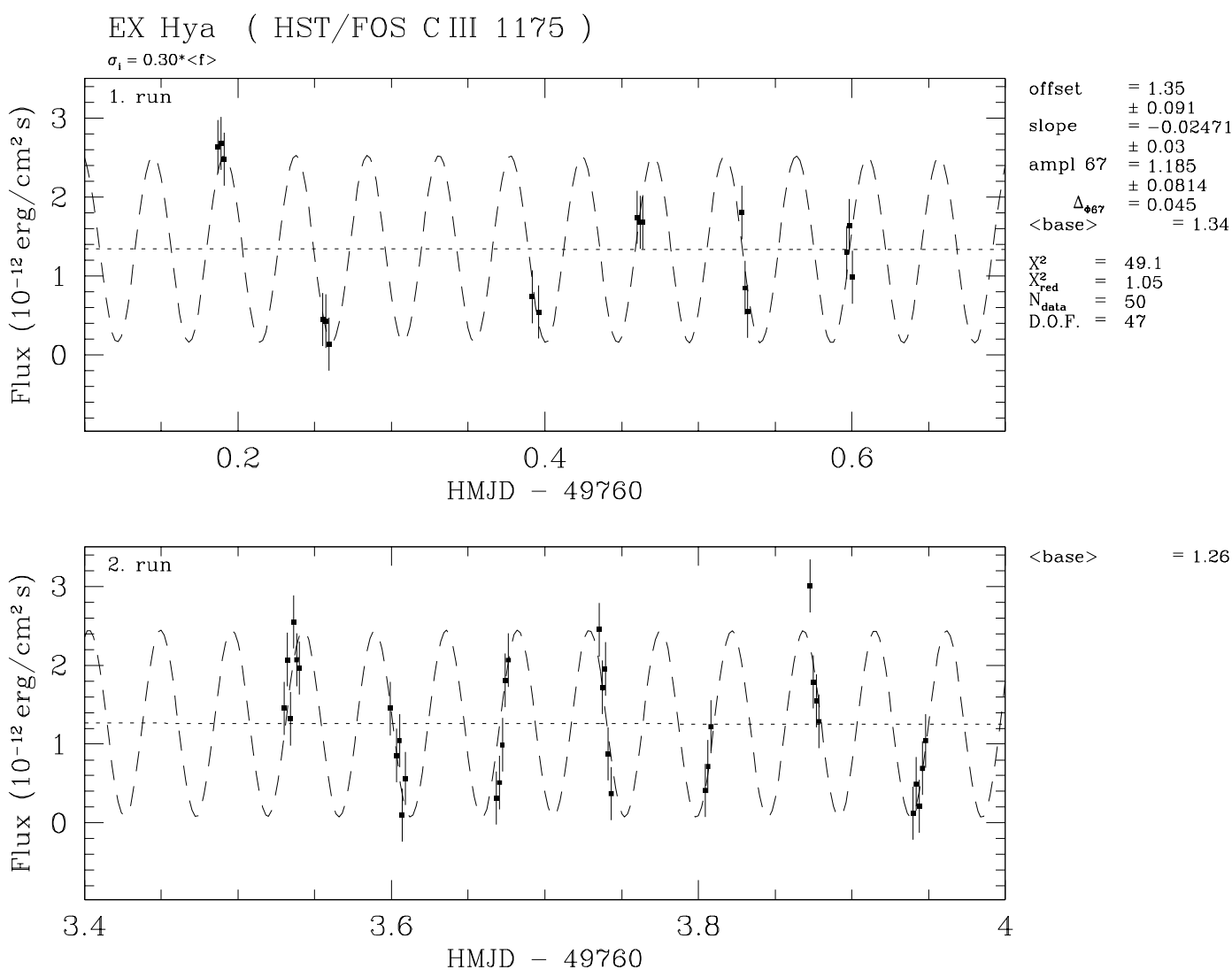

<base $>\quad=1.26$

Abbildung A.15: Fit an HST-Lichtkurve C III 1175;

Modell: $f_{\text {line }}=$ base $+A_{67} \cdot \cos \left(\Phi_{67}-\Delta_{\Phi 67}\right)$ mit base $\equiv C+B \cdot($ HMJD -49760$)$. 


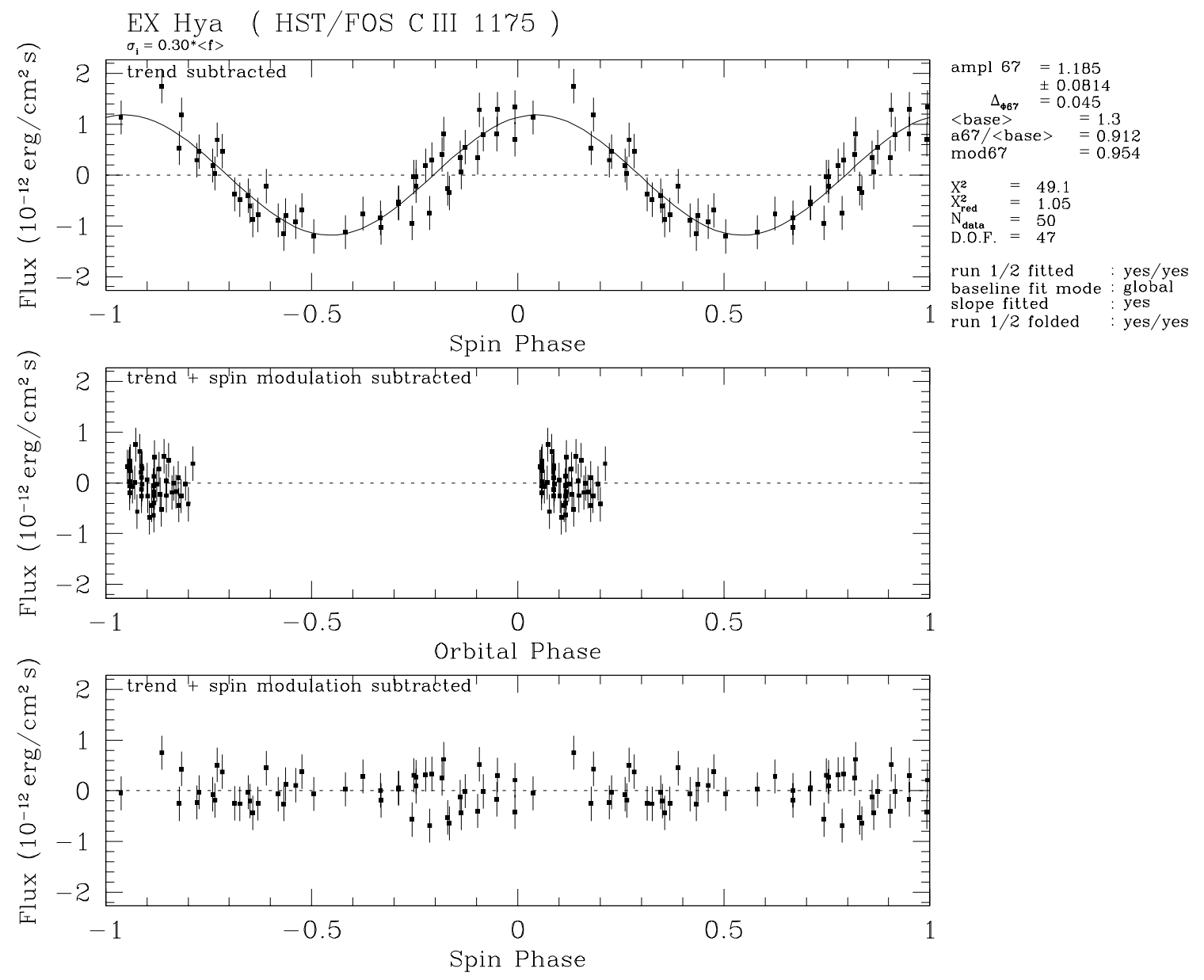

Abbildung A.16: Fit aus Abbildung A.15 über $\Phi_{67}$ bzw. $\Phi_{98}$ gefaltet, lineare Komponente abgezogen. 

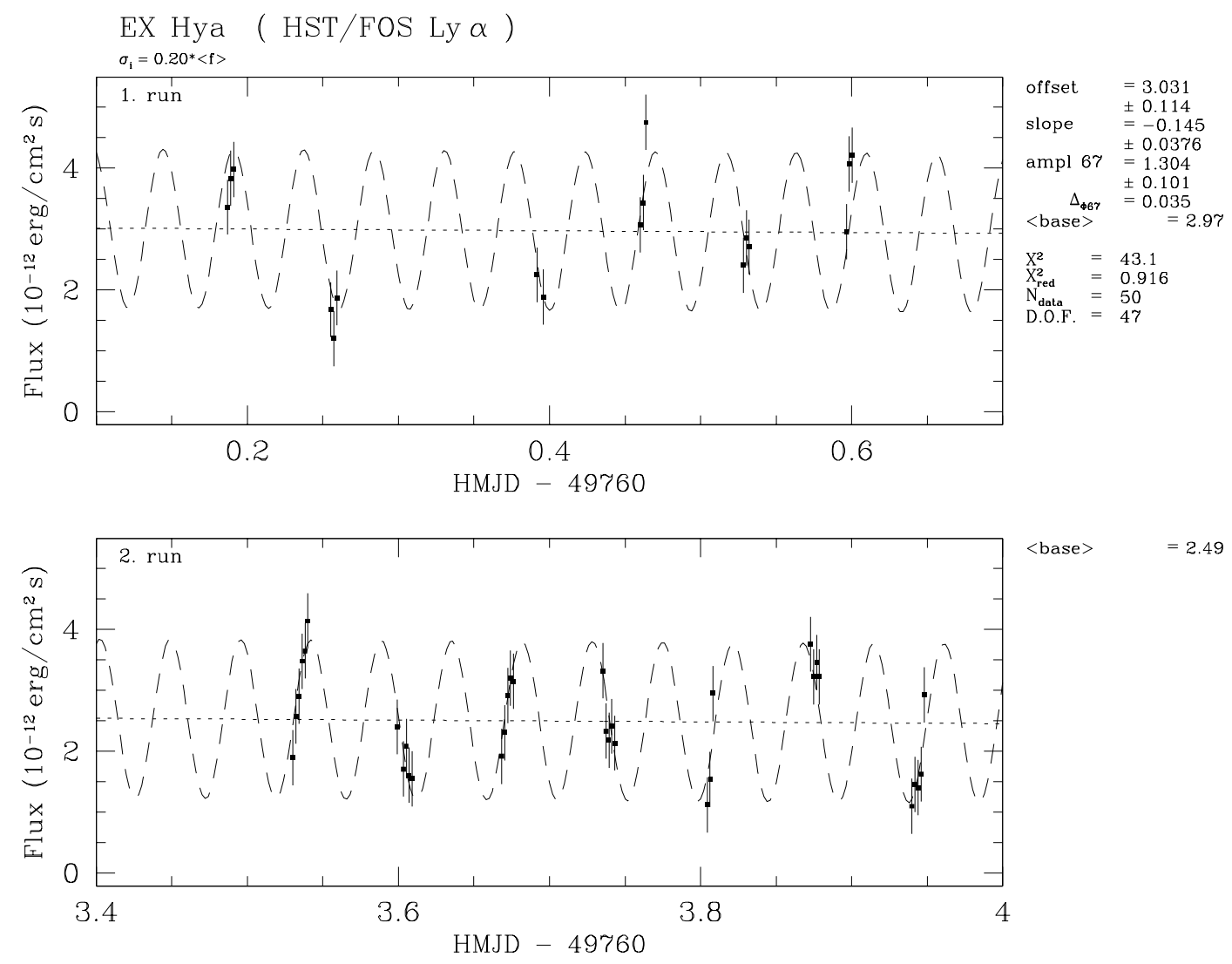

Abbildung A.17: Fit an HST-Lichtkurve Ly $\alpha$;

Modell: $f_{\text {line }}=$ base $+A_{67} \cdot \cos \left(\Phi_{67}-\Delta_{\Phi 67}\right)$ mit base $\equiv C+B \cdot($ HMJD -49760$)$. 


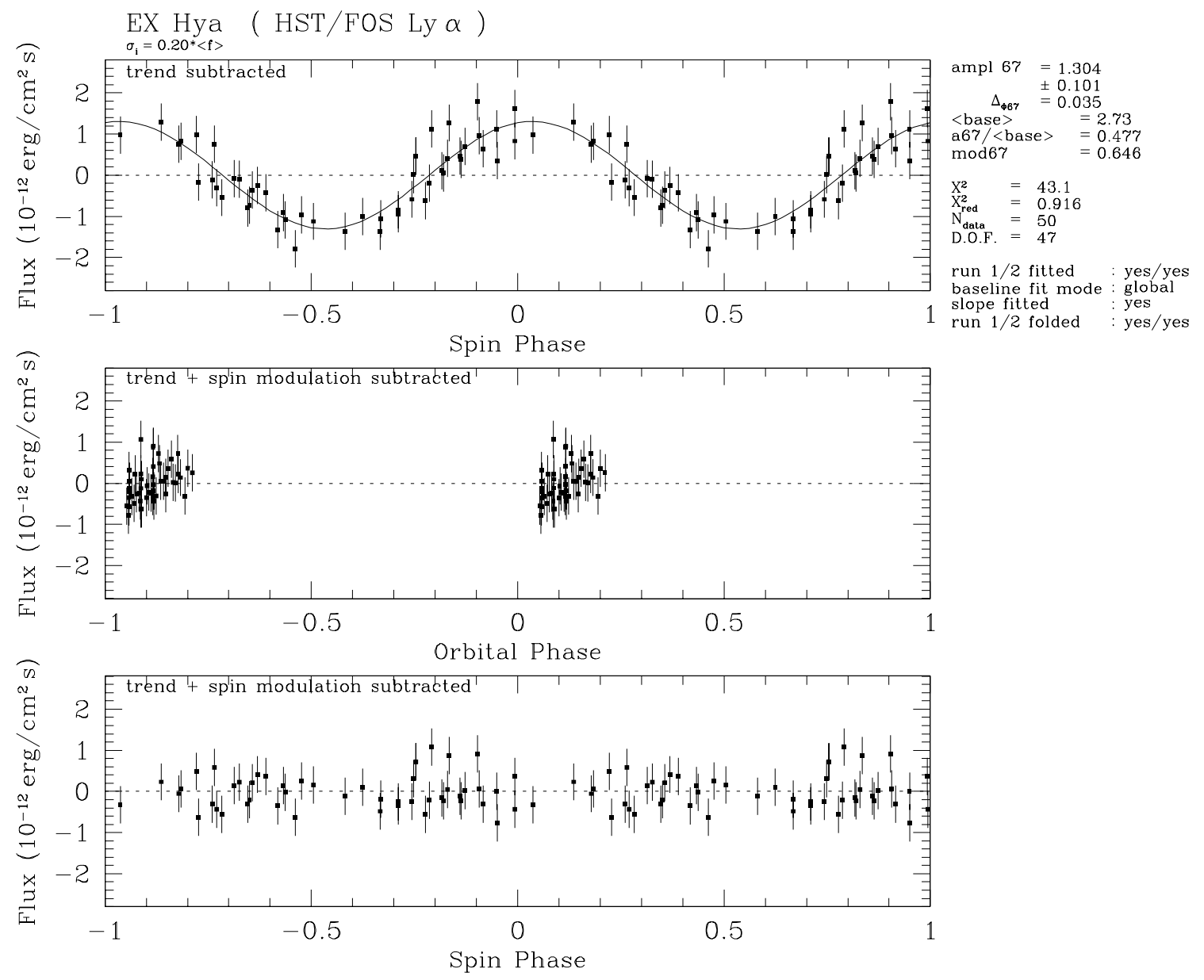

Abbildung A.18: Fit aus Abbildung A.17 über $\Phi_{67}$ bzw. $\Phi_{98}$ gefaltet, lineare Komponente abgezogen. 

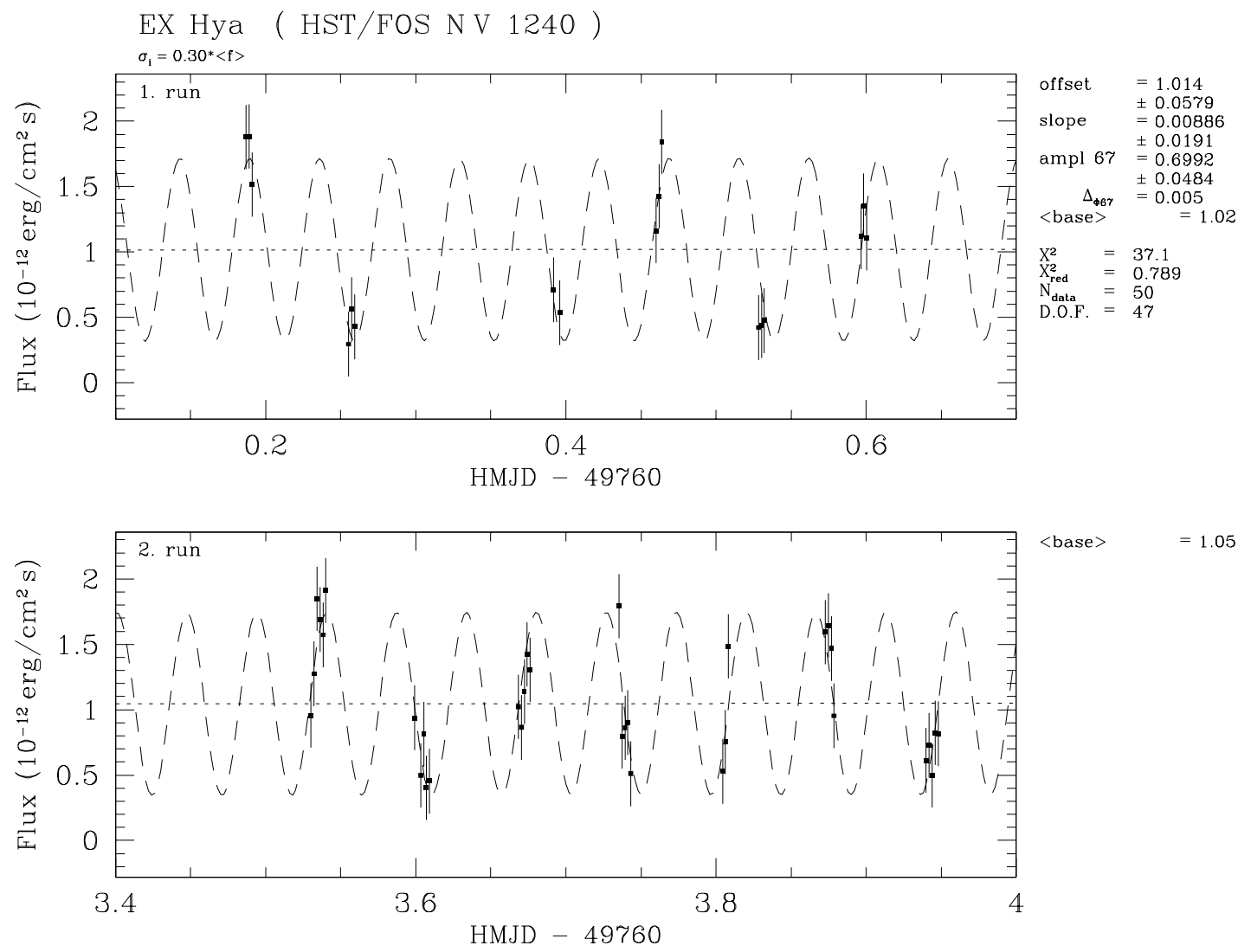

$<$ base $>\quad=1.05$

Abbildung A.19: Fit an HST-Lichtkurve N V 1240;

Modell: $f_{\text {line }}=$ base $+A_{67} \cdot \cos \left(\Phi_{67}-\Delta_{\Phi 67}\right)$ mit base $\equiv C+B \cdot($ HMJD -49760$)$. 


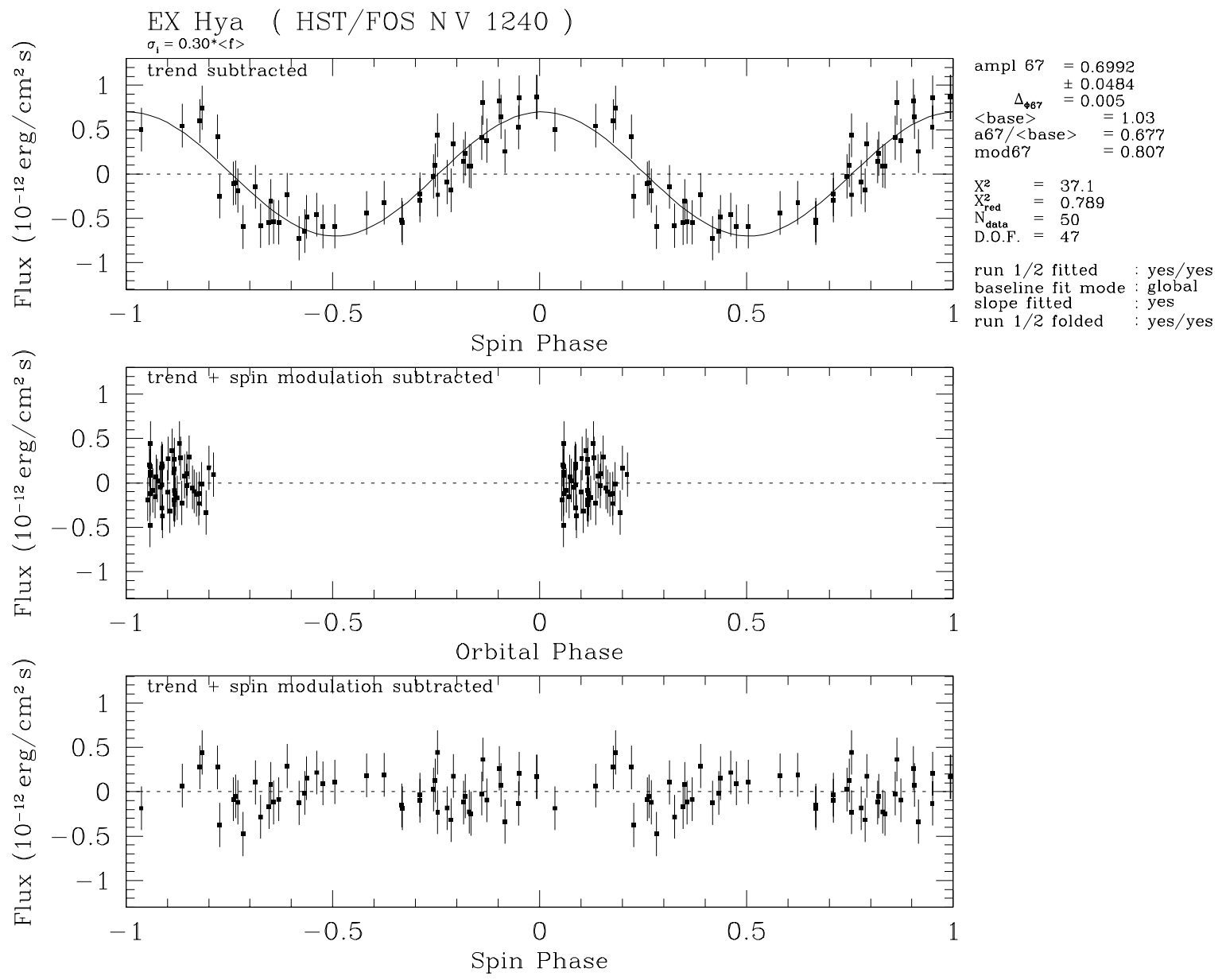

Abbildung A.20: Fit aus Abbildung A.19 über $\Phi_{67}$ bzw. $\Phi_{98}$ gefaltet, lineare Komponente abgezogen. 

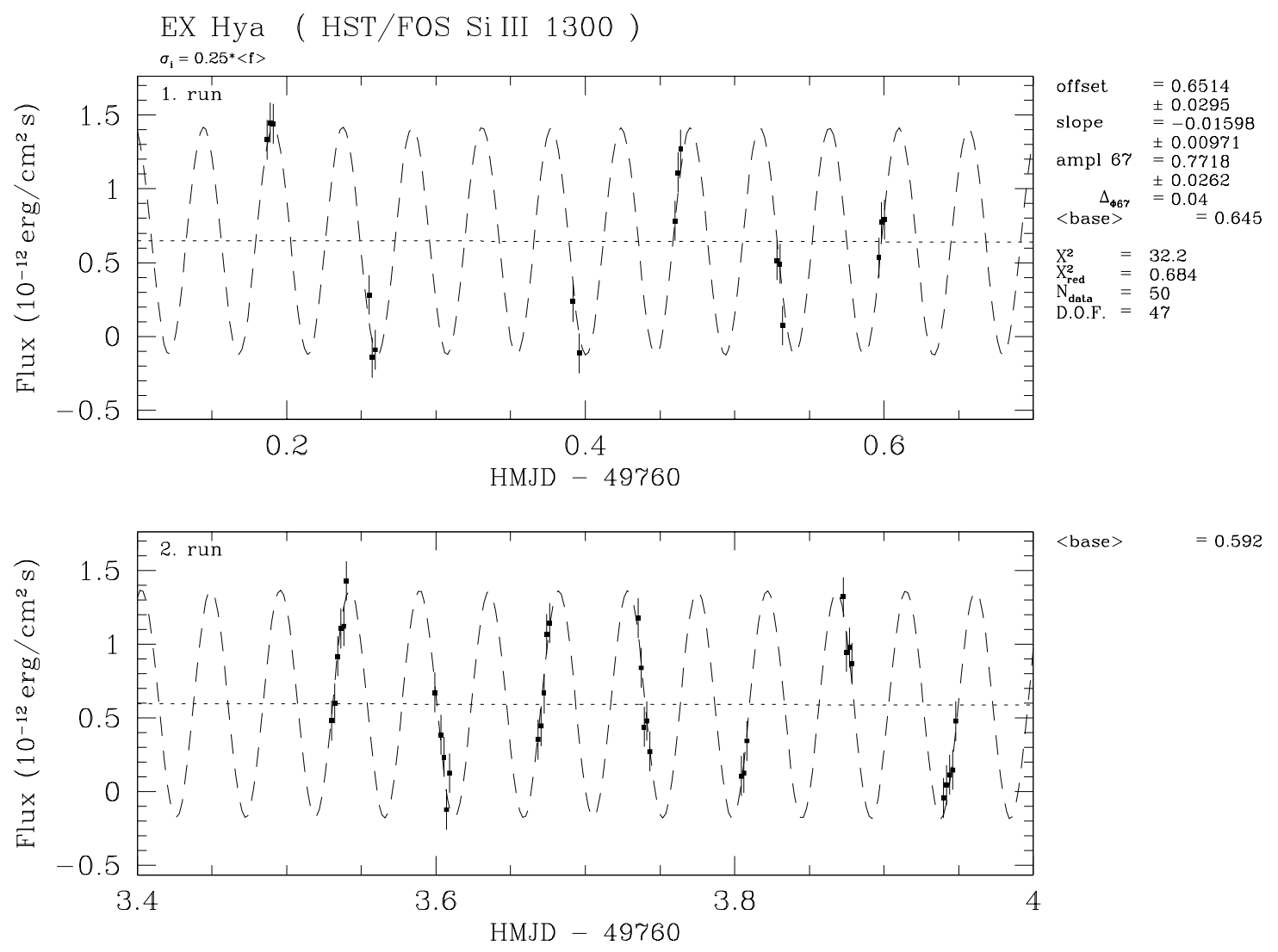

$<$ base $>\quad=0.592$

Abbildung A.21: Fit an HST-Lichtkurve Si III 1300;

Modell: $f_{\text {line }}=$ base $+A_{67} \cdot \cos \left(\Phi_{67}-\Delta_{\Phi 67}\right)$ mit base $\equiv C+B \cdot($ HMJD -49760$)$. 


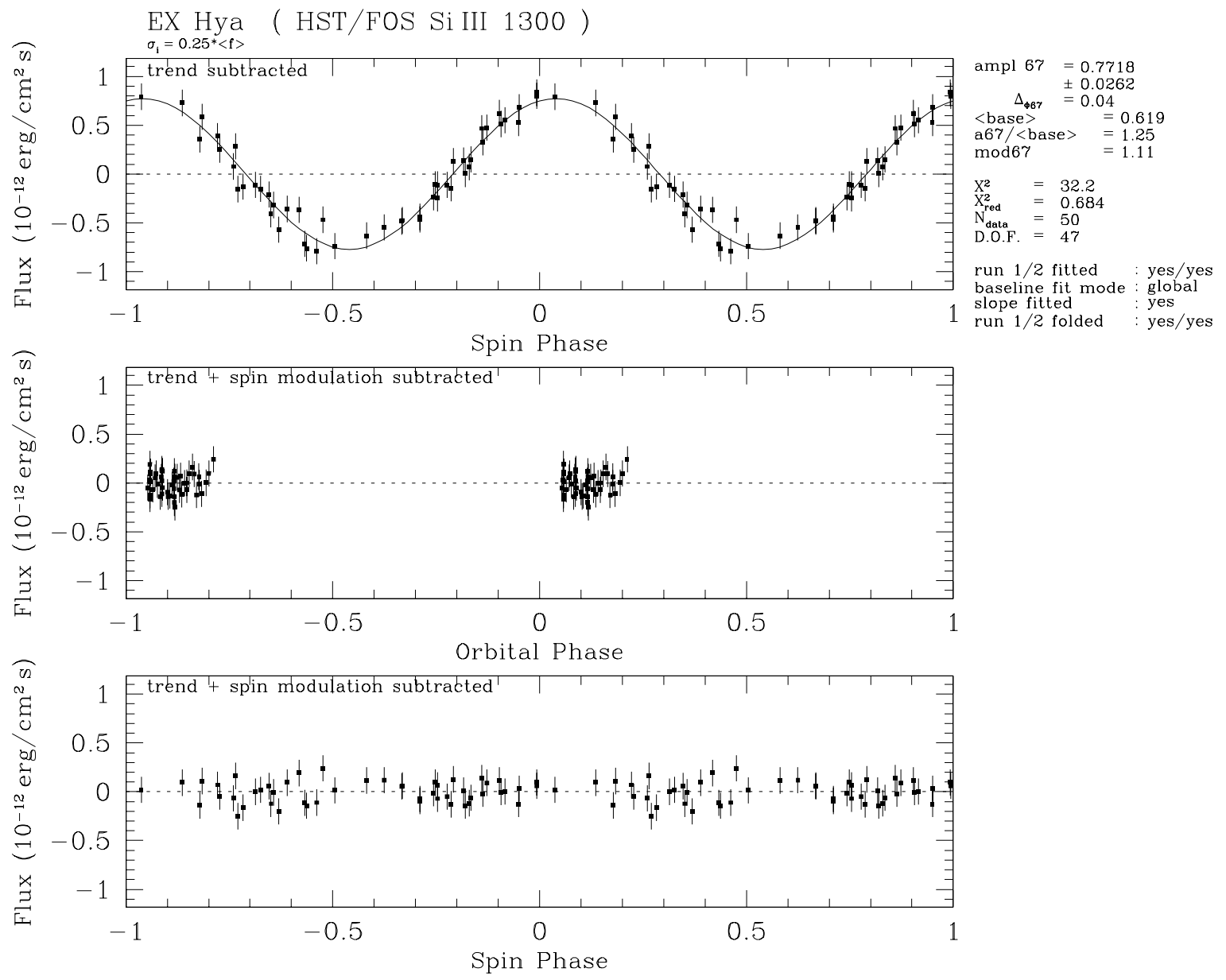

Abbildung A.22: Fit aus Abbildung A.21 über $\Phi_{67}$ bzw. $\Phi_{98}$ gefaltet, lineare Komponente abgezogen. 

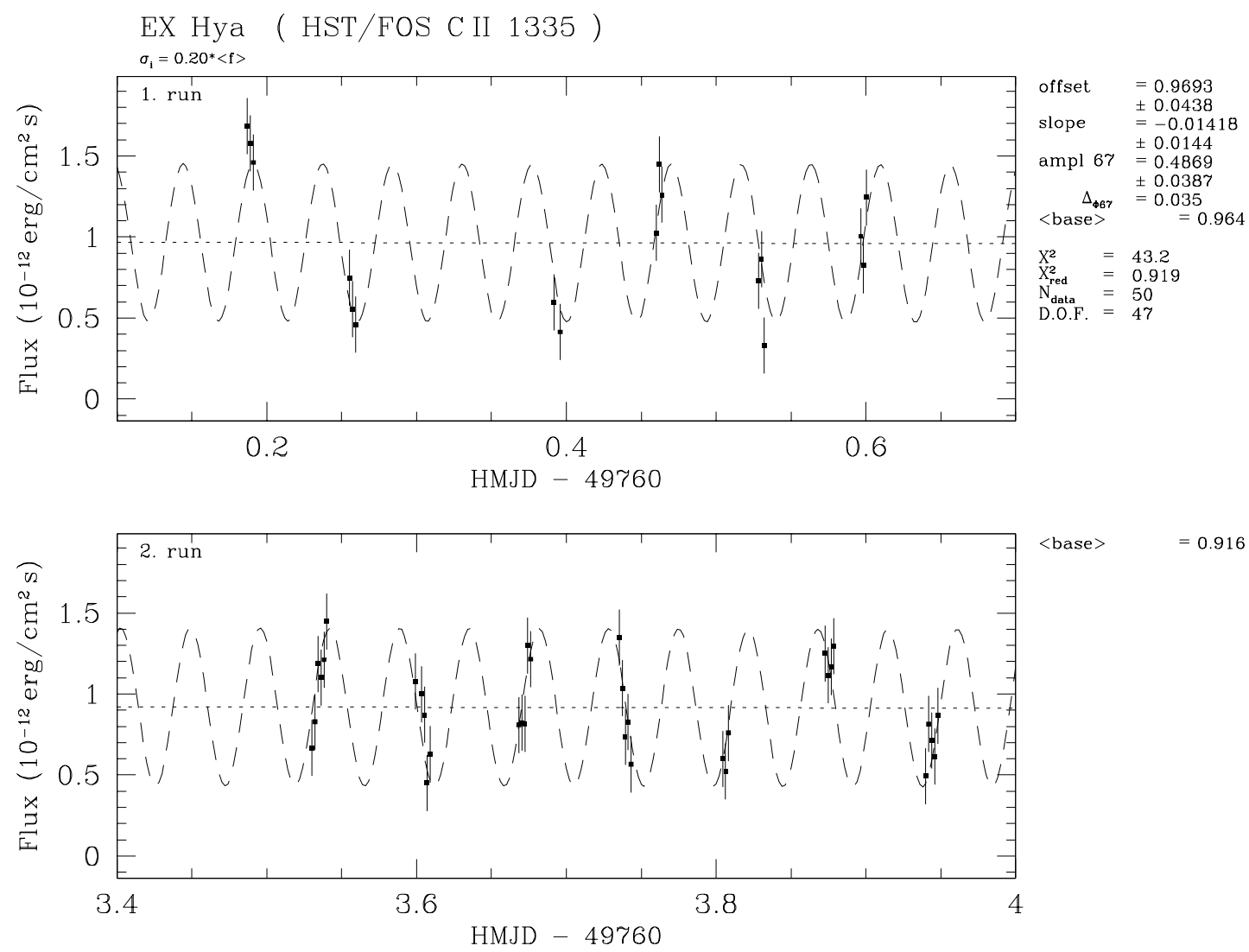

<base $>0.916$

Abbildung A.23: Fit an HST-Lichtkurve C II 1335;

Modell: $f_{\text {line }}=$ base $+A_{67} \cdot \cos \left(\Phi_{67}-\Delta_{\Phi 67}\right)$ mit base $\equiv C+B \cdot($ HMJD -49760$)$. 


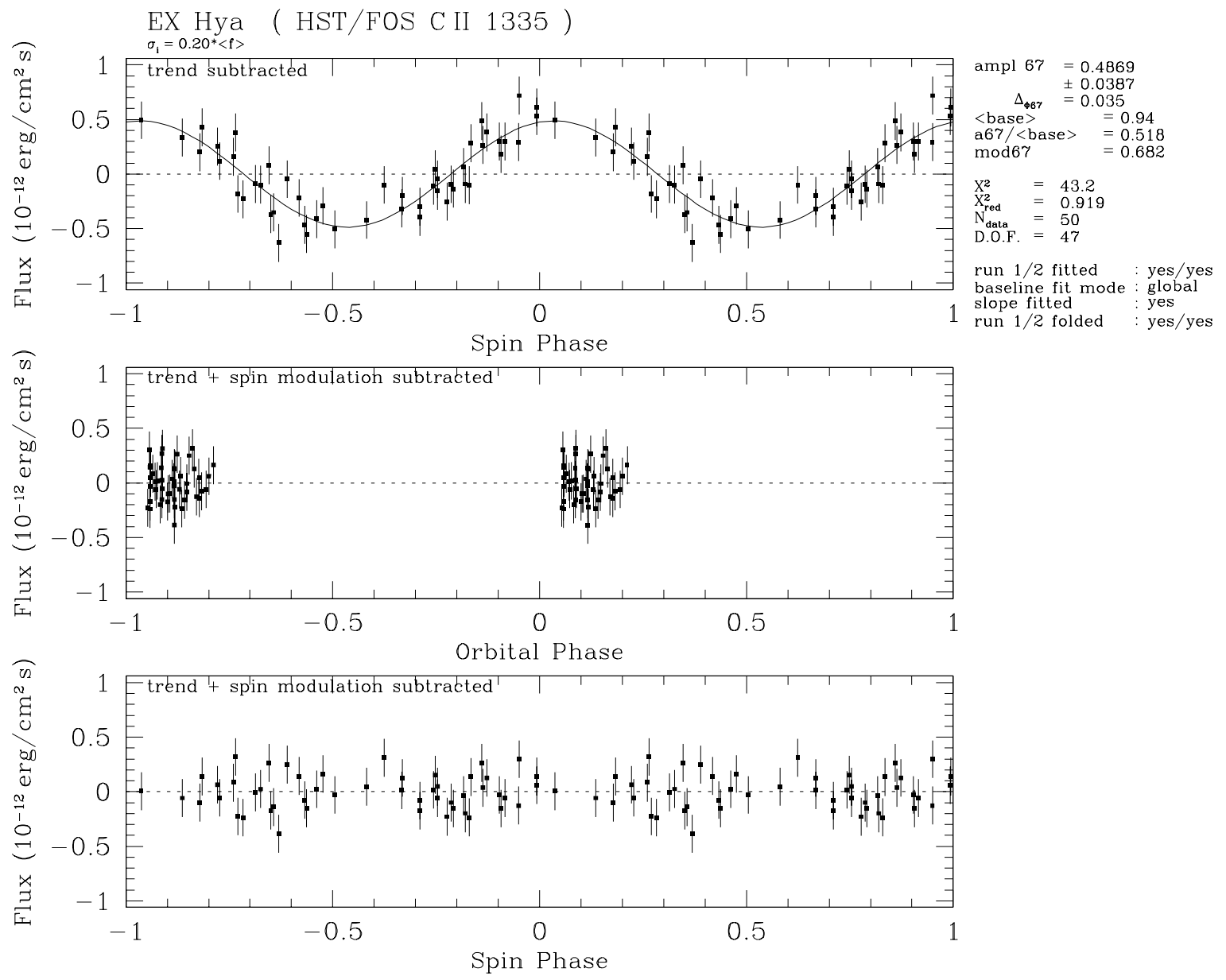

Abbildung A.24: Fit aus Abbildung A.23 über $\Phi_{67}$ bzw. $\Phi_{98}$ gefaltet, lineare Komponente abgezogen. 

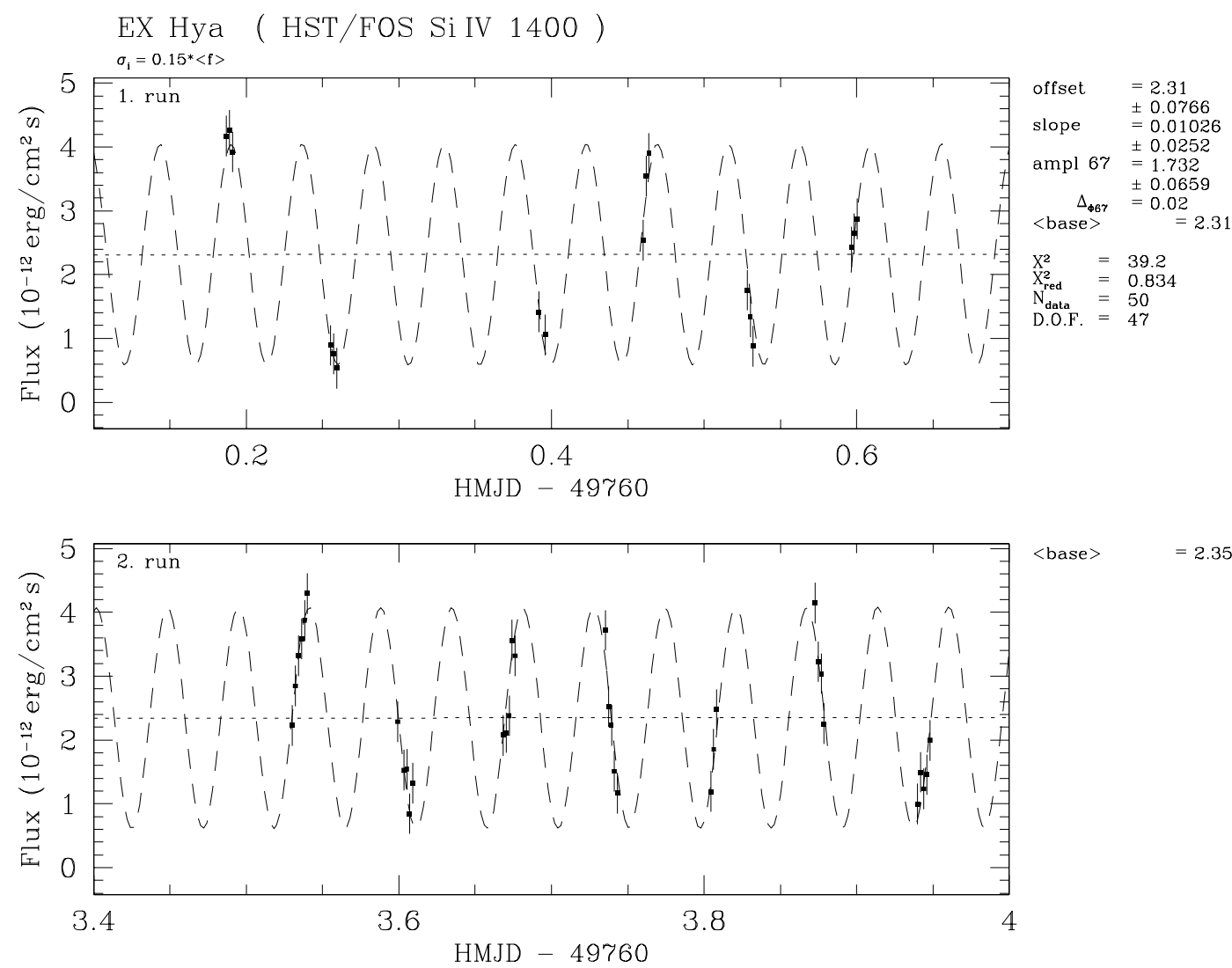

<base $>\quad=2.35$

Abbildung A.25: Fit an HST-Lichtkurve Si IV 1400;

Modell: $f_{\text {line }}=$ base $+A_{67} \cdot \cos \left(\Phi_{67}-\Delta_{\Phi 67}\right)$ mit base $\equiv C+B \cdot($ HMJD -49760$)$. 


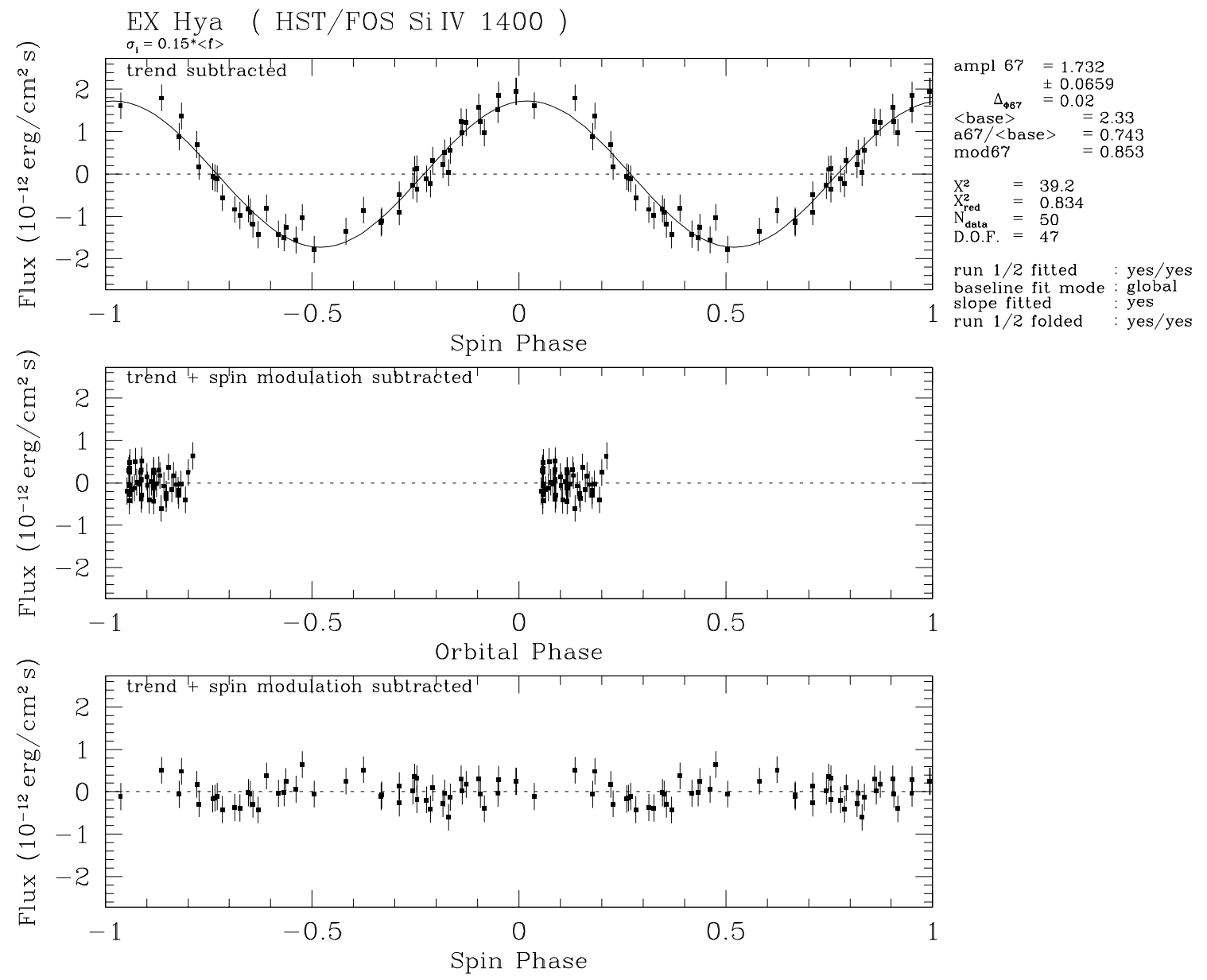

Abbildung A.26: Fit aus Abbildung A.25 über $\Phi_{67}$ bzw. $\Phi_{98}$ gefaltet, lineare Komponente abgezogen. 

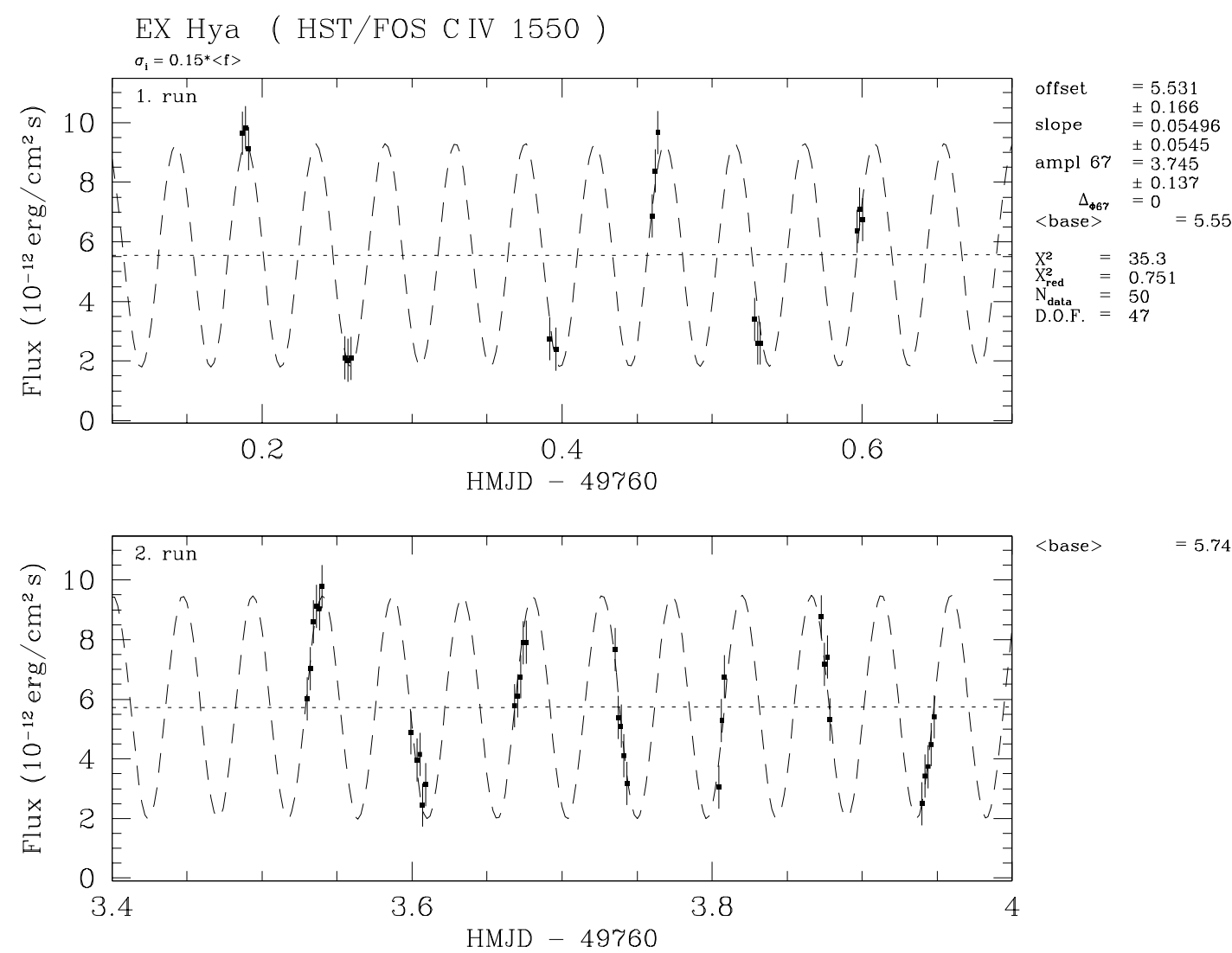

$<$ base $>\quad=5.74$

Abbildung A.27: Fit an HST-Lichtkurve C IV 1550;

Modell: $f_{\text {line }}=$ base $+A_{67} \cdot \cos \left(\Phi_{67}-\Delta_{\Phi 67}\right)$ mit base $\equiv C+B \cdot($ HMJD -49760$)$. 


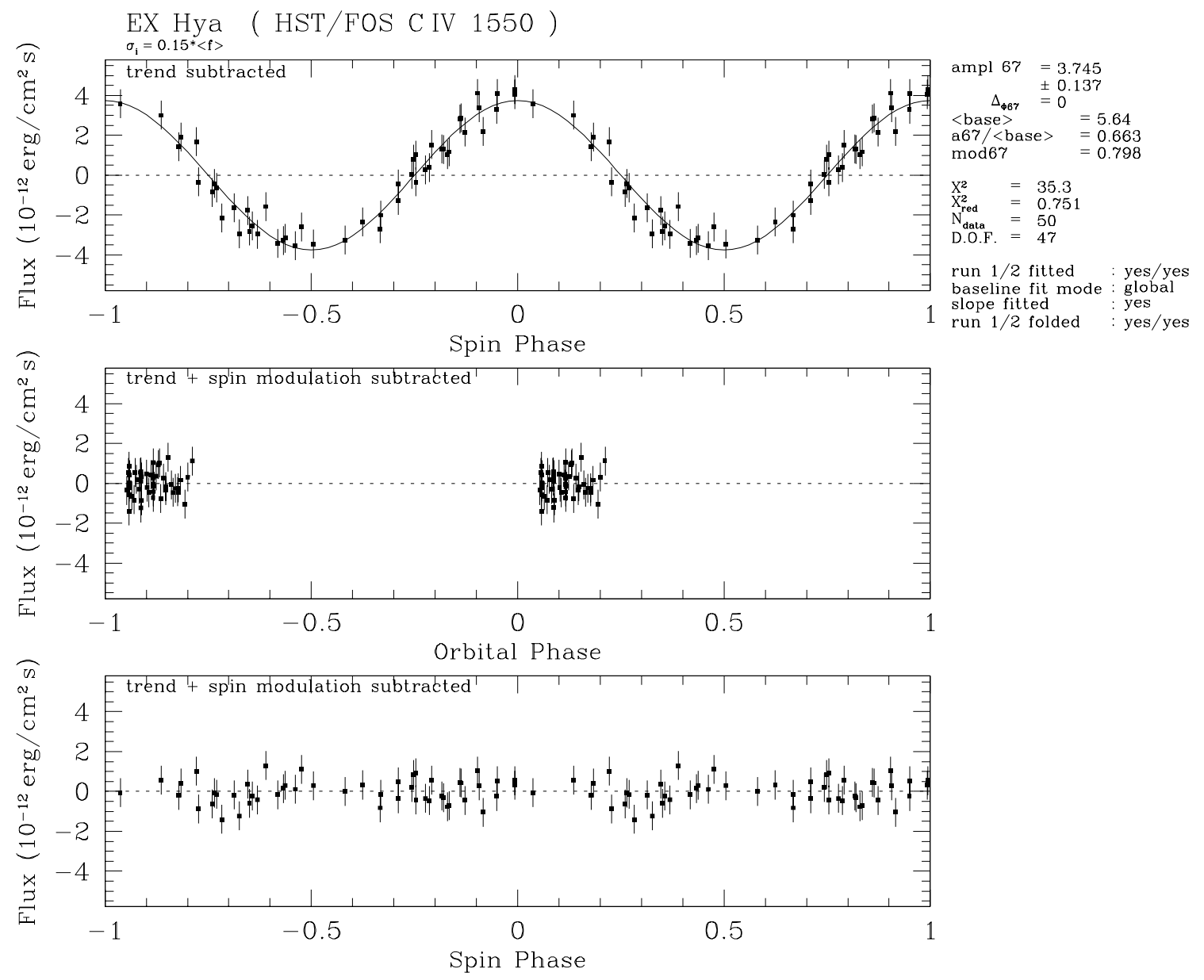

Abbildung A.28: Fit aus Abbildung A.27 über $\Phi_{67}$ bzw. $\Phi_{98}$ gefaltet, lineare Komponente abgezogen. 


\section{A.2 IUE-Lichtkurven}

\section{A.2.1 IUE-Lichtkurven: Kontinuumsflüsse}

Tabelle A.3: Übersicht über die Anpassungen an die IUE-Kontinuumslichtkurven: Definition der Bänder, best-fit-Amplituden, mittlerer Fluß und Modulationsgrad. $A_{67}$ und 〈base〉 sind in Einheiten von $10^{-12} \mathrm{erg} \mathrm{cm}^{-2} \mathrm{~s}^{-1} \AA^{-1}$ angegeben.

\begin{tabular}{llllll}
\hline Nr. & $\lambda \lambda(\AA)$ & $\lambda_{\text {cen }}(\AA)$ & Abbildung & $N_{\text {data }}$ & Bemerkungen \\
\hline 1 & $1255-1285$ & 1270 & A.29 & 29 & \\
2 & $1350-1380$ & 1365 & A.30 & 29 & \\
3 & $1425-1525$ & 1475 & A.31 & 29 & \\
4 & $1575-1625$ & 1600 & A.32 & 29 & \\
5 & $1680-1780$ & 1730 & A.33 & 29 & \\
6 & $1875-1975$ & 1925 & A.34 & 29 & \\
7 & $1250-1380$ & 1315 & A.35 & 29 & enthält Emissionslinien \\
8 & $1250-1950$ & 1600 & A.36 & 29 & enthält Emissionslinien \\
\hline
\end{tabular}

\begin{tabular}{lllllll}
\hline Nr. & $A_{67}$ & $\Delta_{\Phi 67}$ & $\chi_{\text {red }}^{2}$ & $\langle$ base $\rangle$ & $A_{67} /\langle$ base $\rangle$ & $\bmod _{67}$ \\
\hline 1 & $0.0264 \pm 0.0040$ & +0.050 & 0.92 & 0.145 & 0.182 & 0.308 \\
2 & $0.0356 \pm 0.0060$ & +0.015 & 1.77 & 0.156 & 0.228 & 0.372 \\
3 & $0.0211 \pm 0.0052$ & +0.020 & 1.60 & 0.144 & 0.147 & 0.256 \\
4 & $0.0250 \pm 0.0049$ & +0.035 & 2.00 & 0.122 & 0.205 & 0.341 \\
5 & $0.0262 \pm 0.0035$ & +0.025 & 1.36 & 0.103 & 0.255 & 0.407 \\
6 & $0.0283 \pm 0.0039$ & +0.025 & 1.71 & 0.103 & 0.274 & 0.430 \\
7 & $0.0366 \pm 0.0047$ & +0.030 & 0.97 & 0.166 & 0.220 & 0.361 \\
8 & $0.0380 \pm 0.0043$ & +0.030 & 1.12 & 0.141 & 0.270 & 0.425 \\
\hline
\end{tabular}



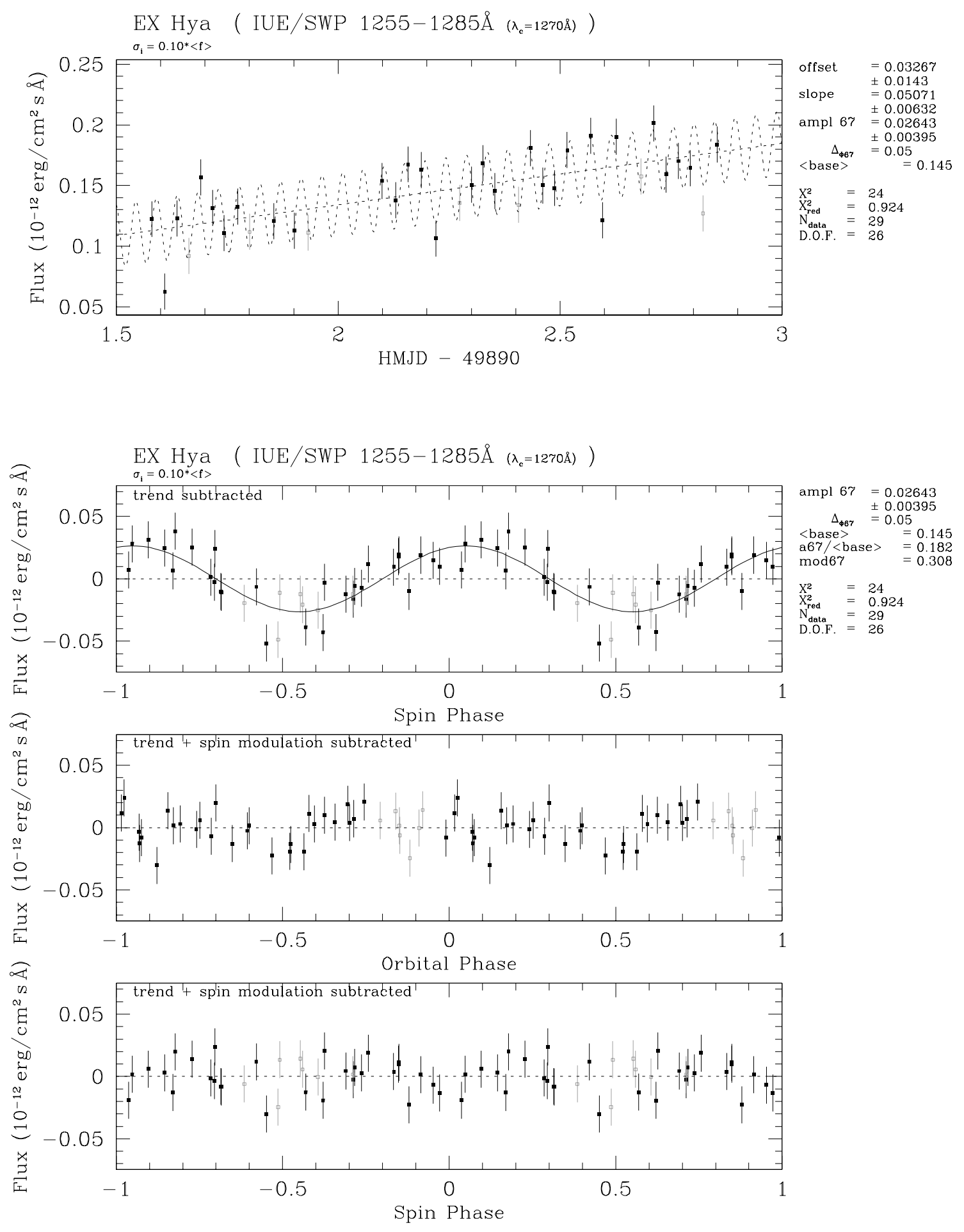

Abbildung A.29: Fit an IUE-Lichtkurve 1255...1285 $\AA\left(\lambda_{\text {cen }}=1270 \AA\right)$;

Modell: $\left\langle f_{\lambda}\right\rangle_{\text {band }}=$ base $+A_{67} \cdot \cos \left(\Phi_{67}-\Delta_{\Phi 67}\right) \quad$ mit $\quad$ base $\equiv C+B \cdot($ HMJD -49890$)$; oben: $\left\langle f_{\lambda}\right\rangle(t)$; unten: $\left\langle f_{\lambda}\right\rangle\left(\Phi_{67}\right)$ und $\left\langle f_{\lambda}\right\rangle\left(\Phi_{98}\right)$, lineare Komponente abgezogen. 

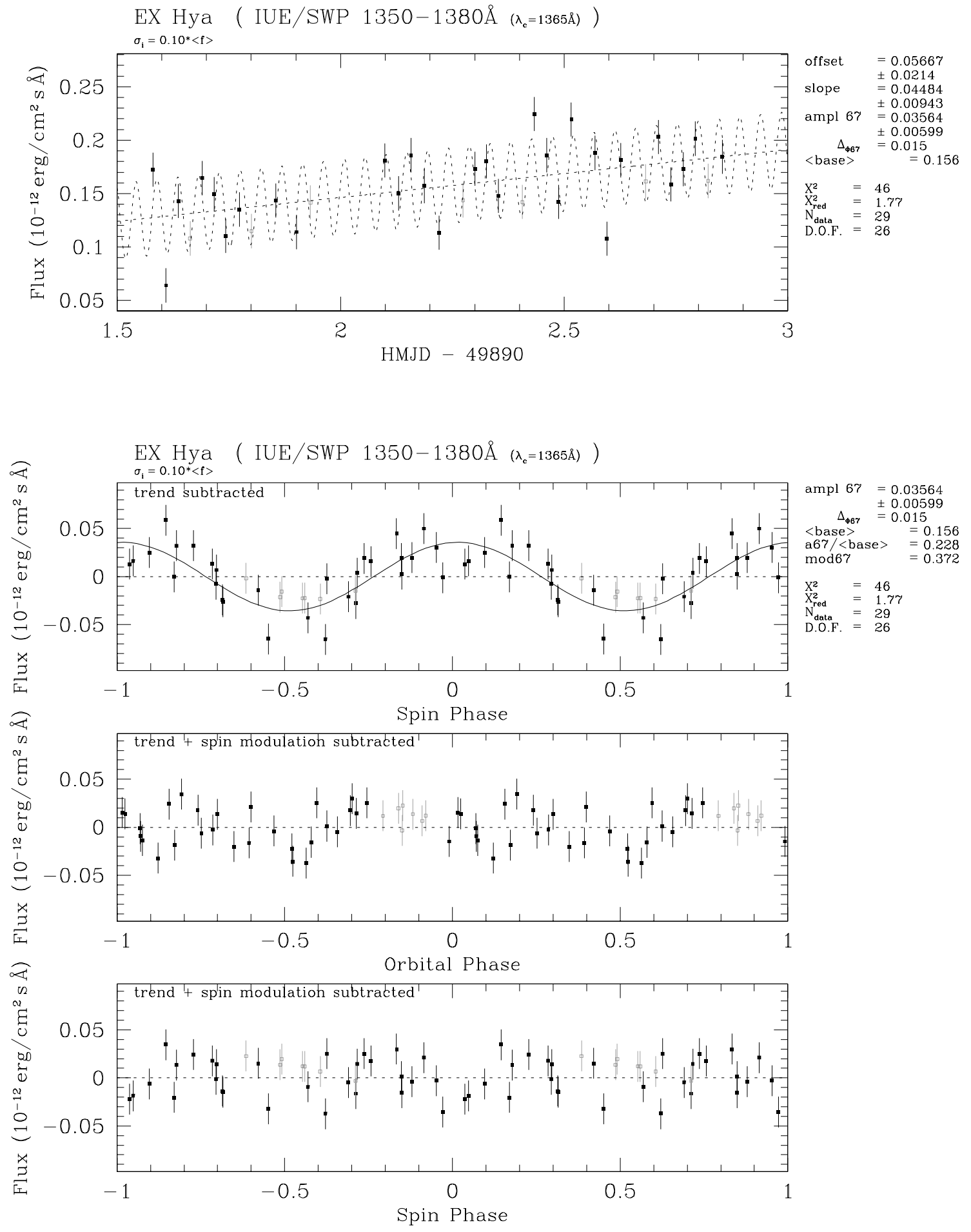

Abbildung A.30: Fit an IUE-Lichtkurve 1350...1380 $\AA\left(\lambda_{\text {cen }}=1365 \AA\right)$;

Modell: $\left\langle f_{\lambda}\right\rangle_{\text {band }}=$ base $+A_{67} \cdot \cos \left(\Phi_{67}-\Delta_{\Phi 67}\right) \quad$ mit $\quad$ base $\equiv C+B \cdot($ HMJD -49890$)$; oben: $\left\langle f_{\lambda}\right\rangle(t)$; unten: $\left\langle f_{\lambda}\right\rangle\left(\Phi_{67}\right)$ und $\left\langle f_{\lambda}\right\rangle\left(\Phi_{98}\right)$, lineare Komponente abgezogen. 

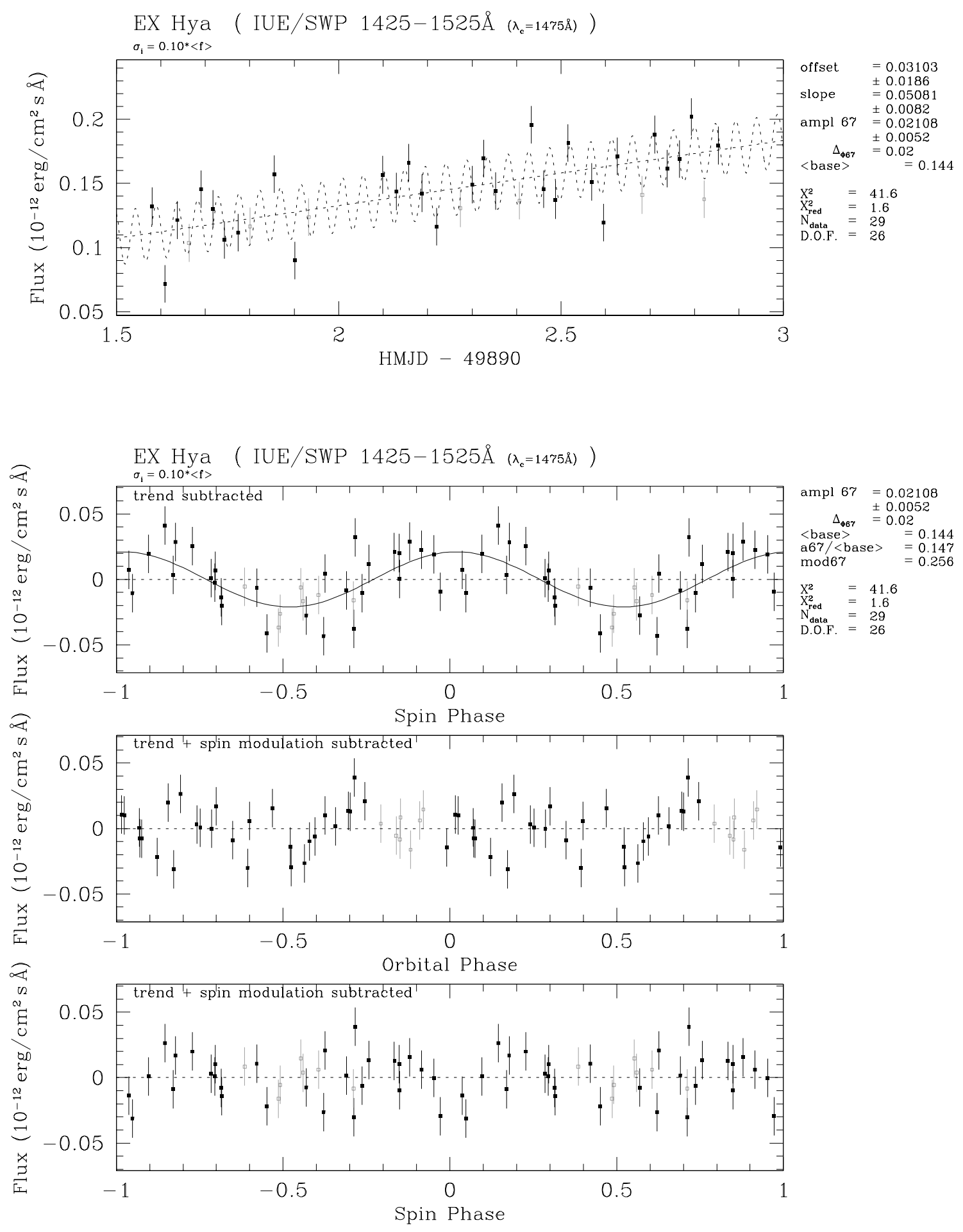

Abbildung A.31: Fit an IUE-Lichtkurve 1425...1525 $\AA\left(\lambda_{\text {cen }}=1475 \AA\right)$;

Modell: $\left\langle f_{\lambda}\right\rangle_{\text {band }}=$ base $+A_{67} \cdot \cos \left(\Phi_{67}-\Delta_{\Phi 67}\right)$ mit $\quad$ base $\equiv C+B \cdot($ HMJD -49890$)$; oben: $\left\langle f_{\lambda}\right\rangle(t)$; unten: $\left\langle f_{\lambda}\right\rangle\left(\Phi_{67}\right)$ und $\left\langle f_{\lambda}\right\rangle\left(\Phi_{98}\right)$, lineare Komponente abgezogen. 

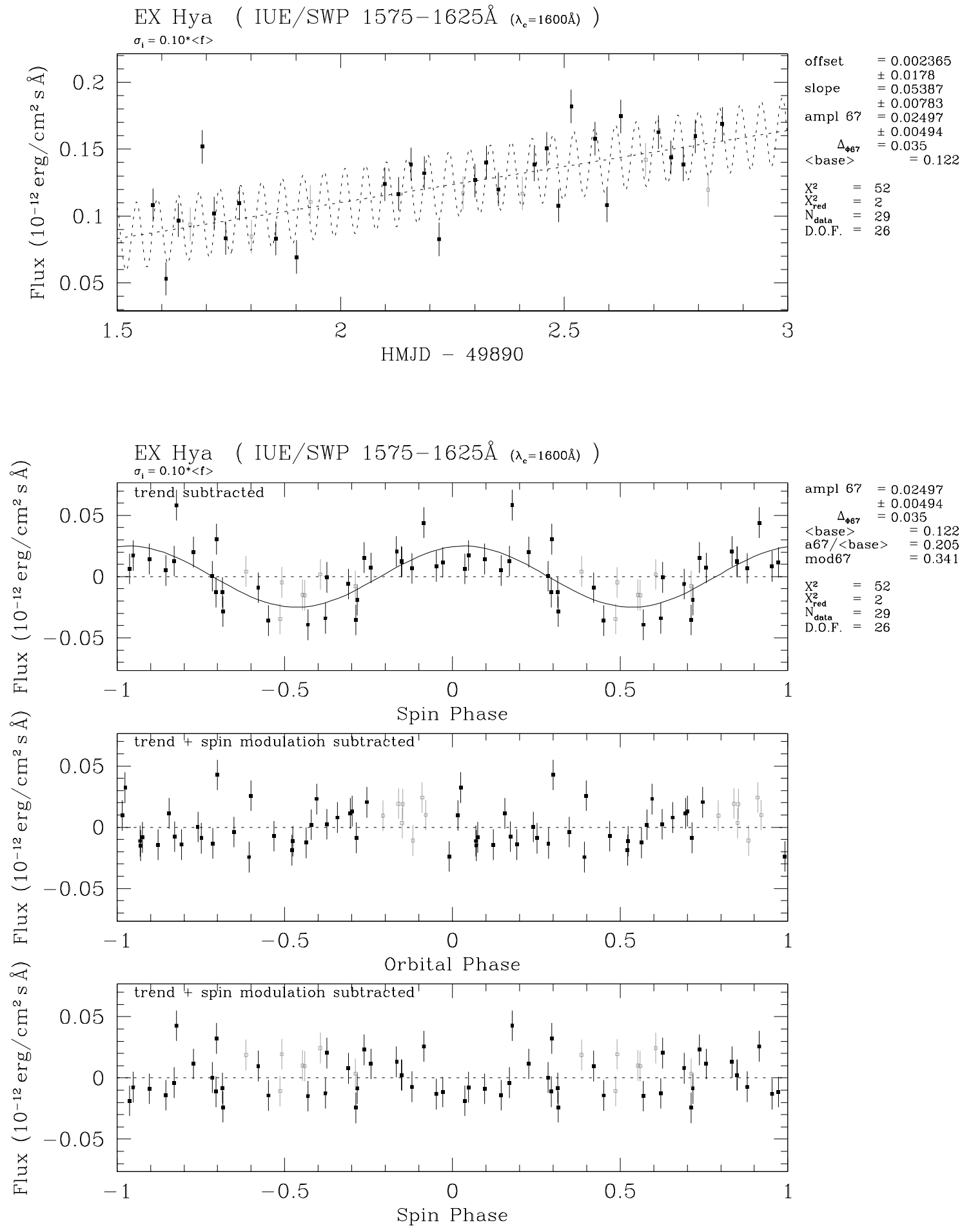

Abbildung A.32: Fit an IUE-Lichtkurve 1575...1625 $\AA\left(\lambda_{\text {cen }}=1600 \AA\right)$;

Modell: $\left\langle f_{\lambda}\right\rangle_{\text {band }}=$ base $+A_{67} \cdot \cos \left(\Phi_{67}-\Delta_{\Phi 67}\right) \quad$ mit $\quad$ base $\equiv C+B \cdot($ HMJD -49890$)$; oben: $\left\langle f_{\lambda}\right\rangle(t)$; unten: $\left\langle f_{\lambda}\right\rangle\left(\Phi_{67}\right)$ und $\left\langle f_{\lambda}\right\rangle\left(\Phi_{98}\right)$, lineare Komponente abgezogen. 

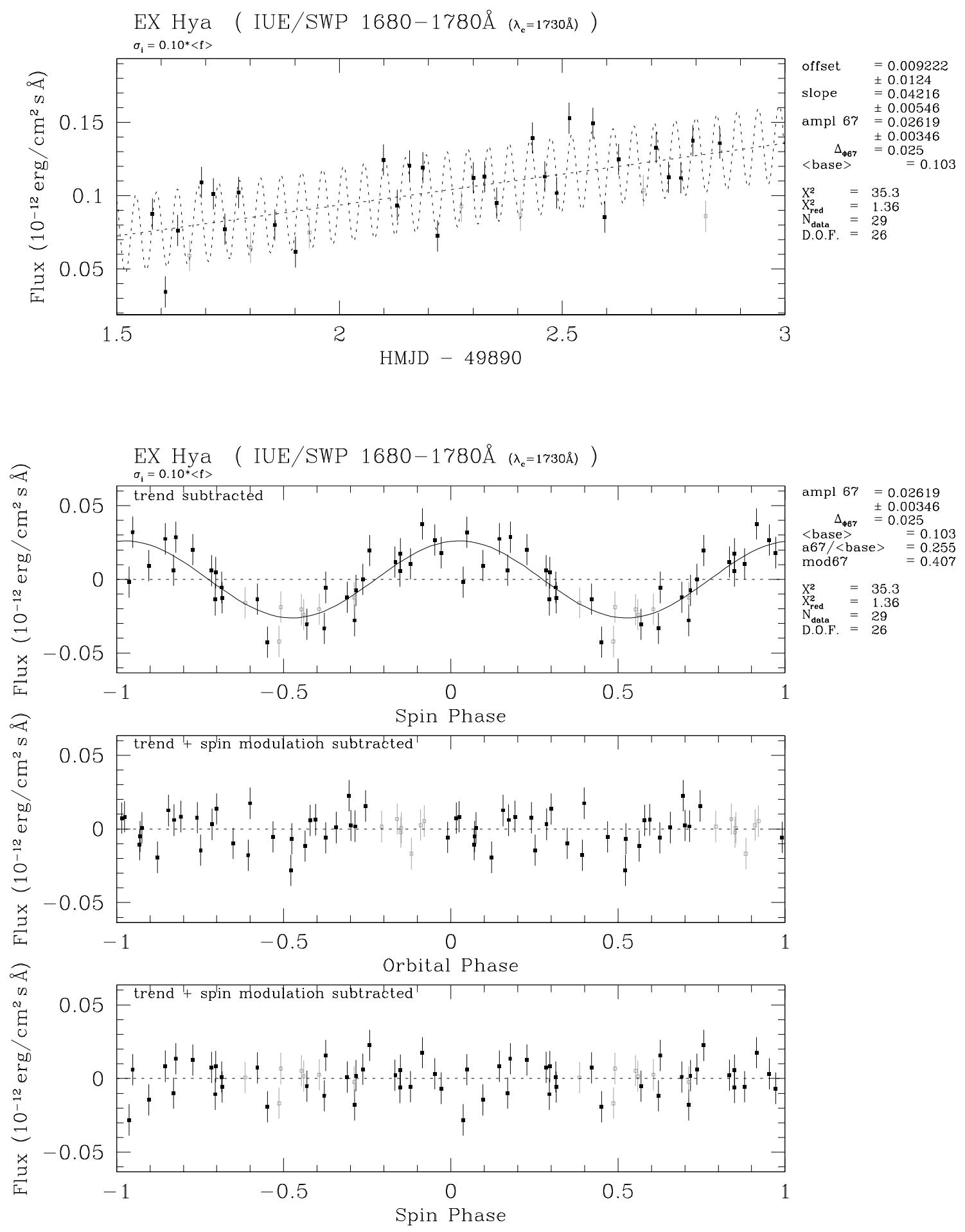

Abbildung A.33: Fit an IUE-Lichtkurve 1680...1780 $\AA\left(\lambda_{\text {cen }}=1730 \AA\right)$;

Modell: $\left\langle f_{\lambda}\right\rangle_{\text {band }}=$ base $+A_{67} \cdot \cos \left(\Phi_{67}-\Delta_{\Phi 67}\right) \quad$ mit $\quad$ base $\equiv C+B \cdot($ HMJD -49890$)$; oben: $\left\langle f_{\lambda}\right\rangle(t)$; unten: $\left\langle f_{\lambda}\right\rangle\left(\Phi_{67}\right)$ und $\left\langle f_{\lambda}\right\rangle\left(\Phi_{98}\right)$, lineare Komponente abgezogen. 

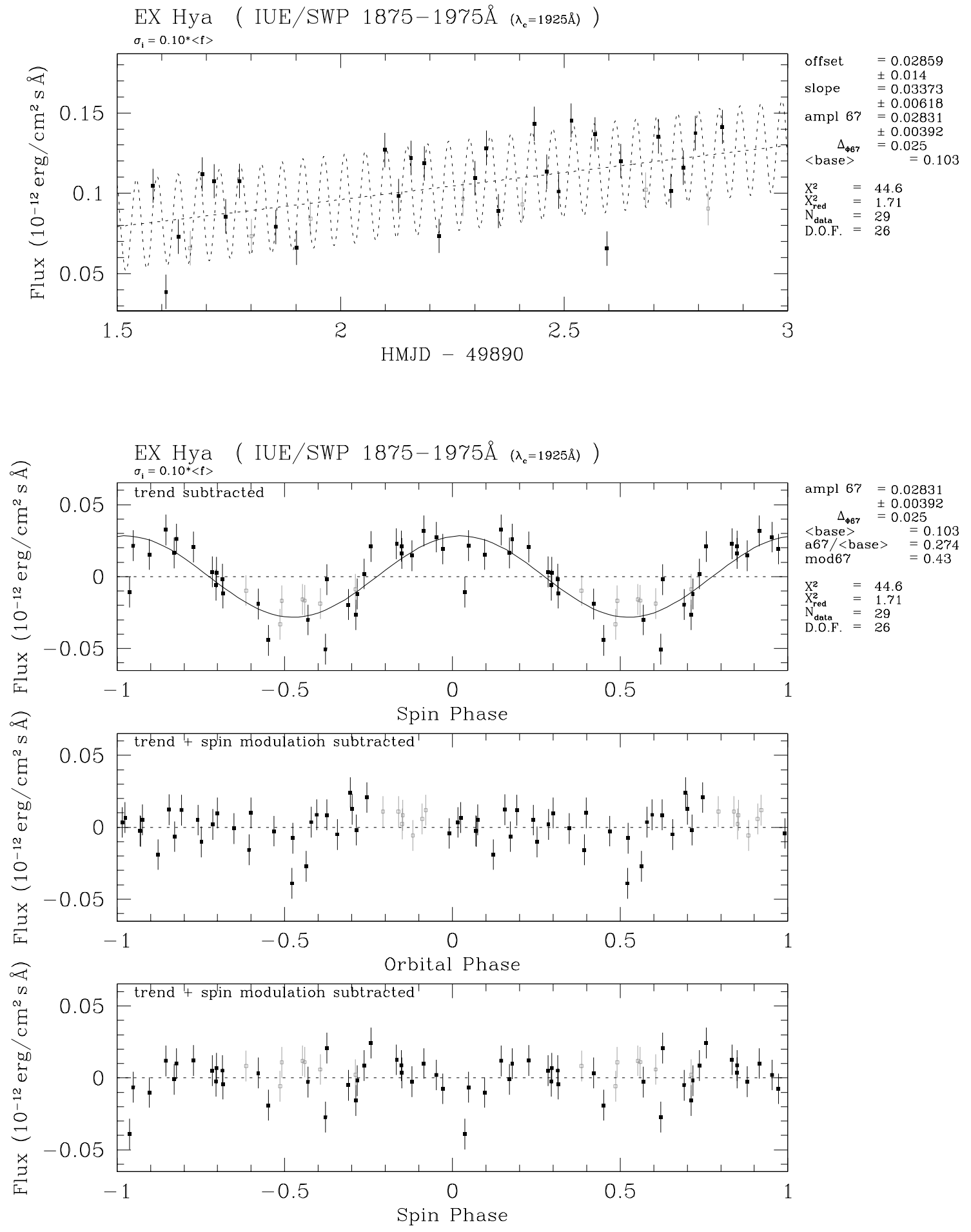

Abbildung A.34: Fit an IUE-Lichtkurve 1875...1975 $\AA\left(\lambda_{\text {cen }}=1925 \AA\right)$;

Modell: $\left\langle f_{\lambda}\right\rangle_{\text {band }}=$ base $+A_{67} \cdot \cos \left(\Phi_{67}-\Delta_{\Phi 67}\right) \quad$ mit $\quad$ base $\equiv C+B \cdot($ HMJD -49890$)$; oben: $\left\langle f_{\lambda}\right\rangle(t)$; unten: $\left\langle f_{\lambda}\right\rangle\left(\Phi_{67}\right)$ und $\left\langle f_{\lambda}\right\rangle\left(\Phi_{98}\right)$, lineare Komponente abgezogen. 

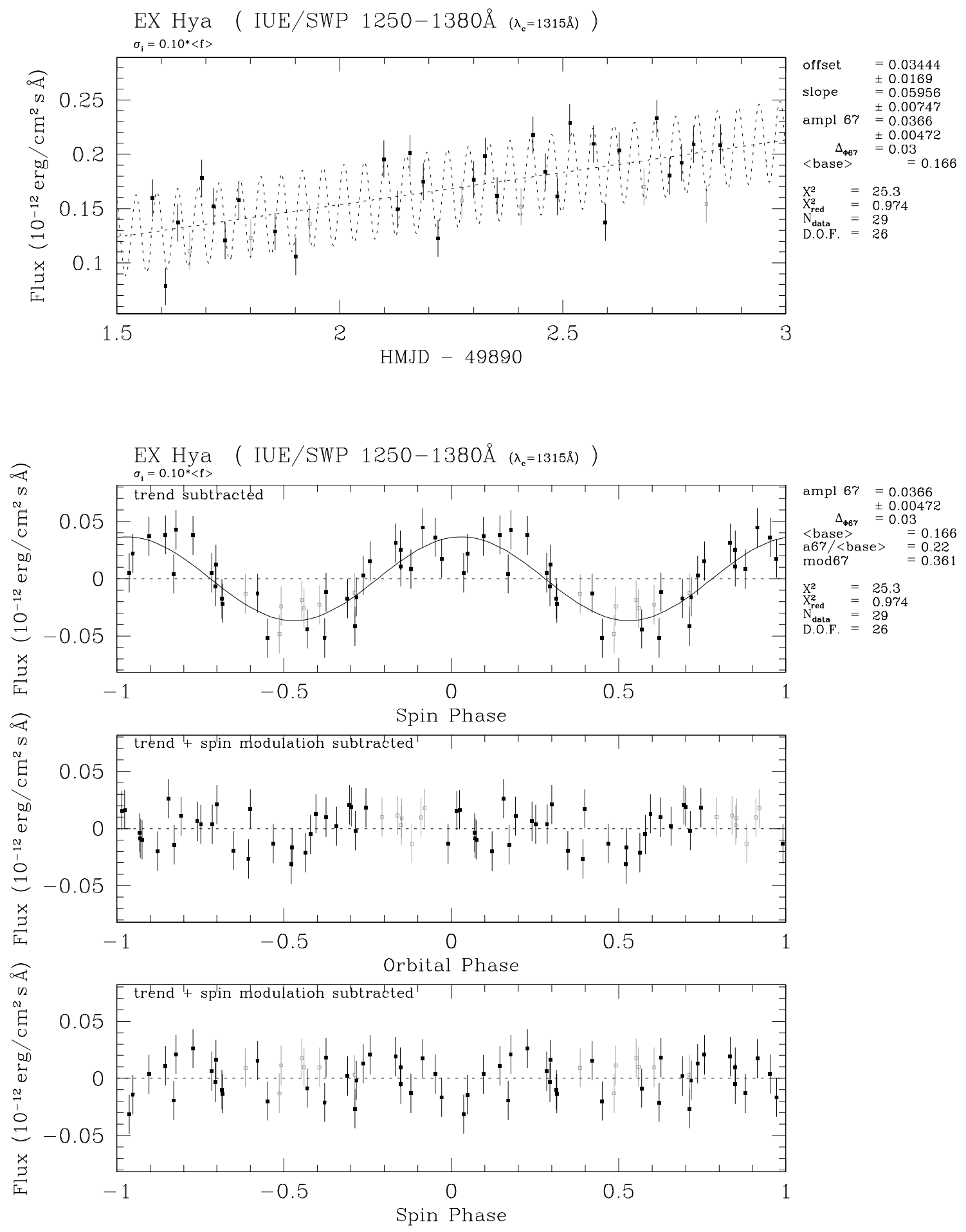

Abbildung A.35: Fit an IUE-Lichtkurve $1250 \ldots 1380 \AA\left(\lambda_{\text {cen }}=1315 \AA\right)$;

Modell: $\left\langle f_{\lambda}\right\rangle_{\text {band }}=$ base $+A_{67} \cdot \cos \left(\Phi_{67}-\Delta_{\Phi 67}\right) \quad$ mit $\quad$ base $\equiv C+B \cdot($ HMJD -49890$)$; oben: $\left\langle f_{\lambda}\right\rangle(t)$; unten: $\left\langle f_{\lambda}\right\rangle\left(\Phi_{67}\right)$ und $\left\langle f_{\lambda}\right\rangle\left(\Phi_{98}\right)$, lineare Komponente abgezogen. 

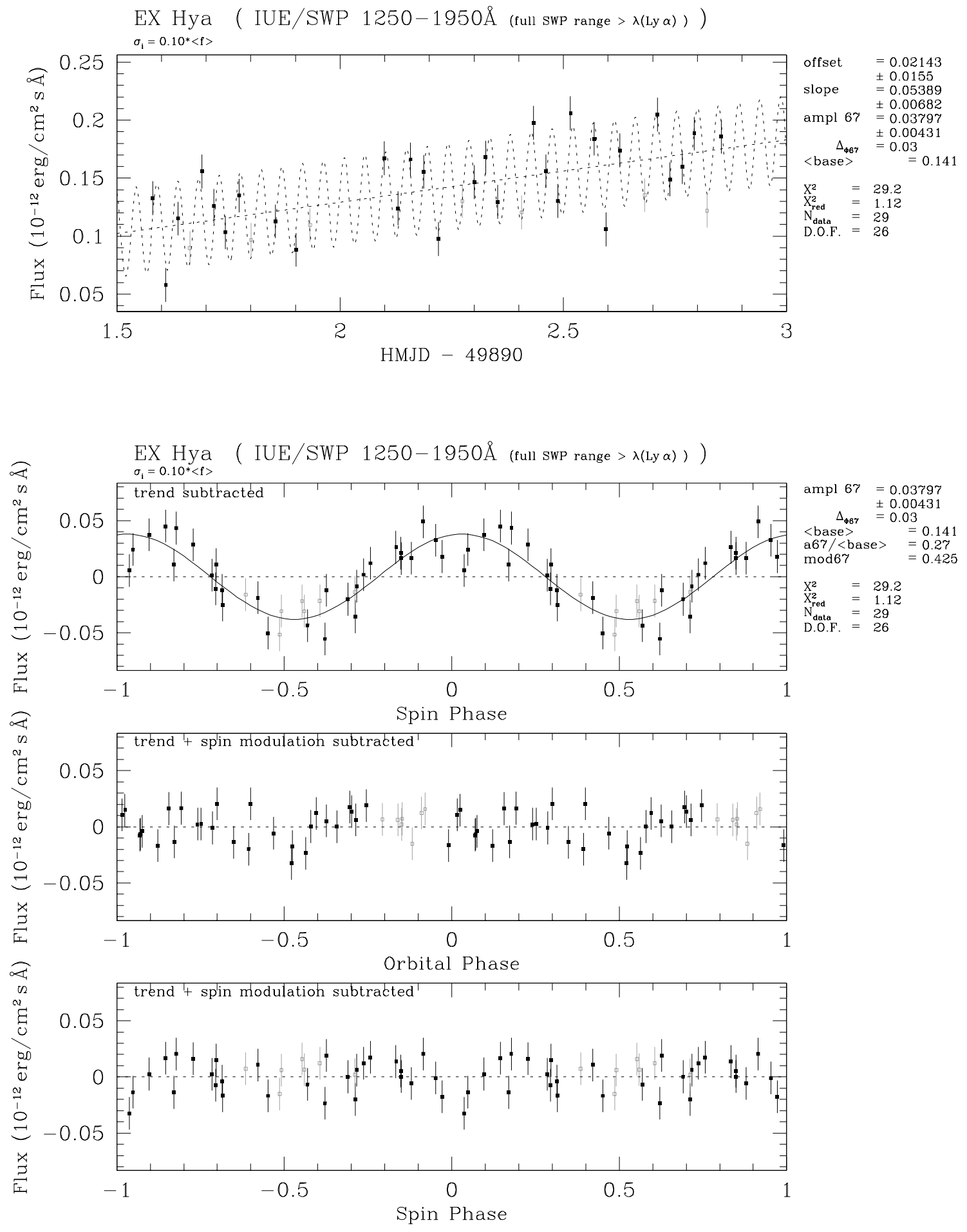

Abbildung A.36: Fit an IUE-Lichtkurve 1250...1950 $\AA\left(\lambda_{\text {cen }}=1600 \AA\right)$; Modell: $\left\langle f_{\lambda}\right\rangle_{\text {band }}=$ base $+A_{67} \cdot \cos \left(\Phi_{67}-\Delta_{\Phi 67}\right) \quad$ mit $\quad$ base $\equiv C+B \cdot($ HMJD -49890$)$; oben: $\left\langle f_{\lambda}\right\rangle(t)$; unten: $\left\langle f_{\lambda}\right\rangle\left(\Phi_{67}\right)$ und $\left\langle f_{\lambda}\right\rangle\left(\Phi_{98}\right)$, lineare Komponente abgezogen. 


\section{A.2.2 IUE-Lichtkurven: Linienflüsse}

Tabelle A.4: Übersicht über die Anpassungen an die IUE-Emissionslinienlichtkurven: best-fit-Amplituden, mittlerer Fluß und Modulationsgrad. $A_{67}$ und $\langle$ base $\rangle$ sind in Einheiten von $10^{-12} \mathrm{erg} \mathrm{cm}^{-2} \mathrm{~s}^{-1}$ angegeben.

\begin{tabular}{lllll}
\hline Nr. & Linie & Abbildung & $N_{\text {data }}$ & Bemerkungen \\
\hline 1 & Si IV 1400 & A.37 & 30 & \\
2 & C IV 1550 & A.38 & 30 & \\
3 & He II 1640 & A.39 & 30 & \\
4 & Al III 1860 & A.40 & 30 & \\
\hline
\end{tabular}

\begin{tabular}{|c|c|c|c|c|c|c|}
\hline Nr. & $A_{67}$ & $\Delta_{\Phi 67}$ & $\chi_{\mathrm{red}}^{2}$ & $\langle$ base $\rangle$ & $A_{67} /\langle$ base $\rangle$ & $\bmod _{67}$ \\
\hline 1 & $1.41 \pm 0.18$ & +0.015 & 1.83 & 2.13 & 0.661 & 0.796 \\
\hline 2 & $3.94 \pm 0.30$ & +0.005 & 1.59 & 5.16 & 0.762 & 0.865 \\
\hline 3 & $0.849 \pm 0.098$ & +0.045 & 2.32 & 0.674 & 1.26 & 1.11 \\
\hline 4 & $0.456 \pm 0.072$ & +0.030 & 2.48 & 0.496 & 0.919 & 0.958 \\
\hline
\end{tabular}



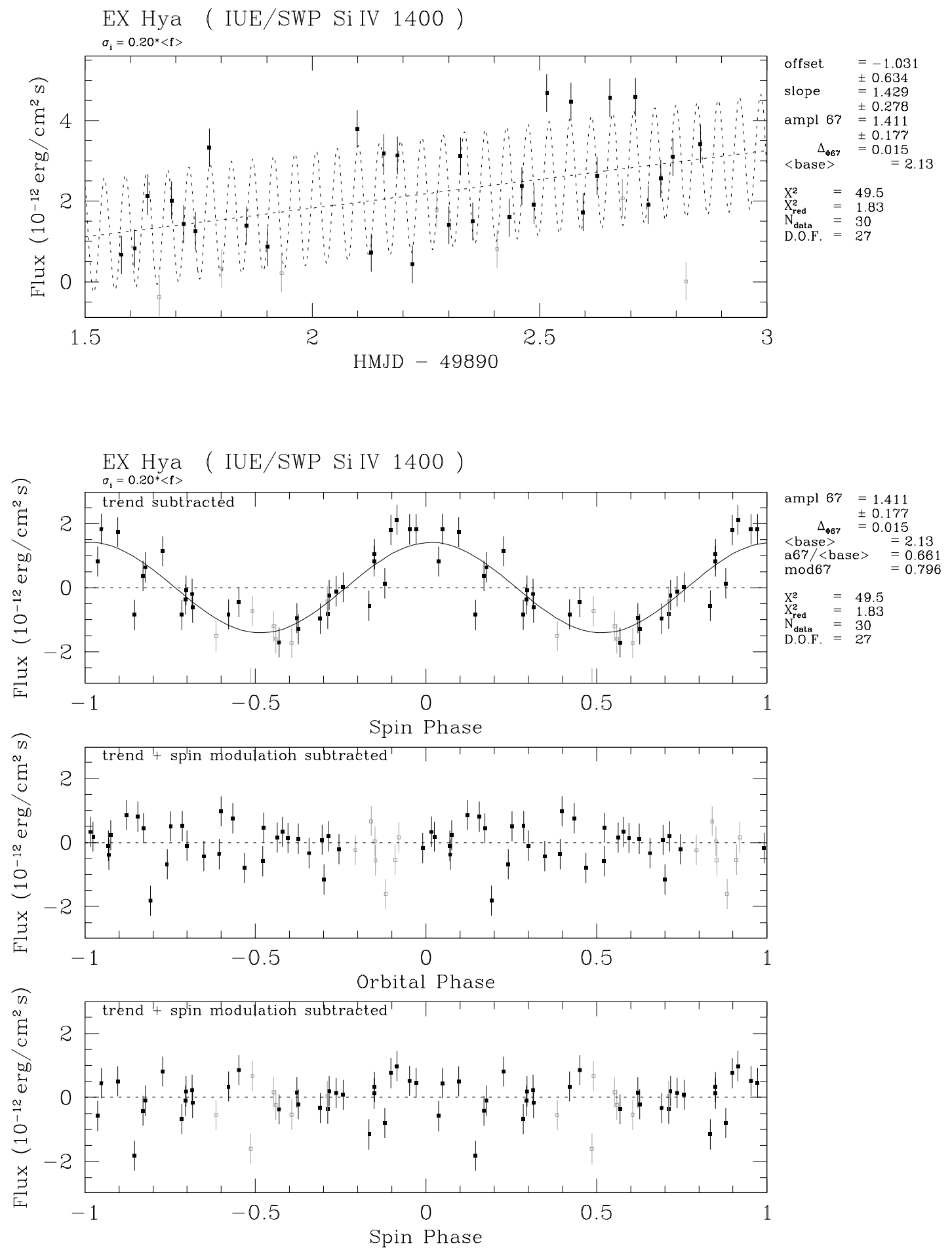

Abbildung A.37: Fit an IUE-Lichtkurve Si IV 1400;

Modell: $f_{\text {line }}=$ base $+A_{67} \cdot \cos \left(\Phi_{67}-\Delta_{\Phi 67}\right)$ mit base $\equiv C+B \cdot($ HMJD -49890$)$; oben: $f(t)$; unten: $f\left(\Phi_{67}\right)$ und $f\left(\Phi_{98}\right)$, lineare Komponente abgezogen. 

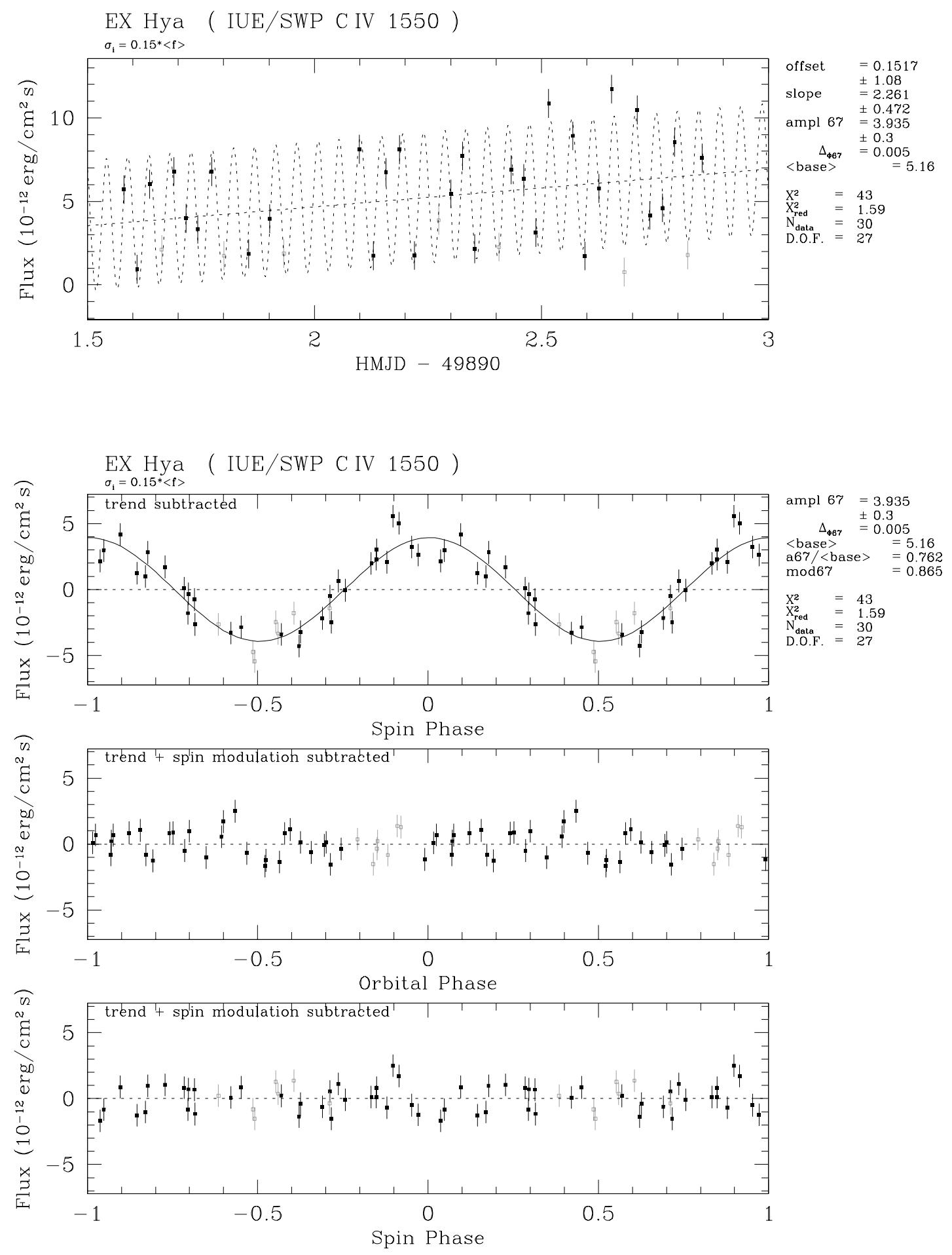

Abbildung A.38: Fit an IUE-Lichtkurve C IV 1550;

Modell: $f_{\text {line }}=$ base $+A_{67} \cdot \cos \left(\Phi_{67}-\Delta_{\Phi 67}\right)$ mit base $\equiv C+B \cdot($ HMJD -49890$)$; oben: $f(t)$; unten: $f\left(\Phi_{67}\right)$ und $f\left(\Phi_{98}\right)$, lineare Komponente abgezogen. 

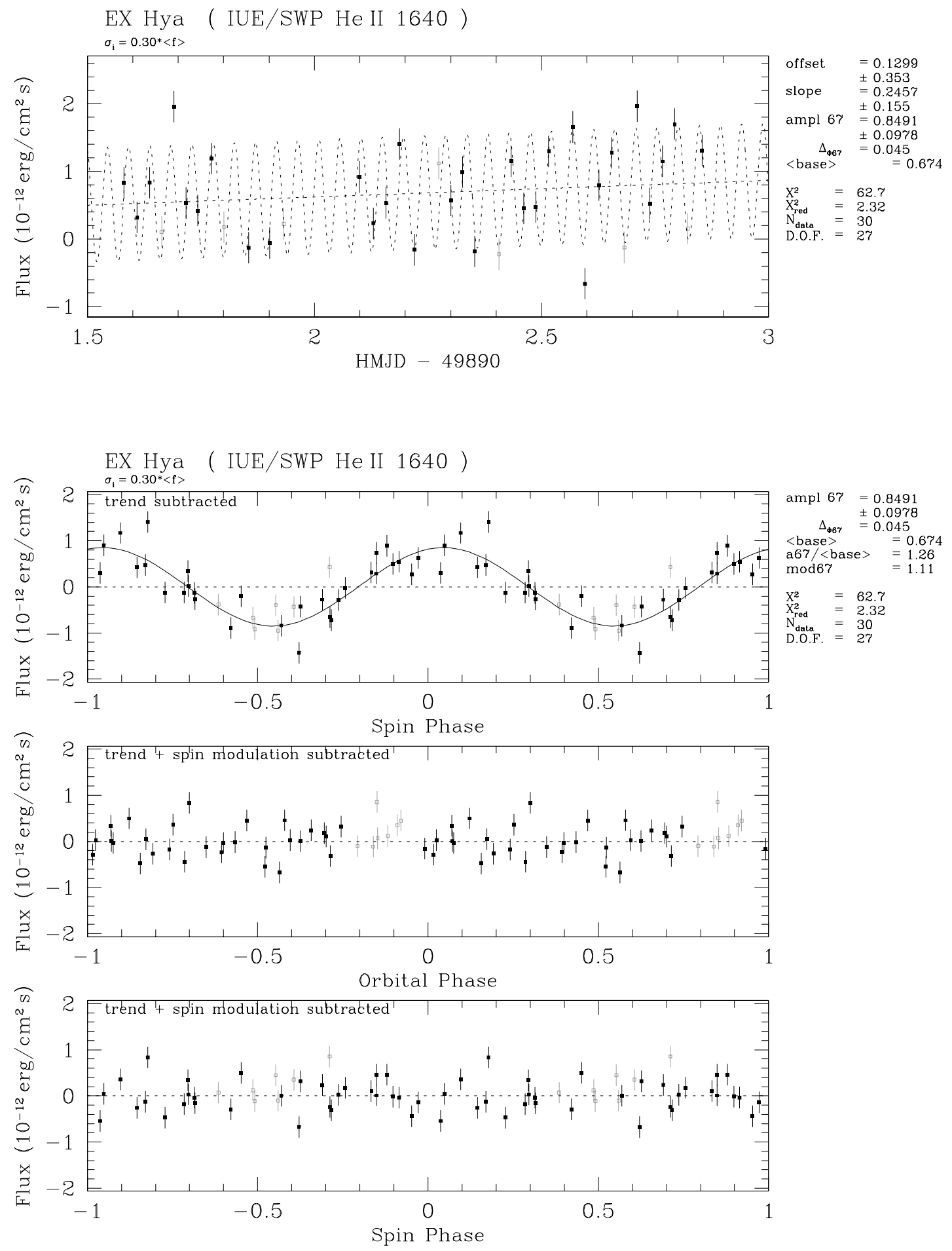

Abbildung A.39: Fit an IUE-Lichtkurve He II 1640;

Modell: $f_{\text {line }}=$ base $+A_{67} \cdot \cos \left(\Phi_{67}-\Delta_{\Phi 67}\right)$ mit base $\equiv C+B \cdot($ HMJD -49890$)$; oben: $f(t)$; unten: $f\left(\Phi_{67}\right)$ und $f\left(\Phi_{98}\right)$, lineare Komponente abgezogen. 

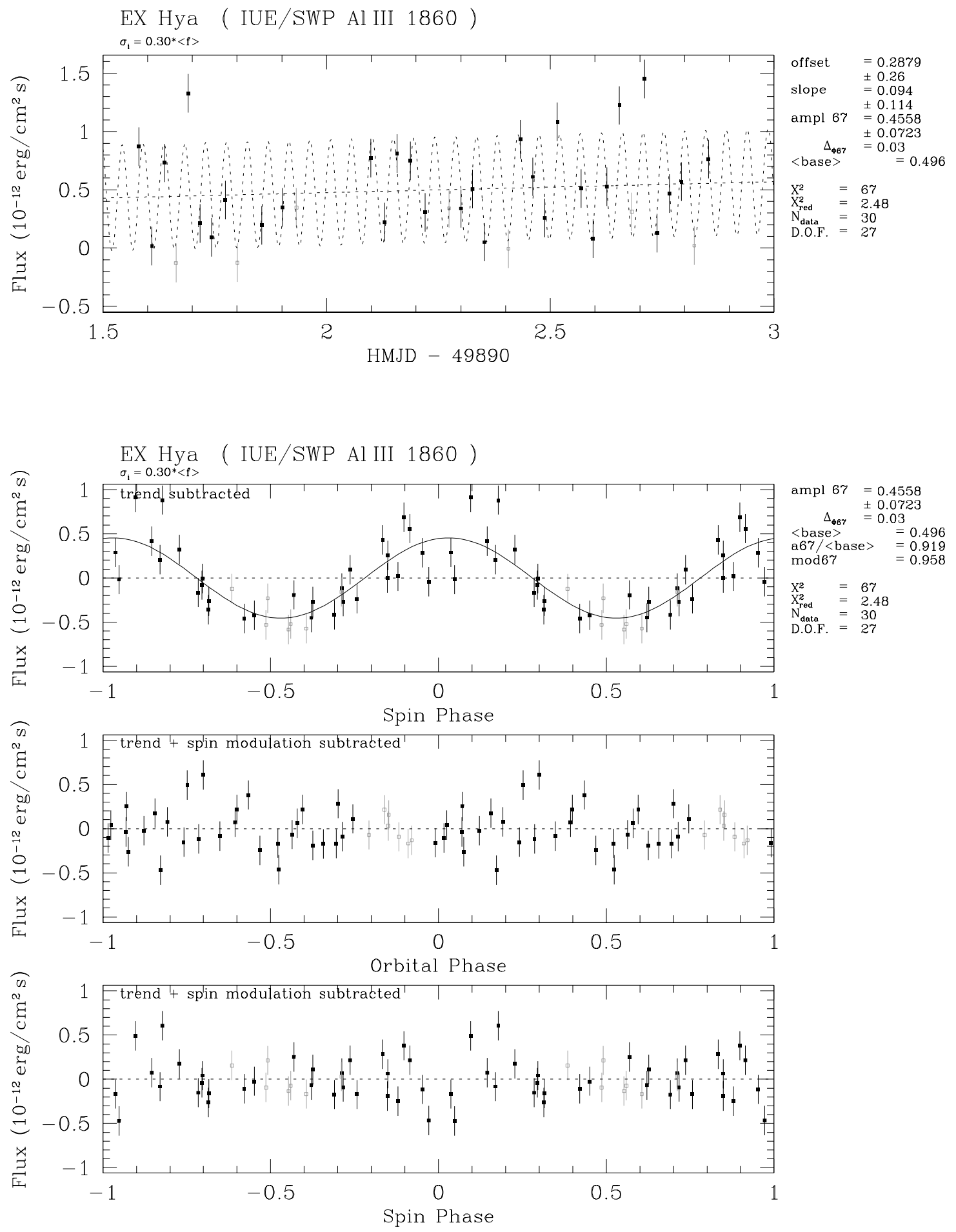

Abbildung A.40: Fit an IUE-Lichtkurve Al III 1860;

Modell: $f_{\text {line }}=$ base $+A_{67} \cdot \cos \left(\Phi_{67}-\Delta_{\Phi 67}\right)$ mit base $\equiv C+B \cdot($ HMJD -49890$)$; oben: $f(t)$; unten: $f\left(\Phi_{67}\right)$ und $f\left(\Phi_{98}\right)$, lineare Komponente abgezogen. 


\section{A.3 EFOSC2-Lichtkurven}

\section{A.3.1 EFOSC2-Lichtkurven: Kontinuumsflüsse}

Tabelle A.5: Übersicht über die Anpassungen an die EFOSC2-Kontinuumslichtkurven: Definition der Bänder, best-fit-Amplituden, mittlerer Fluß und Modulationsgrad. $A_{67}$ und 〈base〉 sind in Einheiten von $10^{-16} \mathrm{erg} \mathrm{cm}^{-2} \mathrm{~s}^{-1} \AA^{-1}$ angegeben.

\begin{tabular}{llllll}
\hline Nr. & $\lambda \lambda(\AA)$ & $\lambda_{\text {cen }}(\AA)$ & Abbildung & $N_{\text {data }}$ & Bemerkungen \\
\hline 1 & $3600-4000$ & 3800 & A.41 & 18 & "U" (langwelliger Teil) \\
2 & $3900-5000$ & 4450 & A.42 & 18 & "B" \\
3 & $5000-6200$ & 5600 & A.43 & 18 & "V" \\
4 & $5800-8000$ & 6900 & A.44 & 18 & "R" \\
5 & $7500-10000$ & 8750 & A.45 & 18 & "I" (kurzwelliger Teil) \\
\hline
\end{tabular}

\begin{tabular}{lllllll}
\hline Nr. & $A_{67}$ & $\Delta_{\Phi 67}$ & $\chi_{\text {red }}^{2}$ & $\langle$ base $\rangle$ & $A_{67} /\langle$ base $\rangle$ & $\bmod _{67}$ \\
\hline 1 & $112 \pm 14$ & +0.030 & 1.01 & 431 & 0.259 & 0.411 \\
2 & $47.3 \pm 8.4$ & +0.035 & 1.22 & 229 & 0.206 & 0.342 \\
3 & $20.7 \pm 4.5$ & +0.050 & 1.23 & 124 & 0.167 & 0.287 \\
4 & $17.8 \pm 3.2$ & +0.040 & 1.00 & 96.5 & 0.184 & 0.312 \\
5 & $9.09 \pm 1.9$ & +0.040 & 0.89 & 59.5 & 0.153 & 0.265 \\
\hline
\end{tabular}



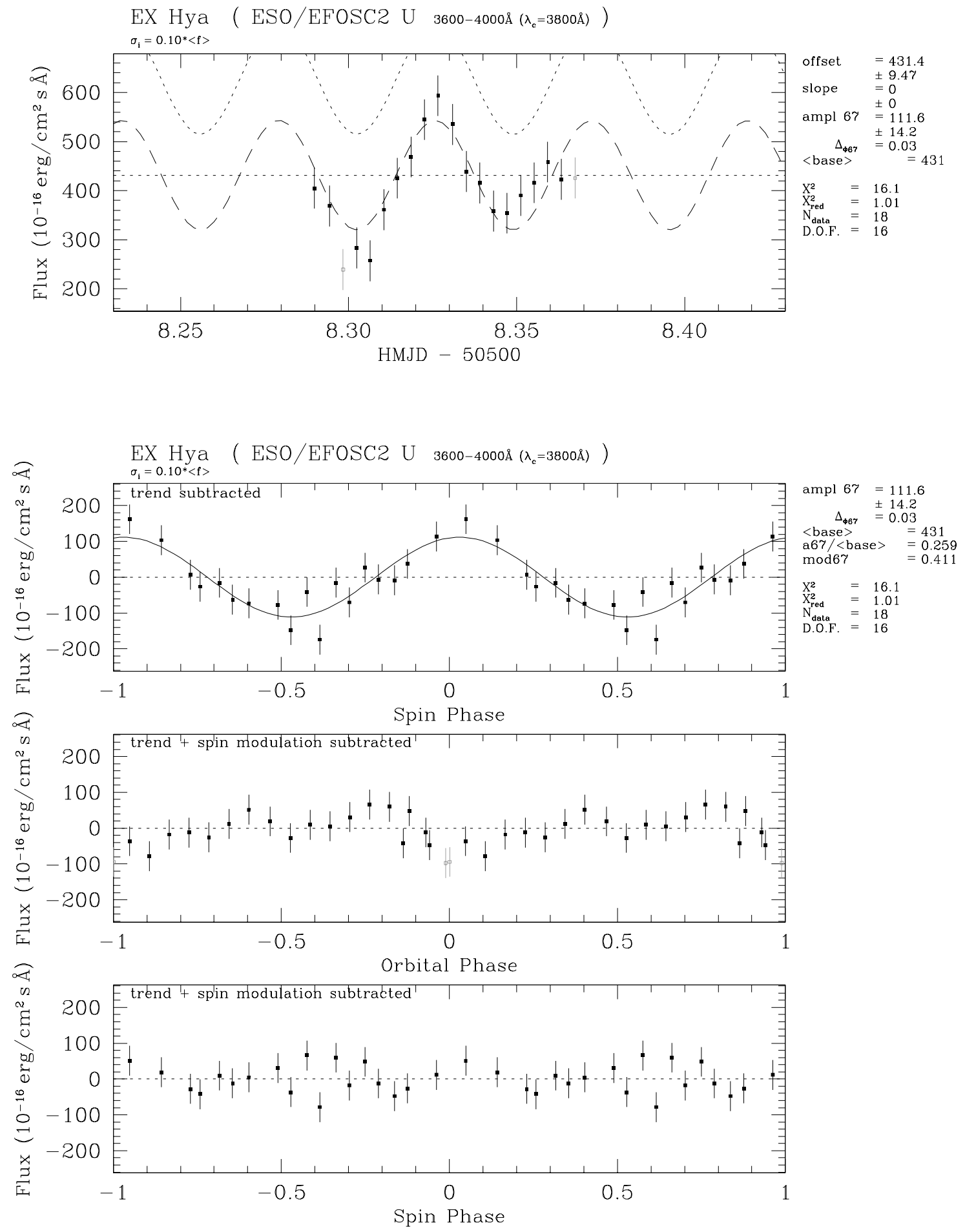

Abbildung A.41: Fit an EFOSC2-Lichtkurve $3600 \ldots 4000 \AA$ ("U"; $\lambda_{\text {cen }}=3800 \AA$ ); Modell: $\left\langle f_{\lambda}\right\rangle_{\text {band }}=C+A_{67} \cdot \cos \left(\Phi_{67}-\Delta_{\Phi 67}\right)$;

oben: $\left\langle f_{\lambda}\right\rangle(t)$; unten: $\left\langle f_{\lambda}\right\rangle\left(\Phi_{67}\right)$ und $\left\langle f_{\lambda}\right\rangle\left(\Phi_{98}\right)$, konstanter Offset abgezogen. 

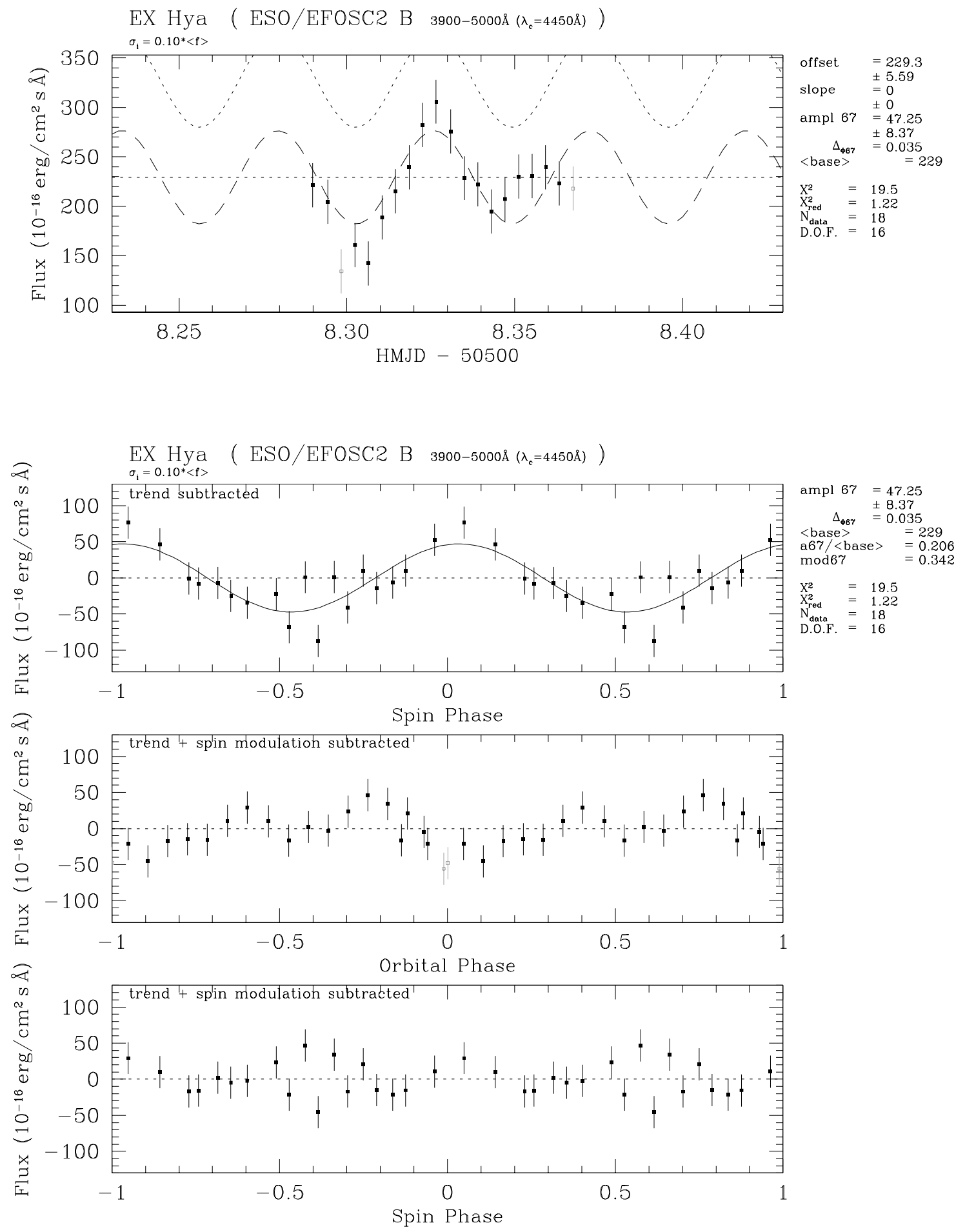

Abbildung A.42: Fit an EFOSC2-Lichtkurve $3900 . . .5000 \AA$ ("B"; $\lambda_{\text {cen }}=4450 \AA$ ); Modell: $\left\langle f_{\lambda}\right\rangle_{\text {band }}=C+A_{67} \cdot \cos \left(\Phi_{67}-\Delta_{\Phi 67}\right)$;

oben: $\left\langle f_{\lambda}\right\rangle(t)$; unten: $\left\langle f_{\lambda}\right\rangle\left(\Phi_{67}\right)$ und $\left\langle f_{\lambda}\right\rangle\left(\Phi_{98}\right)$, konstanter Offset abgezogen. 

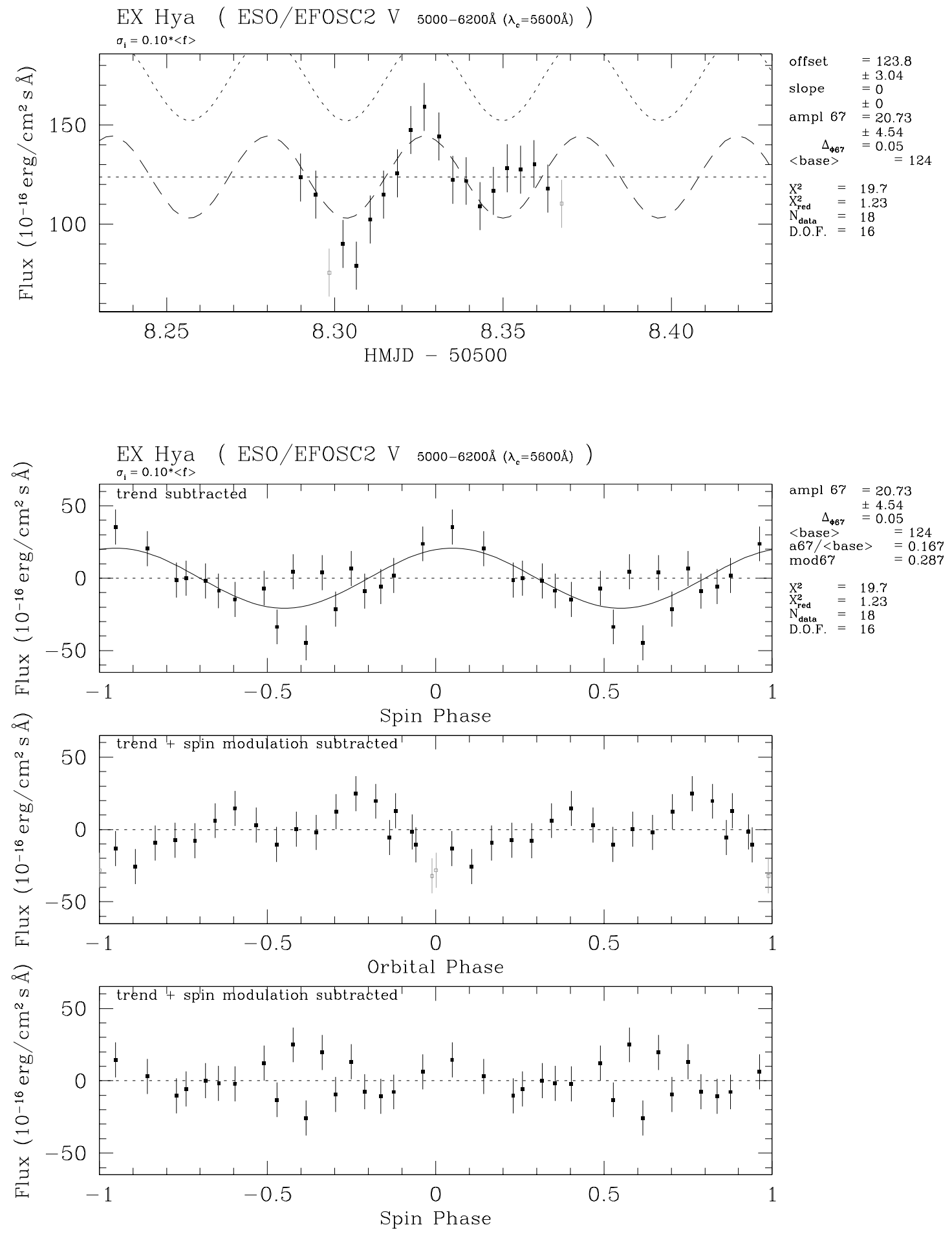

Abbildung A.43: Fit an EFOSC2-Lichtkurve 5000...6200 $\AA$ ("V"; $\lambda_{\text {cen }}=5600 \AA$ ); Modell: $\left\langle f_{\lambda}\right\rangle_{\text {band }}=C+A_{67} \cdot \cos \left(\Phi_{67}-\Delta_{\Phi 67}\right)$;

oben: $\left\langle f_{\lambda}\right\rangle(t)$; unten: $\left\langle f_{\lambda}\right\rangle\left(\Phi_{67}\right)$ und $\left\langle f_{\lambda}\right\rangle\left(\Phi_{98}\right)$, konstanter Offset abgezogen. 

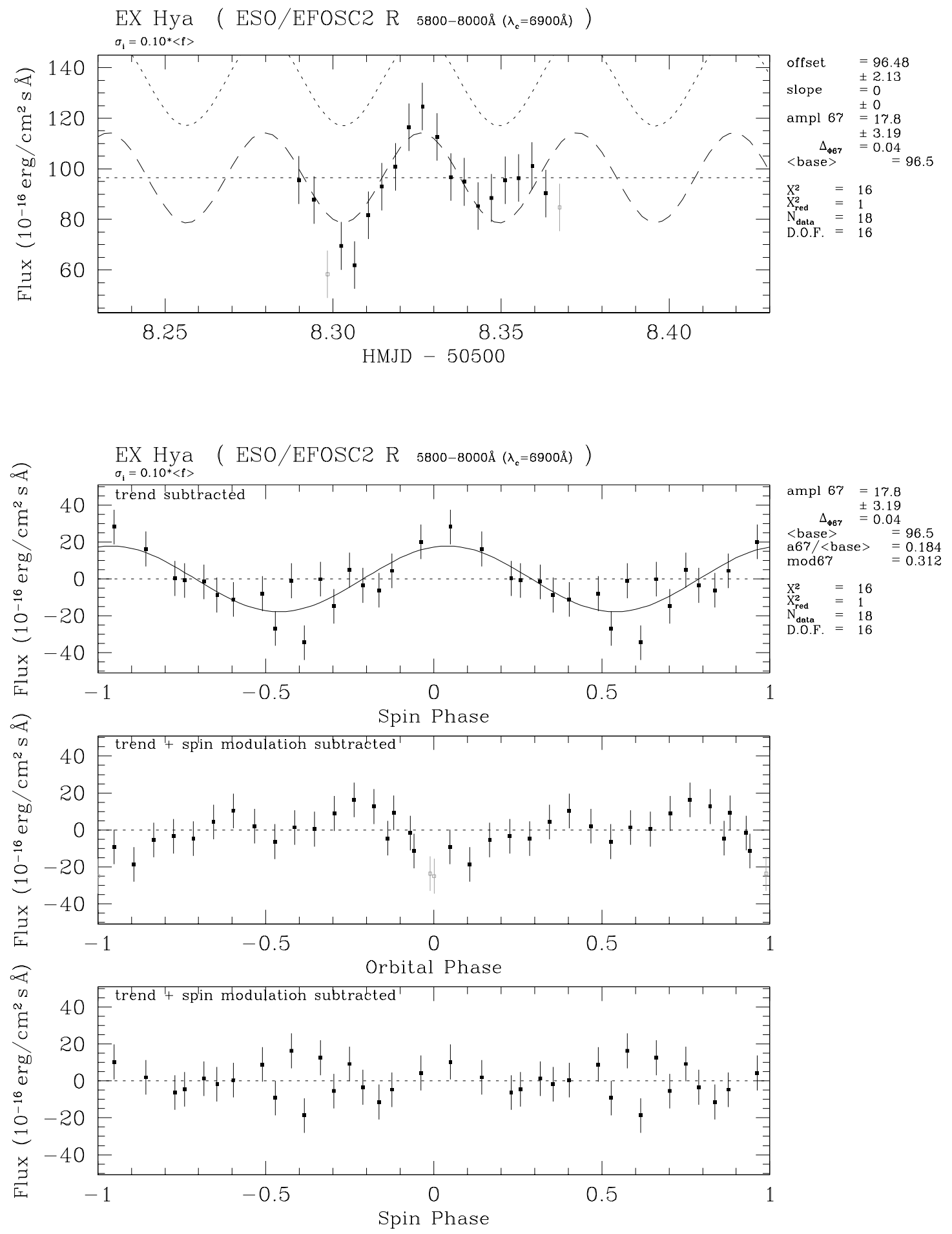

Abbildung A.44: Fit an EFOSC2-Lichtkurve 5800...8000 $\AA$ ("R"; $\lambda_{\text {cen }}=6900 \AA$ ); Modell: $\left\langle f_{\lambda}\right\rangle_{\text {band }}=C+A_{67} \cdot \cos \left(\Phi_{67}-\Delta_{\Phi 67}\right)$;

oben: $\left\langle f_{\lambda}\right\rangle(t)$; unten: $\left\langle f_{\lambda}\right\rangle\left(\Phi_{67}\right)$ und $\left\langle f_{\lambda}\right\rangle\left(\Phi_{98}\right)$, konstanter Offset abgezogen. 

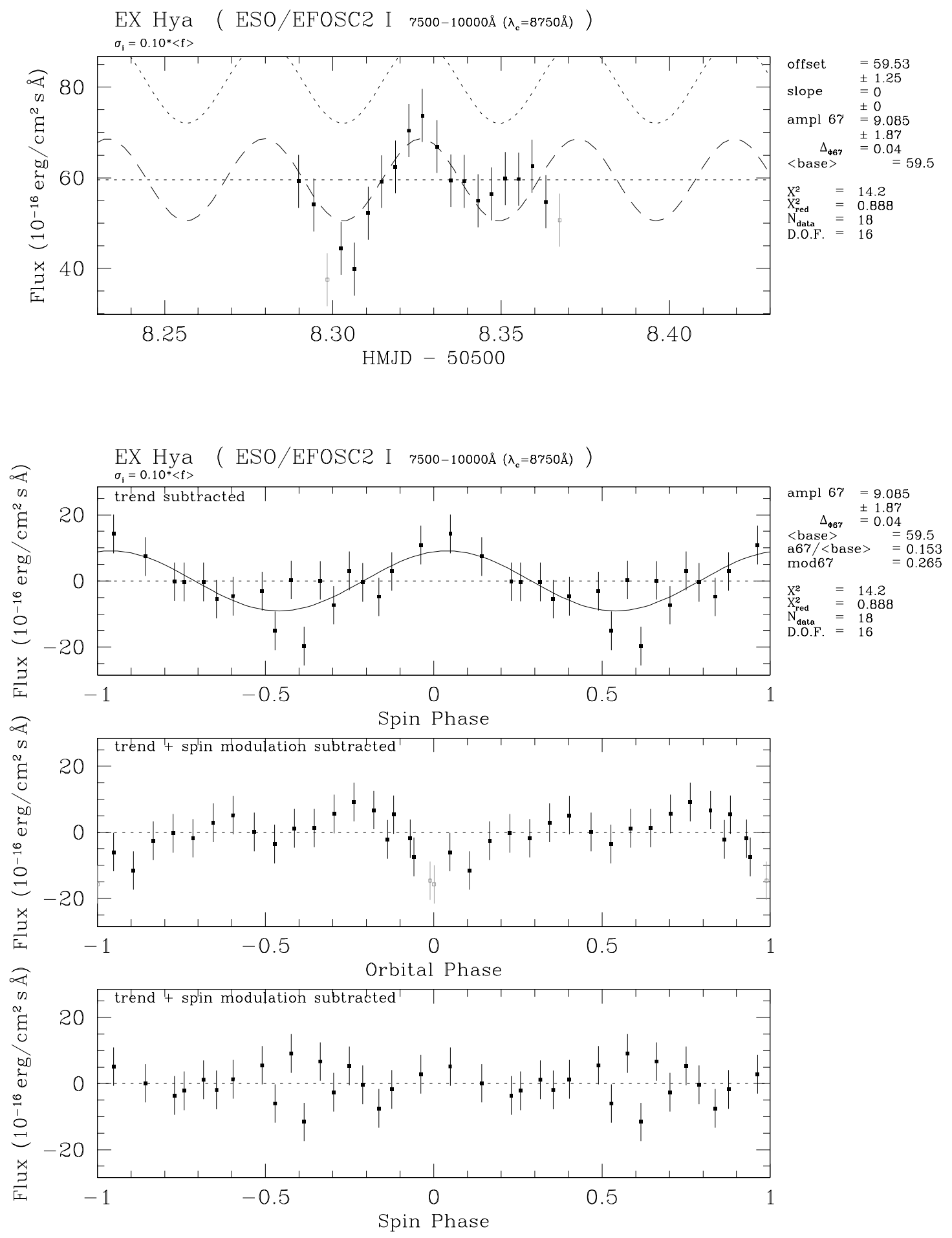

Abbildung A.45: Fit an EFOSC2-Lichtkurve 7500...10000 Å ("I", kurzwelliger Teil; $\left.\lambda_{\text {cen }}=8750 \AA\right)$; Modell: $\left\langle f_{\lambda}\right\rangle_{\text {band }}=C+A_{67} \cdot \cos \left(\Phi_{67}-\Delta_{\Phi 67}\right)$; oben: $\left\langle f_{\lambda}\right\rangle(t)$; unten: $\left\langle f_{\lambda}\right\rangle\left(\Phi_{67}\right)$ und $\left\langle f_{\lambda}\right\rangle\left(\Phi_{98}\right)$, konstanter Offset abgezogen. 


\section{A.3.2 EFOSC2-Lichtkurven: Linienflüsse}

Tabelle A.6: Übersicht über die Anpassungen an die EFOSC2-Emissionslinienlichtkurven: best-fit-Amplituden, mittlerer Fluß und Modulationsgrad. $A_{67}$ und $\langle$ base $\rangle$ sind in Einheiten von $10^{-16} \mathrm{erg} \mathrm{cm}^{-2} \mathrm{~s}^{-1}$ angegeben.

\begin{tabular}{lllll}
\hline Nr. & Linie & Abbildung & $N_{\text {data }}$ & Bemerkungen \\
\hline 1 & He II 4686 & A.46 & 18 & \\
2 & H $\beta$ & A.47 & 18 & \\
3 & HeI 5875 & A.48 & 18 & \\
4 & H $\alpha$ & A.49 & 18 & \\
\hline
\end{tabular}

\begin{tabular}{lllllll}
\hline Nr. & $A_{67}$ & $\Delta_{\Phi 67}$ & $\chi_{\text {red }}^{2}$ & $\langle$ base $\rangle$ & $A_{67} /\langle$ base $\rangle$ & $\bmod _{67}$ \\
\hline 1 & $362 \pm 46$ & +0.025 & 1.52 & 1130 & 0.319 & 0.484 \\
2 & $4108 \pm 280$ & +0.010 & 0.91 & 9030 & 0.455 & 0.625 \\
3 & $891 \pm 58$ & -0.005 & 0.97 & 1830 & 0.486 & 0.654 \\
4 & $2770 \pm 190$ & +0.010 & 0.54 & 8090 & 0.342 & 0.510 \\
\hline
\end{tabular}



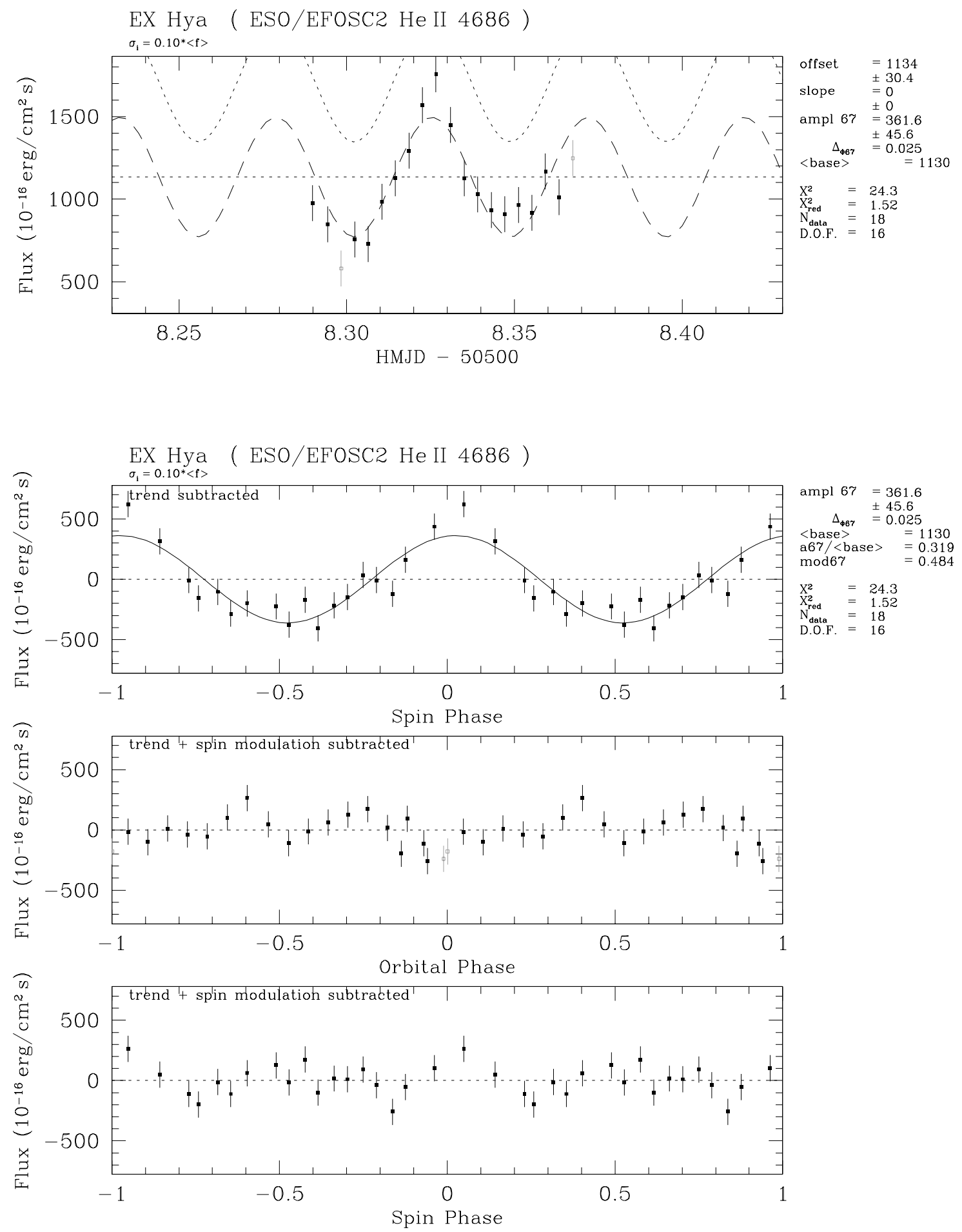

Abbildung A.46: Fit an EFOSC2-Lichtkurve He II 4686;

Modell: $f_{\text {line }}=C+A_{67} \cdot \cos \left(\Phi_{67}-\Delta_{\Phi 67}\right)$;

oben: $f(t)$; unten: $f\left(\Phi_{67}\right)$ und $f\left(\Phi_{98}\right)$, konstanter Offset abgezogen. 

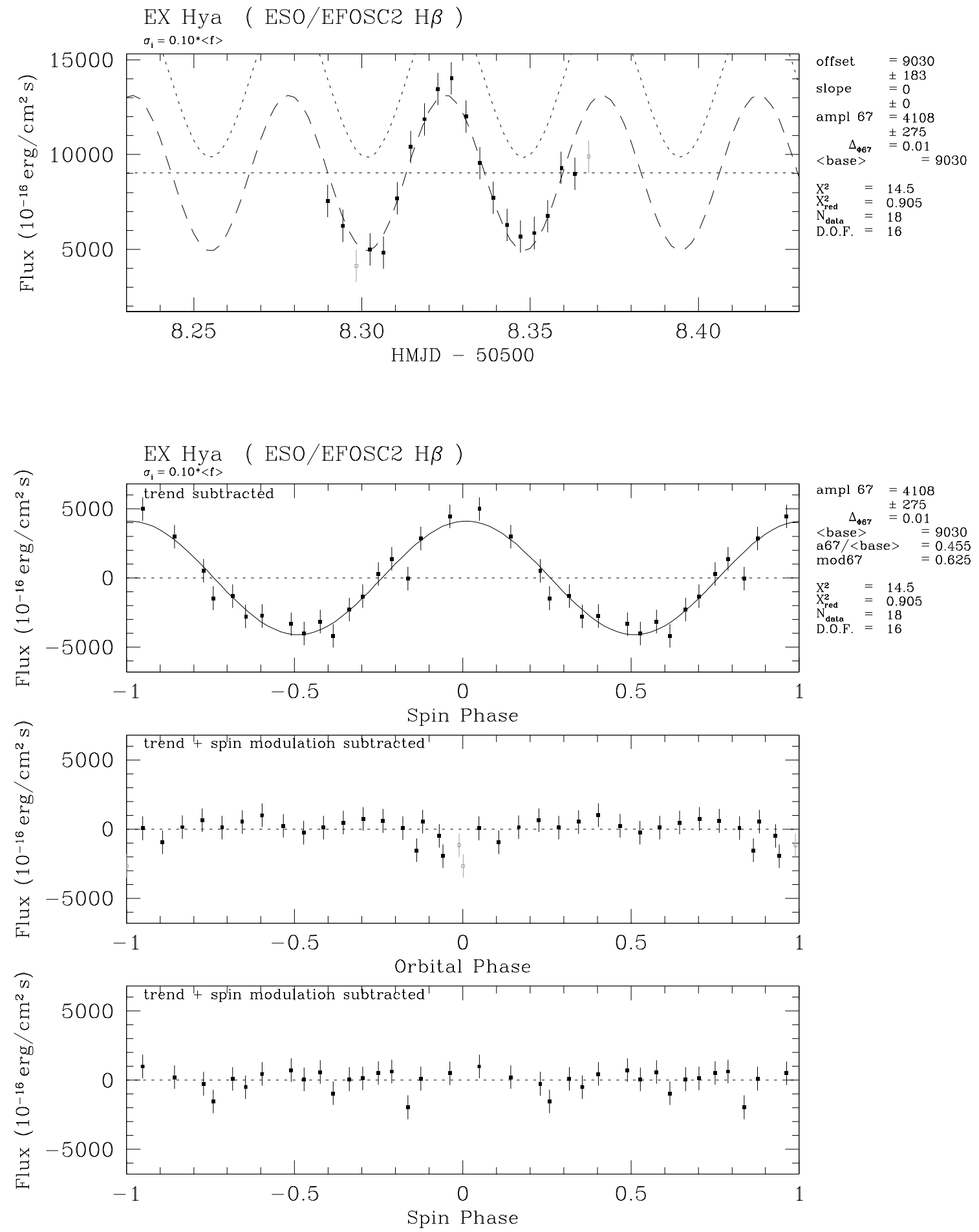

Abbildung A.47: Fit an EFOSC2-Lichtkurve $\mathrm{H} \beta$;

Modell: $f_{\text {line }}=C+A_{67} \cdot \cos \left(\Phi_{67}-\Delta_{\Phi 67}\right)$;

oben: $f(t)$; unten: $f\left(\Phi_{67}\right)$ und $f\left(\Phi_{98}\right)$, konstanter Offset abgezogen. 

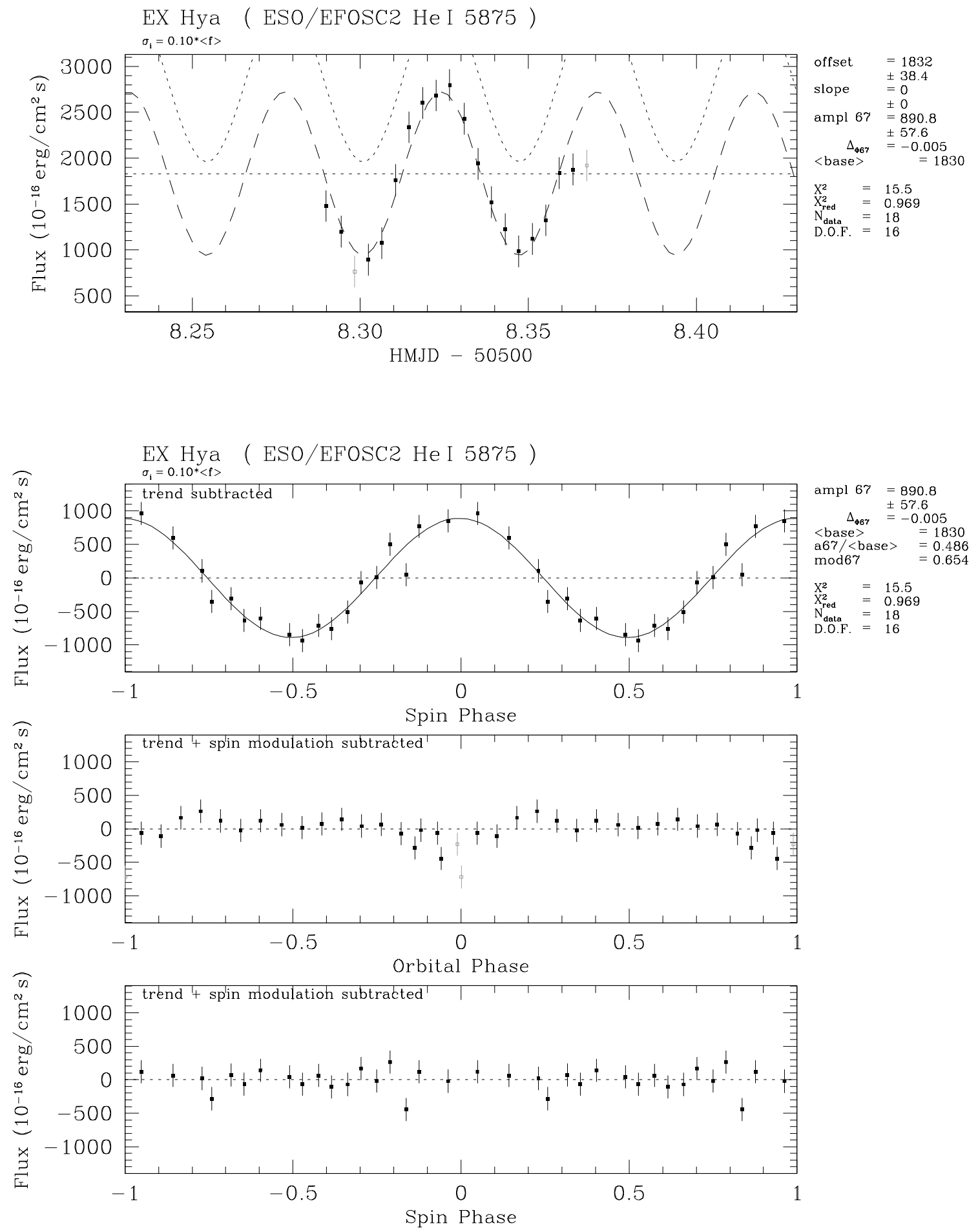

Abbildung A.48: Fit an EFOSC2-Lichtkurve He I 5875;

Modell: $f_{\text {line }}=C+A_{67} \cdot \cos \left(\Phi_{67}-\Delta_{\Phi 67}\right)$;

oben: $f(t)$; unten: $f\left(\Phi_{67}\right)$ und $f\left(\Phi_{98}\right)$, konstanter Offset abgezogen. 

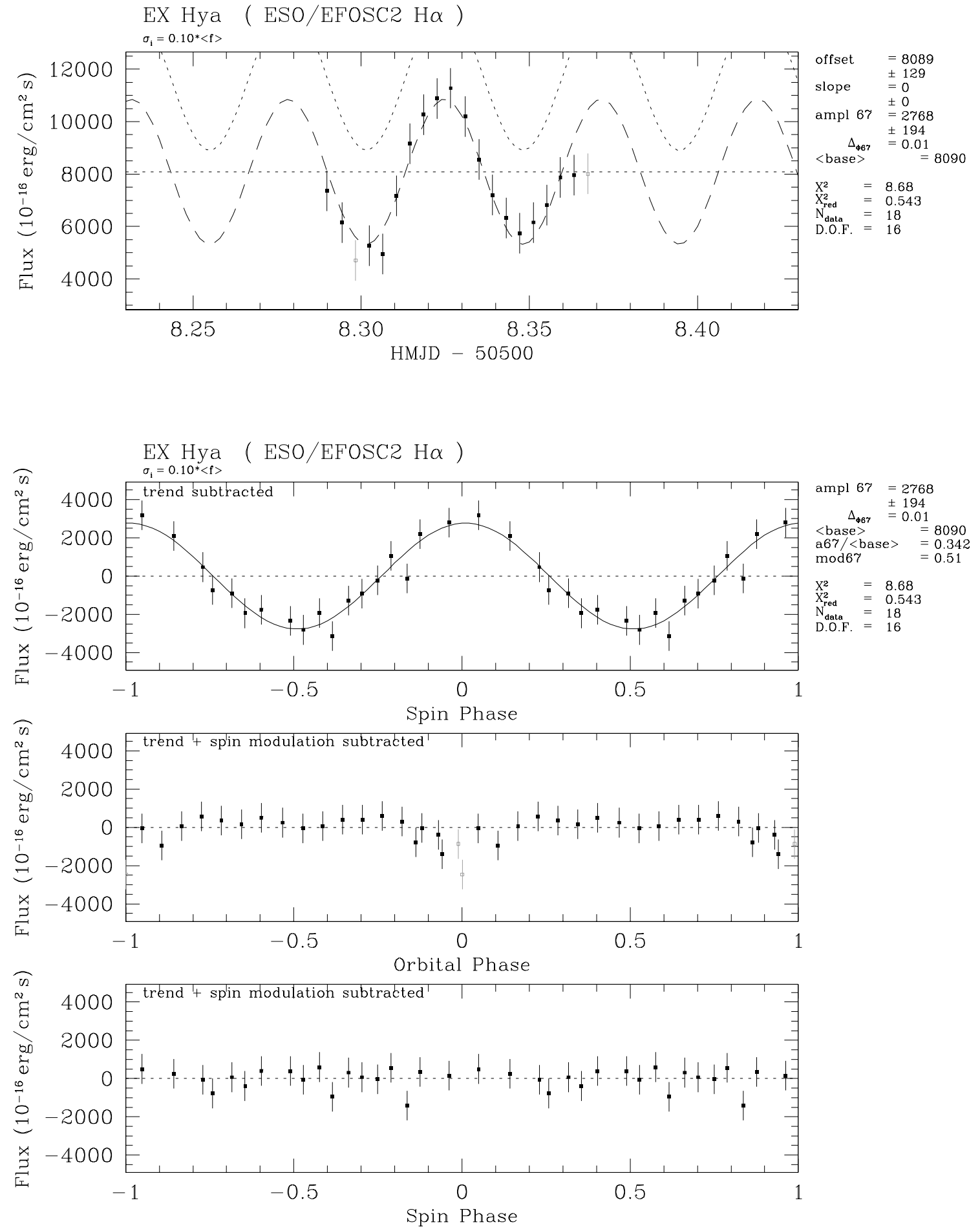

Abbildung A.49: Fit an EFOSC2-Lichtkurve $\mathrm{H} \alpha$;

Modell: $f_{\text {line }}=C+A_{67} \cdot \cos \left(\Phi_{67}-\Delta_{\Phi 67}\right)$;

oben: $f(t)$; unten: $f\left(\Phi_{67}\right)$ und $f\left(\Phi_{98}\right)$, konstanter Offset abgezogen. 


\section{A.4 IRS-Lichtkurven}

\section{A.4.1 IRS-Lichtkurven: Kontinuumsflüsse}

Tabelle A.7: Übersicht über die Anpassungen an die IRS-Kontinuumslichtkurven: Definition der Bänder, best-fit-Amplituden, mittlerer Fluß und Modulationsgrad. $A_{67}, A_{49}$ und 〈base〉 sind in Einheiten von $10^{-16} \mathrm{erg} \mathrm{cm}^{-2} \mathrm{~s}^{-1} \AA^{-1}$ angegeben.

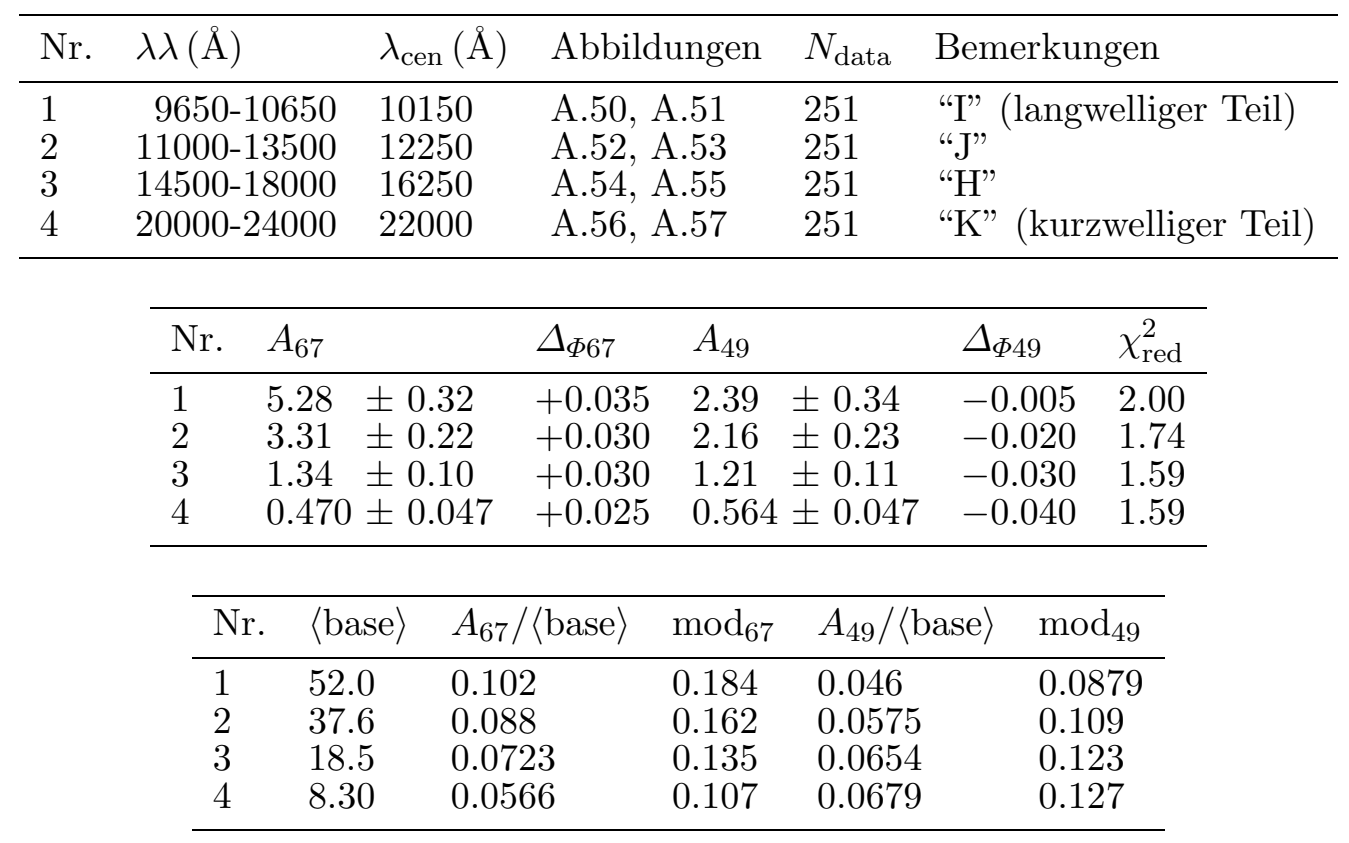



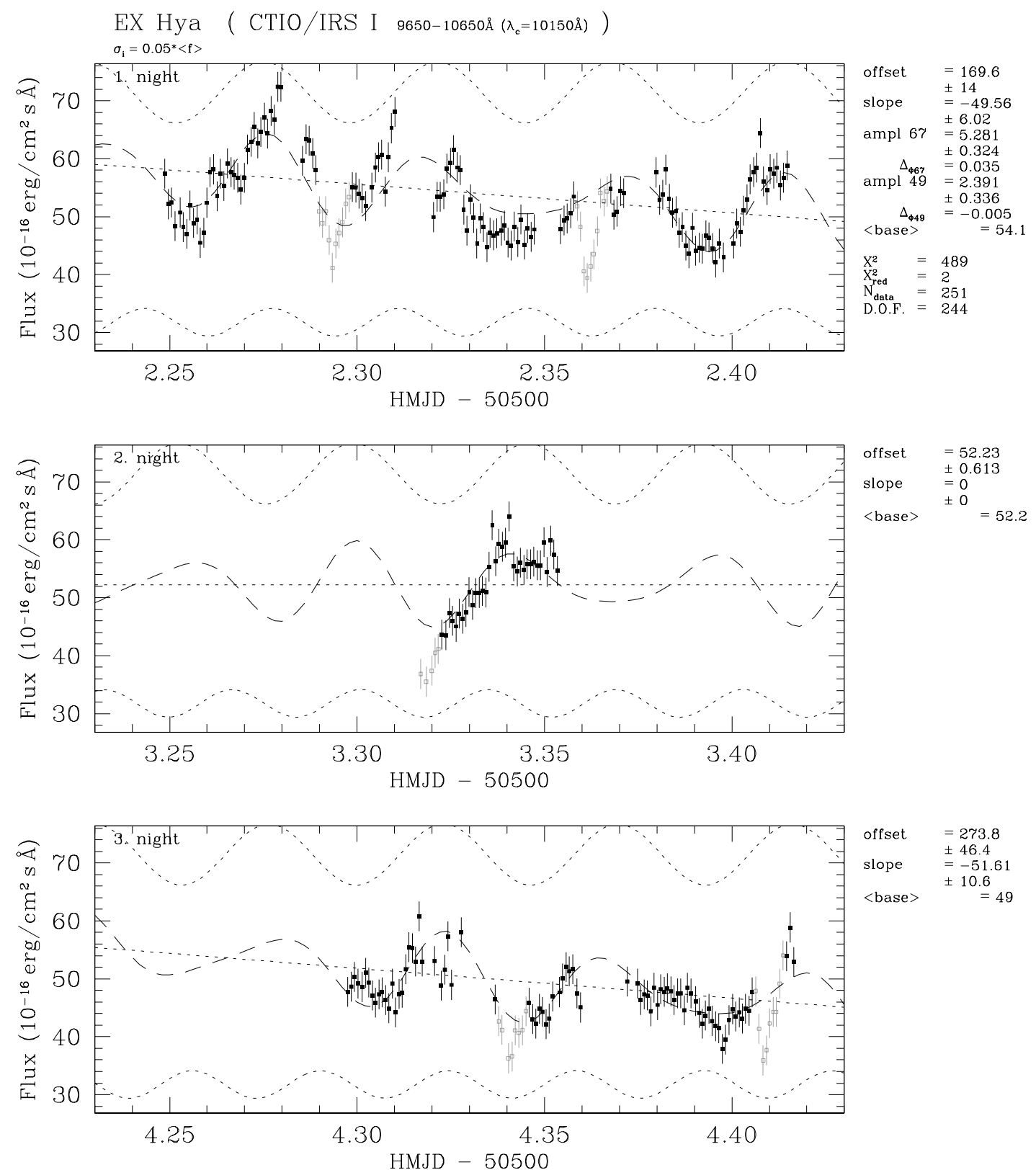

Abbildung A.50: Fit an IRS-Lichtkurve 9650...10650 A ("I", langwelliger Teil; $\left.\lambda_{\text {cen }}=10150 \AA\right)$;

Modell: $\left\langle f_{\lambda}\right\rangle_{\text {band }}=$ base $+A_{67} \cdot \cos \left(\Phi_{67}-\Delta_{\Phi 67}\right)-A_{49} \cdot \cos \left(\Phi_{49}-\Delta_{\Phi 49}\right)$ mit base $\equiv \sum_{i=1}^{3}\left(C_{i}+B_{i} \cdot(\right.$ HMJD -50500$\left.)\right)$. 


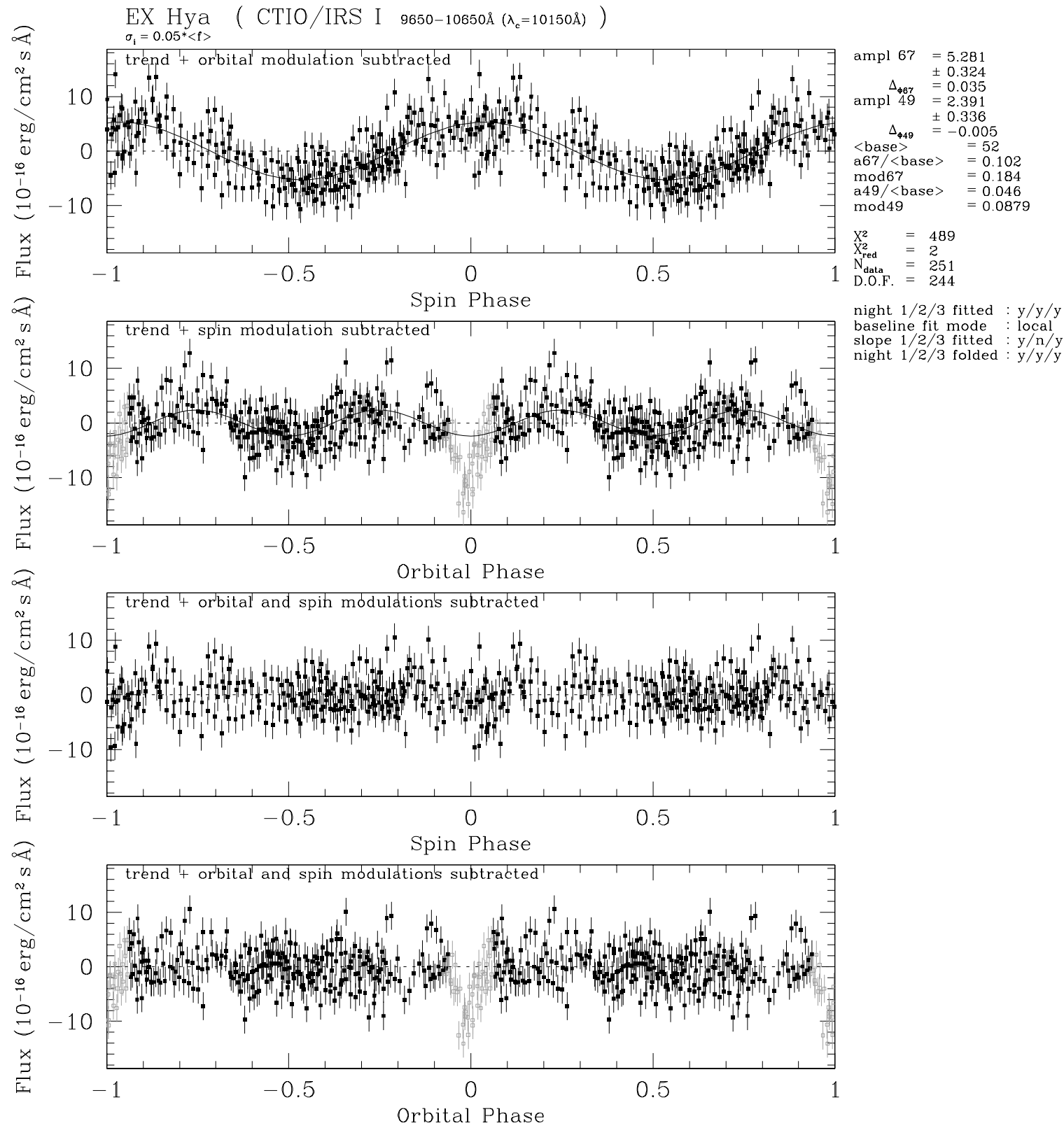

Abbildung A.51: Fit aus Abbildung A.50 über $\Phi_{67}$ bzw. $\Phi_{98}$ gefaltet, lineare Komponenten abgezogen. 

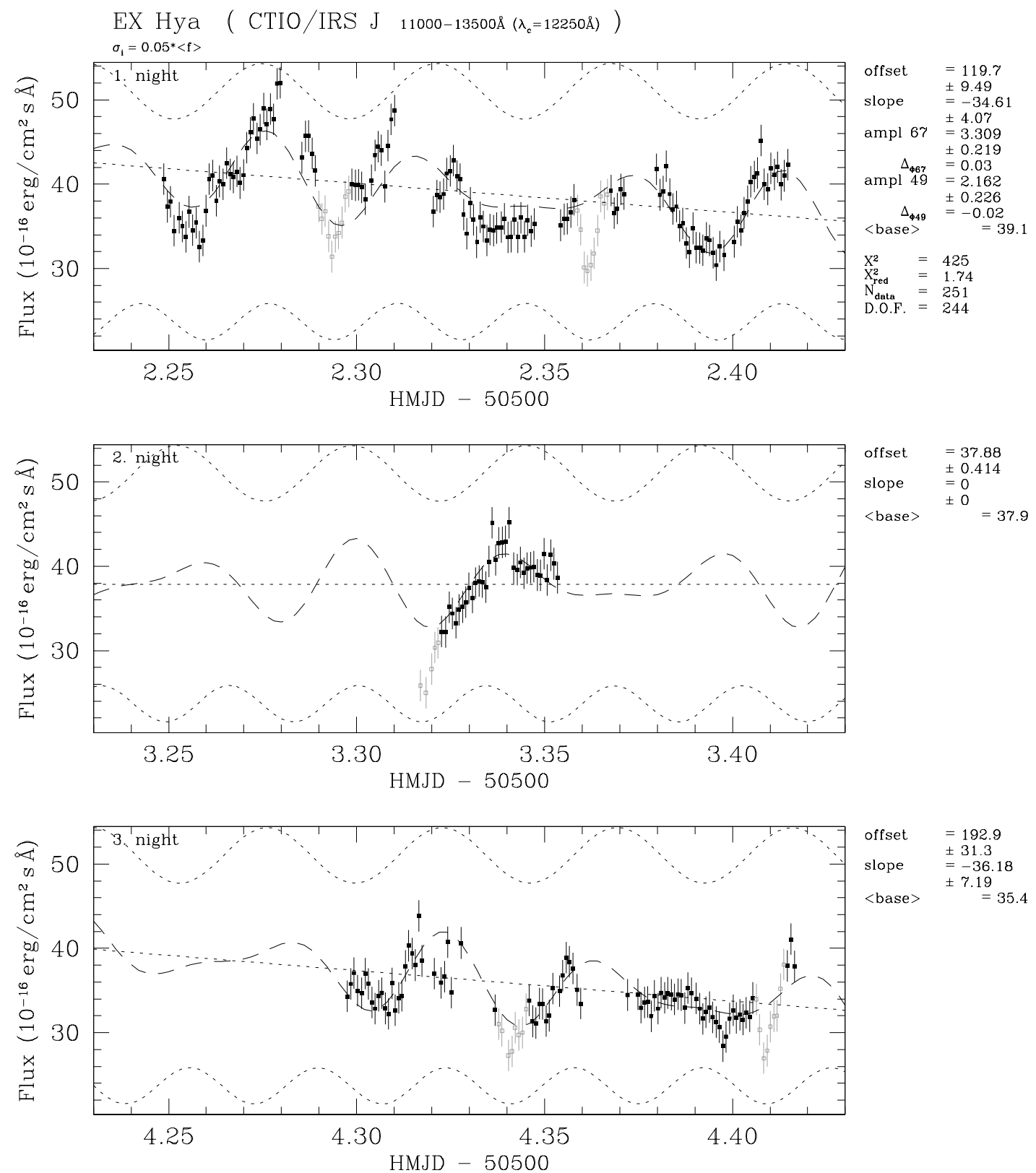

Abbildung A.52: Fit an IRS-Lichtkurve 11000...13500 $\AA$ ("J"; $\lambda_{\text {cen }}=12250 \AA$ );

Modell: $\left\langle f_{\lambda}\right\rangle_{\text {band }}=$ base $+A_{67} \cdot \cos \left(\Phi_{67}-\Delta_{\Phi 67}\right)-A_{49} \cdot \cos \left(\Phi_{49}-\Delta_{\Phi 49}\right)$

mit base $\equiv \sum_{i=1}^{3}\left(C_{i}+B_{i} \cdot(\right.$ HMJD -50500$\left.)\right)$. 


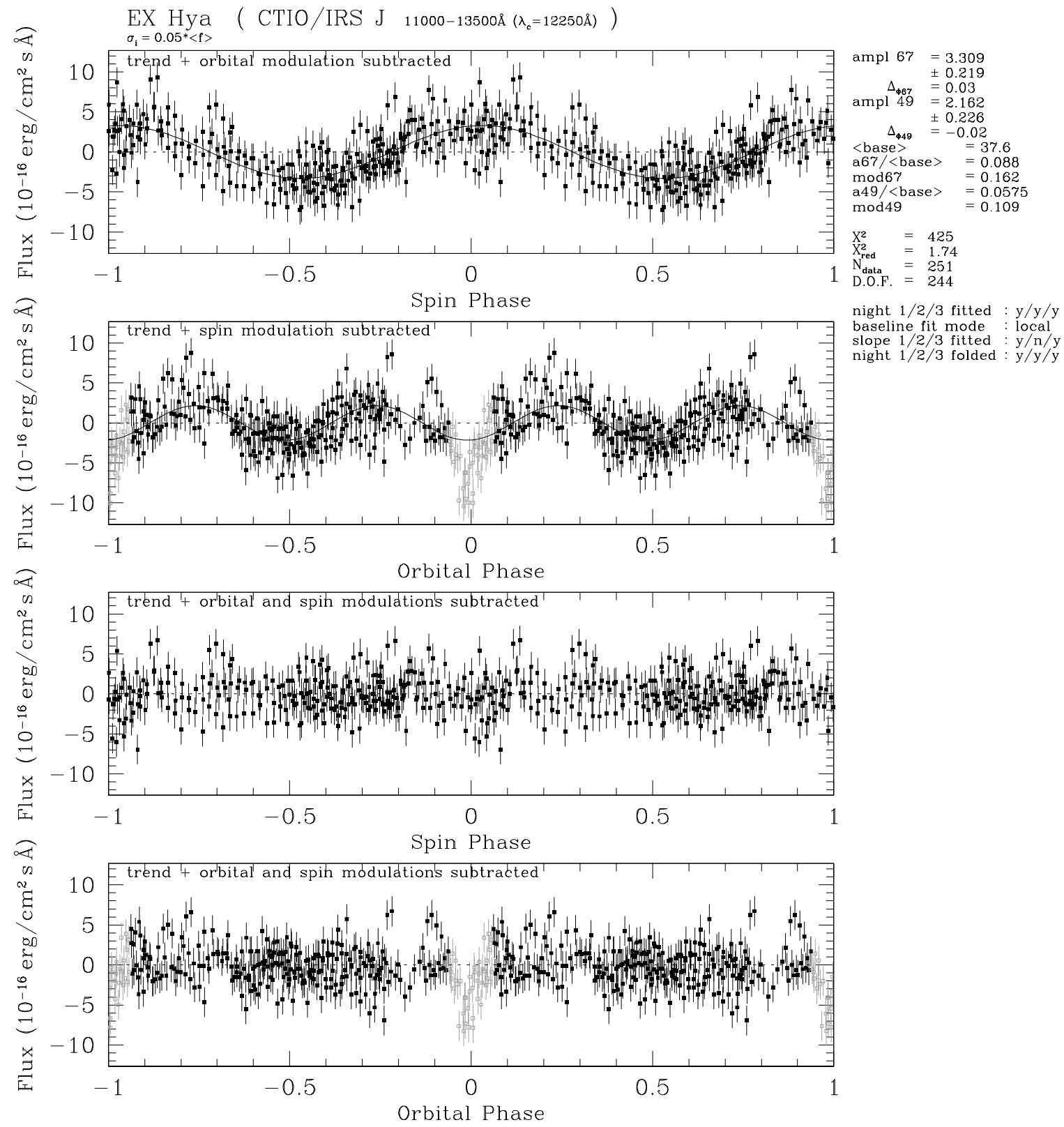

Abbildung A.53: Fit aus Abbildung A.52 über $\Phi_{67}$ bzw. $\Phi_{98}$ gefaltet, lineare Komponenten abgezogen. 

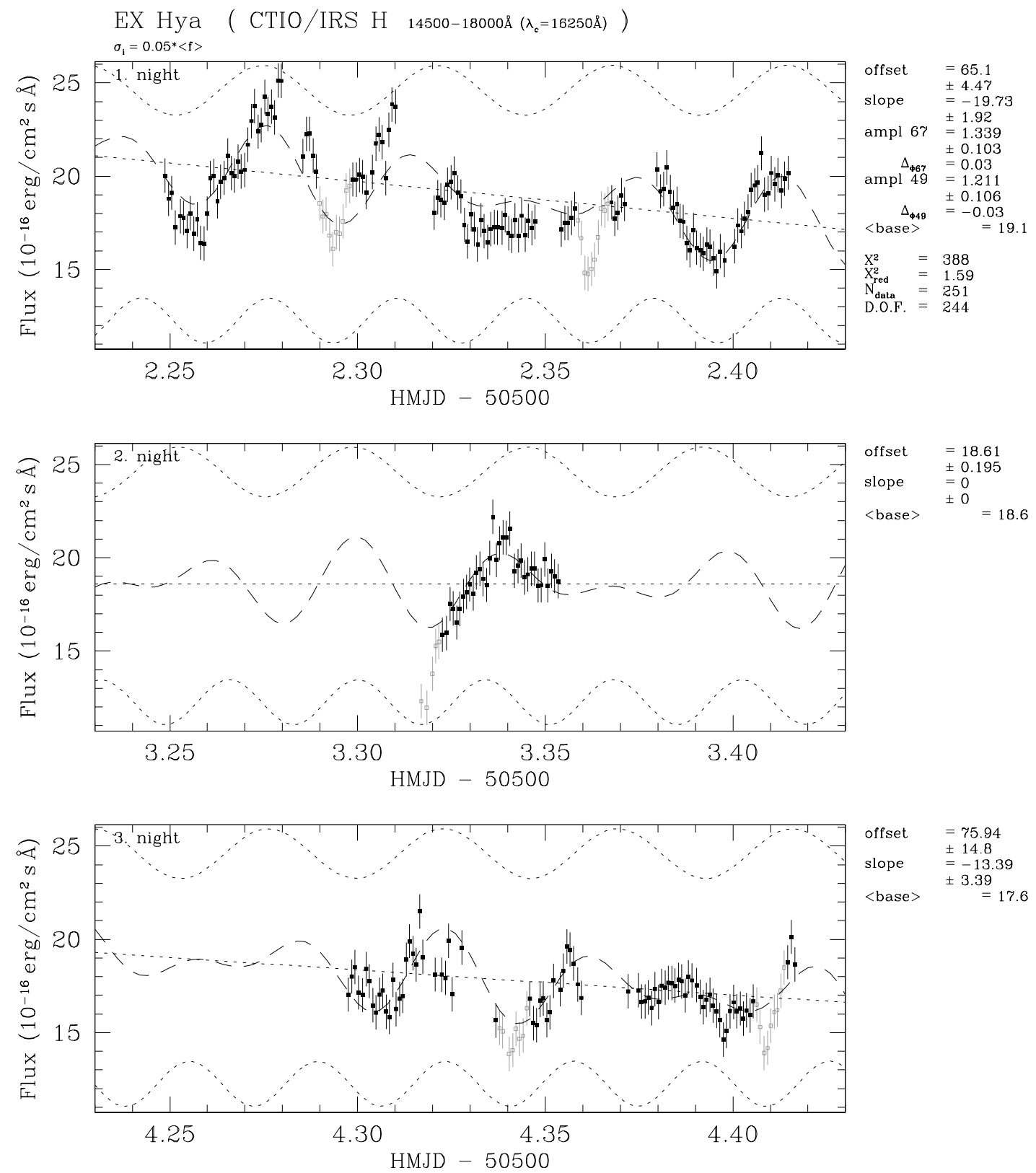

Abbildung A.54: Fit an IRS-Lichtkurve 14500...18000 $\AA$ ("H"; $\lambda_{\text {cen }}=16250 \AA$ );

Modell: $\left\langle f_{\lambda}\right\rangle_{\text {band }}=$ base $+A_{67} \cdot \cos \left(\Phi_{67}-\Delta_{\Phi 67}\right)-A_{49} \cdot \cos \left(\Phi_{49}-\Delta_{\Phi 49}\right)$

mit base $\equiv \sum_{i=1}^{3}\left(C_{i}+B_{i} \cdot(\right.$ HMJD -50500$\left.)\right)$. 


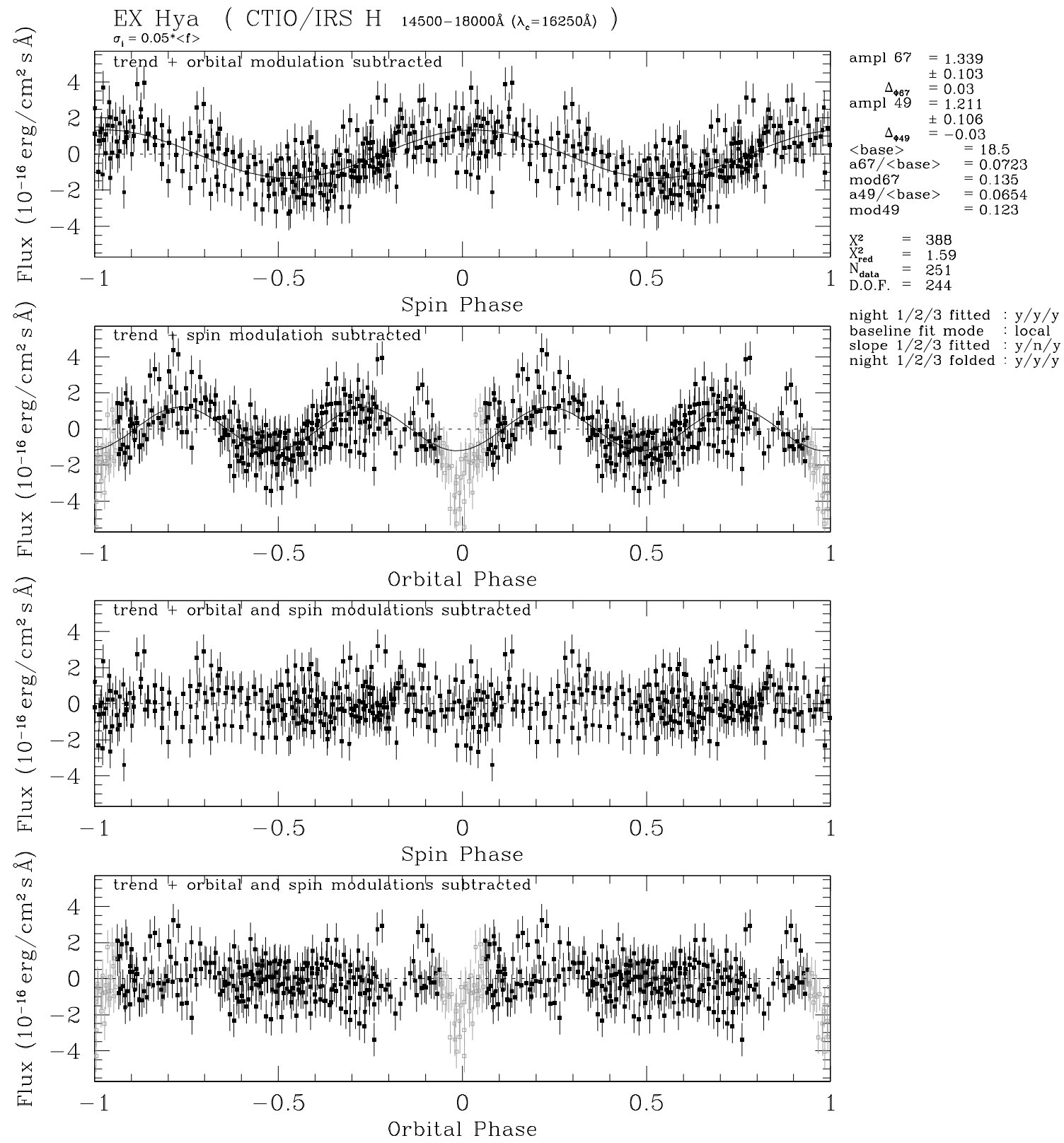

Abbildung A.55: Fit aus Abbildung A.54 über $\Phi_{67}$ bzw. $\Phi_{98}$ gefaltet, lineare Komponenten abgezogen. 

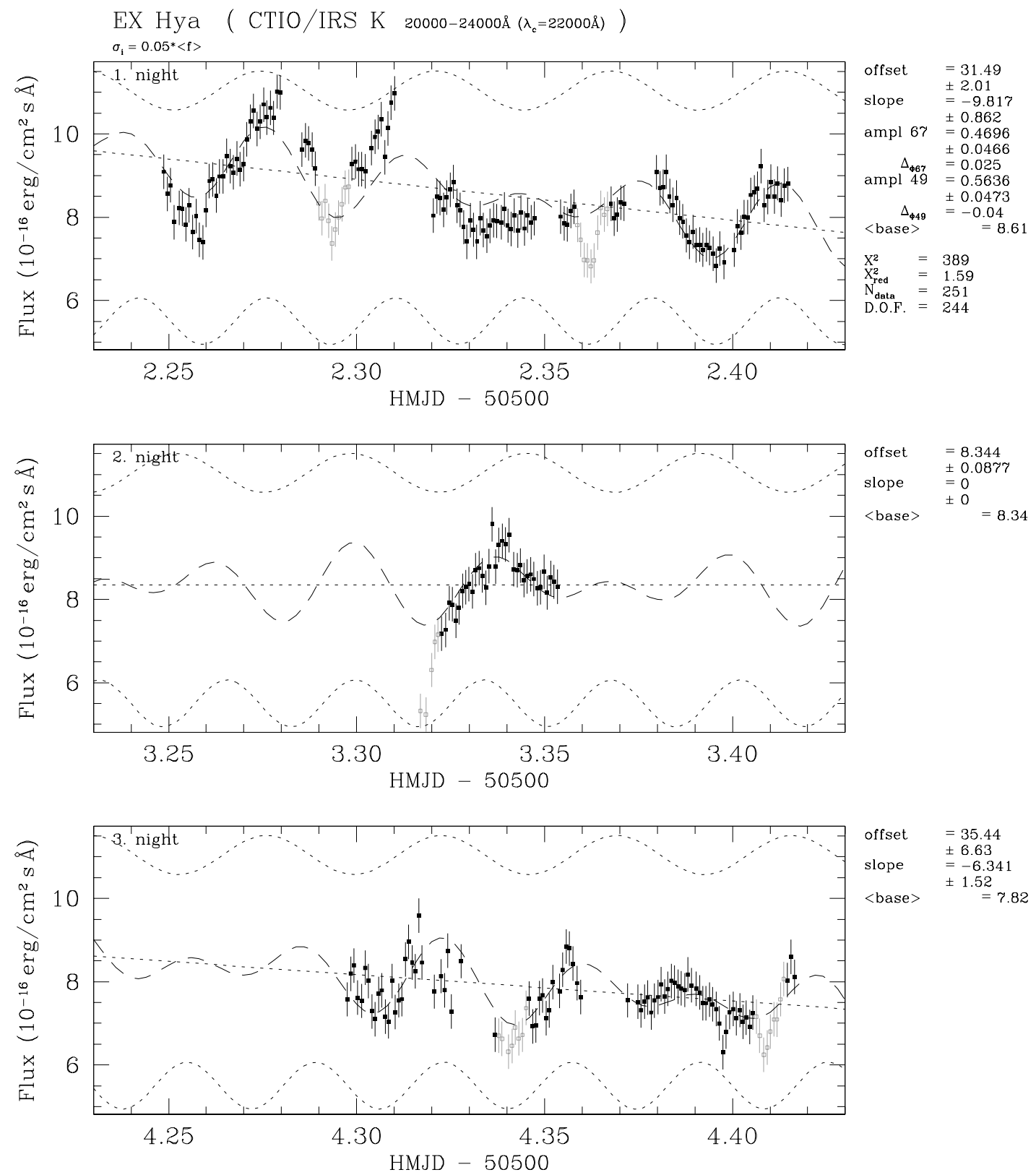

Abbildung A.56: Fit an IRS-Lichtkurve 20000...24000 $\AA$ ("K"; $\lambda_{\text {cen }}=22000 \AA$ );

Modell: $\left\langle f_{\lambda}\right\rangle_{\text {band }}=$ base $+A_{67} \cdot \cos \left(\Phi_{67}-\Delta_{\Phi 67}\right)-A_{49} \cdot \cos \left(\Phi_{49}-\Delta_{\Phi 49}\right)$

mit base $\equiv \sum_{i=1}^{3}\left(C_{i}+B_{i} \cdot(\right.$ HMJD -50500$\left.)\right)$. 


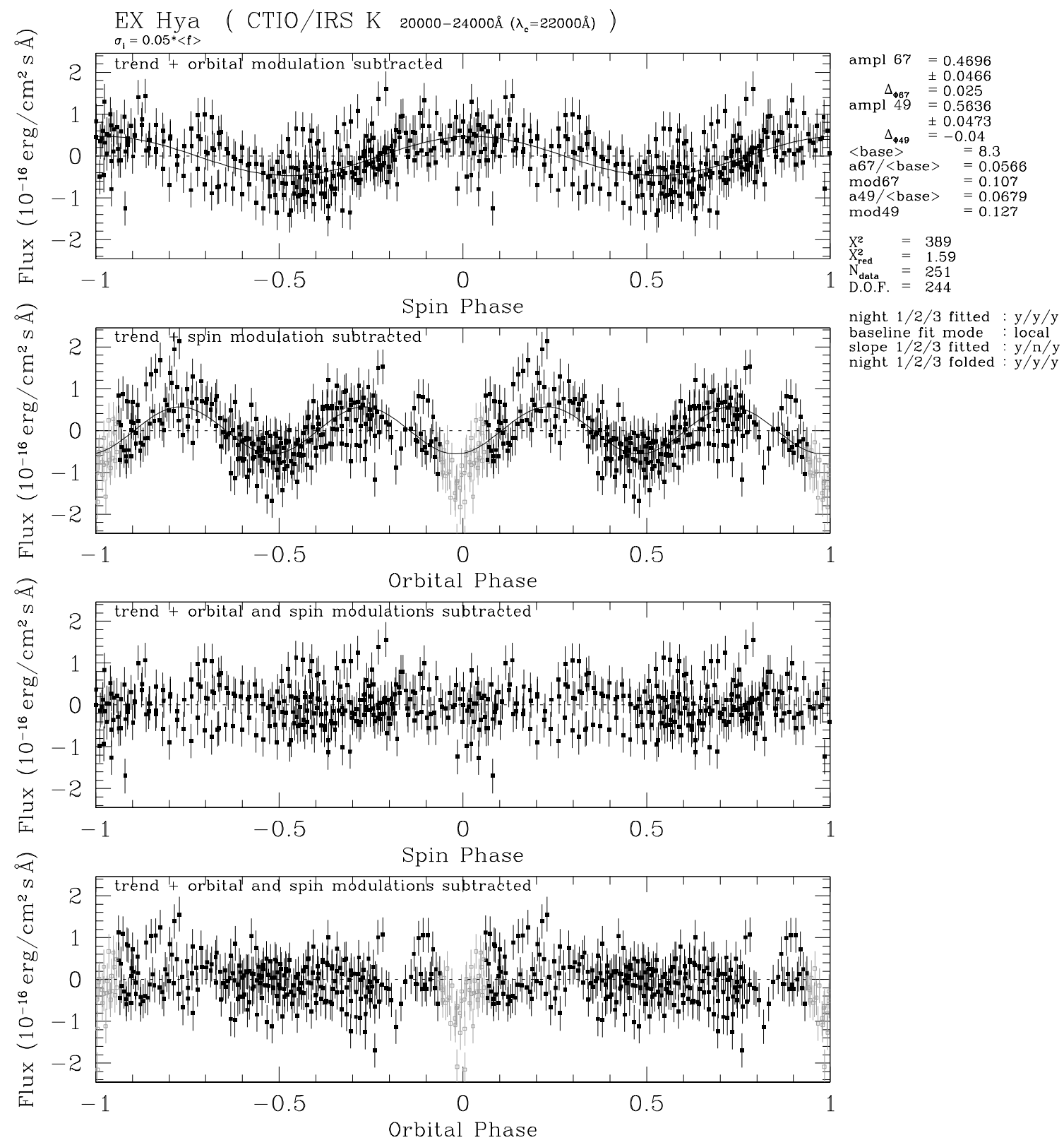

Abbildung A.57: Fit aus Abbildung A.56 über $\Phi_{67}$ bzw. $\Phi_{98}$ gefaltet, lineare Komponenten abgezogen. 


\section{A.4.2 IRS-Lichtkurven: Linienflüsse}

Tabelle A.8: Übersicht über die Anpassungen an die IRS-Emissionslinienlichtkurven: bestfit-Amplituden, mittlerer Fluß und Modulationsgrad. $A_{67}, A_{49}, A_{98}$ und $\langle$ base $\rangle$ sind in Einheiten von $10^{-16} \mathrm{erg} \mathrm{cm}^{-2} \mathrm{~s}^{-1}$ angegeben.

\begin{tabular}{|c|c|c|c|c|}
\hline $\mathrm{Nr}$. & Linie & Abbildungen & $N_{\text {data }}$ & Bemerkungen \\
\hline 1 & $\mathrm{P} \delta$ & A.58, A.59 & 251 & \multirow{4}{*}{ Linien nicht trennbar } \\
\hline 2 & $\mathrm{P} \gamma / \mathrm{He}$ I 10830 & A.60, A.61 & 251 & \\
\hline 3 & $\mathrm{P} \beta^{\prime}$ & A.62, A.63 & 251 & \\
\hline 4 & $\mathrm{~B} \gamma$ & A.64, A.65 & 251 & \\
\hline
\end{tabular}

\begin{tabular}{llllllll}
\hline Nr. & $A_{67}$ & $\Delta_{\Phi 67}$ & $A_{49}$ & $\Delta_{\Phi 49}$ & $A_{98}$ & $\Delta_{\Phi 98}$ & $\chi_{\text {red }}^{2}$ \\
\hline 1 & $412 \pm 14$ & +0.020 & $47.7 \pm 15$ & +0.025 & $139 \pm 17$ & +0.330 & 2.35 \\
2 & $755 \pm 39$ & +0.020 & $284 \pm 39$ & +0.045 & $359 \pm 41$ & +0.215 & 1.83 \\
3 & $334 \pm 15$ & +0.020 & $99.9 \pm 15$ & +0.015 & $157 \pm 17$ & +0.275 & 2.36 \\
4 & $7.64 \pm 4.8$ & +0.000 & $30.0 \pm 4.7$ & -0.025 & $40.5 \pm 5.2$ & +0.240 & 3.09 \\
\hline
\end{tabular}

\begin{tabular}{llllllll}
\hline Nr. & $\langle$ base $\rangle$ & $A_{67} /\langle$ base $\rangle$ & $\bmod _{67}$ & $A_{49} /\langle$ base $\rangle$ & $\bmod _{49}$ & $A_{98} /\langle$ base $\rangle$ & $\bmod _{98}$ \\
\hline 1 & 2060 & 0.200 & 0.333 & 0.0231 & 0.0452 & 0.0674 & 0.126 \\
2 & 6260 & 0.121 & 0.215 & 0.0454 & 0.0869 & 0.0574 & 0.109 \\
3 & 2080 & 0.161 & 0.277 & 0.0480 & 0.0917 & 0.0754 & 0.140 \\
4 & 585 & 0.0131 & 0.0258 & 0.0513 & 0.0976 & 0.0693 & 0.130 \\
\hline
\end{tabular}



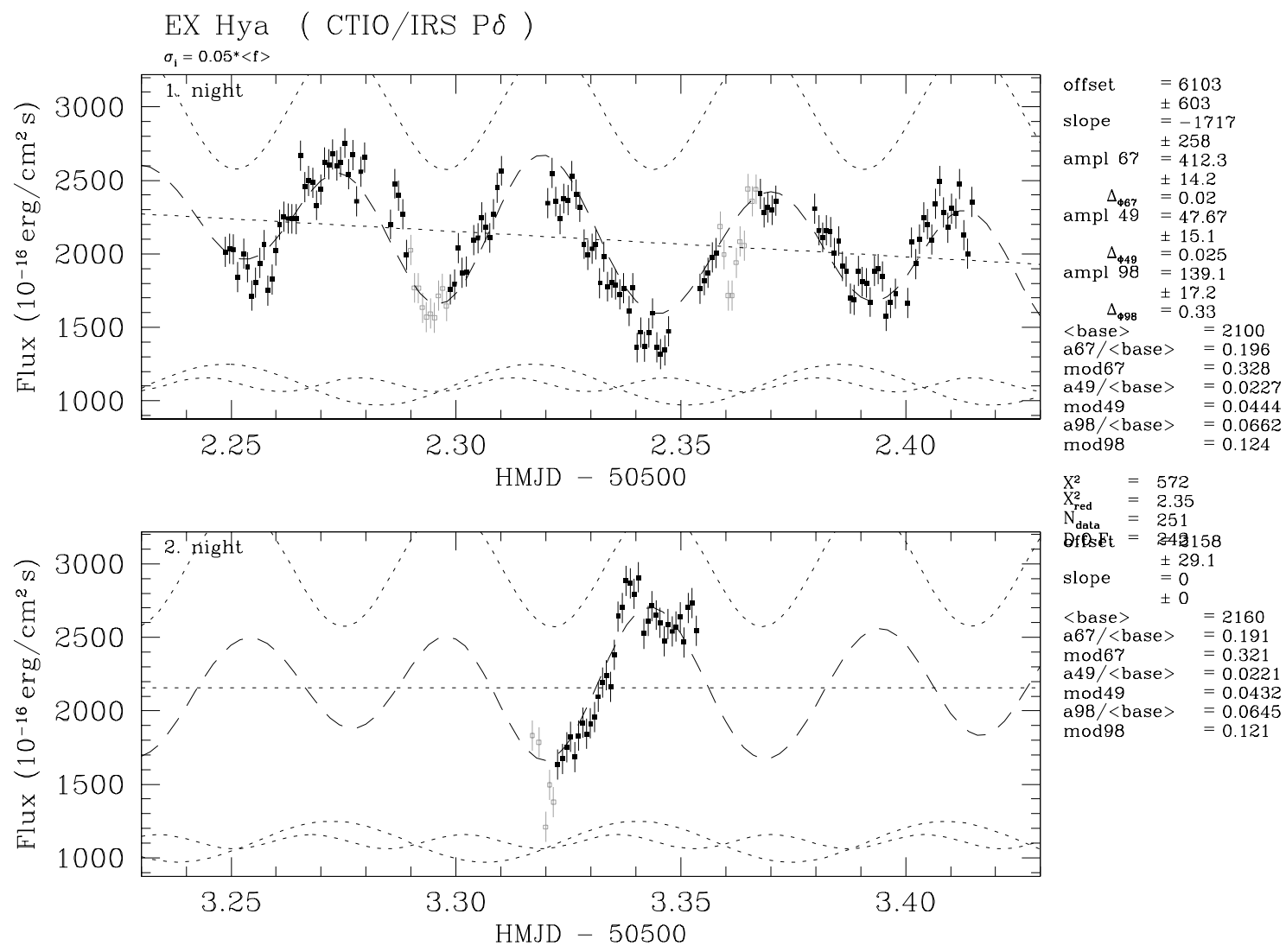

$\begin{array}{ll}\mathrm{X}^{2} & =572 \\ \mathrm{X}_{\text {red }}^{2} & =2.35\end{array}$

$\begin{array}{ll}X_{\text {red }}^{2} & =2.35 \\ \mathrm{~N}_{\text {data }} & =251\end{array}$

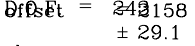

slope $\quad=0$

$<$ base $>\quad=2160$

a $67 /<$ base $>=0.191$

$\bmod 67=0.321$

$\begin{array}{ll}\mathrm{a} 49 /<\mathrm{base}> & =0.0221 \\ \bmod 49 & =0.0432\end{array}$

$\bmod 49=0.0432$

a98/<base $>=0.0645$

$\bmod 98$

$=0.12$
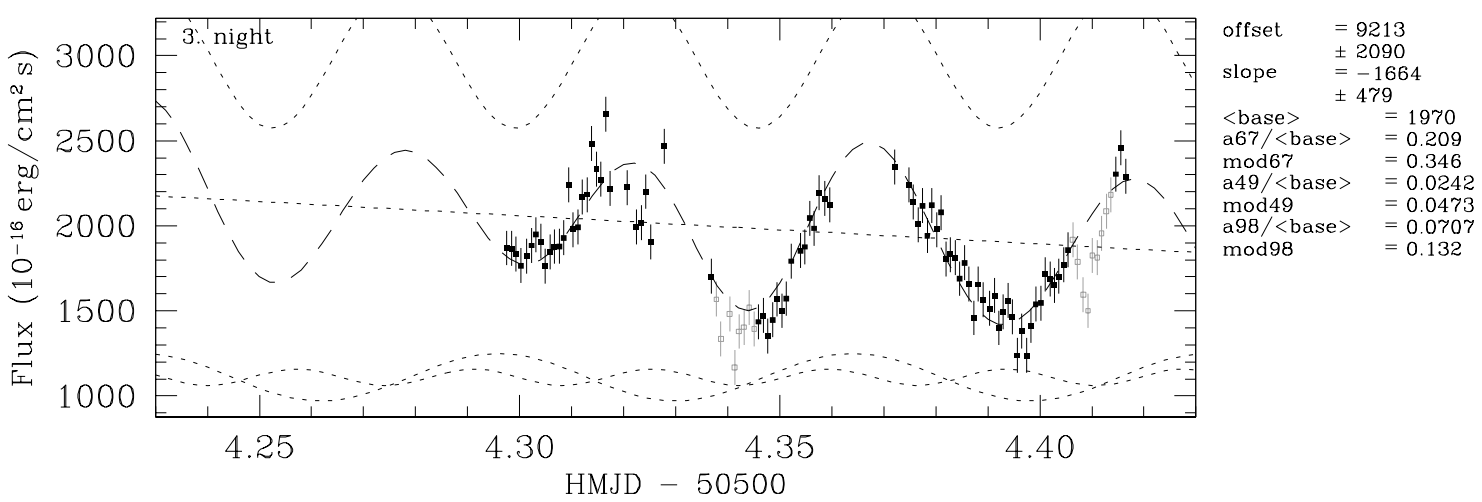

Abbildung A.58: Fit an IRS-Lichtkurve P $\delta$;

Modell:

$f_{\text {line }}=$ base $+A_{67} \cdot \cos \left(\Phi_{67}-\Delta_{\Phi 67}\right)-A_{49} \cdot \cos \left(\Phi_{49}-\Delta_{\Phi 49}\right)+A_{98} \cdot \cos \left(\Phi_{98}-\Delta_{\Phi 98}\right)$

mit base $\equiv \sum_{i=1}^{3}\left(C_{i}+B_{i} \cdot(\right.$ HMJD -50500$\left.)\right)$. 


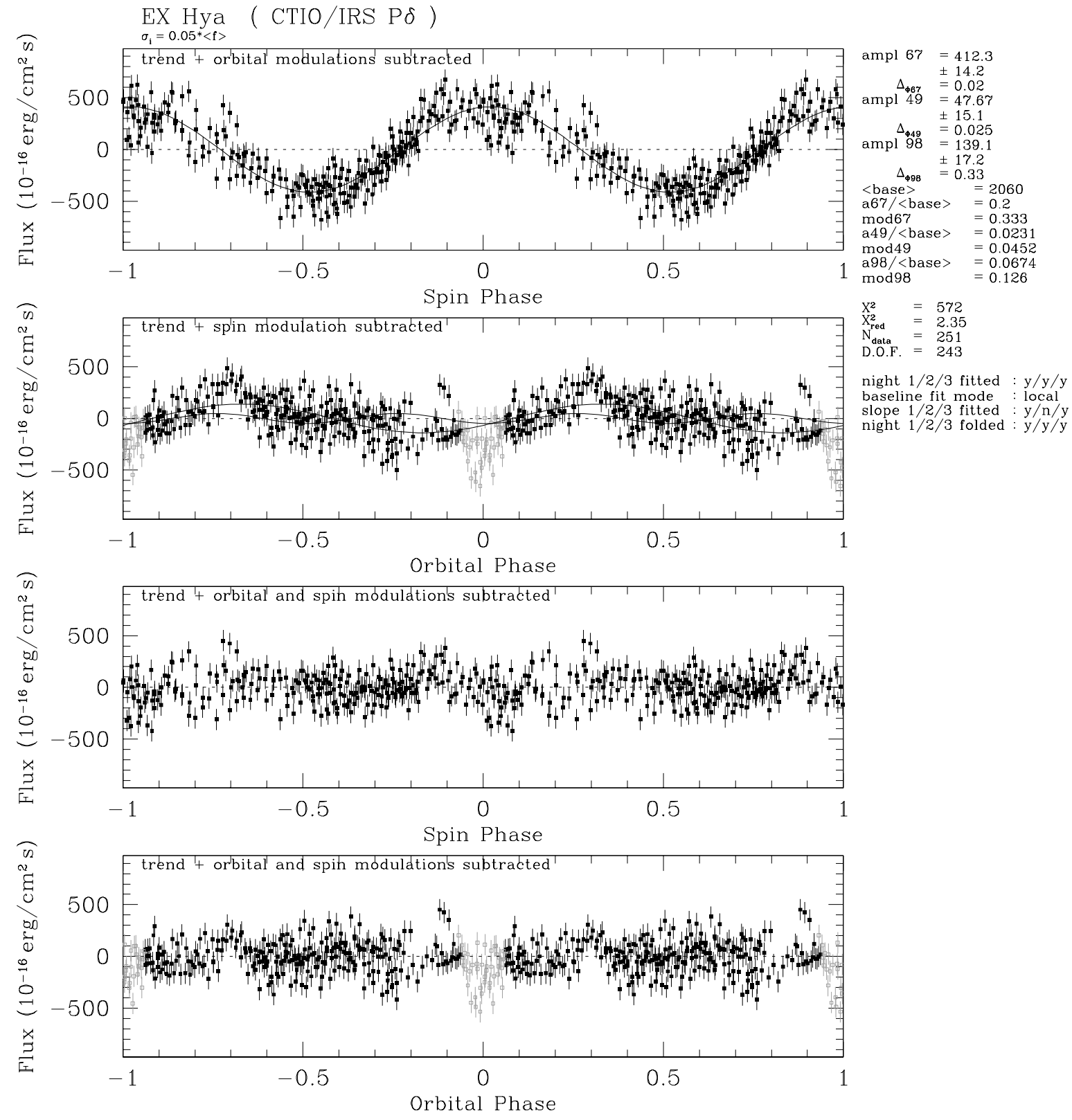

Abbildung A.59: Fit aus Abbildung A.58 über $\Phi_{67}$ bzw. $\Phi_{98}$ gefaltet, lineare Komponenten abgezogen. 

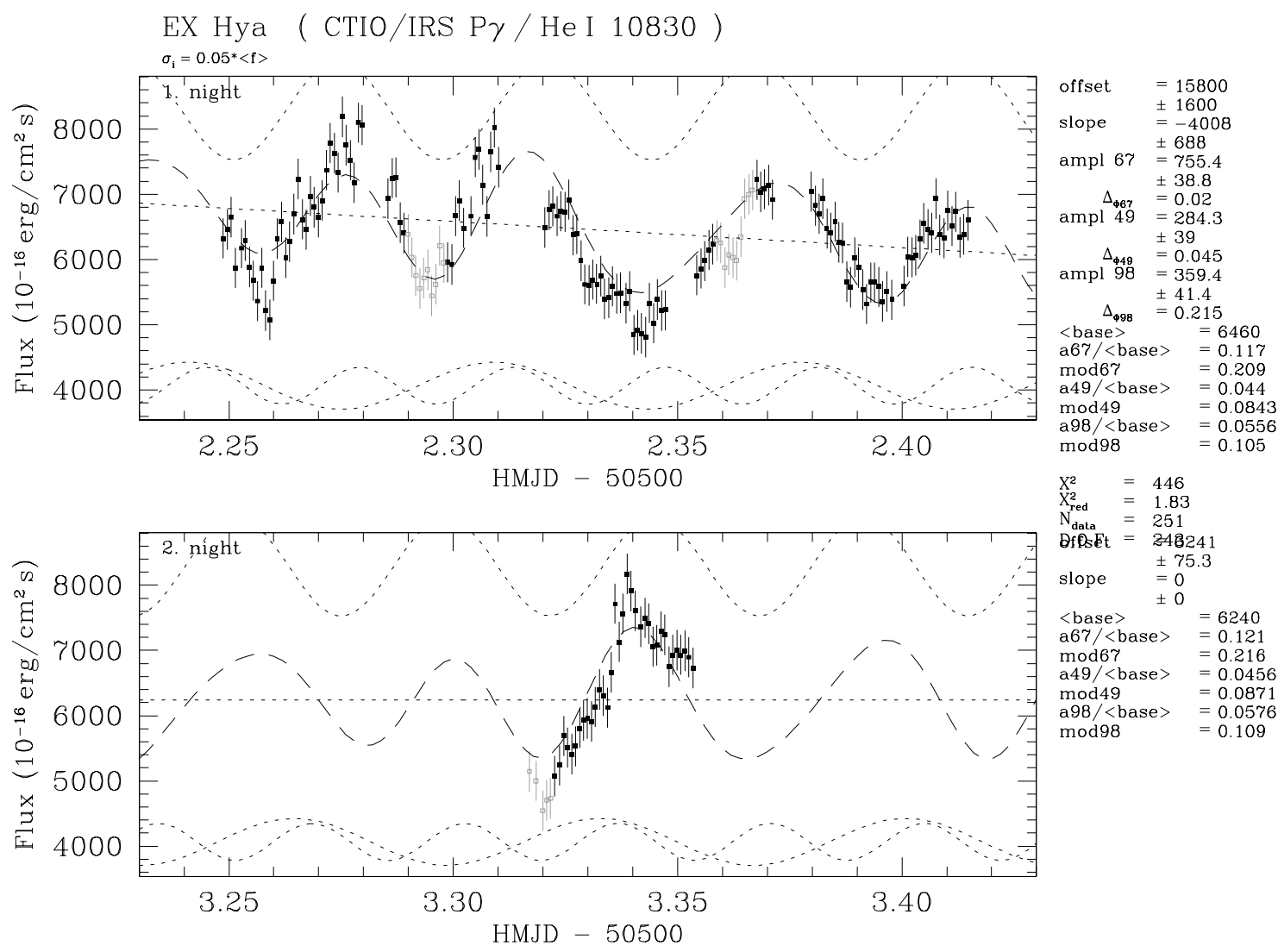

$\begin{array}{ll}\mathrm{X}^{2} & =446 \\ \mathrm{X}_{\text {red }}^{2} & =1.83\end{array}$

$\begin{array}{ll}\mathrm{X}_{\text {red }}^{2} & =1.83 \\ \mathrm{~N}_{\text {data }} & =251\end{array}$

$\begin{aligned} \text { DfOset }= & 248241 \\ & \pm 75.3\end{aligned}$

$\begin{array}{ll} & =0 \\ & \pm 0\end{array}$

$<$ base $>\quad \pm 0=6240$

a67 $/\langle$ base $>=0.121$

$\bmod 67=0.216$

a $49 /<$ base $>=0.0456$

$\bmod 49=0.0871$

$\begin{array}{ll}\bmod 98 & =0.0576 \\ & =0.109\end{array}$
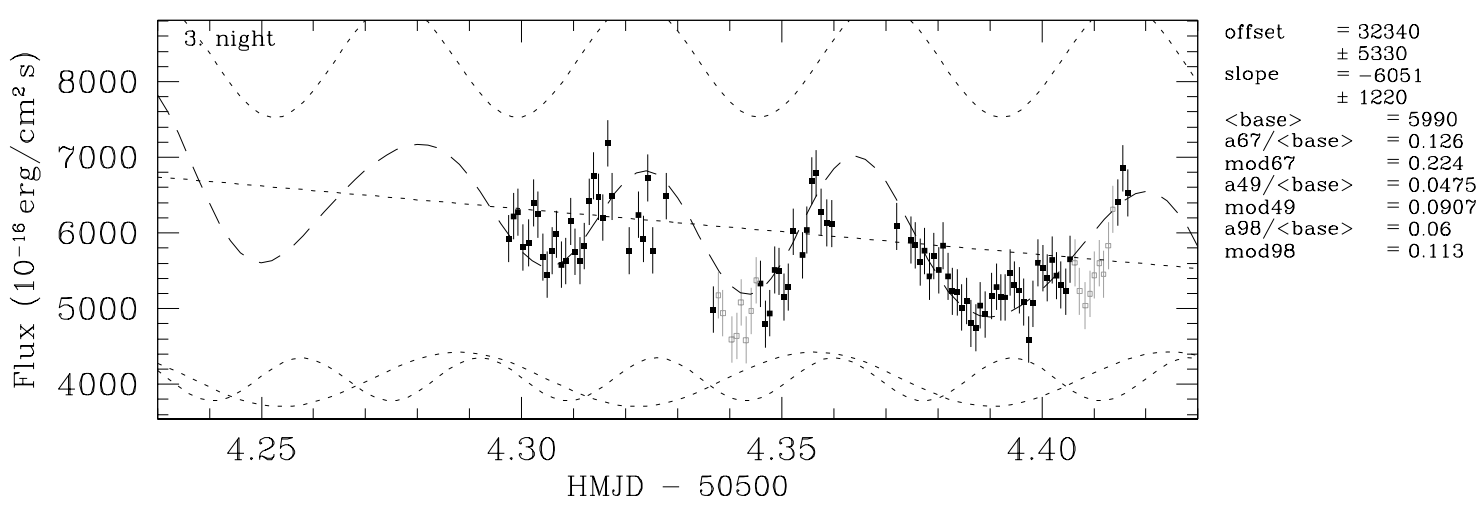

Abbildung A.60: Fit an IRS-Lichtkurve P $\gamma+$ HeI 10830;

Modell:

$f_{\text {line }}=$ base $+A_{67} \cdot \cos \left(\Phi_{67}-\Delta_{\Phi 67}\right)-A_{49} \cdot \cos \left(\Phi_{49}-\Delta_{\Phi 49}\right)+A_{98} \cdot \cos \left(\Phi_{98}-\Delta_{\Phi 98}\right)$

mit base $\equiv \sum_{i=1}^{3}\left(C_{i}+B_{i} \cdot(\right.$ HMJD -50500$\left.)\right)$. 


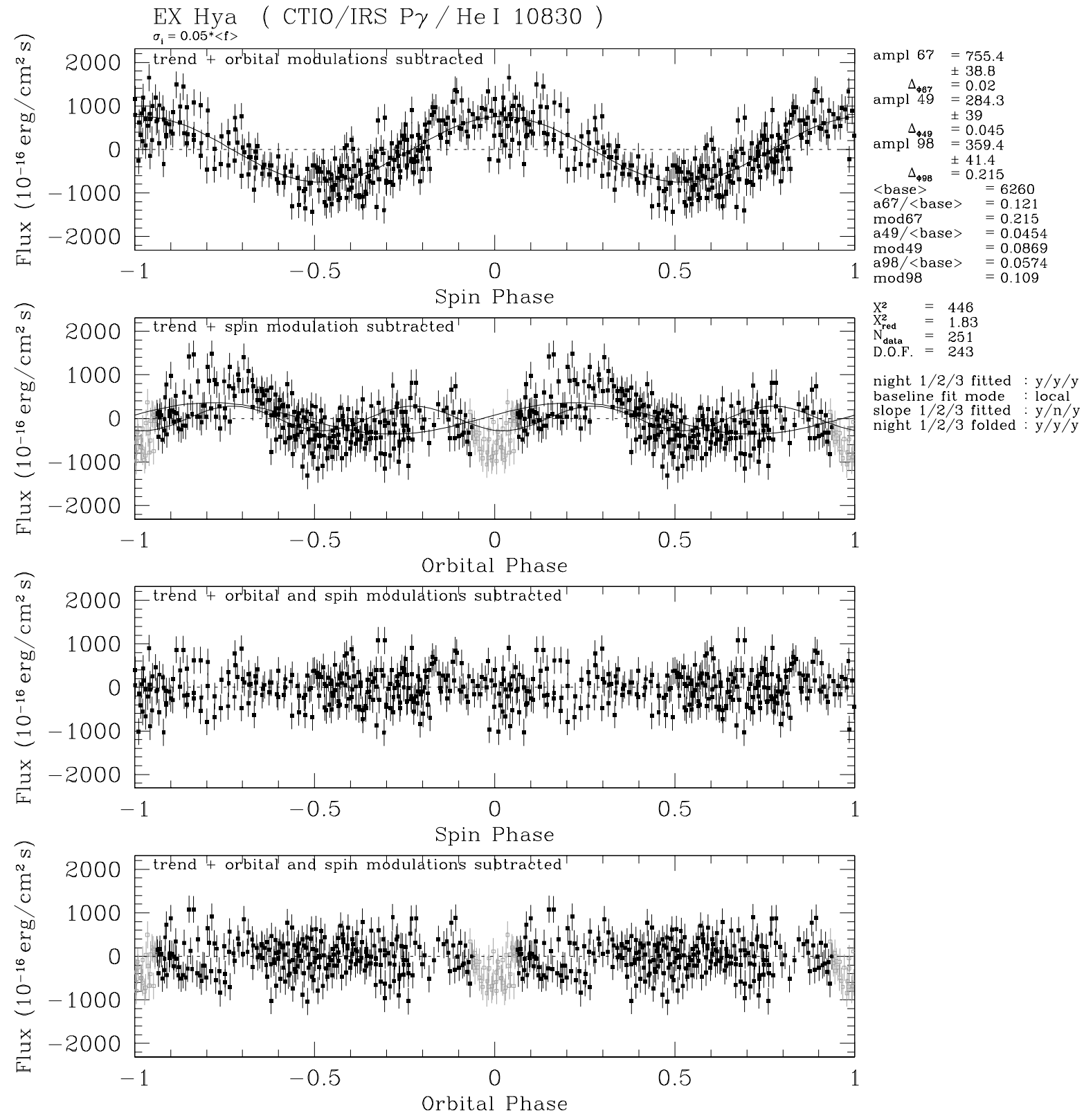

Abbildung A.61: Fit aus Abbildung A.60 über $\Phi_{67}$ bzw. $\Phi_{98}$ gefaltet, lineare Komponenten abgezogen. 

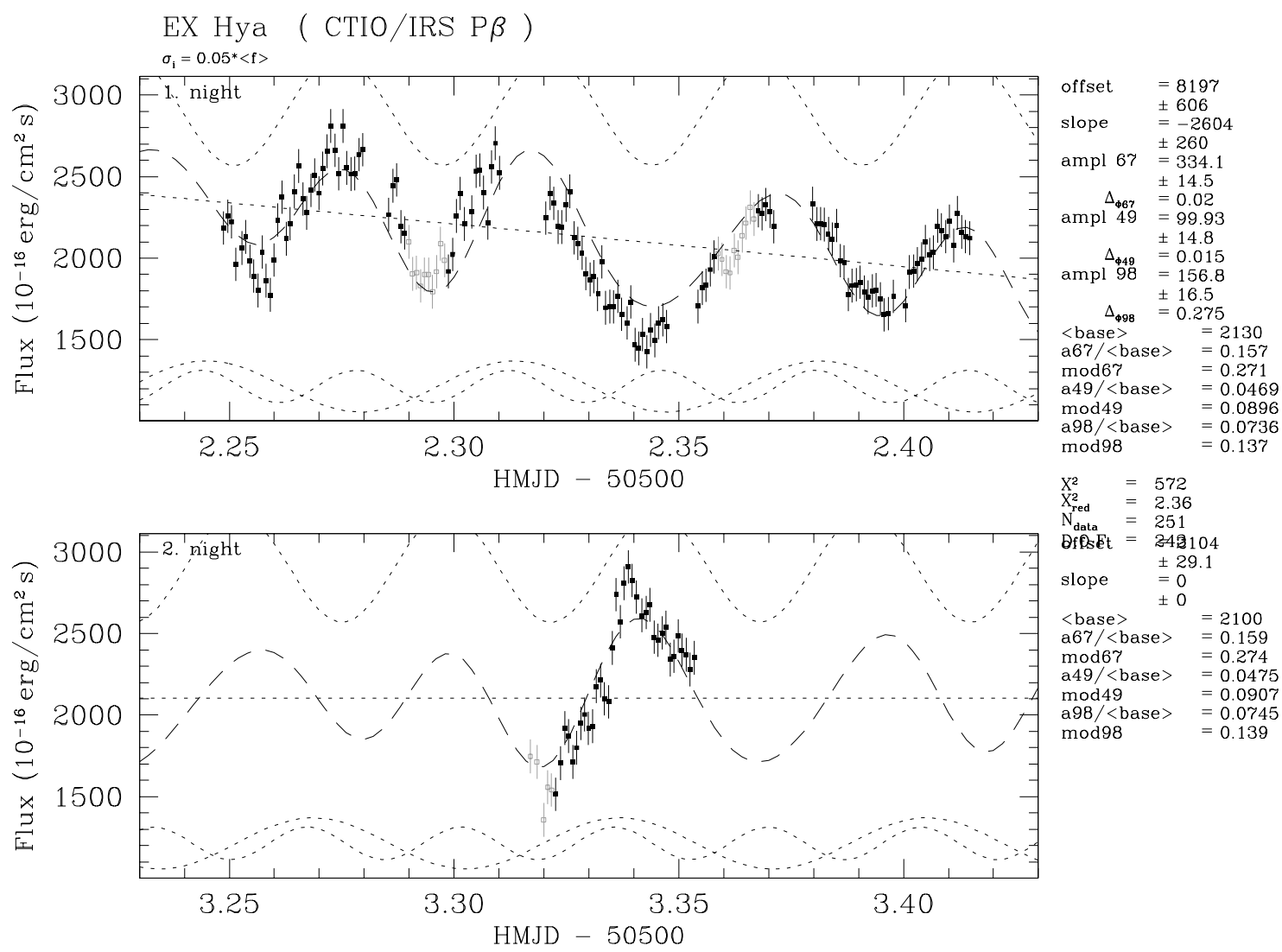

$\begin{array}{ll}\mathrm{X}^{2} & =572 \\ \mathrm{X}_{\text {red }}^{2} & =2.36 \\ \mathrm{~N} & =251\end{array}$ $\mathrm{X}_{\text {red }}=2.36$
$\mathrm{~N}_{\text {data }}=251$
DfDsEt slope $\quad \pm 29.1$ slope $\quad \begin{aligned} & \\ & \\ & \end{aligned}$ <base $>\quad \pm 0=2100$ a67 $/<$ base $>=0.159$ $\bmod 67 \quad=0.274$ a $49 /<$ base $>=0.0475$ $\bmod 49=0.0907$ $\begin{array}{ll}\text { a } 98 /<\text { base }> & =0.0745 \\ \bmod 98 & =0.139\end{array}$
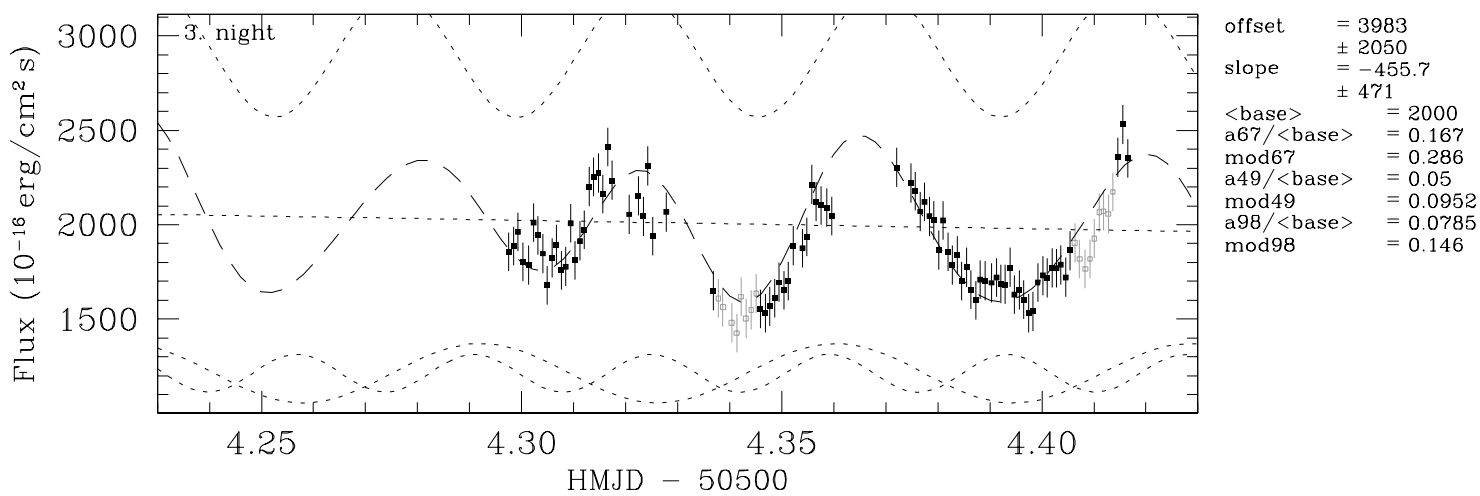

Abbildung A.62: Fit an IRS-Lichtkurve $\mathrm{P} \beta$;

Modell:

$f_{\text {line }}=$ base $+A_{67} \cdot \cos \left(\Phi_{67}-\Delta_{\Phi 67}\right)-A_{49} \cdot \cos \left(\Phi_{49}-\Delta_{\Phi 49}\right)+A_{98} \cdot \cos \left(\Phi_{98}-\Delta_{\Phi 98}\right)$ mit base $\equiv \sum_{i=1}^{3}\left(C_{i}+B_{i} \cdot(\right.$ HMJD -50500$\left.)\right)$. 


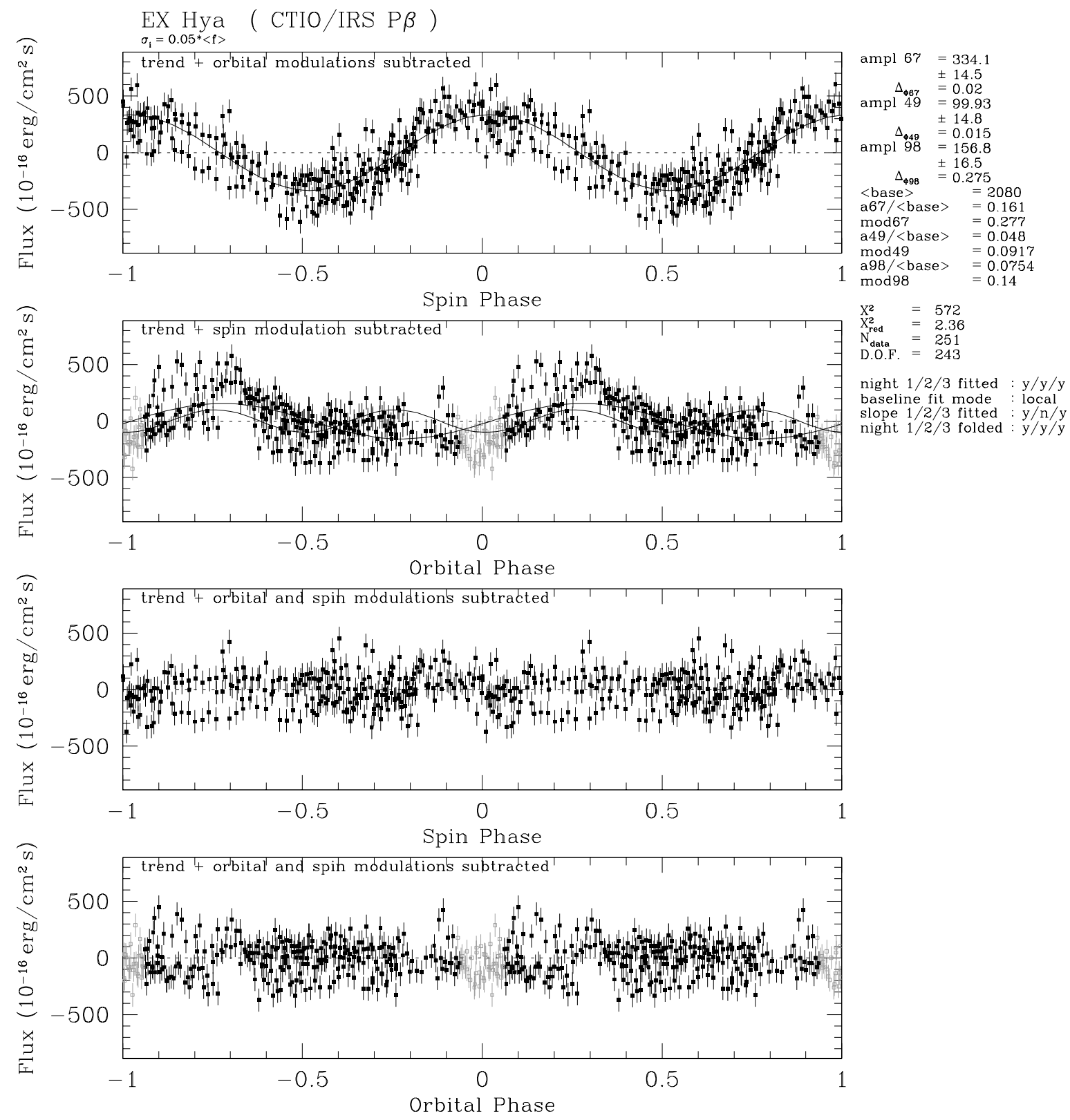

Abbildung A.63: Fit aus Abbildung A.62 über $\Phi_{67}$ bzw. $\Phi_{98}$ gefaltet, lineare Komponenten abgezogen. 

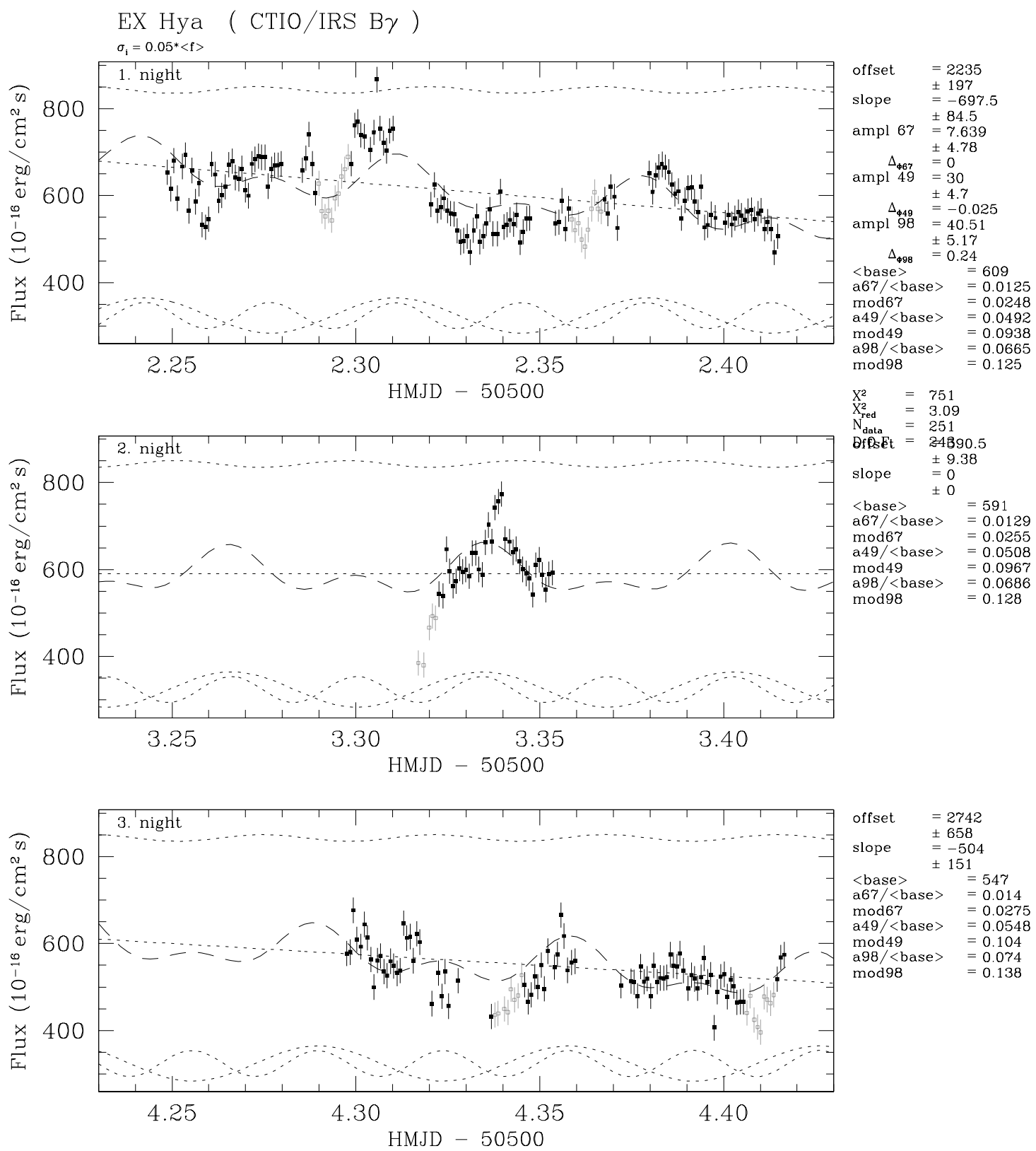

Abbildung A.64: Fit an IRS-Lichtkurve B $\gamma$;

Modell:

$f_{\text {line }}=$ base $+A_{67} \cdot \cos \left(\Phi_{67}-\Delta_{\Phi 67}\right)-A_{49} \cdot \cos \left(\Phi_{49}-\Delta_{\Phi 49}\right)+A_{98} \cdot \cos \left(\Phi_{98}-\Delta_{\Phi 98}\right)$ mit base $\equiv \sum_{i=1}^{3}\left(C_{i}+B_{i} \cdot(\right.$ HMJD -50500$\left.)\right)$. 


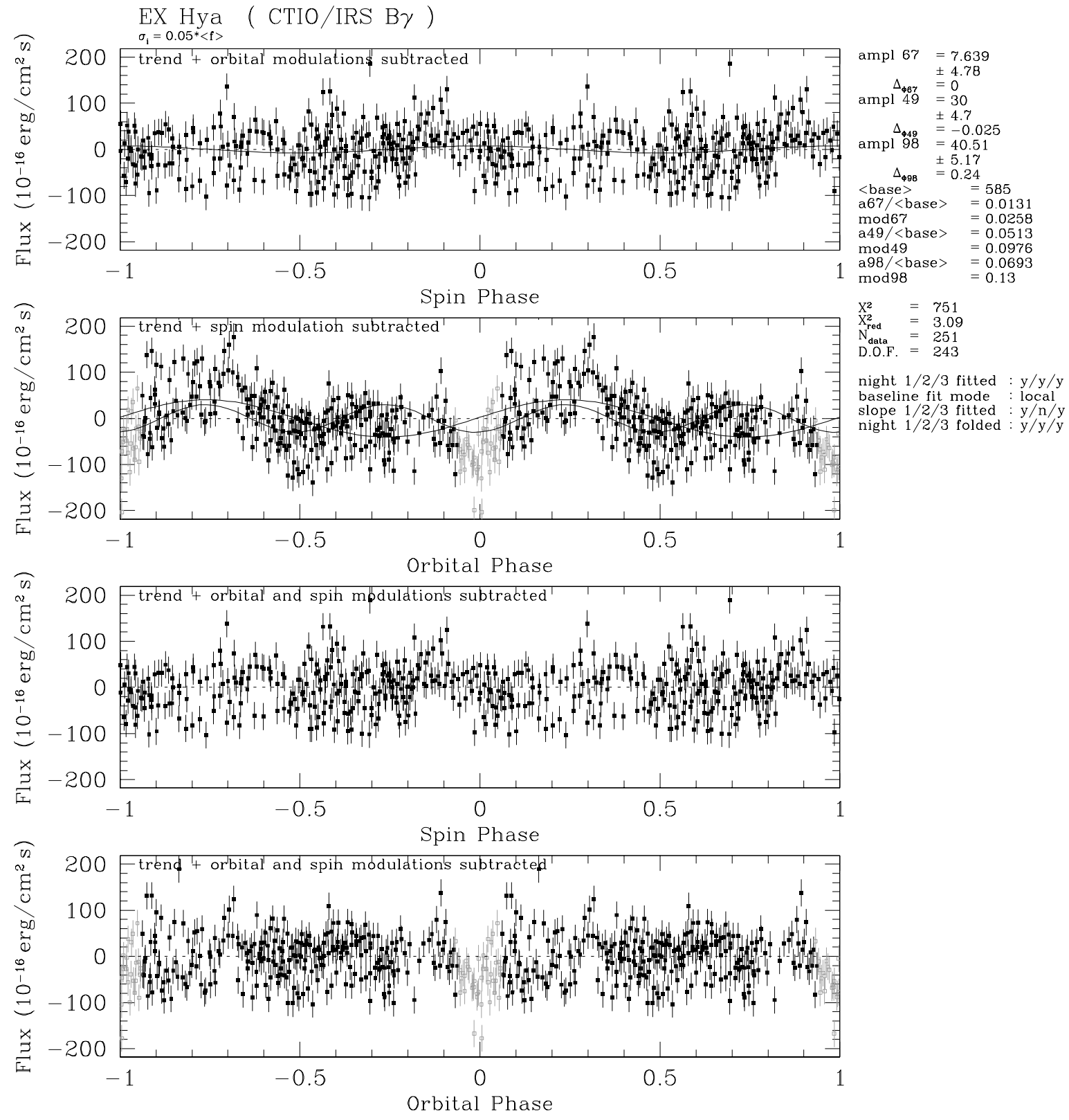

Abbildung A.65: Fit aus Abbildung A.64 über $\Phi_{67}$ bzw. $\Phi_{98}$ gefaltet, lineare Komponenten abgezogen. 


\section{Anhang B}

\section{Reduktion von IRS-Spektren}

In diesem Anhang beschreibe ich das angewandte Verfahren zur Reduktion von Spektren, die mit dem Infrared Spectrometer (IRS) des Cerro Tololo Interamerican Observatory (CTIO) gewonnen wurden (für eine Beschreibung des Instruments s. Depoy et al. 1990). Die Bauart des Instruments und die daraus resultierende Struktur der Daten macht eine Reduktion der Spektren mit den Standardprozeduren, die das verwendete Softwarepaket MIDAS (Munich Image Data Analysis System) der ESO bereithält, in weiten Teilen inpraktikabel. Erschwerend kommt hinzu, daß allgemein bei bodengebundenen Beobachtungen im nahen Infrarot zum einen die thermische Strahlung des Teleskopspiegels das Spektrum bei Wellenlängen $\gtrsim 20000 \AA$ dominiert und zum anderen das Spektrum durch starke atmosphärische Emissionslinien insbesondere des $\mathrm{OH}$-Radikals sowie durch mehrere kräftige Absorptionsbanden des atmosphärischen Wasserdampfs überlagert ist. Für große Teile der Datenreduktion habe ich daher eigene Software in der Programmiersprache C entwickelt, die den besonderen Anforderungen der Daten bzw. des Instruments Rechnung trägt. Die Programme für die verschiedenen Reduktionsschritte wurden dabei unter Benutzung der C-Programmierschnittstelle von MIDAS geschrieben und über Kommandoprozeduren in das Laufzeitsystem eingebunden; sie erlauben daher eine effiziente Bearbeitung aus dem System heraus, wobei das Verhalten jedes Programms bei jedem Aufruf durch Kommandoparameter an die aktuellen Erfordernisse angepaßt werden kann.

Im folgenden Abschnitt beschreibe ich die grundsätzlichen Eigenschaften der mit dem IRS gewonnenen Daten und in Abschnitt B.2 diejenigen Kalibrationsdaten, die zur Extraktion der Nutzdaten aus den aufgenommenen zweidimensionalen Bildern vonnöten sind. In Abschnitt B.3 folgt eine Darstellung der Beobachtungstechnik und der Reduktion der Daten bis zu dem Punkt, an dem die Spektren in eindimensionaler Form vorliegen. Abschnitt B.4 behandelt die Wellenlängen- und Flußkalibration sowie die Korrektur der atmosphärischen Absorption durch Wasserdampf. Dabei ist bei der Beschreibung zweidimensionaler Daten stets von 'Bildern' die Rede. Die Abbildungen, auf die ich mich im Text beziehe, befinden sich gesammelt am Ende dieses Anhangs.

\section{B.1 Struktur der IRS-Daten}

Das IRS in der für die Beobachtungen verwendeten Konfiguration bildet in der aktuellen Bauform das Spektrum im Bereich $\approx 8000 \ldots 24000 \AA$ mit Hilfe eines goldbeschichteten Reflexionsgitters auf einen mit Flüssigstickstoff gekühlten InSb-Detektor mit $256 \times 256$ Pixel ab. Das Gitter weist Linien in zwei aufeinander senkrecht stehenden Richtungen auf ('cross-dispersed grating'). Die eigentliche spektrale Zerlegung des einfallenden Lichts erfolgt dabei durch die Linien in der einen Richtung; durch die Linien senkrecht dazu werden die überlappenden Ordnungen des Spektrums räumlich getrennt in Form schmaler Streifen auf den Detektor abgebildet. Das effektiv erreichbare spektrale Aufösungsvermögen ist 
durch die Größe der Detektorpixel begrenzt und beträgt laut Beobachterhandbuch zum IRS für ein Bildelement von $\Delta x=2$ Pixel Größe $\lambda / \Delta \lambda \approx 560$ in der 3...6. Ordnung und $\approx 320$ in der 7 . Ordnung (vgl. Tabelle B.1). Der Wellenlängenbereich $\approx 18200 \ldots 19300 \AA$ liegt zwischen den Bereichen, die von der 3. und 4. Ordnung überdeckt werden, und ist daher nicht beobachtbar. Da nur der Detektor, nicht aber die Teleskopspiegel und die Zusatzoptik gekühlt sind, dominiert deren thermische Strahlung das Spektrum bei großen Wellenlängen $(\lambda \gtrsim 20000 \AA)$.

Ungefähr $0.2 \%$ der Pixel des Detektors liefern Signale, die aus verschiedenen Gründen für die Auswertung unbrauchbar sind ('bad pixel'; s. Abschnitt B.2). Weiterhin ist der Detektor des IRS ebenso wie optische CCDs empfindlich für Einschläge von Teilchen der kosmischen Strahlung (cosmic ray hits); während einer einminütigen Belichtung treten $\approx 5-10$ solcher Ereignisse ('cosmics') auf.

\section{B.2 IRS-spezifische Kalibrationsdaten}

\section{Lage der Ordnungen}

Um aus den Rohbildern (s. z.B. Abbildung B.7) die Spektren extrahieren zu können, muß die genaue Lage der Ordnungen bekannt sein. Hierfür wurde in jeder Nacht eine Serie von Bildern einer weißen, gleichmäßig beleuchteten Fläche an der Innenseite des Teleskopdoms durch eine kleine Lochblende aufgenommen ('pinhole images'; Abbildung B.1). Nach Mittelung dieser Bilder und einer Median-Filterung des Resultats, die störende Pixelartefakte unterdrückt, wurde die Position jedes pinhole-Streifens, von denen jeder die Lage einer Ordnung wiedergibt, an 16 Stellen entlang der Dispersionsrichtung per Anpassung eines Gaussprofils gemessen (Kreuze in der Abbildung). An die resultierenden Spurpunkte einer Ordnung wurde jeweils ein Polynom dritten Grades angepaßt; die Pixelbereiche jeder Ordnung lassen sich dann durch Offsets $\Delta x_{1}, \Delta x_{2}$ relativ zu diesen Modellen definieren (in Abbildung B.1 angedeutet durch die Kurven links und rechts der pinhole-Streifen), so daß z.B. die gezielte Extraktion aller zu einer bestimmten Ordnung gehörigen Pixel möglich ist. Innerhalb jeder Ordnung steigt die Wellenlänge von oben nach unten an; von Ordnung zu Ordnung nimmt die Wellenlänge von rechts nach links zu. Die genauen Wellenlängenbereiche der Ordnungen sowie das pro Pixel in jeder Ordnung überdeckte Wellenlängenintervall werden in Abschnitt B.4 angegeben.

\section{Defekte Pixel}

Etliche Pixel des IRS-Detektors $(\approx 0.2 \%)$ weisen Defekte auf, aufgrund derer die gelieferten Werte unbrauchbar sind:

— 'Heiße' bzw. 'kalte' Pixel weisen praktisch unabhängig von der aufgenommenen Lichtmenge stets einen etwa konstanten sehr hohen bzw. sehr niedrigen Wert auf.

— 'Überempfindliche' Pixel reagieren sehr viel stärker als 'normale' Pixel auf Licht; sie werden daher schon bei kurzen Belichtungszeiten gesättigt und ändern ihren Wert danach nicht mehr.

- 'Springende' Pixel haben in verschiedenen Bildern, die unter gleichen Bedingungen aufgenommen wurden, erratisch schwankende Werte. 
Alle defekten Pixel müssen bei der Reduktion von Nutzdaten gesondert behandelt werden. Um eine 'Karte' dieser Pixel ('bad pixel map') herzustellen, wurden am Anfang und am Ende jeder Nacht Bilder der oben erwähnten weißen Fläche mit und ohne Beleuchtung ('flatfields' und 'darkfields') mit verschiedenen Belichtungszeiten von $0.5 . .8 \mathrm{~s}$ aufgenommen (Abbildung B.2 sowie Abbildung B.3, die insbesondere auch die thermische Strahlung des Teleskopspiegels zeigt). Um die 'springenden' Pixel identifizieren zu können, wurden immer Serien von typischerweise 20 gleichartigen Bildern aufgenommen. Aus einem (überwiegend manuellen) Vergleich der pro Belichtungszeit gemittelten flatfields, darkfields und ihrer Differenzen (vgl. Abbildung B.4) miteinander sowie mit den mittleren Bildern gleichen Typs bei verschiedenen Belichtungszeiten und den Einzelbildern vor der Mittelung lassen sich alle drei Typen defekter Pixel identifizieren und in einer Karte zusammenfassen, die z.B. den Wert '0' bzw. '1' für ein intaktes bzw. defektes Pixel enthält. Da die so gewonnenen Karten für die drei Nächte keine signifikanten Unterschiede aufweisen, wurde für die Reduktion stets dieselbe, in Abbildung B.5 gezeigte Karte verwendet.

\section{Pixel-zu-Pixel-Variationen der Empfindlichkeit}

Aus den eben schon benutzten Differenzen von flatfields und darkfields (s. Abbildung B.4) lassen sich die kleinskaligen Variationen der Lichtempfindlichkeit des Detektors ableiten. Hierzu wurden aus den Differenzbildern der Aufnahmen mit einer Belichtungszeit von 8s die einzelnen Ordnungen entlang der in Abbildung B.1 durch die Linien angedeuteten Bänder extrahiert. Die in Abbildung B.4 besonders im mittleren Band erkennbaren großskaligen Strukturen beruhen z.T. auf echten Variationen der spektralen Empfindlichkeit, sind z.T. aber auch darauf zurückzuführen, daß das Spektrum der für die Beleuchtung verwendeten Lampe infolge von wellenlängenabhängiger Absorption im Quarzkolben starke intrinsische Variationen aufweist. Auch die abbildende Optik hat gewisse Einflüsse (Vignettierung, Blaze-Winkel des Gitters). Diese großskaligen Strukturen wurden für jede Ordnung durch eine Spline-Funktion in Dispersionsrichtung approximiert. Schließlich wurden die extrahierten Ordnungen durch die Spline-Funktionen dividiert und die sich ergebenden Pixelwerte, die nun überwiegend im Bereich $\approx 0.9 \ldots 1.1$ liegen, wieder an die ursprünglichen Positionen (d.h. diejenigen vor der Extraktion) zurückgeschrieben. Pixel außerhalb der von den Ordnungen überdeckten Bereiche wurden auf den Wert 1 gesetzt.

Die so konstruierten Hochfrequenz-flatfields aus den drei Nächten sind im wesentlichen identisch; für die Reduktion aller Objektspektren wurde daher durchgängig ein mittleres flatfield benutzt, das in Abbildung B.6 dargestellt ist, wobei die Grautöne schwarz bzw. weiß Pixelwerte $\leq 0.95$ bzw. $\geq 1.05$ repräsentieren. Die Abbildung zeigt neben Schleifspuren, die aus dem Herstellungsprozess des Detektors herrühren, im unteren Bildteil auch einen ausgedehnten Kratzer, in dessen Bereich die Empfindlichkeit der Pixel auf $\approx 90 \%$ herabgesetzt ist und der auch in den Abbildungen B.2 und B.4 erkennbar ist. 


\section{B.3 Beobachtungstechnik und Extraktion der Spektren}

\section{Beobachtungstechnik}

Da der insbesondere bei großen Wellenlängen hohe thermische Hintergrund und der in einigen Ordnungen der Spektren hohe Himmelshintergrund bei der Reduktion hinderlich sind, ist es vorteilhaft, beide bei der Reduktion schon möglichst früh größtenteils (zumindest näherungsweise) zu unterdrücken. Hierzu wurde die Spaltlänge, die der Breite der Ordnungen in den Bändern entspricht und beim IRS kontinuierlich einstellbar ist, so gewählt, daß die einzelnen Ordnungen möglichst breit waren, ohne sich jedoch zu überlappen. Bei den Beobachtungen wurden die Objekte dann in stetigem Wechsel an verschiedene Stellen des Spalts positioniert, so daß die Spektren an unterschiedlichen Stellen des Detektors zu liegen kamen (vgl. Abbildung B.7). In der Regel wurde dabei zwischen zwei Positionen A und B ('links' und 'rechts') nach dem Muster ABBAABB... gewechselt, wobei der Wechsel von einer Position zur anderen nur ca. 1 Sekunde in Anspruch nahm.

\section{Näherungsweise Unterdrückung des Himmelshintergrunds}

Wie in Kapitel 4.2.6 erwähnt, wurden von EX Hya in jeder Nacht zwei bis drei Serien von Bildern über jeweils $\approx 1 \mathrm{~h}$ hinweg aufgenommen. Bei der Reduktion wurden die Bilder jeder Serie nach der Position der Objektspektren im Spalt sortiert. Anschließend wurde für jede Position aus allen zugehörigen Bildern durch pixelweise Medianbildung ein mittleres Bild erzeugt. Jedes dieser mittleren Bilder enthält außerhalb des Bereichs, den das Objektspektrum selbst bedeckt, den mittleren Himmelshintergrund dieser Serie von Bildern. Da in jedem mittleren Bild einer Serie per Konstruktion das Spektrum an einer anderen Stelle liegt, liefert das pixelweise Minimum der mittleren Bilder - zumindest dann, wenn sich wenigstens zwei Bilder finden lassen, in denen die Spektren in Spaltrichtung klar getrennt sind - ein Bild des mittleren Himmelshintergrunds der Serie über die gesamte Spaltlänge und ohne einen Beitrag des Objektspektrums. Abbildung B.8 zeigt ein Beispiel hierfür. In Abbildung B.9 ist das linke Bild aus Abbildung B.7 nach Abzug des mittleren Himmels gezeigt.

Der besonders störende thermische Hintergrund am linken Bildrand wird durch dieses Verfahren nahezu vollständig unterdrückt. Die Intensität der atmosphärischen Emissionslinien wird immerhin um einen Faktor $\approx 10$ herabgesetzt. (Zur Verdeutlichung wurden die Grauwerte anders als in Abbildung B.7 gewählt; dies sollte nicht über die wahren Intensitätsverhältnisse hinwegtäuschen.) Die in Abbildung B.9 noch deutlich zu erkennenden Strukturen in der zweiten Ordnung von links beruhen zum einen auf tatsächlicher Variabilität der Linien im Laufe der Beobachtungsserie, werden aber auch durch eine mechanische Drift des Instruments in Dispersionsrichtung im Laufe der Beobachtung hervorgerufen. (Dieser Umstand erfordert später bei der Wellenlängenkalibration noch besondere Aufmerksamkeit.) Es sei noch angemerkt, daß sich durch dieses Vorgehen das separate Abziehen des Dunkelstroms und des Bias, wie es z.B. bei der Reduktion von Einzelaufnahmen optischer CCDs nötig ist, erübrigt, da beide Beiträge in beiden eingehenden Bildern aufgrund der gleichen Belichtungszeit identisch sind. 


\section{Pixelbasierte Korrekturen}

Die folgenden drei Schritte korrigieren die Pixel-zu-Pixel-Variationen der Empfindlichkeit sowie die durch defekte Pixel und cosmics hervorgerufenen Artefakte. Sie führen im Rahmen der in dieser Arbeit erreichbaren Abbildungsqualität zu keiner wesentlichen Veränderung der Bilder; ich verzichte daher auf deren separate Darstellung.

Flatfield-Korrektur. Die Flatfield-Korrektur besteht aus der Division des Bildes aus Abbildung B.9 durch das in Abbildung B.6 dargestellte Hochfrequenz-Flatfield, wodurch das Bild auf das eines Detektors mit räumlich gleichförmiger, allerdings noch glatt wellenlängenabhängiger Empfindlichkeit zurückgeführt wird.

Maskierung defekter Pixel. Die in Abbildung B.5 gezeigte Karte defekter Pixel wird derart angewandt, daß der Wert jedes dort als defekt markierten Pixels durch einen Wert ersetzt wird, der aus den Werten intakter Pixel in einer geeignet zu wählenden Umgebung berechnet wird. (Im Detail: Das Programm mittelt die Werte aller intakten Pixel in der Umgebung $(x \pm \Delta x, y \pm \Delta y)$ um das defekte Pixel bei den Koordinaten $(x, y)$ herum. Findet es dort keine intakten Pixel, kann eine alternative größere Umgebung $\left(x \pm \Delta x_{2}, y \pm \Delta y_{2}\right)$ zum Einsatz kommen. In der Praxis haben sich Umgebungen $(\Delta x, \Delta y)=(1,0)$, d.h. Mittelung des links und rechts benachbarten Pixels, und $\left(\Delta x_{2}, \Delta y_{2}\right)=(1,1)$, d.h. Mittelung aller benachbarten Pixel bewährt.)

Maskierung der cosmics. Zur Entfernung der Spuren, die Einschläge energiereicher kosmischer Teilchen auf dem Detektor hinterlassen, wurde eine modifizierte Version des entsprechenden MIDAS-Standardprogramms für diesen Zweck benutzt. Letzteres identifiziert cosmics anhand der scharfen Form der durch die Teilchen verursachten Intensitätsmaxima im Bild; außerdem muß die Höhe des Intensitätsmaximums einen gewissen globalen Schwellwert überschreiten (' $\kappa$ - $\sigma$-clipping-Algorithmus'). Die von einem cosmic beeinflußten Pixel werden durch Werte ersetzt, die aus denen benachbarter, nicht betroffener Pixel bestimmt werden. Die modifizierte Variante unterscheidet sich hiervon im wesentlichen in folgenden Punkten:

- Der Schwellwert für die Erkennung eines cosmics gilt nicht global, sondern wird lokal aus der Varianz der Pixel in einer vorzugebenden Umgebung des jeweils aktuellen Pixels bestimmt. Dies ist notwendig, da das MIDAS-Standardprogramm einen 'intakten' Himmelshintergrund voraussetzt, der nach dem Abzug des mittleren Himmels (s.o.) nicht mehr vorhanden ist; es läßt sich aber auch nicht auf die ursprünglichen Bilder (s. Abbildung B.7) anwenden, da sich die kleinskalige Struktur der Bilder nicht hinreichend mit einem globalen Schwellwert erfassen läßt und zur Erkennung zahlreicher 'falscher' cosmics führt.

- Ein cosmic kann beim IRS-Detektor offenbar auch zur Entladung eines Pixels führen; solche Pixel haben gelegentlich auch während der nächsten Aufnahmen eine deutlich reduzierte Empfindlichkeit und entsprechend niedrige Werte. Das modifizierte Programm sucht, erkennt und korrigiert daher auch 'negative' cosmics. 


\section{Extraktion der Ordnungen}

Es folgt die Extraktion und 'Begradigung' der einzelnen Ordnungen des Spektrums unter Benutzung der in Abschnitt B.2 bestimmten Form derselben (vgl. Abbildung B.1). Die dort besprochenen Offsets $\Delta x_{1}, \Delta x_{2}$ wurden dabei für jede Serie von Spektren separat bestimmt, da das Instrument während einer Nacht auch eine mechanische Drift in Spaltrichtung aufwies. Dabei wurde darauf geachtet, daß der vom Flatfield erfaßte Bereich nicht überschritten wurde (vgl. Abbildung B.6). Die Pixelwerte wurden dabei senkrecht zur Dispersionsrichtung neu gebinnt. Das Ergebnis dieser Operation ist in Abbildung B.10 im linken Teilbild dargestellt. (Es sei hier angemerkt, daß bei der Konstruktion des Flatfields (s. Abschnitt B.2) die Pixel während der Extraktion der Ordnungen nicht neu gebinnt wurden, um die Pixel-zu-Pixel-Variationen exakt zu erhalten; dies äußert sich in der stufigen Struktur der Ordnungen in Abbildung B.6.)

\section{Bestimmung und Abzug des Himmelshintergrunds}

Der Abzug des noch verbliebenen Himmelshintergrunds vollzieht sich wie folgt:

- Um die Bereiche zu identifizieren, die nur Himmelshintergrund enthalten, wird das Bild mit den extrahierten Ordnungen (Abbildung B.10 links) zunächst zeilenweise gemittelt; anschließend werden die so erhaltenen Profile der fünf Ordnungen gemittelt. Anhand dieses mittleren Profils werden diejenigen Spalten ermittelt, aus denen in jeder Ordnung der Himmelshintergrund bestimmt werden soll. Hierfür wird der Bruchteil $f$ (z.B. $f=0.5$ ) der Spalten mit dem niedrigsten Fluß ausgewählt. Dieser Vorgang ist in Abbildung B.11] veranschaulicht.

- In jeder Ordnung wird nun zeilenweise der Median der Pixelwerte in den ausgewählten Spalten bestimmt und als Himmelshintergrund in dieser Zeile der jeweiligen Ordnung angenommen. Das so konstruierte Bild des Hintergrunds (Abbildung B.10 Mitte) wird schließlich vom Spektrum abgezogen; das resultierende Bild ist im wesentlichen hintergrundfrei, wobei in den Randspalten jeder Ordnung noch Artefakte verbleiben können (Abbildung B.10 rechts).

\section{Extraktion des Objektspektrums}

Als letzter Schritt der Datenextraktion wird für jede Ordnung der Fluß des Objektspektrums aufintegriert. Hierzu werden zunächst (analog zum Verfahren zur Bestimmung der Spalten für den Himmelshintergrund) die zu verwendenden Spalten ermittelt; hier werden allerdings diejenigen Spalten ausgewählt, die zusammengenommen den Bruchteil $g$ (z.B. $g=0.95$ ) des gesamten im Profil vorhandenen Flusses repräsentieren (s. Abbildung B.11). Um Randeffekte, die in den äußersten Spalten jeder Ordnung noch auftreten können, möglichst zu vermeiden (vgl. etwa die Reste des Himmelshintergrunds im rechten Teilbild von Abbildung (B.10), bleiben jeweils die erste und letzte Spalte jeder Ordnung unberücksichtigt. Die so ausgewählten Spalten werden schließlich aufsummiert und abschließend durch Multiplikation mit $1 / g$ auf 1 normiert. Das Resultat ist ein Bild mit $256 \times 5$ Pixel (eine Zeile pro Ordnung, ohne Abbildung) 
Tabelle B.1: Wellenlängenbereiche und Dispersion $\Delta \lambda / \Delta y$ der einzelnen Ordnungen des IRS gemäß der Wellenlängenkalibration (auf Vielfache von 5 bzw. 0.05 gerundet) und Auflösungsvermögen $\lambda / \Delta \lambda$ (für $\Delta y=2$ Pixel) laut Beobachterhandbuch zum IRS. In den Abbildungen B.1 bis B.10 liegt die 3. Ordnung links und die 7. Ordnung rechts; die Wellenlängen nehmen von oben nach unten zu.

\begin{tabular}{crccc}
\hline Ordnung & $\lambda_{\text {start }}[\AA]$ & $\lambda_{\text {end }}[\AA]$ & $\Delta \lambda / \Delta y[\AA /$ Pixel $]$ & $\lambda / \Delta \lambda$ \\
\hline 3. & 19270 & 24225 & 19.40 & 558 \\
4. & 14460 & 18175 & 14.55 & 561 \\
5. & 11570 & 14545 & 11.65 & 559 \\
6. & 9645 & 12125 & 9.70 & 558 \\
7. & 8275 & 10395 & 8.30 & 320 \\
\hline
\end{tabular}

\section{B.4 Kalibration der Spektren}

\section{Korrektur der Drift in Dispersionsrichtung}

Für die Korrektur der in Abschnitt B.3 im zweiten Abschnitt erwähnten mechanischen Drift des IRS in Dispersionsrichtung selbst während einer Serie von Aufnahmen (die im übrigen auch der wesentliche Grund für die in Abbildung B.9 noch vorhandenen Strukturen im Himmelshintergrund ist), lassen sich die ansonsten störenden atmosphärischen Emissionslinien verwenden. Hierzu wurde aus jedem Rohbild (vgl. Abbildung B.7) nach einer Median-Filterung senkrecht zur Dispersionsrichtung, die die Pixelartefakte (defekte Pixel, cosmics) weitestgehend eliminiert, nach dem oben beschriebenen Verfahren die im Bild zweite Ordnung von links extrahiert und das Himmelsspektrum analog zu der im vorletzten Schritt der Datenextraktion angewandten Methode ermittelt. Auf die gleiche Weise wurde als Referenz das besonders gut definierte Himmelsspektrum aus der Beobachtung des Sterns SAO 137387 präpariert. (Dieser Stern wurde auch zur Korrektur der atmosphärischen Absorption bei einem der M-Sterne verwendet, vgl. Tabelle 4.4.) Durch Kreuzkorrelation des Himmelsspektrums mit diesem Referenzspektrum wurde dann für jedes Einzelbild die Drift $\Delta y$ (in Pixel) in Dispersionsrichtung bestimmt. Dies ist in Abbildung $\mathrm{B} .12$ illustriert. Die auftretenden Driftwerte $\Delta y$ können im Extremfall bis zu $\approx 5$ Pixel betragen, entsprechend einer Wellenlängendrift von (je nach Ordnung) 40...200 A, und werden bei der nachfolgend beschriebenen Wellenlängenkalibration berücksichtigt.

\section{Wellenlängenkalibration}

Die Wellenlängenkalibration wurde für jede Ordnung separat durchgeführt. Hierzu wurden aus Spektren einer HeNeAr-Kalibrationslampe, die am Anfang jeder Nacht aufgenommen wurden, die einzelnen Ordnungen nach dem oben beschriebenen Verfahren extrahiert und für jede Ordnung mit den entsprechenden MIDAS-Standardprozeduren eine Dispersionsrelation angepaßt. In allen Fällen erwies sich eine lineare Dispersionsrelation als ausreichend. Die Dispersionsrelationen aus den verschiedenen Nächten unterschieden sich nicht wesentlich, so daß für die Kalibration pro Ordnung eine mittlere Relation benutzt wurde. Tabelle B.1 gibt den von jeder Ordnung überdeckten Wellenlängenbereich sowie die Dispersion in $\AA /$ Pixel an. 
Tabelle B.2: Zur Flußkalibration der IRS-Spektren verwendete Sterne (Spektraltyp und Literaturwerte $m_{\text {Lit }}$ der Helligkeiten (CIT-System) aus Elias et al. 1982; Effektivtemperaturen für die angegebenen Spektraltypen aus Scheffler \& Elsässer 1990). Die gemessenen Helligkeiten $m_{\text {obs }}$ wurden aus den mit der mittleren Response-Funktion flußkalibrierten und auf atmosphärische Absorption korrigierten IRS-Spektren der Sterne bestimmt und beziehen sich auf das Johnson-System.

\begin{tabular}{llclccc}
\hline Stern & SpTyp & $T_{\text {eff }}$ & & \multicolumn{3}{c}{ Band } \\
& & & & $J$ & $H$ & $K$ \\
\hline HD 40335 & \multirow{2}{*}{ A0 } & \multirow{2}{*}{9520} & $m_{\text {Lit }}$ & 6.540 & 6.470 & 6.450 \\
& & & $m_{\text {obs }}$ & 6.552 & 6.515 & 6.423 \\
\hline HD 77281 & \multirow{2}{*}{ A2 } & \multirow{2}{*}{8990} & $m_{\text {Lit }}$ & 7.105 & 7.050 & 7.030 \\
& & & $m_{\text {obs }}$ & 7.101 & 7.091 & 7.000 \\
\hline HD 130163 & \multirow{2}{*}{ A0V } & \multirow{2}{*}{9520} & $m_{\text {Lit }}$ & 6.855 & 6.845 & 6.835 \\
& & & $m_{\text {obs }}$ & 6.823 & 6.881 & 6.789 \\
\hline
\end{tabular}

Bei der Wellenlängenkalibration der Einzelspektren wurde auch deren oben ermittelte Drift $\Delta y$ relativ zum Referenzspektrum berücksichtigt. Das Referenzspektrum selbst hat ebenfalls eine Drift $\Delta y_{\text {ref }}$ relativ zur absoluten Wellenlängenskala. Diese wurde bestimmt, indem die Wellenlängenkalibration probehalber auf den Stern SAO 137387 angewandt wurde, aus dessen Beobachtungen das Referenzspektrum gewonnen wurde. Die anschließende Vermessung der Wasserstoff-Absorptionslinien und ein Vergleich mit den Soll-Wellenlängen lieferten dann den Wert von $\Delta y_{\text {ref }}$; die Summe $\Delta y+\Delta y_{\text {ref }}$ ist dann die Drift des Einzelspektrums relativ zur absoluten Wellenlängenskala.

Es sei abschließend noch angemerkt, daß die Wellenlängenkalibration (anders als bei Beobachtungen z.B. in kleineren Wellenlängenbereichen und mit höherer spektraler Auflösung) nicht allein anhand der atmosphärischen Emissionslinien, d.h. unabhängig von einer externen Kalibrationslampe durchgeführt werden kann, da geeignete Linien im wesentlichen nur in einer Ordnung auftreten (vgl. Abbildung B.7)

\section{Flußkalibration}

Die absolute Flußkalibration im nahen Infrarot stellt ein besonderes Problem dar, da es in der Literatur keine tabellierten Flüsse spektroskopischer Standardsterne in diesem Bereich gibt. Als Ersatz wurden daher Spektren der drei in Tabelle B.2 aufgeführten photometrischen IR-Standardsterne frühen Spektraltyps aufgenommen, deren Emission im Infrarot abgesehen von wenigen Absorptionslinien - in guter Näherung die eines Schwarzkörpers ist. Bis auf HD 77281 wurden die Sterne jeweils in zwei Nächten beobachtet. Zur Konstruktion der spektralen Antwortfunktion des Teleskop-Spektrometer-Systems (ab jetzt kurz 'Response-Funktion') für jede Ordnung wurde wie folgt verfahren:

- Für jeden Stern wurde ein Schwarzkörper-Spektrum $f_{\lambda}(\lambda)$ für die jeweilige Temperatur $T_{\text {eff }}$ berechnet. 
- Für das Schwarzkörper-Spektrum wurden die photometrischen Helligkeiten im J-, Hund K-Band gemäß (4.1) mit den Flußkonstanten aus Tabelle 4.3 (Johnson-System) bestimmt.

- Das Schwarzkörper-Spektrum für jeden Stern wurde so skaliert, daß es die Literaturwerte aus Tabelle B.2 möglichst gut wiedergibt. (Die Unterschiede zwischen den gemessenen Johnson-Helligkeiten und den CIT-Helligkeiten aus der Tabelle sind hierbei vernachlässigbar.)

- Die beobachteten Spektren der Standardsterne wurden in der in den vorigen Abschnitten beschriebenen Weise bis hin zur Wellenlängenkalibration reduziert.

- Mit den entsprechenden MIDAS-Standardprozeduren wurde aus den konstruierten Standardspektren und den beobachteten Spektren für jede Ordnung die zugehörige Response-Funktion berechnet.

Alle so erhaltenen Response-Funktionen sind in Abbildung B.13 dargestellt. Die stark strukturierte Form rührt daher, daß die mittlere atmosphärische Absorption durch Wasserdampf bereits in die Response-Funktionen einbezogen wurde. Hierdurch muß bei der unten beschriebenen Korrektur der Absorption in den Objektspektren nur noch der deutlich geringere variable Anteil der Absorption behandelt werden. Man beachte auch, daß der beobachtbare Fluß im Bereich $\approx 18000 \ldots 19000 \AA$ aufgrund der Absorption fast auf Null abfällt, wodurch die durch die Bauart des IRS bedingte Lücke in den Spektren in eben diesem Bereich keinen nennenswerten Verlust darstellt.

Aus den durchgezogenen Kurven wurde schließlich durch Mittelung eine mittlere Response-Funktion für jede Ordnung erzeugt, die dann für die Kalibration aller Spektren verwendet wurde. (Die gepunkteten Kurven in der Abbildung entsprechen der Beobachtung von HD 130163 aus der dritten Nacht, die aufgrund der atmosphärischen Bedingungen verfälscht sind; sie wurden bei der Mittelung nicht berücksichtigt.) Als Kontrolle sind in Tabelle B.2 noch die (Johnson-)Helligkeiten der Standardsterne angegeben, wie sie aus deren IRS-Spektren nach der Flußkalibration mit der mittleren Response-Funktion erhalten wurden. Dabei wurde die oben erwähnte Lücke in den Spektren mit interpolierten Werten aufgefüllt und das Spektrum bis $\approx 26000 \AA$ extrapoliert, um Verfälschungen der K-Helligkeit zu vermeiden. Während die J-Helligkeiten die Literaturwerte im Mittel gut wiedergeben, weichen die $\mathrm{H}-$ bzw. K-Helligkeiten systematisch $\mathrm{um} \approx+0.04 \mathrm{mag}$ bzw. $\approx-0.04 \mathrm{mag}$ davon ab. Ich führe dies darauf zurück, daß das angenommene Schwarzkörper-Spektrum das wahre Spektrum der Sterne nur näherungsweise repräsentiert.

\section{Zusammenführung der Ordnungen zum Gesamtspektrum}

Die bisher getrennt vorliegenden, wellenlängen- und flußkalibrierten Ordnungen der Spektren werden mit prinzipiell demselben Verfahren zum Gesamtspektrum verknüpft, das in Kapitel 4.2.2 zur Verknüpfung der optischen und Infrarotspektren der M-Sterne verwendet wurde (Mittelung mit gleitenden Gewichten im Überlappbereich der Ordnungen), wobei die Randpixel jeder Ordnung verworfen wurden. Von den beobachteten M-Sternen und den für die Korrektur der atmosphärischen Absorption verwendeten Sternen wurden jeweils mehrere (typischerweise 10) Spektren hintereinander aufgenommen, die nach erfolgter Zusammenführung der Ordnungen gemittelt wurden. 
Abbildung B.14 zeigt unten das Spektrum aus dem rechten Teilbild von Abbildung B.10 nach erfolgter Wellenlängen- und Flußkalibration und darüber (vertikal versetzt) dasselbe Spektrum nach Verknüpfung der Ordnungen. Die geringen Flußdifferenzen in den Überlappbereichen der einzelnen Ordnungen demonstrieren zum einen die Güte der entsprechenden Teile der Response-Funktion relativ zueinander und rechtfertigen zum anderen das hier angewandte Verknüpfungsverfahren.

\section{Korrektur der atmosphärischen Absorption}

Als letzter Schritt der Reduktion erfolgt die Korrektur der atmosphärischen Absorption. Wie in Abschnitt 4.1.1 erwähnt, wurden zeitnah zu jedem Objekt je 10...20 Spektren eines im selben Himmelsgebiet stehenden F- oder G-Sterns aufgenommen (s.a. Tabelle 4.4). Solche Sterne haben im nahen Infrarot intrinsisch näherungsweise glatte Kontinua und erlauben es daher, den Einfluß der Absorption zu bestimmen bzw. eine Korrekturfunktion hierfür abzuleiten, die aufgrund der zeitlichen und räumlichen Nähe auch auf das Spektrum des 'Nutzobjekts' angewandt werden kann.

Die Spektren der Vergleichssterne wurden zunächst wie in den vorigen Abschnitten beschrieben reduziert und anschließend mit einem Medianfilter geglättet. Für die geglätteten Spektren wurde der Verlauf des unabsorbierten Kontinuums bestimmt, indem an die Flüsse $f(\lambda)$ aller nicht von Absorption betroffenen Punkte ein Polynom $P(\lambda)$ hohen (6...8.) Grades angepaßt und alle Punkte mit $f(\lambda)<P(\lambda)$ verworfen wurden. Nach einigen (3...5) Iterationen dieses Prozesses ist $P(\lambda)$ ein gute Näherung für die obere Einhüllende des Spektrums, mit der das unabsorbierte Spektrum hinreichend gut über die von Absorption betroffenen Gebiete hinweg modelliert werden kann (glatte Kurve in Abbildung B.15). Zusammen mit dem ursprünglichen, absorbierten Spektrum ergibt sich daraus die gesuchte Korrekturfunktion in den Absorptionsbanden (unterste Kurve in der Abbildung; Wertebereich der Kurve $\approx 1 \ldots 1.25$ ). Die Korrektur des Objektspektrums erfolgt dann einfach durch Multiplikation mit der Korrekturfunktion (s. Abbildung B.16). Im Fall von EX Hya wurden jeweils die beiden vor und nach der betreffenden Serie von Spektren ermittelten Korrekturfunktionen auf den Zeitpunkt der aktuellen Aufnahme interpoliert (vgl. Abschnitt 4.2.6).

Abschließend sei noch auf zwei Punkte hingewiesen:

- Da die mittlere atmosphärische Absorption bereits in der Response-Funktion berücksichtigt ist (s.o.), kann sie durch das Korrekturverfahren unter Umständen auch überkorrigiert werden. Dies ist im Einzelfall zu prüfen und kann z.B. durch einen Skalierungsfaktor bei der Anwendung der Korrekturfunktion berichtigt werden.

- Bei besonders starker Absorption kann der Fluß im Einflußbereich der Absorptionsbanden auf Werte nahe Null sinken (vgl. die Response-Funktion bei $\approx 14000 \AA$ in Abbildung B.13). In diesen Fällen führt das angewandte Korrekturverfahren zu starkem Rauschen in den betroffenen Gebieten (s. z.B. die Spektren der M-Sterne in Abbildung 4.11). 


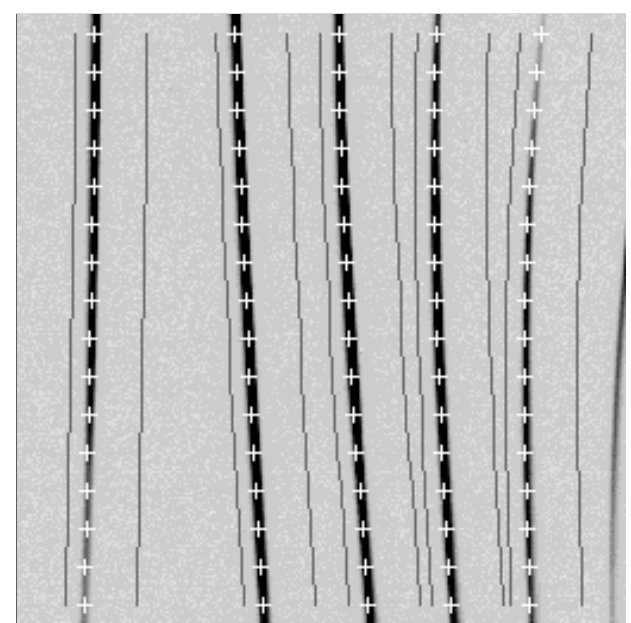

Abb. B.1: Lage der Ordnungen

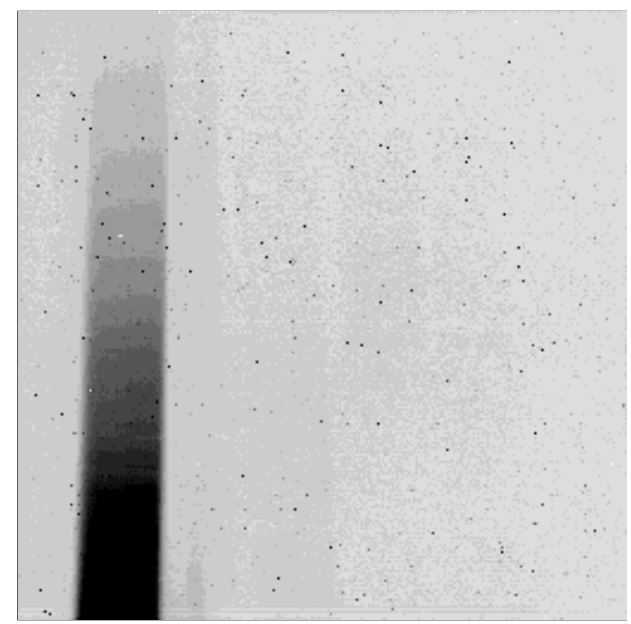

Abb. B.3: Darkfield

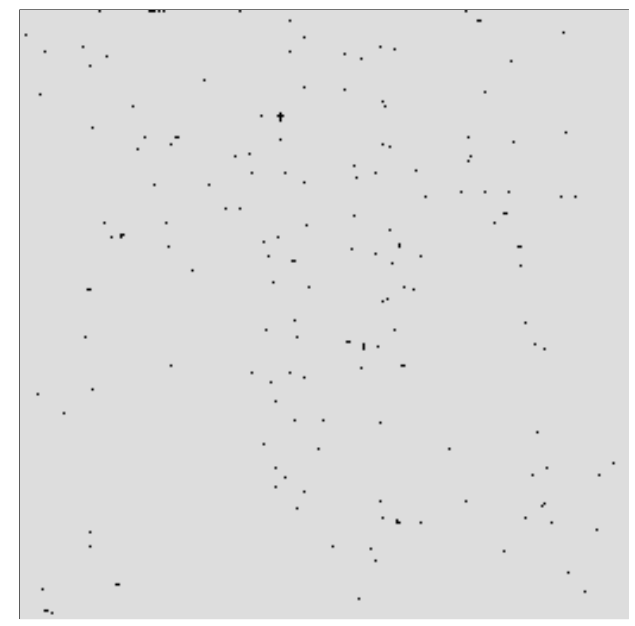

Abb. B.5: Karte defekter Pixel

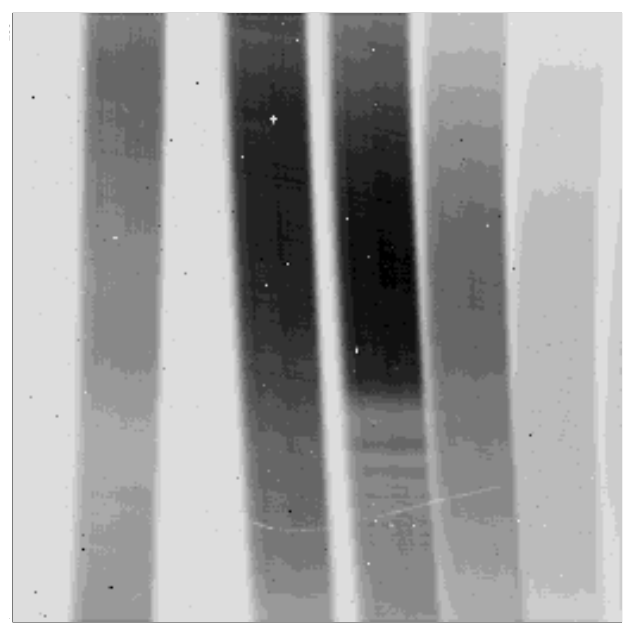

Abb. B.2: Flatfieldspektrum

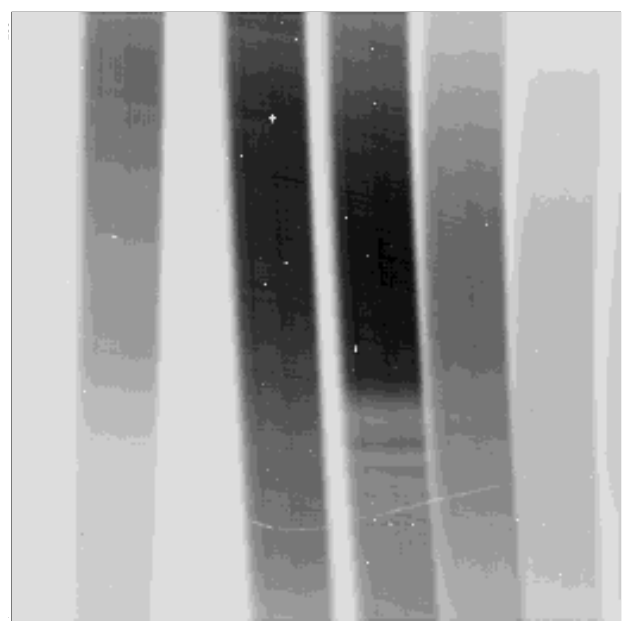

Abb. B.4: Flatfield - Darkfield

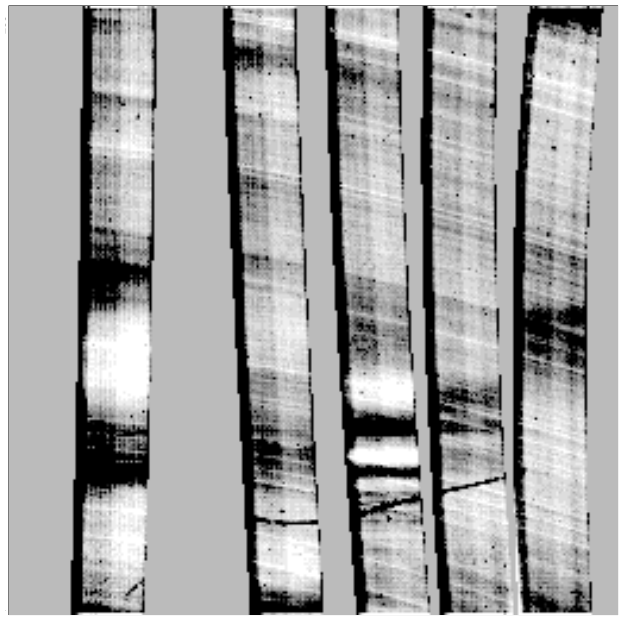

Abb. B.6: Hochfrequenz-Flatfield 

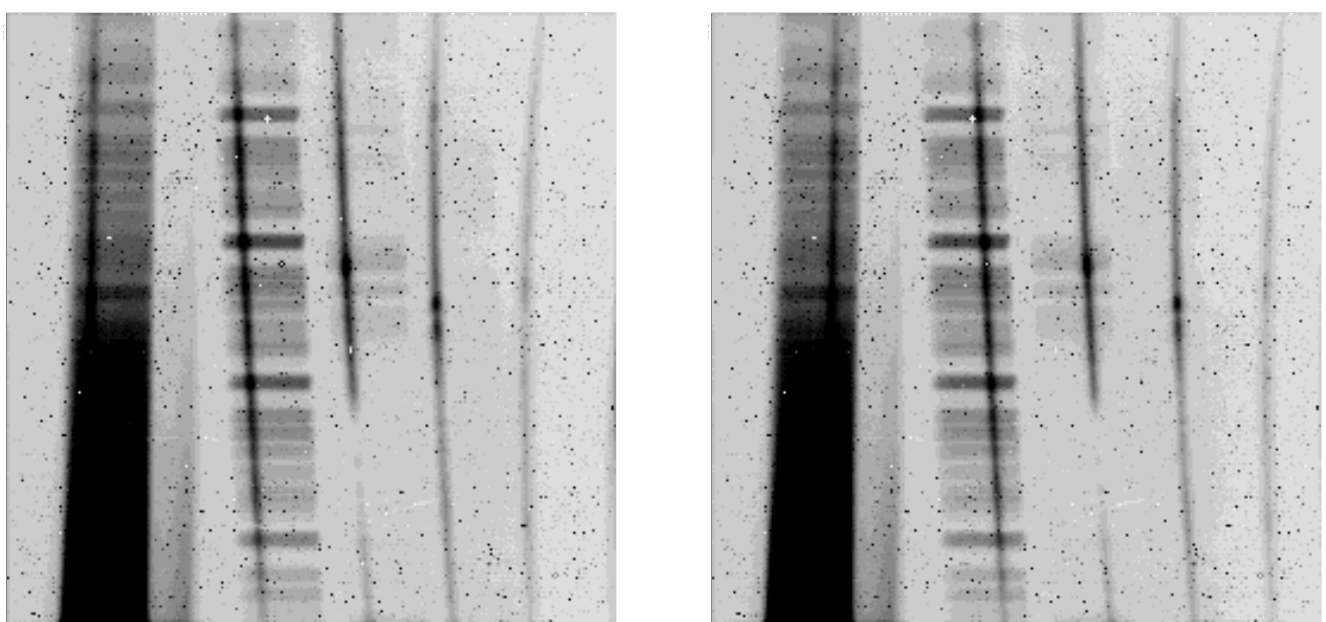

Abb. B.7: Beobachtungstechnik: Objektspektrum an verschiedenen Positionen

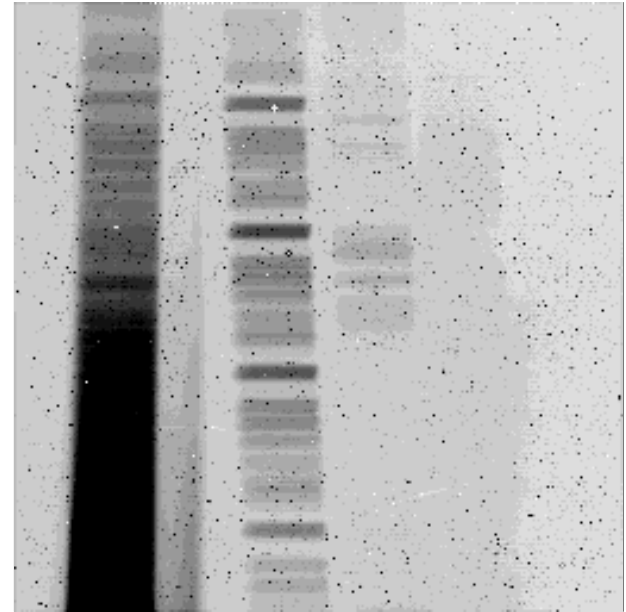

Abb. B.8: Mittleres Himmelsspektrum einer Serie von Spektren

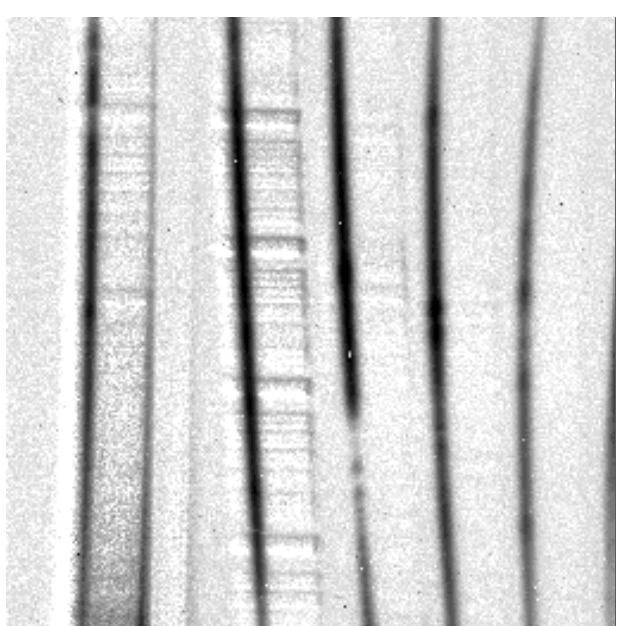

Abb. B.9: Objektspektrum nach Abzug des mittleren Himmelsspektrums
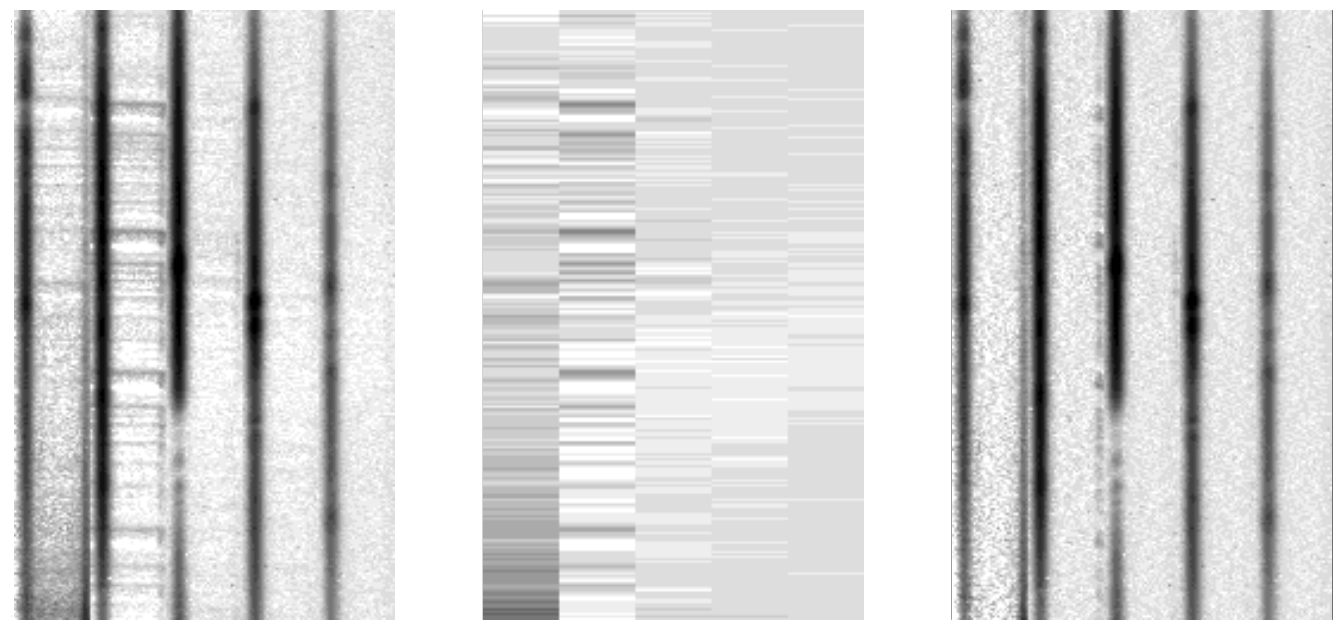

Abb. B.10: Extraktion der Ordnungen und Abzug des Himmelshintergrunds 

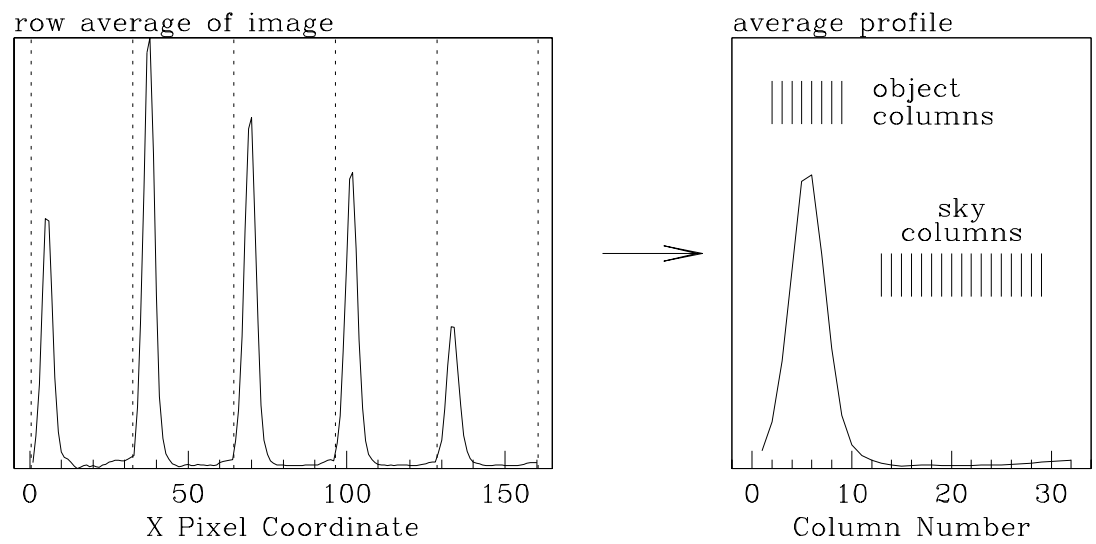

Abb. B.11: Auswahl der Spalten des Himmels- bzw. Objektspektrums
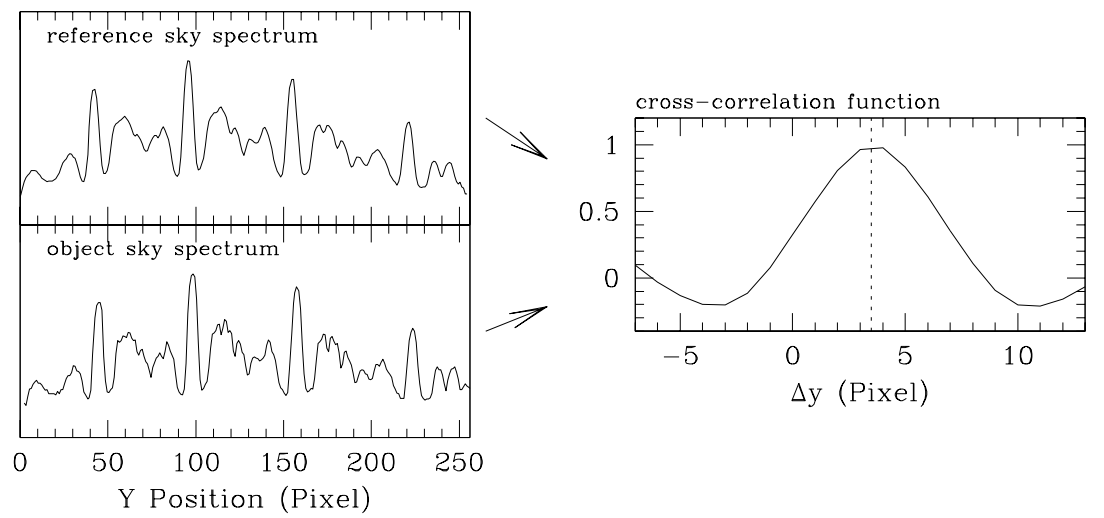

Abb. B.12: Bestimmung der mechanischen Drift in Dispersionsrichtung

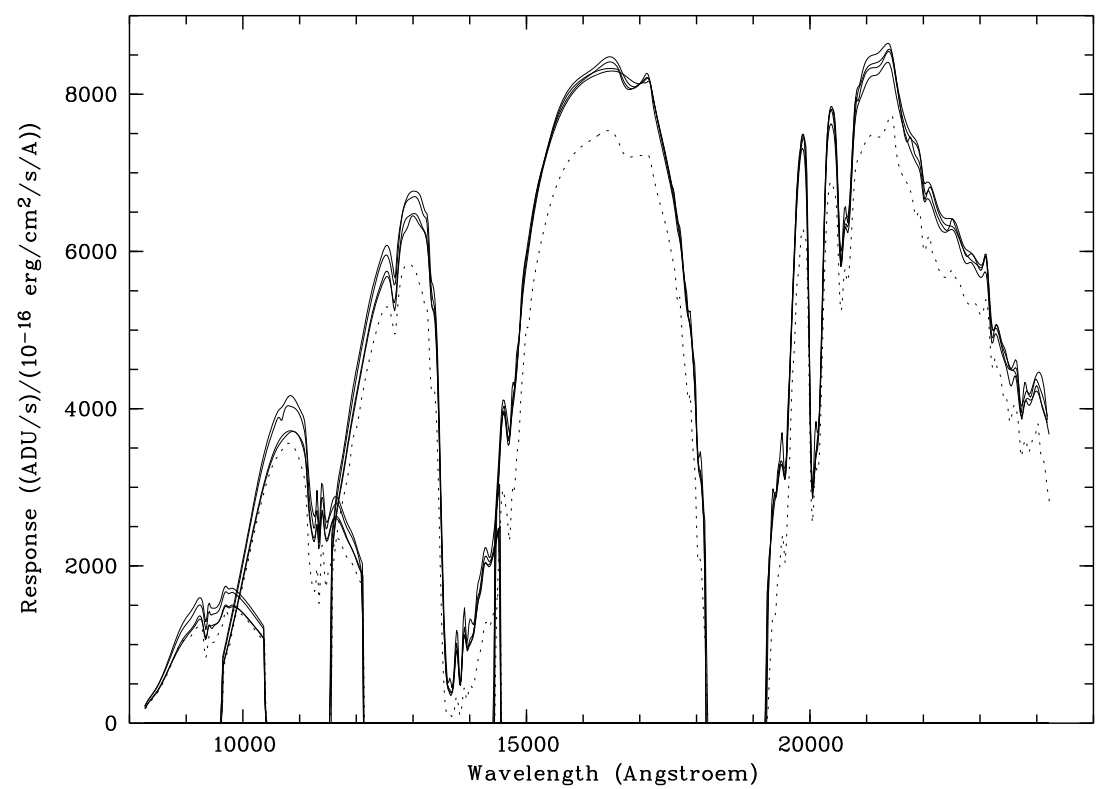

Abb. B.13: Response-Funktionen für die Ordnungen der IRS-Spektren. 


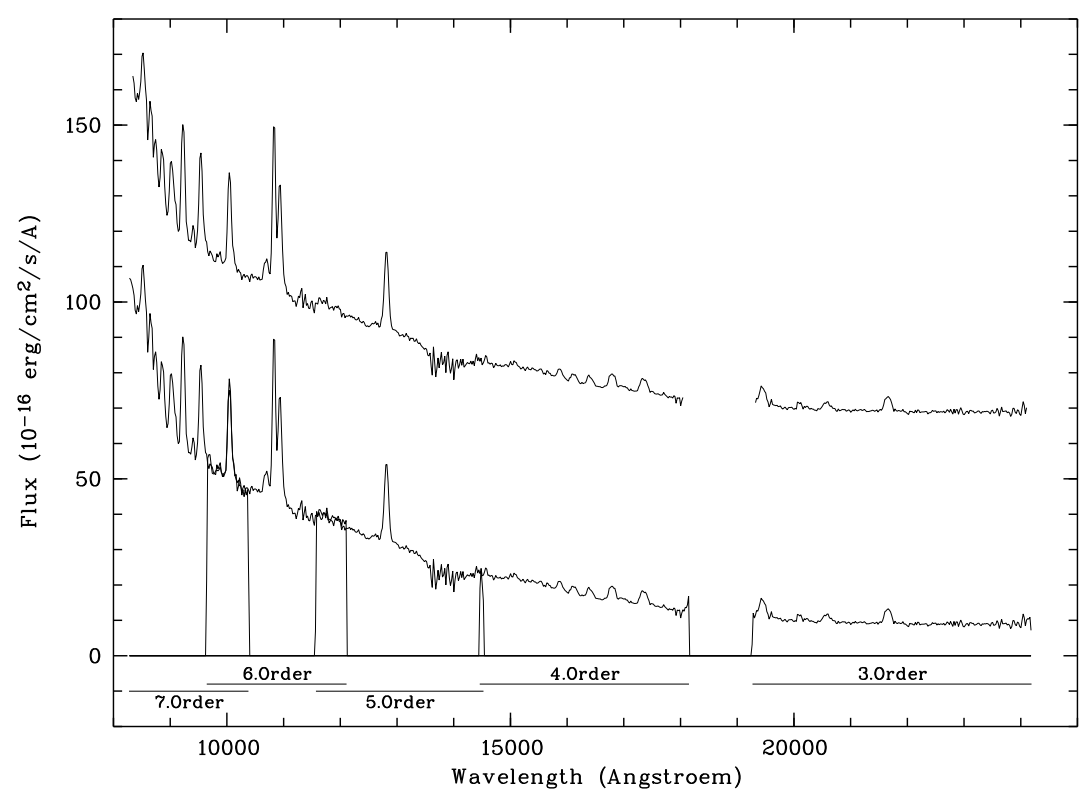

Abb. B.14: Verknüpfung der Ordnungen zum Gesamtspektrum. 


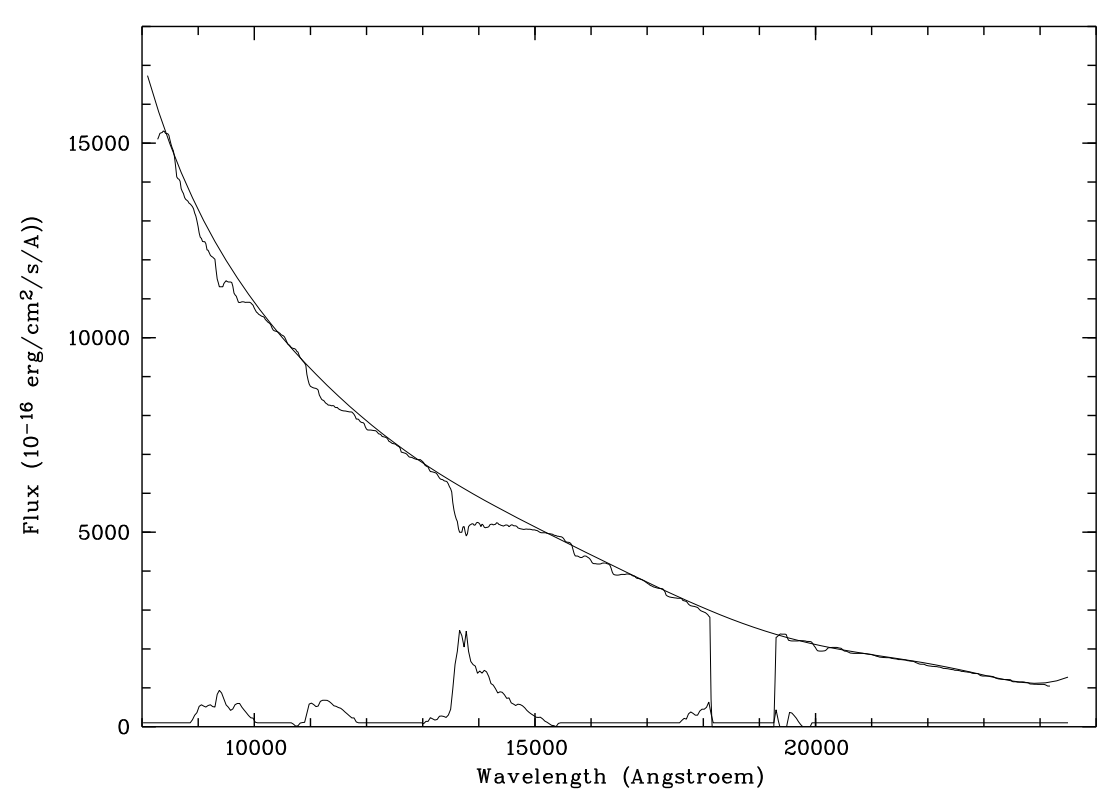

Abb. B.15: Konstruktion einer atmosphärischen Korrekturfunktion

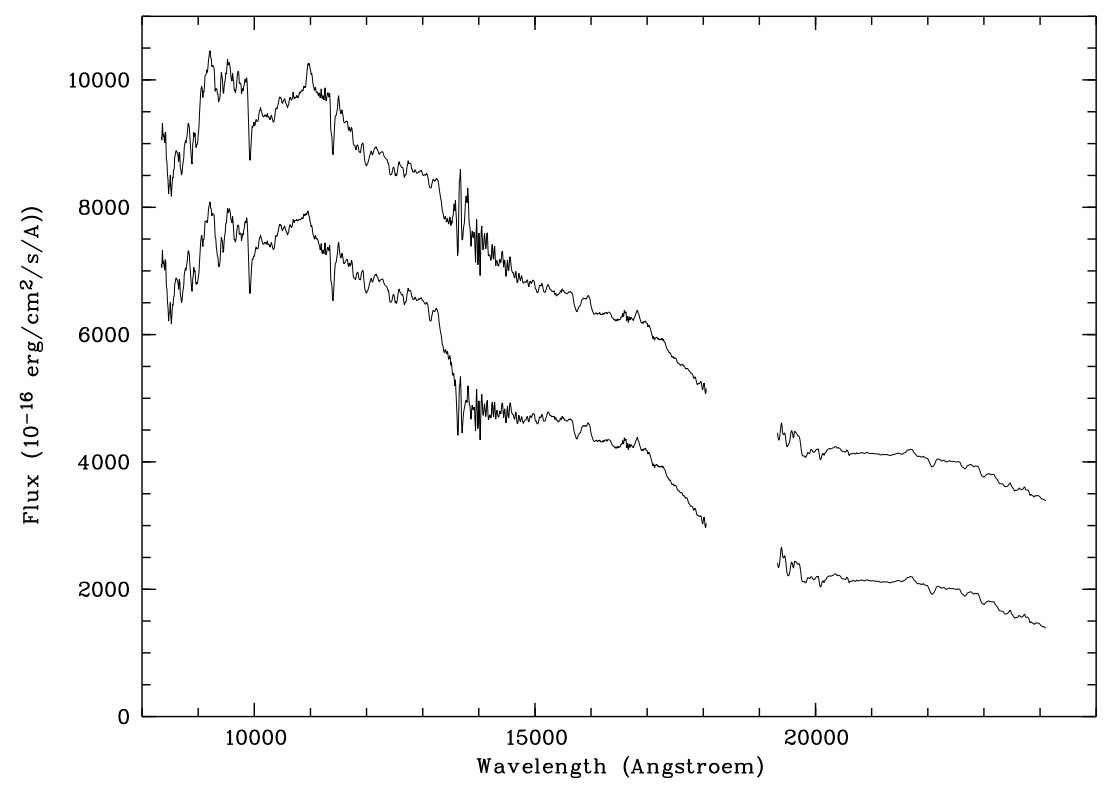

Abb. B.16: Atmosphärische Korrektur eines IRS-Spektrums 


\section{Literaturverzeichnis}

Allan, A., Hellier, C., Beardmore, A. 1998, MNRAS 295, 167

Bailey, J. 1981, MNRAS 197, 31

Baraffe, I., Chabrier, G., Allard, F. et al. 1995, ApJ 446, L35

Barnes, T.G., Evans, D.S. 1976, MNRAS 174, 489

Bath, G.T., Pringle, J.E., Whelan, J.A.J. 1980, MNRAS 190, 185

Bergeron, P., Saffer, R.A., Liebert, J. 1992, ApJ 394, 228

Berriman, G., Szkody, P., Capps, R.W. 1985, MNRAS 217, 327

Beuermann, K. 1997, in Perspectives in High Energy Astronomy and Astrophysics, ed. Agraval, P., Proc. of the Int. Coll. to Commemorate the Golden Jubilee of the Tata Inst. of Fund. Res.

Beuermann, K. 2000a, New Astr. Rev. 44, 93

Beuermann, K. 2000b, A\&A, in Vorbereitung

Beuermann, K. 2000c, private Mitteilung

Beuermann, K., Baraffe, I., Hauschildt, P. 1999, A\&A 348, 524

Beuermann, K., Burwitz, V. 1995, ASP Conf. Ser. 85, 99

Beuermann, K., Osborne, J.P. 1984, Proc. Symp. X-ray Astronomy '84, Bologna, eds. Oda, M., Giacconi, R., Beuermann, K., Osborne, J.P. 1985, Space Sci. Rev. 40, 117

Beuermann, K., Osborne, J.P. 1988, A\&A 189, 128

Beuermann, K., Weichhold, M. 1999, ASP Conf. Ser. 157, 283

Beuermann, K., Woelk, U. 1996, IAU Coll. 158, 199

Beuermann, K. Baraffe, I., Kolb, U. et al. 1998, A\&A 339, 518

Beuermann, K. Wheatley, P., Ramsay, G. et al. 2000, A\&A 354, L49

Bond, I.A., Freeth, R.V. 1988, MNRAS 232, 753

Burwitz, V. 1997, Dissertation, Georg-August-Universität, Göttingen

Caillault, J.-P., Patterson, J. 1990, AJ 100, 825

Campbell, C.G. 1985, MNRAS 215, 509

Campbell, C.G. 1989, MNRAS 236, 475

Campbell, C.G. 1990, MNRAS 244, 367

Córdova, F.A., Riegler, G.M. 1979, MNRAS 188, 103

Córdova, F.A., Mason, K.O., Kahn, S.M. 1985, MNRAS 212, 447

Cowley, A.P., Hutchings, J.B., Crampton, D. 1981, ApJ 246, 489

Cropper, M.S. 1986, MNRAS 222, 225

Cropper, M.S. 1990, Space Sci. Rev. 54, 195

Cropper, M.S., Wu, K., Ramsay, G. et al. 1999, MNRAS 306, 684

Depoy, D.L., Gregory, B., Elias, J. et al. 1990, PASP 102, 1433

Dhillon, V.S., Marsh, T.R. 1995, MNRAS 275, 89

Dhillon, V.S., Marsh, T.R., Duck, S.R. et al. 1997, MNRAS 285, 95

Eggleton, P.P. 1983, ApJ 268, 368

Elias, J.H., Frogel, J.A., Matthews, K. et al. 1982, AJ 87, 1029

Ezuka, H., Ishida, M. 1999, ApJS 120, 277

Fischer, A., Beuermann, K. 2000, A\&A, eingereicht 
Frank, J., King, A., Raine, D. 1985, Accretion Power in Astrophysics

(Cambridge Astrophysics Series)

Fruscione, A., Hawkins, I., Jelinsky, P. et al. 1994, ApJS 94, 127

Fujimoto, R., Ishida, M. 1997, ApJ 474, 774

Gänsicke, B.T. 1997, Dissertation, Georg-August-Universität, Göttingen

Gänsicke, B.T. 2000, Rev. Mod. Astr. 13, 151

Gänsicke, B.T., Beuermann, K., de Martino, D. 1995, A\&A 303, 127

Gilliland, R.L. 1982, ApJ 258, 576

Greeley, B.W., Blair, W.P., Long, K.S. et al. 1997, ApJ 488, 419

Haberl, F., Motch, C. 1995, A\&A 297, L37

Hameury, J.-M., King, A.R., Lasota, J.-P. 1986, MNRAS 218, 695

Heise, J., Mewe, R., Kruszewski, A. et al. 1987, A\&A 183, 73

Hellier, C. 1996, IAU Coll. 158, 143

Hellier, C., Sproats, L.N. 1992, IBVS 3724

Hellier, C., Mason, K.O., Rosen, S.R. et al. 1987, MNRAS 228, 463

Hellier, C., Garlick, M.A., Mason, K.O. 1993, MNRAS 260, 299

Hellier, C., Kemp, J., Naylor, T. et al. 2000, MNRAS 313, 703

Hill, K.M., Watson, R.D. 1984, PASAu 5, 532

Hurwitz, M., Sirk, M., Bowyer, S. et al. 1997, ApJ 477, 390

Ishida, M., Makishima, K., Mukai, K. et al. 1994, MNRAS 266, 367

Jablonski, F., Busko, I.C. 1985, MNRAS 214, 219

Kahabka, P., van den Heuvel, E.P.J. 1997, Ann. Rev. Astr. Astrophys. 35, 69

Kaitchuck, R.H., Hantzios, P., Kakaletris, P. et al. 1987, ApJ 317; 765

Kim, Y., Beuermann, K. 1995, A\&A 298, 165

Kim, Y., Beuermann, K. 1996, A\&A 307, 824

King, A.R. 1988, Quart. Journ. Roy. Astr. Soc. 29, 1

King, A.R. 1993, MNRAS 261, 144

King, A.R., Lasota, J.P. 1979, MNRAS 188, 653

King, A.R., Wynn, G.A. 1999, MNRAS 310, 203

Kippenhahn, R., Weigert, A. 1991, Stellar Structure and Evolution, 2. Aufl. (Springer-Verl.)

Kley, W. 1991, A\&A 247, 95

Kolb, U. 1995, ASP Conf. Ser. 85, 440

Kolb, U. 1996, IAU Coll. 158, 433

Kolb, U., Baraffe, I. 1999, MNRAS 309, 1034

Kolb, U., Baraffe, I. 2000, New Astr. Rev. 44, 99

Kolb, U., Stehle, R. 1996, MNRAS 282, 1454

Kraft, R.P. 1962, ApJ 135, 408

Kraft, R.P., Krzeminski, W. 1962, Annual Report of the Director Mt Wilson and Palomar Observatories 1961-1962, 20

Kraft, R.P., Mathews, J., Greenstein, J.L. 1962, ApJ 136, 312

Krautter, J., Buchholz, W. 1990, in Accretion Powered Compact Binaries, ed. Mauche, C. (Cambridge Univ. Press) 
Kruszewski, A., Mewe, R., Heise, J. et al. 1981, Space Sci. Rev. 30, 221

Krzeminski, W., Kraft, R.P. 1964, ApJ 140, 921

Kube, J., Gänsicke, B.T., Beuermann, K. 2000, A\&A 356, 490

Kuijpers, J., Pringle, J.E. 1982, A\&A 114, L4

Kurucz, R.L., Bell, B. 1995, Kurucz CD-ROM No. 23, Atomic Line List

(Harvard-Smithsonian Center for Astrophysics); online unter http://cfa-www.harvard.edu/amdata/ampdata/kurucz23/sekur.html

Lamb, D.Q., Masters, A.R. 1979, ApJ 234, 117

Lamb, D.Q., Melia, F. 1988, in Polarization of Circumstellar Origin, eds. Coyne, G.V. et al. (Vatican Observatory)

Lamb, F.K., Aly, J.-J., Cook, M.C. et al. 1983, ApJ 274, L71

Leggett, S.K. 1992, ApJS 82, 351

Leggett, S.K. Allard, F., Berriman, G. et al. 1996, ApJS 104, 117

Litchfield, S.J., King, A.R. 1990, MNRAS 247, 200

Lub, J., Pel, J.W. 1977, A\&A, 54, 137

Mason, K.O., Rosen, S.R., Hellier, C. 1988, Adv. Space Res. 8, 293

Mauche, C. 1999, ApJ 520, 822

McDermott, P.N., Taam, R.E. 1989, ApJ 342, 1019

Morfill, G.E., Trümper, J., Tenorio-Tagle, G. et al. 1984, A\&A 139, 7

Mukai, K., Ishida, M., Osborne, J.P. et al. 1998, ASP Conf. Ser. 137, 554

Mumford, G.S. 1967, ApJS 15, 1

Nariai, K., Nomoto, K. 1979, IAU Coll. 53, 525

Nauenberg, M. 1972, ApJ 175, 417

Nomoto, K. 1982, ApJ 253, 798

Norton, A.J., Watson, M.G. 1989, MNRAS 237, 853

Paczyński, B. 1971, Ann. Rev. Astr. Astrophys. 9, 183

Patterson, J. 1984, ApJS 54, 443

Patterson, J., Raymond, J.C. 1985, ApJ 292, 550

Patterson, J., Lamb, D.Q., Fabbiano, G. et al. 1984, ApJ 279, 785

Plavec, M., Kratochvil, P. 1964, Bull. Astr. Czech. 15, 165

Popham, R. 1997, ApJ 478, 734

Prialnik, D., Kovetz, A. 1995, ApJ 445, 789

Pringle, J.E. 1985, in Interacting Binary Stars, eds. Pringle, J.E., Wade, R.A.,

(Cambridge University Press)

Pringle, J.E. 1988, MNRAS 230, 587

Ramsay, G. 2000, MNRAS 314, 403

Ramsay, G., Mason, K.O., Cropper, M. et al. 1994, MNRAS 270, 692

Ramseyer, T.F. 1994, ApJ 425, 243

Rappaport, S., Verbunt, F., Joss, P.C. 1983, ApJ 275, 713

Reinsch, K., Burwitz, V., Beuermann, K. et al. 1994, A\&A 291, L27

Ritter, H. 1985, A\&A 148, 207

Ritter, H., Kolb, U. 1998, A\&AS 129, 83

Rosen, S.R., Mason, K.O., Córdova, F.A. 1988, MNRAS 231, 549 
Rosen, S.R., Mason, K.O., Mukai, K. et al. 1991, MNRAS 249, 417

Rousseau, T., Fischer, A., Beuermann, K. et al. 1996, A\&A 310, 526

Scheffler, H., Elsässer, H. 1990, Physik der Sterne und der Sonne, 2. Aufl. (BI-Wiss.-Verl.)

Schmidt, G.D., Liebert, J., Stockman, H.S. 1995, ApJ 441, 414

Schwartz, D.A., Fabbiano, G., Griffiths, R.E. et al. 1979, AJ 84, 1560

Shakura, N.I., Sunyaev, R.A. 1973, A\&A, 24, 337

Sherrington, M.R., Lawson, P.A., King, A.R. et al. 1980, MNRAS 191, 185

Siegel, N., Reinsch, K., Beuermann, K. et al. 1989, A\&A 225, 97

Singh, J., Swank, J. 1993, MNRAS 262, 1000

Sion, E.M. 1991, AJ 102, 295

Sion, E.M. 1995, ApJ 438, 876

Smith, R.C. 2000, private Mitteilung

Smith, R.C., Collier-Cameron, A., Tucknott, D.S. 1993, Ann. Israel Phys. Soc. 10, 70

Sterken, C., Vogt, N., Freeth, R. et al. 1983, A\&A 118, 325

Stockman, H.S., Schmidt, G.D., Lamb, D.Q. 1988, ApJ 332, 282

Stockman, H.S., Schmidt, G.D., Berriman, G. et al. 1992, ApJ 401, 628

Tylenda, R. 1981, Acta Astronomica 31, 127

Tylenda, R. 1988, A\&A 197, 225

van Teeseling, A. 1998, ASP Conf. Ser. 137, 385

van Teeseling, A., Kaastra, J.S., Heise, J. 1996, A\&A, 312, 186

van Teeseling, A., Hessman, F.V., Romani, R.W. 1999, A\&A 342, L45

Verbunt, F. 1984, MNRAS 209, 227

Verbunt, F. 1987, A\&AS 71, 339

Verbunt, F., Zwaan, C. 1981, A\&A 100, L7

Vogt, N., Krzeminski, W., Sterken, C. 1980, A\&A 85, 106

Wade, R.A. 1982, AJ 87, 1558

Warner, B. 1983, IAU Coll. 72, 155

Warner, B. 1986, MNRAS 219, 347

Warner, B. 1987, MNRAS 227, 23

Warner, B. 1995, Cataclysmic Variable Stars (Cambridge University Press)

Warner, B., McGraw, J.T. 1981, MNRAS 196, Short Comm. 59

Watson, M.G., Sherrington, M.R., Jameson, R.F. 1978, MNRAS 184, 79

Wiese, W.L., Smith, M.W., Glennon, B.M. 1966, Atomic Transition Probabilities,

Vol. I: H through Ne (Nat. Stand. Ref. Data Ser., Nat. Bur. Stand. (U.S.), 4)

Wiese, W.L., Smith, M.W., Glennon, B.M. 1969, Atomic Transition Probabilities,

Vol. II: Na through Ca (Nat. Stand. Ref. Data Ser., Nat. Bur. Stand. (U.S.), 22)

Williams, R.E. 1980, ApJ 235, 939

Woelk, U., Beuermann, K. 1992, A\&A 256, 498

Woelk, U., Beuermann, K. 1993, A\&A 280, 169

Woelk, U., Beuermann, K. 1996, A\&A 306, 232

Wood, M. 1994, IAU Coll. 147, 612

Wynn, G.A., King, A.R. 1995, MNRAS 275, 9 


\title{
Abkürzungsverzeichnis
}

\author{
AAT Anglo-Australian Telescope \\ ASCA Advanced Satellite for Cosmology and Astrophysics \\ CDS Centre de Données astronomiques de Strasbourg \\ CTIO Cerro Tololo Interamerican Observatory (auf dem Cerro Tololo, Chile) \\ CV cataclysmic variable (star) \\ EFOSC ESO Faint Object Spectrograph and Camera \\ ESO European Southern Observatory \\ EUV extreme ultraviolet $(\approx 100-912 \AA)$ \\ EUVE Extreme Ultraviolet Explorer \\ EXOSAT European X-ray Observatory Satellite \\ FOS $\quad$ Faint Object Spectrograph (an Bord von $\rightarrow$ HST) \\ FUV far ultraviolet $(\approx 912-1200 \AA)$ \\ FWHM full width at half maximum \\ GINGA japanischer astronomischer Röntgensatellit ( = ASTRO-C) \\ HST Hubble Space Telescope \\ HUT Hopkins Ultraviolet Telescope (auf der Astro-2 Space Shuttle-Mission) \\ IP intermediate polar \\ IRS $\quad$ Infrared Spectrometer (am 4 m-Teleskop des $\rightarrow$ CTIO) \\ ISM interstellares Medium \\ ISO Infrared Space Observatory \\ IUE International Ultraviolet Explorer \\ LWP Long Wave Prime camera (an Bord von $\rightarrow$ IUE) \\ LWR Long Wave Redundant camera (an Bord von $\rightarrow$ IUE) \\ MIDAS Munich Image Data Analysis System \\ ORFEUS Orbiting Retrievable Far and Extreme Ultraviolet Spectrometer \\ PHOT-S niedrigaufösendes Spektrophotometer an Bord von $\rightarrow$ ISO \\ RXTE Rossi X-ray Timing Explorer \\ SBRC Santa Barbara Research Corporation (Entwickler des $\rightarrow$ IRS-Detektors) \\ SIMBAD Set of Identifications, Measurements and Bibliography for Astronomical \\ Data (vom $\rightarrow$ CDS betriebene astronomische Datenbank) \\ $\mathrm{SSS} \rightarrow \mathrm{SUSO}$ \\ SUSO supersoft (X-ray) source \\ SWP Short Wave Prime camera (an Bord von $\rightarrow$ IUE) \\ TNR Thermonuclear Runaway, Ursache eines Nova-Ereignisses \\ ZAMS Zero Age Main Sequence
}





\section{Naturkonstanten und astronomische Maßeinheiten}

Naturkonstanten

\begin{tabular}{llll}
\hline Konstante & Symbol & im SI-System & im cgs-System \\
\hline Boltzmann-Konstante & $k_{\mathrm{B}}$ & $1.3805 \cdot 10^{-23} \mathrm{~J} \mathrm{~K}^{-1}$ & $1.3805 \cdot 10^{-16} \mathrm{erg} \mathrm{K}^{-1}$ \\
Gravitationskonstante & $G$ & $6.674 \cdot 10^{-11} \mathrm{~m}^{3} \mathrm{~kg}^{-1} \mathrm{~s}^{-2}$ & $6.674 \cdot 10^{-8} \mathrm{~cm}^{3} \mathrm{~g}^{-1} \mathrm{~s}^{-2}$ \\
Plancksches W.-quantum & $h$ & $6.6256 \cdot 10^{-34} \mathrm{~J} \mathrm{~s}$ & $6.6256 \cdot 10^{-27} \mathrm{erg} \mathrm{s}$ \\
Elektronenmasse & $m_{\mathrm{e}}$ & $9.1095 \cdot 10^{-31} \mathrm{~kg}$ & $9.1095 \cdot 10^{-28} \mathrm{~g}$ \\
Protonenmasse & $m_{\mathrm{p}}$ & $1.67265 \cdot 10^{-27} \mathrm{~kg}$ & $1.67265 \cdot 10^{-24} \mathrm{~g}$ \\
Masse des H-Atoms & $m_{\mathrm{H}}$ & $1.6736 \cdot 10^{-27} \mathrm{~kg}$ & $1.6736 \cdot 10^{-24} \mathrm{~g}$ \\
Lichtgeschwindigkeit & $c$ & $2.99792 \cdot 10^{8} \mathrm{~m} \mathrm{~s}^{-1}$ & $2.99792 \cdot 10^{10} \mathrm{~cm} \mathrm{~s}^{-1}$ \\
Elementarladung & $e$ & $1.602 \cdot 10^{-19} \mathrm{~A} \mathrm{~s}^{-8} \mathrm{~A} \mathrm{~s}^{-2} \mathrm{~K}^{-4}$ & $5.67 \cdot 10^{-5} \mathrm{erg} \mathrm{cm}^{-2} \mathrm{~s}^{-1} \mathrm{~K}^{-4}$ \\
\hline
\end{tabular}

(1) $1 \mathrm{erg}=1 \mathrm{~g} \mathrm{~cm}^{2} \mathrm{~s}^{-2}=10^{-7} \mathrm{~J} ; \quad 1 \mathrm{dyn}=1 \mathrm{~g} \mathrm{~cm}^{-1} \mathrm{~s}^{-2}=10^{-5} \mathrm{~N} ; \quad 1 \mathrm{dyn} \mathrm{cm}^{-2}=0.1 \mathrm{~Pa}\left(=10^{-6} \mathrm{bar}\right)$

In der Astronomie übliche Maßeinheiten

\begin{tabular}{lllll}
\hline Größe & Einheit & Symbol & im SI-System & im cgs-System \\
\hline Masse & Sonnenmasse & $M_{\odot}$ & $1.9891 \cdot 10^{30} \mathrm{~kg}$ & $1.9891 \cdot 10^{33} \mathrm{~g}$ \\
Länge & Sonnenradius & $R_{\odot}$ & $6.96 \cdot 10^{8} \mathrm{~m}$ & $6.96 \cdot 10^{10} \mathrm{~cm}$ \\
Leuchtkraft & Sonnenleuchtkraft & $L_{\odot}$ & $3.866 \cdot 10^{26} \mathrm{~W}$ & $3.866 \cdot 10^{33} \mathrm{erg} \mathrm{s}^{-1}$ \\
Entfernung & Parsec & $1 \mathrm{pc}$ & $3.088 \cdot 10^{16} \mathrm{~m}$ & $3.088 \cdot 10^{18} \mathrm{~cm}$ \\
Spektraler Fluß & Jansky & $1 \mathrm{Jy}$ & $10^{-26} \mathrm{~W} \mathrm{~m}^{-2} \mathrm{~Hz}^{-1}$ & $10^{-23} \mathrm{erg} \mathrm{cm}^{-2} \mathrm{~s}^{-1} \mathrm{~Hz}^{-1}$ \\
Wellenlänge & Angstrœm & $1 \AA$ & $10^{-10} \mathrm{~m}$ & $10^{-8} \mathrm{~cm}$ \\
Photonenenergie & Elektronenvolt & $\mathrm{eV}$ & $1.602 \cdot 10^{-19} \mathrm{~J}$ & $1.602 \cdot 10^{-12} \mathrm{erg}$ \\
Magnetfeld & Gauss & $1 \mathrm{G}$ & $10^{-4} \mathrm{~T}$ & \\
\hline
\end{tabular}





\section{Danksagungen}

Ich möchte an dieser Stelle den vielen Personen danken, die es mir ermöglicht haben, diese Arbeit in der hier vorliegenden Form anzufertigen.

An erster Stelle gebührt mein Dank Herrn Prof. Dr. K. Beuermann, der mit seiner Themenstellung den Grundstein der Arbeit gelegt und mir in zahlreichen Diskussionen viele Einsichten in die Zusammenhänge der Astrophysik im allgemeinen und in die Physik kataklysmischer Veränderlicher im besonderen vermittelt hat. Die zahlreichen Diskussionen mit ihm, seine Kritik, Anregungen, Hilfestellungen, Berichtigungen und Kommentare hatten wesentlichen Anteil am Gelingen der Arbeit.

Meinem Kollegen Herrn Dr. Klaus Reinsch danke ich für seine stetige Bereitschaft, physikalische Fragen zu erörtern, mir bei den Tücken von VMS und UNIX zu helfen, seine Erfahrung mit mir zu teilen, seine Geduld, wenn ich ihm zusätzliche Arbeit aufbürdete, die Durchsicht des Manuskripts und die anschließenden Diskussionen und nicht zuletzt auch für die netten Grünkohlessen, die wir gemeinsam veranstaltet haben. Vielen Dank auch an Herrn Dr. Boris Gänsicke, der mir für diese Arbeit sein Programm zur Berechnung optisch dünner Spektren zur Verfügung gestellt und sogar noch an meine Bedürfnisse angepaßt hat. Herrn Prof. Dr. F. Kneer möchte ich dafür danken, daß er sich bereit erklärt hat, als Koreferent für diese Arbeit zu fungieren, was ihn sicher so manche Stunde lang beschäftigen wird.

Weiterhin möchte ich allen weiteren derzeitigen und auch ehemaligen Mitarbeiterinnen und Mitarbeitern an der Sternwarte danken, die stets für eine angenehme und freundliche Arbeitsatmosphäre gesorgt haben. Der Platz reicht nicht aus, sie alle namentlich zu erwähnen; sie mögen es mir bitte nicht übelnehmen. Die 'unsichtbaren Geister', die in sicher mühseliger und nie endender Arbeit das Rechnernetzwerk der Sternwarte am Laufen halten, sollten aber sicher nicht unerwähnt bleiben.

Schließlich danke ich meinen Eltern, die mir mein Studium finanziell erst ermöglicht haben und so auch einen nicht geringen Beitrag zu dieser Arbeit geleistet haben, und meinen Freunden, die mich - obwohl ich mich viel zu wenig um sie gekümmert habe - immer noch nicht vergessen haben. In ganz besonderem Maße danke ich meiner Frau Bettina, die mir in den vergangenen Jahren Liebe und Wärme gegeben hat, mich moralisch gestützt hat, wenn es nicht mehr weiterzugehen schien, und nicht zuletzt in den letzten Monaten alleine dafür gesorgt hat, daß der Kühlschrank immer voll war (was ich ihr oft genug durch Abwesenheit bis tief in die Nacht gedankt habe). Des weiteren hat sie die Mühe auf sich genommen, das fertige Manuskript zu korrigieren. Ohne sie wäre ich vermutlich nicht imstande gewesen, die Arbeit in dieser Form zum Abschluß zu bringen. Außerdem möchte ich schon jetzt meinem vor wenigen Tagen geborenen Sohn Alexander dafür danken, daß er sicher zu verhindern wissen wird, daß ich mich in den nächsten Wochen langweile.

Diese Arbeit wurde im Rahmen der Verbundforschung unter der Projektbezeichnung "IRZyklotronspektroskopie akkretierender magnetischer Weißer Zwerge" (50 OR 9610 4) vom Deutschen Zentrum für Luft- und Raumfahrt e.V. (DLR) finanziell unterstützt. 



\section{Lebenslauf}

Name: $\quad$ Stephan Eisenbart, geb. Brandt

Gebutsdatum: 15. Dezember 1966

Geburtsort: Flensburg

Nationalität: Deutscher

Familienstand: Seit 24. Mai 1996 verheiratet mit Dr. jur. Bettina Eisenbart

Ein Kind: Alexander Eisenbart, geb. 10. September 2000

\section{Ausbildung und Wehrdienst:}

Schule: $\quad$ Aug. 1972 - Juli 1976 Grund- und Hauptschule in Harrislee

Aug. 1976 - Juli 1985 Altes Gymnasium in Flensburg

Mai $1985 \quad$ Erwerb der Hochschulreife

Wehrdienst: Okt. 1985 - Sep. 1988 Neumünster, Idar-Oberstein, Flensburg

Studium: Okt. $1988 \quad$ Beginn des Diplomstudiengangs Physik an der

Georg-August-Universität zu Göttingen

Nov. $1990 \quad$ Erwerb des Vordiploms

Okt. 1990 - Nov. 1995 Hauptstudium mit dem Schwerpunkt Astrophysik an der Georg-August-Universität zu Göttingen

Nov. 1995

1996 - Sep. 2000 Abschluß des Studiums und Erwerb des Diploms Aufbaustudium an der Georg-August-Universität zu Göttingen und Anfertigung der vorliegenden Dissertation

Diplomarbeit (im September 1995 abgeschlossen):

Betreuer: Prof. Dr. K. Beuermann

Universität: Physikalische Fakultät der Georg-August-Universität zu Göttingen

Titel: $\quad$ Stellare Röntgenquellen in ausgewählten Feldern der LMC 
